NASA Contractor Report 4290

\title{
Absolute Wind Measurements in the Lower Thermosphere of Venus Using Infrared Heterodyne Spectroscopy
}

Jeffrey J. Goldstein

University of Pennsylvania

Philadelphia, Pennsylvania

Prepared for

Goddard Space Flight Center under Grant NGT 39-090-800

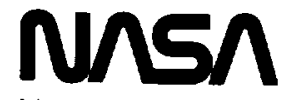

National Aeronautics and Space Administration

Office of Management Scientific and Technical Information Division

1990 
The author was: 1) a Visiting Astronomer at the Infrared Telescope Facility which is operated by the University of Hawaii under contract to the National Aeronautics and Space Administration, and 2) a Guest Investigator, National Solar Observatory, a division of the National Optical Astronomy Observatories, which is operated by the Association of Universities for Research in Astronomy, under contract with the National Science Foundation.

This work was supported by NASA Graduate Student Researcher Grant NGT 39-090-800. 


\section{ABSOLUTE WIND MEASUREMENTS IN THE LOWER THERMOSPHERE \\ OF VENUS USING INERARED HETERODYNE SPECTROSCOPY}

Jeffrey Jay Goldstein

A DISSERTATION

in

ASTRONOMY AND ASTROPHYSICS

Presented to the Faculties of the University of Pennsylvania in Partial Fulfillment of the Requirements for the Degree of Doctor of Philosophy.

1989
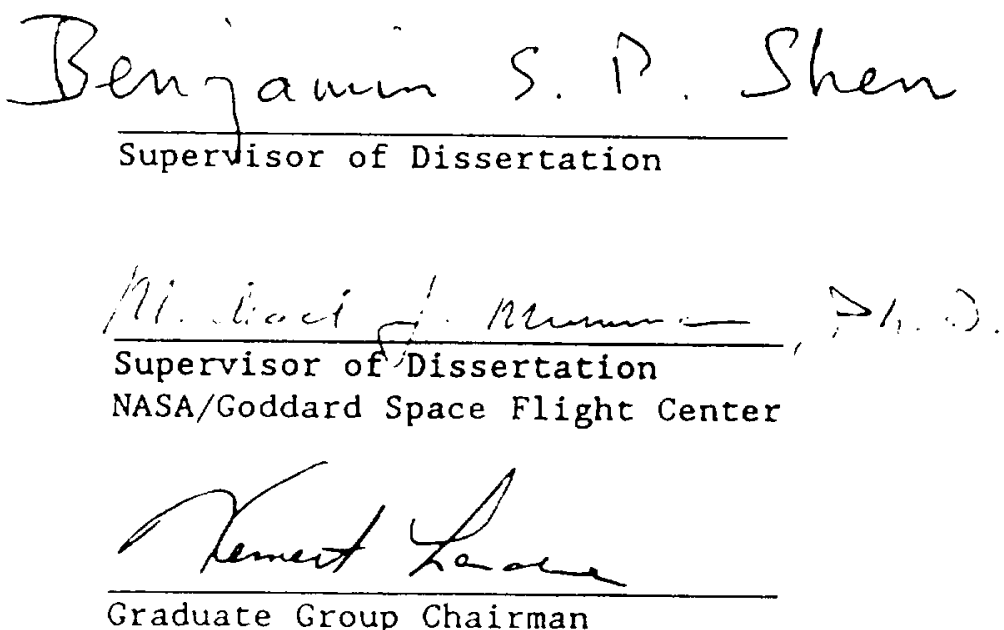
ABSTRACT

Absolute Wind Measurements in the Lower Thermosphere of Venus Using Infrared Heterodyne Spectroscopy

Jeffrey Jay Goldstein

Supervisor, University of Pennsylvania: Benjamin S.P. Shen Supervisor, NASA/Goddard Space Flight Center: Michael J. Mumma

In the atmosphere of Venus, observed diurnal and latitudinal temperature gradients over altitude imply a transition from retrograde zonal to subsolar-antisolar circulation at $-100 \mathrm{~km}$ altitude. Descent probe in situ investigations and orbital remote sensing have, to date, provided no direct measurements of winds above the cloud-tops $(-65 \mathrm{~km})$. Early ground-based heterodyne observations at 110 and $-75 \mathrm{~km}$ (Betz et al. 1977b; Betz 1977) yielded dynamically unreasonable wind velocities, possibly due to laser local oscillator drift and/or offset.

The first absolute wind velocities above the cloud-tops were obtained using NASA/Goddard Space Flight Center infrared heterodyne spectrometers at the NASA IRTF and the McMath Solar Telescope, December 1985 to March 1987, during four planetary phases spanning 828 of a synodic period. Beam-integrated Doppler displacements in the nonthermal emission core of ${ }^{12} \mathrm{C}^{16} \mathrm{O}_{2} 10.33 \mu \mathrm{m} \mathrm{R}(8)$ (obtained at sub-Doppler resolution: $\lambda / \Delta \lambda=10^{7}$ ), sampled the line of sight projection of the 
lower thermospheric wind field (100-120 km). A field-usable Lamb-dip laser stabilization system, developed for spectrometer absolute frequency calibration to $₫ 0.1 \mathrm{MHz}$, allowed S/N-limited line of sight velocity resolution at the $1 \mathrm{~m} / \mathrm{s}$ level. Ephemerides accurate to $<1 \mathrm{~m} / \mathrm{s}$ provided removal of the Venus/Earth-station topocentric Doppler shift. The spectrometer's diffraction-limited beam (1.7 arc-second HPBW at McMath, 0.9 arc-second HPBW at IRTF), and 1-2 arc-second seeing, provided the spatial resolution necessary for circulation model discrimination. Qualitative analysis of beam-integrated winds definitively established a dominant subsolar-antisolar circulation in the lower thermosphere. A retrograde zonal component was also suggested. Beam-integrated winds were modelled with a $100 \times 100$ grid over the beam, incorporating beam spatial rolloff and across-the-beam gradients in non-thermal emission intensity, line of sight projection geometry, and horizontal wind velocity. Horizontal wind velocity was derived from a 2-parameter model wind field comprised of subsolarantisolar and zonal components. Best-fit models indicated a dominant subsolar-antisolar flow with $120 \mathrm{~m} / \mathrm{s}$ cross-terminator winds and a retrograde zonal component with a $25 \mathrm{~m} / \mathrm{s}$ equatorial velocity (assuming wind field temporal stability over the synodic period). The subsolarantisolar component is consistent with the Bougher et al. (1986) 2-dimensional hydrodynamical model for $110 \mathrm{~km}$. Detection of a significant zonal component indicates upper thermospheric super-rotation may be coupled to, hence pumped by, cloud level super-rotation. Early heterodyne mesospheric wind measurements (Betz et al. 1977b) were re-examined based on laser stability test results. A reinterpretation 
is consistent with superposition of a $94 \pm 6 \mathrm{~m} / \mathrm{s}$ zonal super-rotation and a $35 \pm 6 \mathrm{~m} / \mathrm{s}$ antisolar-subsolar return flow at $-75 \mathrm{~km}$. A review of all dynamical indicators above the cloud-tops allowed development of an integrated and self-consistent picture of circulation in the $70-200 \mathrm{~km}$ range. Finally, historical visual observations of solar phase-locked structure were reviewed and discussed in light of the heterodyne results. 

TABLE OF CONTENTS

page

Preface: WHY STUDY PLANETARY WINDS

P.1. A Philosophical Preamble................... xxiii

P.2. Comparative Planetology: Real Benefits............ xxiv

P.3. Planetary Winds and Circulation Models............ xxvi

P.4. Venusian Atmospheric Nomenclature.............. xxiii

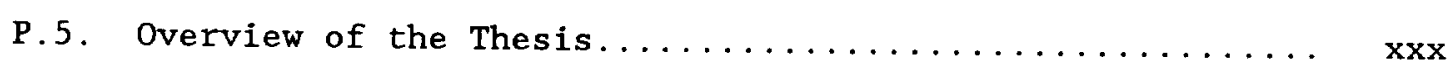

P.6. Acknowledgments...................... xxxiii

Chapter 1: INTRODUCTION

1.1. Statement of the Problem - Venusian Atmospheric Thermal

Structure and Dynamical Implications.............. 1

1.1.1. Atmospheric Thermal structure.............. 2

1.1.2. Dynamical Implications - Mesosphere $(-65-100 \mathrm{~km}$

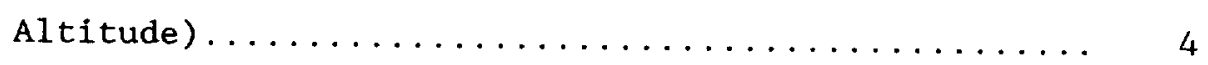

1.1.3. Dynamical Implications - Thermosphere/Cryosphere

$(100-150 \mathrm{~km}$ altitude $) \ldots \ldots \ldots \ldots \ldots \ldots \ldots \ldots \ldots \ldots \ldots \ldots \ldots \ldots$

1.1.4. Summary $\ldots \ldots \ldots \ldots \ldots \ldots \ldots \ldots \ldots \ldots \ldots \ldots \ldots$

1.2. Methods of Direct Wind Velocity Determination in the

Venusian Atmosphere..................... 8

1.2.1. Imaging $\ldots \ldots \ldots \ldots \ldots \ldots \ldots \ldots \ldots \ldots \ldots \ldots \ldots$

1.2.2. Doppler and Interferometric Tracking........... 9

1.2.3. Anemometric Measurements................ 11

1.2.4. Spectroscopy $\ldots \ldots \ldots \ldots \ldots \ldots \ldots \ldots \ldots \ldots \ldots \ldots \ldots \ldots$

ix 
1.3. Infrared Heterodyne Spectroscopic Wind Velocity

Determination.

Chapter 2: VENUSIAN ATMOSPHERIC CIRCULATION

2.1. Overview of Circulation in The Venusian

Troposphere/Mesosphere (Surface to $100 \mathrm{~km}$ ).......... 17

2.1.1. Gross Circulation Below the Cloud Tops........... 17

2.1.2. Longitudinal (Diurnal) variation.............. 18

2.1.3. Latitudinal Variation.................. 19

2.1.4. Cyclostrophic Balance.................. 20

2.1.5. Mean Meridional (Hadley) Circulation........... 23

2.1.6. Maintaining the Zonal Super-Rotation............ 24

2.2. Dynamical Models of the Venusian Thermosphere/Cryosphere.. 29

2.2.1. Early Circulation Models................ 29

2.2.2. Dynamical Models................... 32

2.3. $10 \mu \mathrm{m}$ Non-Thermal Emission Modelling.............. 39

Chapter 3: IR HETERODYNE SPECTROMETER OPERATION

3.1. Basic Theory of Operation.................... 43

3.2. A Quantitative Look at Coherent Detection............ 46

3.2.1. Generation of an Intermediate Frequency......... 46

3.2.2. Diffraction-Limited operation............. 47

3.2.3. Signal-to-Noise.................... 54

3.3. Optical Front End Layout................... 62

3.4. Radio Frequency Back End Layout................ 67

3.5. Front End Alignment / Target Pointing............. 71 
Chapter 4: THE LAMB DIP STABILIZATION SYSTEM

4.1. Basic Laser Operation.................... 79

4.2. The Character of the Gain Curve................ 82

4.3. The Character of the Cavity Etalon............... 85

4.4. Power Peak Stabilization................... 88

4.5. Lamb Dip Theory....................... 90

4.5.1. Basic Theory..................... 90

4.5.2. The Lamb Dip FWHM................... 97

4.5.3. Lamb Dip Frequency Shift Mechanisms............. 100

4.6. The Lamb Dip Cell......................... 104

4.7. Overview of the Lamb Dip Stabilization System......... 117

4.7.1. Introduction. .................. 117

4.7.2. Undifferentiated Mode................. 121

4.7.3. First Derivative Mode.................. 125

4.7.4. Lock Mode...................... 127

4.7.5. Power Peak Stabilization............... 132

4.8. Time Series Analysis of Laser Stability............ 133

4.8.1. Concept....................... 133

4.8.2. Power Peak Time Series................ 138

4.8.3. Lamb Dip Time Series.................. 138

Chapter 5: OVERVIEW of OBSERVATIONS $\ldots \ldots \ldots \ldots \ldots \ldots \ldots \ldots \ldots$

5.1. Choice of Emission Line as Dynamical Probe............ 143

5.2. Adopted Nomenclature....................... 145

5.3. Beam-Integrated Wind Velocities............... 146

5.3.1. December $1985 \ldots \ldots \ldots \ldots \ldots \ldots \ldots \ldots \ldots \ldots \ldots$ 


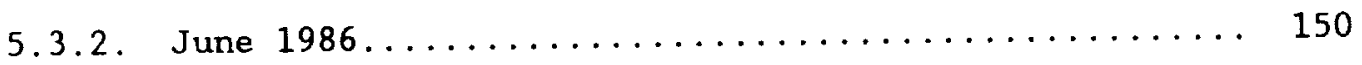

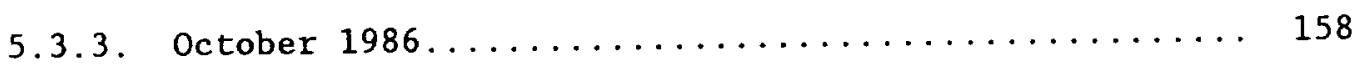

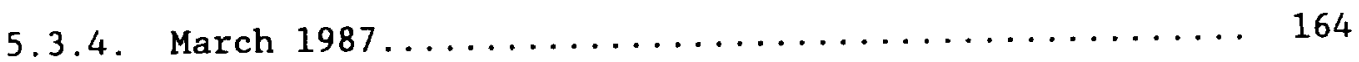

Chapter 6: RESULTS I - QUALITATIVE INTERPRETATION OF THE OBSERVED BEAM-INTEGRATED WIND VELOCITIES

6.1. Velocity Shift Effects.................. 169

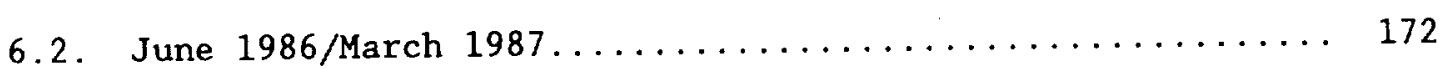

6.3. December $1985 \ldots \ldots \ldots \ldots \ldots \ldots \ldots \ldots \ldots \ldots \ldots \ldots \ldots \ldots \ldots$

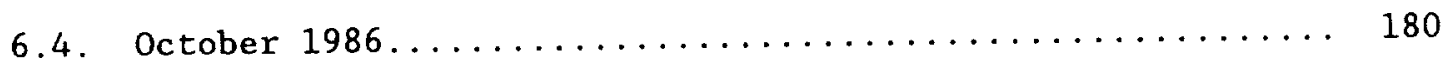

Chapter 7: RESULTS II - WIND FIELD RETRIEVAL FROM KINEMATICAL FITS TO THE BEAM-INTEGRATED WIND VELOCITIES

7.1. Program BEAMINT ....................... 183

7.1.1. Pointing Uncertainty $(\Gamma)$ Considerations.......... 183

7.1.2. Beam Character Considerations.............. 191

7.1.3. BEAMINT Beam Modelling................. 195

7.2. Kinematical Fits to a Two-Component Circulation Model.... 197

Chapter 8: THE BETZ ET AL. HETERODYNE RESULTS - A REINTERPRETATION

8.1. Laser Stability and Implications............... 229

8.2. Mesospheric Winds..................... 233

8.3. Mass Continuity in the Subsolar-Antisolar Cell........ 246

8.4. Lower Thermospheric Winds.................. 251 
Chapter 9: CONCLUSION

9.1. The Lower Thermospheric Heterodyne Results........... 255

9.2. An Integrated Picture.................. 257

9.2.1. Summary of Dynamical Indicators........... 257

9.2.2. Reassessing the Co Observations............. 259

9.2.3. Gross Circulation Above the Cloud Tops........... 264

Chapter 10: FUTURE DIRECTIONS

10.1. Venus - A Search for the Subsolar-Antisolar Return Branch and a Mesospheric Meridional Component............. 269

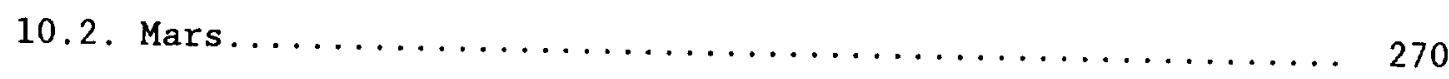

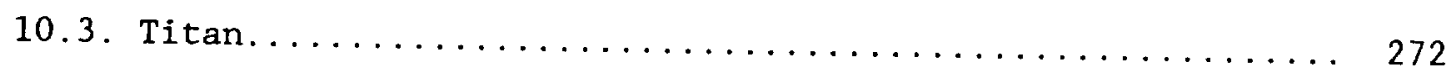

10.4. Other Planetary Atmospheres................ 276

10.5. Laboratory IR Heterodyne Spectroscopy............ 277

Appendix 1: DATA ACQUISITION AND ANALYSIS ALGORITHMS

A1.1. Correction for system offset................ 279

A1.2. Correction for Terrestrial Absorption............. 284

A1.3. Correction for Differential Doppler Shifts........... 288

A1.4. Line Fitting....................... 296

A1.5. Beam-Integrated Line of Sight Wind Velocity Determination. 299

A1.6. Reduction of Time Series Data................. 302

Appendix 2: PROGRAM BEAMINT THEORETICAL DEVELOPMENT

A2.1. Introduction. $\ldots \ldots \ldots \ldots \ldots \ldots \ldots \ldots \ldots \ldots \ldots \ldots \ldots \ldots \ldots$

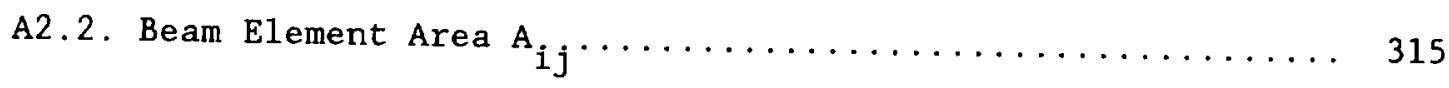

A2.3. Beam Element Centroid Determination.............. 316 
A2.4. Beam Element Weighting $\Phi_{i j} \ldots \ldots \ldots \ldots \ldots \ldots \ldots \ldots \ldots \ldots \ldots$

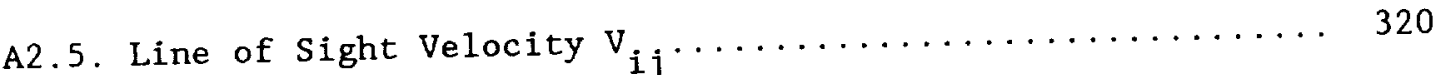

A2.5.1. Zonal Components $v_{x i}(i, j)$ and $T_{x}(i, j) \ldots \ldots \ldots \ldots 320$

A2.5.2. Subsolar-Antisolar Component $v_{x 2}(i, j) \ldots \ldots \ldots \ldots \ldots 22$

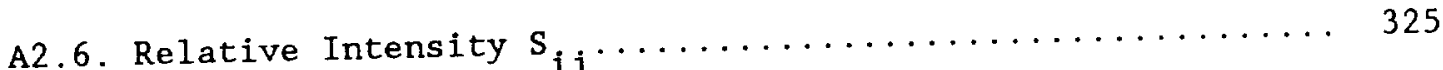

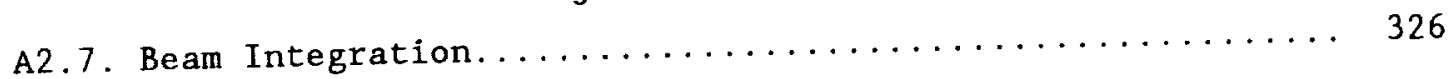

Appendix 3: THE VENUSIAN ATMOSPHERE - A HISTORICAL REVIEW TO 1962

A3.1. Early Telescopic Observations................. 343

A3.2. Photographic Detection of Rotation.............. 348

A3.3. Spectroscopic Detection of Rotation.............. 351

A3.4. Radiometric Observations of the Cloud Layer........... 352

A3.5. Radar Observations....................... 353

A3.6. Radiometric Observations at Long Wavelengths......... 355

Appendix 4: HISTORICAL OBSERVATIONS OF PHASE-LOCKED STRUCTURE

A4.1. The Lowellian Markings on Venus.............. 359

A4.2. The Modern Resurrection of the Lowellian Markings....... 369

A4.3. The Lowellian Markings and the Heterodyne Results - A

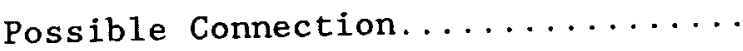

References $\ldots \ldots \ldots \ldots \ldots \ldots \ldots \ldots \ldots$

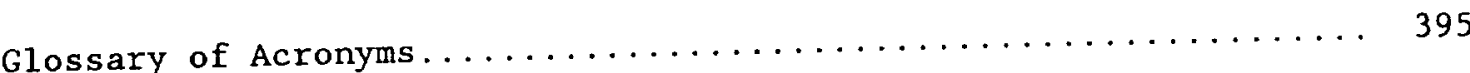




\section{LIST OF TABLES}

page

Table 5.1: December 1985 Observed Beam-Integrated Wind Velocities........................... 149

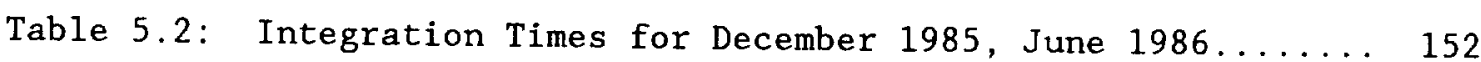

Table 5.3: June 1986 Observed Beam-Integrated Wind Velocities... 155

Table 5.4: October 1986 Observed Beam-Integrated Wind Velocities......................... 160

Table 5.5: Integration Times for October 1986, March 1987..... 163

Table 5.6: March 1987 Observed Beam-Integrated Wind Velocities... 167

Table 7.1: December 1985 Goodness-of-Fit Parameter S Over the Mode1 space........................ 205

Table 7.2: October 1986 Goodness-of-Fit Parameter S Over the Model space......................... 213

Table 7.3: March 1987 Goodness-of-Fit Parameter S Over the Model space........................ 219

Table 7.4: June 1986 Goodness-of-Fit Parameter S Over the Model space........................ 225

Table 8.1: Betz et al. Mesospheric Time Series............. 239

Table A1.1: Lamb Dip Stabilization Time Series Analysis........ 308

Table A1.2: Power Peak Stabilization Time Series Analysis....... 309 
Figure P.I: Venusian Temperature Profile and Atmospheric

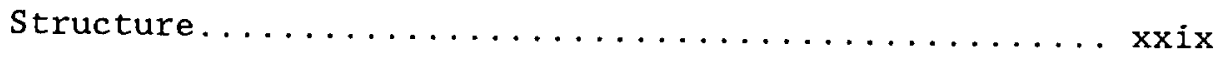

Figure 2.1: P. Lowe11's Circulation Mode1, 1910............ 31

Figure 2.2: Thermospheric Circulation Model (Bougher

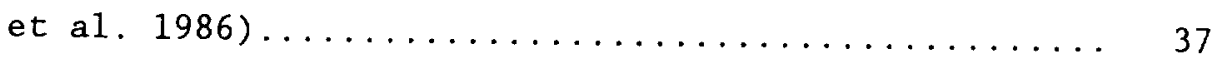

Figure 3.1: Generation of a Double Sideband Spectrum......... 45

Figure 3.2: Local Oscillator Wavefront Geometry for Diffraction-Limited Heterodyne Detection.......... 49

Figure 3.3: Source Wavefront Geometry for Diffraction-Limited Heterodyne Detection.................... 51

Figure 3.4: Heterodyne Detector Spatial Contribution Function... 53

Figure 3.5: Heterodyne Detector Beam Shape Function.......... 53

Figure 3.6: Source / Coherent Receiver observing Geometry....... 58

Figure 3.7: Theoretical Heterodyne Detection Signal-to-Noise for Blackbody Sources.................... 61

Figure 3.8: Infrared Heterodyne Spectrometer Optical Front End Layout......................... 63

Figure 3.9: Optical Front End Beam-Throw Geometry.......... 65

Figure 3.10: Generation of a Spectrum in the High Resolution Filter Bank....................... 68

Figure 3.11: Radio Frequency Back End Layout............... 70

Figure 3.12: Radio Frequency Detection Sequence............ 70

xvii 
Figure 3.13: A Typical Guiding Template................ 73

Figure 3.14: The McMath Solar Telescope................. 75

Figure 3.15: Optical Layout of the NASA Infrared Telescope

Facility........................ 77

Figure 4.1: The Laser Local Oscillator Cavity............ 80

Figure 4.2: The Diffraction Grating / Piezoelectric

Translator Mount.................... 80

Figure 4.3: A Cavity Etalon Standing Wave............. 83

Figure 4.4: A Laser Gain Profile.................. 83

Figure 4.5: Adjacent Laser Gain Profiles.............. 83

Figure 4.6: Piezoelectric Translator Cross-Section........... 87

Figure 4.7: A Laser Gain Profile with a Lamb Dip........... 87

Figure 4.8: ${ }^{12} \mathrm{C}^{16} \mathrm{O}_{2}$ Energy Level Diagram.................. 92

Figure 4.9: Generation of the Lamb Dip.............. 94

Figure 4.10: Lamb Dip Cell Size Versus Pressure............ 107

Figure 4.11: $4.3 \mu \mathrm{m}$ F1uorescence Collisional De-excitation

Pathways........................ 109

Figure 4.12: The Lamb Dip Cell in Cross-Section............ 113

Figure 4.13: The Lamb Dip Cel1 / Dewar Configuration........... 114

Figure 4.14: A Photograph of the Lamb Dip Package............ 115

Figure 4.15: A Photograph of the Lamb Dip Package Within the

Laser Cavity..................... 116

Figure 4.16: The Overall Laser stabilization system.......... 118

Figure 4.17: A Gain Profile for a Cavity Detuned from the

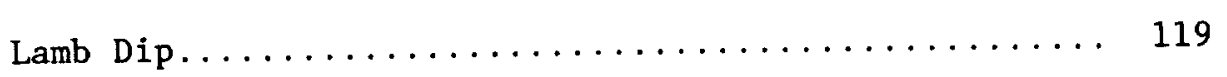


Figure 4.18: Piezoelectric Translator / Laser Frequency

Modulation Phasing..................... 119

Figure 4.19: The Phase of the $4.3 \mu \mathrm{m}$ Fluorescence Signal....... 122

Figure 4.20: Lamb Dip Stabilization System Undifferentiated

Mode............................ 123

Figure 4.21: Lamb Dip Stabilization System First Derivative

Mode.......................... 126

Figure 4.22: The Lamb Dip First Derivative Signa1........... 128

Figure 4.23: A Schematic Diagram of the Laser Stabilizer........ 129

Figure 4.24: Lamb Dip Stabilization System Laser Lock Mode...... 131

Figure 4.25: Power Peak Stabilization Lock Mode............. 134

Figure 4.26: Lamb Dip and Power Peak Stabilization Time Series... 137

Figure 5.1: Investigation Overview of Venusian Aspect

Geometries and Beam Positions............... 144

Figure 5.2: December 1985 Venus Aspect Geometry at McMath...... 147

Figure 5.3: December 1985 Data Acquisition at McMath......... 151

Figure 5.4: June 1986 Venus Aspect Geometry at McMath......... 154

Figure 5.5: June 1986 Data Acquisition at McMath (First Half)... 156

Figure 5.6: June 1986 Data Acquisition at McMath (Second Half)... 157

Figure 5.7: October 1986 Venus Aspect Geometry at IRTF........ 159

Figure 5.8: October 1986 Data Acquisition at IRTF.......... 162

Figure 5.9: March 1987 Venus Aspect Geometry at IRTF......... 165

Figure 5.10: March 1987 Data Acquisition at IRTF............ 168

Figure 6.1: Comparison Between June 1986 and March 1987 Aspect

xix 
Geometries...................... 173

Figure 6.2: June 1986/March 1987 wind Velocities........... 175

Figure 6.3: December 1985 wind Velocities............... 179

Figure 6.4: October 1986 wind Velocities................ 181

Figure 7.1: Beam Center Locator................... 184

Figure 7.2: Beam Center Uncertainty December $1985 \ldots \ldots \ldots \ldots \ldots \ldots$

Figure 7.3: Beam Center Uncertainty June $1986 \ldots \ldots \ldots \ldots \ldots \ldots \ldots$

Figure 7.4: Beam Center Uncertainty October $1986 \ldots \ldots \ldots \ldots \ldots \ldots$

Figure 7.5: Beam Center Uncertainty March $1987 \ldots \ldots \ldots \ldots \ldots \ldots \ldots 7$

Figure 7.6: Non-thermal Emission Model ............... 193

Figure 7.7: Program BEAMINT Geometry................ 194

Figure 7.8: Model Fits: December $1985 \ldots \ldots \ldots \ldots \ldots \ldots \ldots \ldots \ldots$

Figure 7.9: Model Fits: October $1986 \ldots \ldots \ldots \ldots \ldots \ldots \ldots \ldots \ldots$

Figure 7.10: Mode1 Fits: March 1987................. 214

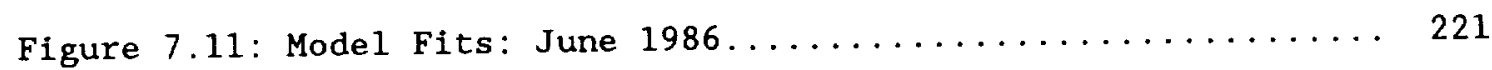

Figure 8.1: Betz et a1. Mesospheric Observing Geometry........ 235

Figure 8.2: Betz et al. Mesospheric Time Series............. 241

Figure 8.3: Betz et al. Zero-Corrected Mesospheric Time Series... 243

Figure 8.4: Contribution Function for ${ }^{13} \mathrm{C}^{16} \mathrm{O}_{2} 892 \mathrm{~cm}^{-1} \mathrm{P}(26) \ldots 245$

Figure 8.5: Cross-Section of the Subsolar-Antisolar Ce11....... 247

Figure 8.6: Antisolar-to-Subsolar Return velocity............ 249

Figure 8.7: Betz et al. Thermospheric Observing Geometries...... 252

Figure 9.1: Proposed Circulation Model for Venus........... 265 
Figure 10.1: June 1986 Mars Aspect Geometry at McMath........ 273

Figure 10.2: IRHS Mars Spectrum, McMath, June $1986 \ldots \ldots \ldots \ldots \ldots 274$

Figure Al.1: Beam Configuration for a Planetary Target....... 280

Figure A1.2: Beam Configuration for a Calibration Blackbody..... 280

Figure Al.3: A Lunar Scan...................... 289

Figure A1.4: Terrestrial Optical Depth as Derived from Lunar

Data............................. 289

Figure A1.5: Removing a Differential Doppler Shift.......... 293

Figure A1.6: Venus Data Reduction................. 295

Figure A1.7: A PLOTOBS Line Fit to Venusian Non-Thermal Emission. 298

Figure A1.8: Time Series Optical Front End Layout........... 303

Figure A1.9: A PLOTOBS Line Fit to White Cell-Generated

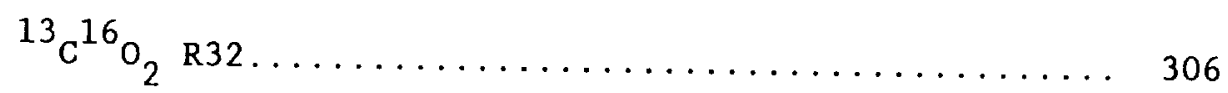

Figure A2.1: Venus Beam Aspect Geometry.............. 312

Figure A2.2: Transformation from Planetary Latitude and Longitude to Plane-of-Sky Coordinates.......... 318

Figure A2.3: SS-AS Circulation Aspect Geometry............ 324

Figure A4.1: Drawings by P. Lowell from Visual Observations

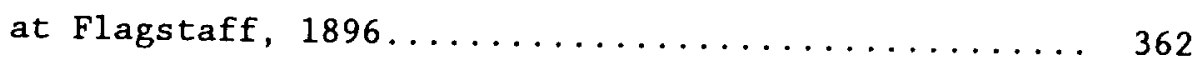

Figure A4.2: Drawings by D.A. Drew and P. Lowell from Visual Observations at Flagstaff, $1896 \ldots \ldots \ldots \ldots \ldots \ldots \ldots$

Figure A4.3: Map of Venus by P. Lowell from Visual Observations

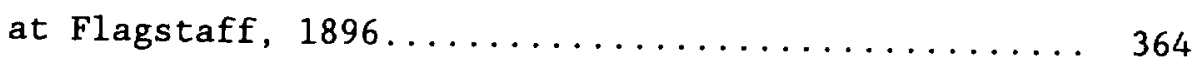


Figure A4.4: Drawings by A.E. Douglass and D.A. Drew from

Visual Observations at Flagstaff, April 1898..... 366

Figure A4.5: Drawings by P. Lowell from Visual Observations at

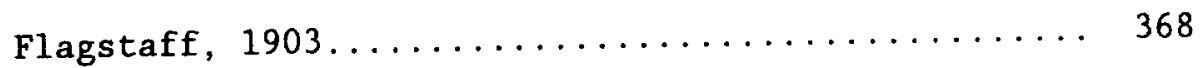

Figure A4.6: Drawings by J. Camus from Visual Observations,

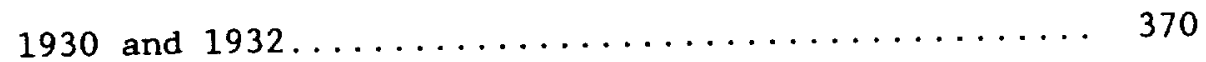

Figure A4.7a: Yellow Light Composite Drawings of Venus by A.

Dollfus at Pic-du-Midi, $1948-53 \ldots \ldots \ldots \ldots \ldots \ldots \ldots 2$

Figure A4.7b: Planisphere, by A. Dollfus, from Yellow Light

Composite Drawings................... 372

Figure A4.8: Comparison Between Visual Observations and Blue

Light Photographic Composites............. 374

Figure A4.9: Drawings of Venus by G. Mottoni, 1921, and A.

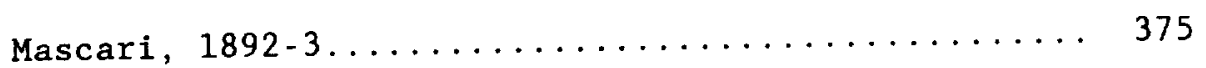

Figure A4.10: Comparison Between a Drawing of Venus by R.M.

Baum, 1951, and Dollfus Planisphere......... 378

Figure A4.11: Convection Cell Model of Venusian Circulation

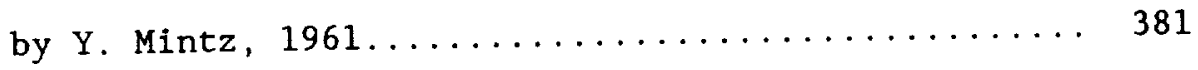

xxii 


\section{Preface: Why Study Planetary Winds}

\section{P.1. A PHILOSOPHICAL PREAMBLE}

A more appropriate first question might be, "Why study other planets?" A first motivation derives from the obvious - the simple need to know, integral to the human spirit, is a powerful driving force in science, as in any exploratory endeavor. To gain upon any aspect of the universe is both intellectually and emotionally satisfying, defining ever more precisely our place within it. T.S. Eliot wrote;

\footnotetext{
"We shall not cease from exploration. And the end of all our exploring will be to arrive where we started and know the place for the very first time."
}

In this same vein, a second more subtle motivation arises - the study of other planets is truly a study of our own planet Earth. Such is a fundamental precept of comparative planetary studies. Furthermore, comparative planetology holds that Earth is but one member of a planetary family rather than the prototype from whose mold the other planets were cast. The latter anthropocentric approach offers a narrowed study of terrestrial processes manifestly constrained by Earth's characteristic location in space, chemical composition and distribution, spin/orbital dynamics, geologic activity, and overall phase of planetary evolution. Such an approach may fail to reveal a phenomenology vital for a deeper understanding of many natural processes involved. Yet these same processes, under the vastly freer constraints offered by the diversity of worlds, often build upon one another in fundamentally different ways - often resulting in distinctly aterrestrial phenomenology. Therefore, at its best, planetary 
phenomenological comparison can serve as the primary probe of, even reveal, processes perhaps masked or long since inactive here on Earth, provide insight into process dependence and interdependence, and in fact establish the environmental regimes in which these processes operate.

Obviously the sheer level of scientific scrutiny afforded Earth enhances our understanding of other planetary environments far more often than the reverse. Yet such a "scrutiny gradient" does not preclude information transfer in the reverse sense - as indicated by the following examples.

\section{P.2. COMPARATIVE PLANETOLOGY: REAL BENEFITS}

Earth's biosphere is today under attack on a considerable number of fronts as a direct result of man's technological advance. Atmospheric injection of chlorofluorocarbons and hydrocarbons, deforestation, the burning of fossil fuels, and chemical pollution are now being tied with increasing confidence to ozone depletion, global warming, desertification, acid rain, and destruction of ecosystems worldwide. From this vantage point, it is imperative we understand the cause and effect relationship between human action and biome response so that our technology may be guided reasonably. To this end, Venus/Earth comparative planetological studies have already reaped benefits.

For a world so similar to Earth in mass, density, composition and distance from the Sun, it is hard to believe Venus could exhibit such marked departures from the terrestrially familiar, apparently due to 
subtle differences in initial condition.

1. The in situ determination that the dramatic Venusian temperatures derive from an extreme Greenhouse Effect (see Avduevsky et al. 1971, Pollack et al. 1980) has provided insight into the corresponding terrestrial case and the effects, present and future, of man's industrialization on the Earth's heat budget. Venusian Greenhouse models have reproduced the observed temperature profile (Seiff 1983) providing quantitative confirmation of theory and an accounting of relevant physical processes. Terrestrial Greenhouse models, called upon to discern subtle global temperature variation, thereby rest on tested ground (Kahn 1982).

2. The importance of chlorine photochemistry was first identified in the atmosphere of Venus (Prinn 1971). Venus studies thereby helped construct the theoretical framework from which were derived the specifics of photochemical destruction of terrestrial ozone (Kahn 1982; Molina and Rowland 1974). Similarly, an understanding of photooxidation pathways for sulfur species $\left(10^{5}\right.$ times more plentiful in the Venusian atmosphere) to concentrated $\mathrm{H}_{2} \mathrm{SO}_{4}$ might provide insight into similar mechanisms producing acid rain on Earth (Prinn and Fegley 1987).

3. The presence of nitrates in the Venusian atmosphere prove the existence of abiological nitrogen fixing pathways (Kahn 1982) while, conversely, the absence of abundant $\mathrm{O}_{2}$ in planetary atmospheres points to the importance of biological $\mathrm{O}_{2}$ production here on Earth (Kahn 1982).

Extending comparative planetology to the rest of the solar 
system - studies of the pristine surfaces of the Moon and Mercury, as we11 as meteorites, provide windows on the earliest epochs of Earth development - their mark long since erased from the terrestrial landscape through erosive forces and geologic activity (Head 1981). (Similarly, the solar wind/Venus interaction may provide insight into the solar wind/Earth atmospheric interaction during past periods of terrestrial magnetic field reversal (Kahn 1982)).

Plate tectonic theory is afforded support as the apparent absence of plate tectonics on Mars can account for the enormous size of Martian volcanoes (Carr et al. 1984).

Studies of carbonaceous chondritic meteorite composition and asteroidal/cometary physical characteristics and spatial distribution provided the basis for Alvarez et al. (1980, 1984) to conclude extraterrestrial impact as the cause of the Cretaceous-Tertiary mass extinction. Catastrophic shaping of the biosphere, routinely dismissed over the last century for lack of obvious terrestrial evidence, is now recognized.

\section{P.3. PLANETARY WINDS AND CIRCULATION MODELS}

Models of observed terrestrial phenomena may be characterized by the scale they address ranging from the planetary or global to the very local. While model testing, regardless of the associated scale, may benefit from comparison with observed phenomena on other worlds, those models of global scale are particularly dependent on such comparative planetology since they are by definition world models. Circulation Models (CM's) are one such class.

xxvi 
Earth's meteorological complexity derives from a variety of sources including: large-scale topographic relief, water-land thermal contrast, obliquity-induced seasonal variation, variable cloud cover, biogenic effects and rapid rotation. On Venus, these sources are either nonexistent or minimal (Prinn and Fegley 1987), allowing CM theory to be tested at a more basic level and in fundamentally different meteorological regimes (e.g., Earth quasi-geostrophic circulation as opposed to Venusian quasi-cyclostrophic circulation; see Chapter 2). Such CM testing may be extended to Mars, providing a second Earth-1ike regime (Prinn and Fegley 1987), and Titan, whose characteristics borrow from both Earth and Venus (Hunten et al. 1984), establishing a quite comprehensive meteorological database.

As a gauge of our understanding regarding Earth meteorology, it is interesting to note that present Numerical Weather Prediction Models (NWPM's) provide only 6.5-day reasonable forecast accuracy (for the Northern Hemisphere), twice that of the early 1970's (Curran 1987). Today, NWPM's derive the wind field, an important input parameter field, from geostrophic balance using temperature, pressure and humidity data (Curran 1987). However, geostrophic balance generally does not hold on the small scale, now important due to increased NWPM resolution, or in the tropics. It is therefore believed that further advance in forecast capability mandates the acquisition of wind profiles with $100 \mathrm{~km}$ horizontal resolution, $1 \mathrm{~km}$ vertical resolution, $-6 \mathrm{hr}$ sampling and $1-5 \mathrm{~m} / \mathrm{s}$ wind accuracies (Baker and Curran 1985).

CM testing on Venus has reached a similar impasse. While limited Venusian wind information has been acquired by in situ descent probes, 
balloons, orbiting platforms, and ground-based facilities, only one set of (suspect) wind measurements exists above the $65 \mathrm{~km}$ level (Betz 1977, 1982). Yet, CM's predict a transition between two fundamentally different large-scale circulation patterns at $-90-100 \mathrm{~km}$ altitude (Schubert 1983). While radiometric data exist for this altitude regime, it is not enough that observed temperature (and pressure) fields are CM-consistent. The predicted and observed vector wind fields must be compared. To this end, the need for high altitude Venusian wind measurements is clear. While Doppler LIDAR sounding of the Earth's atmosphere has been proposed to provide the wind profiles needed by NWPM's (Curran 1987), the only avenue for Venusian upper atmospheric wind sounding, to date, is provided by this work.

Through comparative meteorology, models for the very mechanism of weather are put to the test on the global scale. Validation of model predictions concerning other planetary atmospheres provides for a comprehensive understanding of meteorology in general. And it is the observational data, specifically in this case measurements of the planetary wind field, that allow such model validation.

\section{P.4. VENUSIAN ATMOSPHERIC NOMENCLATURE}

To provide the thesis with both a necessary convention and some semblance of conformity to existing literature, the delineation of Venusian atmospheric levels as provided in Figure P.I has been adopted. The temperature profile is from seiff (1983). The altitude boundaries for the troposphere (generally characterized by a near adiabatic lapse rate), the mesosphere (associated with a strongly subadiabatic lapse 


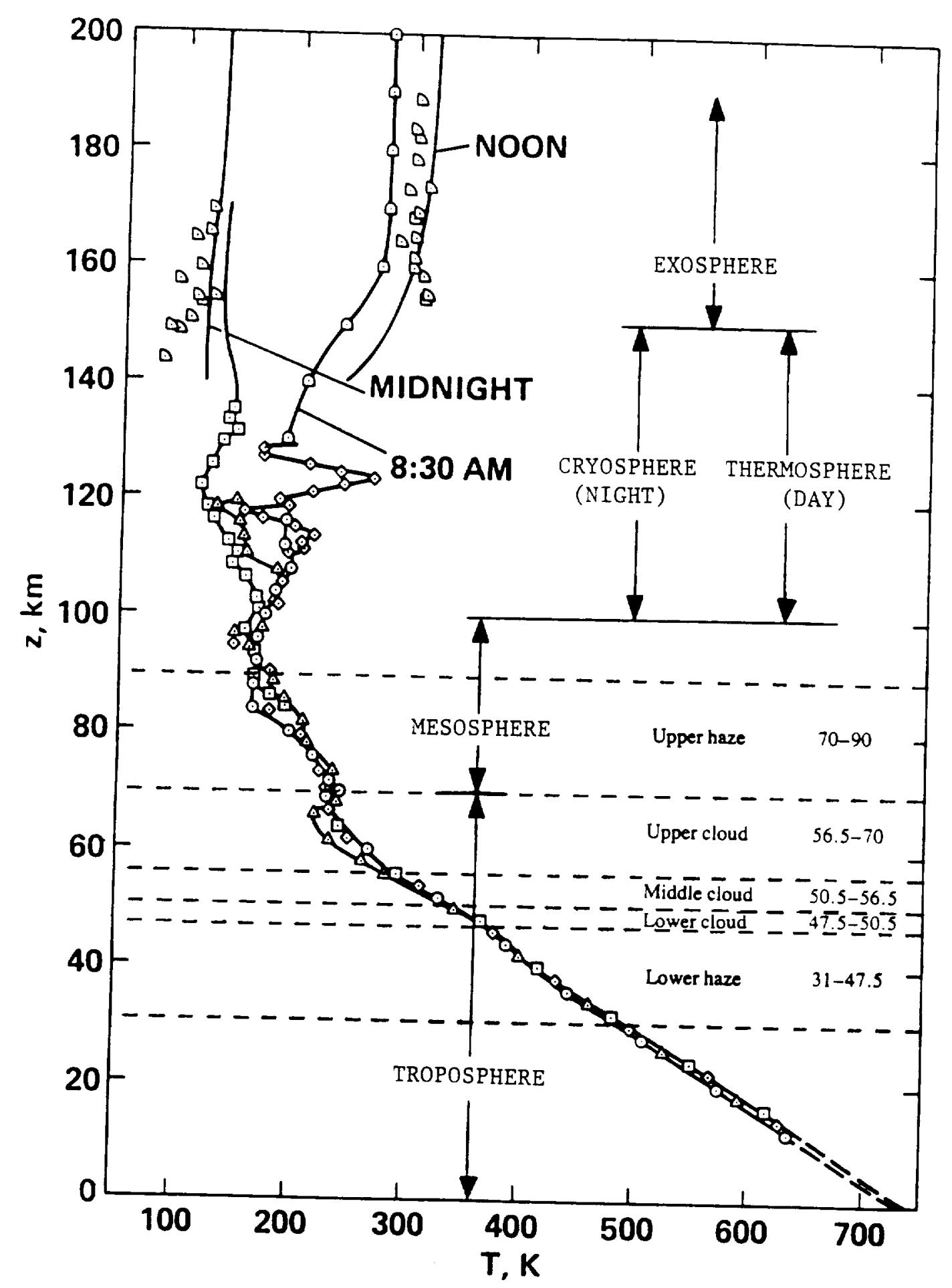

Figure P.1. Venus Vertical Temperature and Atmospheric Structure 
rate and no diurnal variation), the thermosphere/cryosphere (exhibiting a strong diurnal temperature variation below the exobase) and the exosphere are reasonably consistent with those recommended by Hunten et al. (1983). The altitude regimes for the various cloud layers are taken from Esposito et al. (1983).

\section{P.5. OVERVIEW OF THE THESIS}

The thesis has been divided into ten chapters and four appendices. Chapter 1 provides a statement of the problem at hand: the need for direct and absolute Venusian wind measurements in the 100-150 km altitude range, and the means by which this challenge has been met through absolute frequency stabilized IR heterodyne spectroscopy.

Chapter 2 is a review of observations and theory pertaining to Venusian atmospheric dynamics below $100 \mathrm{~km}$ altitude (troposphere and mesosphere), and from 100 to $150 \mathrm{~km}$ (thermosphere/cryosphere). Such a comprehensive approach allows consideration of the thesis results within the broader context of overall Venusian circulation. Recent hydrodynamical models of the thermosphere/cryosphere, particularly Bougher et al. (1986), are reviewed at length. Models for the $\mathrm{CO}_{2} 10$ $\mu \mathrm{m}$ non-thermal thermospheric emission (used in the present work as the spectroscopic probe of upper atmospheric dynamics) are also investigated.

Chapter 3 covers all facets of IR heterodyne spectrometer operation (except the specifics of the laser local oscillator subsystem). Heterodyne principles, theory of operation, description of subsystems, telescope-specific characteristics, and field operation are 
discussed in detail.

Chapter 4 gives the specifics of the laser local oscillator and the laser frequency stabilization system, the latter developed to allow acquisition of absolute line of sight wind velocities. Topics covered include: the character of the laser cavity and gain environment, saturated resonance absorption (Lamb dip) theory, laser stabilization theory, a detailed review of the stabilization hardware, and a comparison of past and present laser stabilization systems used for heterodyne determination of Venusian winds.

Chapter 5 provides an overview of observations made during the four observing periods: December 1985 at McMath, June 1986 at McMath, October 1986 at IRTF, and March 1987 at IRTF. Earth/Venus aspect geometry, beam shape, spatial and temporal coverage, seeing, and integration times are detailed for each of the four observing periods. The derived line of sight, beam-integrated wind velocities are presented with their associated systematic and random errors.

Chapter 6 provides a qualitative analysis of the observed beam-integrated wind velocities, allowing determination of the dominant circulation at $100-120 \mathrm{~km}$ altitude.

Chapter 7 provides a quantitative determination of the global wind field at $100-120 \mathrm{~km}$ altitude. Presented is a cursory overview of program BEAMINT - developed to generate line of sight beam-integrated winds from a model horizontal wind field. The BEAMINT-generated beamintegrated winds are then compared to the observed data, allowing model discrimination. (The specifics of BEAMINT are given in Appendix 2.)

Chapter 8 gives a detailed review of the pioneering wind 
measurements made by Betz et al. (1976, 1977a,b; Betz 1977, 1982;

Johnson et al. 1976). Discussed are the dynamical problems associated with the early derived wind velocities, the system-inherent shortcomings they allude to, the successful means of correction adopted by the present investigation, and an attempt to tie both data sets together.

Chapter 9 addresses the results and conclusions of the present heterodyne investigation. BEAMINT-derived best fit horizontal wind fields are compared with the 2 -dimensional circulation model by Bougher et al. (1986) at $110 \mathrm{~km}$ altitude. The heterodyne results, along with results of all other dynamical studies above the clouds to date, are then integrated into a self-consistent model of Venusian circulation from $70-200 \mathrm{~km}$ altitude.

Appendix 1 presents the data reduction specifics including: removal of systematic offsets, terrestrial absorption, and Earth rotation, line-fitting, beam-integrated wind velocity determination, and error analysis. Reduction of laser stabilization system time series data is also covered.

Appendix 2 provides the detailed theory of BEAMINT-generated beam-integrated wind velocity, including modelling of across-the-beam gradients in: line of sight projection geometry, beam shape, emission intensity, and horizontal wind field.

Appendix 3 provides a rather complete historical overview of Venusian observations over the last 300 years. Beyond the fascinating history associated with Venus as a target of ground-based study, the rationale for presenting a detailed account of the early work is 
two-fold. First, prior to the application of radio and radar astronomy to Venus studies in the late $1950^{\prime} \mathrm{s}, 300$ years of Venusian observation was necessarily restricted to the character and dynamics of the Venusian atmosphere - specifically, the cloud decks. Second, in light of both past and the present heterodyne results, as well as Venusian upper atmospheric circulation model (CM) predictions, one particular class of visual observations indicating phase-locked structure becomes particularly intriguing. Appendix 4 surveys the history of this structure and discusses a possible connection with the heterodyne results.

\section{P.6. ACKNOWLEDGEMENTS}

I am grateful to Drs. Michael J. Mumma, Theodor Kostiuk, and Drake Deming at NASA/Goddard Space Flight Center, and Dr. David Zipoy, at the Astronomy Program, University of Maryland, for their insight into the problems at hand, from instrument development to data acquisition to modelling. Special thanks go to Mr. Fred Espenak for software support and to Mr. James Faris for support in instrument development. Additional thanks go to Drs. David Glenar, David Buhl and Uli Kauf1, for technical assistance, and to Tilak Hewagama, and Bashar Rizk for aid in data acquisition. 
$\longrightarrow$ 


\section{CHAPTER 1: INTRODUCTION}

1.1. STATEMENT OF THE PROBLEM - VENUSIAN ATMOSPHERIC THERMAL STRUCTURE AND DYNAMICAL IMPLICATIONS

Early IR heterodyne wind measurements (Betz 1977, 1982; Betz et al. 1976, 1977b), and microwave studies of global Co distribution (Clancy and Muhleman 1985a,b), have been interpreted as resulting from subsolar-to-antisolar circulation (SS-AS) in the lower thermosphere (95-120 km altitude), consistent with the best available hydrodynamical models (Bougher et al. 1986). These results, however, are far from definitive. At lower levels $(12-65 \mathrm{~km})$ a dominant retrograde zonal super-rotation has been firmly established (see Schubert 1983), and may have been seen penetrating to $90 \mathrm{~km}$ (Clancy and Muhleman 1985a,b). Near $100 \mathrm{~km}$ altitude, a transition from super-rotation to SS-AS flow as the dominant circulation is thus suggested. (Zonal super-rotation may also be important above $150 \mathrm{~km}$ altitude; see, e.g., Keating et al. 1980, Brinton et al. 1980.)

The present investigation (Goldstein et al. 1985, 1987a,b) seeks to definitively establish the relative involvement of zonal and SS-AS components in the $100-120 \mathrm{~km}$ altitude regime, and to re-examine all dynamical indicators from $70-200 \mathrm{~km}$, in the hope of presenting an integrated and self-consistent picture of circulation as a function of altitude.

It is the task of circulation models to reconcile observed spatial fields of various atmospheric parameters, e.g., temperature, pressure and density of atmospheric species, with observed dynamics. Direct wind measurement in as yet dynamically unprobed or poorly probed regions of 
the Venusian atmosphere thereby serves as a powerful test of model validity and provides an expanded database upon which subsequent models may be formulated. Models based on observed atmospheric thermal structure point to decidedly different dynamical regimes above and below $100 \mathrm{~km}$ altitude, yet there is only one direct but poorly calibrated set of wind measurements above the cloud tops $(-65 \mathrm{~km})$ to date - emphasizing the critical need for precise wind information.

\subsubsection{Atmospheric Thermal Structure}

Thermal structure in the Venusian atmosphere has been studied in situ from Venera (Avduevsky et al. 1983), Vega (Sagdeev et al. 1986c) and Pioneer Venus (PV) (Seiff 1983) descent probes, and Vega balloon-borne gondolas (Sagdeev et al. 1986b). Temperature remote sensing has also been undertaken from Mariner flyby craft (Barath et al. 1964, Sagan and Pollack 1969, Dunne and Burgess 1978), Pioneer Venus 1 (Seiff 1983) and Venera (Yakovlev et al. 1976) orbiters, and ground-based facilities (see Taylor et al. 1983).

Descent probes have provided direct temperature measurements below the cloud tops $(-65 \mathrm{~km}$ altitude to the surface; Seiff 1983) at selected entry points (coverage: 0:07-13:42 Local Venusian Time (LVT), $38^{\circ} \mathrm{S}-60^{\circ} \mathrm{N}$ lat.; Colin 1983, Sagdeev et a1. 1986). Simultaneous multiprobe entry, as in the case of the four Pioneer Venus 2 probes, has provided limited data on the latitudinal and longitudinal (diurnal) thermal contrast, with altitude, characterizing the atmosphere at a specific time (Seiff 1983). The Vega balloon-borne investigations yielded moderate duration monitoring of atmospheric parameters ( -2 days each) at a nominal 53.6 
$\mathrm{km}$ float altitude, covering $-7.3^{\mathrm{h}}$ in LVT over approximately latitude-constant, near equatorial float paths (Sagdeev et al. 1986). The thermal structure of the neutral atmosphere above the clouds has been obtained only indirectly - following from other measured atmospheric parameters, e.g., atmospheric density, probe deceleration, index of refraction and distribution of neutral gas species. On-orbit experiments providing such temperature determinations include: Venera 9 and 10 orbiter radio occultation (Yakovlev et al. 1976), PV Orbiter Radio Occultation (ORO; 38-86 km alt.; Kliore and Patel 1980), the PV Orbiter IR Radiometer (OIR; 60-140 km alt.; Taylor et a1. 1980), PV Orbiter Atmospheric Drag (OAD; $140-190 \mathrm{~km}$ alt.; Keating et al. 1980) and the PV Orbiter Neutral Mass Spectrometer (ONMS; $150-250 \mathrm{~km} \mathrm{alt.;}$ Niemann et al. 1980). These on-orbit remote sensing experiments have provided both long term and global-scale atmospheric coverage.

Experiments performed during probe entry include accelerometer-measured atmospheric drag aboard Venera (65-110 km alt.; Avduevsky et al. 1983) and PV entry probes (LAS and SAS; surface to $126 \mathrm{~km}$; Seiff et al. 1980) and the PV multiprobe Bus Neutral Mass Spectrometer (BNMS; $130-650 \mathrm{~km}$ alt.; von Zahn et al. 1980).

Figure P.1 (Seiff 1983) provides a canonical Venus temperature profile indicating gross structure from the surface to $200 \mathrm{~km}$ altitude, as derived from PV ONMS, BNMS, OAD, LAS and SAS.

Observed longitudinal (diurnal) temperature variation $\left(\Delta \mathrm{T}_{\mathrm{D}}\right.$ ) below $100 \mathrm{~km}$ is small $\left(12-20 \mathrm{~km}\right.$ alt., $\Delta \mathrm{T}_{\mathrm{D}}<5 \mathrm{~K} ; 20-35 \mathrm{~km}$ alt., $\Delta \mathrm{T}_{\mathrm{D}}<1 \mathrm{~K} ; 35-65$ $\mathrm{km}$ alt., mean $\Delta \mathrm{T}_{\mathrm{D}}-0 \mathrm{~K}, \Delta \mathrm{T}_{\mathrm{D}}$ oscillation with altitude; $65-100 \mathrm{~km}$ alt., mean $\Delta T_{D} \sim$ a few $\mathrm{K}$ with temporal variability of $\leq 10 \mathrm{~K}$ amplitude; Seiff 
1983).

Observed latitudinal temperature variation below the clouds $(<-45$ $\mathrm{km}$ altitude) is also small - a $-5 \mathrm{~K}$ poleward decrease from $30-60^{\circ}$ latitude, and $-5 \mathrm{~K}$ poleward increase from $0-30^{\circ}$ latitude, at fixed altitude. However, in the 0.2-2.0 bar range within the cloud decks (45-60 km altitude), 25-40 $\mathrm{K}$ equator-to-pole decreases have been observed (as measured along isobaric surfaces; Seiff 1983). In the $-70-90 \mathrm{~km}$ altitude range, the latitudinal temperature gradient is reversed, with observed equator-to-pole increases of as much as $40 \mathrm{~K}$ (Seiff 1983, Schubert 1983).

Above $100 \mathrm{~km}$ altitude, a strong diurnal temperature gradient exists, forming a dayside thermosphere $(-300 \mathrm{~K}$ at the exobase) and a nightside cryosphere (100-130 $\mathrm{K}$ at the exobase) (Seiff 1983; see Fig. P.1). The resulting diurnal pressure variation is dramatic. At $190 \mathrm{~km}$ altitude $P_{\text {noon }} / P_{\text {midnight }}=10^{3}$, with a 100 -fold decrease just within the $1.5^{\mathrm{h}}$ to either side of the terminator (Schubert 1983).

No seasonal variation is expected due to a nearly circular orbit $\left(e=0.007\right.$; Colin 1983) and an obliquity of $2.6^{\circ}$ (Colin 1983; really $177.4^{\circ}$ owing to a retrograde rotation).

1.1.2. Dynamical Implications - Mesosphere ( $-65-100 \mathrm{~km}$ Altitude) Below $100 \mathrm{~km}$ altitude, the apparent near absence of diurnal temperature contrast implies spin axisymmetric circulation. Numerous ground- and space-based observations have established zonal super-rotation (with $-100 \mathrm{~m} / \mathrm{s}$ equatorial cloud-top winds) as the primary component from 12 to $65 \mathrm{~km}$ altitude, while a mean meridional 
(Hadley) component, though present, is associated with order of magnitude reduced wind speeds (Schubert 1983). Existence of Hadley circulation is best established within the clouds at $-45-65 \mathrm{~km}$ altitude, consistent with the observed strong latitudinal temperature gradient. Models assuming cyclostrophic balance (see Chapter 2) and based on observed latitudinal temperature gradients, predict the increasing zonal winds with altitude observed from 12 to $65 \mathrm{~km}$ for 30-60 ${ }^{\circ}$ latitude (Schubert 1983). These models further predict maximal zonal wind velocity near $70 \mathrm{~km}$ and, from 70 to $90 \mathrm{~km}$, decreasing zonal winds with a possible concomitant dramatic increase in mean meridional circulation (Taylor et al. 1980, E1son 1979, Limaye 1985). Such enhanced meridional flow would be associated with a direct Hadley cell just above the cloud-level cell (though circulating in the opposite sense), consistent with the observed poleward warming in this region. However, the virtual absence of wind measurements in the mesosphere, -65-100 km, precludes the most basic tests of these predictions.

To date, the only direct mesospheric wind measurements, from $\sim 10$ $\mu \mathrm{m}{ }^{13} \mathrm{C}^{16} \mathrm{O}_{2}$ absorption line observations, indicate $-90 \mathrm{~m} / \mathrm{s}$ zonal super-rotation at $75-85 \mathrm{~km}$ altitude (Betz et al. 1977b, Betz 1977, 1982). However, this predominantly equatorial investigation (unsuited as a probe of the meridional component) seems to have been associated with rather large systematic and random errors. A consistent mean vertical wind velocity of $-45 \mathrm{~m} / \mathrm{s}$ (100 mph), with up to $40 \mathrm{~m} / \mathrm{s}$ "real" fluctuations, was observed near inferior conjunction (disk-center) - which is dynamically unreasonable (see, e.g., Clancy and Muhleman 1985a,b). The technique employed - infrared heterodyne 
spectroscopy - is the same as used in this work, though improvements in spatial resolution, $S / N$ and, particularly, frequency stability have been effected.

The only other indicators of mesospheric dynamics are speculative at best. The distribution of ${ }^{12} \mathrm{C}^{16} \mathrm{O}$ in the $80-90 \mathrm{~km}$ altitude range, from microwave spectroscopy (Clancy and Muhleman 1985a,b), has been interpreted as circulation induced, resulting from a superposition of zonal super-rotation and a subsolar-to-antisolar axisymmetric flow (after the failure of purely photochemical/vertical eddy diffusion models). PV Orbiter UV photopolarimetry suggests super-rotation at -80 km altitude (Limaye 1984).

1.1.3. Dynamical Implications - Thermosphere/Cryosphere (100-150 km Altitude)

The thermosphere/cryosphere temperature gradient should drive a subsolar-to-antisolar circulation with strong cross-terminator winds and a lower-level return branch. The simplest flow would be subsolar-to-antisolar axisymmetric.

The sole wind measurements in the thermosphere were again obtained from infrared heterodyne spectroscopy (Betz 1977, 1982; Betz et al. $1976,1977 \mathrm{~b})$. Observations of $-10 \mu \mathrm{m}{ }^{12} \mathrm{C}^{16} \mathrm{O}_{2}$ non-thermal emission, at $109 \mathrm{~km}$ altitude, have indicated SS-AS circulation with $-130 \mathrm{~m} / \mathrm{s}$ cross-terminator winds. Unfortunately, as with the mesospheric measurements, dynamically unreasonable vertical wind velocities $(30 \mathrm{~m} / \mathrm{s})$ were inferred, rendering the results speculative. (The sole observing period, out of four, indicating high velocity 
cross-terminator winds also indicated the suspect $45 \mathrm{~m} / \mathrm{s}$ mesospheric vertical velocities.)

${ }^{12} \mathrm{C}^{16} \mathrm{O}$ microwave observations at $-95 \mathrm{~km}$ altitude ( $\mathrm{Clancy}$ and

Muhleman 1985a,b) show a nightside bulge centered near midnight, interpreted as resulting from SS-AS circulation with no zonal component.

Above $-150 \mathrm{~km}$ altitude, a post-noon maximum in temperature (Keating et al. 1980) and post-midnight maxima in neutral $\mathrm{H}$ density (Brinton et al. 1980), He density (Niemann et al. 1980), and UV airglow (Stewart and Barth 1979) have been observed. These point to a retrograde zonal component to the wind field with $\sim 50-100 \mathrm{~m} / \mathrm{s}$ equatorial winds at $150 \mathrm{~km}$ altitude (see, e.g., Mayr et a1. 1980). Consistent with this picture, the evening terminator pressure gradient is nearly twice the morning terminator gradient (Schubert 1983).

\subsubsection{Summary}

Above the cloud decks, thermal, pressure, and specie density fields, together with the early heterodyne results, provide only a speculative picture of circulation, e.g.:

decreasing zonal wind speed with altitude above $-70 \mathrm{~km}$, with a possible concomitant increase in the meridional component - SS-AS circulation becoming important by possibly $80-90 \mathrm{~km}$. Nearly pure SS-AS flow exists at $95-120 \mathrm{~km}$, while at higher altitudes (>150 $\mathrm{km}$ ?), zonal super-rotation again becomes important. (The antisolar-to-subsolar directed return branch required by mass continuity is not addressed in the literature.) 
1.2. METHODS OF DIRECT WIND VELOCITY DETERMINATION IN THE VENUSIAN ATMOSPHERE

Venusian atmospheric wind velocities have been obtained from ground-based (e.g., Boyer and Camichel 1965, 1967; Boyer and Guerin 1966, 1969; Smith 1967) and space-based (Limaye and Suomi 1981; Rossow et al. 1980) UV imaging, ground-based IR imaging (Allen and Crawford 1984), ground-based spectroscopy (e.g., Guinot and Feissel 1968; Traub and Carleton 1975, 1979; Betz et al. 1976, 1977b; Betz 1977, 1982), Doppler and Differential Very Long Baseline Interferometric (DVLBI) tracking of descent probes (Kerzhanovich and Marov 1983, Counselman et a1. 1980) and balloon-borne gondolas (Preston et al. 1986), and from descent probe (Avduevsky et al. 1976) and balloon-borne anemometers (Kremnev et al. 1986) and acoustic sensors (Moroz 1983).

\subsubsection{Imaging}

The observed structure in UV images of Venus has been attributed to UV absorbers within the cloud decks near $65 \mathrm{~km}$ altitude (Esposito et al. 1983). Such structure may be used as a tracer of cloud-level dynamics, assuming it is co-moving with the atmosphere. However, spatial and temporal variability in observed structure may result from, e.g., the propagation of planetary waves or the large-scale creation/destruction of the responsible atmospheric absorbers (Young 1975).

While ground-based UV imaging can resolve only planetary-scale structure, the in situ Mariner 10 TV Camera (Limaye and Suomi 1981) and 
PV Orbiter Cloud Photopolarimeter (OCPP) (Rossow et al. 1980) investigations additionally allowed tracking of large numbers of small-scale features. In situ imaging suggests only the small-scale structure is co-moving with the atmosphere. The large-scale structure results from planetary waves moving slowly with respect to the bulk atmospheric motion (Belton et al. 1976a,b).

Polarization variability observed by PV OCPP in photopolarimetry mode (see Fimmel et al. 1983), may reflect dynamics within the haze layer at $-5 \mathrm{mb}(80 \mathrm{~km}$ altitude; Limaye 1984). Variable IR structure has been found in images at $1.5-2.5 \mu \mathrm{m}$, the long wavelengths possibly probing down to clouds at $48 \mathrm{~km}$ altitude (Allen and Crawford 1984 , Allen 1987).

\subsubsection{Doppler and Interferometric Tracking}

Doppler and DVLBI tracking of Venera, Vega and PV descent probes and Vega balloon-borne gondolas has provided in-situ wind measurements from $-65 \mathrm{~km}$ altitude to the surface. The Doppler technique involves continuous single antenna monitoring of transmissions from the probe's onboard reference oscillator. Observed Doppler shifts in the reference frequency provide the instantaneous line of sight velocity between probe and receiving antenna. The receiving antenna may be located at an Earth ground station (Venera 4-8, Vega 1,2; see Kerzhanovich and Marov 1983, Preston et al. 1986, respectively), aboard a Venus atmospheric entry vehicle (PV entry probe/Multiprobe Bus uplink; Counselman et al. 1980, Seiff et al. 1980), or aboard a Venus orbiter or flyby spacecraft (Venera 9-14; Kerzhanovich and Marov 1983). 
Accurate ephemerides and receiver trajectory modelling allow determination of the receiver's motion with respect to the Venusian topocentric coordinates at the probe's location. With respect to the local Venusian surface, the line of sight component of the probe's velocity is therefore determined. Independently, the probe's local vertical velocity may be obtained from in situ temperature and pressure measurements as a function of elapsed descent time. Together, the local vertical and line of sight velocities determine the probe's horizontal velocity component in the direction of the receiver.

Below $55 \mathrm{~km}$ altitude, aerodynamic modelling indicates that horizontal velocity of freefalling descent probes and local horizontal wind velocity are the same to within $1 \mathrm{~m} / \mathrm{s}$ (Counselman et al. 1980). With an appropriate choice of receiver/descent probe aspect geometry, Doppler tracking can therefore provide either near zonal or near meridional horizontal wind profiles with altitude. Comparison of measured and modelled probe descent velocity yields vertical wind velocity.

The Doppler-determined, line of sight velocity has been coupled with Very Long Baseline Interferometric Differencing (DVLBI) between probe and space-based receiver to provide the probe's 3 -dimensional space velocity (e.g., PV descent probes, Vega balloons; Counselman et al. 1980, Preston et al. 1986, respectively). The probe's transverse velocity components are obtained using a minimum of 3 non-colinear antennas. Transforming to probe-local coordinates yields wind profiles, with altitude, at $1 \mathrm{~m} / \mathrm{s}$ resolution (Counselman et al. 1980). 


\subsubsection{Anemometric Measurements}

Surface winds have been measured by anemometers on the Venera 9 and 10 landers (Moroz 1983), and evaluated using acoustic sensors on Veneras 13 and 14 (Moroz 1983). Vertical-looking anemometers fitted to the Vega balloon-borne gondolas have provided gondola-relative vertical wind velocities along the entire float trajectory (Kremnev et al. 1986).

\subsubsection{Spectroscopy}

To date, the only spectroscopic determinations of Venusian winds have been ground-based (see Appendix 3). Most recently, Traub and Carlton (1975, 1979) used a PEPSIOS spectrometer to determine limb-to-terminator differential winds, and Betz et al. (1976, 1977b; Betz 1977, 1982) employed IR heterodyne spectroscopy (IRHS) to determine "absolute" line of sight wind components.

The PEPSIOS investigation used primarily the $-8708 \AA \quad \therefore \nu_{3} \mathrm{P} 16 \quad \mathrm{CO}_{2}$ absorption line found in the reflected solar spectrum (produced by Venusian atmospheric $\mathrm{CO}_{2}$ above the reflecting layer). The Fraunhofer $6717 \AA \mathrm{Ca}$ line was also used. The differential Doppler shifts observed in $1 \mathrm{imb} /$ terminator observational pairs, aligned equatorially near maximum elongation, yielded the zonal wind velocity of the cloud decks (Traub and Carlton 1979).

IRHS detection of Doppler shifts in Venusian atmospheric $10 \mu \mathrm{m}$ ${ }^{13} \mathrm{C}^{16} \mathrm{O}_{2}$ and ${ }^{12} \mathrm{C}^{16} \mathrm{O}_{2}$ lines (Betz et al. 1976, 1977b; Betz 1977, 1982) allowed determination of beam-integrated line of sight wind velocities at various locations on the planet. These velocities, containing line 
of sight Venus rotation (negligible) and wind components, provided both horizontal and vertical wind velocity information. Meridional circulation was poorly investigated. IRHS mating to the $81 \mathrm{~cm}$ McMath Auxiliary Telescope, Kitt Peak, Arizona, provided a 3-4 arc-second (HPBW) diffraction-limited beam resulting in seeing-excluded planetary disk-to-beam ratios of 2.5-14.5 for the observed Venusian phases.

The ${ }^{12} \mathrm{C}^{16} \mathrm{O}_{2}$ lines, seen in emission, have been modelled as non-LTE near-IR solar pumped emission (Johnson et al. 1976, Deming and Mumma 1983) centered at $109 \mathrm{~km}$ altitude (Deming and Mumma 1983).

These lines, with a solar zenith angle dependent emission intensity, serve only as probes of the illuminated hemisphere.

The ${ }^{13} \mathrm{C}^{16} \mathrm{O}_{2}$ lines, seen in absorption against the planetary cloud-top $-10 \mu \mathrm{m}$ continuum, have a weighting function peak near $-75 \mathrm{~km}$ altitude (Betz 1977). Formed planet-wide (unlike spectral features used in previous investigations), they serve as spatially unrestricted probes of atmospheric parameters and dynamics in the extended line-forming region above the cloud tops.

To date, the early IR heterodyne spectroscopic measurements and the PV OCPP polarimetry represent the only wind velocity determinations above the clouds.

An overview of system requirements for ground-based $10 \mu \mathrm{m}$ spectroscopic wind measurement, and how they are met by IR heterodyne spectroscopy, is now presented.

1.3. INFRARED HETERODYNE SPECTROSCOPIC WIND VELOCITY DETERMINATION The optically thin $-10 \mu \mathrm{m}{ }^{12} \mathrm{C}^{16} \mathrm{O}_{2}$ non-thermal emission, contained 
in an atmospheric layer centered at $5 \mu \mathrm{b}(109 \mathrm{~km}$ with a $-10 \mathrm{~km}$ HWHM;

Deming and Mumma 1983), is used in the present work as a spectral probe of Venusian lower thermospheric dynamics. $A{ }^{12} \mathrm{C}^{16} \mathrm{O}_{2}$ pressure broadening coefficient of $7.5 \mathrm{MHz} /$ Torr (FWHM) and a temperature near $230 \mathrm{~K}$ insures a thermal line profile with a FWHM of $\Delta \nu=49 \mathrm{MHz}$. A spectral resolving power $\lambda / \Delta \lambda=1.2 \times 10^{6}$, independent of wavelength, is therefore required.

IR heterodyne spectroscopy (IRHS) provides the only avenue to such Doppler-limited spectral resolution. The NASA/GSFC spectrometers sample the 9-12 $\mu \mathrm{m}$ band in either of two modes, low resolution (25 $\mathrm{MHz}$ channels) or high resolution ( $5 \mathrm{MHz}$ channels), yielding $\lambda / \Delta \lambda$ of $1.2 \times 10^{6}$ and $6 \times 10^{6}$, respectively.

While wind measurement precision depends on the spectral resolving power, wind measurement accuracy depends on the ability to determine the line centroid absolute frequency. To obtain Doppler velocities at the $1 \mathrm{~m} / \mathrm{s}$ level from $\sim 10 \mu \mathrm{m}$ spectral features, absolute frequency centroiding to $0.1 \mathrm{MHz}$, or one part in $3 \times 10^{8}$, is necessary.

The GSFC spectrometers photomix the source spectrum and a continuous-wave $\mathrm{CO}_{2}$ laser local oscillator, the latter nearly frequency coincident with the $10 \mu \mathrm{m}$ spectral feature under investigation (within $1.6 \mathrm{GHz}$; see Kostiuk and Mumma 1983). Photomixing produces a double-sideband difference spectrum which contains a radio band "image" of the spectral feature. The difference spectrum is detected in the $0-1.6 \mathrm{GHz}$ effective bandpass of a HgCdTe detector whose output is fed to a contiguous RF filter bank for digitization at sub-Doppler resolution. After correcting for terrestrial absorption, 
system-induced offsets, and a differential Doppler shift over the integration period, fitting a Gaussian to the digitized radio image allows $\mathrm{S} / \mathrm{N}-1$ imited centroid frequency determination in the radio. An integration time between 8 and 40 minutes yields radio image centroiding to $2-5 \mathrm{~m} / \mathrm{s}$, depending on the beam position's solar zenith angle. Adding the laser frequency to the radio image centroid frequency yields the absolute frequency of the $10 \mu \mathrm{m}$ spectral feature. The laser frequency must be known to better than $\pm 0.1 \mathrm{MHz}$ to obtain absolute Doppler velocities to $\pm 1 \mathrm{~m} / \mathrm{s}$.

While $\mathrm{CO}_{2}$ laser transition frequencies are known to $0.05-0.1 \mathrm{MHz}$ (Freed et al. 1980), the laser will maintain gain, hence significant output, over much of the $-170 \mathrm{MHz}$ (FWHM) range in the laser gain profile. The laser's instantaneous operating position on the gain profile is dictated by laser cavity length. Length variation of just 1 $\mu \mathrm{m}$, due to, e.g., cavity thermal expansion/contraction, introduces a "pseudo wind" of $240 \mathrm{~m} / \mathrm{s}$. The need for laser frequency stabilization is thus clear.

The early heterodyne wind measurements (Betz 1977), made with a passive feedback system, did not provide laser stabilization to an absolute frequency standard, e.g., the laser transition rest frequency. The derived winds were associated with what appears to be an unpredictable systematic offset at the tens of $\mathrm{m} / \mathrm{s}$ level (see Chapter 8), consistent with a lack of spectrometer absolute frequency calibration. For the present investigation, an active feedback system was developed utilizing the saturated resonance (Lamb-dip) technique (Freed and Javan 1970), allowing stabilization to the laser transition 
rest frequency. Stability tests indicated an undetectable laser frequency offset from the transition rest frequency and rms fluctuations at the test's detection threshold. Absolute Doppler velocities at the $\pm 1 \mathrm{~m} / \mathrm{s}$ level were therefore achieved.

IRHS is a coherent detection process dictating a diffractionlimited field of view (FOV). When properly mated to the telescope, the spectrometer FOV is the telescope's diffraction limit. Spectrometer mating to the 1.52 meter McMath Solar Telescope, Kitt Peak, Arizona, and the 3 meter NASA Infrared Telescope Facility instrument (IRTF), Mauna Kea, Hawai, provides diffraction-limited beams of 1.7 and 0.9 arc-seconds (HPBW), respectively. Over the Venusian phase cycle, planetary disk size varies from 10 to 60 arc-seconds (Espenak 1987) which allows a seeing-excluded disk-to-beam ratio up to 65 .

The frequency shift between the ${ }^{12} \mathrm{C}^{16} \mathrm{O}_{2} 9-11 \mu \mathrm{m}$ band spectral feature formed in the target planetary atmosphere, and the transition's rest frequency, provides the target planet/Earth station Doppler velocity. Often the atmospheric transition of interest and the laser transition are the same - the line position in the spectrometer bandpass thus dictated solely by the Doppler velocity. The bandpass, corresponding to a $16 \mathrm{~km} / \mathrm{s}$ range in Doppler velocity, allows detection of such a line regardless of Venus phase (the Venus/Earth relative velocity is always $< \pm 14 \mathrm{~km} / \mathrm{s}$; Espenak 1986). Observing periods are chosen, however, which center the line in the bandpass, yielding optimum line-fitting. Accurate ephemerides allow removal of the Earth/target-planet rotation and barycentric translation components to $<1 \mathrm{~m} / \mathrm{s}$. The residual Doppler shift identifies the line of sight wind 
velocity of the planetary atmospheric parcel within the beam.

The 0.9-1.7 arc-second diffraction-limited beam, the $S / N$ achievable on Venus, and use of the Lamb-dip stabilization system, allowed production of high spatial resolution line of sight wind field maps, at four phases spanning 828 of a synodic period, with $2-5 \mathrm{~m} / \mathrm{s}$ absolute velocity resolution. Knowledge of the Earth/Venus/Sun aspect geometry allowed reconstruction of the planetary-scale wind field in the lower thermosphere centered at $110 \mathrm{~km}$ altitude. 


\section{CHAPTER 2: VENUSIAN ATMOSPHERIC CIRCULATION}

A comprehensive overview of Venusian atmospheric dynamics is presented below, providing a framework within which the present heterodyne results may be assessed and integrated with other dynamical indicators to form a self-consistent model of Venusian circulation.

\subsection{OVERVIEW OF CIRCULATION IN THE VENUSIAN TROPOSPHERE/MESOSPHERE} (SURFACE TO $100 \mathrm{KM}$ )

\subsubsection{Gross Circulation Below the Cloud Tops}

High velocity winds (relative to the solid planet) are not unique to Venus. Earth's subtropical jet streams (at $-40,000$ feet), embedded in the deep mid-latitude westerlies, can attain speeds exceeding 160 m/s (350 mph; Considine 1976, Hess 1959, Prinn and Fegley 1987). Yet Earth's easterly tradewinds in the tropics represent a large reservoir of oppositely-directed angular momentum (Hess 1959). The angular momenta carried by Earth's opposing winds tend to cancel - the atmospheric total only a few percent in excess of that expected for an atmosphere co-rotating with the surface (Schubert 1983, Hide et al. 1980, Prinn and Fegley 1987). On Venus, however, the atmosphere between 10 and $70 \mathrm{~km}$, en masse, is super-rotating (rotating faster than the co-rotating frame), with the excess atmospheric angular momentum a factor of ten higher than expected for co-rotation (Schubert 1983).

Furthermore - maximal angular momentum for a co-rotating atmosphere is found in the equatorial region. Assuming conservation of angular momentum, transport of an equatorial parcel to higher latitudes will produce an angular momentum excess at the parcel's new location, 
inducing super-rotation. Consistent with this view, higher latitudes on Earth, in general, are indeed super-rotating while the equatorial region is actually sub-rotating (Hess 1959, Schubert 1983). On Venus however, a strongly super-rotating equatorial region defies explanation - what is the source of its large excess angular momentum?

From Venera (Kerzhanovich and Marov 1983, Kerzhanovich and Limaye 1985) and Pioneer Venus (PV) (Counselman et al. 1980) descent probe tracking, the Venusian retrograde zonal wind speed is known to increase from near 0 at $10 \mathrm{~km}$ altitude to $-100 \mathrm{~m} / \mathrm{s}(220 \mathrm{mph})$ at the $-60 \mathrm{~km} \mathrm{level}$, consistent with a $4.3^{\mathrm{d}}$ periodicity. The angular momentum density is maximal at $-20 \mathrm{~km}$ altitude where the characteristic super-rotation period is $-12^{\mathrm{d}}$ (Schubert 1983). IR imaging at $1.5-2.5 \mu \mathrm{m}$ indicates a period of $5.4 \pm 0.1^{d}$ (Allen and Crawford 1984), the longward wavelengths probing down to clouds at $-40 \mathrm{~km}$ altitude (Allen 1987).

\subsubsection{Longitudinal (Diurnal) Variation}

Probes entering close to the subsolar meridian (Veneras 9, 10 and 12) indicated only $-50 \mathrm{~m} / \mathrm{s}$ winds at $-60 \mathrm{~km}$ (Schubert 1983, Kerzhanovich and Marov 1983) which imply, together with both Earth and spacecraft based UV imaging (Boyer 1973; Limaye and Suomi 1981, Limaye et al. 1982) and spectroscopic results (Traub and Carleton 1975), wind deceleration prior to and acceleration after local noon. While the Vega 1 and 2 balloon-borne equatorial investigations $\left(+8.1^{\circ}\right.$ and $-7.5^{\circ}$ latitude) were well suited for detection of longitudinal (diurnal) variation in zonal wind speed, they never entered the subsolar meridian region. Atmospheric insertion of both craft near local 
midnight, with 46 hours of continuous operation at the $53-54 \mathrm{~km}$ float altitude, provided $30 \%$ circumferential coverage before transmission termination at solar zenith angles of $-60^{\circ}$ and $-55^{\circ}$ for Vega 1 and 2 , respectively (Sagdeev et al. 1986). Assuming a constant velocity zonal wind field, the observed balloon radial velocities were consistent with $69.3 \pm 1 \mathrm{~m} / \mathrm{s}$ (Vega 1) and $66.4 \pm 1 \mathrm{~m} / \mathrm{s}$ (Vega 2) mean zonal wind speeds, $5-10 \mathrm{~m} / \mathrm{s}$ faster than previous probe results for float altitude (Andreev et al. 1986, Sagdeev et al. 1986). Deviations from constant zonal flow were $<4 \mathrm{~m} / \mathrm{s}$ except for a Vega $2 \sim 60 \mathrm{~m} / \mathrm{s}$ anomaly over Aphrodite Terra (Preston et al. 1986). During the anomaly, the balloon's vertical velocity never exceeded $2 \mathrm{~m} / \mathrm{s}$. Both craft (though deployed 4 days apart) indicated a sinusoidal spatial variation in zonal wind speed with a $3 \mathrm{~m} / \mathrm{s}$ peak near $3^{\mathrm{h}} 30^{\mathrm{m}}$ local time, possibly indicative of a thermal tide (Preston et al. 1986, Blamont et al. 1986). Solar-fixed wavenumber- 2 structure observed by the PV Orbiter IR Radiometer (OIR) at $65-100 \mathrm{~km}$ altitude has been identified as a thermal tide (Schofield and Taylor 1983, Taylor et. al 1980). (Note that OIR performed the Venus Orbiter Radiometric Temperature Experiment or VORTEX.)

\subsubsection{Latitudinal Variation}

Regarding latitudinal variation, Mariner $10 \mathrm{UV}$ imaging of the cloud tops indicated a mid-latitude super-rotation considerably faster than expected for cloud-top solid body super-rotation at the equatorial rate (Limaye and Suomi 1981, Travis 1978). The enhanced mid-latitude super-rotation culminated in strong jets (110 m/s) asymmetrically located with respect to the equator at $+35^{\circ}$ and $-50^{\circ}$ latitude (Schubert 
1983).

The in situ wind profiles derived from the four PV entry probes (entry latitiudes $+4.4^{\circ},+59.3^{\circ},-31.7^{\circ}$ and $-28.7^{\circ} ; \operatorname{Col}$ in 1983) provided limited latitudinal contrast with altitude. All four probes were far enough from the noon meridian so as not to reflect the strong near-noon longitudinal variation in the zonal wind. One can envision the atmosphere below the clouds as a series of concentric atmospheric shells each undergoing solid body super-rotation at a rate set by the PV Large probe entering near the equator at $+4.4^{\circ}$. The Day and Night probes, entering near $-30^{\circ}$, indicated such solid body super-rotation from $20-40 \mathrm{~km}$ altitude (Schubert 1983). Above $40 \mathrm{~km}$ there was an observed excess super-rotation. The North probe entering near $+60^{\circ}$ indicated solid body super-rotation no higher than $20 \mathrm{~km}$. At $55 \mathrm{~km}$ the super-rotation was twice as great as expected for the atmospheric solid body case, consistent with mid-latitude jets. The Venera 8 descent probe may have detected a jet at $50 \mathrm{~km}$ altitude, $10^{\circ} \mathrm{S}$ latitude (Limaye 1985).

In contrast, PV Orbiter Cloud Photopolarimeter (OCPP) UV imaging, two months after the entry probe measurements (Schubert 1983), indicated nearly solid body cloud top super-rotation between $\pm 60^{\circ}$ latitude (the latitudinal limits for both Mariner 10 and PV UV imaging investigations; Rossow et al. 1980).

\subsubsection{Cyclostrophic Balance}

For latitudes greater than $\pm 30^{\circ}$, the observed wind profiles with altitude are consistent with circulation models invoking cyclostrophic 
balance (Seiff et al. 1980, Schubert et al. 1980, Schubert 1983). Such a condition, first proposed by Leovy (1973), implies that a zonally circulating atmospheric parcel experiences a balance between the pressure gradient force directed poleward (assuming 1ittle or no longitudinal temperature gradient, consistent with observation) and the equatorward component of centrifugal force. Unlike the Earth, Coriolis forces are relatively unimportant on Venus due to the slow Venusian solid planet rotation. Earth large-scale circulation, e.g., the mid-latitude westerlies and tropical easterlies, operate in a quite different dynamical regime, experiencing quasi-geostrophic balance, whereby the poleward pressure gradient force is balanced by the Coriolis force (Prinn and Fegley 1987).

Coupling cyclostrophic balance with hydrostatic equilibrium yields the Thermal Wind Equation, dictating an increasing zonal wind speed with altitude in the presence of a poleward decreasing latitudinal temperature gradient (Schubert 1983). Assuming cyclostrophic balance, with a wind profile anchored by the low altitude wind measurements, and the latitudinal temperature gradient as a function of altitude obtained from the PV Orbiter Radio Occultation (ORO) (Newman et al. 1984) and VORTEX (Taylor et al. 1980) experiments, the wind profile can be extrapolated to higher altitudes. The result: above $60 \mathrm{~km}$ the wind speed continues to increase, reaching a maximum of $-130 \mathrm{~m} / \mathrm{s}$ (290 $\mathrm{mph}$ ) near $70 \mathrm{~km}$ where it begins a steady decrease to possibly insignificant levels by $90-100 \mathrm{~km}$ (Seiff et al. 1980, Seiff 1982, Schubert 1983). Newman et al. (1984) obtained pronounced, possibly meandering $\left( \pm 50-70^{\circ}\right.$ latitude) jets at the cloud-tops and above, consistent with observation 
$(-150 \mathrm{~m} / \mathrm{s}$ at $31.7 \mathrm{mb},-70 \mathrm{~km} ;-100 \mathrm{~m} / \mathrm{s}$ at $135 \mathrm{mb},-63 \mathrm{~km})$, using a cyclostrophic balance model and an ORO-derived temperature field. Employing a larger ORO database, Walterscheid et al. (1985) obtained similar jet characteristics.

It is important to note an apparent contradiction: while the PV ORO data, assuming cyclostrophic balance, implies mid-latitude jets (as does the limited PV entry probe results and Mariner $10 \mathrm{UV}$ imaging), PV OCPP images indicated near solid body super-rotation of the cloud tops. (PV OCPP also suggests $-65 \pm 25 \mathrm{~m} / \mathrm{s}$ super-rotation of the upper haze layer at $5 \mathrm{mb}(-80 \mathrm{~km}$; Limaye 1984)).

The $-90 \mathrm{~m} / \mathrm{s}$ zonal super-rotation indicated by the ground-based IR heterodyne ${ }^{13} \mathrm{C}^{16} \mathrm{O}_{2}$ absorption line observations (Betz et al. 1977b; Betz 1977, 1982; see Chapter 1), at $75-85 \mathrm{~km}$ altitude, is consistent with cyclostrophic balance. However, large heterodyne-derived vertical wind velocities $(-45 \mathrm{~m} / \mathrm{s}$ upwelling; $40 \mathrm{~m} / \mathrm{s}$ fluctuations) may be indicative of large systematic errors (see Chapter 8). Vega 1 and 2 observed vertical velocities typically $\sim 0.5 \mathrm{~m} / \mathrm{s}$ and maximally $2.5-3.5$ $\mathrm{m} / \mathrm{s}$, with Vega 2 experiencing large and prolonged vertical velocities over Aphrodite Terra's $5 \mathrm{~km}$ altitude terrain (Blamont et al. 1986). Mixing length theory applied to the convective zone in the middle cloud layer (Vega 1 and 2 float altitudes were situated at the top of this zone) yields expected vertical velocities of $\sim 1 \mathrm{~m} / \mathrm{s}$ (Blamont et al. 1986). Venera results point to turbulence-induced vertical velocities $<3 \mathrm{~m} / \mathrm{s}$ (Kerzhanovich and Marov 1983). The heterodyne derived vertical velocities thus seem dynamically unreasonable ( $\mathrm{Clancy}$ and Muhleman 1985b; see Chapter 8). 
Ground-based ${ }^{12} \mathrm{C}^{16} \mathrm{O}$ microwave observations $(-80-90 \mathrm{~km}$ alt.; Clancy and Muhleman $1985 \mathrm{a}, \mathrm{b})$, indicating an $8.5^{\mathrm{h}}$ Local Venusian Time maximum, have been interpreted as resulting from superposition of -50 $\mathrm{m} / \mathrm{s}$ zonal super-rotation ( $90 \mathrm{~km}$ upper cutoff alt.) and a subsolar-toantisolar flow (penetrating down to the $80 \mathrm{~km}$ level and characterized by $4 \mathrm{~cm} / \mathrm{s}$ vertical wind speeds).

\subsubsection{Mean Meridional (Hadley) Circulation}

In the troposphere and mesosphere ( $<100 \mathrm{~km} \mathrm{alt.)} \mathrm{sma11} \mathrm{diurnal}$ temperature gradients (see Fig. P.1) imply axisymmetric circulation with respect to the spin axis. Besides the zonal flow, reasonably consistent with this view, latitudinal temperature gradients should drive a mean meridional circulation. Mariner 10 (Limaye and Suomi 1981) and PV OCPP (Limaye et a1. 1982, Rossow et al. 1980) UV imaging as well as PV entry probe Differential Very Long Baseline Interferometric (DVLBI) tracking (Counselman et al. 1980) indicate a cloud-level meridional circulation with rising air near the equator, poleward transport at the cloud tops $(65-70 \mathrm{~km})$, sinking at the poles and equatorward return flow near the base of the clouds (see Limaye 1985). This is consistent with the poleward decrease in temperature observed by ORO (Newman et al. 1984) and VORTEX (Taylor et al. 1980) at cloud leve1. DVLBI-derived mean meridional wind speeds were typically a few $\mathrm{m} / \mathrm{s}$ and maximally $10 \mathrm{~m} / \mathrm{s}$ (Limaye 1985).

While Mariner 10 and PV OCPP imaging have identified a global cell in each hemisphere, they seem, unexpectedly, to be located asymmetrically about the equator with the northern hemisphere cell 
extending down to $-25^{\circ} \mathrm{S}$ latitude (Schubert 1983).

The limited spatial and temporal coverage afforded by PV DVLBI entry probe tracking cannot be expected to discern properties of the steady-state meridional circulation, yet many probes allude to a stacking of Hadley cells below cloud level (Limaye 1985, Schubert 1983). An indirect cell (transport from low to high temperature regions) directly beneath, and apparently driven by the cloud-level cell seems indicated. A surface cell has been postulated on theoretical grounds (Schubert et al. 1980, Rossow 1982).

Above the clouds, dynamical models based on PV VORTEX-derived thermal wind fields have suggested a direct cell (Taylor et al. 1980, Limaye 1985) consistent with the observed poleward increase in temperature in the $75.95 \mathrm{~km}$ regime (Taylor et al. 1980, Schofield and Taylor 1983). Meridional velocities in the equatorward upper branch of the cell may attain speeds of $-120 \mathrm{~m} / \mathrm{s}$ at the $90-100 \mathrm{~km}$ altitude level (Taylor et al. 1980, Elson 1979, Kerzhanovich and Limaye 1985).

\subsubsection{Maintaining the Zonal Super-Rotation}

The mystery of the Venusian zonal super-rotation lies not so much in its dynamical characterization as in its maintenance. Assuming the present situation is steady state, two fundamental but not necessarily independent problems still persist.

Problem 1 - there remains no definitive picture as to how en masse atmospheric super-rotation is maintained against expected drag forces from the underlying slowly rotating solid planet. Either the atmosphere is essentially decoupled from the solid planet, or angular 
momentum must be pumped into the atmosphere. The latter might proceed through the action of solar torques acting on atmospheric thermal tides (Gold and Soter 1971, Dobrovolskis and Ingersoll 1980), or more subtly, from below, through some interaction with the Venusian surface (Covey et a1. 1986, Schubert 1983).

Problem 2 - below the clouds, the observed angular momentum distribution per unit mass (increasing equatorward and with altitude), under the action of the observed cloud level Hadley cell, should result in depletion of equatorial angular momentum. As this is not observed, other processes not yet understood must be operating.

The following discussion provides a cursory overview of theories concerning the origin and maintenance of Venusian atmospheric super-rotation.

Solar torques acting on the solid planet tidal bulge remove retrograde angular momentum, which should result in a $1: 1$ spin-orbit lock in $10^{8}$ years (Dobrovolskis and Ingersoll 1980). As such has not occurred, an additional torque on the solid planet seems necessary. A possible solar torquing of the atmospheric thermal tide may act to both balance the torque on the solid body tide (through drag) and pump atmospheric super-rotation. Additionally, a near resonance between the Venusian rotation period (with respect to the Venus/Earth line) and the Venusian synodic period implies Earth-induced torques might also be involved.

Super-rotation from solar torques on the atmospheric semi-diurnal thermal tide would require temperature minima just prior to local noon 
and midnight (temperature maxima just after local noon and midnight). At the cloud tops, ground-based observations (Ainsworth and Herman 1978, Apt and Goody 1979, Apt et al. 1980) and PV VORTEX results (Taylor et al. 1980, Schofield and Taylor 1983) indicate just the reverse $\left(\mathrm{T}_{\min }-3 \mathrm{AM}, \mathrm{T}_{\max } \sim 9 \mathrm{PM} ; \mathrm{T}_{\max }\right.$ prior to noon and midnight, respectively), implying tidal-induced reduction of retrograde angular momentum (see Schubert 1983). (Interestingly, a thermal tide eastward phase tilt with altitude, evident in the VORTEX results $(65-100 \mathrm{~km}$ altitude; see Fig. 10a, Schofield and Taylor 1983), provides the requisite phase for tidal pumping of the super-rotation by $85 \mathrm{~km}$ altitude.)

Pumping of the super-rotation from below, through interaction with the solid planet, might be ongoing, or may have been sequential, e.g., early atmospheric spin-up and corresponding solid planet spin-down followed by essentially a decoupling of the two angular momentum reservoirs (see Covey et al. 1986, Schubert 1983). Clearly, if atmosphere and solid planet are now decoupled, barring effects of solar gravitational torquing, all mechanisms operating in the atmosphere serve only to redistribute atmospheric angular momentum. Eddies, mean meridional circulation and planetary-scale waves (PSW's) prove theoretically viable as mechanisms for angular momentum redistribution in both latitude and altitude (Schubert 1983).

As proposed by Gierasch (1975), the mean meridional (Hadley) circulation could transport retrograde angular momentum aloft in the equatorial region followed by a poleward transport at cloud levels. However, the resulting depletion of equatorial angular momentum, and 
the inability of the meridional circulation to replenish it, warranted an equatorward angular momentum transport mechanism. Gierasch (1975) thought eddies might be responsible. Rossow and Williams (1979) showed the requisite eddies could be generated through barotropic instability. Covey et al. (1986) employed a high spatial resolution General Circulation Model to test this theory. The conclusion: the mechanism might maintain, but cannot establish, a strongly super-rotating atmosphere. While PV OCPP imaging indicates poleward transport of angular momentum by the poleward branch of the cloud level Hadley cell, there is no evidence of equatorward transport by eddies (Limaye and Suomi 1981, Limaye et al. 1982, Limaye 1985).

The identification of mid-latitude jets in Mariner 10 images and their apparent absence in PV OCPP images seems to indicate vacillating circulation over time. Rossow et al. (1980) have suggested the semi-permanence of the mid-latitude jets at the cloud tops results from sequential involvement of the mean meridional circulation and eddies. The poleward transport effected by the meridional circulation results in mid-latitude buildup of angular momentum, producing jets consistent with Mariner 10 imaging. Eddies eventually develop through barotropic instability, causing angular momentum redistribution latitudinally, providing the near solid body cloud top super-rotation observed by PV OCPP imaging.

Limaye (1985) offers an alternative view - that temporal changes in cloud top circulation implied by Mariner 10 and PV OCPP imaging are not real. Both investigations may have been probing different altitudes due to temporal variation in the altitude of the cloud tops. The fact 
that mid-latitude jets are predicted by cyclostrophic balance models using PV ORO data supports this contention (Newman et al. 1984). The circulation may in fact have long term temporal stability. During the PV nominal mission, evidence for aerosol loading above the clouds (Limaye 1985, Deming et al. 1982) implies OCPP probing at cloud top altitudes slightly higher than Mariner 10, particularly in the midlatitudes (Limaye 1985). If the jet core lies below the cloud tops (whose altitude remains poorly established) both imaging investigations become consistent with temporally stable circulation (Limaye 1985). Also consistent with this view is the departure from solid body atmospheric super-rotation (at high latitudes) indicated by the PV entry probes at relatively low $(-55 \mathrm{~km})$ altitudes (Schubert 1983).

A variety of classes of planetary-scale waves (PSW's) have been identified in the Venusian atmosphere. Possible means of PSW generation include solar thermal forcing, convection (known to be important within the cloud decks and probably near the surface) and large-scale topographic relief (Prinn and Fegley 1987).

Some examples: The $Y$, reverse $C$ and "psi" global features apparent in Mariner 10 (Belton et al. 1976a,b) and PV (Rossow et al. 1980) UV imaging (as well as ground-based images) are three common patterns resulting from the action of internal gravity waves (Schubert 1983). The Vega 2 zonal and vertical wind speed anomalies over Aphrodite Terra are consistent with topographic forcing of gravity waves (Andreev et a1. 1986, Blamont et al. 1986, Preston et al. 1986). Vega long term sinusoidal variation in zonal wind speed (Preston et al. 1986), and the already mentioned ground-based and PV VORTEX derived radiometric 
fields, indicate the presence of a semidiurnal thermal tide - a solar forced, vertically propagating gravity wave.

Fels and Lindzen (1974) showed the thermal tide can be an important mechanism for the redistribution of angular momentum, though no observational evidence exists supporting this contention (Schubert 1983). The interaction between the thermal tide and the mean meridional circulation has been investigated by, e.g., Young and Pollack (1977), Williams and Holloway (1982), Hou and Goody (1985), and most recently, Baker and Leovy (1987). In the Baker and Leovy (1987) model (assuming an initial altitude-dependent solid-body zonal super-rotation) the resulting steady state zonal velocity variation with altitude and latitude reasonably matches the dynamical observables such as jet strength and location, mean zonal speed, thermal tide amplitude, and vertical wavelength.

\subsection{DYNAMICAL MODELS OF THE VENUSIAN THERMOSPHERE/CRYOSPHERE}

\subsubsection{Early Circulation Models}

An early solar-driven circulation model was proposed by Edmund Halley (1686) as an explanation of Earth's easterly trade winds. Halley compiled a (largely) mariners' account of the global wind field within the tropics, which indicated predominantly northeasterly and southeasterly winds north and south of the equator, respectively. Halley's model - a solar insolation-induced pressure gradient, coupled with the Sun's westward diurnal motion and annual variation in declination, produced the observed easterlies. Halley in fact considered the easterlies as a low-level return branch to what would 
be a subsolar-to-antisolar (SS-AS) circulation in the Sun-fixed frame. SS-AS circulation has been characterized in the literature as "Halley circulation" (see Covey et a1. 1986).

It was apparently Percival Lowell (1896a) who first proposed a SS-AS circulation model for Venus. SS-AS circulation naturally followed from Lowell's belief that Venus was a solar-synchronous rotator (first proposed by Schiaparelli (1890, 1895); see Appendices 3 and 4). Lowell's circulation, shown on Figure 2.1 (Lowel1 1910), is consistent with the thermosphere/cryosphere temperature gradient observed above $100 \mathrm{~km}$ altitude (see Fig. P.1).

After half a century of conflicting photographic, spectroscopic and photometric determinations of the Venusian rotation period (see Appendix 3), the radar discovery of a slow retrograde solid planet rotation $\left(243^{\mathrm{d}}\right)$, in $1961-67$, helped bring renewed interest to SS-AS circulation models. Some notable examples: Mintz (1961) proposed a low-level SS-AS circulation, comprised of multiple convection cells, as an explanation of visual observations made by Dollfus (1953, 1955 , 1961) at Pic-du-Midi (see Appendices 3 and 4). Mintz (1962) further calculated SS-AS horizontal velocity as a function of surface temperature and pressure, and temperature lapse rate, obtaining 0.08 to $180 \mathrm{~m} / \mathrm{s}$. Mahoney (1962) modelled a SS-AS cell with a direct branch above $10 \mathrm{~km}$ altitude and $35 \mathrm{~m} / \mathrm{s}$ maximum velocities. Sagan and Kellogg (1963) considered a dust composition for the Venusian clouds and proposed SS-AS circulation as a means of maintaining dust aloft. Finally, Goody and Robinson (1966) forwarded a SS-AS model to explain $-600 \mathrm{~K}$ temperatures observed at $\lambda=2$ to $20 \mathrm{~cm}$, assuming emission from 


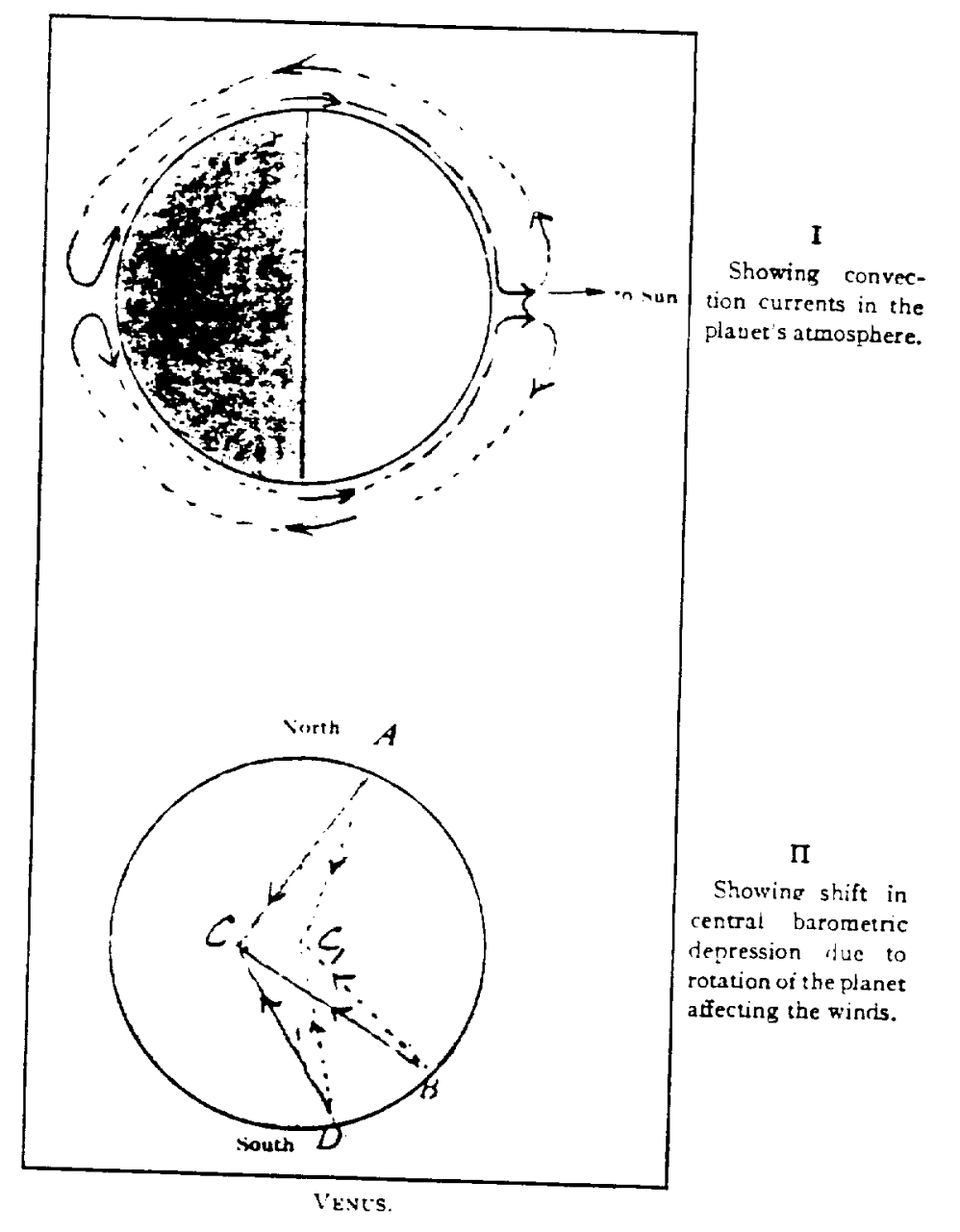

Figure 2.1. Subsolar-to-Antisolar Circulation Lowell 1896

ORIGINAL PACE IS OF POOR QUALITY 
the base of the clouds and, contrary to Greenhouse theories, an atmosphere totally opaque to both solar and IR radiation.

\subsubsection{Dynamical Models}

Any hydrodynamical model for the thermosphere/cryosphere must reproduce the associated thermal, pressure, (specie) density, and wind fields. Only observational properties of the first three are known in any detail to date.

Prior to the Pioneer Venus (PV) encounter, Dickinson and Ridley (1977) formulated a 2-D hydrodynamical model of the thermosphere, hereafter DRM, assuming a symmetric subsolar-to-antisolar circulation (independent variables: SZA and altitude). DRM assumed a three component atmosphere consisting of $\mathrm{CO}_{2}$ and its photodissociation products $C O$ and 0 . The model was fully self-consistent, and coupled photochemistry with dynamics, allowing feedback between the thermal, (specie) density, and wind fields from -95 to $200 \mathrm{~km}$ altitude $(0.1$ mbar to $\left.5 \times 10^{-6} \mu \mathrm{bar}\right)$. A global thermal balance was maintained between solar EUV, UV and near-IR heating and thermal IR cooling, the latter dominated by $\mathrm{CO}_{2}$ collisional excitation by $\mathrm{O}$ with subsequent $15 \mu \mathrm{m}$ radiative cooling. (A 1 -D thermal model by Dickinson (1976) established the mechanisms and relative involvements for these thermal processes. The resulting mean global thermal profile was used by DRM to generate near-IR heating and $15 \mu \mathrm{m}$ cooling rates globally.) The local thermal balance also included effects of molecular conduction and large-scale hydrodynamical transport. Small-scale eddy mixing was neglected as its effects lacked a reasonable quantitative description. 
Empirical model fits to early Pioneer Venus data (associated with solar maximum) revealed a significant number of DRM inconsistencies:

1. The globally averaged temperature above $100 \mathrm{~km}$ was cooler than predicted, by maximally $35 \mathrm{~K}$ (at the exobase near $150 \mathrm{~km}$;

Dickinson and Bougher 1986).

2. DRM predicted a warmer thermosphere than observed - at the exobase, the observed and predicted subsolar temperatures were $300 \mathrm{~K}$ and $335 \mathrm{~K}$, respectively (Dickinson and Bougher 1986).

3. DRM predicted a far warmer cryosphere than observed, brought on primarily by subsidence-induced adiabatic heating suffered by the strong cross-terminator winds. At the exobase, the maximum discrepancy was $100 \mathrm{~K}$ for $\mathrm{SZA}=\sim 150^{\circ}$ (Dickinson and Bougher 1986).

4. DRM predicted nightside enhancement (along isobars) of $O$ and CO mixing ratios ([O] and [CO]) resulting from dayside $\mathrm{CO}_{2}$ photodissociation followed by rapid global-scale transport across the terminator ( $[0] \equiv n_{0} / \sum_{n_{i}}$, where $n_{i}$ is species $i$ number density). At $5 \times 10^{-3} \mu$ bar $(-135 \mathrm{~km})$ predicted $[0]_{\mathrm{SZA}=180} /[0]_{\mathrm{SZA}=0}$ was $-10: 1$ (Dickinson and Ridley 1976). The PV-modelled distributions of [0] and [ $\mathrm{CO}$ ] (along isobars below $150 \mathrm{~km}$ ) indicated dayside enhancements (see, e.g., Dickinson and Bougher 1986).

5. Indirect evidence for retrograde zonal super-rotation above $150 \mathrm{~km}$ altitude $(-50-100 \mathrm{~m} / \mathrm{s}$ equatorial wind speed, e.g., see Mayr et al. 1980) included PV observations of: post-midnight maxima in neutral $\mathrm{H}$ density (Brinton et al. 1980), He density (Niemann et al. 1980), and UV airglow (Stewart and Barth 1979), 
and a post-noon maximum in temperature (Keating et al. 1980). Such asymmetries could not be modelled by a symmetric SS-AS flow (Bougher et al. 1986).

The DRM-associated wind field had the following characteristics:

1. Model horizontal velocities, maximal across the terminator, steadily increased with altitude reaching $-350 \mathrm{~m} / \mathrm{s}$ at the exobase $(-150 \mathrm{~km})$, assuming a low EUV heating efficiency of 10\%. A higher EUV heating efficiency ( $30 \%$ ) enhanced predicted horizontal velocities by -258 . At $-118 \mathrm{~km}$, the early-modelled altitude of peak $10 \mu \mathrm{m}{ }^{12} \mathrm{C}^{16} \mathrm{O}_{2}$ non-thermal emission (Johnson et al. 1976; see Section 2.3), DRM predicted $150 \mathrm{~m} / \mathrm{s}$ cross-terminator horizontal winds (low EUV case), reasonably consistent with the $130 \mathrm{~m} / \mathrm{s}$ reported by Betz et al. (1977b). More detailed modelling, however, has placed the non-thermal emission peak at $109 \mathrm{~km}$ (Deming and Mumma 1983) where DRM predicted only $75 \mathrm{~m} / \mathrm{s}$ cross-terminator velocities.

2. Model vertical velocities (assuming low EUV efficiency) were maximum on the terminator's nightward side, above $120 \mathrm{~km}$, due to subsidence caused by the dramatic cross-terminator pressure gradient. A $3 \mathrm{~m} / \mathrm{s}$ maximum occured at the exobase. Vertical velocities were doubled for the high EUV efficiency case.

A post Pioneer Venus 2-D hydrodynamical model $(95-210 \mathrm{~km})$ was developed by Bougher et al.(1986), henceforth BEAM, using essentially the same formalism as DRM. The new 1-D model of the mean global 
thermal field (Dickinson and Bougher 1986) incorporated Pioneer Venus based: solar EUV and UV fluxes, distributions of the DRM atmospheric species (including $\mathrm{N}_{2}$ ), and model atmospheres. The new EUV flux was twice Dickinson's (1976), inhibiting generation of a lower global mean temperature field. Conversely, $15 \mu \mathrm{m}$ cooling was enhanced due to [0] double the previous value (Dickinson 1976), and a 20 -fold increase in the rate constant for $\mathrm{O}-\mathrm{CO}_{2} \mathrm{~V}-\mathrm{T}$ collisions. To model $15 \mu \mathrm{m}$ cooling more accurately, a highly nonlinear function of temperature, the 1-D global mean model was partitioned into day and night models. Partial solar heating was provided on the nightside to simulate dynamical heating from, e.g., near-terminator subsidence induced adiabatic compression and thermal advection. Models were adjusted to reproduce the observed thermosphere/cryosphere diurnal variation. The improved $15 \mu \mathrm{m}$ cooling rate was obtained as a function of SZA by appropriate non-linear interpolation between the day and night profiles.

The DRM-predicted high temperature cryosphere, and nightside buildup of [0] and [CO], were circulation induced. BEAM relied on a weakened circulation to obtain larger dayside [0] and [CO], and a larger thermosphere/cryosphere temperature gradient. Circulation was attenuated through wave drag due to both eddy viscosity and Rayleigh friction. Wave drag was not included in a self-consistent manner due to a lack of coupling physics to wave phenomena in the Venusian atmosphere.

In addition, BEAM investigated effects of eddy diffusion, eddy conduction and varied solar EUV absorption efficiency. Compared to the large-scale circulation, eddy diffusion was found to play a minor 
role in determining composition fields. Effects of eddy conduction were found negligible. The BEAM final reference model, with 10 EUV absorption efficiency, yielded exospheric temperatures for the thermosphere and cryosphere of $304 \mathrm{~K}$ and $113 \mathrm{~K}$ respectively, in agreement with PV empirical models (Hedin et al. 1983). EUV efficiencies $>10-128$ could not be balanced by any known cooling mechanism, though values of 12-188 have been proposed (Fox and Dalgarno 1981, Hollenbach et a1. 1985).

Along the $7 \times 10^{-4} \mu$ bar isobar $\left(-150 \mathrm{~km}\right.$ at $\mathrm{SZA}=0^{\circ},-135 \mathrm{~km}$ at $\mathrm{SZA}=180^{\circ}$ ) the $[0]_{\mathrm{SZA}=0}$ predicted by the $\mathrm{PV}$ empirical model (Hedin et a1. 1983), BEAM, and DRM are: $\sim 0.3,-0.18$, and -0.09 ; and for $[0]_{S Z A=180}$ : $\sim 0.15,-0.20$, and -0.75 . BEAM's weakened circulation boosted dayside [0] by a factor of 2 and dramatically reduced the nightside bulge (DRM nightside [0] is five times greater than observed). The empirical model and BEAM-predicted $[\mathrm{CO}]_{\mathrm{SZA}=0}$ are: -0.17 , and -0.15 ; and for $[\mathrm{CO}]_{\mathrm{SZA}=180}$ are: -0.05 , and -0.045 - in excellent agreement. In general, BEAM values were within 30-508 of the PV empirical model.

Wave drag limited the BEAM wind field to maximum cross-terminator velocities of $-230 \mathrm{~m} / \mathrm{s}$ at the exobase, almost half the DRM prediction. BEAM-generated horizontal wind speed versus $S Z A$ at $110 \mathrm{~km}$ altitude, for solar maximum conditions, is given on Figure 2.2 (Bougher et al. 1986; Bougher 1987). The wind field is essentially symmetric about the vertical 1 ine $S Z A=-82^{\circ}$. For $S Z A<90^{\circ}$, the horizontal wind speed can be represented by a linear fit with 0 and $121 \mathrm{~m} / \mathrm{s}$ winds at the subsolar point and across the terminator, respectively (at $\mathrm{SZA}=90^{\circ}$ the linear fit departs from the BEAM-predicted $110 \mathrm{~m} / \mathrm{s}$ wind speed). BEAM-derived 


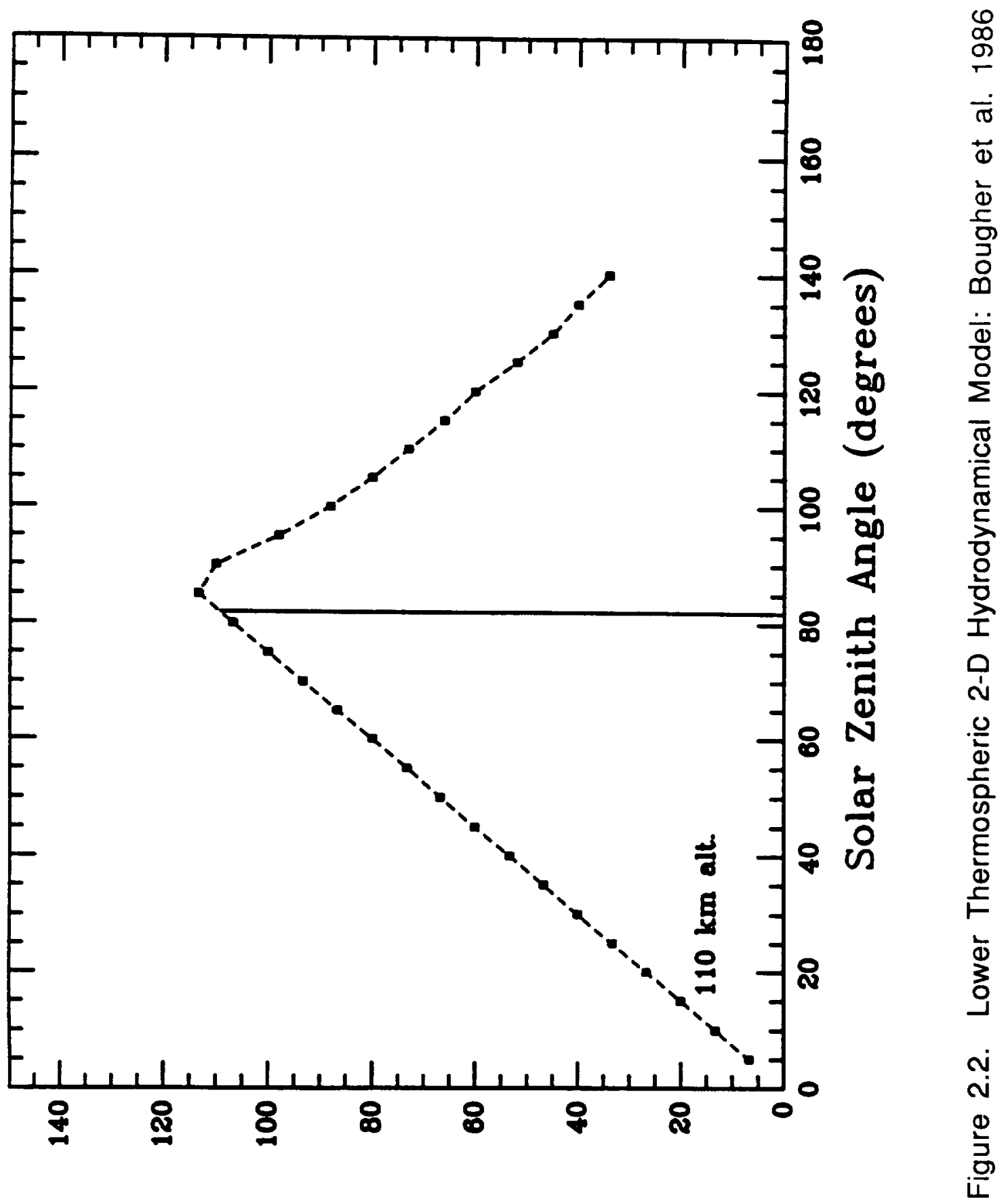

(s/w) pəəds puṭ [вұนozṭoH 
solar cycle variation of the wind field is negligible below $-120 \mathrm{~km}$.

BEAM (as DRM) could not address the observed composition, thermal, and airglow asymmetries above $150 \mathrm{~km}$. Mayr et al. (1980, 1985) employed models which included a wind field zonal component, yet were highly parameterized, thus not self-consistent. The Mayr et al. (1980) 2-D model employed Pioneer Venus-derived global mean composition and temperature fields and adopted DRM UV heating and photodissociation rate parameterizations. Treatment of IR heating and $15 \mu \mathrm{m}$ cooling rates were less detailed than in DRM. UV heating efficiency was equivalent to the DRM 108 case. Diurnal composition fields were determined by global circulation and vertical eddy diffusion. Specie densities, as functions of Venus Local Time, were calculated along the equator at 150 $\mathrm{km}$ altitude for varied zonal wind speeds and vertical eddy diffusion coefficients (taken constant with altitude). An eddy coefficient of $2.5 \times 10^{7} \mathrm{~cm}^{2} / \mathrm{sec}$ and a $40-80 \mathrm{~m} / \mathrm{s}$ zonal equatorial velocity yielded diurnal density variations in $\mathrm{CO}_{2}, \mathrm{O}$, $\mathrm{He}$, and $\mathrm{H}$, and diurnal thermal contrast, in reasonable agreement with the observed. The associated SS-AS cross-terminator winds were $200 \mathrm{~m} / \mathrm{s}$ at $150 \mathrm{~km}$ altitude - yet only $20 \mathrm{~m} / \mathrm{s}$ at $110 \mathrm{~km}$.

A 3-D model (Mayr et al. 1985), based on the 2-D formalism, also included DRM formulations for IR heating and $15 \mu \mathrm{m}$ cooling, and an altitude-dependent vertical eddy diffusion coefficient (inversely proportional to the square root of the density). The UV heating efficiency was $30 \%$. The best fit model composition and thermal fields yielded $300 \mathrm{~m} / \mathrm{s}$ SS-AS cross-terminator velocities at the exobase and a $75 \mathrm{~m} / \mathrm{s}$ (equatorial) solid body atmospheric super-rotation. SS-AS winds 
near the base of the thermosphere $(-110 \mathrm{~km})$ were not significantly enhanced over the 2-D predictions (Mayr 1989).

\section{3. $10 \mu \mathrm{m}$ NON-THERMAL EMISSION MODELLING}

The ${ }^{12} \mathrm{C}^{16} \mathrm{O}_{2}$ non-thermal core emission in the $10 \mu \mathrm{m} \sum_{\mathrm{u}}{ }^{+} 00^{\circ} 1$ $\sum_{\mathrm{g}}{ }^{+}\left[10^{\circ} 0,02^{\circ} 0\right]_{\mathrm{I}, \mathrm{II}}$ bands was first observed in the atmospheres of Mars and Venus by Betz et al. (1976), and modelled by Johnson et al. (1976). The line shapes were Gaussian with widths corresponding to temperatures of $-170 \mathrm{~K}$ and $-200 \mathrm{~K}$, respectively. Mars observations of the P12 through P28 rotational lines in the $10.4 \mu \mathrm{m}$ I-band indicated rotational temperatures consistent with the thermal widths. The emission intensities, however, were 5 to 15 times greater than expected for a blackbody at the associated temperatures.

A systematic study of emission strength versus beam position was undertaken on Venus near maximum western elongation with the subsolar point on the eastern limb (Johnson et al. 1976). Observations indicated that the emission was approximately constant along lines of constant solar insolation, increased dramatically near the subsolar limb, and was absent on the unilluminated hemisphere. Coupling these observations with an observed 6-fold enhanced Venusian emission intensity over Mars' led to the conclusion that the emission was solar pumped.

A host of mechanisms were rejected including: scattering of solar $10 \mu \mathrm{m}$ radiation, indirect pumping through solar XUV electronic excitation, and direct pumping of the $10 \mu \mathrm{m} \sum_{u}{ }^{+} 00^{\circ} 1$ upper state by $4 \mu \mathrm{m}$ solar radiation (Johnson et al. 1976). Two mechanisms were proposed 
involving absorption of near-IR solar radiation $(<3 \mu \mathrm{m})$ followed by $V-V$ collisional exchange of $\nu_{3}$ quanta to the $\sum_{u}^{+} 00^{\circ} 1 \nu_{3}$ fundamental level. The first mechanism relied on direct pumping of the $\mathrm{CO}_{2}$ near-IR bands. The second relied on absorption by trace atmospheric $\mathrm{H}_{2} \mathrm{O}$ in bands within $40 \mathrm{~cm}^{-1}$ of $\mathrm{CO}_{2}$ near-IR bands, allowing $\mathrm{V}-\mathrm{V}$ collisional transfer of $\nu_{3}$ quanta.

Johnson et al. (1976) showed radiative de-excitation of the $\sum_{\mathrm{u}}{ }^{+} 00^{\circ} 1$ level along the $10 \mu \mathrm{m}$ branch $\left(\mathrm{A}=0.67 \mathrm{~s}^{-1}\right)$ is not quelched by collisional de-excitation for densities $\rho<10^{13} \mathrm{~cm}^{-3}$. The $4 \mu \mathrm{m}$ pathway to the ground state, while prefered $\left(A=500 \mathrm{~s}^{-1}\right)$, is blocked by radiative trapping due to $4 \mu \mathrm{m}$ mean free paths of only a few meters at $\rho=10^{13} \mathrm{~cm}^{-3}$. Conversely, the atmosphere is optically thin at $10 \mu \mathrm{m}$ due to underpopulation of the $\sum_{\mathrm{g}}^{+}\left[10^{\circ} 0,02^{\circ} 0\right] \mathrm{I}$, II lower states (at $\mathrm{T}=-200 \mathrm{~K}$ ).

Mechanism 1 was evaluated considering absorption from only the strongest line in each of the eight strongest near-IR $\mathrm{CO}_{2}$ bands. Calculated $10 \mu \mathrm{m}$ emission strength was within $20 \%$ of the observed, with peak emission at $120 \mathrm{~km}$ altitude $\left(\rho=10^{13} \mathrm{~cm}^{-3}\right)$ for Venus. The estimated effect of including all weaker line absorption was a 4 -fold enhancement in emission intensity.

Mechanism 2 was evaluated assuming absorption in three near-IR $\mathrm{H}_{2} \mathrm{O}$ bands. Calculated $10 \mu \mathrm{m}$ emission strength matched the observed for a $1: 2800 \mathrm{H}_{2} \mathrm{O}$ to $\mathrm{CO}_{2}$ abundance ratio, consistent with Mars observations. Whether the Venusian atmospheric water vapor content was sufficient for mechanism 2 was uncertain.

Adopting the Johnson et al. (1976) $10 \mu \mathrm{m}$ emission mechanism, Deming and Mumma (1983) solved the non-LTE radiative transfer problem 
considering near-IR absorption by all CO 2 lines from $2000 \mathrm{~cm}^{-1}$ to 9500 $\mathrm{cm}^{-1}(1.1$ to $5 \mu \mathrm{m})$, as listed on the 1973 AFCRL tape (McClatchey et al. 1973). Some $2 \times 10^{4}$ lines, including all $\mathrm{CO}_{2}$ isotopic species, were considered. Direct pumping of the $10 \mu \mathrm{m} \sum_{\mathrm{u}}{ }^{+} 00^{\circ} 1$ upper state via ground state absorption of solar $4 \mu \mathrm{m}$ radiation, neglected by Johnson et al. (1976), was also included.

Deming and Mumma (1983) confirmed absorption in the near-IR 2-3 $\mu \mathrm{m}$ bands $\left(3000-4500 \mathrm{~cm}^{-1}\right)$ was the dominant $\sum_{u}{ }^{+} 00^{\circ} 1$ excitation mechanism. In addition it was found that bands in the 1.1 to $1.7 \mu \mathrm{m}$ range contributed an additional $30 \%$, and direct solar pumping of the $\sum_{\mathrm{u}}{ }^{+} 00^{\circ} 1$ level via the $4 \mu \mathrm{m}$ pathway was significant - even dominant at higher altitudes. Peak emission occured at $109 \mathrm{~km}$ with half power altitudes of 101 and $120 \mathrm{~km}$. Comparison to observed Venus and Mars emission (Deming et al. 1983) indicated model-predicted fluxes 1308 and 1008 of observed, respectively. Optical depth calculations indicated the $10 \mu \mathrm{m}$ emission was optically thin along line of sight column lengths through the emitting shell, even for near limb observations. The $10 \mu \mathrm{m}$ intensity was found approximately proportional to line of sight column length and the cosine of the beam position's solar zenith angle. Stimulated emission proved to be a negligible effect. The model additionally confirmed the claimed population inversion in the $10 \mu \mathrm{m}$ band (Mumma et al. 1981) at high altitudes. The inversion was ubiquitous and insensitive to the atmospheric model used. The Johnson et al. (1976) $\mathrm{H}_{2} \mathrm{O}$ pumping mechanism was not considered. 


\section{CHAPTER 3: IR HETERODYNE SPECTROMETER OPERATION}

\subsection{BASIC THEORY OF OPERATION}

An IR heterodyne spectrometer (IRHS) maps an IR source spectral region, containing the spectral feature(s) under investigation, into the radio band where it may be digitized into bins a few $\mathrm{MHz}$ wide and stored in a computer for real time or future data manipulation. This is achieved by photomixing the source signal with an IR laser, a nearly monochromatic and coherent local oscillator (LO), in an IR photomixer. The photomixed signal or intermediate frequency (IF) is a beat spectrum characteristic of the frequency difference between source and LO, and whose spectral bandpass is dictated by the frequency response of the photomixer and its preamplifier.

The IF is amplified and sampled by a contiguous radio frequency (RF) filter bank with typically a 0 to $\sim 2 \mathrm{GHz}$ bandpass. Variation in LO frequency $\left(\nu_{10}\right)$ allows the RF bandpass to view different regions of the source's spectrum. Ideally, a local oscillator with continuously

variable $\nu_{10}$ is desired. To date, however, such devices are inherently noisy, as with the tunable diode laser (TDL) (Mumma et al. 1975, Glenar et al. 1982). Investigations where high $\mathrm{S} / \mathrm{N}$ is mandatory, e.g., mapping planetary wind fields, thereby preclude use of TDL systems.

The Goddard Space Flight Center (GSFC) IRHS employs low pressure $\mathrm{CO}_{2}$ continuous-wave laser local oscillators (Kostiuk et al. 1977, Kostiuk and Mumma 1983, Mumma et al. 1978, Glenar et al. 1986). Such LO's can provide at least discontinuous variation in $\nu_{10}$ through selection of different ro-vibrational laser lines, and different isotopic form of $\mathrm{CO}_{2}$ used as the working gas. Taken together, the 
photomixer bandwidth about each of the candidate lines (considering all available isotopes) covers $15 \%$ of the $9-12 \mu \mathrm{m}$ operating band of these lasers (Kostiuk and Mumma 1983).

The typical filter bank employed in the GSFC IRHS consists of 64 $25 \mathrm{MHz}$ filters. Doppler-limited spectral lines associated with planetary atmospheres and the Sun have FWHM's of order $50 \mathrm{MHz}$ and 400 $\mathrm{MHz}$, respectively, making possible a complete sampling of such lines by the $0-1.6 \mathrm{GHz}$ filter bank bandpass.

If $I(\nu)$ is the source spectral distribution in the IR and $I\left(\nu^{\prime}\right)$ is the distribution in the IF then, $\nu^{\prime}=\nu-\nu{ }_{10}$ and the mapping takes the form $I(\nu) \rightarrow I\left(\nu-\nu_{10}\right)$. In fact, as the photomixer does not distinguish between $\nu-\nu_{10}$ and $\nu_{10}-\nu$, the mapping necessarily generates a double sideband (DSB) spectrum equal to the sum of the two single sidebands (SSB). Hence:

$$
\mathrm{I}(\nu)+\mathrm{I}\left(2 \nu_{10}-\nu\right) \rightarrow \mathrm{I}\left(\nu-\nu_{10}\right)
$$

Often the spectral feature under investigation lies completely to one side of the LO (with the other SSB a flat continuum). If the DSB spectrum $S / N$ allows continuum level determination, subtracting $1 / 2$ the DSB continuum yields the SSB source spectrum in the IF (Fig. 3.1). Once the DSB spectrum has been digitized, converting to SSB in the IF, and carrying out the inverse mapping, knowing $\nu_{10}$, generates the source spectrum in the IR at sub-Doppler resolution.

For lines formed in planetary atmospheres, $25 \mathrm{MHz}$ resolution is rather coarse, with a particular line resolved by $\leq 3$ filters (or channels). A high resolution filter bank (HRB) is thus employed 


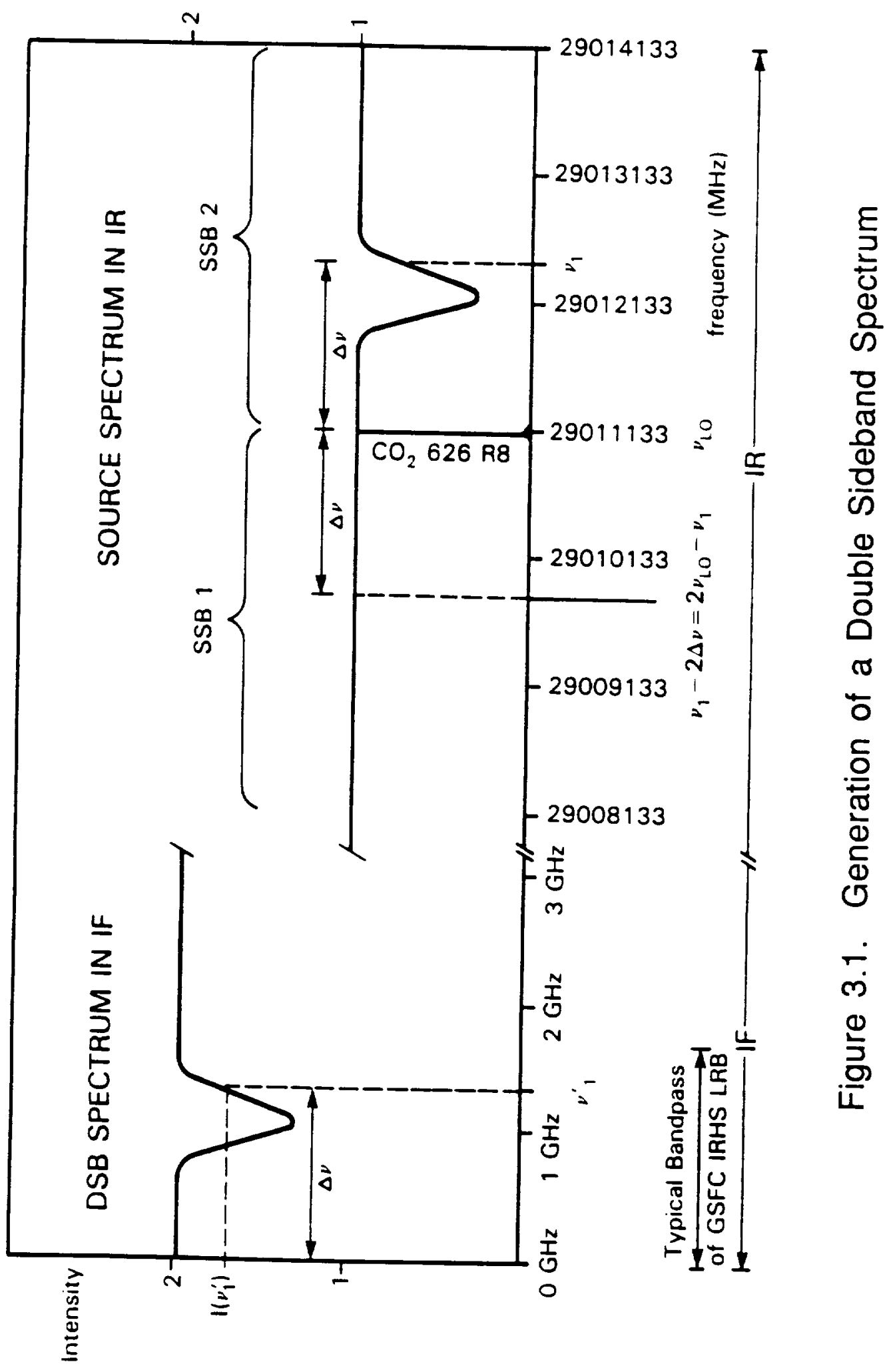


consisting of $645 \mathrm{MHz}$ filters with a $320 \mathrm{MHz}$ overall bandwidth. The $\mathrm{HRB}$ may be tuned to any region of the $1.6 \mathrm{GHz}$ low resolution filter bank (LRB) bandpass. This is accomplished by heterodyning the IF with a continuously tunable radio frequency local oscillator (RFLO). At $-10 \mu \mathrm{m}, 5 \mathrm{MHz}$ resolution corresponds to $\Delta \lambda=10^{-6} \mu \mathrm{m}$, thus $\lambda / \Delta \lambda=10^{7}$.

\subsection{A QUANTITATIVE LOOK AT COHERENT DETECTION}

\subsubsection{Generation of an Intermediate Frequency}

The theory of coherent detection (employing an IRHS) has been covered in the literature in detail by Blaney (1975), Cohen (1975) and Abbas et al. (1976). A first-order treatment is presented here to provide a basic understanding of heterodyne principles.

Consider a detector whose generated photocurrent $i(t)$ is proportional to the flux incident on its surface. The behavior of such a detector is given by:

$$
i(t)=c \int E_{T}(r, t)^{*} \cdot E_{T}(r, t) d A,
$$

where $\mathrm{E}_{\mathrm{T}}(\mathbf{r}, \mathrm{t})$ is the total electric field at position $\mathbf{r}$ on the detector surface at time $t, c$ is a constant, ${ }^{*}$ denotes the complex conjugate, and the integral is carried out over the surface of the detector.

IR heterodyne spectroscopy relies on photomixing the source signal with an IR laser local oscillator, thus:

$$
E_{T}(r, t)=E_{10}(r, t)+E_{S}(r, t)
$$

where the 10 and $s$ terms are the LO and source contributions, respectively. 
Consider a coherent and linearly polarized LO with an electric field given by:

$$
E_{10}(r, t)=E_{10}(r) \exp \left(-j \omega_{10} t\right)
$$

where $E_{10}(r)$ is the spatial part of the field and $\omega_{10}=2 \pi \nu_{10}$. In addition, consider a source frequency $\omega$ with electric field:

$$
E_{S}(r, t)=E_{S}(r) \exp (-j \omega t) .
$$

Substituting Eqs. 3.3, 3.4 and 3.5 into 3.2 and rearranging yields:

$i(t)=i_{D C}+C \int\left[E_{10}(r) \cdot E_{S}(r){ }^{*} \exp \left(-j\left(\omega_{10}-\omega\right) t\right)+E_{10}(r)^{*} \cdot E_{S}(r) \exp \left(j\left(\omega_{10}-\omega\right) t\right)\right] d A$

where:

$$
i_{D C}=C \int\left[E_{10}(r)^{*} \cdot E_{10}(r)+E_{S}(r)^{*} \cdot E_{S}(r)\right] d A .
$$

$i_{D C}$ has no time varying contribution and represents the average photocurrent generated by all radiation incident on the detector over a time $r>2 \pi /\left(\omega_{10}-\omega\right)$. Eq. 3.6 indicates $i(t)$ varies with the difference or intermediate (IF) frequency (thus providing heterodyne detection), and only the LO's polarization is detected in the source signal.

\subsubsection{Diffraction-Limited Operation}

Coherent detection also dictates a field-of-view (FOV) matched to the telescope's diffraction limit. (At $10 \mu \mathrm{m}$, the $1.5 \mathrm{~m}$ (60-inch) primary of the McMath Solar Telescope on Kitt Peak yields 1.6" (FWHM) spatial resolution.) Diffraction-limited operation as an outgrowth of Section 3.2.1 can be shown by employing a simple interference model. 
Consider an aperture that is a horizontal slit of width $D$ and infinite length located a distance $\mathrm{Z}$ from the detector plane (Fig. 3.2). A plane parallel Lo wavefront is directed axially upon the slit. Using Huygen's Principle, wavefronts between the slit and the detector are generated by the interference of cylindrical wavelets from point sources comprising the wavefront at $\mathrm{Z}=0$. Also, assume a detector plane at $2>D$, insuring Fraunhofer diffraction results.

Let a given point source be located a distance $X$ from the axis and have spatial extent $d x$. Define $r$ as the displacement vector from point source $A$, at $X=0$, to point $P$ on the detector. The spatial part of the electric field at $P$, due to the point source at distance $X$, is:

$$
\mathrm{dE}_{10}(r)=\frac{\mathrm{c}_{10}}{\mathrm{D}(\mathrm{r}-\mathrm{X} \sin \theta)} \exp \left(j \mathrm{k}_{10}(r-\mathrm{X} \sin \theta)\right) \mathrm{dX} \text {, }
$$

where wavenumber $k_{10}$ is given by $k_{10}=2 \pi / \lambda_{10}$, $r$ denotes the magnitude of $r, \theta$ is the angle between $r$ and the optical axis, and $c_{10}$ is a constant vector. In the case of Fraunhofer diffraction, $(r-X \sin \theta)-r$. This substitution may be made in the denominator, but not in the exponential term, if $k_{10} X \sin \theta$ is of order $\pi$. The latter is true for $\operatorname{smal1} \theta$, at $10 \mu \mathrm{m}$, for $\mathrm{X} \geq 1 \mathrm{~mm}$.

The spatial part of the electric field $E_{10}(r)$ at $P$, due to all sources from $-D / 2$ to $D / 2$, is obtained by integrating Eq. 3.8, yielding:

$$
E_{10}(r)=c_{10}\left(\frac{p}{r}\right) \exp \left(j k_{10} r\right) \text { where: } p=\frac{\sin \left(\left(k_{10} D / 2\right) \sin \theta\right)}{\left(k_{10} D / 2\right) \sin \theta} \text {. }
$$

Consider the same situation for a plane parallel wavefront 


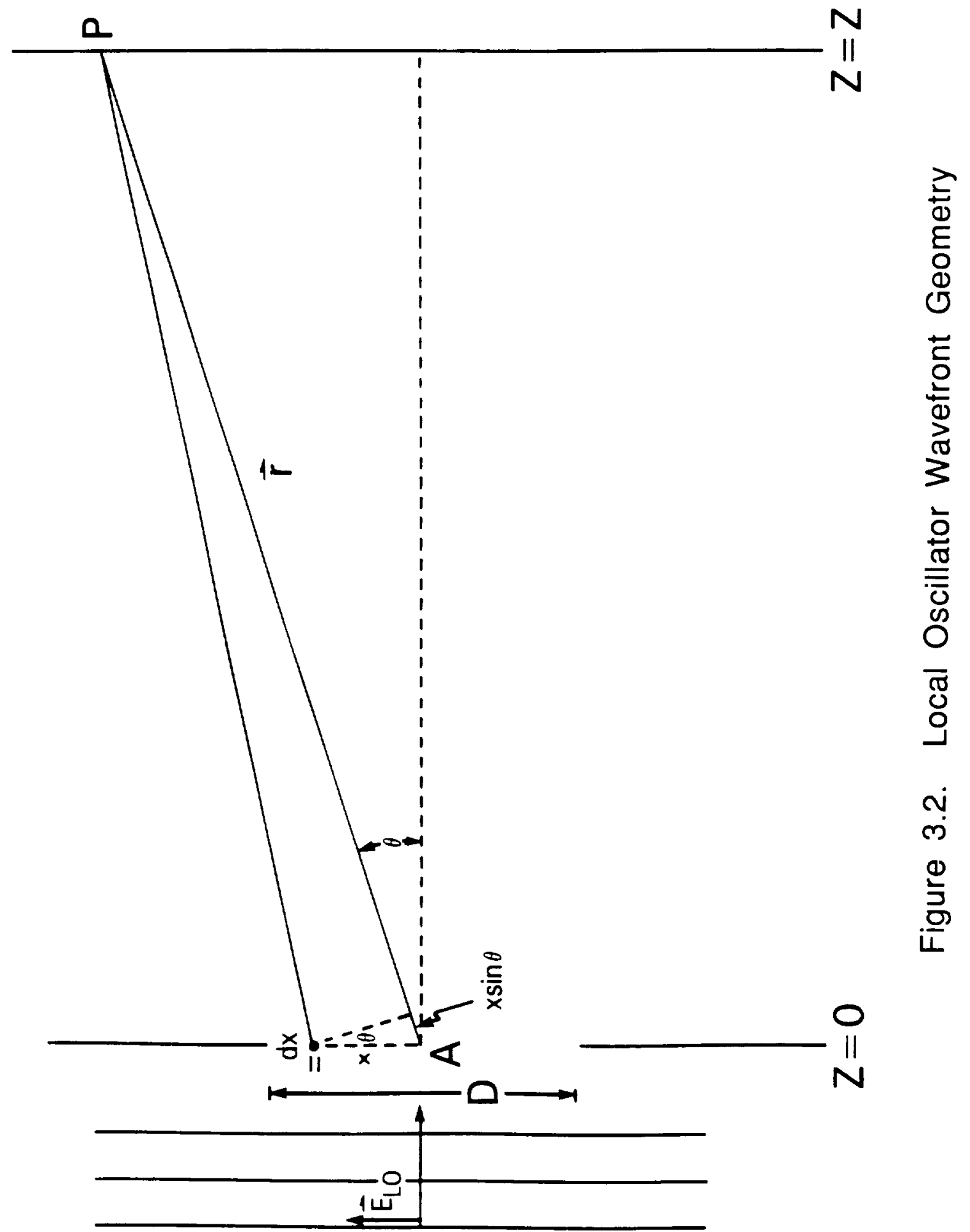


associated with $E_{S}(r, t)$ inclined at an angle $\alpha$ to the axis (Fig. 3.3). A point source at distance $X$ provides an electric field at $P$ given by:

$$
d E_{s}(r)=\frac{c_{s}}{D(r-X \sin \theta+X \sin \alpha)} \exp (j k(r-X \sin \theta+X \sin \alpha)) d X,
$$

Using the same arguments regarding Fraunhofer diffraction as before, and integrating over $\mathrm{X}$, yields:

$$
E_{s}(r)=c_{s}\left(\frac{q}{r}\right) \exp (j k r) \text { where: } q=\frac{\sin ((k D / 2)(\sin \theta-\sin \alpha))}{(k D / 2)(\sin \theta-\sin \alpha)} \text {. }
$$

The spatial dependence of $i(t)$ over the detector surface can now be determined. Substituting Eqs. 3.9 and 3.11 into 3.6, and making use of the relations $\mathrm{r}=\mathrm{z} / \cos \theta$ and $\mathrm{dA}=(\mathrm{Lr} / \cos \theta) \mathrm{d} \theta$, where $\mathrm{L}$ is the horizontal length of the detector, one obtains:

$$
i(t, \alpha)=i_{D C}+c_{10} \cdot c_{s}\left(\frac{2 c L}{z}\right) \int \cos \left(\left(k_{10}-k\right) r-\left(\omega_{10}-\omega\right) t\right) p q d \theta
$$

Here $i(t, \alpha)$ is the total source-generated photocurrent between $\alpha$ and $\alpha+d \alpha$. For small $\theta$ the cosine term may be taken from the integral, yielding:

$$
i(t, \alpha)=i_{D C}+c_{10} \cdot c_{S}\left(\frac{2 c L}{z}\right) \cos \left(\left(k_{10}-k\right) z-\left(\omega_{10}-\omega\right) t\right) F(\alpha),
$$

where:

$$
F(\alpha)=\int \frac{\sin \left(\left(k_{10} D / 2\right) \theta\right)}{\left(k_{10} D / 2\right) \theta} \frac{\sin ((k D / 2)(\theta-\alpha))}{(k D / 2)(\theta-\alpha)} d \theta
$$

For a given $\alpha$, the integrand of Eq. 3.14 represents the spatial 


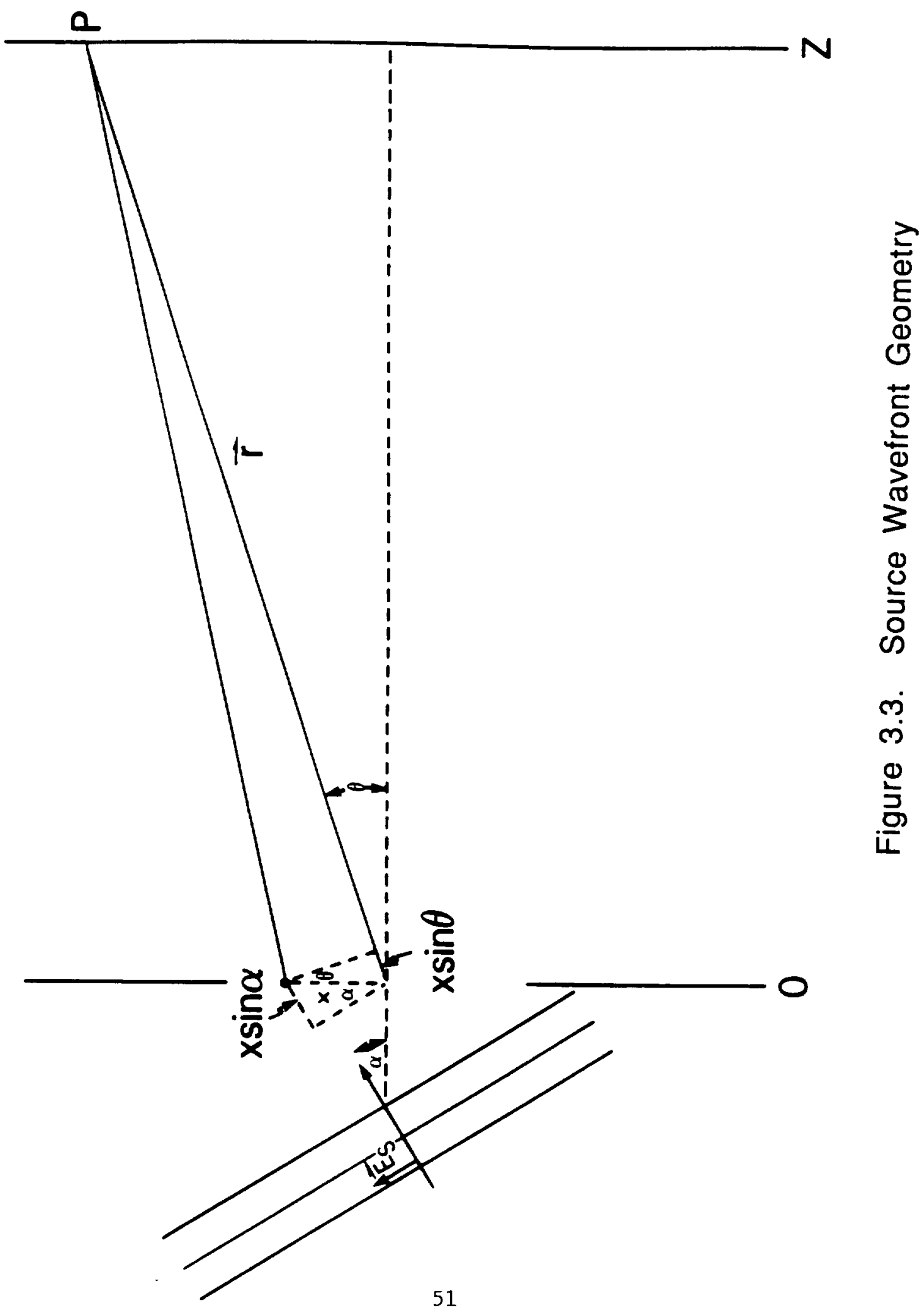


contribution function $\rho(\theta, \alpha)$ over the detector for the time varying component of $i(t, \alpha)$. Figure 3.4 is a plot of $\rho(\theta, \alpha)$ from $\theta=-2 \lambda / D$ to $2 \lambda / D$, for $\alpha=0, \lambda / 2 \mathrm{D}$ and $\lambda / \mathrm{D}$. For small $\alpha$, regions of the detector far from the axis yield insignificant contributions to the photocurrent. (A negative $\rho(\theta, \alpha)$ implies an oppositely directed electric field which dictates an oppositely directed photocurrent.)

For a uniform infinitely extended emitter as source, $F(\alpha)$ represents the beam shape and explicitly defines what the detector can "see". Figure 3.5 is a plot of $F(\alpha)$ from $\alpha=-5 \lambda / D$ to $5 \lambda / D$. The FWHM of the beam is $\Delta \alpha \sim \lambda / D$. Coherent detection thereby provides a beam at the diffraction limit.

For the more complex case of a circular aperture, the diffraction limit is still found to be $\Delta \alpha=1.22 \lambda / D$ FWHM. This result is embodied in the Antenna Theorem (Seigman 1966) which states the solid angle FOV $(\Omega)$ of a coherent detector is given by $\Omega-\lambda^{2} / A$, where $A$ is the collecting area of the detector. Both relations may be recognized as equivalent if one notices that $A=\pi D^{2} / 4$ and $\Omega=\pi \Delta \alpha^{2} / 4$.

An IRHS properly mated to a telescope has an FOV at the telescope's diffraction 1imit. In Figure 3.3, imagine the collimated source beam to the left of the aperture is a product of a telescope's various optical components. The detector's solid angle FOV $(\Omega)$ receives radiation collected within a necessarily different solid angle $\Omega_{t}$ as seen by the primary. $\Omega_{t}$, the quantity of interest, defines the FOV in the sky to which the detector is sensitive. A proper mating of detector and telescope occurs when light rays intercepting the edge of the aperture in Figure 3.3 also intercept the edge of the primary. 


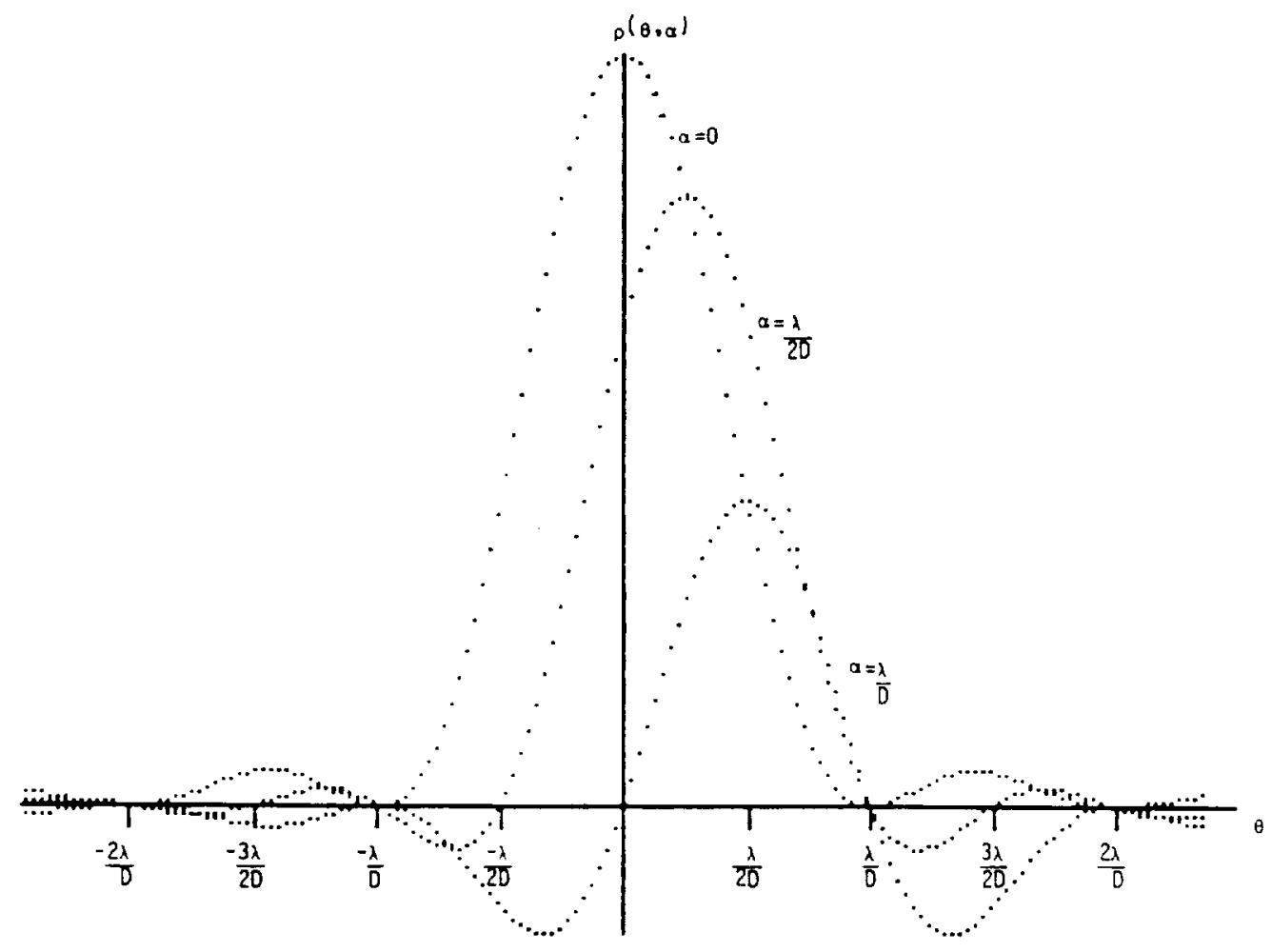

Figure 3.4. Heterodyne Spatial Contribution Function

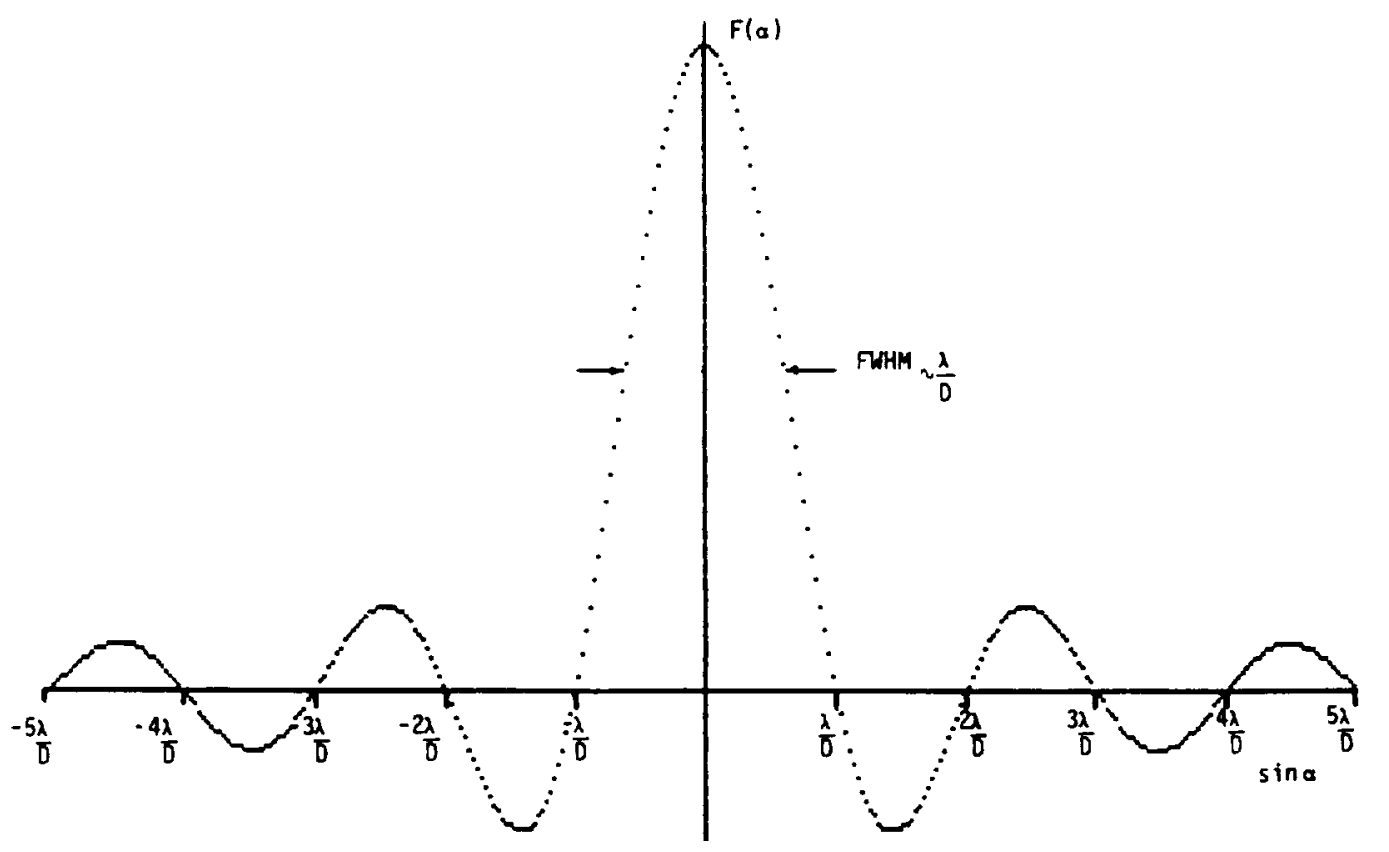

Figure 3.5. Heterodyne Beam-Shape Function 
When this criterion is met, the following relation holds:

$$
\frac{\Omega}{\Omega_{t}}=\frac{A_{t}}{A}
$$

where $A_{t}$ is the primary's collecting area. The product $\Omega A$, called the etendue, is a constant, and from the Antenna Theorem equals $\lambda^{2}$.

Solving Eq. 3.15 for $\Omega_{t}$ gives:

$$
\Omega_{t}=\frac{\Omega A}{A_{t}}=\frac{\lambda^{2}}{A_{t}}
$$

which is the diffraction limit of the telescope.

\subsubsection{Signal-to-Noise}

To determine the signal-to-noise ratio ( $/ N$ ) expected for heterodyne detection, one would like to express the DC and time varying components of the photocurrent in terms of the incident LO and source power. The dot products in Eq. 3.6 may be replaced using the transform:

$$
E_{10}(r) \cdot E_{s}(r)^{*} \equiv E_{10} E_{s}[\exp (j \Phi)]
$$

where:

$$
E_{10}=\left|E_{10}(r)\right| \text { and } \quad E_{s}=\left|E_{s}(r)\right|
$$

yielding:

$$
i(t)=c \iint\left(E_{10}{ }^{2}+E_{s}^{2}\right) d x d y+(2 c) \cos \left(\left(\omega_{10}-\omega\right) t-\Phi\right) \int E_{10} E_{s} d x d y,
$$

with $\mathrm{dxdy}=\mathrm{dA}$. If $\mathrm{E}_{10}(\mathrm{r})$ and $\mathrm{E}_{\mathbf{S}}(\mathbf{r})$ are matched across the detector then $E_{10}$ and $E_{s}$ are of the form:

$$
E_{10}=U(f(x, y)) \quad E_{s}=V(f(x, y)),
$$


where $U$ and $V$ are constants. Substituting Eq. 3.20 into 3.19 gives:

$i(t)=c\left(U^{2}+V^{2}\right) \iint f^{2}(x, y) d x d y+(2 c u V) \cos \left(\left(\omega_{10}-\omega\right) t-\Phi\right) \iint f^{2}(x, y) d x d y$

If $\mathrm{P}_{10}$ and $\mathrm{P}_{S}$ are the $\mathrm{LO}$ power and source power incident on the detector, then the total number of photons incident per second $\mathrm{N}_{\nu}$ is:

$$
\mathrm{N}_{\nu}=\left(\mathrm{P}_{10}+\mathrm{P}_{\mathrm{s}}\right) / \mathrm{h} \nu,
$$

where $h$ is Planck's constant. Define the detector's quantum efficiency $\eta$ as the number of electrons generated per incident photon. If $\mathrm{e}$ is the charge per electron, then the average total photocurrent $i_{D C}$ is:

$$
i_{\mathrm{DC}}=\mathrm{eN} \mathrm{e}=\left(\frac{\mathrm{e} \eta}{\mathrm{h} \nu}\right)\left(\mathrm{P}_{10}+\mathrm{P}_{\mathrm{s}}\right)
$$

Comparison of Eq. 3.23 with the DC portion of Eq. 3.21 allows determination of $\mathrm{Uf}(\mathrm{x}, \mathrm{y})$ and $\mathrm{Vf}(\mathrm{x}, \mathrm{y})$ in terms of $\mathrm{P}_{10}$ and $\mathrm{P}_{\mathrm{S}}$. Substitution of these forms into Eq. 3.21 gives:

$$
\left.i(t)=\frac{e \eta}{h \nu}\left(P_{10}+P_{s}\right)+\frac{2 e \eta\left(P_{10}\right.}{h \nu} P_{s}\right)^{1 / 2} \cos \left(\left(\omega_{10}-\omega\right) t-\Phi\right)
$$

The time varying portion of Eq. 3.24, ${ }^{i}{ }_{I F}$, represents the photocurrent contribution due to coherent detection. The heterodyne signal, after square law detection, is proportional to $\left\langle i_{I F}{ }^{2}\right\rangle$ where a time average has been taken over a period $\tau=2 \pi /\left(\omega_{10}-\omega\right)$, thus:

$$
{ }_{i \mathrm{IF}}^{2}>=\frac{4}{\tau}\left(\frac{\mathrm{e} \eta}{\mathrm{h} \nu}\right)^{2}\left(\mathrm{P}_{10} \mathrm{P}_{\mathrm{s}}\right) \int_{0}^{\tau} \cos ^{2}\left(\left(\omega_{10}-\omega\right) t-\Phi\right) \mathrm{dt}
$$

Integrating yields: 


$$
{ }^{<i F}{ }^{2}>=2\left(\frac{e \eta}{h \nu}\right)^{2} \mathrm{P}_{10} \mathrm{P}_{\mathrm{s}}
$$

In a coherent detector, the dominant noise contribution is shot noise in the photocurrent. If $i_{I F} \ll i_{D C}$, then only the $i_{D C}$ shot noise contribution need be considered. This condition is fulfilled if $\mathrm{P}_{10} \gg \mathrm{P}_{\mathrm{S}}$ and corresponds to laser shot noise-1imited operation. Such operation maximizes $\mathrm{S} / \mathrm{N}$ (as shown below) and is easily achievable using $\mathrm{CO}_{2}$ laser local oscillators.

The heterodyne noise level is proportional to $\left\langle i_{n}{ }^{2}\right\rangle$ where $i_{n}$ is the shot noise photocurrent given by:

$$
\left\langle i_{n}^{2}>=2 e i_{D C}^{B}\right.
$$

where $B$ is the bandwidth of the detector channel. Substituting Eq. 3.23 into 3.27 gives:

$$
<i_{n}^{2}>=\frac{2 e^{2} B \eta}{h \nu}\left(P_{1 o}+P_{s}\right) .
$$

The $\mathrm{S} / \mathrm{N}$ is given by $\left\langle i_{\mathrm{IF}}{ }^{2}>/<i_{\mathrm{n}}{ }^{2}\right\rangle$. Thus from Eq. 3.26 and 3.28 :

$$
\mathrm{S} / \mathrm{N}=\frac{\eta}{h \nu \mathrm{B}}\left(\frac{\mathrm{P}_{\mathrm{s}}}{1+\mathrm{P}_{\mathrm{s}} / \mathrm{P}_{10}}\right) .
$$

Clearly, if $\mathrm{P}_{10}>\mathrm{P}_{S}$, the $\mathrm{S} / \mathrm{N}$ is maximized for a given $\mathrm{P}_{S}$. For laser shot noise-limited operation, Eq. 3.29 becomes:

$$
\mathrm{S} / \mathrm{N}=\frac{\eta \mathrm{P}_{\mathrm{S}}}{h \nu \mathrm{B}} .
$$

The condition under which the assumption $\mathrm{P}_{10} \gg \mathrm{P}_{\mathrm{S}}$ is invalid has been explored in detail by Abbas et al. (1976) and Betz (1977). Typically, $\mathrm{P}_{10}$ for the IRHS is of order $1 \mathrm{~mW}$. For $\mathrm{P}_{10}$ and $\mathrm{P}_{\mathrm{S}}$ to be 
comparable at $10 \mu \mathrm{m}$, a $2000 \mathrm{~K}$ source temperature is required. Planetary investigations do not fall within this regime.

Spectral lines formed in planetary atmospheres are viewed against a planetary continuum which may be taken as Planckian. $\mathrm{S} / \mathrm{N}$ on the continuum is therefore a natural measure of IRHS sensitivity, and with the Planck function $B_{\nu}(T)$, may be readily found. $B_{\nu}(T)$ is given by:

$$
B_{\nu}(T)=\frac{2 h \nu^{3}}{c^{2}}(\exp [h \nu / k T]-1)^{-1}
$$

where $c$ is the speed of light and $k$ is Boltzmann's constant.

Figure 3.6 depicts a coherent receiver with collecting area $A$, and diffraction-limited beam $\Omega$, a distance $R$ from the emitting region of a planetary atmosphere. Define $\Omega_{r}$ as the solid angle subtended by the receiver at the location of the planet. In addition, define $A_{S}$ as the area of the planetary atmosphere within the receiver's diffraction-limited beam. The following relations hold:

$$
\Omega_{r}=A / R^{2} \quad \text { and } \quad A_{S}=R^{2}
$$

The source power $\left(\mathrm{P}_{S}\right)$ incident on the receiver may be obtained by integrating $B_{\nu}(T)$ over $A_{S}, \Omega_{r}$, and receiver bandwidth $B . B_{\nu}(T)$ may be taken as constant over the range of integration to yield (assuming detection of one polarization):

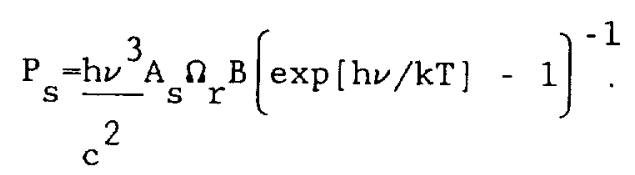

Substituting Eq. 3.32 into 3.33 gives: 


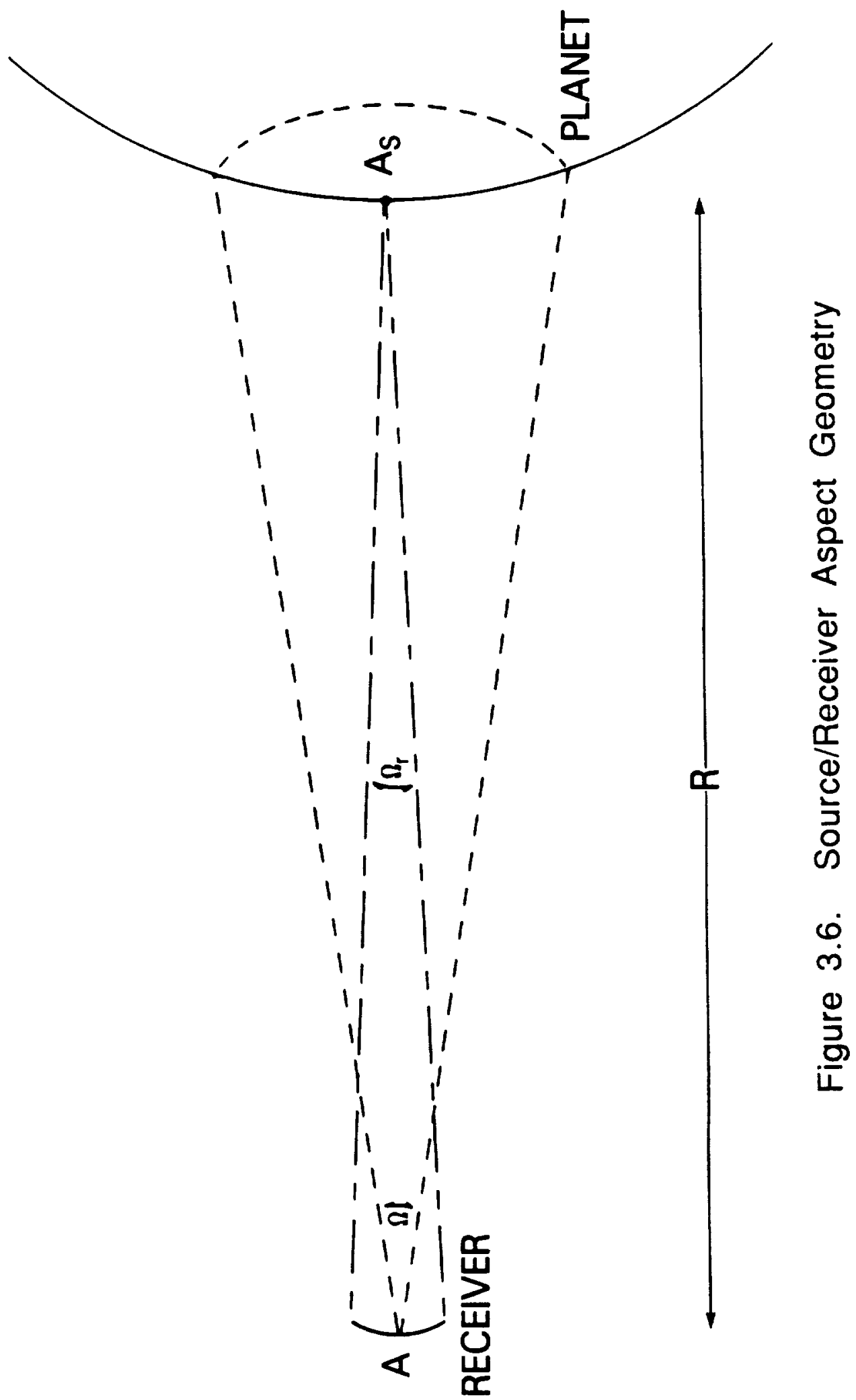




$$
\mathrm{P}_{\mathrm{s}}=\frac{\mathrm{h} \nu^{3}}{\mathrm{c}^{2}} \mathrm{~A} \Omega \mathrm{B}(\exp [\mathrm{h} \nu / \mathrm{kT}]-1)^{-1}
$$

Using the Antenna Theorem, and the relation $\nu \lambda=c$, Eq. 3.34 reduces to:

$$
P_{S}=h \nu B(\exp [h \nu / k T]-1)^{-1}
$$

(Since Eq. 3.35 is independent of telescope aperture, employing a larger telescope does not enhance signal power assuming the source fills the diffraction-limited beam.) Substituting Eq. 3.35 into Eq. 3.30 yields the $\mathrm{S} / \mathrm{N}$ on the continuum:

$$
\mathrm{S} / \mathrm{N}=\eta(\exp [\mathrm{h} \nu / \mathrm{kT}]-1)^{-1}
$$

The post detection $S / N$, employing a square-law detector, scales with $(\mathrm{B} \tau)^{1 / 2}$ where $\tau$ is the integration time (Betz 1977). The post detection $\mathrm{S} / \mathrm{N}$ is thus:

$$
\mathrm{S} / \mathrm{N}=\eta[\mathrm{B} \tau]^{1 / 2}(\exp [\mathrm{h} \nu / \mathrm{kT}]-1)^{-1}
$$

Eq. 3.37 assumes detection of only one polarization and a SSB spectrum.

During actual IRHS operation, a variety of factors act to degrade the $\mathrm{S} / \mathrm{N}$ from the ideal case. Adopting the convention of Abbas et al. (1976), all degradation factors may be incorporated within a single term $\Delta$, with Eq. 3.37 becoming:

$$
\mathrm{S} / \mathrm{N}=\underset{\Delta}{2} \eta[\mathrm{B} \tau]^{1 / 2}(\exp [\mathrm{h} \nu / \mathrm{kT}]-1)^{-1} .
$$

Normal field operation implies $\Delta$ contains at least a factor of 2 for heterodyne detection of a single source polarization and a factor of 2 
for optical chopping between a source and reference beam with a $50 \%$ duty cycle. Additional degradation may be attributed to attenuation of the source signal by the IRHS optics, the effects of the various noise components, and a decreasing quantum efficiency with increasing IF due to a sensitivity roll-off in the photomixer and its matched IF preamp. The optimal $\triangle$ achieved by the GSFC IRHS varies from 7 near $\nu^{\prime}=0$ to 20 near $\nu^{\prime}=1.6 \mathrm{GHz}$ (Kostiuk and Mumma 1983).

Figure 3.7 (Kostiuk and Mumma 1983) is a plot of $\mathrm{S} / \mathrm{N}$ versus $\lambda$ for a variety of blackbody sources where a bandwidth $\mathrm{B}=25 \mathrm{MHz}$, an integration time $\tau=3600 \mathrm{sec}$, and a $\Delta=7$ are assumed. Also indicated are some planet-specific temperature ranges that may be probed at $10 \mu \mathrm{m}$ which offer $S / N$ on the continuum conducive for high resolution spectroscopic studies. 


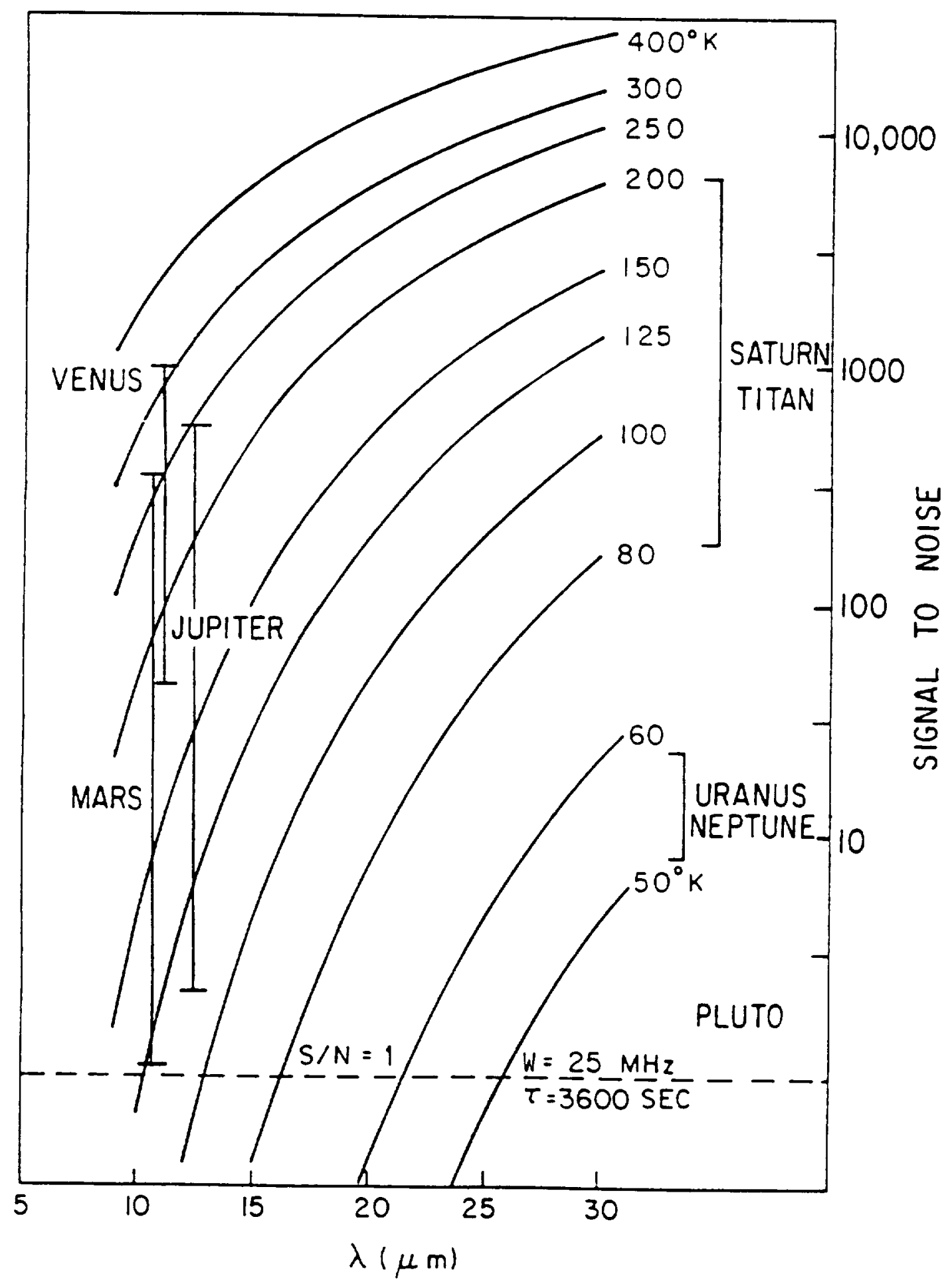

Figure 3.7. Spectral Intensity of Blackbody Sources 


\subsection{OPTICAL FRONT END LAYOUT}

The optical front end of the GSFC IRHS consists of a HgCdTe IR photomixer, the laser local oscillator, calibration blackbodies, a visual guiding system and all optical elements necessary to provide IR photomixing at the detector plane. The front end is mated at Coudé to either the $1.5 \mathrm{~m}$ (60-inch) F/60 McMath Solar Telescope on Kitt Peak, Arizona, or the $3 \mathrm{~m}$ (120-inch) F/120 NASA Infrared Telescope Facility (IRTF) instrument on Mauna Kea, Hawaii. The IF output of the photomixer is fed into an RF back end for spectral analysis. Figure 3.8 shows the front end layout for the McMath spectrometer. The IRTF front end layout is basically the same.

The output of the laser local oscillator is intercepted by beam splitter $\mathrm{ml}$. The transmitted beam, used as a probe of laser output power, is focused by lens Ll onto a high speed pyroelectric detector (see Chapter 4).

The reflected beam at $\mathrm{ml}$ is first attenuated, to obtain an optimum performance photocurrent in the HgCdTe photomixer, and then expanded, to provide a uniform field over the surface of off-axis parabola $m 9$. The attenuator contains a ZnSe window, mounted at the Brewster angle, which can be rotated with respect to the beam polarization. The McMath beam expander consists of lenses $L 3$ and L2 with a 10:1 focal length ratio (a reflective optics expander is used at IRTF). Flip mirror m3 allows determination and periodic monitoring of the $\mathrm{CO}_{2}$ laser line with a spectrum analyzer.

The source signal from the telescope intercepts a dichroic which transmits 208 in the visible and reflects 968 in the IR. A TV camera 


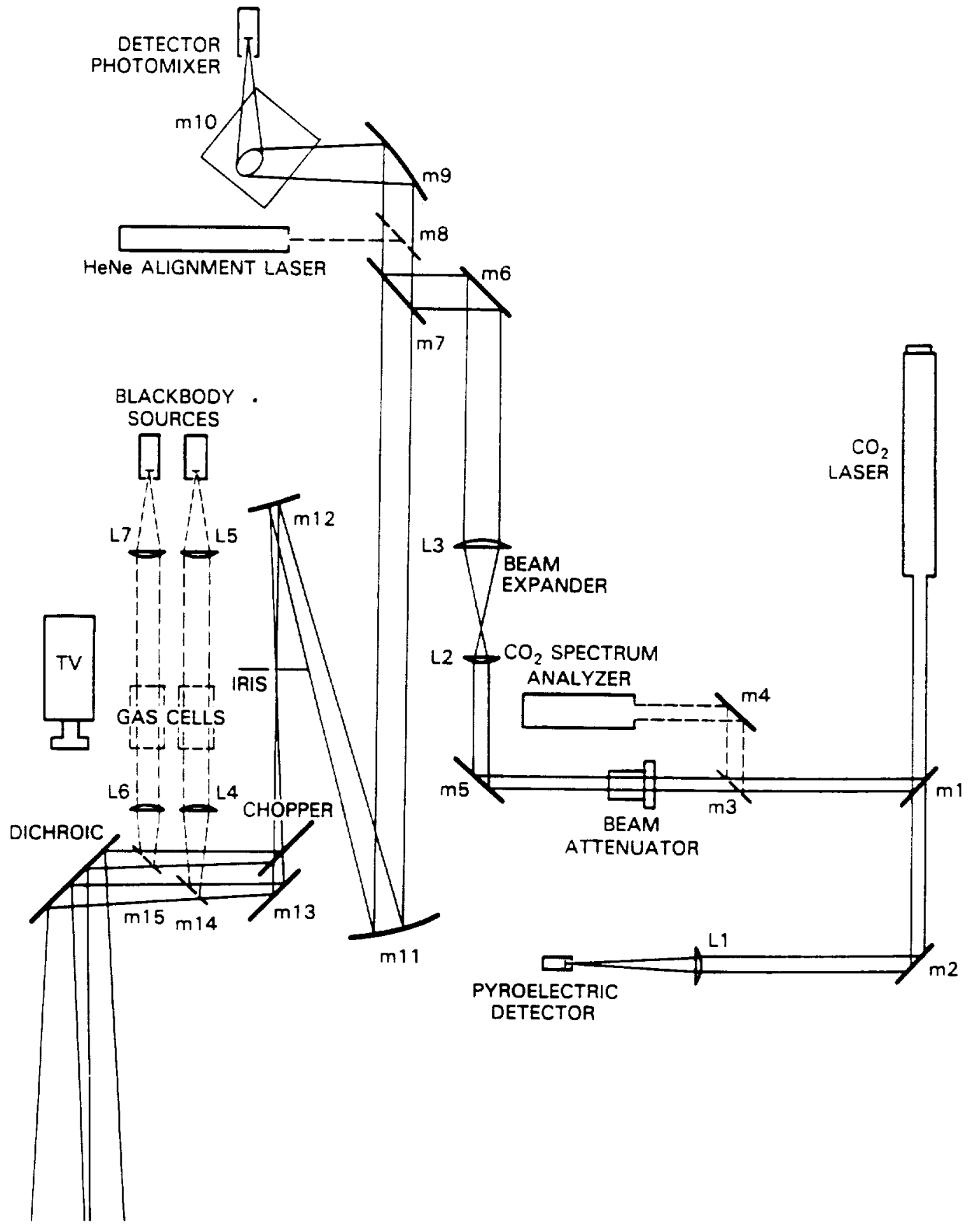

Figure 3.8. The GSFC IR Heterodyne Spectrometer Optical Front End 
located behind the dichroic monitors the position of the target planet with respect to the IR optical axis of the instrument (defined by a cross hair in the TV field-of-view). Flat m13 samples the source beam (s) aligned with the TV camera. A rotating butterfly chopper samples the reference beam $(r)$ which may be located up to $60^{\prime \prime}$ off the $s$ beam for the McMath configuration. The telescope focal plane is located at the system iris.

Figure $3.9 \mathrm{~A}$ details the front end beam throw elements. Shown are rays from both the $s$ beam, aligned with the telescope axis, and the $r$ beam, off axis by angle $\theta$. Points $A$ and $B$ behind the dichroic, separated by distance $d$, mark the foci of the respective beam centers in the absence of the front end optical elements. Points A and B along the instrument axis are the actual foci. (The apparent displacements of the $s$ and $r$ beams are exaggerated.) The beam throw geometry of Figure $3.9 \mathrm{~B}$ implies that the separation distance between chopper and fixed flat dictate $d$. The choice of $d$, coupled with the telescope focal plane image scale, fixes the beam throw $\theta$.

The McMath focal plane image scale is 0.4 arc-minutes per $\mathrm{cm}$. With a maximum mirror separation of $3.5 \mathrm{~cm}$ for McMath, a maximum beam throw of 1 arc-minute is achieved. The chopper/fixed-mirror assembly for the IRTF front end provides a maximum $\mathrm{d}$ of $1 \mathrm{~cm}$. An IRTF focal plane image scale at Coudé of 0.0955 arc-minutes per cm provides a maximum beam throw of only $5.7^{\prime \prime}$.

Venus at inferior conjunction has the largest planetary disk, reaching a 63" diameter during favorable conjunctions. The McMath IRHS $r$ beam can sample sky regardless of $s$ beam position on such a disk. 


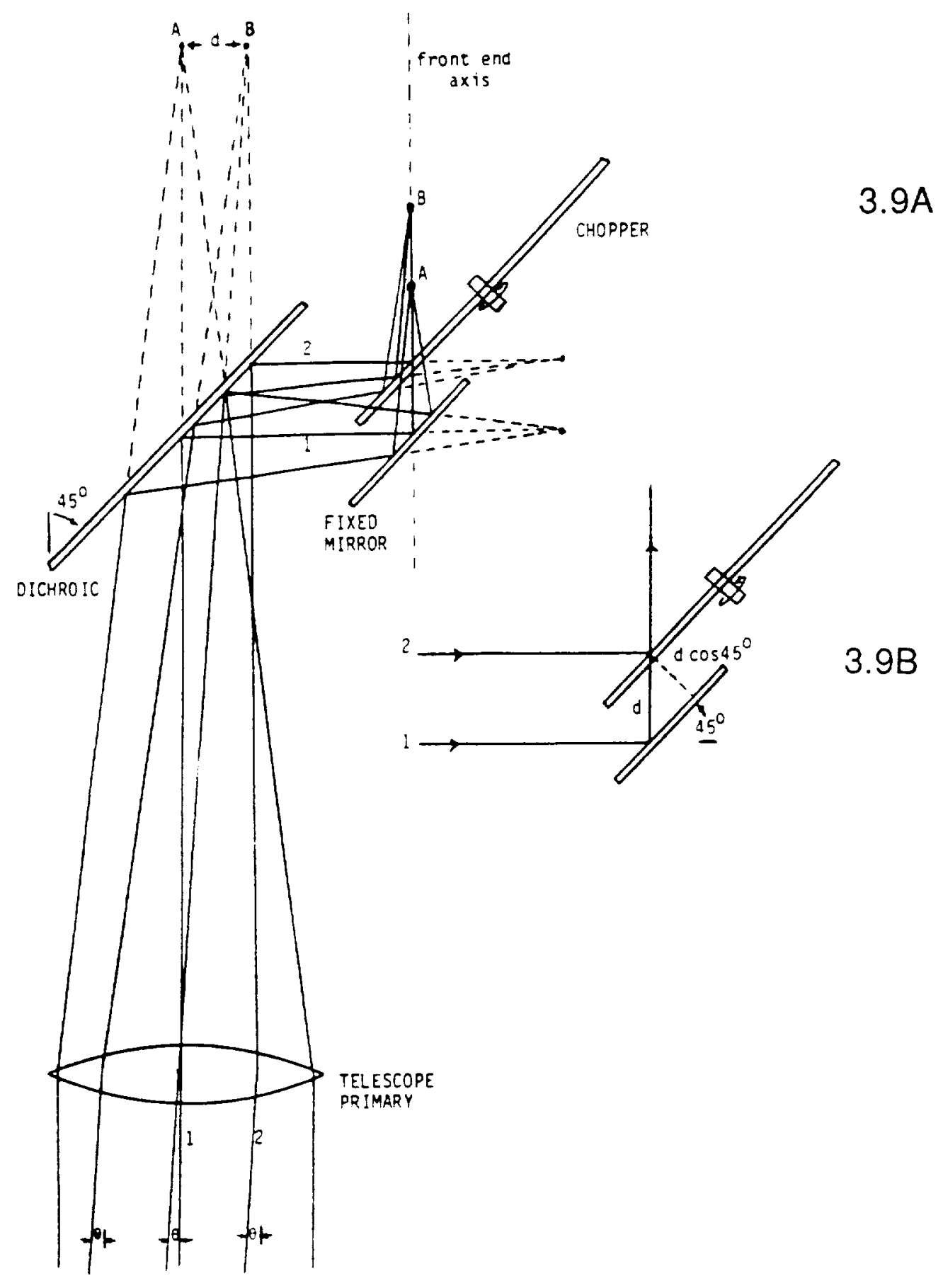

Figure 3.9. Optical Front End Beam Throw 
However, the IRTF front end chopper can only be used in conjunction with planetary disks smaller than $-10^{\prime \prime}$. To observe larger disks at the IRTF, the Facility wobbling secondary is employed, providing a beam throw up to 6 arc-minutes at a frequency $\leq 40 \mathrm{~Hz}$ (synchronized to the IRHS system clock).

Flat m12 directs the $s$ and $r$ beams to off-axis parabola mll, the latter providing collimation. Beam splitter $m 7$ combines the $s$ and $r$ beams with the LO output. Off-axis parabola $\mathrm{m} 9$ focuses the resultant beam onto the detector plane. Flat m10 directs the resultant beam vertically into the down-looking $\mathrm{N}_{2}$ dewar containing the $\mathrm{HgCdTe}$ detector.

Calibration blackbody sources may be substituted for either or both the $s$ and $r$ beams employing flip mirrors $\mathrm{m} 14$ and $\mathrm{m} 15$. Lenses L5 and L7 collimate the blackbody beams allowing generation of absorption lines with the insertion of low pressure gas cells. Such unshifted spectral lines allow periodic monitoring of Lo stability (see Chapter 4). Lenses L4 and L6 focus the blackbody beams onto the telescope focal plane at the system iris.

The alignment of any optical element may be judged with respect to the instrument axis by introducing a HeNe laser with flip mirror m8. (The axis is defined by the system iris and a second transportable rail mounted iris.)

The photomixer, a high speed HgCdTe IR photodiode mounted in a liquid nitrogen dewar at $77 \mathrm{~K}$, is provided by D.L. Spears at M.I.T. Lincoln Laboratory. Detailed reviews of the theory and fabrication of these devices are covered in the 1iterature by Spears (1977, 1981). 
Optimal heterodyne quantum efficiencies are obtained by operating the photodiode in a reverse-bias mode near $500 \mathrm{mV}$ with a laser-induced photocurrent of $-3 \mathrm{ma}$. The photodiode and its matched IF preamp provide heterodyne quantum efficiencies as high as 0.80 at $0 \mathrm{MHz}$ and 0.25 at $1.5 \mathrm{GHz}$ in the IF (Spears 1981).

\subsection{RADIO FREQUENCY BACK END LAYOUT}

The radio frequency ( $R F$ ) back end performs digitization of the source spectral distribution in the IF over a pre-determined bandpass, allowing computer storage and manipulation of data. The bandpass is provided via a contiguous series of equal bandwidth filters. The filter bandwidth is dependent upon the investigation-specific spectral resolution desired.

The bandpass of a filter bank may view any portion of the IF by heterodyning the latter with a frequency tuneable RF local oscillator (RFLO). Given RFLO frequency $\nu_{r f}$, and a region of the IF from $\nu^{\prime}$ o to $\nu^{\prime}{ }_{1}$, then heterodyning provides a beat spectrum from $\nu_{\mathrm{rf}}{ }^{-\nu^{\prime}}$ o to $\nu_{\mathrm{rf}}{ }^{-\nu^{\prime}}{ }_{1}$ (Fig. 3.10).

The present GSFC IRHS system has two separate filter banks. The low resolution bank (LRB) consists of $6425 \mathrm{MHz}$ bandwidth filters. LRB operation, without an RFLO, provides coverage of the IF from 0 to 1600 MHz. The high resolution bank (HRB) consists of $645 \mathrm{MHz}$ bandwidth filters with a bandpass from 1287.5 to $1607.5 \mathrm{MHz}$. The HRB, with RFLO mixing capability, may be tuned to cover any $320 \mathrm{MHz}$ sector of the IF (Fig. 3.10).

Each bank has an associated amplifier section where the IF is 


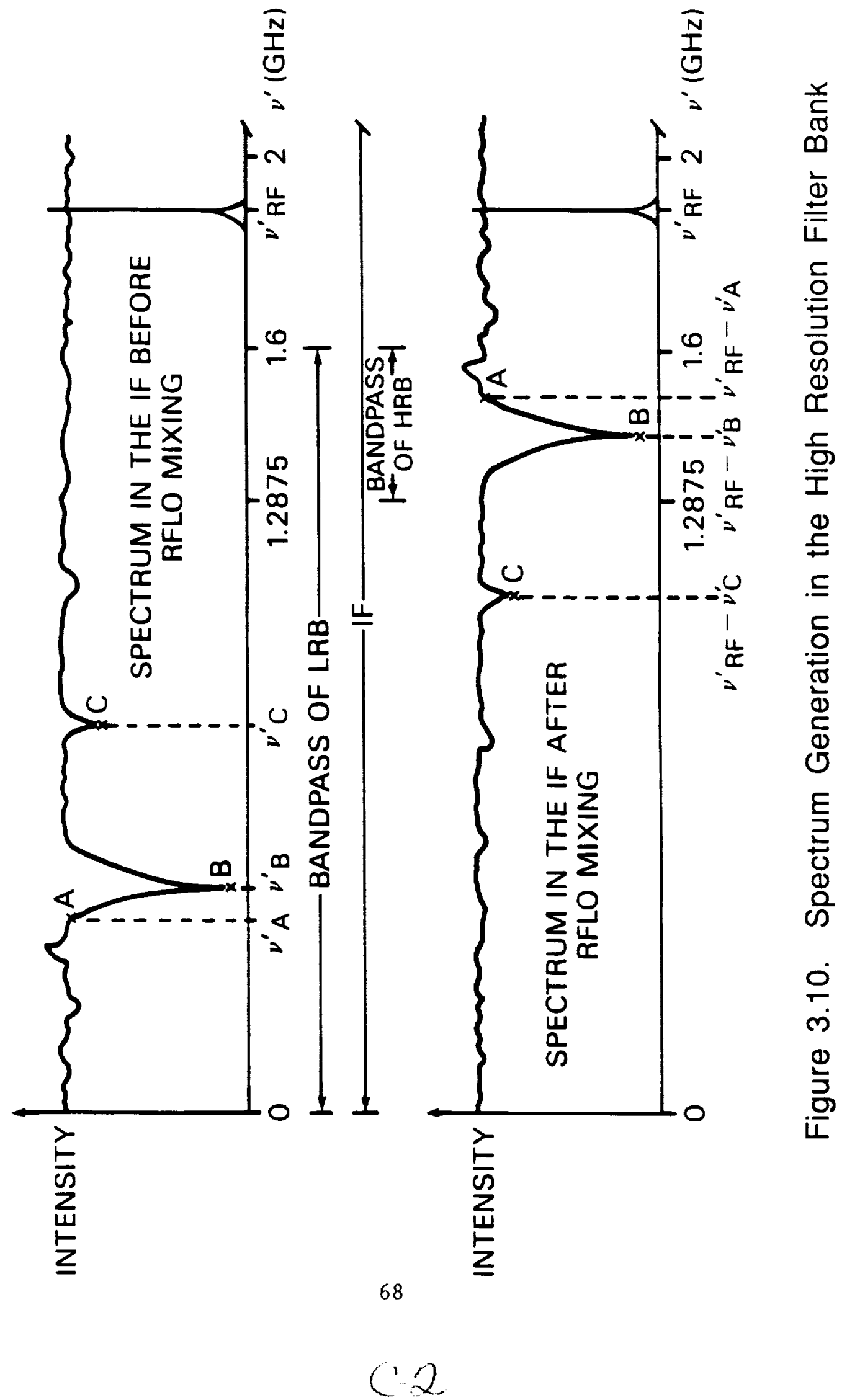


amplified then power split and distributed to 8 racks of 8 filters each (Fig. 3.11). Prior to distribution to the 64 HRB filters, the IF is also heterodyned with an RFLO. Each filter has an RF detector providing an instantaneous output voltage proportional to applied RF power. The outputs from the RF detectors are fed to an analog multiplexer (AMUX) where all 128 channels are simultaneously integrated for a prescribed interval. The AMUX integrator voltages are then fed to a digital multiplexer (DMUX) where two A/D converters, one for each filter bank, digitize the signals.

The IF signal power is dominated by a post-detection noise level with the actual source contribution only a few percent at best. Beam chopping, accomplished either externally to the IRHS (e.g., the IRTF wobbling secondary) or through use of the front end chopper, allows removal of the noise level during data reduction. Data acquisition from two distinct beams is therefore necessary, requiring the filter bank have knowledge of the fundamental chop frequency. To achieve this a system clock (a variable frequency square wave generator) is located within the filter bank. The clock output signal is fed to the DMUX chassis where the characteristic clock period ${ }^{\top}{ }_{\mathrm{cl}}$ is expanded by $5 \times 10^{4}$ to an active chop period $\tau_{\mathrm{ch}}$. The DMUX chassis then provides a phasing signal $\tau_{\text {ph }}$, to whatever beam chopping device is being employed, allowing synchronization to $\tau_{\mathrm{ch}}$. For the 3 -phase McMath front end chopper $\tau_{\mathrm{ph}}=6 \tau_{\mathrm{ch}}$, and the optimal chop frequency is $\sim 10-30 \mathrm{~Hz}$. The associated range in clock frequency is $-0.5-1.5 \mathrm{MHz}$.

The AMUX integrates for almost $(1 / 2) \tau_{\mathrm{ch}}$ before the integrator voltages are first frozen and then sequentially read by the DMUX A/D's. 


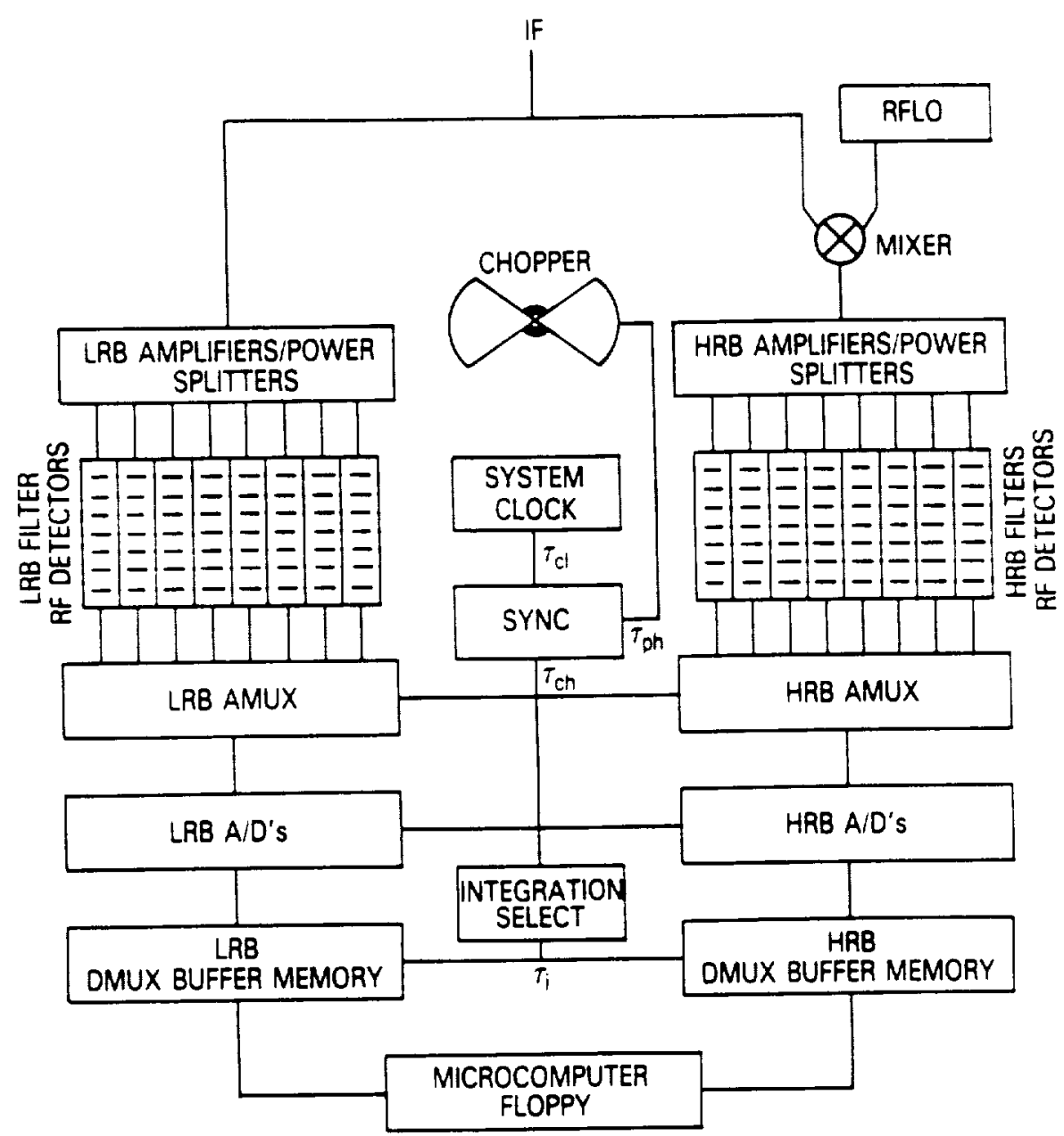

Figure 3.11. The RF Back End

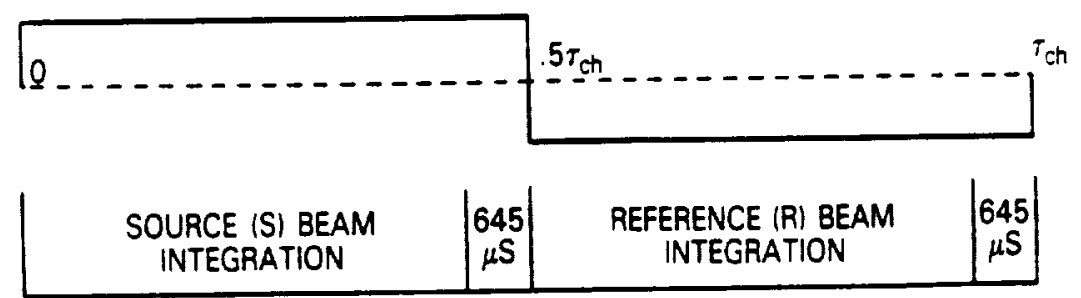

Figure 3.12. RF Detection Sequence 
Reading one channel takes $-10 \mu \mathrm{s}$, thus one $A / D$ will read an entire filter bank in $640 \mu \mathrm{s}$. The second A/D simultaneously reads the other filter bank. After DMUX storage of the digitized integrator voltages, integrators are reinitialized and the second half of the chop period begins. Only $-640 \mu \mathrm{s}$ at the tail end of each $(1 / 2) r_{\mathrm{ch}}$ is down time for the system. The sequence is repeated during the second half of ${ }{ }_{\mathrm{ch}}$ for the second beam (Fig. 3.12).

The DMUX does not dump the digitized IF for both beams directly to the computer but rather integrates for a preset number of chop periods within a buffer memory. One can select an integration time $\tau_{i}$ in units of $256 \times{ }^{\mathrm{ch}} \mathrm{ch}$. Initialization of integrators, DMUX memory dump and the start of an integration are accomplished via the computer console.

In general, in the absence of an incoming IF, the RF detectors have non-zero output voltages. A zero-level reading of all detectors is thus warranted for generation of a digitized IF spectral distribution. Prior to integration, the zero levels are measured, stored in the DMUX, and after $\tau_{i}$, read to disk along with the IF spectra for both beams.

\subsection{FRONT END ALIGNMENT / TARGET POINTING}

A TV system allows continuous monitoring of telescope pointing and target aspect via the TV camera behind the dichroic (see Fig. 3.8). A videotape record is made of the target's image (with the UT date and time superimposed) over the observing period. A cross hair in the TV field-of-view marks the center of the diffraction-limited IR beam. Alignment of the cross hair reticle is critical for any planetary wind 


\section{field mapping.}

Alignment of the monitoring system is begun by introducing the HeNe laser to the front end optics. A flat placed between the dichroic and telescope retroreflects the alignment beam into the camera where its position may be centered on the cross hair. The HeNe beam, however, is too diffuse for alignment to the required pointing accuracy $\left(<1^{\prime \prime}\right)$, and there is no guarantee that the visible and IR beams are colinear. Final alignment may be achieved by training the telescope on an IR point source such as VY CMa, $\alpha$ Ori or o Cet. The broad-band heterodyne signal (displayed on a lock-in amplifier) is peaked on the point source through motion of the telescope. The cross hair reticle is then centered on the visible image on the TV monitor. Differential refraction as a function of air mass must necessarily cause misalignment of the IR and visible beams. A handle on the magnitude of this effect was obtained at KPNO in June 1986. Seeing was good enough during this period to clearly discern the limbs of Venus and Mars in the broad-band heterodyne signal. Mars at opposition was commonly associated with $<1 "$ seeing. By rastering the Martian limb across the TV monitor cross hair, the visible-IR alignment could be determined to 1". Tests performed from 2-3 air masses over two nights failed to detect any secular misalignment of the beams at the 1 " level.

Two modes of guiding are employed in the field. Prior to the observing period, knowledge of the video monitor image scale, and the target planet's disk size, allow generation of acetate templates which provide planetary aspect (see Figure 3.13). Guiding circles are drawn on the templates for each of the target beam positions. Once the 


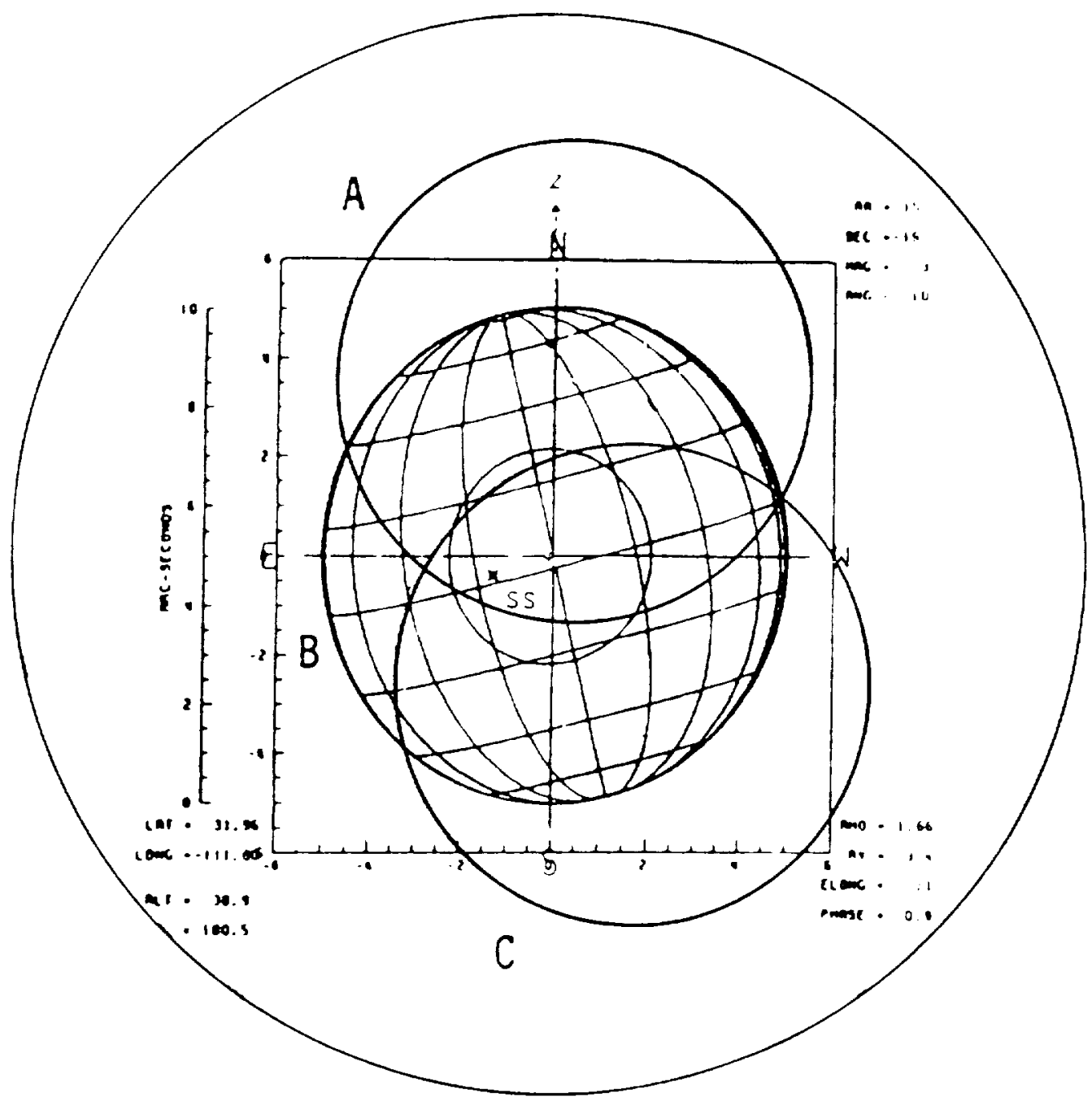

Figure 3.13. Typical Guiding Template: the template origin is aligned with the TV monitor cross hair. The planetary disk is held within one of three guiding circles (ss locates the subsolar point).

circle $\mathrm{A}: 40^{\circ} \mathrm{S}$ - subsolar meridian

circle B: disk center

circle C: $40^{\circ} \mathrm{N}$ - subsolar meridian 
template is affixed to the TV monitor, the observer maintains the planetary disk within a specific guiding circle via a telescope drive paddle. This technique allows continuous correction for telescope track rate drift and low frequency image motion. Due to image rotation in the TV field-of-view, periodic slewing of the telescope N-S or E-W determines whether template rotation is needed.

At the IRTF, computer control allows more passive guiding. Once a zero point is established on the planetary disk, the telescope may be offset to any desired beam position. Tracking rates at Coudé are good to $\pm 2 \mathrm{~m} / \mathrm{hr}$, thus the stability and reproducibility of beam positions depend upon the frequency of zero point realignment.

The McMath Solar Telescope, shown in Figure 3.14 (Pierce 1964), is located on Kitt Peak, Arizona, at an elevation of $2073 \mathrm{~m}$ (6800 ft). The telescope's $2 \mathrm{~m}$ ( 80 -inch) heliostat flat is mounted on a column 30 meters above ground level. The number 2 mirror, the $1.52 \mathrm{~m}$ ( 60 -inch) primary, is located at the end of a 155 meter shaft, 80 meters vertically below the heliostat. The $f / 60$ beam reflected from the $90 \mathrm{~m}$ focal length primary is intercepted at ground level by the number 3 mirror, a $1.2 \mathrm{~m}$ (48-inch) flat that can project the beam into one of three Coudé ports. One port is occupied by the McMath FTS while another is occupied by the GSFC IRHS. The total path length from heliostat to telescope focal plane is $240 \mathrm{~m}$. At $10 \mu \mathrm{m}$, the diffraction-limited beam size is $1.6 "$ (FWHM) in right ascension. In declination, the projected heliostat aperture $P$ goes as:

$$
\mathrm{P}=(\mathrm{D} / 2 / 2)(\cos \delta / 2-\sin \delta / 2),
$$




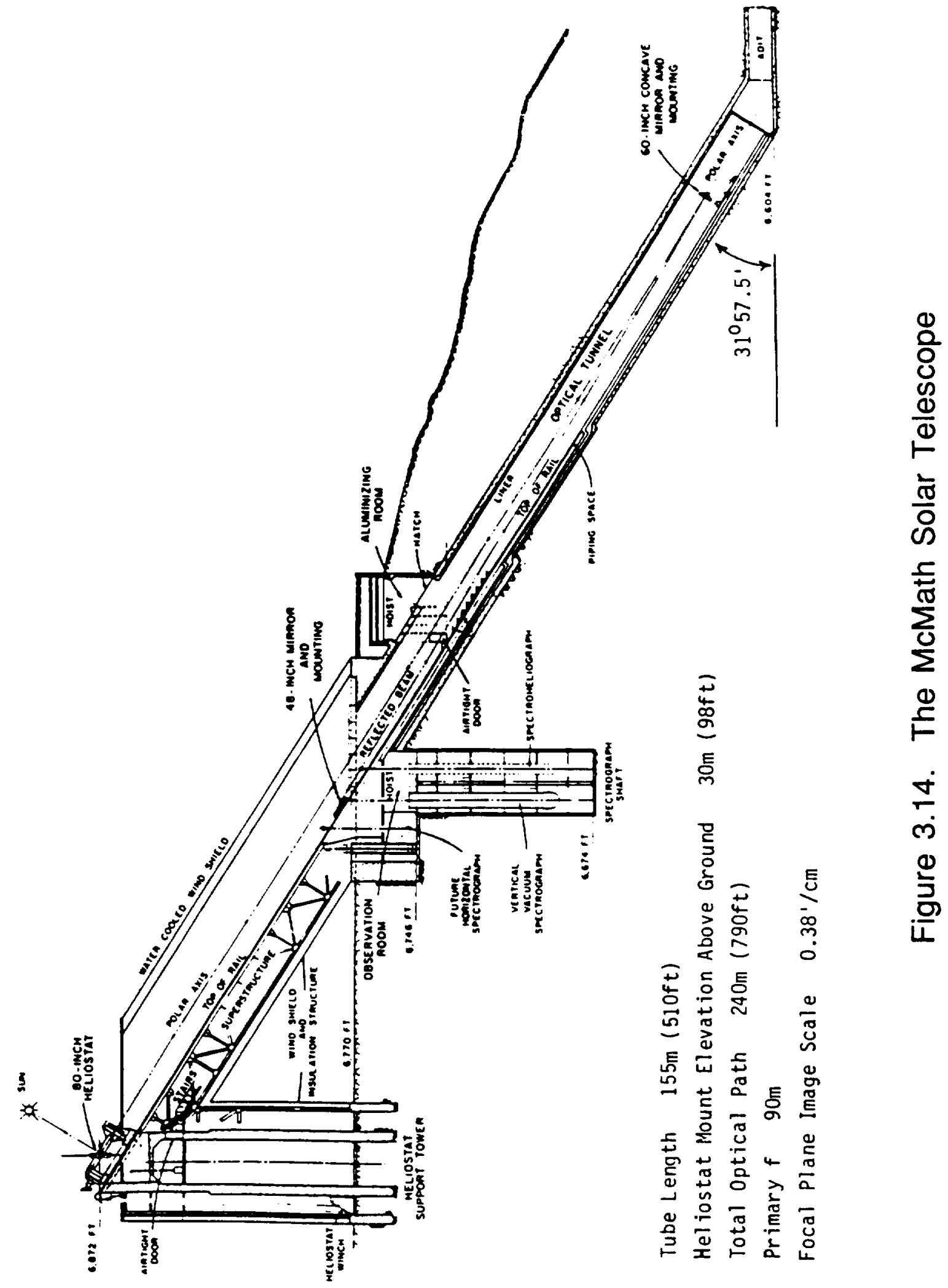


where $D$ is the heliostat diameter and $\delta$ is the target planet's declination. A range in $\delta$ from $-\pi / 6$ to $\pi / 6$ corresponds to a range in $P$ from $0.87 \mathrm{D}$ to $0.5 \mathrm{D}$. For $\mathrm{P}<0.75 \mathrm{D}$, the heliostat no longer fully illuminates the primary. The resulting diffraction-1imited beam size in declination varies from 1.6 to $2.5 "$ (FWHM) for the same declination range.

The NASA IRTF is located on Mauna Kea, on the island of Hawaii, at an elevation of $4100 \mathrm{~m}(13,600 \mathrm{ft})$. The atmosphere above the site has typically $\leq 1 \mathrm{~mm}$ total condensible $\mathrm{H}_{2} \mathrm{O}$ vapor, 688 of the nights are photometric for at least 6 hours, and $<1 "$ seeing is the norm (Cruikshank 1984). Figure 3.15 shows the optical arrangement for the IRTF $3 \mathrm{~m}$ telescope (IRTF Observer's Manual 1985). The Coudé secondary provides an $\mathrm{f} / 120$ beam with a 3 arc-minute field-of-view to a series of four flats contained within the telescope assembly. The last of these flats directs the beam into the Coudé room where a fifth flat couples the beam to the IRHS front end. The diffraction-1imited field-of-view for the telescope is 0.8 " FWHM. 


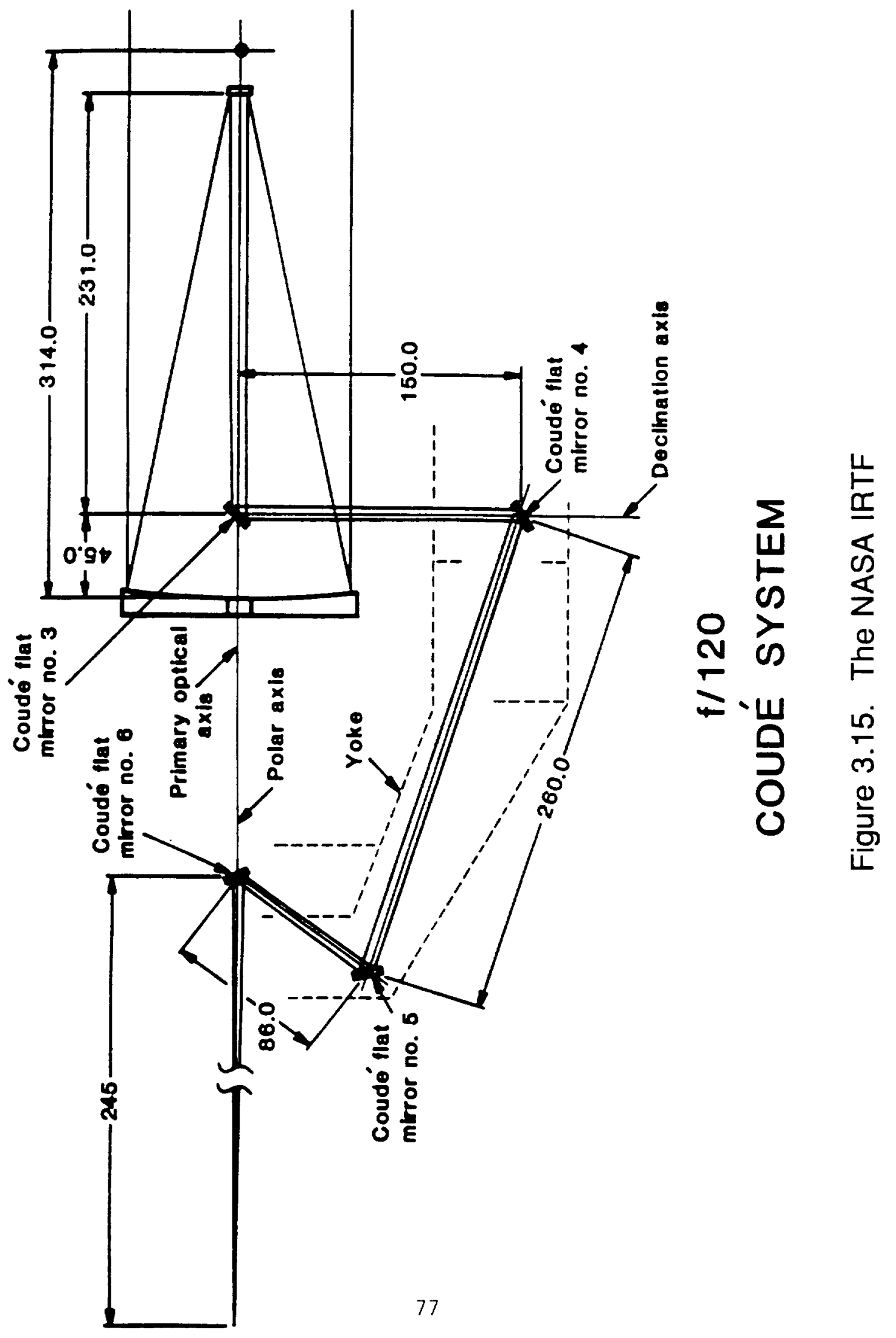





\section{CHAPTER 4: THE LAMB DIP STABILIZATION SYSTEM (LDSS)}

\subsection{BASIC LASER OPERATION}

Typical $\mathrm{CO}_{2}$ laser local oscillators employed in Goddard IRHS systems consist of a laser gain cell axially mounted between a $\sim 5 \%$ transmittance (T) output coupler and a diffraction grating. The grating and output coupler are located on gimballed mounts connected by 4 1-inch diameter rods of either stainless steel or Invar and whose lengths dictate the fixed length of the laser cavity etalon. Rod materials are picked for their low expansion coefficients, minimizing cavity length variation with temperature. In addition to the gain ce11, the length of the cavity allows accommodation of a number of other intracavity devices (Fig. 4.1).

Either the output coupler or diffraction grating is seated on a piezoelectric translator (PZT) within the gimballed mount (Fig. 4.2).

The fixed length of the laser cavity may thus be varied in a controlled manner by applying a known potential difference across the PZT.

With respect to the cavity optical axis, the diffraction grating's gross tilt allows retroreflection of the first order maximum for axially incoming 9-11 $\mu \mathrm{m}$ photons. This bandpass contains the

$\sum_{\mathrm{u}}{ }^{+} 00^{\circ} 1-\sum_{\mathrm{g}}^{+}\left[10^{\circ} 0,02^{\circ} 0\right]_{\mathrm{I}}$ and $\sum_{\mathrm{u}}^{+} 00^{\circ} 1-\sum_{\mathrm{g}}^{+}\left[10^{\circ} 0,02^{\circ} \mathrm{O}\right]_{\mathrm{I}} \mathrm{CO}_{2}$ laser transitions of interest. Within the laser gain cell, each $\mathrm{CO}_{2}$ ro-vibrational downward transition is characterized by emission with a $-170 \mathrm{MHz}$ FWHM Voigt profile due to Doppler and pressure broadening. By varying the tilt of the diffraction grating, one selects which ro-vibrational laser line shall be associated with photons allowed multiple reflections within the cavity. Such photons, on average, 


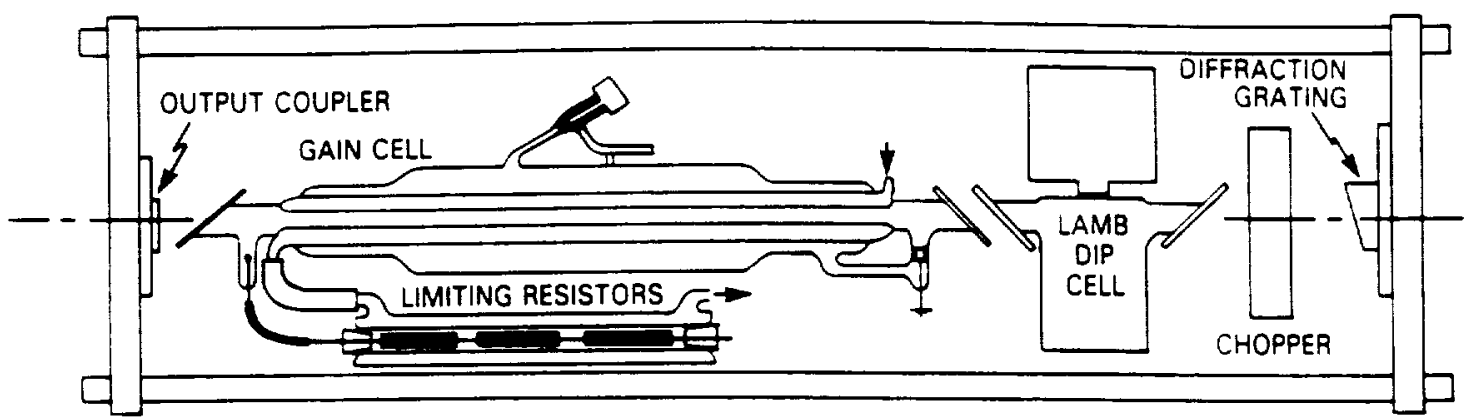

Figure 4.1. The Laser Cavity

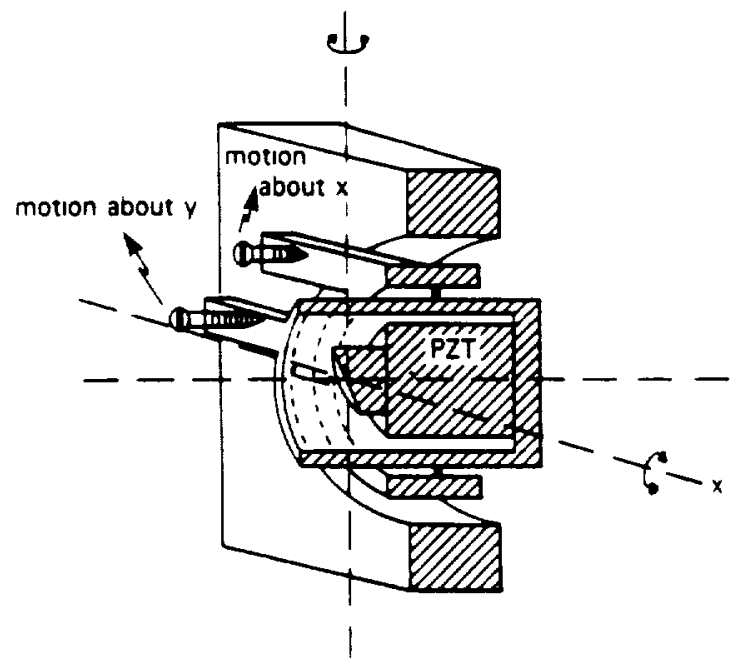

Figure 4.2. The Diffraction Grating Mount and PZT 
undergo $1 / \mathrm{T}$ or 20 circuits of the cavity before exiting through the output coupler. This multiple pass condition allows enough gain through stimulated emission to support only the laser line profile favored by the grating, which is designated the gain profile or gain curve of the laser.

Photon trajectories along the cavity axis necessarily pass through a variety of media such as air, the gain cell's plasma environment, and the windows associated with all intracavity devices. Within medium $i$, with index of refraction $\mathrm{n}_{i}$, a photon whose free-space wavelength is $\lambda_{10}$, is characterized by a wavelength $\lambda_{i}=\lambda_{10} / n_{i}$. If $x_{i}$ represents the path length along the axis within medium $i$, then the number of wavelengths $k$ maintained within the etalon is given by:

$$
\mathrm{k}=\sum\left[\mathrm{x}_{i} / \lambda_{i}\right]
$$

The etalon's effective length $L$ is defined as the length necessary to yield $k$ wavelengths of $\lambda_{10}$, or:

$$
\mathrm{L}=\mathrm{k} \lambda_{10} .
$$

Substituting Eq. 4.1 into 4.2 gives:

$$
\mathrm{L}=\sum\left(\mathrm{x}_{i} \lambda_{10} / \lambda_{i}\right)=\sum\left(\mathrm{x}_{i} \mathrm{n}_{i}\right)
$$

$L$ is a function of both the fixed length of the individual media as well as their indices of refraction. (Note that $\sum \mathrm{x}_{i}$ defines the fixed length of the cavity.)

The cavity etalon will support a standing wave such that effective cavity length is given by:

$$
\mathrm{L}=\mathrm{p} \lambda_{10} / 2=\mathrm{pc} / 2 \nu_{10},
$$


where $\lambda_{10}$ is the supported wavelength, $\nu_{10}$ is the associated standing wave frequency and $p$ is an integer (Fig. 4.3).

The standing wave is not monochromatic, but has a narrow Lorentzian profile $\left(<10 \mathrm{KHz}\right.$ FWHM) centered on $\nu_{10}$. The etalon spoils laser gain for all but the photons within this narrow Lorentzian (Fig. 4.4). By varying effective cavity length with the PZT, one can thus tune the laser output frequency (centered on $\nu_{10}$ ) over the entire gain curve.

Substituting Eq. 4.3 into 4.4 yields:

$$
\nu_{10}=\mathrm{pc} / 2\left(\sum \mathrm{x}_{i} \mathrm{n}_{i}\right)
$$

From the standpoint of a planetary wind investigation, where knowledge of $\nu_{10}$ is critical for a Doppler shift determination, Eq. 4.5 embodies the basic problem - variation in any of the path lengths or indices of refraction provides variation in $\nu_{10}$. Such variations occur continuously during system operation due to changing thermodynamic conditions throughout the laser cavity environment.

\subsection{THE CHARACTER OF THE GAIN CURVE}

In general, two effects preclude a gain curve centered on the laser transition rest frequency $\left(\nu_{1 t}\right)$.

\section{The Gain Cell Pressure Shift}

The pressure associated with the laser plasma produces a shift in line frequency. Perturbation of a molecule's energy states by collision yields resonance radiation whose frequency (on average) differs slightly from the unperturbed case (SooHoo 1984). 


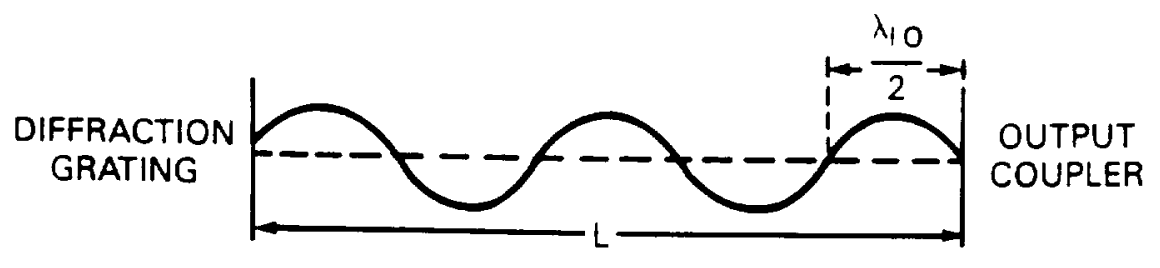

Figure 4.3. A Cavity Etalon Standing Wave

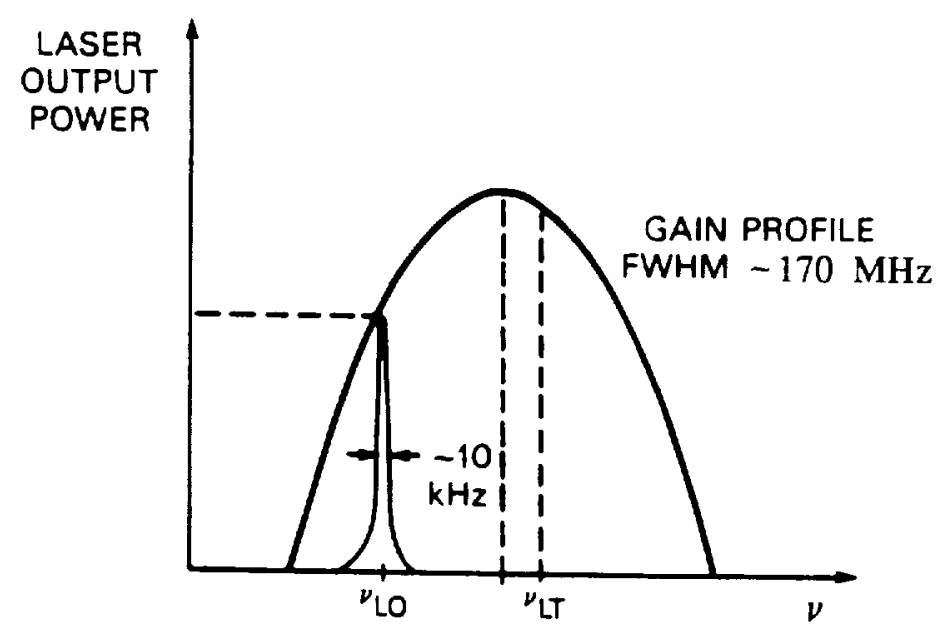

Figure 4.4. The Laser Gain Profile

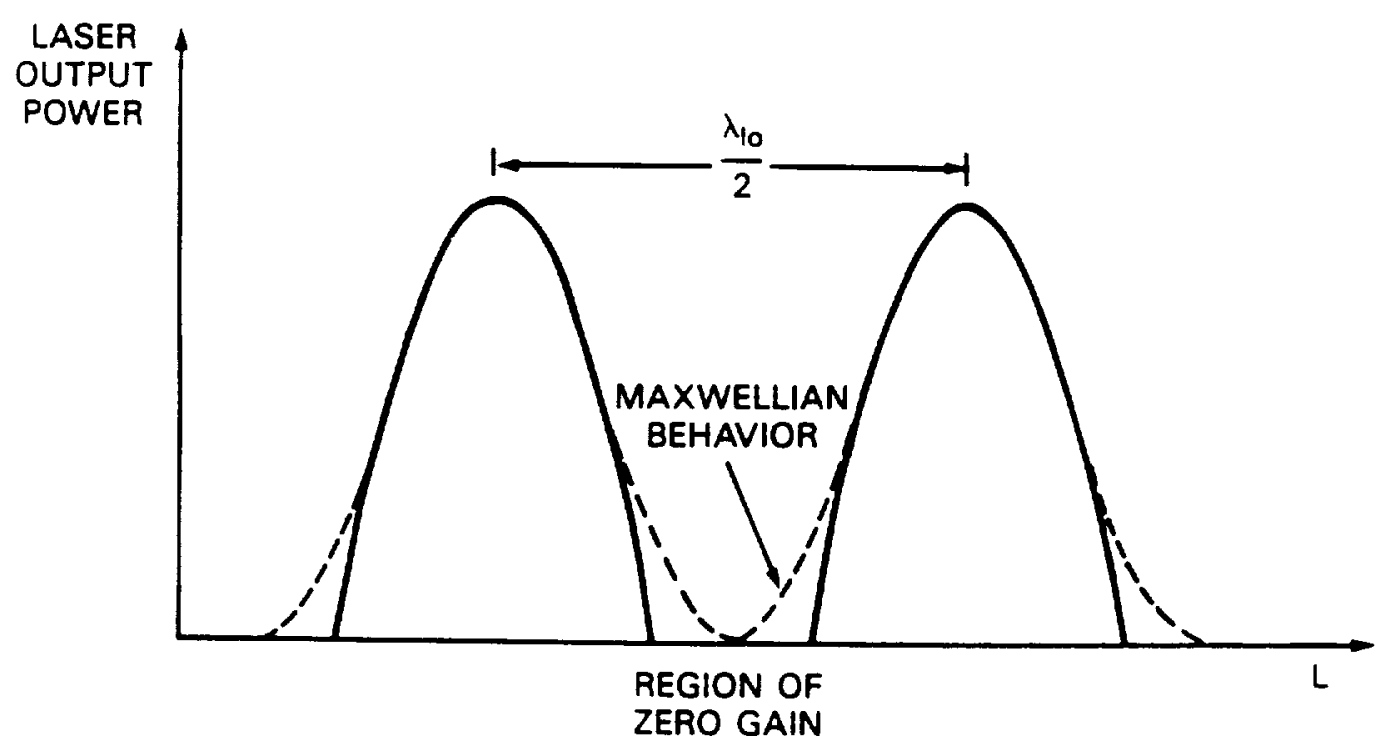

Figure 4.5. Adjacent Gain Profiles 
For the $\mathrm{CO}_{2}-\mathrm{CO}_{2}$ interaction, experimental determination of the pressure shift using saturated resonance techniques have yielded shifts (to the red) of 100-300 KHz/Torr (Freed and Javan 1970, Woods and Jolliffe 1976), and most recently by SooHoo (1984; SooHoo et al. 1985), of $80 \pm 50 \mathrm{kHz} /$ Torr for pressures from 1 to 10 Torr. The laser gain cell maintains a mix of $\mathrm{CO}_{2}$ and four other gases at a total pressure of 16 Torr. The pressure shift in the lasing radiation is thus dictated by both $\mathrm{CO}_{2}-\mathrm{CO}_{2}$ and $\mathrm{CO}_{2}$-foreign molecule collisions. Adopting the results of SooHoo (1984), a redshift of $1.3 \pm 0.8 \mathrm{MHz}$ is expected for pure $\mathrm{CO}_{2}$ at 16 Torr. In the presence of the foreign gas interaction a $\pm 1.0 \mathrm{MHz}$ added uncertainty is not unreasonable (Hillman et a1. 1977).

\section{Laser Cavity Effects on the Gain Profile}

The gain profile is also affected by the laser's spatial mode and the level of simultaneous cavity support for other laser transitions. Such gain profile dependence ultimately rests on the specific laser configuration, the intracavity components, and, most critically, on the cavity alignment.

Changes in cavity alignment will result from cavity assembly/disassembly, long term thermal variation of the cavity rods during Lo operation, and adjustment of output coupler and PZT verniers. The latter is normally associated with LO performance optimization in the field (predominantly during daily system power-up for GSFC LO's).

Variation of cavity alignment can frequency transport the 
gain profile peak a few MHz to either side of the transition rest frequency. Such behavior has been observed in GSFC LO's and is documented in the literature (SooHoo 1984; SooHoo et al. 1985).

In general, combined pressure shift and cavity alignment effects yield a gain profile shift, either red (more likely) or blue, up to possibly a few $\mathrm{MHz}\left(10^{\prime} \mathrm{s}\right.$ of $\mathrm{m} / \mathrm{s}$ at $\left.10 \mu \mathrm{m}\right)$ from $\nu_{1 \mathrm{t}}$. During the course of a days observations, with minimal Lo adjustment, the gain profile shift can be quite constant (in the mean). Such stability was observed spanning 11 hours by Deming et al. (1986).

\subsection{THE CHARACTER OF THE CAVITY ETALON}

The variation in $\nu_{10}$ with effective cavity length may be found by combining Eq. 4.4 and its differential form with the relation $\nu_{10} \lambda_{10}=\mathrm{c}$ to yield:

$$
\mathrm{d} \nu_{10}=\left(\mathrm{c} / \lambda_{10} \mathrm{~L}\right) \mathrm{dL}
$$

On consideration of the terms in Eq. 4.3 one finds that, to first order, the cavity's effective length equals its fixed length. For $\mathrm{L} \sim 1.25 \mathrm{~m}$ and $\lambda_{10}-10 \mu \mathrm{m}$, Eq. 4.6 reduces to $\mathrm{d} \nu_{10} / \mathrm{dL}=24 \mathrm{MHz} / \mu \mathrm{m}$.

If one varies the fixed length by more than $\lambda_{10} / 2$, the cavity will be tuned over more than one gain profile (Fig. 4.5). The gain profile separation is just $\lambda_{10} / 2$ or $-5 \mu \mathrm{m}$. With respect to a single gain profile, such a dL corresponds to $\mathrm{d} \nu_{10}=120 \mathrm{MHz}$.

Adjacent gain profiles do not converge on one another smoothly as might be expected for Maxwellian behavior of the emitting molecules. Too few emitters populate the high energy tails of the $\sum_{u}{ }^{+} 00^{\circ} 1$ 
vibrational state distribution to allow overall gain by stimulated emission. As one tunes the etalon toward the wings of the gain profile one would thus expect a rapid drop to zero laser output power (see Figure 4.5). However, for a broad gain profile (relative to profile separation), adjacent profiles can significantly overlap, as is the case for the cavity constructed (170 MHz profile FWHM, $120 \mathrm{MHz}$ profile separation).

Since the directly measurable quantity associated with the PZT is the applied potential difference $E$, not its contraction in $\mu \mathrm{m}$, a more useful relation than $\mathrm{d} \nu_{10} / \mathrm{dL}$ is $\mathrm{d} \nu_{10} / \mathrm{dE}$. Dividing Eq. 4.6 by $\mathrm{dE}$ yields:

$$
\mathrm{d}_{10} / \mathrm{dE}=\left(\mathrm{c} / \lambda_{10} \mathrm{~L}\right) \mathrm{dL} / \mathrm{dE}
$$

The PZT used in the Goddard IRHS's, the Lansing 21.938, consists of 12 piezoelectric wafers arranged in two stacks, the bias stack and the modulation stack, with 10 and 2 wafers respectively. Within a stack, wafers are connected in parallel (Fig. 4.6). An applied negative going $\mathrm{E}$ causes a contraction of approximately $0.8 \mu \mathrm{m}$ per 1000 $V$ per wafer (though this value may vary slightly from stack to stack). Maximal recommended applied voltage is $-1500 \mathrm{~V}$. A $-12 \mu \mathrm{m}$ cavity length variation is therefore achievable using the bias stack, allowing tuneability over -2.5 gain profiles for $10 \mu \mathrm{m}$ radiation. Small-scale cavity modulation, if desired, may be carried out employing the modulation stack.

Monitoring the laser output with a power meter while sweeping the bias stack through two gain profiles allows one to determine the peak 


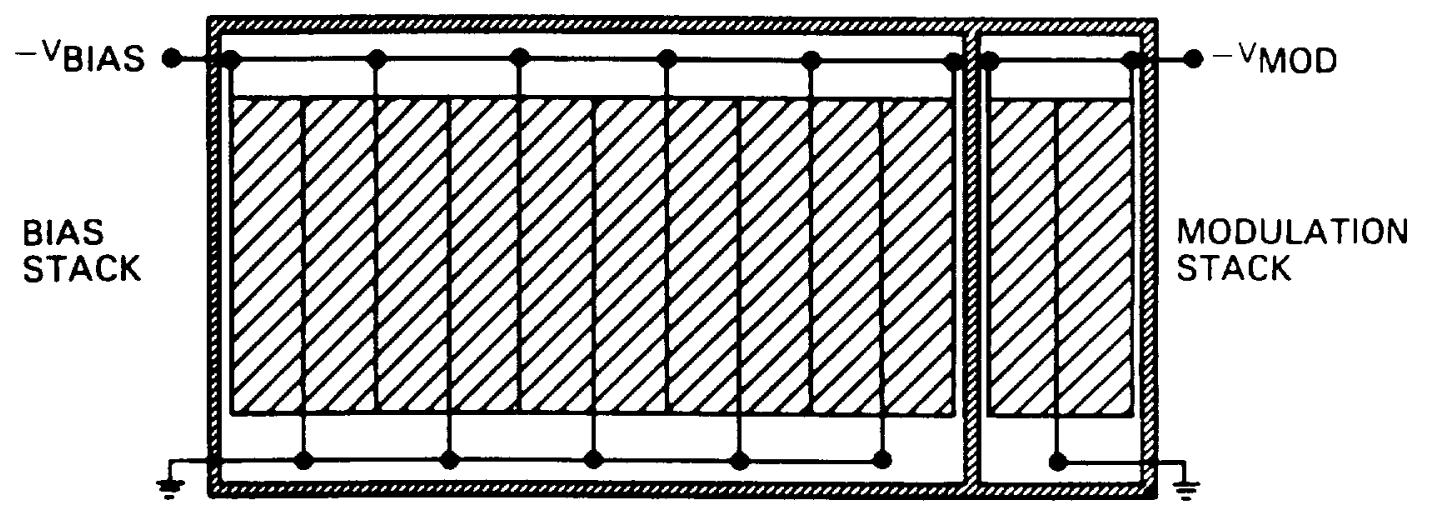

Figure 4.6. PZT Cross-Section

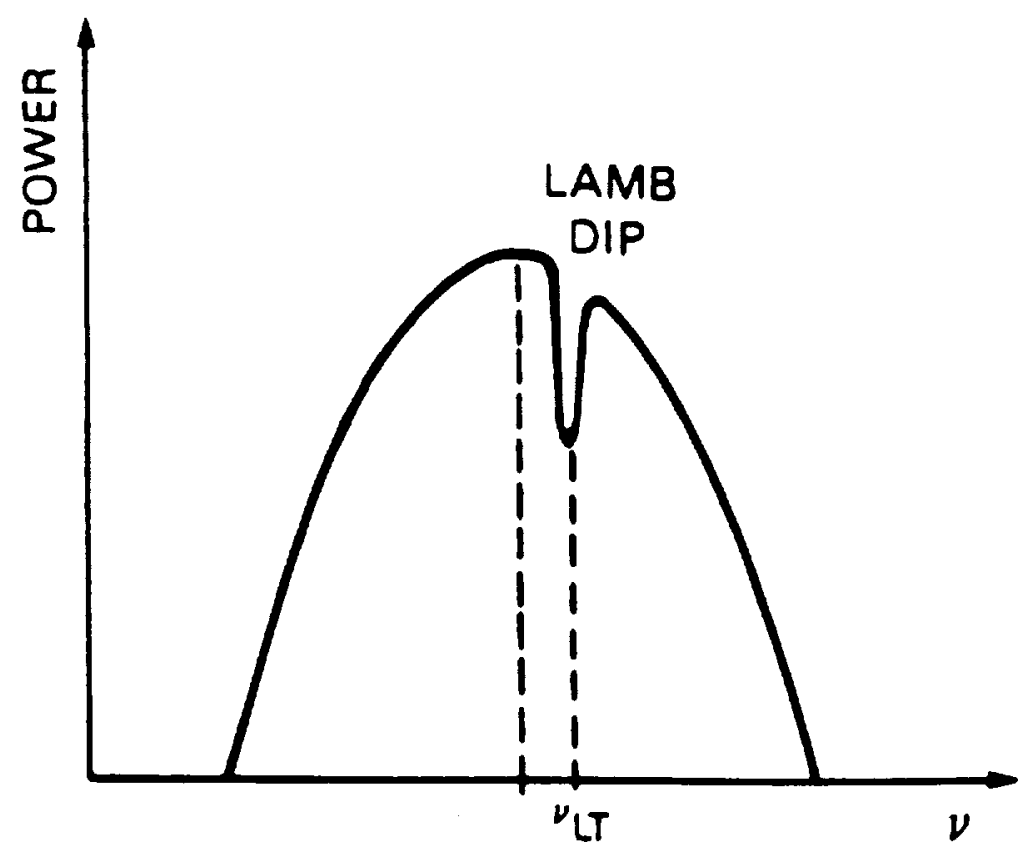

Figure 4.7. The Saturated Resonance (Lamb) Dip 
positions in applied potential difference. If $\Delta \mathrm{E}$ represents the measured potential difference between two gain peaks, corresponding to $\mathrm{dL}=\lambda_{10} / 2$, then substitution into the right side of Eq. 4.7 gives:

$$
\mathrm{d} \nu_{10} / \mathrm{dE}=\mathrm{c} / 2 \mathrm{~L} \Delta \mathrm{E}
$$

Though not directly obvious, Eq. 4.8 is dependent upon the specific laser line used as different lines necessarily have different values of $\Delta \mathrm{E}$. For $\lambda_{10}-10 \mathrm{um}$ and $\mathrm{L}=1.25 \mathrm{~m}, \Delta \mathrm{E}$ corresponds to $-625 \mathrm{~V}$, thus $\mathrm{d} \nu_{10} / \mathrm{dE}=0.2 \mathrm{MHz} / \mathrm{V}$.

Use of different PZT's or variation in $L$ or $\lambda_{10}$ lead to departures in the above derived quantities. Hence, a careful experimental determination of these values is warranted for proper characterization of the soon to be discussed Lamb dip and its associated laser stabilization system.

\subsection{POWER PEAK STABILIZATION}

After digitization of the spectral feature in the IF, the IR source spectrum is obtained by shifting the IF in frequency space by $\nu_{10}$. A spectral resolution of $\lambda / \Delta \lambda=10^{7}$, and the achievable $\mathrm{S} / \mathrm{N}$, allow determination of the IF line center frequency to order $0.1 \mathrm{MHz}(1 \mathrm{~m} / \mathrm{s}$ at $10 \mu \mathrm{m})$. Measurement of a planetary wind field to order $1 \mathrm{~m} / \mathrm{s}$ thus requires that $\nu_{10}$ be:

1. stable to $<0.1 \mathrm{MHz}$. A drift in etalon length of just $0.04 \mu \mathrm{m}$, corresponding a $1 \mathrm{MHz}$ drift in $\nu_{10}$, would render wind measurements to better than $10 \mathrm{~m} / \mathrm{s}$ impossible.

2. known to $<0.1 \mathrm{MHz}$. A planetary wind investigation requires 
acquisition of absolute Doppler shifts, thus requiring an absolute determination of $\nu_{10}$.

A common and easily achieved means of LO stabilization fixes $\nu_{10}$ at the peak of the gain profile. Yet the peak's frequency position $\left(\nu_{\text {lop }}\right)$ is subject to drift, due to variation in either cavity alignment or gain cell pressure. As long as the drift timescale is much greater than the integration time per data scan, intermittent determination of

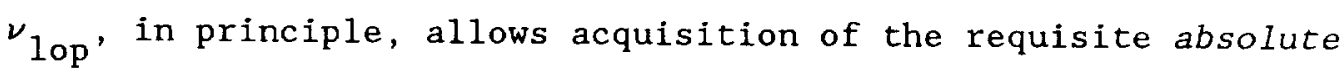
Doppler shifts. This may be accomplished by intermittently (or simultaneously) monitoring a gas cell generated reference line in the field, throughout data acquisition. Given the spectral feature's line center frequency $\nu_{o}$, and the measured value in the IF $\nu_{1 c}$, then $\nu_{1 o p}$ is given by: $\nu_{1 \mathrm{op}}=\nu_{\mathrm{o}} \pm \nu_{1 \mathrm{c}}$. In practice, however, this technique has a number of problems:

i. as $\nu_{10 p}$ can be associated with significant drift (a few $\mathrm{MHz}$; see Section 4.8), nearly continuous monitoring of the reference line, during data acquisition, is essential.

ii. laboratory lines whose $\nu_{0}$ is within an IF bandwidth of $\nu_{\text {lop }}$ are rare. (Conversely, a substantial overlap of the source and reference lines would preclude simultaneous monitoring.)

iii. $\nu_{0}$ of the laboratory line must be known to $<\sim 0.05 \mathrm{MHz}$ for a $10 \mu \mathrm{m}$ wind investigation at the $1 \mathrm{~m} / \mathrm{s}$ level. This criterion constrains candidate lines to an even smaller group than that associated with condition ii.

iv. the $170 \mathrm{MHz}$ (FWHM) gain profile is rather broad if one considers stability to $0.1 \mathrm{MHz}$ essential. Typical stabilization servo 
circuits modulate the etalon length to sense variation in laser output power, allowing determination of the system's instantaneous operating position on the gain profile. A broad peak allows the system to operate significantly far from peak power before the servo circuitry senses correction is necessary. Even in the absence of $\nu_{\text {lop }}$ drift, significant fluctuations in $\nu_{10}$ about $\nu_{1 \text { op }}$ are therefore possible.

The problems associated with power peak stabilization would be removed if the laser could be stabilized absolutely by locking to a frequency-narrow, known absolute frequency standard. The standing wave saturated resonance technique, discussed in Section 4.5, provides such an avenue by generating a small absorption feature, called the Lamb dip, on the gain curve at $\nu_{1 t}$ (Fig. 4.7). The line center of the feature is independent of laser environment and cavity alignment (for the system constructed), and the rest frequencies $\left(\nu_{1 t}\right)$ for the $P$ and $R$ branches of the $\sum_{\mathrm{u}}{ }^{+} 00^{\circ} 1-\sum_{\mathrm{g}}{ }^{+}\left[10^{\circ} 0,02^{\circ} 0\right] \mathrm{I}$ and $\sum_{\mathrm{u}}{ }^{+} 00^{\circ} 1-\sum_{\mathrm{g}}{ }^{+}\left[10^{\circ} 0,02^{\circ} 0\right] \mathrm{II}$ laser transitions of $\mathrm{CO}_{2}$ are known to better than $0.1 \mathrm{MHz}$ for a variety of isotopic forms (Freed et a1. 1980). In addition, the Lamb dip should have the laser's Lorentzian FWHM, as dictated by the cavity etalon, allowing stabilization to much better accuracy than achievable using the comparatively flat peak of the gain profile.

\subsection{LAMB DIP THEORY}

\subsubsection{Basic Theory}

The standing wave saturated resonance technique requires 
introduction of a low pressure gas cell (hereafter designated the "Lamb dip cell" or LDC) to a laser's standing wave field. The laser's working gas and the LDC gas are usually the same.

The standing wave field may be generated outside the laser cavity through beam retroreflection. (Cavity re-entry is prevented through slight misalignment of the retroreflected beam or through polarization rotation.) Intracavity operation, as with the GSFC LDC (see Fig. 4.1), makes use of the standing wave field already present in the cavity etalon, though such operation is associated with far higher power leve1s.

Designate the oppositely directed beams within the cavity as $a^{+}$ and $\mathrm{a}^{-}$. Within the LDC, a molecule will resonantly absorb only radiation at $\nu_{1 t}$, as seen from the molecule's rest frame. A laser detuned from $\nu_{1 t}$ can produce resonant absorption if the molecule's along-the-axis velocity component $V_{\text {axis }}$ induces a Doppler shift in the lasing photons from $\nu_{10}$ to $\nu_{1 t}$.

Figure 4.8 is a ${ }^{12} \mathrm{C}^{16} \mathrm{O}_{2}$ vibrational energy level diagram showing the $9.4 \mu \mathrm{m} \sum_{\mathrm{g}}^{+}\left[10^{0} 0,02^{0} 0\right]_{\mathrm{II}}-\sum_{\mathrm{u}}{ }^{+} 00^{0} 1$ and $10.4 \mu \mathrm{m} \sum_{\mathrm{g}}^{+}\left[10^{0} 0,02^{0} 0\right]_{\mathrm{I}}$ $\sum_{u}{ }^{+} 00^{0} 1$ laser bands. LDC molecules excited to the $\sum_{u}{ }^{+} 00^{0} 1$ state, after random walking out of the beam, are constrained by LDC design parameters (see Section 4.6) to radiatively de-excite to the ground state on a timescale ${ }^{\mathrm{rad}}=441^{-1} \mathrm{~s}$, (assuming an Einstein $\mathrm{A}=441 \mathrm{~s}^{-1}$; Deming and Mumma 1983). This transition is by far the dominant radiative de-excitation pathway $\left(A=0.2 \mathrm{~s}^{-1}\right.$ for both the 9.4 and $10.4 \mu \mathrm{m}$ bands; Murray et al. 1974), and is characterized by $4.3 \mu \mathrm{m}$ emission. Due to collisional coupling of the $\sum_{u}{ }^{+} 00^{0} 1$ rotational levels, emission 

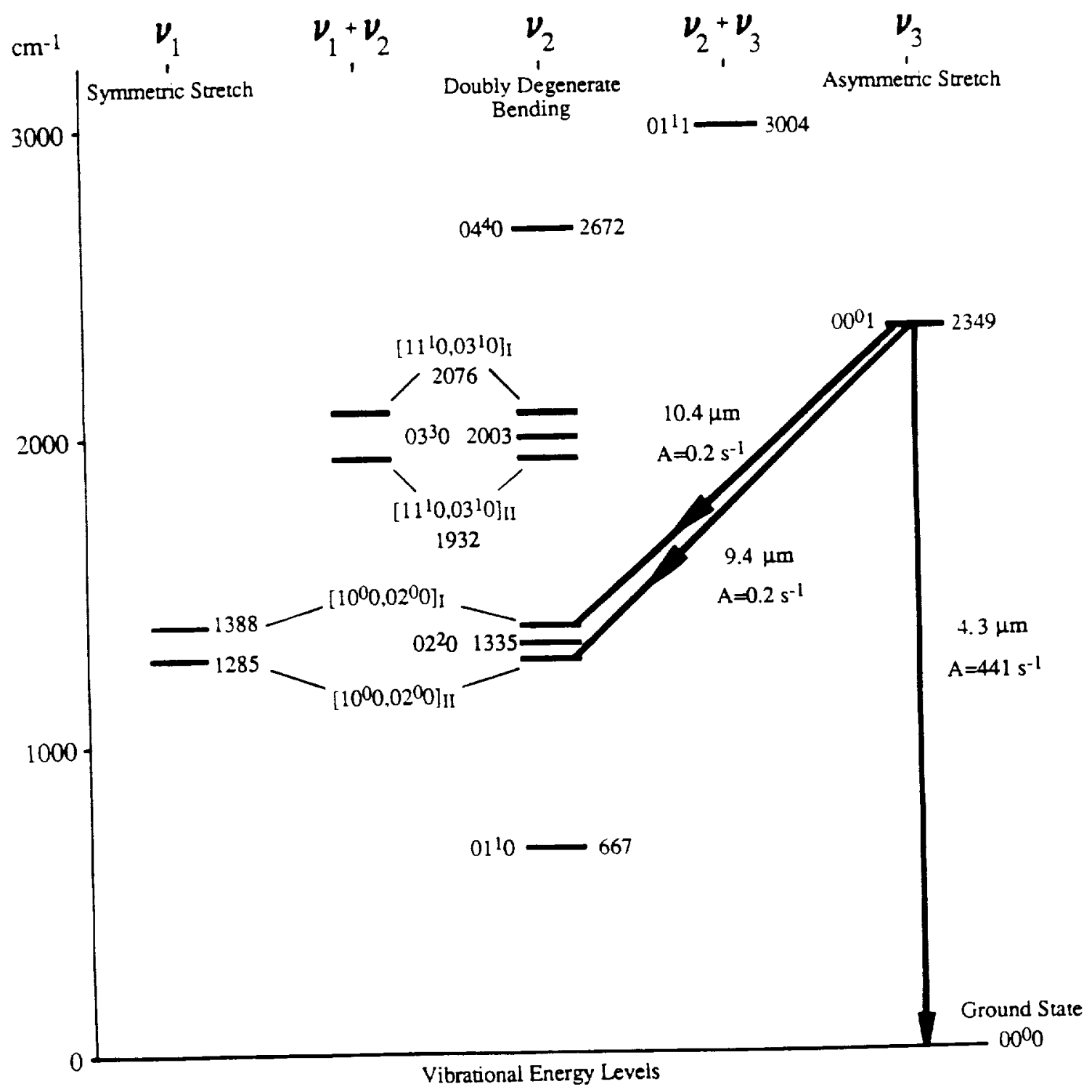

Figure 4.8. Saturated Resonance Energetics for $12 \mathrm{C}^{16 \mathrm{O}_{2}}$ 
takes place over both the $4.3 \mu \mathrm{m} P$ and $R$ rotational bands.

The top plate of Figure 4.9a is a laser gain profile (in general, offset from $\nu_{1 t}$ ), with the laser detuned redward of $\nu_{1 t}$. Frequency $\nu$ is in units of $\Delta \nu_{D} / \sqrt{2}$, where $\Delta \nu_{D}$ is the Doppler halfwidth to $1 / e$. The laser's Lorentzian power distribution is shown in the center plate of the Figure. The lower plate provides the $V_{\text {axis }}$ distribution for LDC molecules in the $\sum_{\mathrm{g}}^{+}\left[10^{0} 0,02^{0} 0\right]_{\mathrm{I} \text {, II }}$ rotational level of interest. $V_{\text {axis }}$ is in corresponding units of the one-dimensional r.m.s. velocity $[\mathrm{kT} / \mathrm{m}]^{1 / 2}$, where $\mathrm{k}$ is Boltzmann's constant, $\mathrm{T}$ is the cell temperature and $\mathrm{m}$ is the molecular weight of ${ }^{12} \mathrm{C}^{16} \mathrm{O}_{2}$.

Photons comprising the $\mathrm{a}^{+}$and $\mathrm{a}^{-}$beams appear Doppler shifted to $\nu_{1 t}$ for molecules with $V_{\text {axis }}=+\left[\left(\nu_{10} / \nu_{1 t}\right)-1\right] c$ and $-\left[\left(\nu_{10} / \nu_{1 t}\right)-1\right] c$, respectively, where positive $V_{\text {axis }}$ is in the direction of the ${ }^{+}$beam. At $10 \mu \mathrm{m}$, this corresponds to $\mathrm{V}_{\text {axis }}=10 \mathrm{~m} / \mathrm{s}$ per $1 \mathrm{MHz}$ detuning from $\nu_{1 \mathrm{t}}$. Resonant absorption thus depopulates two velocity groups, symmetrically located about $\nu_{1 t}$, and characterized by the laser's Lorentzian width (for power levels up to saturation). The two "holes burned" in the $V_{\text {axis }}$ distribution quickly lead to $4.3 \mu \mathrm{m}$ emission, which is detected by a broadband InSb detector.

Figure $4.9 \mathrm{~b}$ provides the same situation for a laser tuned closer to $\nu_{1 t}$. The associated increases in laser power and resonant absorber number density lead to enhanced $4.3 \mu \mathrm{m}$ emission.

On Figure $4.9 \mathrm{c}$, the laser is finally tuned to $\nu_{1 t}$. The absorber population is maximal with $\mathrm{V}_{\text {axis }}=0$, and laser power is nearly maximal (not maximal, in general, due to gain curve offset from $\nu_{1 t}$ ). However, the two absorber populations associated with the $\mathrm{a}^{+}$and $\mathrm{a}^{-}$beams have 


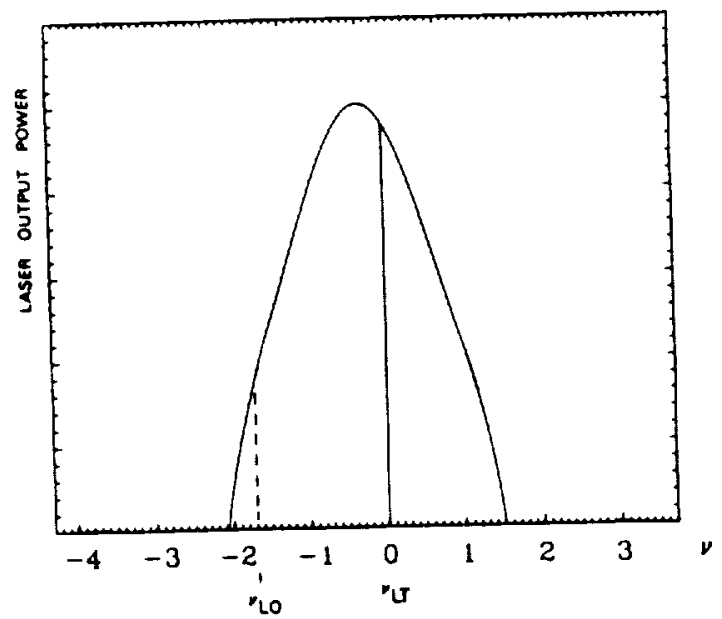

Gain Curve

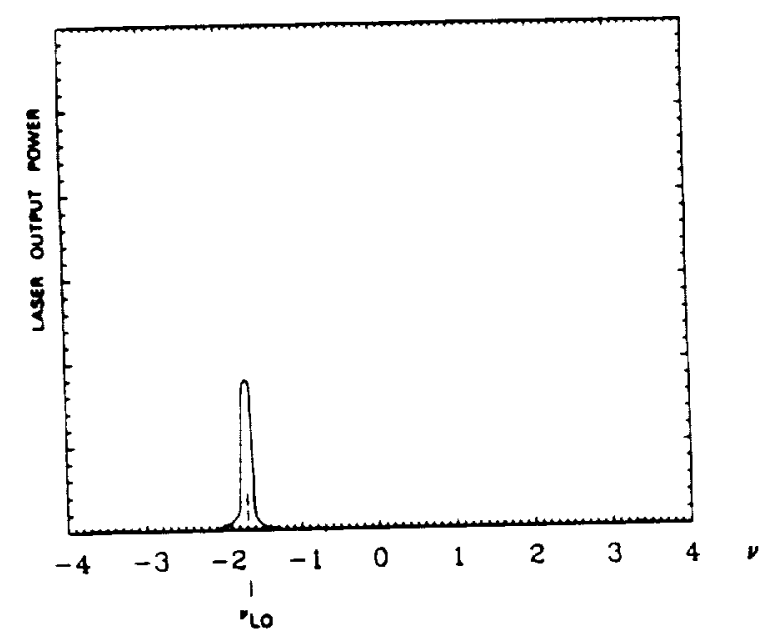

\section{Lorentzian}

Laser Profile

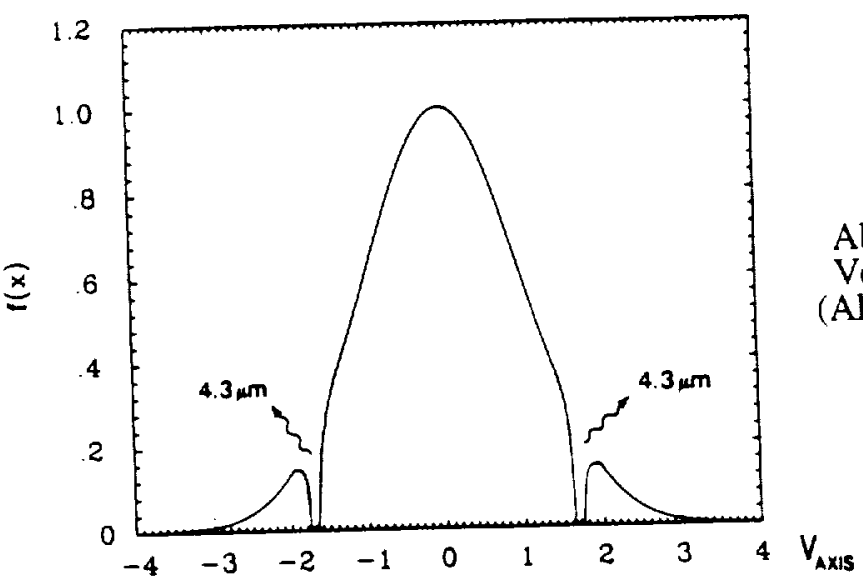

Figure $4.9 \mathrm{a}$ 


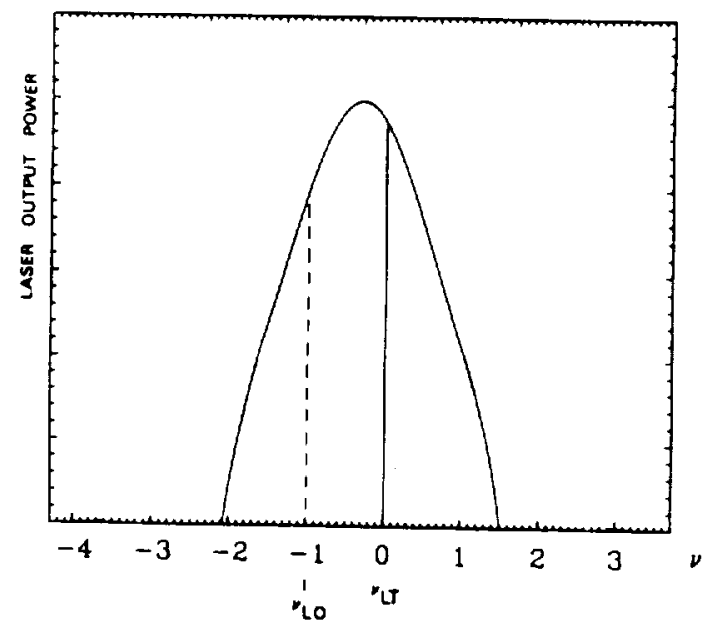

Gain Curve

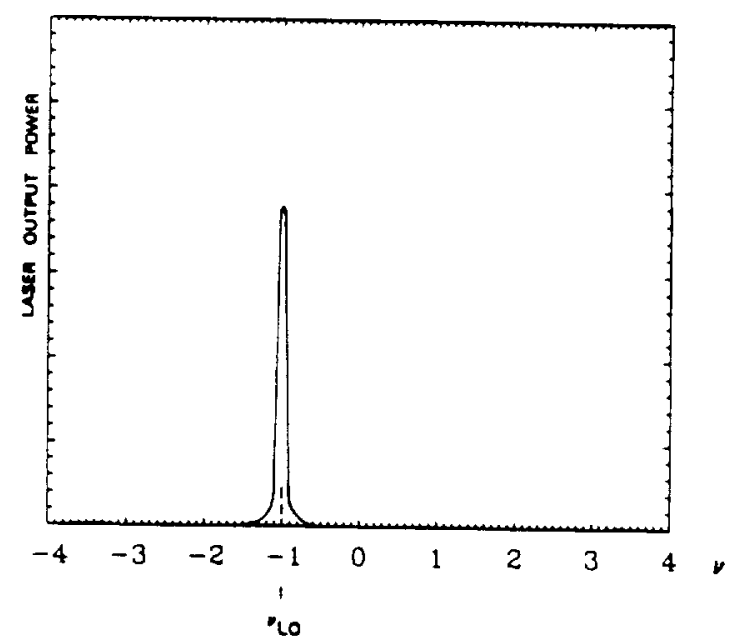

Lorentzian

Laser Profile

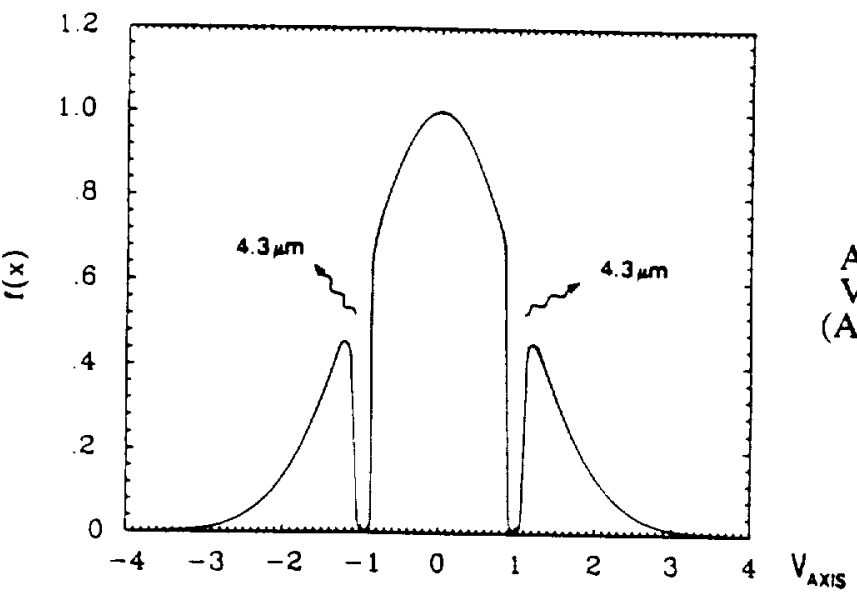

Figure $4.9 b$ 


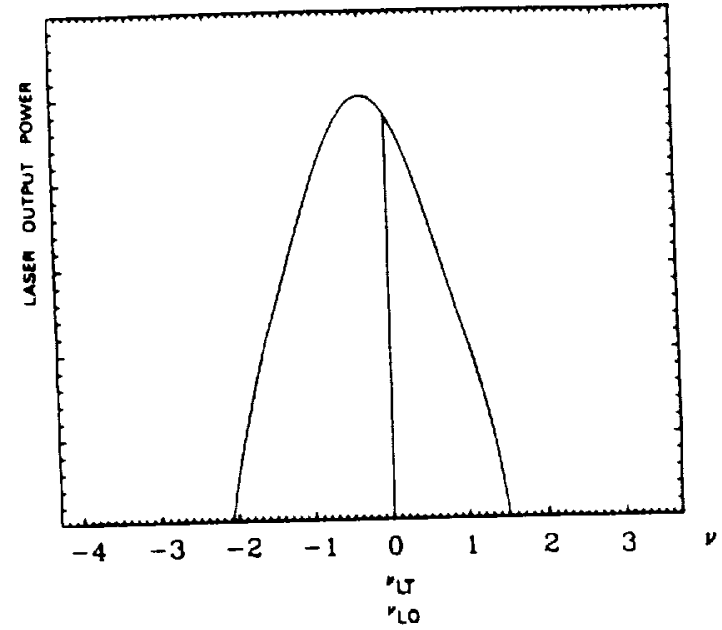

\section{Gain Curve}

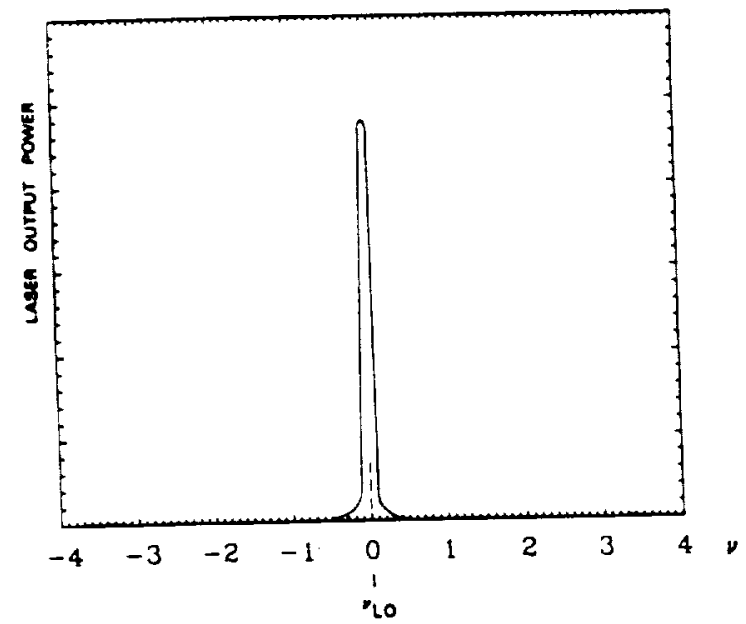

\section{Lorentzian \\ Laser Profile}

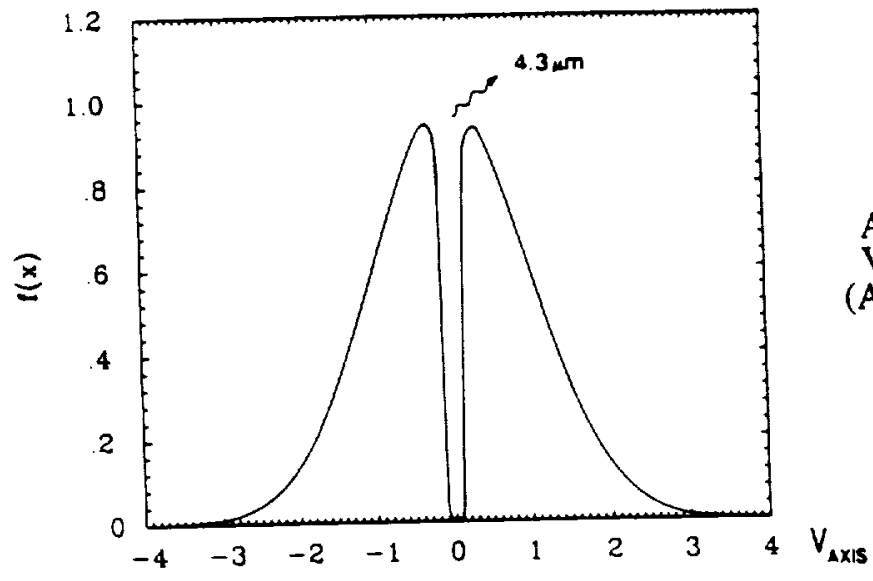

Figure $4.9 \mathrm{c}$ 
now coalesced into a single population. The resulting $4.3 \mu \mathrm{m}$ signal therefore suffers a diminution, compared to its value just before laser tuning to $\nu_{1 t}$ (when both populations had not yet coalesced).

As one tunes the laser cavity across the entire $10 \mu \mathrm{m}$ gain profile, the $4.3 \mu \mathrm{m}$ signal power traces a gain profile with a resonant dip at $\nu_{1 t}$ (the Lamb dip) as shown on Figure 4.7. In general, due to $10 \mu \mathrm{m}$ gain profile asymmetry about $\nu_{1 t}$, the $4.3 \mu \mathrm{m}$ signal power for the laser detuned blueward of $\nu_{1 t}$ will be different than for the laser detuned redward by the same amount. The result is a $4.3 \mu \mathrm{m}$ gain profile with an assymetrically located Lamb dip (see Fig. 4.7).

\subsubsection{The Lamb Dip FWHM}

The GSFC intracavity LDC produces a 3 to $4 \mathrm{MHz}$ FWHM Lamb dip, considerably broader than the laser line width. Possible Lamb dip broadening mechanisms include: pressure broadening, transit time broadening, acoustical broadening, misalignment broadening, and power broadening.

i. A $\mathrm{CO}_{2}$ pressure broadening coefficient of $7.5 \mathrm{MHz} / \mathrm{Torr}$ (FWHM) implies 150 to $300 \mathrm{KHz}$ broadening (FWHM) for the 20 to $40 \mathrm{mTorr}$ nominal pressures in the Lamb dip cell. A 400 mTorr pressure would be necessary for $3 \mathrm{MHz}$ broadening.

ii. Transit time broadening results from the interaction between absorber and laser field as the absorber transits the beam. The Uncertainty Principle gives $\Delta \nu_{t t} \Delta \mathrm{T}_{r}=[2 \pi]^{-1}$, where $\mathrm{T}_{\mathrm{r}}$ is the FWHM transit time across the beam, and $\Delta \nu_{t t}$ is the FWHM transit time broadening (Letokhov and Chebotayev 1977). $T_{r}$ is given by: 
$\mathrm{T}_{\mathrm{r}}=2 \mathrm{r}_{\mathrm{o}} / \mathrm{v}$, where $\mathrm{r}_{\mathrm{o}}$ is the HWHM beam radius and $\mathrm{v}$ is the r.m.s. absorber velocity. $\Delta \nu_{t t}=13.1 \mathrm{KHz}$ for ${ }^{12} \mathrm{C}^{16} \mathrm{O}_{2}$ at room temperature $\left(v=4.1 \times 10^{4} \mathrm{~cm} / \mathrm{s}\right)$, with $r_{0}=-0.25 \mathrm{~cm}$ for GSFC LO's.

iii. Acoustical pickup-induced high frequency modulation of the cavity length will produce laser frequency broadening. A peak-to-peak modulation amplitude of $0.12 \mu \mathrm{m}$ will cause $3 \mathrm{MHz}$ (FWHM) broadening. Significant attempts at laser cavity vibrational isolation were undertaken, including: floating the cavity optical bench on dampening pads and construction of a cavity isolation enclosure. No change in the Lamb dip FWHM was observed.

iv. If the $a^{+}$and $a^{-}$laser beams through the Lamb dip cell are not spatially coincident, broadening will result. For cells external to the laser cavity, misalignment of the beams is used to prevent the return beam from re-entering the laser cavity (polarization rotation may also be used). However, intracavity cells, like the one developed for this investigation, are not associated with misalignment broadening.

v. The holes burned in the Lamb dip cell absorber population by the $\mathrm{a}^{+}$and $\mathrm{a}^{-}$laser beams will no longer have the beam FWHM for laser powers beyond the hole saturation level. In this regime, increasing laser power will burn wider holes in the absorber population, causing power broadening. A power broadened Lamb dip results as the cavity is tuned to $\sim \nu_{1 t}$ where the two holes coalesce.

SooHoo (1984) observed an increasing Lamb dip FWHM (from 
0.94 to $1.09 \mathrm{MHz}$ ) with increasing laser output power (from 0.3 to 1.8 watts), using an external cell at 30 mTorr pressure. Pressure broadening and misalignment broadening, the only other significant broadening effects for his system, contributed 0.23 $\mathrm{MHz}$ and $-0.35 \mathrm{MHz}$ to the dip FWHM, respectively. The latter assumes $-70 \mathrm{KHz}$ per milliradian of misalignment (SooHoo et al. 1985), and $\sim 5$ milliradians of misalignment (SooHoo 1984). Power broadening was therefore claimed significant.

Mechanisms i through iv above cannot account for the observed 3-4 MHz FWHM dip obtained using the GSFC intracavity cell. An indication of power broadening involvement (mechanism $v$ ) may be obtained by assuming a linear relationship between dip FWHM and laser power, a relation borne out to first order by the SooHoo (1984) measurements. (This assumption, however, may be invalid when extrapolating to intracavity circulating power levels.)

The circulating power through the GSFC intracavity cell is typically 40 Watts (in one direction) corresponding to 2 Watts of laser output power and a $5 \%$ transmittance output coupler. A least squares fit to the four SooHoo (1984) 30 mTorr FWHM measurements: 0.94, 1.00, 1.03, $1.09 \mathrm{MHz}$ (from SooHoo (1984) Fig. 27), corresponding to power levels of $0.3,0.7,1.1$ and 1.8 watts, respectively, yields a $4.4 \mathrm{MHz}$ FWHM dip for 40 watts (after removing misalignment broadening). This is consistent with the GSFC observed Lamb dip FWHM.

SooHoo et al. (1985) obtained a power and misalignment broadening-limited $0.7 \mathrm{MHz}$ FWHM Lamb dip with their external cell at 10 mTorr pressure. Significantly narrower dips could be achieved by 
decreasing the laser power density through beam expansion and employing an intracavity cell (SooHoo et al. 1985). While narrower Lamb dips would facilitate a higher precision lock to $\nu_{1 t}$, the $3-4 \mathrm{MHz}$ FWHM dip achieved with the GSFC intracavity cell, and the associated servo feedback electronics, provide a lock to $\nu_{1 t} \pm 0.04 \mathrm{MHz}(0.4 \mathrm{~m} / \mathrm{s}$ at 10 $\mu \mathrm{m}$ ) for a 20 minute integration (see section 4.8). This is already at the level of uncertainty in ephemeris-derived planet-planet line of sight velocity. Fortunately, a Venus wind field map to order $1 \mathrm{~m} / \mathrm{s}$ is certainly adequate.

\subsubsection{Lamb Dip Frequency Shift Mechanisms}

A number of mechanisms will cause a Lamb dip frequency departure from $\nu_{1 t}$. They have been fully investigated by SooHoo et al. (1985; SooHoo 1984) and include: pressure shifting, the combined effects of transit time and wavefront curvature, the second order Doppler shift, absorber recoil, and power slope error.

i. The best determination of the $\mathrm{CO}_{2}-\mathrm{CO}_{2}$ pressure shift was made by SooHoo (1984; SooHoo et al. 1985) using a two channel heterodyne system with both lasers stabilized to the Lamb dip. For pressures between 20 and $120 \mathrm{mTorr}, 43.8$ to $87.2 \mathrm{kHz} /$ Torr blueshifts were observed for four $\mathrm{CO}_{2}$ isotopic forms. Little $\mathrm{J}$ dependence was found and statistical differences between the I and II bands were not significant.

For the ${ }^{12} \mathrm{C}^{16} \mathrm{O}_{2} 967.7 \mathrm{~cm}^{-1} \mathrm{R}(8)$ line, used in the Venusian wind investigation, a pressure shift of $62.1 \pm 18.1 \mathrm{KHz} / \mathrm{Torr}$ is expected (SooHoo et al. 1985). At a nominal pressure of 20 to 
40 mTorr, the GSFC intracavity Lamb dip cell should therefore produce a total shift of only $1.2 \pm 0.4$ to $2.5 \pm .7 \mathrm{KHz}$ (1.2 to 2.5 $\mathrm{cm} / \mathrm{s}$ at $10 \mu \mathrm{m})$, blueward.

ii. The frequency shift due to transit time and wavefront curvature has been investigated in detail by Bordé et a1. (1976; see SooHoo et al. 1985). The shift was parametrized in terms of the relaxation parameter $\left(\mathrm{p}_{r}\right)$ and the transit time $\left(\mathrm{T}_{r}\right)$, where the former is defined as the product of $\mathrm{T}_{r}$ and the Lamb dip FWHM. The frequency shift decreases with increasing $P_{r}$. For GSFC LO's, $\mathrm{T}_{\mathrm{r}}=1.2 \times 10^{-5} \mathrm{~s}$ and the Lamb dip FWHM is $-4 \mathrm{MHz}$, thus $\mathrm{p}_{\mathrm{r}}=49$. For $\mathrm{p}_{\mathrm{r}}=10$, Bordé et al. (1976) found a shift of $-7.5 \mathrm{KHz}(7.5 \mathrm{~cm} / \mathrm{s}$ at $10 \mu \mathrm{m})$. For $\mathrm{p}_{\mathrm{r}}=49$, the associated shift should be significantly less than $1.5 \mathrm{KHz}(1.5 \mathrm{~cm} / \mathrm{s})$, again negligible.

iii. The second order Doppler shift is redward and given by: $-v^{2}[2 c \lambda]^{-1}$, where $v$ is the r.m.s. absorber velocity, $c$ is the speed of light and $\lambda$ is the transition rest wavelength (SooHoo et a1. 1985). $v=4.1 \times 10^{4} \mathrm{~cm} / \mathrm{s}$ for the room temperature Lamb dip cell with a ${ }^{12} \mathrm{C}^{16} \mathrm{O}_{2}$ fill, yielding a second order Doppler shift of only $28 \mathrm{~Hz}(0.028 \mathrm{~cm} / \mathrm{s})$ for $10 \mu \mathrm{m}$ transitions.

iv. The recoil shift is given by: $\pm h\left[2 m \lambda^{2}\right]^{-1}$, where $h$ is Planck's constant and $\mathrm{m}$ is the absorber mass (SooHoo et al. 1985). The recoil shift is only $\pm 45.1 \mathrm{~Hz}(0.045 \mathrm{~cm} / \mathrm{s})$ for ${ }^{12} \mathrm{C}^{16} \mathrm{O}_{2} 10 \mu \mathrm{m}$ transitions.

v. In general, the Lamb dip will not be coincident with the peak of the gain profile, resulting in an asymmetric dip. Superposition of the Lamb dip onto a non-zero gain profile slope causes a dip 
frequency departure from $\nu_{1 t}$, termed power slope error (PSE) (SooHoo et al. 1985). The PSE may be red or blue.

SooHoo (1984) modelled the PSE as proportional to the cell pressure squared and decreasing with increasing laser power (for a given $\nu_{1 t}$ offset from gain peak). For a 0.3 watt power level, the model yielded PSE's of $-7.9,1.17$ and $0.1 \mathrm{MHz}$ for 140,80 and 30 mTorr, respectively (as taken from Fig. 40 SooHoo 1984). A 50 $\mathrm{MHz}$ gain profile FWHM and a $1 \mathrm{MHz} \nu_{1 t}$ offset from gain peak were assumed. A power level of 3 watts yielded PSE's of -2.2 and 0.7 $\mathrm{MHz}$ for 140 and $80 \mathrm{mTorr}$, respectively.

SooHoo's (1984) pressure shift measurements made in the presence of a PSE (the uncorrected pressure shift) were found, as expected, to be red or blue depending on diffraction grating tilt, hence $\nu_{1 t}$ offset dependent. The PSE was therefore easily capable of masking the cell-associated pressure shift for pressures $>-40$ mTorr (see Fig. 46 SooHoo 1984) and laser power levels from 0.3 to 3 watts. However, the magnitude of the observed PSE was significantly less than the model predictions (even with $\nu_{1 \mathrm{t}}$ offsets from gain peak typically up to $2 \mathrm{MHz}$ (SooHoo 1988; see SooHoo et al. 1985)).

As SooHoo's external cell was associated with low power levels, a means of PSE removal was mandatory for pressure shift measurements. This was accomplished by sensing the $10 \mu \mathrm{m}$ laser output and $4.3 \mu \mathrm{m}$ fluorescence signals simultaneously. Through diffraction grating adjustment, with the laser locked to the Lamb dip, the gain profile peak was positioned coincident with the 
dip, thereby removing the PSE.

The PSE, for a given $\nu_{1 t}$ offset from gain peak, was obtained by comparing the uncorrected pressure shift to the actual

pressure shift. A typical blueward uncorrected pressure shift data set (taken from Fig. 41 SooHoo 1984) yields PSE's of only $14.8,8.1$ and $3.9 \mathrm{KHz}$ for power levels of $0.5,1$ and 2 watts respectively, with an 80 mTorr cell pressure. At $30 \mathrm{mTorr}$ and 2 watts of power, the uncorrected and corrected pressure shifts were indistinguishable, implying an undetectable PSE at the $2 \mathrm{KHz}$ leve1 $(2 \mathrm{~cm} / \mathrm{s}$ at $10 \mu \mathrm{m})$.

As pointed out by SooHoo (1988), the $\mathrm{CO}_{2}$ absolute frequency line atlas by Freed et al. (1980), for seven isotopic forms, was made before the PSE effect was recognized. The data set was acquired with a two-channel heterodyne system of the same basic design as SooHoo's (Freed et a1. 1980), with $50 \pm 15$ mTorr Lamb dip cell pressures (Bradley et al. 1986). Standard deviations in ${ }^{12} \mathrm{C}^{16} \mathrm{O}_{2}$ and ${ }^{13} \mathrm{C}^{16} \mathrm{O}_{2}$ absolute frequencies were typically 50-100 $\mathrm{KHz}$, reflecting predominantly the absolute frequency zero point uncertainty in the 9.33 and $10.18 \mu \mathrm{m}{ }^{12} \mathrm{C}^{16} \mathrm{O}_{2}$ calibration lines (measured by Evenson et a1. 1973). However, the directly measured heterodyne difference frequencies, bearing the effects of PSE, were an order of magnitude more reproducible. A revised line atlas, for 9 isotopic forms (Bradley et al. 1986), was compiled using the old data set in conjunction with a very precise absolute determination of ${ }^{12} \mathrm{C}^{16} \mathrm{O}_{2} 10.18 \mu \mathrm{m} \mathrm{R}(30)$ (Petersen et al. 1983). The new standard deviations reflected, 
amoung other things, the recognized effects of PSE. Typical ${ }^{12} \mathrm{C}^{16} \mathrm{O}_{2}$ and ${ }^{13} \mathrm{C}^{16} \mathrm{O}_{2}$ standard deviations were $<5 \mathrm{KHz}$.

Thus, for the GSFC intracavity cell, the typical 40 watts of circulating power implies a PSE significantly less than $5 \mathrm{KHz}$ ( 5 $\mathrm{cm} / \mathrm{s}$ at $10 \mu \mathrm{m}$ ) for a cell pressure of $30 \mathrm{mTorr}$.

For the GSFC system, the combined frequency offset effects should therefore shift the Lamb dip from $\nu_{1 t}$ by $<0.1 \mathrm{~m} / \mathrm{s}$.

\subsection{THE LAMB DIP CELL}

The Lamb dip cell (LDC) design was based on a set of first-order constraints, as presented below. The success of this approach eliminated the need for detailed balancing models to optimize cell geometry.

An intracavity, low pressure LDC was most desirable, rendering power slope error and the cell-associated pressure shift/broadening negligible. Intracavity operation warranted a compact design, which was facilitated by room temperature operation and a sealed cell configuration. The need for temperature control and flow system fixtures was thereby removed.

The LDC holds pure $\mathrm{CO}_{2}$ of the same isotopic form as the gain cell working gas. Within the $\mathrm{LDC}, 10 \mu \mathrm{m}$ laser emission excites $\mathrm{CO}_{2}$ molecules from the $\sum_{\mathrm{g}}{ }^{+}\left[10^{0} 0,02^{\circ} 0\right]_{\mathrm{I}, \text { II }}$ to the $\sum_{\mathrm{u}}{ }^{+} 00^{0} 1$ state (see Fig. 4.9), forming $\mathrm{CO}_{2}{ }^{*}$. The Lamb dip is detected in the subsequent $4.3 \mu \mathrm{m}$ spontaneous emission. This emission can be spoiled by $\mathrm{CO}_{2}{ }^{*}$ collisional de-excitation with either the cell wall or other $\mathrm{CO}_{2}$ molecules. 
Therefore, through variation of design parameters, the $4.3 \mu \mathrm{m}$ spontaneous de-excitation timescale $\tau_{\mathrm{rad}}=2.27 \times 10^{-3} \mathrm{~s}$ (for an Einstein $A=441 \mathrm{~s}^{-1}$ ) must be constrained as the characteristic timescale of the system.

$$
{ }^{12} \mathrm{C}^{16} \mathrm{O}_{2}{ }^{*} \text { has an r.m.s. velocity } \mathrm{v}_{\mathrm{rms}}=4.1 \times 10^{4} \mathrm{~cm} / \mathrm{s} \text {, at room }
$$

temperature $(\mathrm{T}=293 \mathrm{~K})$, thereby traversing a distance $\left(\mathrm{d}=\mathrm{v}_{\mathrm{rms}}{ }^{\tau} \mathrm{rad}\right)$ of $96.7 \mathrm{~cm}$ before spontaneously emitting at $4.3 \mu \mathrm{m}$. This distance is necessarily folded into a random walk due to $\mathrm{CO}_{2}{ }^{*}-\mathrm{CO}_{2}$ collisions. To minimize $\mathrm{CO}_{2}{ }^{*}$ de-excitation with the cell wall, the cell radius $\mathrm{R}$ must be greater than the random walk distance $\mathrm{d}$ (assuming $\mathrm{CO}_{2}{ }^{*}$ excitation near the center of the LDC). In the absence of $\mathrm{CO}_{2}{ }^{*}-\mathrm{CO}_{2}$ collisions, $\mathrm{d}$ is $96.7 \mathrm{~cm}$, far too large for an intracavity LDC. Yet the more $\mathrm{CO}_{2}{ }^{*}-\mathrm{CO}_{2}$ collisions introduced to decrease $\mathrm{d}$, the greater the chance of $\mathrm{CO}_{2}{ }^{*}-\mathrm{CO}_{2}$ collisional de-excitation. Assume for the moment that the latter does not take place.

\section{Minimizing $\mathrm{CO}_{2}{ }^{*} / \mathrm{cell}$ wall collisional de-excitation}

The random walk distance is given by:

$$
\mathrm{d}=\mathrm{n}^{1 / 2} \mathrm{~L}_{\mathrm{mf}}
$$

where $\mathrm{n}$ is the number of collisions suffered over $\tau_{\text {rad' }}$, and $\mathrm{L}_{\mathrm{mf}}$ is the $\mathrm{CO}_{2}{ }^{*}$ mean free path. But:

$$
\mathrm{v}_{\mathrm{rms}}{ }^{\tau} \mathrm{rad}^{\mathrm{nL}} \mathrm{mf} \text {. }
$$

Substituting Eq. 4.10 into 4.9, and noting that $R \geq d$, yields:

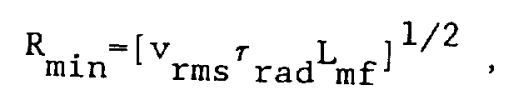

where $R_{\text {min }}$ is the minimum LDC radius for a given $\mathrm{CO}_{2}$ environment 
within the cell. $L_{m f}$ is given by:

$$
\mathrm{L}_{\mathrm{mf}}=[\mathrm{N} \sigma]^{-1}
$$

where $\mathrm{N}$ is the $\mathrm{CO}_{2}$ number density and $\sigma$ is the $\mathrm{CO}_{2}$ collision cross-section. $N$ may be obtained from the ideal gas law:

$$
\mathrm{P}=\mathrm{NkT} \text {, }
$$

where $P$ is the gas pressure, $k$ is Boltzmann's constant, and $T$ is the gas temperature. Substituting Eqs. 4.12 and 4.13 into 4.11 yields:

$$
R_{\min }=\left(\frac{v_{\mathrm{rms}^{\top} \mathrm{rad}^{\mathrm{kT}}}}{\mathrm{Po}}\right)^{1 / 2}
$$

The molecular collision cross-section is given by (see Rosenberg 1977, Dushman 1962):

$$
\sigma=2^{1 / 2} \pi \delta^{2}
$$

where $\delta$ is the molecular collision diameter, usually defined in terms of the Lennard-Jones potential energy function (see Rosenberg 1977, Taylor and Bitterman 1969).

For ${ }^{12} \mathrm{C}^{16} \mathrm{O}_{2}$ at room temperature, $\sigma=6.92 \times 10^{-15} \mathrm{~cm}^{2}$ (assuming a $3.947 \times 10^{-8} \mathrm{~cm}$ molecular collision diameter; Taylor and Bitterman 1969), and Eq. 4.14 reduces to:

$$
\mathrm{R}_{\min }=13 \cdot 0 / \mathrm{P}^{1 / 2}
$$

for $R_{\min }$ in $\mathrm{cm}$ and $P$ in mTorr.

Figure 4.10 is a plot of $R_{\text {min }}$ versus $P$, as given by Eq.

4.16. A reasonably sized intracavity cell is associated with 


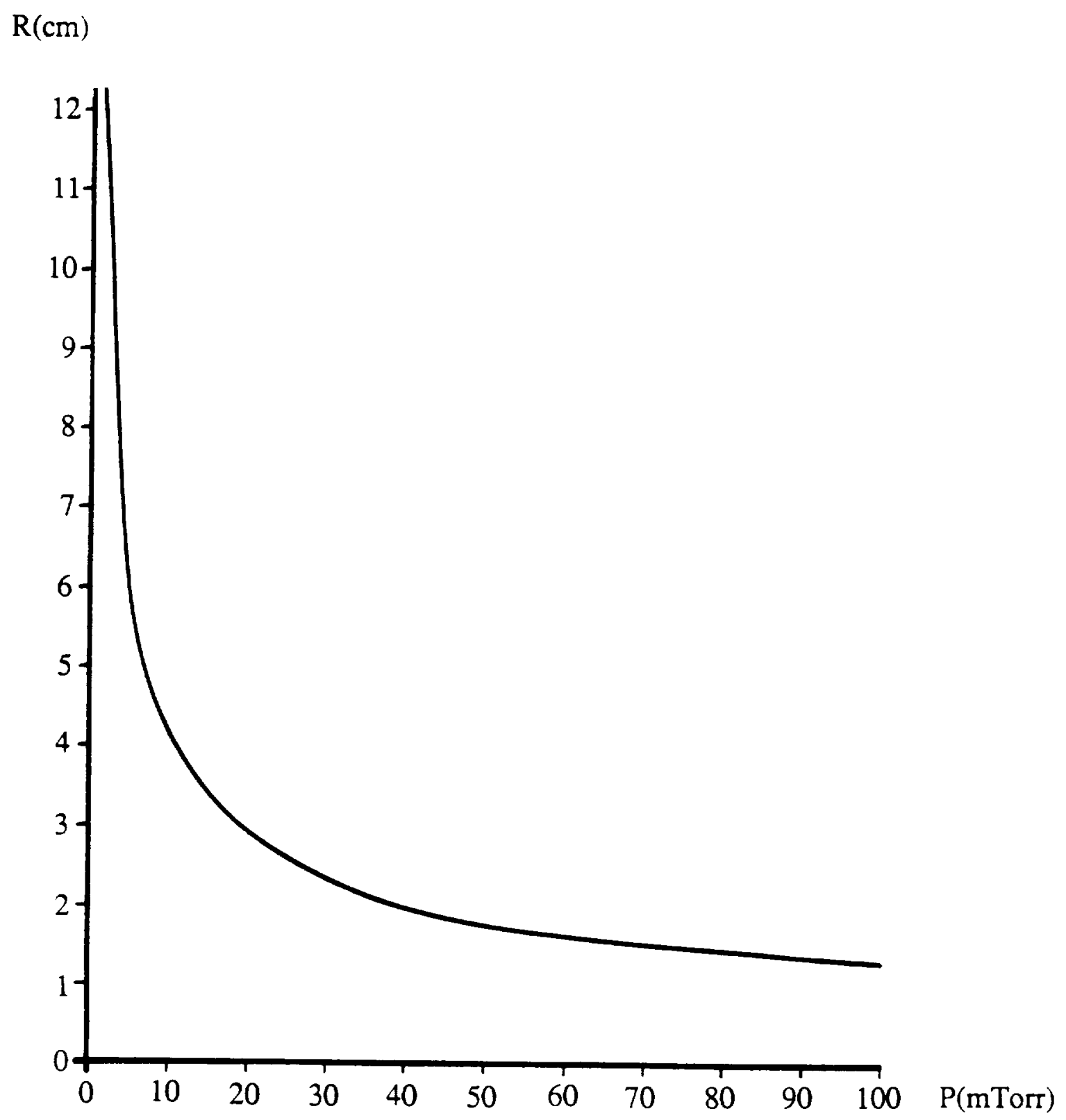

Figure 4.10. LDC Minimum Radius Versus Pressure 
pressures $>10$ mTorr. Conversely, LDC operation at the lowest possible pressure is desired to maintain power slope error, the pressure shift and pressure broadening at negligible levels. In particular, pressure broadening becomes significant, against an already power broadened Lamb dip ( $3-4 \mathrm{MHz}$ FWHM), at pressures as low as 100 mTorr. Though stabilization to $<1 \mathrm{~m} / \mathrm{s}$ is attained in the unavoidable presence of intracavity power broadening, the additional effect of pressure broadening should and can be avoided.

2. $\mathrm{CO}_{2}{ }^{*}-\mathrm{CO}_{2}$ collisional de-excitation A $\mathrm{CO}_{2}{ }^{*}-\mathrm{CO}_{2}$ collision does not always result in de-excitation. The kinetic rate constant $K_{r}$ is defined by:

$$
\mathrm{K}_{\mathrm{r}}=\sigma \mathrm{v}_{\mathrm{rms}} / \mathrm{f}
$$

where $f$ is the average number of collisions before collisional relaxation (Taylor and Bitterman 1969). Figure 4.11 shows the three fastest collisional relaxation pathways from the $\sum_{u}{ }^{+} 00^{0}{ }_{1}$ state. All three have roughly the same magnitude $\mathrm{K}_{\mathrm{r}}$ (Taylor and Bitterman 1969). For the $\sum_{u}{ }^{+} 00^{0} 1$ to $\sum_{g}{ }^{+}\left[10^{0} 0,02^{\circ} 0\right]$ II pathway, at room temperature, $\mathrm{K}_{\mathrm{r}}=4-6 \times 10^{-15} \mathrm{~cm}^{3} \mathrm{~s}^{-1}$ (see Fig. 14 in Taylor and Bitterman 1969). Plugging into Eq. 4.17 gives $\mathrm{f}=4.7 \times 10^{4}$ to $7.1 \times 10^{4}$.

The number of collisions $(\eta)$ suffered by $\mathrm{CO}_{2}{ }^{*}$, before spontaneously emitting at $4.3 \mu \mathrm{m}$, may be obtained as a function of cell pressure by combining Eqs. 4.10, 4.12 and 4.13:

$$
\eta=\left(\frac{\mathrm{v}_{\mathrm{rms}}{ }^{\tau} \mathrm{rad}^{\sigma}}{\mathrm{kT}}\right) \mathrm{P} .
$$




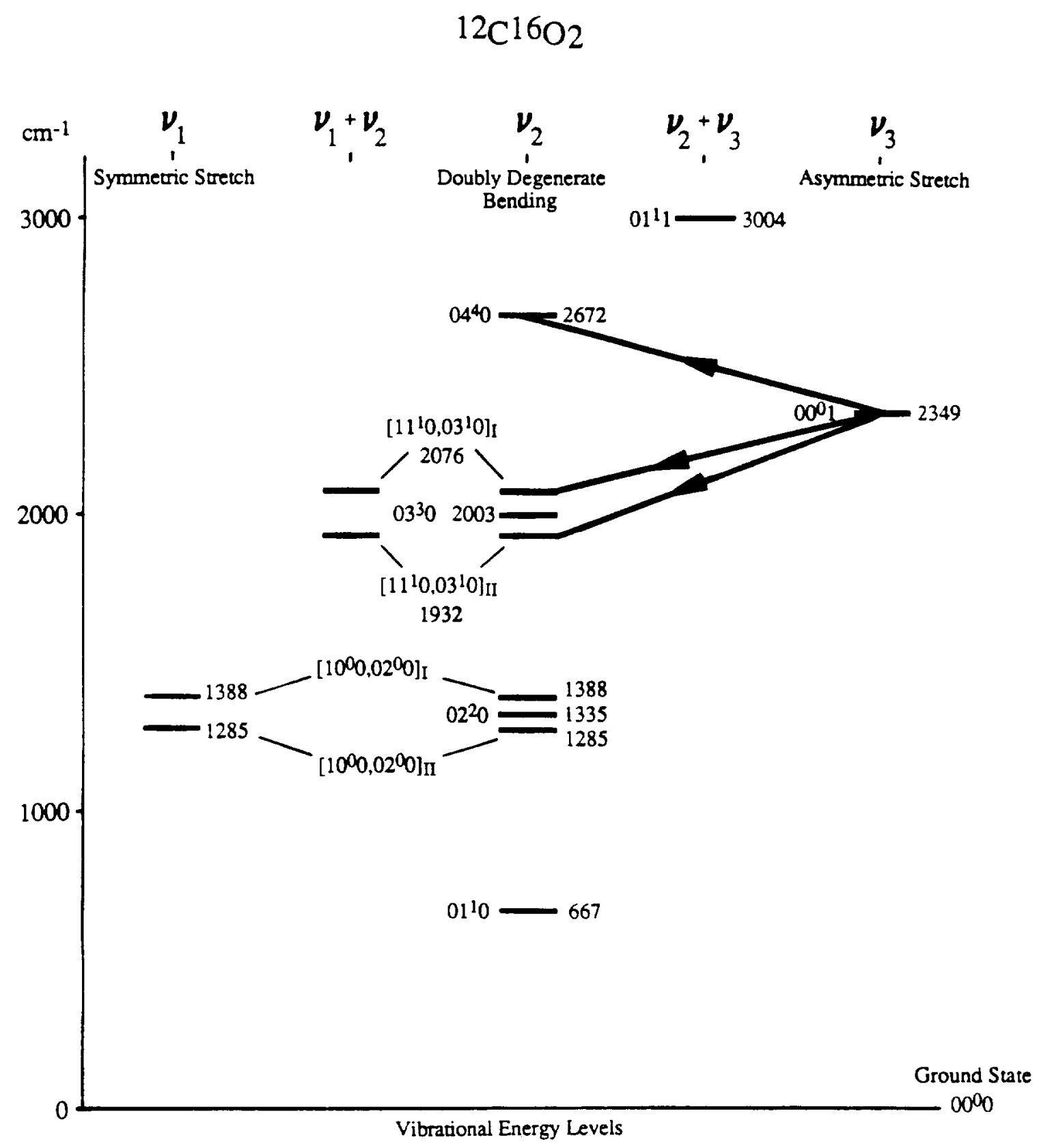

Figure 4.11. 0001 Collisional De-Excitation Pathways 
A room temperature cell, at $\mathrm{P}<100 \mathrm{~m}$ Torr (133 dynes $/ \mathrm{cm}^{2}$ ), gives $\eta<2100$. Since $\eta$ is at least 20 -fold smaller than $f$, $\mathrm{CO}_{2}{ }^{*}-\mathrm{CO}_{2}$ collisional de-excitation is negligible.

3. LDC optical depth at $4.3 \mu \mathrm{m}$

A determination of LDC optical depth at $4.3 \mu \mathrm{m}\left(\tau_{4.3}\right)$ is important. Large $\tau_{4.3}$ will lead to radiative trapping. A resulting phase $1 \mathrm{ag}$ in the detected $4.3 \mu \mathrm{m}$ signal will inhibit its effectiveness as an instantaneous probe of laser output frequency. While a phase lag will not introduce a Lamb dip frequency offset, it will decrease efficiency of the stabilization electronics (SooHoo et al. 1985).

Over a characteristic LDC radius $R_{\min }, \tau_{4.3}$ is given by:

$$
\tau_{4.3}=\mathrm{Sf}_{\mathrm{D}}\left(\nu-\nu_{\mathrm{o}}\right) \mathrm{R}_{\mathrm{min}} \mathrm{P}
$$

where $S$ is the integrated absorption coefficient and $f_{D}\left(\nu-\nu_{0}\right)$ is the normalized Doppler line shape function. At line center,

$$
\begin{aligned}
& f_{D}\left(\nu-\nu_{0}\right)=f_{D}(0) \text { with: } \\
& \qquad f_{D}(0)=\lambda_{0}[m / 2 \pi k T]^{1 / 2},
\end{aligned}
$$

where $\lambda_{0}$ is the transition wavelength, $k$ is Boltzmann's constant, $\mathrm{m}$ is the molecular mass of ${ }^{12} \mathrm{C}^{16} \mathrm{O}_{2}$ and $\mathrm{T}$ is the LDC temperature. Substituting Eqs. 4.20 and 4.14 into 4.19 yields $\tau_{4.3}$ as a function of $P$ :

$$
\tau_{4.3}=\mathrm{S} \lambda_{\mathrm{o}}\left(\frac{\mathrm{mv}_{\mathrm{rms}}{ }^{\top} \mathrm{rad}}{2 \pi \sigma}\right) \mathrm{P}^{1 / 2} .
$$

Due to collisional coupling of the $\sum_{u}{ }^{+} 00^{0} 1$ rotational 
levels, $\mathrm{CO}_{2}{ }^{*}$ will give rise to $4.3 \mu \mathrm{m}$ emission over both $\mathrm{P}$ and $\mathrm{R}$ branches. It is therefore of interest to determine $r_{4.3}$ for the most optically thick line. $\mathrm{P}(16)$ is associated with the largest $\mathrm{S}=3.455 \times 10^{-18} \mathrm{~cm} / \mathrm{molecule}$, at $296 \mathrm{~K}$. With the appropriate substitutions, Eq. 4.21 reduces to:

$$
\tau_{4.3}=[0.44] \mathrm{P}^{1 / 2}
$$

where $\mathrm{P}$ is in mTorr. Eq. 4.22 provides the optical depth at line center, for the most optically thick line in the $4.3 \mu \mathrm{m}$ band,

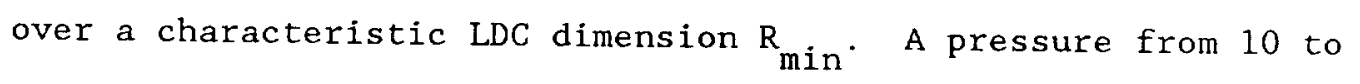
$50 \mathrm{mTorr}$ corresponds to $\tau_{4.3}=1.4$ to 3.1 .

For $\tau_{4.3} \leq 1$, characterizing negligible radiative trapping, the lag time between excitation to $\mathrm{CO}_{2}{ }^{*}$ and $4.3 \mu \mathrm{m}$ signal detection is $\sim \tau$ rad. Stabilization to the Lamb dip warrants cavity length modulation at some frequency $\nu_{\bmod }$ (see Section 4.7). Thus, as long as $1 / \nu_{\bmod }>r \operatorname{rad}\left(\nu_{\bmod }<<A=441 \mathrm{~s}^{-1}\right)$, the phase lag will be negligible.

From these first-order considerations, a $L D C \mathrm{R}_{\min }=2.9 \mathrm{~cm}$, corresponding to a -20 mTorr cell pressure and $r_{4.3}=2$, seems most suitable for intracavity operation. A cell with these general characteristics was constructed, based on a configuration developed by D. Zipoy from the University of Maryland Astronomy Program. An optimal pressure of 20-30 mTorr was experimentally determined for the cell.

Maintaining low pressures within a small, sealed LDC is difficult. Low levels of desorption can have dramatic effects on cell pressure. A glass construction, encorporating a ballast chamber, was found to 
provide the requisite hold time. A 1 mTorr per hour maximum $\mathrm{CO}_{2}$ desorption rate was achieved with an initial pressure of 20 mTorr.

Over the course of a typical 12 hour observation period, a 20-30 mTorr pressure was thus maintained.

Figure 4.12 is a cross-section of the LDC. The cell arms are made of 0.5 inch (i.d.) tubing ground to a $10 \mu \mathrm{m}$ Brewster angle of $22.6^{\circ}$. ZnSe arm windows (chosen for their $10 \mu \mathrm{m}$ transmittance) were side-sealed to the cell using a low desorption epoxy.

The ballast volume is made from $51 \mathrm{~mm}(0 . \mathrm{d}$.$) tubing, its top ground$ flat to accommodate a sapphire window used as the $4.3 \mu \mathrm{m}$ signal port. The sapphire window's lower face is located $1.25 \mathrm{~cm}$ from the beam axis. Figure 4.13 shows the LDC, its intracavity support structure, and the down-looking, liquid nitrogen cooled dewar containing an InSb detector. The detector is located in the base of a 1-3/16 inch diameter dewar port, with the detector plane $0.56 \mathrm{~cm}$ above the dewar window's outer face. A narrow band filter $(0.2 \mu \mathrm{m}$ bandwidth) centered at $4.3 \mu \mathrm{m}$ and a $120^{\circ}$ FOV heat shield are incorporated into the detector assembly.

Figure 4.14 shows the LDC assembly under normal cavity operation. The LDC filler valve, not shown on Figure 4.12, is to the cell's right. The output coupler is at far left.

Figure 4.15 provides a more extended view of the laser cavity. Shown are the LDC (1), Dewar (2), LDC filler valve (3), output coupler (4), intracavity chopper (5), gain cell (6), and cavity rods (7). The cavity is enclosed in an acetate box providing laser environment isolation from acoustical pickup and fluctuations in temperature and air density at Coudé. 


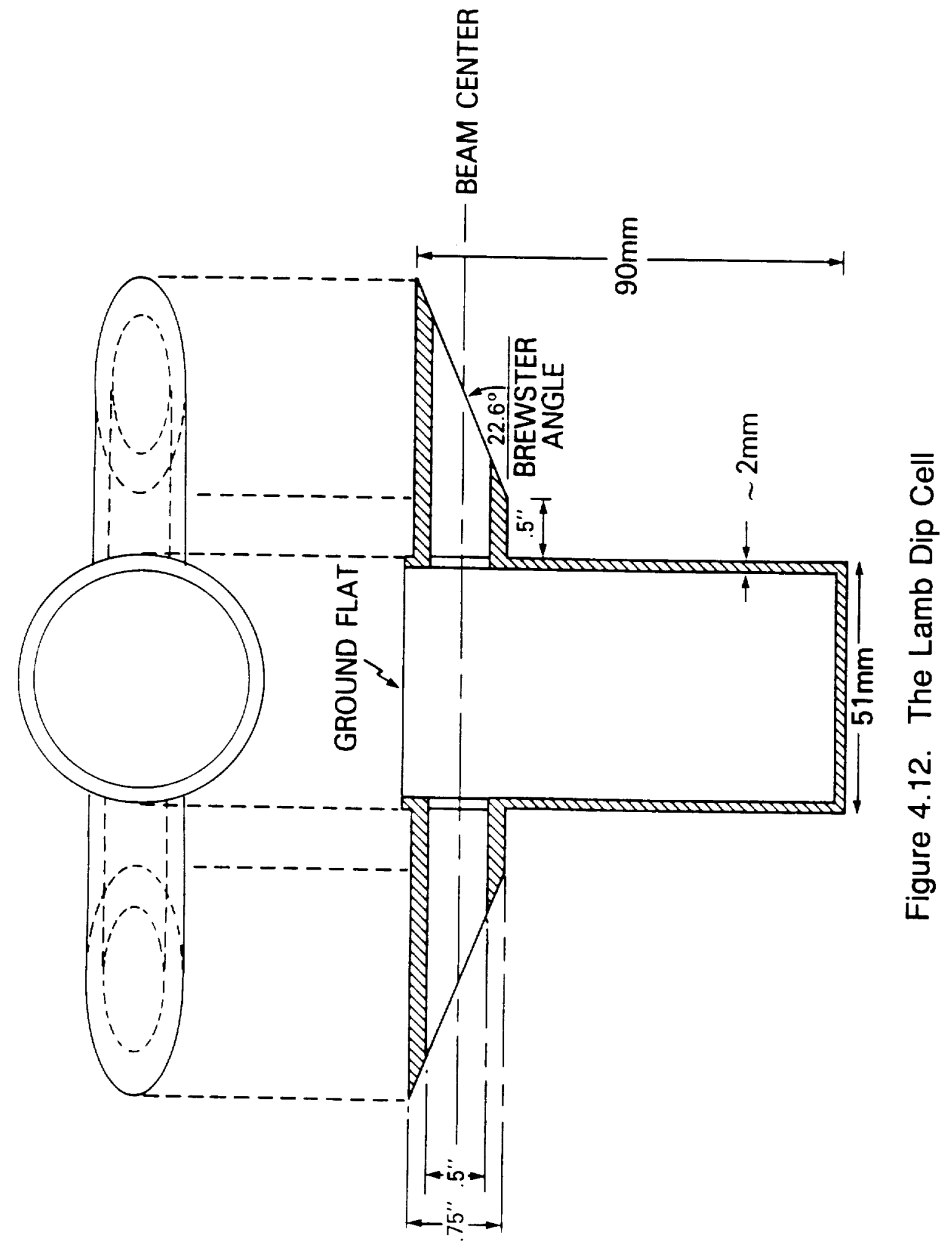




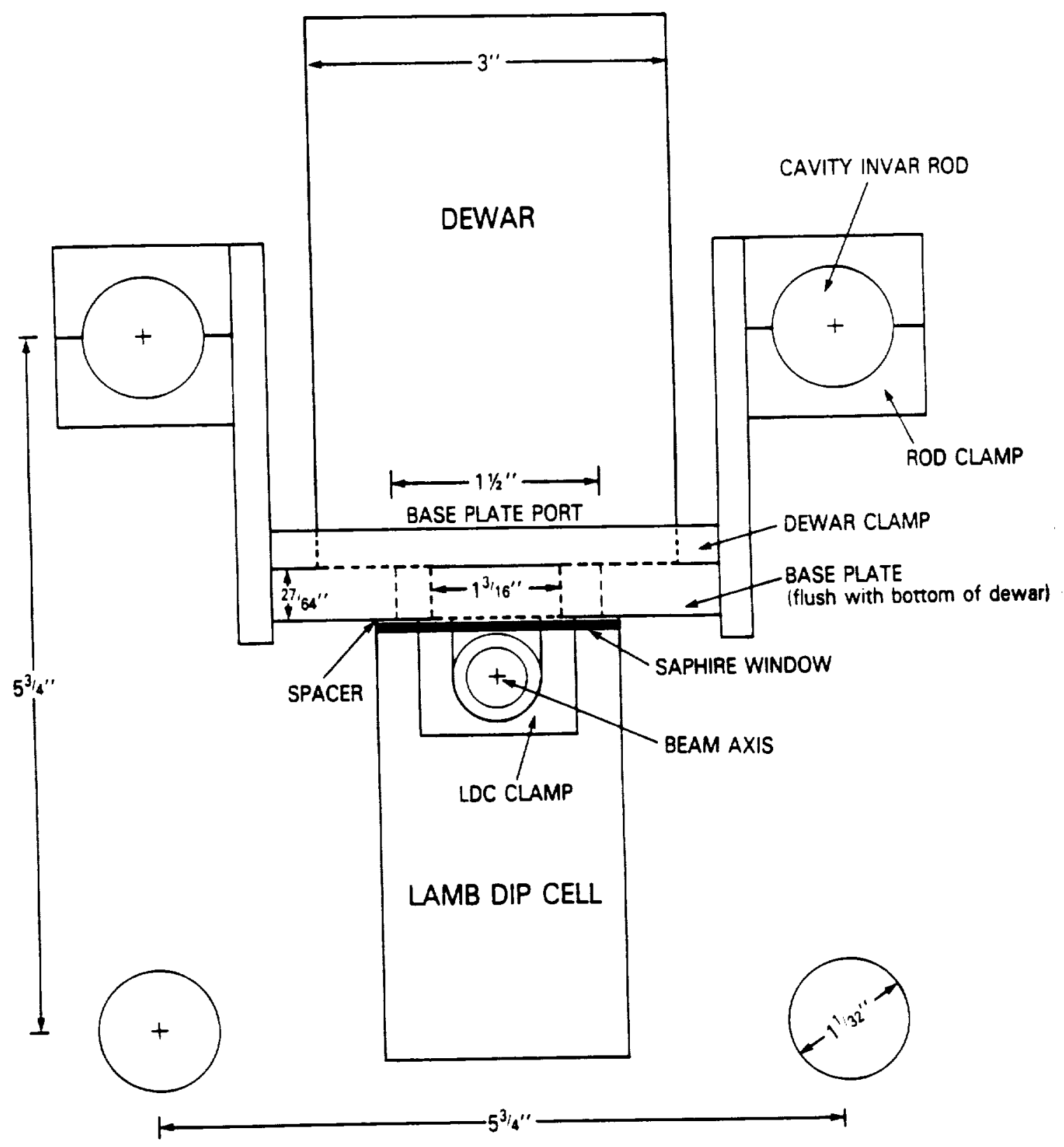

Figure 4.13. Lamb Dip Cell/Dewar Configuration 


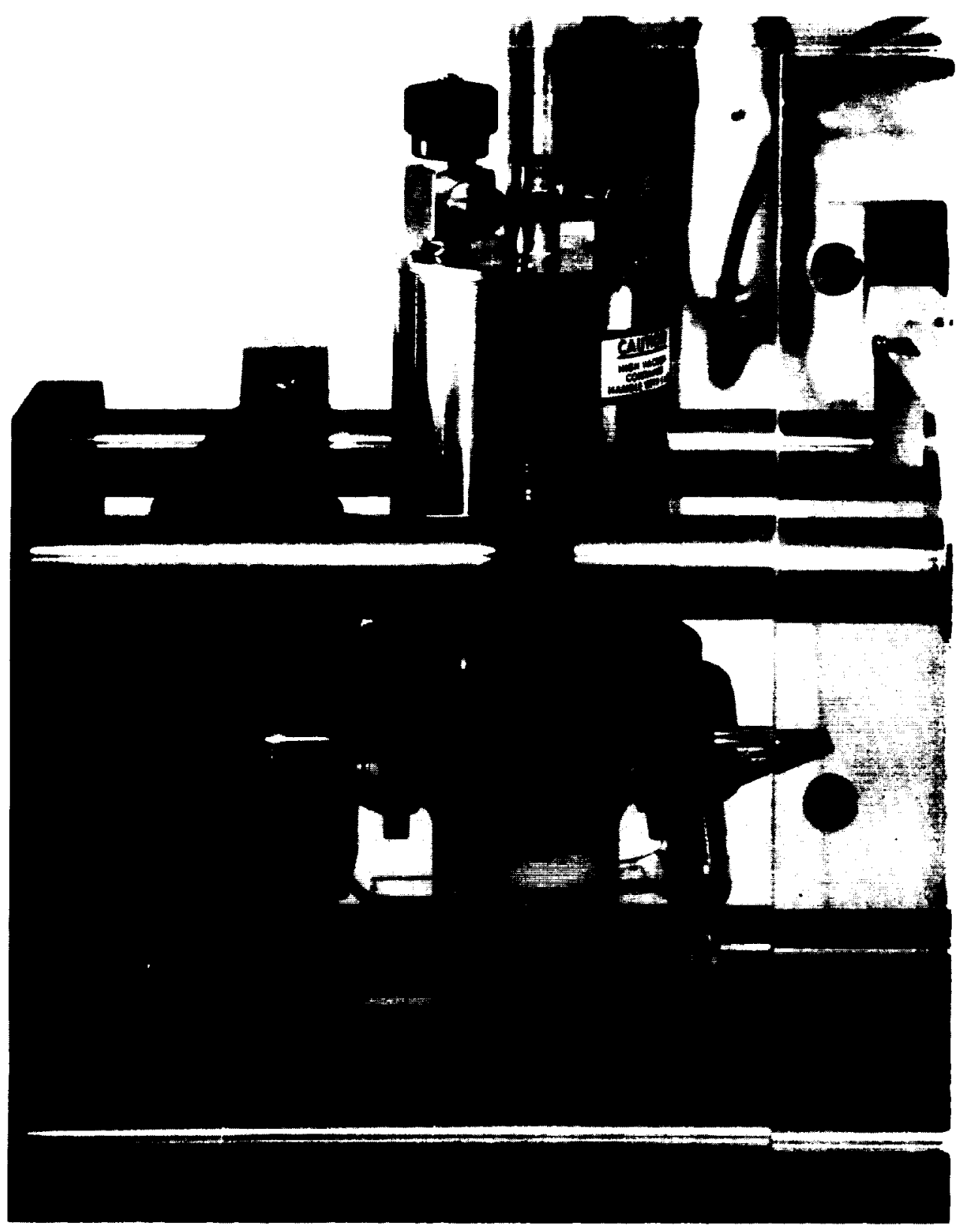

Figure 4.14 
ORIGINAL PAGE

BLACK AND WHITE PHOIOGRAPH

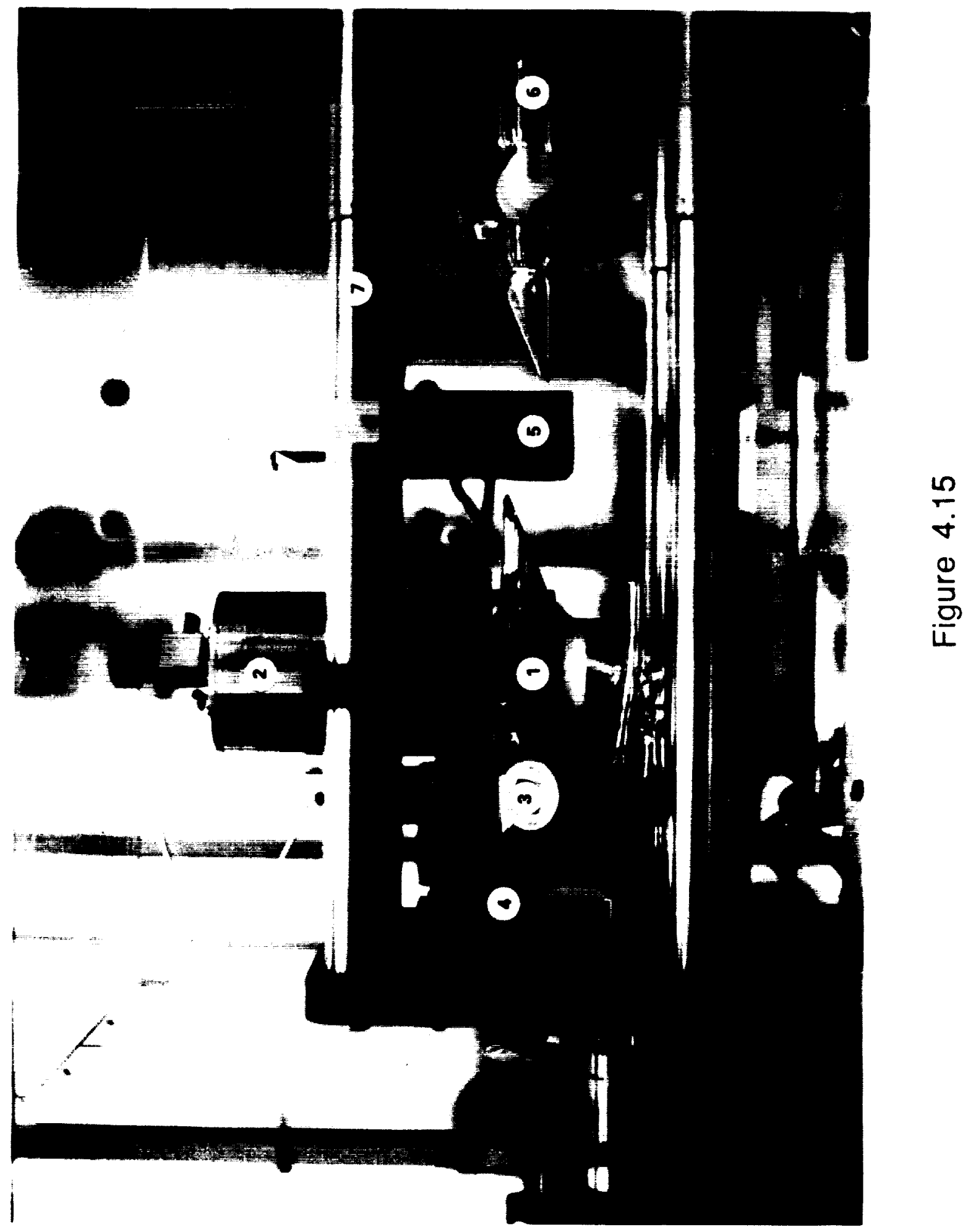




\subsection{OVERVIEW OF THE LAMB DIP STABILIZATION SYSTEM (LDSS)}

\subsubsection{Introduction}

The overall LDSS is shown in Figure 4.16. The apparent complexity is due solely to the number of operating modes integrated into the system. Through use of the Mode Selector, one may choose either power peak or Lamb dip stabilization and can perform a variety of diagnostic tests to determine operational readiness. The versatility gained from such an integrated system allows one to rapidly pinpoint problems in the field and, if necessary, implement an alternate form of stabilization with minimum down time.

Before undertaking a detailed discussion of the individual operating modes, an overview of stabilization requirements is necessary.

Lamb dip stabilization of the cavity etalon may be accomplished if a DC signal proportional to the negative of the $4.3 \mu \mathrm{m}$ gain profile's slope is continuously fed to the PZT. Consider Figure 4.17, with the cavity tuned to a point on the Lamb dip's high frequency side characterized by positive slope. A negative feedback signal, added to the negative DC bias necessarily present on the PZT for normal operation, will cause further PZT contraction. The associated lengthening of the cavity provides support for a standing wave with lower frequency $\nu_{10}$, thereby driving the cavity toward the Lamb dip. For a cavity tuned to a point on the dip's low frequency side, characterized by negative slope, a positive feedback DC signal will drive the cavity toward higher $\nu_{10}$, again in the direction of the dip. A cavity tuned to the Lamb dip, a point characterized by zero slope, 


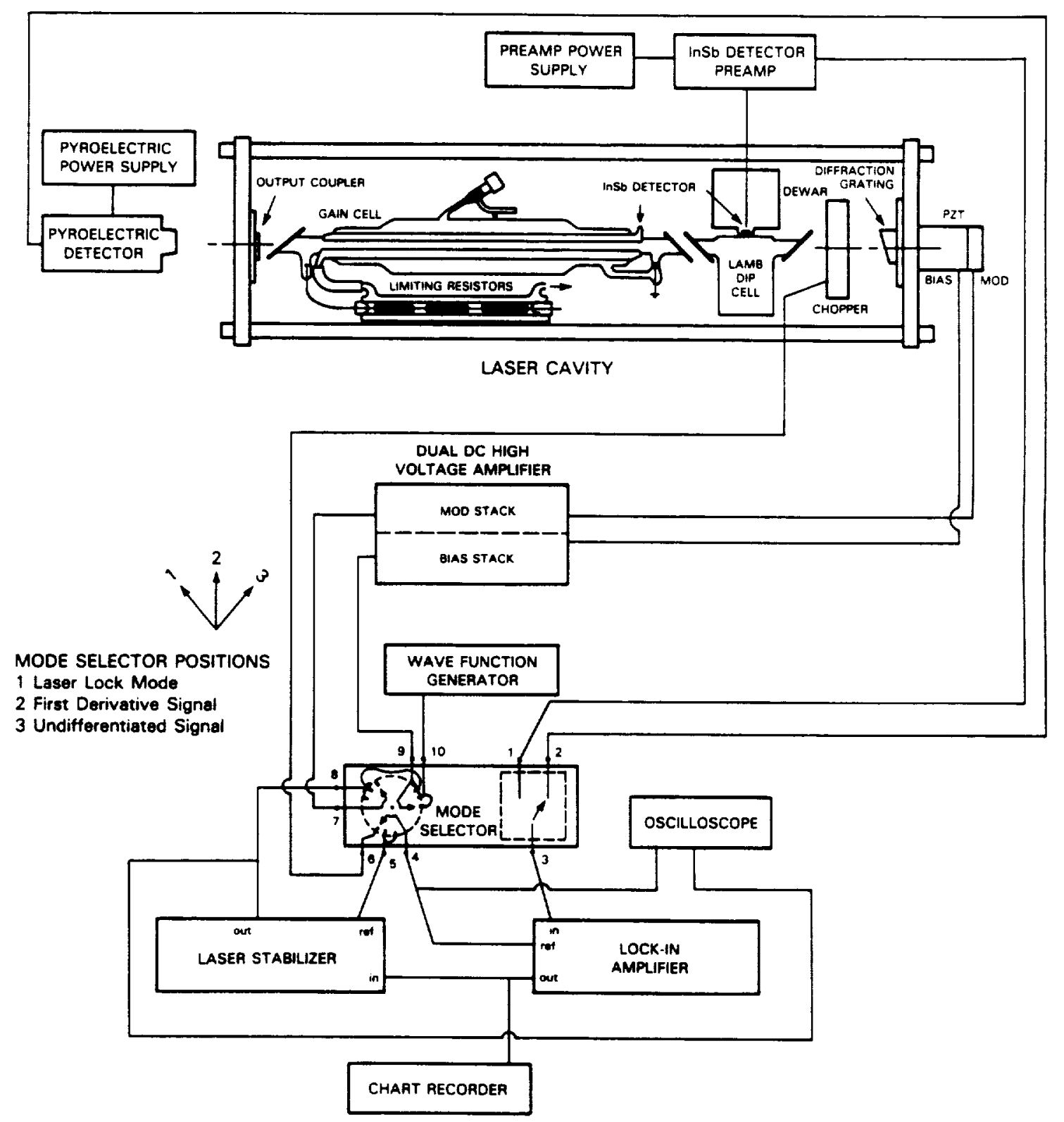

Figure 4.16. The Overall Laser Stabilization System 


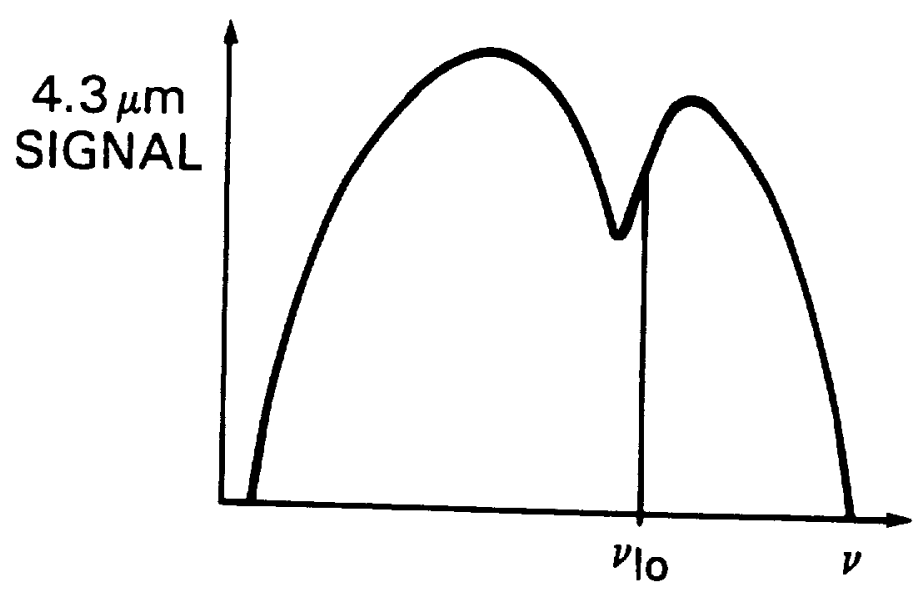

Figure 4.17
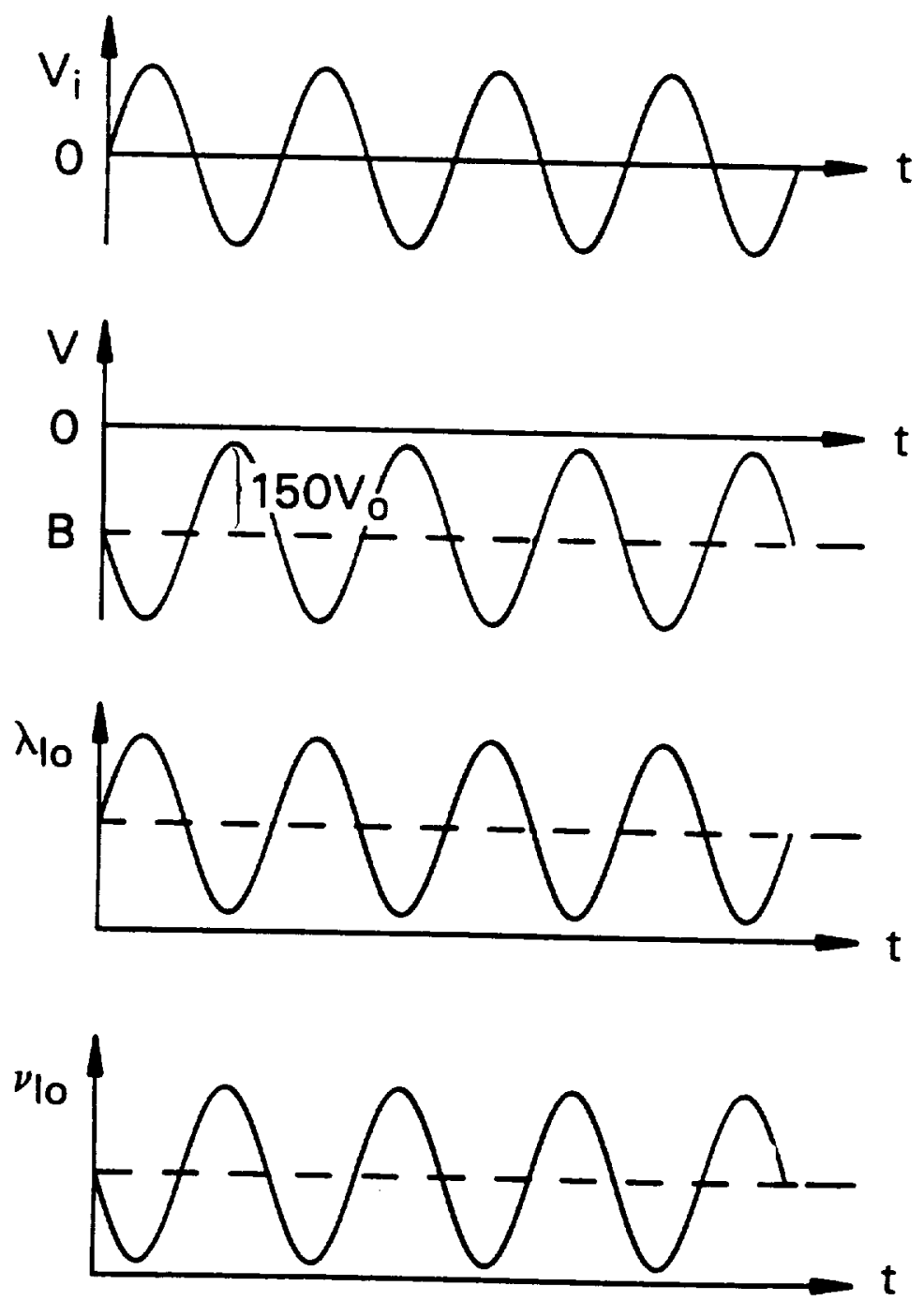

Figure 4.18 
obviously requires zero feedback signal. For a cavity tuned initially outside the Lamb dip, the above stabilization criteria will drive the cavity down the gain profile's side, to stabilize at a trough between adjacent profiles.

If one pursues the same logic for a feedback signal proportional to the slope of the gain profile, stabilization to one of the Lamb dip's two shoulders will be accomplished, both of which are not absolute frequency standards and are subject to the effects of pressure shifting and variation of cavity alignment.

The system employs a DC high voltage amplifier, the Physik model P-263 triple supply, which provides an output voltage to the PZT. The output voltage is the sum of an input signal inverted and amplified 150-fold and a negative-going DC offset voltage. (The offset ranges from 0 to -1000 volts insuring only negative biasing of the PZT.) An offset is selected, employing a control knob on the amplifier's front plate, to center the gain profile in the $12 \mu \mathrm{m}$ contraction range of the PZT. Output voltage to the PZT may be read directly from the digital voltmeter (DVM) also located on the front plate. The digital display is crucial for operation of the LDSS, providing a direct indication of the locked condition, the nature of the locked condition and a determination of the Lamb dip width.

Consider the cavity effects of a small sinusoidal modulation to the PZT via the high voltage DC amplifier. For an amplifier input signal given by $V_{i}=V_{0}$ sinwt, the potential difference across the PZT is $\mathrm{V}=\mathrm{B}-150 \mathrm{~V}_{\mathrm{o}}$ sinwt, where $\mathrm{B}$ is the negative going $\mathrm{DC}$ offset voltage. $\mathrm{B}$ is adjusted so $|\mathrm{B}|>>150 \mathrm{~V}_{0}$, insuring negative PZT biasing (Fig. 4.18). PZT 
contraction with increased negative-going applied voltage allows a correspondingly longer cavity to support a standing wave with larger $\lambda_{10}$. Over a small frequency interval, $\Delta \lambda_{10}$ is directly proportional to $-\Delta \nu_{10}$. Thus, (see Figure 4.18) the modulated input to the DC amplifier is $180^{\circ}$ out of phase with the induced modulation in local oscillator frequency $\nu_{10}$. In Figure 4.19 , the $4.3 \mu$ modulation is seen as either in phase or $180^{\circ}$ out of phase with modulation in local oscillator frequency, depending on which side of the dip the cavity is tuned.

\subsubsection{Undifferentiated Mode}

The first test of system readiness is direct generation of the Lamb dip in the $4.3 \mu \mathrm{m}$ fluorescence signal. This is accomplished employing LDSS Undifferentiated Mode via the mode selector box. The presence of a symmetric dip, with good $\mathrm{S} / \mathrm{N}$, implies proper function of the dewar-detector package, proper optical alignment of the cavity etalon and all intracavity devices, and a LDC pressure close to operational parameters.

Figure 4.20 provides the system overview for LDSS undifferentiated mode. The InSb detector signal is fed to a preamplifier and in turn to the input of a lock-in amplifier. An intracavity bow-tie chopper, whose reference square wave is fed to the lock-in, allows instantaneous detection of the $4.3 \mu \mathrm{m}$ signal above the detector noise floor.

The bias and modulation stacks of the PZT are connected to two of the three outputs of the Physik triple DC amplifier. A wavefunction generator, with a sawtooth output, is connected to the input of the bias stack's high voltage amplifier. With appropriate choice of 


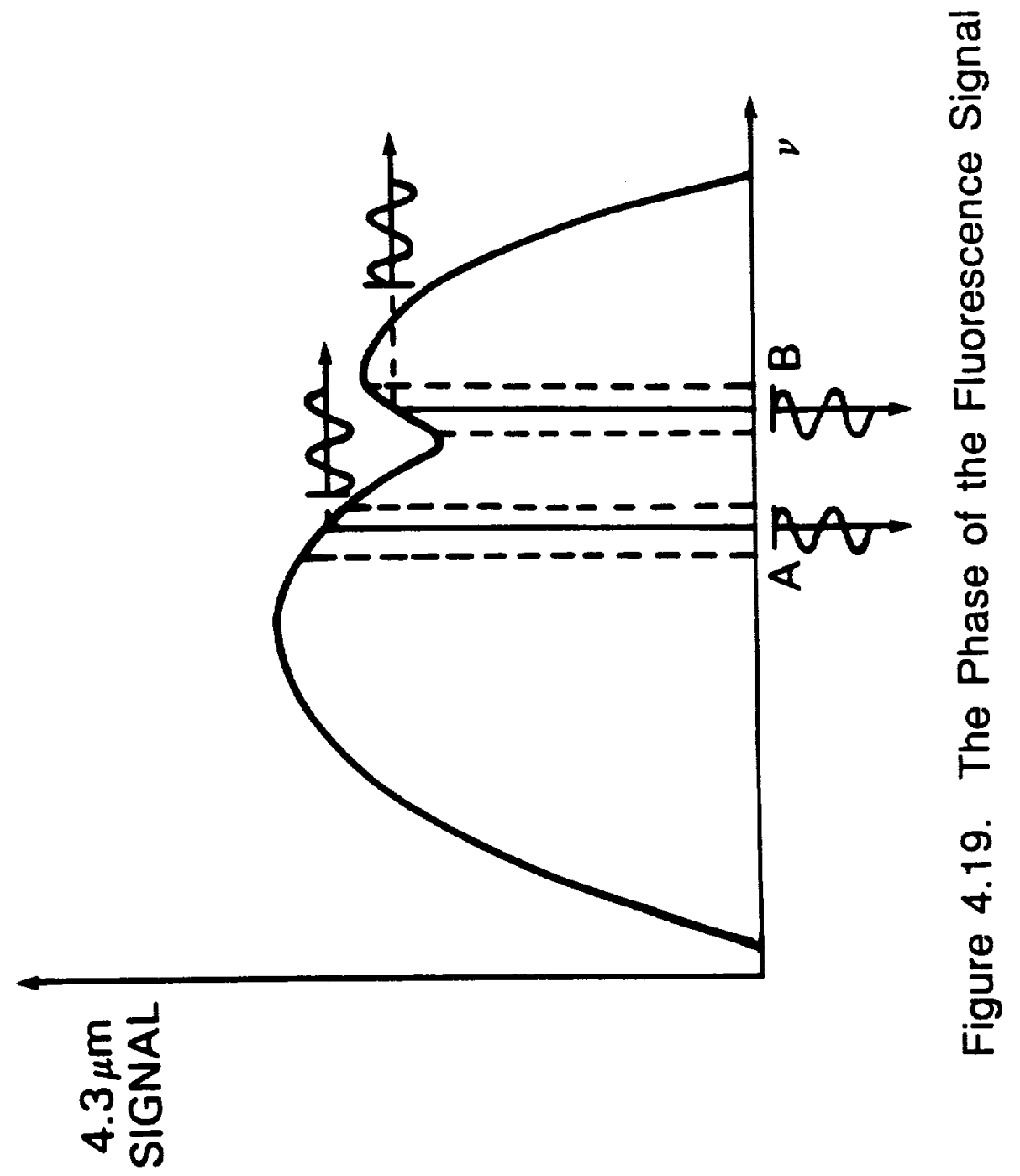




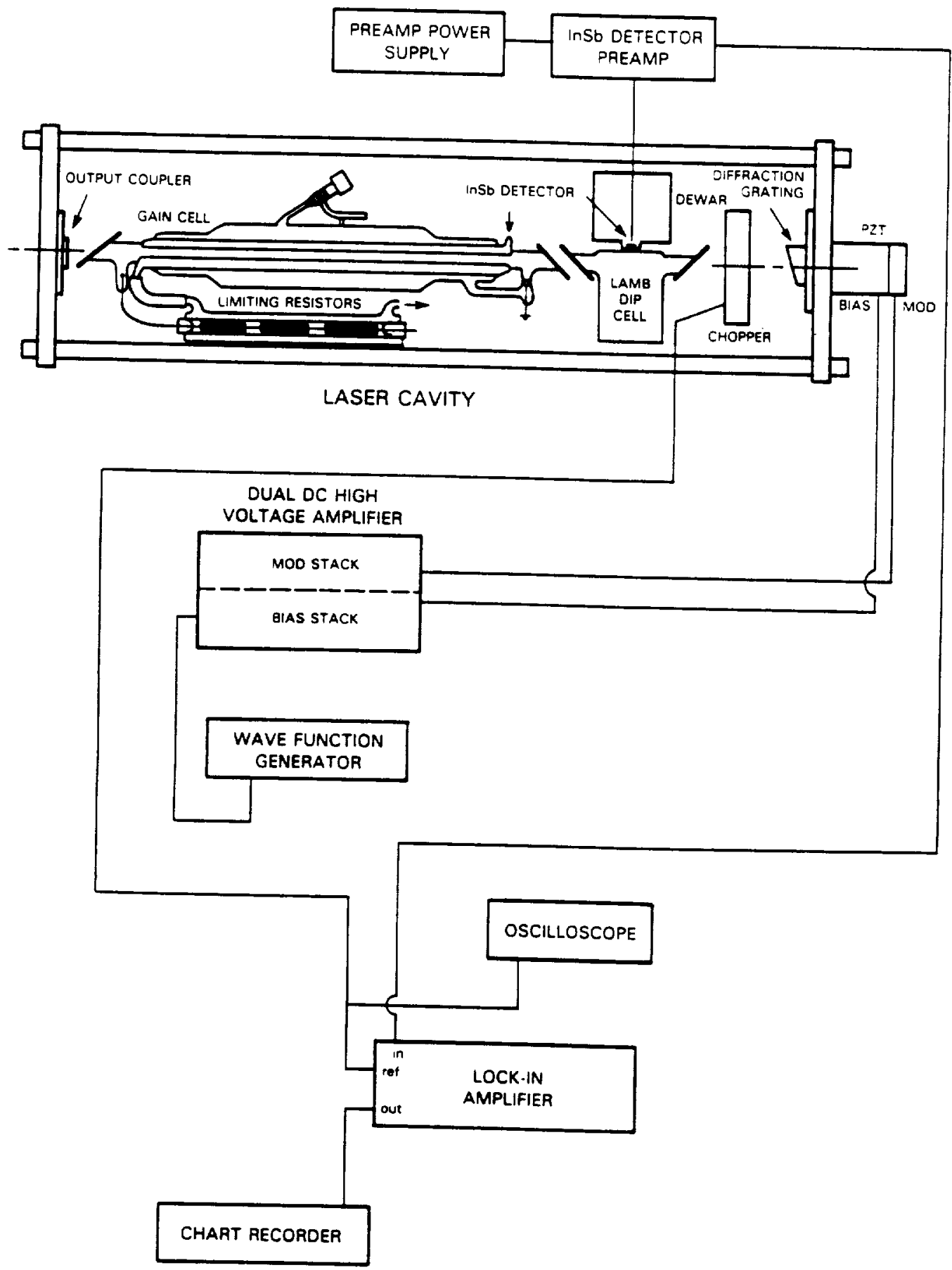

Figure 4.20. LDSS Undifferentiated Mode 
frequency and amplitude on the wavefunction generator, and DC offset voltage on the bias stack amplifier, the cavity may be made to ramp slowly through the $4.3 \mu \mathrm{m}$ gain profile symmetrically about the Lamb dip, or through up to -3 gain profiles. A chart recorder, connected to the output of the lock-in amplifier, records the $4.3 \mu \mathrm{m}$ signal.

Manual ramping may be accomplished by turning off the wavefunction generator and varying the bias stack amplifier's negative going DC offset. The same manual variation of the modulation stack, with only 2 PZT wafers compared to the 10 comprising the bias stack, allows 5 -fold finer resolution.

Employing the wavefunction generator, one can determine the cavity's $\mathrm{d} \nu_{10} / \mathrm{dE}$ by slowly ramping through as large an etalon length change as possible. Measurement of the bias stack applied voltages (using the high voltage amplifier DVM) at at least two widely separated points on the chart recorder output, and substitution into Eq. 4.8, yields $\mathrm{d} \nu_{10} / \mathrm{dE}$. Once $\mathrm{d} \nu_{10} / \mathrm{dE}$ is determined, one can precisely measure the Lamb dip FWHM by ramping in the region of the dip, at a smaller amplitude, and again measuring the bias voltages at two points on the chart recorder output.

At an optimal pressure of $25 \mathrm{mTorr}$, the intracavity LDC provides a 3-4 MHz FWHM dip, resulting from power broadening by -40 watts of circulating power within the cavity (see Section 4.5.2). At pressures > 100 mTorr the dip becomes noticeably smeared out due to a 7.5 $\mathrm{MHz} /$ Torr (FWHM) pressure broadening coefficient (for pure $\mathrm{CO}_{2}$ ).

While undifferentiated mode may be used as a powerful diagnostic of system readiness, it cannot be used for stabilization. The 
intracavity chopper allows only a 508 laser local oscillator duty cycle, which is unacceptable for heterodyne operation.

\subsubsection{First Derivative Mode}

As shown in Section 4.7.1, Lamb dip stabilization requires a DC signal to the PZT proportional to the negative of the $4.3 \mu \mathrm{m}$ gain profile's first derivative. Direct detection of the first derivative's sign may be accomplished by modulating the cavity and eliminates the need for an intracavity chopper.

Figure 4.21 is an overview of LDSS First Derivative Mode. The Laser Stabilizer is a specialized servo circuit whose task is to maintain the correct DC voltage to the PZT. Discussed in detail in Section 4.7.4, its sole function during first derivative operation is to provide a sinusoidal modulation to the modulation stack via the DC high voltage amplifier. Typical stabilizer modulated output is $150 \mathrm{mV}$ $\mathrm{p}-\mathrm{p}$ at a frequency of $80 \mathrm{~Hz}$, corresponding to $22.5 \mathrm{~V} \mathrm{p}-\mathrm{p}$ at the modulation stack $(0.9 \mathrm{MHz}$ p-p cavity modulation). A reference square wave, in phase with the laser stabilizer modulated output, is fed to the lock-in amplifier. As before, the InSb detector signal is fed to the lock-in input, and the lock-in output is fed to a chart recorder. The lock-in now effectively compares the $4.3 \mu \mathrm{m}$ signal generated in both half-cycles of the cavity modulation. The resultant signal is proportional to the first derivative of the $4.3 \mu \mathrm{m}$ gain profile. As in undifferentiated mode, either manual ramping of cavity length or use of the wavefunction generator in conjunction with the bias stack will generate the Lamb dip on the chart recorder, though now in the first 


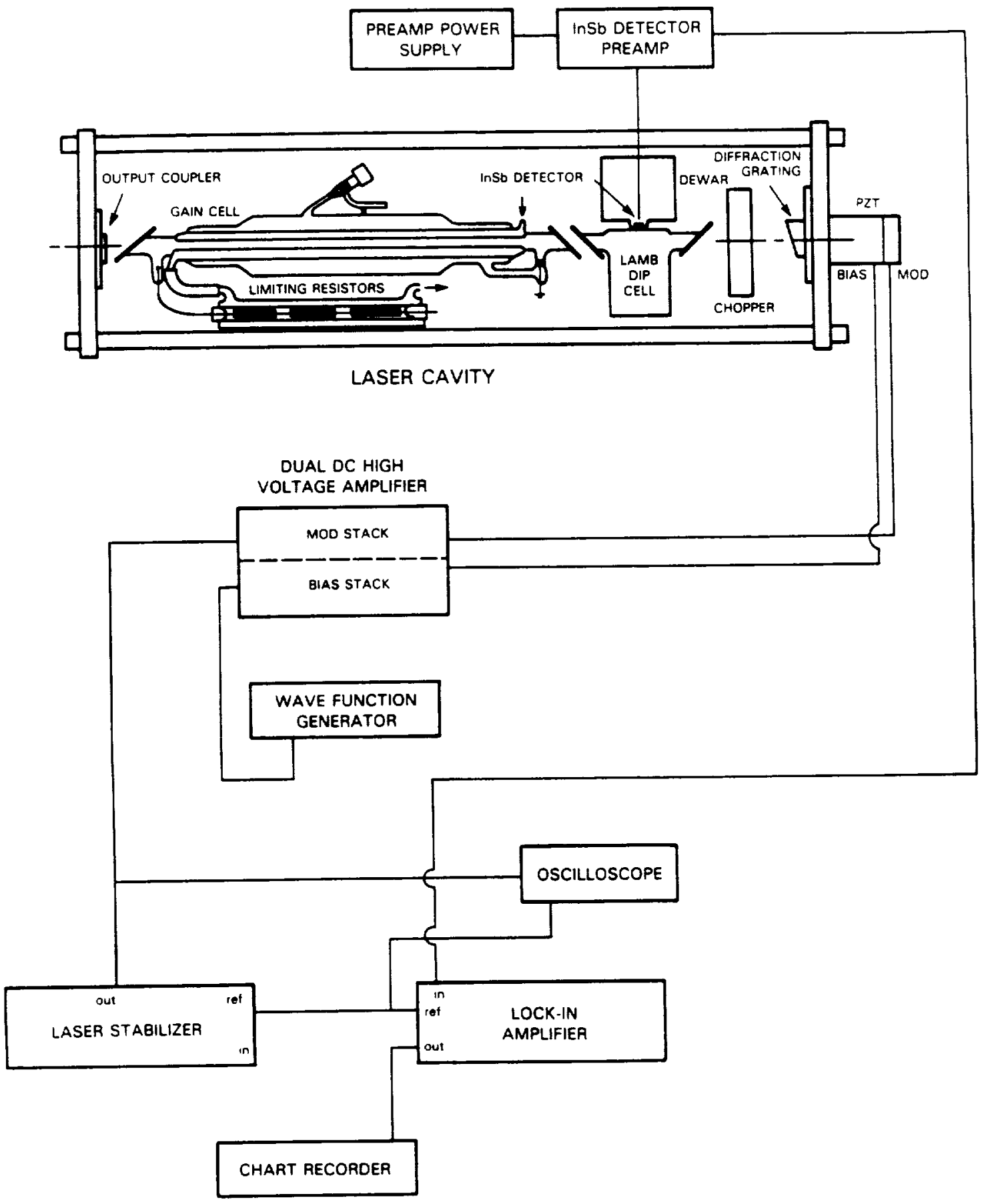

Figure 4.21. LDSS First Derivative Mode 
derivative signal (Fig. 4.22). As a result of lock-in amplifier phasing, the first derivative signal may be inverted with respect to the $4.3 \mu \mathrm{m}$ gain profile.

The etalon must be located within the Lamb dip prior to initiating stabilization, or the cavity will be driven to a trough between adjacent gain profiles. The ability to position the cavity manually at the first derivative's middle zero crossing is therefore essential. To this end, first derivative mode allows familiarization of the user with the dip's appearance in the first derivative signal. Initial ramping with the wavefunction generator is worthwhile as it provides a feel for the expected signal without the distortion associated with manual ramping .

Effective Lamb dip stabilization to the first derivative signal warrants good $S / N$ which can also be assessed in first derivative mode.

\subsubsection{Lock Mode}

The function of the laser stabilizer servo circuit is two-fold: i) provide a modulation signal to the cavity and, $i i)$ maintain an appropriate negative-going feedback voltage to the PZT, allowing cavity tracking of the Lamb dip. Based on an early power peak stabilization design by D.A. Glenar, the laser stabilizer constructed requires only the bias stack for Lock Mode. To accomplish this, the DC feedback and modulation signals are coadded.

Figure 4.23 is a schematic of the laser stabilizer, housing an 8038 waveform generator and four 741 operational amplifiers. The 8038's sinusoidal output is amplified by 741-II and fed to $741-$ I where 


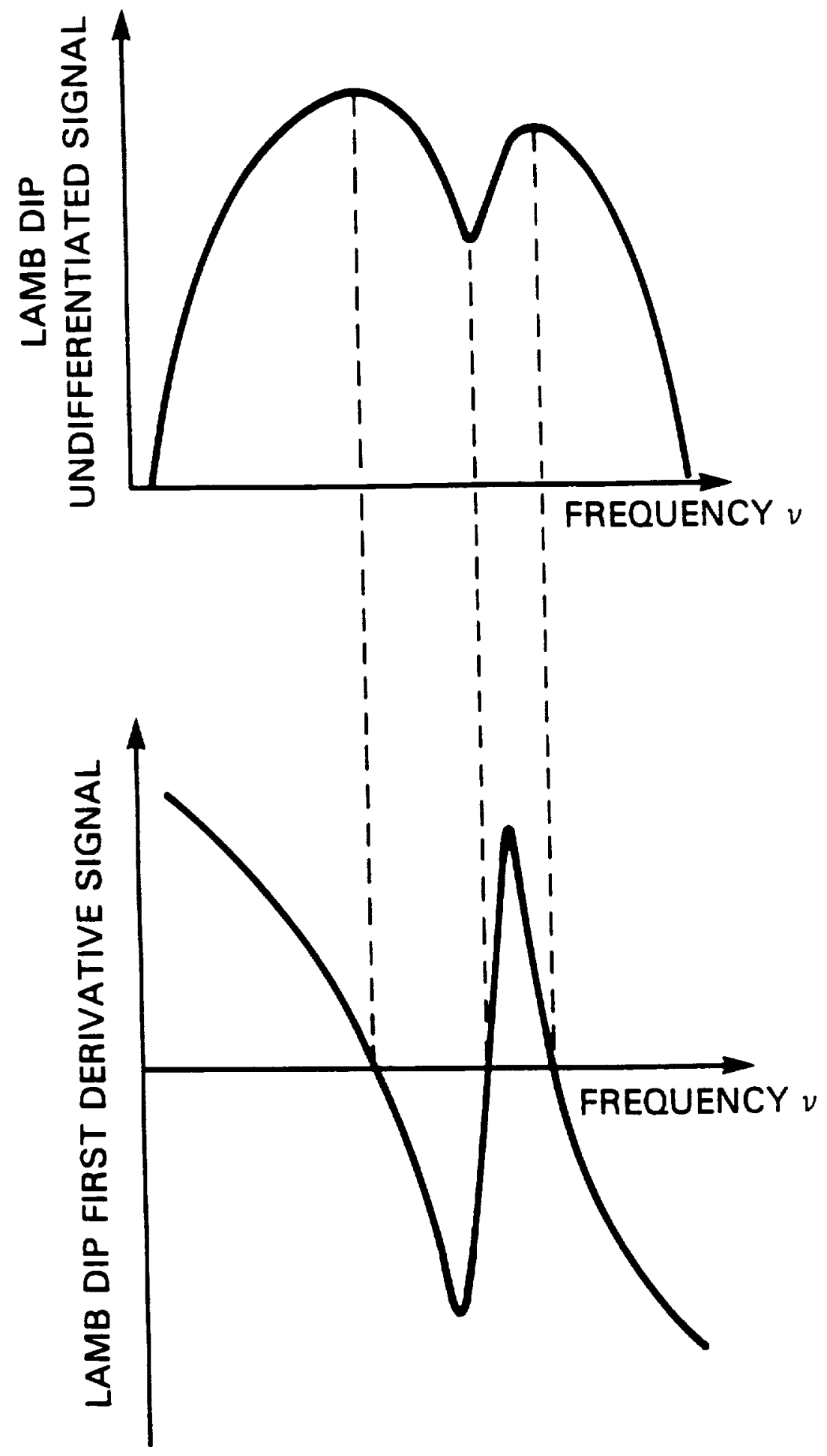

Figure 4.22. The Lamb Dip First Derivative Signal 


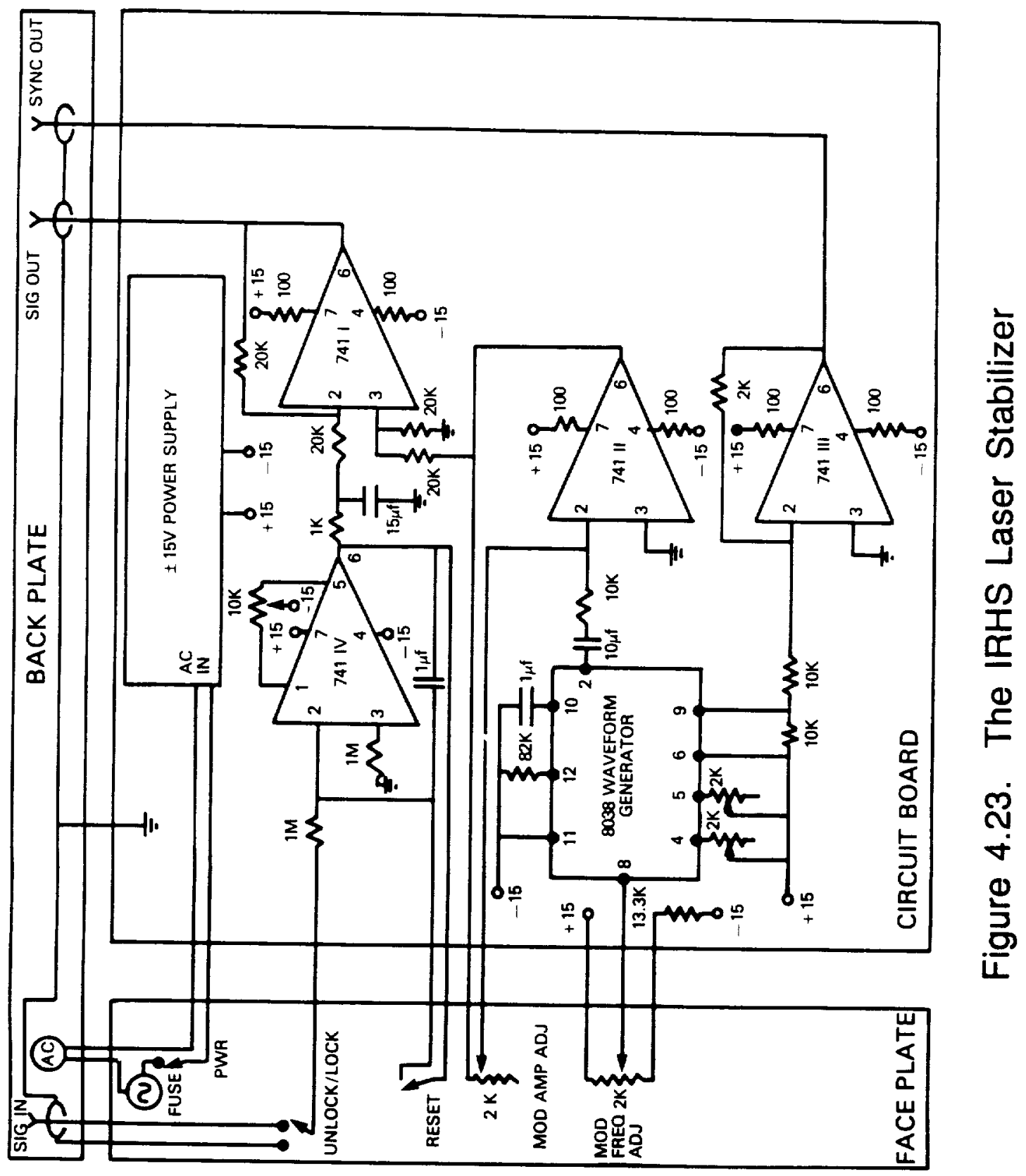


it is coadded with the DC feedback signal. The output of 741-II is therefore the AC portion of SIG OUT and, employing potentiometers on the front plate of the unit, has a $6-200 \mathrm{~Hz}$ frequency range and $0-0.68$ V p-p amplitude range. The 8038's square wave output is amplified by 741-III and serves as the SYNC OUT reference, in phase with the modulation.

As in first derivative mode, the lock-in amplifier output is zero for a cavity tuned to the Lamb dip. The lock-in output serves as the SIG IN input to the stabilizer's 741 -IV integrator. Employing a $1 \mu \mathrm{f}$ low leakage capacitor, the integrator DC output will vary only if the cavity is detuned from the dip. Coadded to the modulation signal at $741-I$, the integrator DC output is proportional to the DC component of SIG OUT, the latter acting as the servo feedback signal to the PZT.

The laser stabilizer may be isolated from the lock-in's output signal using the "lock/unlock" switch, and a pushbutton allows resetting of the integrator capacitor.

Figure 4.24 provides an overview of LDSS Lock Mode. Note that, unlike first derivative mode (see Fig. 4.21), the modulation stack is not used.

To initiate stabilization, the laser stabilizer is set to "unlock" and the integrator capacitor is reset. The stabilizer output signal to the PZT, via the high voltage amplifier, thus consists of only the modulated component. Typical stabilizer modulated output in lock mode is $30 \mathrm{mV} \mathrm{p}-\mathrm{p}$, corresponding to $4.5 \mathrm{~V}$ p-p on the bias stack $(0.9 \mathrm{MHz} \mathrm{p}-\mathrm{p}$ cavity modulation). While observing the first derivative signal on the chart recorder, the cavity is manually positioned near the Lamb dip's 

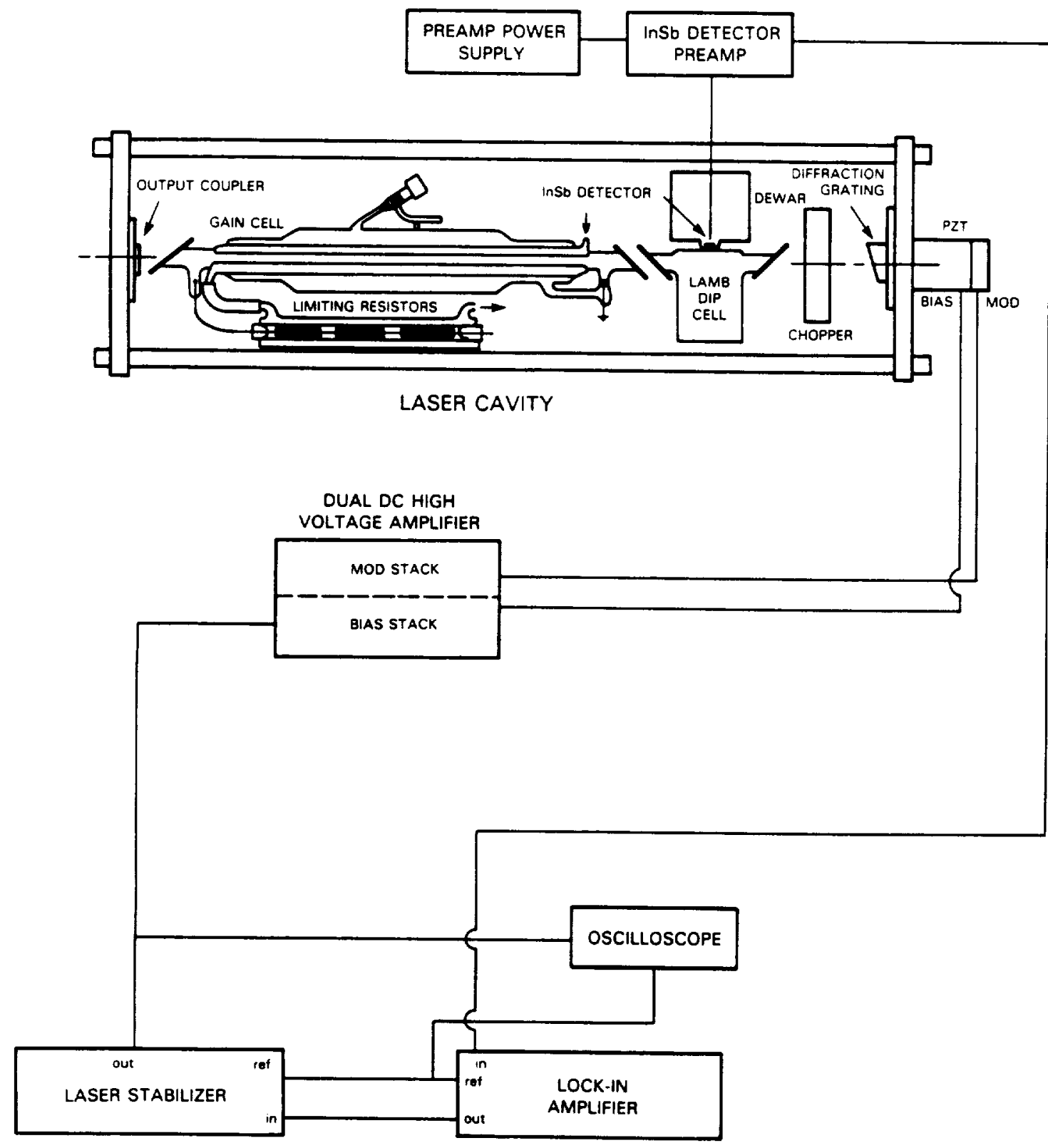

Figure 4.24. LDSS Lock Mode 
middle zero crossing using the bias stack's high voltage amplifier DC offset. Stabilization is achieved by setting the stabilizer to "lock". However, if lock-in amplifier phasing is incorrect, stabilization to one of the dip's two shoulders will result.

Prior to initiating stabilization, the lock-in may be phased by simultaneously feeding a sinusoidal signal (from the wavefunction generator) to the lock-in input and reference ports. Phasing is correct when the lock-in output is maximized negative. To understand why, consider again Figure 4.19. At Point $A$ in the Figure, with the cavity tuned to the dip's low frequency side, $4.3 \mu \mathrm{m}$ signal modulation is $180^{\circ}$ out of phase with modulation in laser frequency. But, from Figure 4.18 , the latter is $180^{\circ}$ out of phase with the laser stabilizer's modulated output. The lock-in amplifier's input is therefore in phase with the lock-in's reference input. Since the gain profile's slope is negative at Point A, a more positive feedback signal must be supplied to the PZT for stabilization to the dip. The laser stabilizer must therefore provide a more negative DC signal due to the inversion at the high voltage amplifier. This will only be accomplished if the lock-in output is negative for Point A.

The same sinusoidal signal fed to the lock-in input and reference ports mimics Point $A$, thus requiring negative peaking of lock-in output for proper phasing.

\subsubsection{Power Peak Stabilization}

Stabilization to power peak may also be achieved with the LDSS. A small amount of $10 \mu \mathrm{m}$ laser emission is routed to a pyroelectric 
detector (the Laser Precision K-1050-2), providing direct measurement of the $10 \mu \mathrm{m}$ gain profile (see Fig. 3.8). The mode selector's "Lamb dip/power peak" switch determines whether the $4.3 \mu \mathrm{m}$ signal or pyroelectric detector output will serve as the lock-in amplifier's input signal (Fig. 4.25). The pyroelectric detector requires the same lock-in phasing and stabilizer modulation as used in Lamb dip lock mode (thereby providing a quick means of Lamb dip lock-in phasing).

To lock to power peak, the stabilizer is unlocked and reset, the cavity is tuned to the peak of the $10 \mu \mathrm{m}$ gain profile with the aid of a laser power meter, and the stabilizer is set to lock.

While power peak stabilization cannot be used for wind measurements, its incorporation into the LDSS provides the versatility necessary for the LDSS to become a permanent spectrometer subsystem.

\subsection{TIME SERIES ANALYSIS OF LASER STABILITY}

4.8.1. Concept

A sound interpretation of the Venus wind measurements warranted a test of LDSS absolute stability. To this end, a laboratory-generated reference line, of known absolute frequency, was monitored by the IRHS for an extended period. During such a time series, absolute variations in LO frequency are detected from induced shifts in the reference line's IF line center frequency.

A $10 \mu \mathrm{m}$ wind investigation, with absolute measurements at the 1 $\mathrm{m} / \mathrm{s}$ level, dictates Lo absolute stability to $<0.1 \mathrm{MHz}$. A time series reference line with an absolute frequency known to $<0.1 \mathrm{MHz}$ is therefore warranted. The absolute line center frequencies for the 


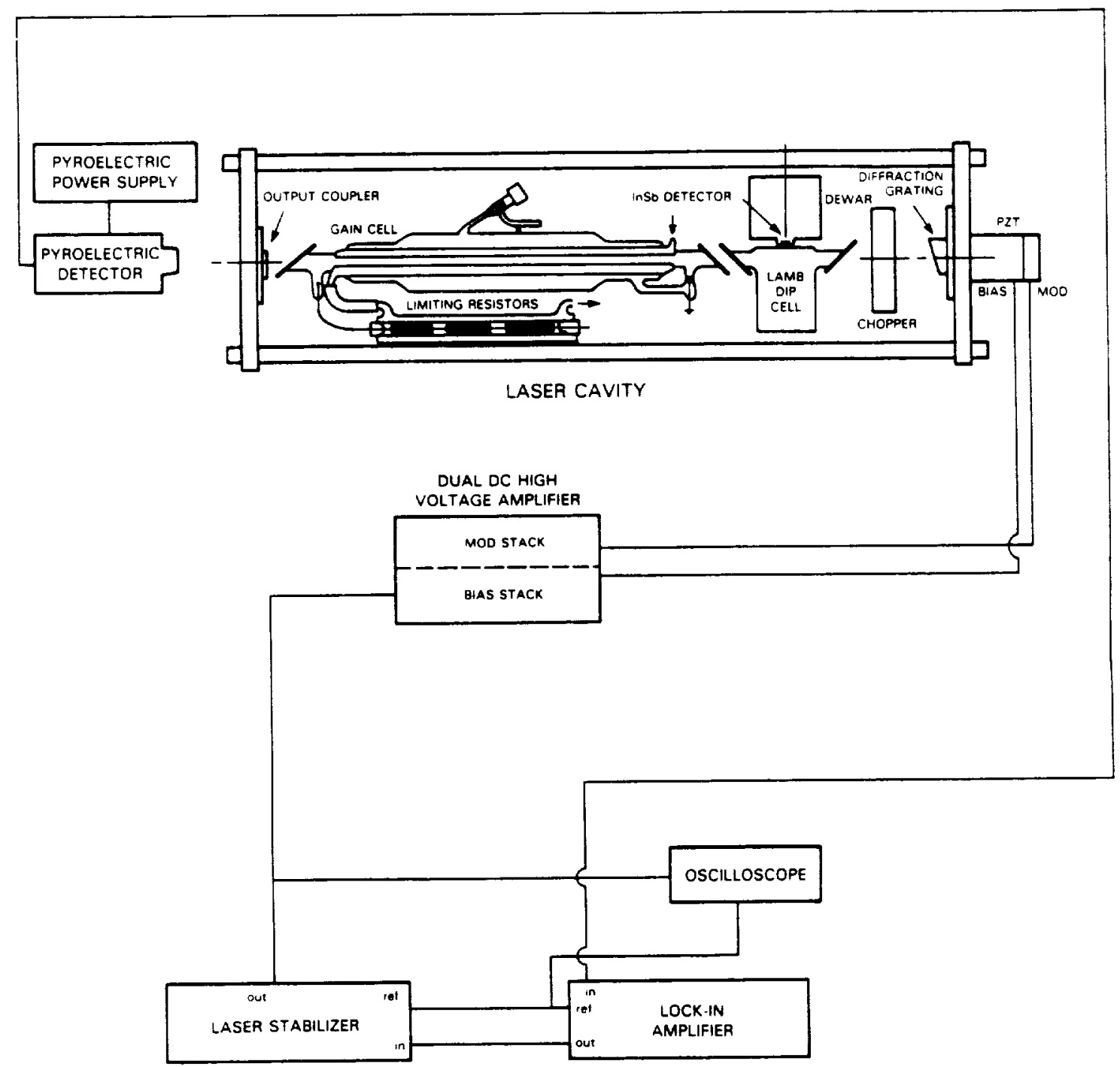

Figure 4.25. Power Peak Stabilization Lock Mode 
$\sum_{\mathrm{u}}{ }^{+} 00^{\circ} 1-\sum_{\mathrm{g}}{ }^{+}\left[10^{\circ} 0,02^{\circ} 0\right]_{\mathrm{I}}$ and $\sum_{\mathrm{u}}{ }^{+} 00^{\circ} 1-\sum_{\mathrm{g}}{ }^{+}\left[10^{\circ} 0,02^{\circ} 0\right]{ }_{\mathrm{II}} \mathrm{CO}_{2}$ laser transitions have been determined by Freed et al. (1980) to typically $0.05-0.10 \mathrm{MHz}$ for 7 isotopic forms. A two-channel heterodyne system was used to derive difference frequencies between two Lamb dip locked lasers (Freed et al, 1980). The difference frequencies were calibrated using the 9.33 and $10.18 \mu \mathrm{m}{ }^{12} \mathrm{C}^{16} \mathrm{O}_{2}$ transition absolute frequencies determined by Evenson et al. (1973). Fourteen overlaps exist within the $1.6 \mathrm{GHz}$ bandwidth of the IRHS, 13 of which involve the more exotic isotopic forms ${ }^{12} \mathrm{C}^{18} \mathrm{O}_{2}$ and ${ }^{16} \mathrm{o}^{12} \mathrm{C}^{18} \mathrm{o}$.

The simplest means of reference line generation is to place an absorption cell in front of one of the IRHS blackbodies. $\mathrm{CO}_{2}$ has a very small integrated absorption coefficient, $-10^{-23} \mathrm{~cm} / \mathrm{mol}$ at $296 \mathrm{~K}$, necessitating either a long path length cell or a high pressure cell environment. The latter, however, will effect an unwanted redward pressure shift in line center frequency of $0.08 \pm .05 \mathrm{MHz} / \mathrm{Torr}$, for pressures from 1 to 10 Torr (SooHoo 1984). A $1.0 \mathrm{~m}$ White cell (the Spectra Physics LO-3 cell; see ref.) was therefore employed allowing a multi-pass path length up to $100 \mathrm{~m}$ at $4 \mathrm{~m}$ increments.

The local oscillator was stabilized to the $9.6 \mu \mathrm{m}{ }^{12} \mathrm{C}^{16} \mathrm{O}_{2}$ P28 line $(31159508.1635 \pm .0457 \mathrm{MHz}), 375.5 \mathrm{MHz}$ redward of the $9.6 \mu \mathrm{m}{ }^{13} \mathrm{C}^{16} \mathrm{o}_{2}$ R32 line $(31159883.6641 \pm .0603 \mathrm{MHz}$ ) generated in the White cell (see Freed et al. 1980). With a $40 \mathrm{~m}$ path length, a pressure of $0.7-1.0$ Torr and a blackbody temperature of $1000 \mathrm{~K}$, a roughly $35 \% 1$ ine was generated, pressure shifted in theory by $\sim 0.1 \mathrm{MHz}$ (adopting SooHoo's (1984) results), yielding sufficient signal-to-noise in the IF to centroid the 1 ine to $1 \mathrm{~m} / \mathrm{s}$. 
Though a longer path length would decrease the pressure shift and/or provide a deeper line allowing better centroid determination in the IF, a $40 \mathrm{~m}$ length was found to be optimal. Greater lengths were associated with unacceptable degradation of blackbody intensity due to multiple reflections in the White cell. The resulting lower signal-to-noise on the continuum admitted poorer line center determinations in the IF.

A $63.2 \pm 18.1 \mathrm{KHz} /$ Torr blueward pressure shift associated with the Lamb dip cell (for the ${ }^{12} \mathrm{C}^{16} \mathrm{O}_{2}$ II band at pressures <100 mTorr; SooHoo et al. 1985) yields only a $1.58 \pm 0.45 \mathrm{KHz}$ shift in the Lamb dip $(1.58 \pm 0.45 \mathrm{~cm} / \mathrm{sec}$ at $10 \mu \mathrm{m})$ for a pressure of $25 \mathrm{mTorr}$, and is thus neglected.

Both Lamb dip and power peak stabilization temporal stability were measured directly. The latter provides a measure of the gain cell pressure shift and cavity alignment effects, as well as the locking accuracy to a feature as broad as the laser gain profile.

The acquisition and reduction of time series data are covered in detail in Appendix 1.

Figure 4.26 is a plot of both Lamb dip and power peak stabilization time series. The ordinate gives the local oscillator frequency departure from the laser transition rest frequency (as given by Freed et al. 1980). Each datum is a two minute integration with $1 \sigma$ error bars representing the combined $\mathrm{S} / \mathrm{N}$ - and RFLO-induced random error contributions. This corresponds to $\pm 0.12 \mathrm{MHz}$ and $\pm 0.11 \mathrm{MHz}$ for the Lamb dip and power peak time series respectively. 


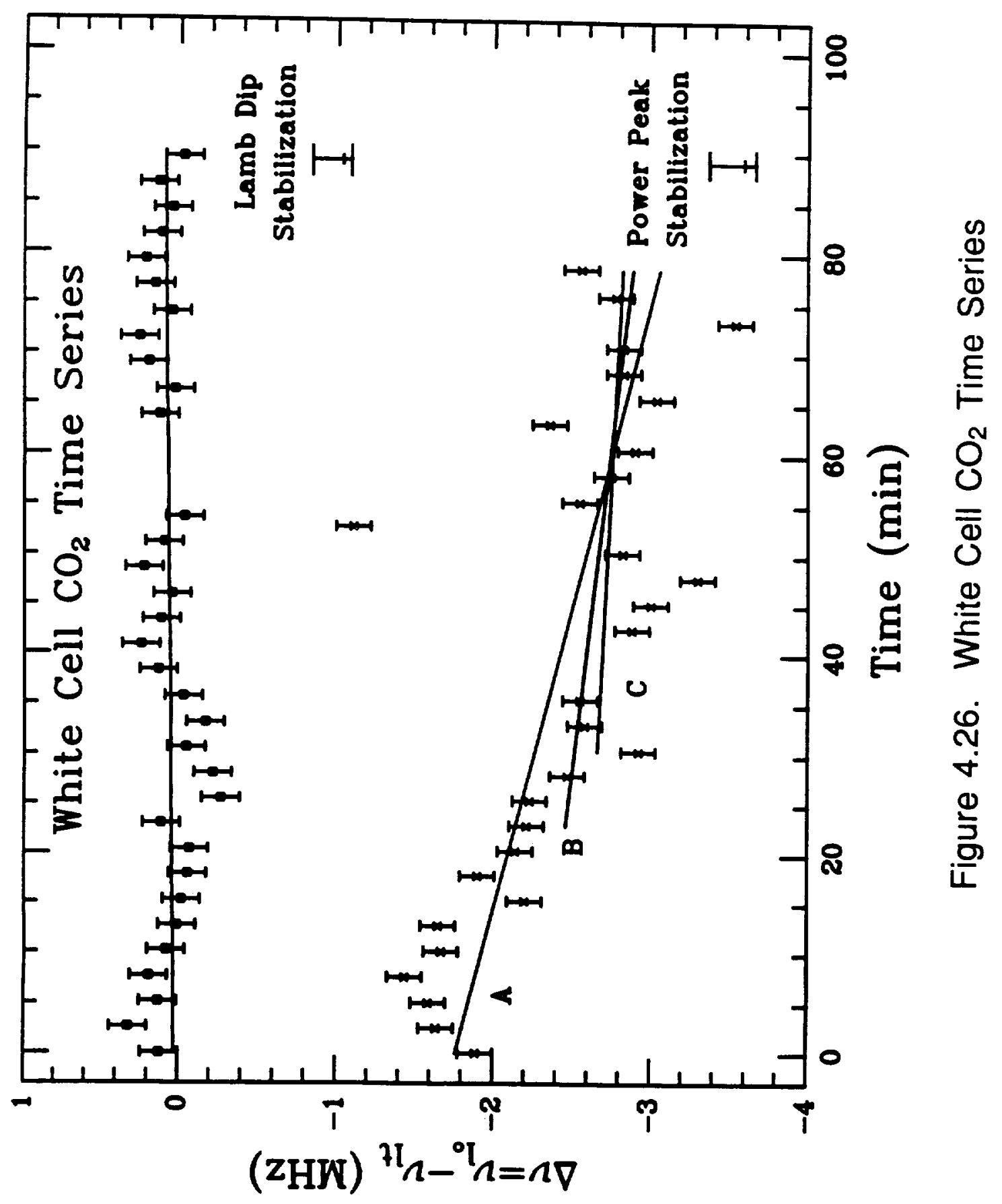




\subsubsection{Power Peak Time Series}

The most noticeable feature concerning the power peak time series is the near monotonic decrease in $\Delta \nu$ over the first -40 minutes. It is not unreasonable that this represents an equilibration time for both cavity thermal variation and the lasing medium within the gain cell. Linear least squares fits $A, B$ and $C$ are associated with points 1 to 30,10 to 30 and 13 to 30 , respectively. Fit $C$, starting 30 minutes into the time series and approximately 1.5 to 2 hours after laser power-up, has a slope statistically indistinguishable from zero possibly indicating steady state operation. (LO frequency stability $(\sim 1 \mathrm{MHz}$, or $10 \mathrm{~m} / \mathrm{s}$ at $10 \mu \mathrm{m})$, for a relatively undisturbed power peak stabilized laser, has been observed over many hours by Deming et al. (1986)). The standard deviation about fit C is $\sigma_{\Delta \nu}=0.51 \mathrm{MHz}$. This is $5 \sigma$ above the combined $\mathrm{S} / \mathrm{N}$ and RFLO induced random error. Real fluctuations in the laser frequency are thus clearly indicated. Also from fit $C,\langle\Delta \nu\rangle=-2.7 \mathrm{MHz}$, equivalent to a redward gain cell "pressure shift" of $170 \mathrm{KHz} /$ Torr, 208 larger than surmised by Hillman et al. (1977), but a factor of $\sim 3$ greater than reported by Betz (1977). The observed monotonic behavior in $\Delta \nu$ is consistent with a gain profile only partially dependent on the pressure shift.

\subsubsection{Lamb Dip Time Series}

The linear least squares fit associated with the Lamb dip time series has a slope of $7.0 \times 10^{-4} \pm 8.6 \times 10^{-4} \mathrm{MHz} / \mathrm{min}$, statistically indistinguishable from zero. The standard deviation about the least squares fit $\left(\sigma_{\Delta \nu}=0.13 \mathrm{MHz}\right)$ is consistent with that derived from the $\mathrm{S} / \mathrm{N}$ 
and RFLO induced random error contributions. This implies that real local oscillator departures from the laser transition frequency may actually be a negligible contribution to the observed r.m.s. fluctuations.

An underlying modulation for at least the first hour is apparent. Such long period modulation is the signature expected from an uncorrected thermal variation in cavity length. This drift was traced to the White cell and is therefore decoupled from normal laser operation. Small fringes were observed in the IF whose amplitude was sensitive to small-scale variation of the White cell length. These fringes, asymmetrically located about the line center in the IF, cause a shift in the center of gravity of the line. Long term thermal variation of the White cell length necessarily leads to an apparent variation in $L 0$ frequency.

The laser is stable to at least $\sigma_{10}=0.13 \mathrm{MHz}$ over a two minute integration. For a typical 20 minute integration on Venus this corresponds to $\sigma_{10}=0.04 \mathrm{MHz}$.

Over the 1.5 hour time series, considering only the random error contributions, $\Delta \nu=0.06 \pm .02 \mathrm{MHz}$ which reflects a statistically significant offset in the data. However, a systematic offset of $0.06 \pm .14 \mathrm{MHz}$ is expected from the White cell pressure shift and uncertainties in the laser transition and White cell generated line frequencies. This systematic error is provided by the error bar located directly under the Lamb dip caption at the right of the Figure. While, strictly speaking, a systematic uncertainty of $\pm .14 \mathrm{MHz}$ will not admit determination of even the sense of the pressure shift, given 
the observed $\langle\Delta \nu\rangle, \Delta \nu=+0.06 \mathrm{MHz}$ is exactly what is expected from a White cell at 0.7 Torr, assuming SooHoo's pressure shift results. Since:

i) the laser should be oscillating at the laser transition frequency $\nu_{1 t}$ (a negligible pressure shift is associated with the Lamb dip cell nominally held at 20 mTorr pressure) and,

ii) the time series yields the expected local oscillator frequency $\left(\nu_{10}\right)$ departure from $\nu_{1 t}$, due to the white cell pressure shift, with no indication of any additional systematic offset then,

$\nu_{10} \equiv \nu_{1 t}$, with a standard deviation of $\sigma_{10}=0.13 /(\tau / 2)^{1 / 2} \mathrm{MHz}$, will be assumed throughout the wind investigation where $\tau$ is the integration time in minutes.

With Lamb dip stabilization, conditions in the isolated and passive environment of the Lamb dip cell are monitored as a means of probing laser output frequency. Power peak stabilization, however, relies on sensing operational departure from the gain profile's peak, whose position in frequency space follows changes in cavity alignment and gain cell thermodynamic conditions. The power peak stabilized laser is at least 5 times more unstable than the Lamb dip stabilized case, the latter's stability being set only by the detection threshold of the experiment. This is not unreasonable as the laser gain profile is more than an order of magnitude broader than the Lamb dip. More critical from the standpoint of a wind investigation, the gain peak offset from $\nu_{1 t}$ corresponds to a $27 \mathrm{~m} / \mathrm{s}$ Doppler shift at $10 \mu \mathrm{m}$. The fact that the offset is variable implies that its removal, without constant monitoring of a laboratory line, is not possible and throws 
into serious question the accuracy of line of sight wind speeds obtained with such a system. These considerations have direct bearing on the pioneering heterodyne wind measurements in the Venusian mesosphere and lower thermosphere made by Betz et a1. (1976, 1977b; Betz 1977, 1982). 



\section{CHAPTER 5: OVERVIEW OF OBSERVATIONS}

Figure 5.1 provides an overview, to scale, of the four investigation-specific planetary aspect geometries, spanning 828 of a synodic period, and characterized by a five-fold variation in planetary disk size. The $1.5 \mathrm{~m}$ (main) McMath Solar Telescope was used for the December 1985 and June 1986 observing periods. The NASA $3 \mathrm{~m}$ Infrared Telescope Facility instrument was used October 1986 and March 1987. The small circles represent the McMath 1.7 arc-second and IRTF 0.9 arc-second diffraction-limited half power beam widths (HPBW) at the various locations sampled.

The line of sight wind velocity associated with each scan set reflects dynamical contributions from all regions intercepted by the beam. For limb scans, and small disk-to-beam ratios, the intercepted region may be a substantial portion of the planet, hence the measured quantity is a beam-integrated wind velocity. A qualitative comparison of beam-integrated winds, for different planetary aspects, can be an effective determinant of gross circulation. However, modelling of the beam-integrated winds is warranted for a quantitative derivation of the global wind field responsible. Both these approaches are explored. Data reduction algorithms used to generate the beam-integrated wind velocities are covered in Appendix 1.

\subsection{CHOICE OF EMISSION LINE AS DYNAMICAL PROBE}

The ${ }^{12} \mathrm{C}^{16} \mathrm{O}_{2} 10.33 \mu \mathrm{m} \mathrm{R}(8)$ line was used exclusively for all wind measurements. In the Venusian atmosphere, $\mathrm{CO}_{2}$ number densities in the collisionally-coupled rotational levels are thermally distributed as a 


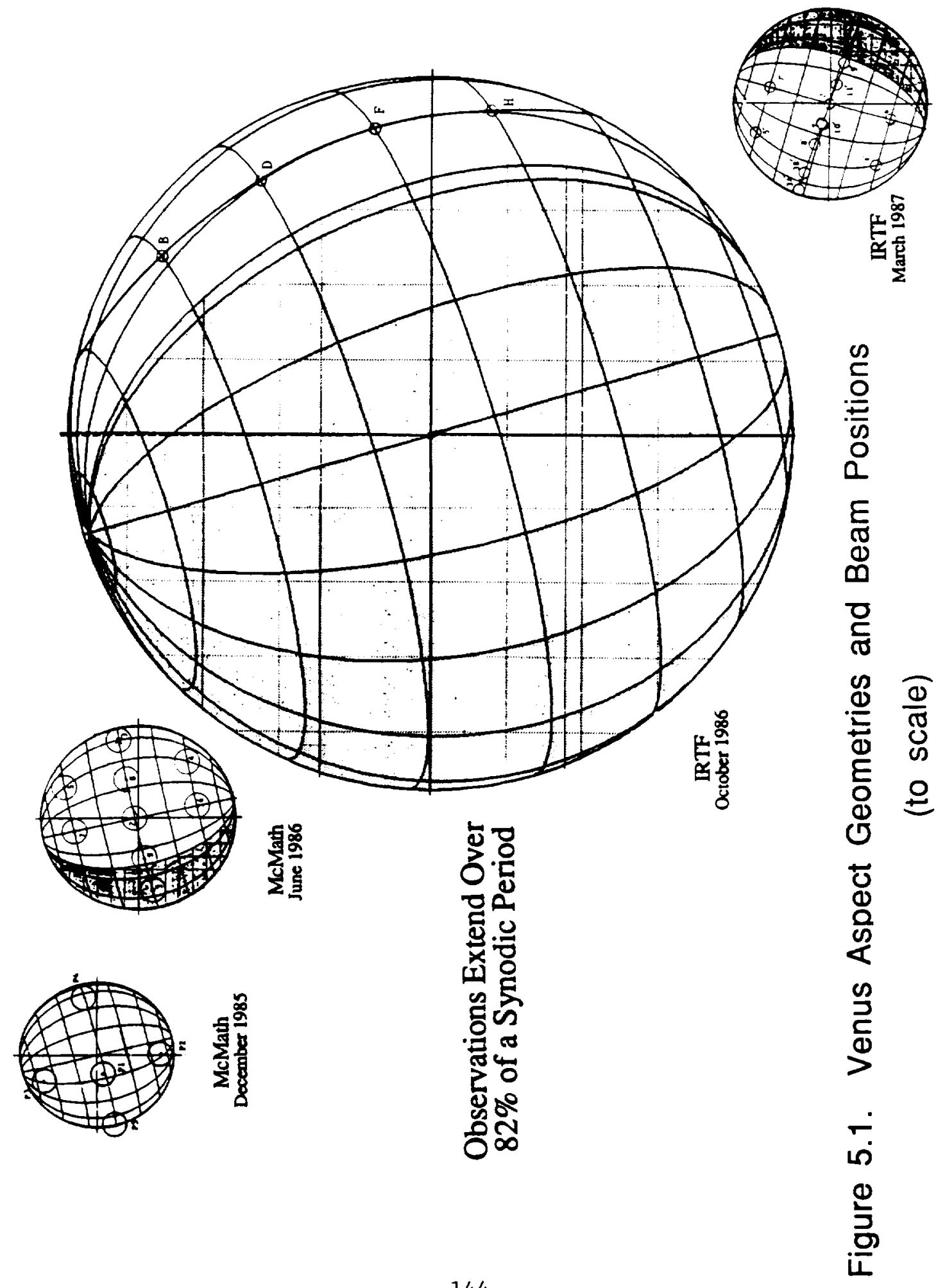


function of $\mathrm{J}$ (Deming et al. 1983). The maximum number density (hence maximum signal strength) occurs at $J_{\max }=0.93 \mathrm{~T}^{1 / 2}-0.5$, where $\mathrm{T}$ is the gas kinetic temperature (see Lang 1980). $\mathrm{J}_{\max }=12$ for $\mathrm{T}=200 \mathrm{~K}$, the approximate non-thermal emitting region temperature $(100-120 \mathrm{~km}$ altitude) for solar zenith angles (SZA) $<90^{\circ}$ (see Figure P.1). The $\mathrm{R}(8)$ laser transition was both near the thermal peak of the rotational distribution and within $1.6 \mathrm{GHz}$ of the rest frequency of $\mathrm{NH}_{3} \mathrm{sQ}(2,2)$. A small $\mathrm{NH}_{3}$ absorption cell placed in front of the IRHS calibration blackbody (see Fig. 3.8) thereby allowed field monitoring of laser frequency stability, though not simultaneously with Venus data acquisition (the $\mathrm{NH}_{3} \mathrm{sQ}(2,2)$ and Venus ${ }^{12} \mathrm{C}^{16} \mathrm{O}_{2} \mathrm{R}(8)$ lines could not be located simultaneously in the high resolution filterbank without significant overlap).

As tests of laser frequency stability, the $\mathrm{NH}_{3}$ time series were superseded by the $\mathrm{CO}_{2}$ laboratory time series (see Chapter 4), the latter providing a measure of absolute stability $\left(\mathrm{CO}_{2}\right.$ transition rest frequencies are precisely known (to $<0.05 \mathrm{MHz}$ ), while those for $\mathrm{NH}_{3}$ are not).

\subsection{ADOPTED NOMENCLATURE}

Beam positions, and the positions of the Subsolar and Subearth Points, will be given in Venus latitude $\left(0^{\circ}\right.$ to $90^{\circ} \mathrm{N}, 0^{\circ}$ to $\left.90^{\circ} \mathrm{S}\right)$ and longitude $\left(0^{\circ}\right.$ to $180^{\circ} \mathrm{E}, 0^{\circ}$ to $\left.180^{\circ} \mathrm{W}\right)$. As defined by the IAU, the north pole of Venus is above the orbital plane. Longitude is measured from the Subearth Meridian (thus Earth-fixed), which is convenient for a wind field projected along the line of sight. (Note - moving eastward 
in longitude is motion against the retrograde planetary rotation).

Hereafter, all dates and times are in Universal Time (UT), unless otherwise stated.

Beam-center pointing uncertainty was determined in the field from tests of: 1) IR $(10 \mu \mathrm{m})$ and visible (tracking) beam misalignment, and 2) guiding mode accuracy (see Sections 3.5 and 7.1.1). The quoted pointing uncertainty reflects a reasonable worst case magnitude for combined systematic and random components. Quantitative deconvolution of both components was, in general, not possible.

Atmospheric seeing (in arc-seconds) refers to the time-averaged FWHM deflections of the Venus limb observed on the television monitor. Scan sets associated with $>2$ arc-second seeing were (in general) discarded.

Planetary aspects (such as those in Fig. 5.1) were generated by Fred Espenak, Code 693, NASA/Goddard Space Flight Center, using physical data found in the "Report of the IAU Working Group on Cartographic Coordinates and Rotational Elements of the Planets and Satellites" by Davies et al. (1983).

\subsection{BEAM-INTEGRATED WIND VELOCITIES}

\subsubsection{December 1985}

Observations 4 December 1985 ( $11^{\circ} \mathrm{W}$ elongation, 0.98 phase), 46 days prior to superior conjunction, yielded six beam-integrated line of sight wind velocities, distributed over the five beam positions indicated on Figure 5.2. (Note $E$ and $W$ on Fig. 5.2 refer to the Earth observer's sky and not the surface of Venus.) A Venus declination of 


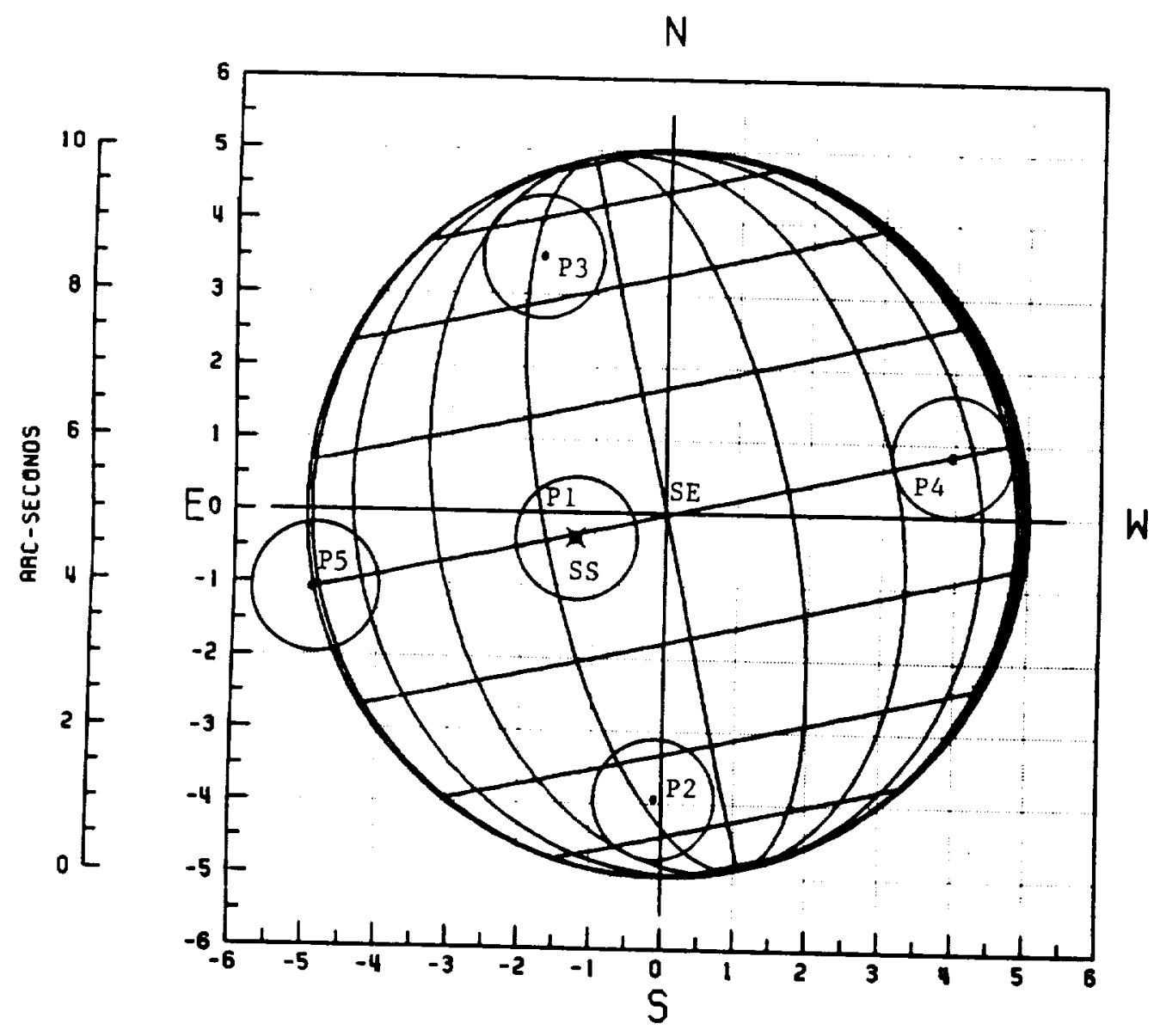

P1 Subsolar Point

P2 $50^{\circ} \mathrm{S}$ - Subsolar Meridian

P3 $50^{\circ} \mathrm{N}$ - Subsolar Meridian

P4 Equator $-55^{\circ} \mathrm{E}$ of Subearth Meridian

P5 Equator $-90^{\circ} \mathrm{W}$ of Subearth Meridian

Figure 5.2. Venus Beam Positions December 1985 McMath Solar Telescope 
$-19.5^{\circ}$ allowed full illumination of the McMath primary, providing a circular 1.7 arc-second (HPBW) diffraction-1imited beam (circles on Fig. 5.2). A 10 arc-second planetary diameter yielded a seeing-excluded disk-to-beam ratio of 6.25. Beam pointing uncertainty was 1 arc-second. The Subearth Point (denoted SE) was located at $0.4^{\circ} \mathrm{N}$ latitude, and by definition, $0^{\circ}$ longitude. The Subsolar Point (denoted SS) was located at $0.5^{\circ} \mathrm{S}$ latitude, $15.1^{\circ} \mathrm{W}$ longitude. Beam position P1 was centered on SS. Positions $\mathrm{P} 2$ and $\mathrm{P} 3$ were centered at $50^{\circ} \mathrm{S}$ and $50^{\circ} \mathrm{N}$ latitude along the Subsolar Meridian. Positions P4 and P5 were centered on the equator at $55^{\circ} \mathrm{E}$ and $90^{\circ} \mathrm{W}$ longitude.

Table 5.1 provides the six beam-integrated wind velocities for December 1985. The following quantities appear in each of the 8 columns :

Column 1 scan set beam position (refer to Fig. 5.2)

Column 2 scan set designation

Column 3 ephemeris-derived topocentric line of sight velocity of Venus $\left(V_{T}\right)$ in $\mathrm{km} / \mathrm{s}$ (positive for recession)

Column 4 high resolution filterbank (HRB) line center position $(\nu$,$) , together with \mathrm{S} / \mathrm{N}$-induced HRB line center uncertainty $\left(\sigma_{1 c}\right)$, both in $5 \mathrm{MHz}$ channels (see Sections 3.4 and A1.5)

Column 5 radio frequency local oscillator (RFLO) frequency $\left(\nu_{\mathrm{rf}}\right)$, and RFLO standard deviation ( $\sigma_{\mathrm{rf}}$ ) both in $\mathrm{MHz}$ (see Sections 3.4 and Al.5)

Column 6 laser local oscillator frequency uncertainty $\left(\sigma_{10}\right)$, in $\mathrm{MHz}$, based on total integration time per scan set (see Sections 4.8 and Al.5)

Column 7 Percent contribution to the wind velocity variance $\left(\sigma_{\mathrm{W}}{ }^{2}\right)$. The three subcolumns (labelled RF, LC, and LO) represent the percent contributions from $\sigma_{\text {rf }}, \sigma_{1 \mathrm{~g}}$, and $\sigma_{10}$ ' respectively. Fluctuations are assumed uncorrelated (see Section A1.5) 


\section{Table 5.1}

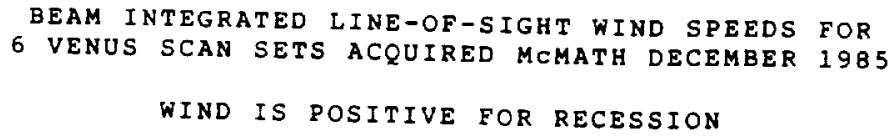

\begin{tabular}{|c|c|c|c|c|c|c|c|c|c|c|c|c|c|c|}
\hline \multirow{2}{*}{$\begin{array}{l}\text { BM } \\
\text { P T } \\
\text { P1 }\end{array}$} & \multirow{2}{*}{$\begin{array}{l}\text { SCN } \\
\text { SET } \\
\text { VO2 }\end{array}$} & \multirow{2}{*}{$\begin{array}{c}\mathrm{V}_{\mathrm{T}} \\
(\mathrm{Km} / \mathrm{s}) \\
3.1472\end{array}$} & \multicolumn{3}{|c|}{$\begin{array}{l}v_{l c} \pm \sigma_{1 c} \\
\text { (channels) }\end{array}$} & \multicolumn{3}{|c|}{$v_{x f \pm{ }_{(M H z)}^{\sigma}}$} & \multirow{2}{*}{$\begin{array}{c}\sigma_{10} \\
(M H 2) \\
.05\end{array}$} & \multicolumn{3}{|c|}{$\begin{array}{c}\% \sigma_{W}^{2} \\
\text { CONTRIB. } \\
\text { RF LC LO }\end{array}$} & \multicolumn{2}{|l|}{ 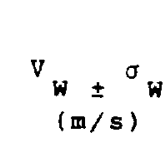 } \\
\hline & & & 34.628 & \pm & .095 & 1762.91 & \pm & .03 & & 0 & 99 & 1 & $2.2 \pm 5$ & 5 \\
\hline P1 & V0 6 & 3.4272 & 28.895 & \pm & .135 & 1762.60 & \pm & .04 & .05 & 0 & 99 & 1 & $15.3 \pm 7$ & 7 \\
\hline P 2 & vol & 3.1789 & 33.193 & \pm & .105 & 1762.96 & \pm & .03 & .04 & 0 & 99 & 1 & $45.2 \pm 5$ & 5 \\
\hline P 3 & V01 & 3.1219 & 34.259 & \pm & .090 & 1763.11 & \pm & .19 & .05 & 15 & 84 & 1 & $48.7 \pm 5$ & 5 \\
\hline P4 & V05 & 3.3205 & 30.618 & \pm & .135 & 1762.83 & \pm & .08 & .04 & 1 & 98 & 0 & $35.3 \pm 7$ & 7 \\
\hline P 5 & vo 4 & 3.2431 & 31.195 & \pm & .085 & 1762.93 & \pm & .04 & .03 & 1 & 99 & 1 & $83.9 \pm$ & \\
\hline
\end{tabular}


Column 8 beam-integrated line of sight wind velocity $\left(\mathrm{V}_{\mathrm{W}}\right)$ (positive for recession) and wind velocity standard deviation $\left(\sigma_{\mathrm{W}}\right)$, both in $\mathrm{m} / \mathrm{s}$ (see Section Al.5)

Column 7 provides a straightforward means of identifying limiting sources of r.m.s. wind velocity error. For all four observing periods, $\mathrm{S} / \mathrm{N}$-induced line center uncertainty $\left(\sigma_{1 c}\right)$ accounts for typically 958 (always $>80 \%$ ) of the wind velocity variance $\left(\sigma_{\mathrm{W}}{ }^{2}\right)$.

As indicated on Table 5.1, uncertainties in ${ }^{12} \mathrm{C}^{16} \mathrm{O}_{2} 10.33 \mu \mathrm{m} \mathrm{R}(8)$ rest frequency $( \pm 0.0523 \mathrm{MHz}$, or $0.523 \mathrm{~m} / \mathrm{s}$ at $10 \mu \mathrm{m}$; Freed et al. 1980), and ephemeris-derived Venus topocentric velocity $( \pm 1 \mathrm{~m} / \mathrm{s})$, provide an overall wind velocity systematic error of $\pm 1.5 \mathrm{~m} / \mathrm{s}$ (as is the case for all four observing periods, except two scan sets acquired October 1986).

Figure 5.3 provides the UT and Local Times associated with December 1985 scan set acquisition. Vertical bars bounding the "Venus" caption are the 2.9 air mass limits.

Scan sets V01 through V05 were associated with 1 arc-second seeing. Seeing for V06 was 2 arc-seconds. Five additional scan sets, four acquired 3 December 1985, and one acquired immediately following V06, were discarded due to $\geq 3$ arc-second seeing conditions.

The upper half of Table 5.2 provides the number of scans, and corresponding total integration time (in minutes), for each December 1985 scan set. The integration time per scan was $90^{\mathrm{S}}$.

\subsubsection{June 1986}

Observations 15 through 27 June $1986\left(-37^{\circ} \mathrm{E}\right.$ elongation, -0.76 phase), starting 73 days prior to greatest eastern elongation, yielded 


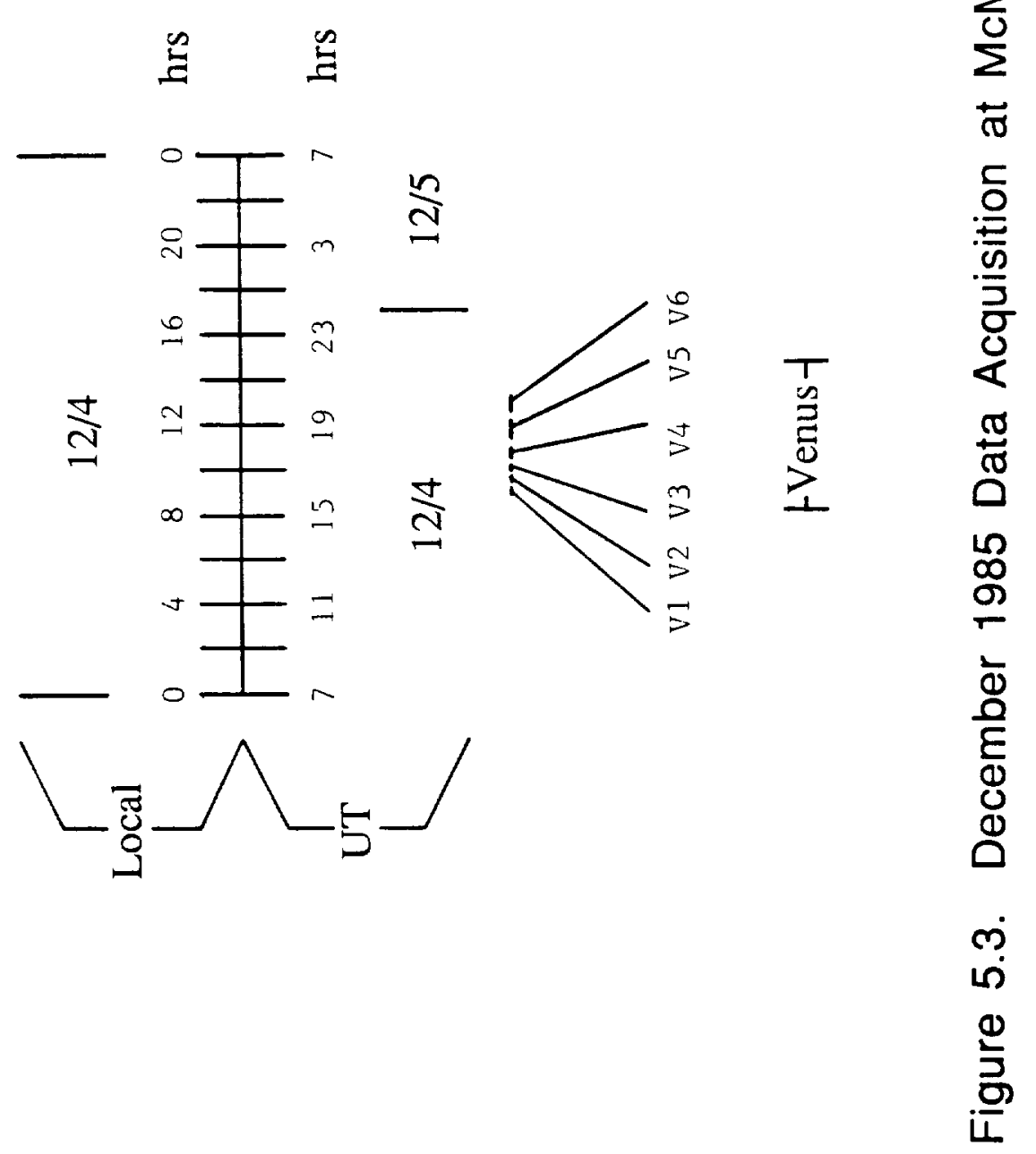


Table 5.2. Integration Time for Venus Scan Sets

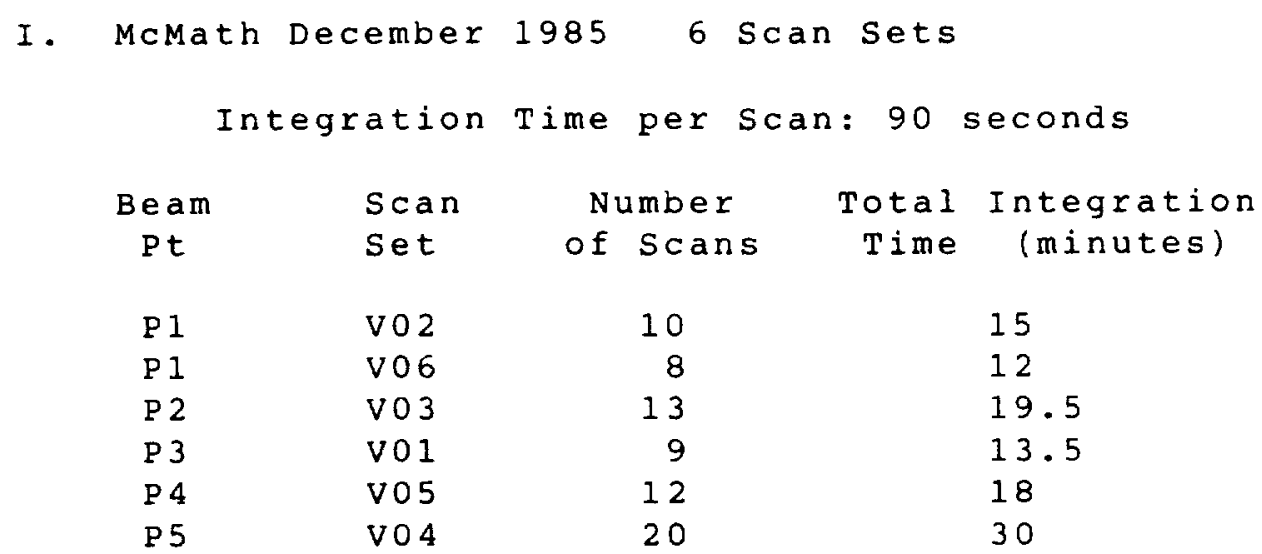

II. McMath June $1986 \quad 19$ Scan Sets

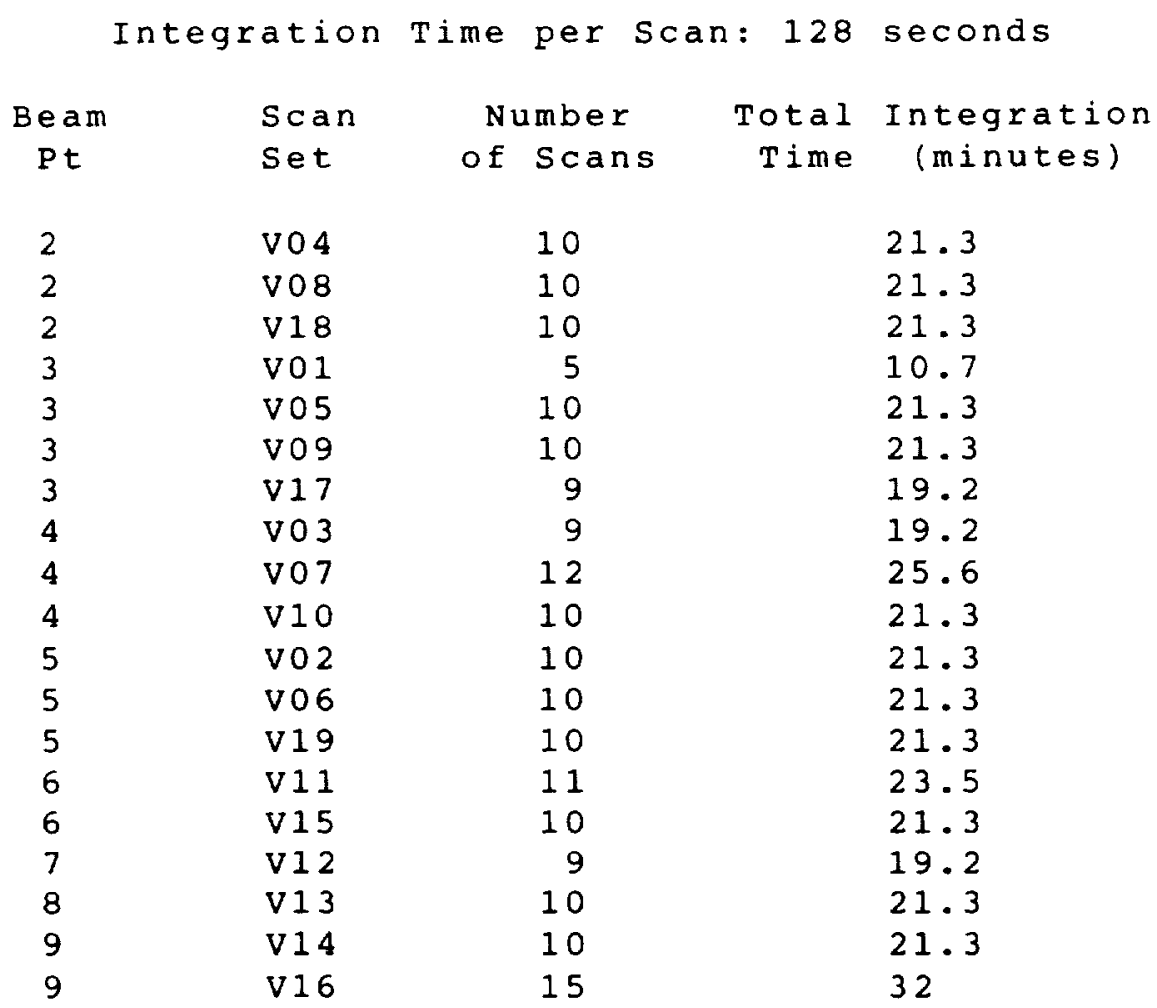


19 beam-integrated line of sight wind velocities distributed over the 8 beam positions indicated on Figure 5.4. A Venus declination of $+20^{\circ}$ caused vignetting of the McMath primary (see Section 3.5), resulting in an elliptical diffraction-1imited beam 1.7 arc-seconds in right ascension (R.A.) by 2.2 arc-seconds in declination (HPBW). A 14 arc-second planetary diameter yielded a seeing-excluded disk-to-beam ratio (in R.A.) of 8.75 . Beam pointing uncertainty was 1 arc-second. Over the course of the observing period, the Subearth Point migrated from $2^{\circ} \mathrm{S}$ to $1.6^{\circ} \mathrm{S}$ latitude. The Subsolar Point migrated from $2.3^{\circ} \mathrm{S}$ latitude, $55.6^{\circ} \mathrm{E}$ longitude, to $1.8^{\circ} \mathrm{S}$ latitude, $60.5^{\circ} \mathrm{E}$ longitude. Beam position 2 was centered at the equator/Subearth meridian intersection, near SE. Beam position 3 was centered on SS. Positions 8 and 9 were centered on the equator at $25^{\circ} \mathrm{E}$ and $25^{\circ} \mathrm{W}$ longitude. Positions 6 and 7 were centered at $40^{\circ} \mathrm{S}$ and $40^{\circ} \mathrm{N}$ latitude along the Subearth Meridian. Positions 4 and 5 were centered at $40^{\circ} \mathrm{S}$ and $40^{\circ} \mathrm{N}$ latitude along the $40^{\circ} \mathrm{E}$ longitude meridian.

Table 5.3 provides the 19 beam-integrated wind velocities for June 1986.

The UT and Local Times associated with June 1986 scan set acquisition are provided on Figure 5.5 (First Half: 15 to 18 June) and Figure 5.6 (Second Half: 25 to 27 June). Lunar scan sets L1 through L4 (Fig. 5.5) and L5 through L9 (Fig. 5.6) were used for terrestrial absorption removal (see Section 5.2). Mars scan sets M1 through M10 (Fig. 5.5) and M11 through M20 (Fig. 5.6) were acquired near 1986 Mars opposition (see Chapter 10). On Figures 5.5 and 5.6, the vertical bars bounding the "Venus", "Moon" and "Mars" captions are the 2.9 air mass 


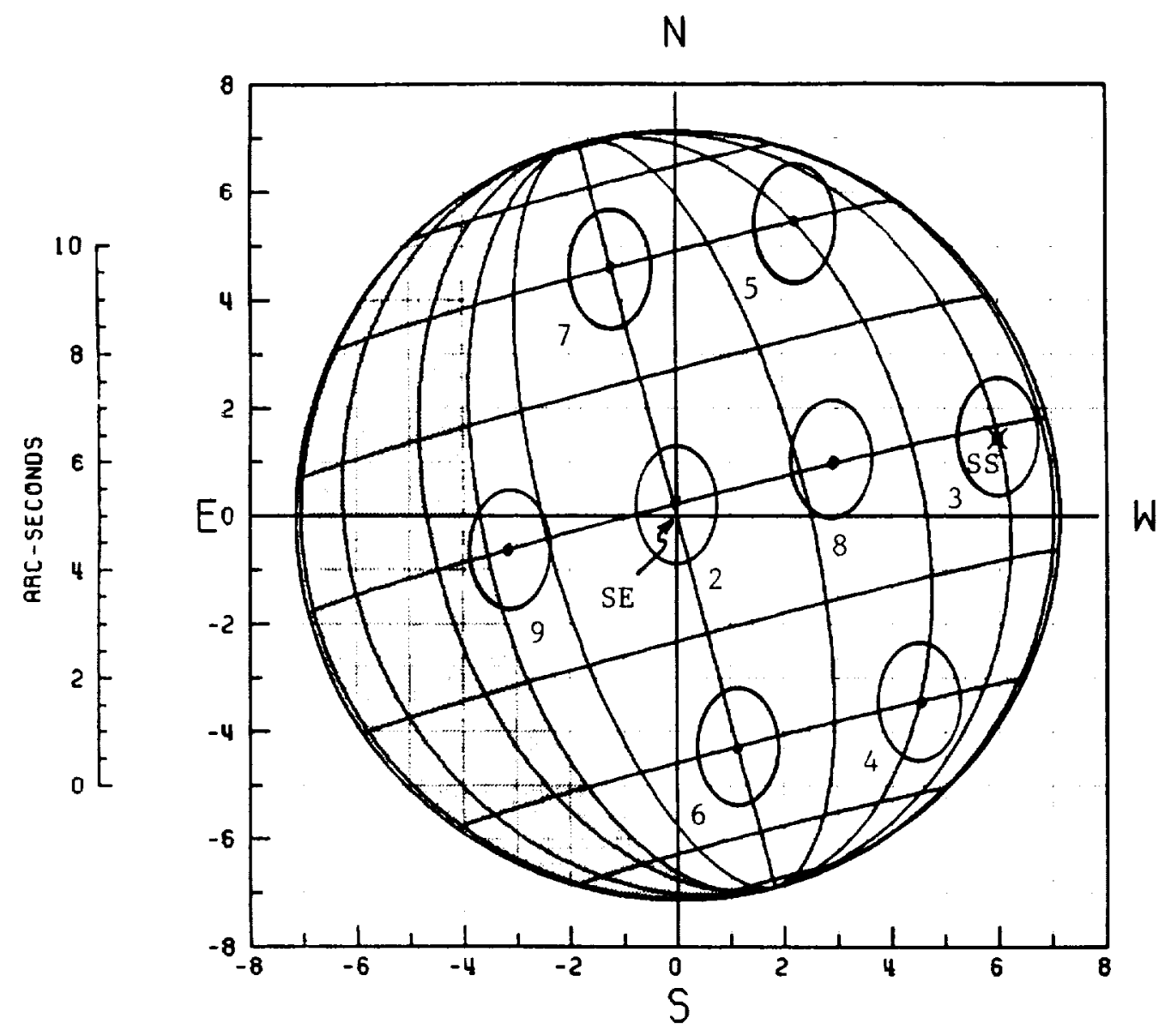
2 Subearth Point
3 Subsolar Point
$440^{\circ} \mathrm{S}-40^{\circ} \mathrm{E}$ of Subearth Meridian
$540^{\circ} \mathrm{N}-40^{\circ} \mathrm{E}$ of Subearth Meridian
$640^{\circ} \mathrm{S}$ - Subearth Meridian
$740^{\circ} \mathrm{N}$ - Subearth Meridian
8 Equator $-25^{\circ} \mathrm{E}$ of Subearth Meridian
9 Equator $-25^{\circ} \mathrm{W}$ of Subearth Meridian

Figure 5.4. Venus Beam Positions June 1986

\section{McMath Solar Telescope}




\section{Table 5.3}

BEAM INTEGRATED LINE-OF-SIGHT WIND SPEEDS FOR 19 VENUS SCAN SETS ACQUIRED MCMATH JUNE 1986

WIND IS POSITIVE FOR RECESSION

LO ISOTOPE AND LINE: CO2626 RB

LO FREQUENCY: $29011133.014 \mathrm{MHZ}$

SYSTEMATIC ERROR:

EPHEMERIS (VT) ERROR: $\pm 1 \mathrm{~m} / \mathrm{s}$

CO2 626 RB REST FREQUENCY ERROR: $\pm .0523 \mathrm{MHz}$

TOTAL SYSTEMATIC ERROR: $\pm 1.5 \mathrm{~m} / \mathrm{s}$

\begin{tabular}{|c|c|c|c|c|c|c|c|c|c|c|c|c|c|c|}
\hline \multirow{2}{*}{$\begin{array}{l}\text { BM } \\
\text { PT } \\
2\end{array}$} & \multirow{2}{*}{$\begin{array}{l}\text { SCN } \\
\text { SET } \\
\text { VO4 }\end{array}$} & \multirow{2}{*}{$\begin{array}{c}V_{T} \\
(\mathrm{~km} / \mathrm{s}) \\
-11.6679\end{array}$} & \multicolumn{3}{|c|}{$\begin{array}{l}v_{1 c} \pm{ }^{\sigma} 1 \mathrm{c} \\
(c h a n n e l s)\end{array}$} & \multicolumn{3}{|c|}{${ }_{(M H z)}^{v_{\text {rf }}}{ }^{\sigma}$ rf } & \multirow{2}{*}{$\begin{array}{c}\sigma_{10} \\
(M H z) \\
.04\end{array}$} & \multicolumn{3}{|c|}{$\begin{array}{c}\% \sigma_{\mathrm{W}}^{2} \\
\operatorname{conTRIB} .\end{array}$} & \multicolumn{2}{|c|}{$v_{(m / s)}$} \\
\hline & & & 41.243 & \pm & .055 & 2619.96 & \pm & .02 & & 1 & 97 & 2 & $4.2 \pm$ & \pm \\
\hline 2 & V08 & -11.7268 & 42.462 & \pm & .041 & 2632.89 & \pm & .03 & .04 & 2 & 94 & 4 & -7.5 & \pm \\
\hline 2 & V1B & -12.0343 & 39.868 & \pm & .056 & 2650.03 & \pm & .05 & .04 & 3 & 95 & 2 & $-11.1 \pm$ & \pm \\
\hline 3 & VOI & -11.8881 & 36.857 & \pm & .056 & 2620.10 & \pm & .03 & .06 & 1 & 95 & 4 & $-3.6 \pm$ & \pm \\
\hline 3 & vos & -11.6050 & 42.496 & \pm & .030 & 2619.96 & \pm & .06 & .04 & 13 & 81 & 6 & $6.1 \pm$ & \pm \\
\hline 3 & vo9 & -11.6796 & 43.946 & \pm & .0 .60 & 2632.71 & \pm & .11 & .04 & 12 & 87 & 2 & $23.8 \pm$ & \pm 3 \\
\hline 3 & V17 & -12.0649 & 40.156 & \pm & .075 & 2650.22 & \pm & .07 & .04 & 3 & 95 & 1 & $32.4 \pm$ & \pm 4 \\
\hline 4 & vo3 & -11.7587 & 39.958 & \pm & .065 & 2620.13 & \pm & .11 & .04 & 10 & 88 & 1 & $26.9 \pm$ & \pm 4 \\
\hline 4 & V07 & -11.4485 & 45.878 & \pm & .054 & 2619.88 & \pm & .06 & .04 & 5 & 94 & 2 & $25.1=$ & \pm 3 \\
\hline 4 & $v 10$ & -11.5897 & 45.602 & \pm & .043 & 2632.47 & \pm & .05 & .04 & 5 & 92 & 3 & $22.0 \pm$ & \pm 2 \\
\hline 5 & VO2 & -11.8101 & 38.863 & \pm & .055 & 2620.11 & \pm & .04 & .04 & 2 & 96 & 2 & $21.9 \pm$ & \pm 3 \\
\hline 5 & v06 & -11.5355 & 44.169 & \pm & .038 & 2619.91 & \pm & .06 & .04 & 9 & 87 & 4 & 23.5 & \pm 2 \\
\hline 5 & V19 & -12.0093 & 41.111 & \pm & .069 & 2649.92 & \pm & .04 & .04 & 1 & 97 & 1 & 29.2 & \pm 4 \\
\hline 6 & V11 & -11.9928 & 45.967 & \pm & .040 & 2674.63 & \pm & .06 & .04 & 8 & 89 & 3 & $8.3=$ & \pm 2 \\
\hline 6 & $\vee 15$ & -11.9700 & 41.425 & \pm & .052 & 2649.41 & \pm & .10 & .04 & 13 & 85 & 2 & $11.4=$ & \pm 3 \\
\hline 7 & V12 & -11.9537 & 46.907 & \pm & .050 & 2674.59 & \pm & .05 & .04 & 4 & 94 & 3 & 18.2 & \pm 3 \\
\hline 8 & V13 & -11.9242 & 47.130 & \pm & .029 & 2674.55 & \pm & .05 & .04 & 10 & 84 & 6 & $0.6=$ & \pm 2 \\
\hline 9 & V14 & -11.8933 & 48.555 & \pm & .116 & 2674.49 & \pm & .03 & .04 & 0 & 99 & 0 & $44.0=$ & \pm 6 \\
\hline 9 & $V 16$ & -11.9506 & 42.461 & \pm & .155 & 2649.27 & \pm & .14 & .03 & 3 & 97 & 0 & 47.0 & \pm 8 \\
\hline
\end{tabular}




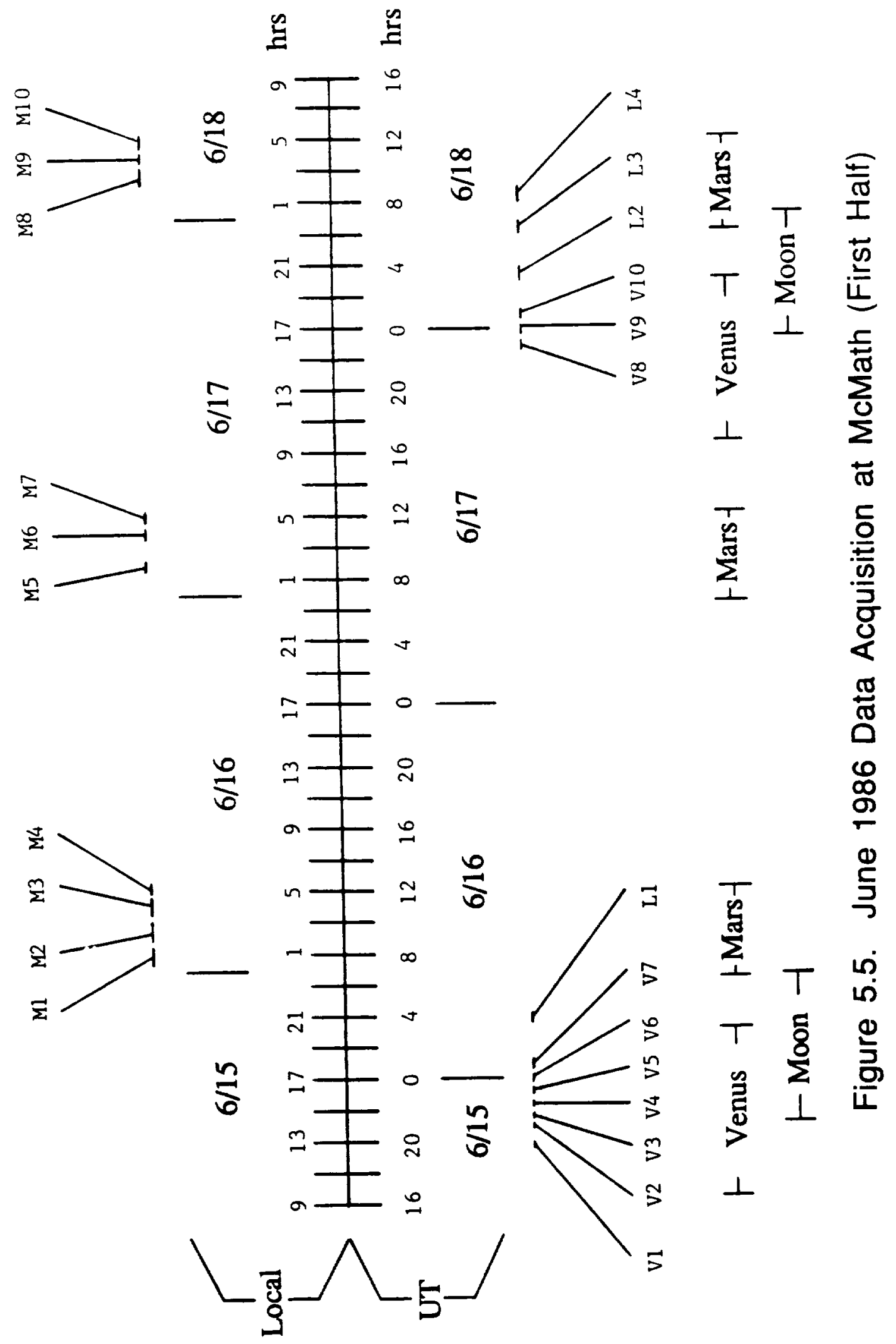




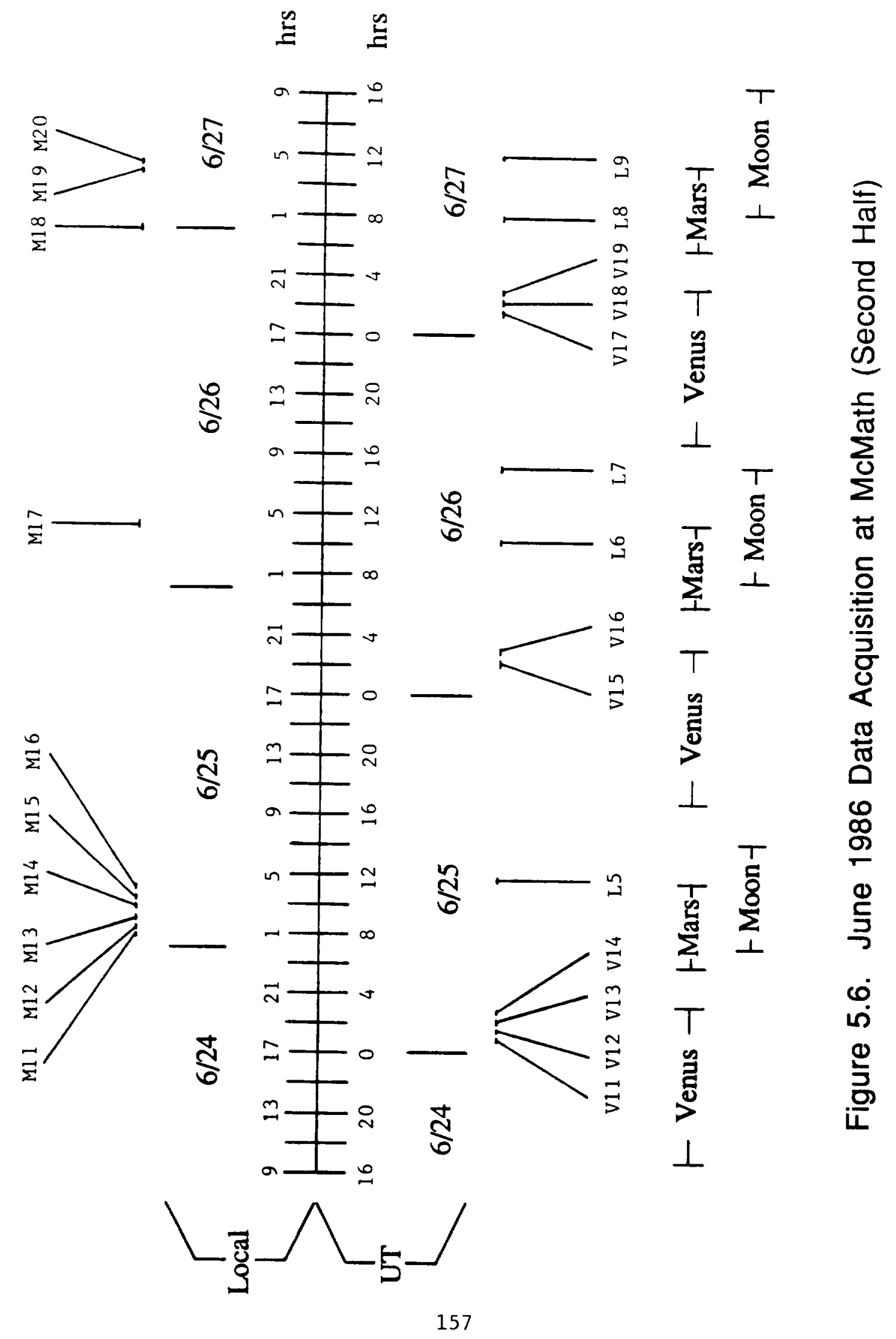


1imits.

1 arc-second seeing was associated with scan sets V1 through V6, V13, V14, and V17 through V19. 2 arc-second seeing was associated with sets V7 through V10, V11, V12, V15 and V16. Four scan sets were discarded due to $\geq 3$ arc-second seeing, including: two immediately following V7, one following V10 and one just prior to V15.

The lower half of Table 5.2 provides the number of scans, and total integration time, per scan set. The integration time per scan was $128^{s}$.

\subsubsection{October 1986}

Observations 11 through 14 October 1986 ( $30^{\circ} \mathrm{E}$ elongation, 0.15 phase), starting 25 days prior to inferior conjunction, yielded eight beam-integrated line of sight wind velocities, distributed over the four beam positions indicated on Figure 5.7. A 48 arc-second planetary diameter, and 0.9 arc-second (HPBW) IRTF diffraction-limited beam, yielded a seeing-excluded disk-to-beam ratio of 60 .

The Subearth Point wAs located at $8^{\circ} \mathrm{N}$ latitude. The Subsolar Point was located at $2^{\circ} \mathrm{N}$ latitude, $135^{\circ} \mathrm{E}$ longitude. Beam positions $\mathrm{B}$, $D, F$ and $H$ were chosen equidistant from the Subsolar Point. Positions $\mathrm{D}, \mathrm{F}$ and $\mathrm{H}$ were nominally centered at $20^{\circ} \mathrm{N}, 0^{\circ} \mathrm{N}$ and $20^{\circ} \mathrm{S}$ latitudes, respectively, on the $60^{\circ} \mathrm{E}$ longitude meridian. Position B was nominally centered at $40^{\circ} \mathrm{N}$ latitude, $65^{\circ} \mathrm{E}$ longitude.

Table 5.4 provides the 8 beam-integrated wind velocities for October 1986.

The LSI $11 / 23$ field computer's clock, driven by line frequency 


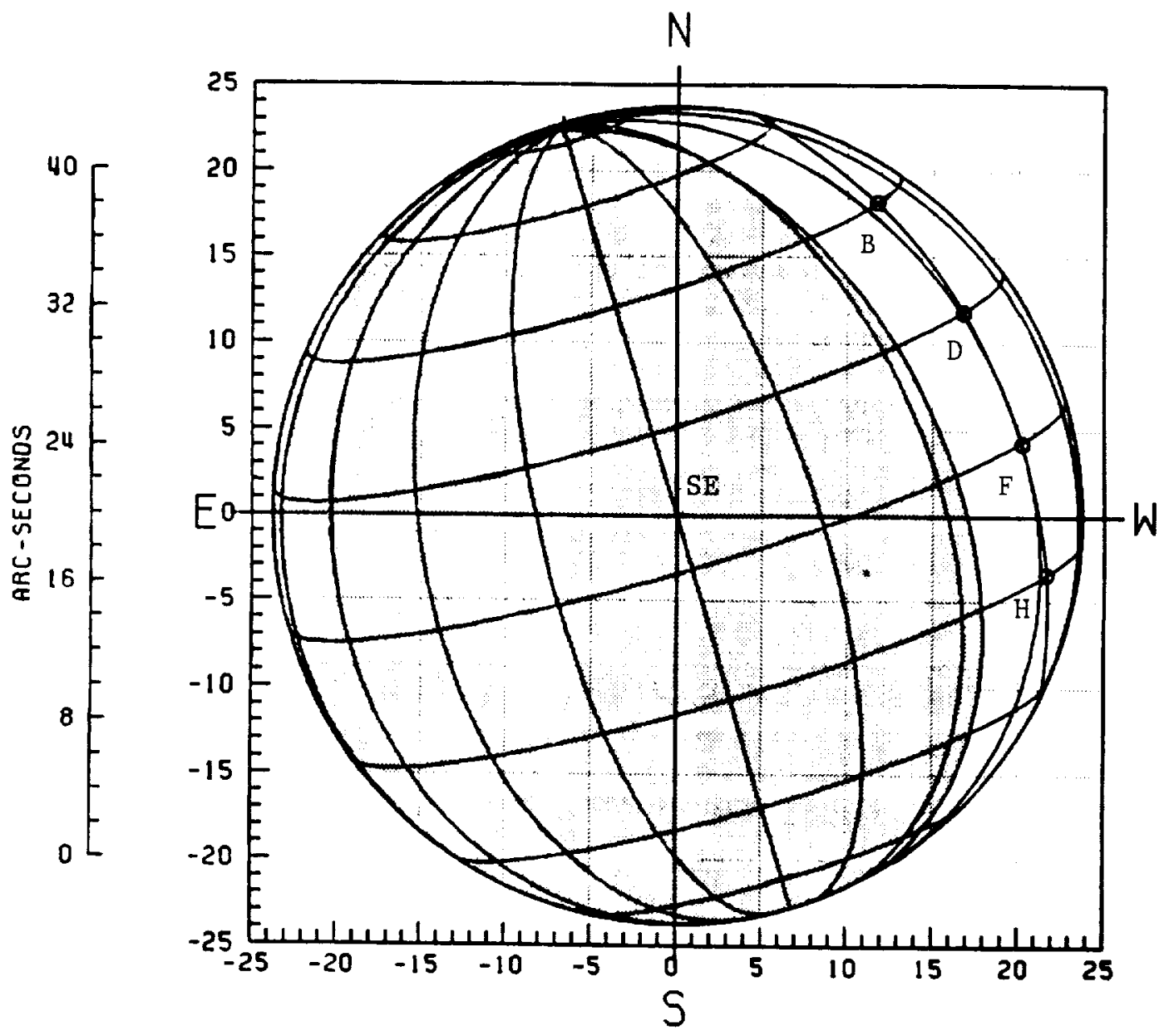

B $40^{\circ} \mathrm{N}-65^{\circ} \mathrm{E}$ of Subearth Meridian

D $20^{\circ} \mathrm{N}-60^{\circ} \mathrm{E}$ of Subearth Meridian

F Equator $-60^{\circ} \mathrm{E}$ of Subearth Meridian

H $20^{\circ} \mathrm{S}-60^{\circ} \mathrm{E}$ of Subearth Meridian

Figure 5.7. Venus Beam Positions October 1986 NASA IRTF 


\section{Table 5.4}

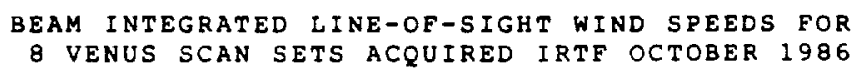

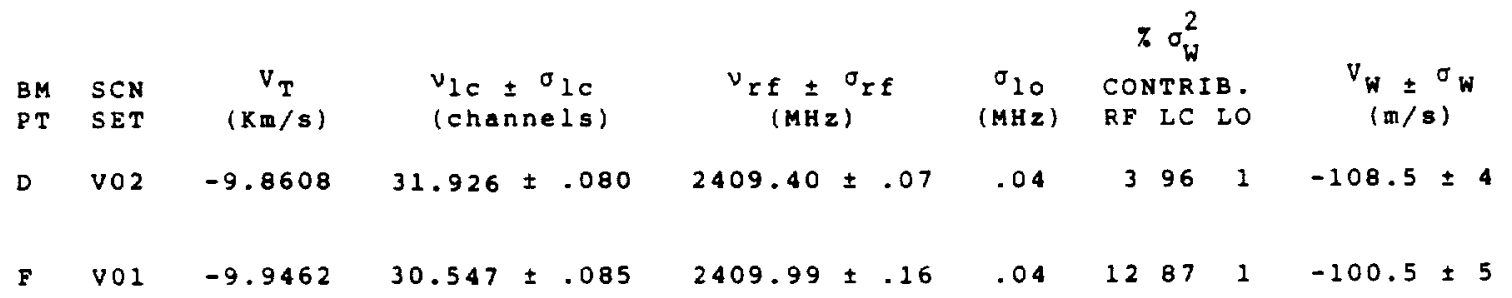

SYSTEMATIC ERROR:

EPHEMERIS (VT) ERROR: $\pm 1 \mathrm{~m} / \mathrm{s}$

CO2 626 R8 REST FREQUENCY ERROR: $\pm .0523 \mathrm{MHz}$

TOTAL SYSTEMATIC ERROR: $\pm 1.5 \mathrm{~m} / \mathrm{s}$

\begin{tabular}{|c|c|c|c|c|c|c|c|c|c|c|c|c|c|c|}
\hline \multirow{2}{*}{$\begin{array}{l}\text { BM } \\
\text { P I } \\
B\end{array}$} & \multirow{2}{*}{$\begin{array}{l}\text { SCN } \\
\text { SET } \\
\text { VOB }\end{array}$} & \multirow{2}{*}{$\begin{array}{c}V_{T} \\
(\mathrm{Km} / \mathrm{s}) \\
-9.3576\end{array}$} & \multicolumn{3}{|c|}{$\begin{array}{l}v_{1 c} \pm \sigma_{1 c} \\
\text { (channels) }\end{array}$} & \multicolumn{3}{|c|}{$v_{(M H Z)}^{v_{I f}} \sigma_{I f}$} & \multirow{2}{*}{$\begin{array}{c}\sigma_{10} \\
(\mathrm{MHz}) \\
.04\end{array}$} & \multicolumn{3}{|c|}{$\begin{array}{c}z \sigma_{W}^{2} \\
\text { CoNTRIB. }\end{array}$} & \multicolumn{2}{|c|}{$\begin{array}{c}V_{W \pm \sigma_{W}}{ }_{(m / 8)} \\
\end{array}$} \\
\hline & & & 42.384 & \pm & .106 & 2412.17 & \pm & .05 & & 1 & 99 & 1 & -100.0 & \pm 6 \\
\hline D & vo6 & -9.6254 & 36.290 & \pm & .065 & 2408.06 & \pm & .06 & .04 & 3 & 95 & 2 & -104.6 & \pm 3 \\
\hline$F$ & vo 4 & -9.8823 & 31.634 & \pm & .060 & 2408.51 & \pm & .08 & .04 & 7 & 92 & 2 & -92.9 & \pm 3 \\
\hline $\mathbf{F}$ & v07 & -9.4691 & 40.152 & \pm & .075 & 2412.32 & \pm & .04 & .04 & 1 & 98 & 1 & -105.4 & \pm 4 \\
\hline H & vo 3 & -9.7736 & 33.627 & \pm & .060 & 2409.02 & \pm & .14 & .04 & 18 & 81 & 2 & -103.9 & \pm 3 \\
\hline H & v05 & -9.7493 & 34.191 & \pm & .060 & 2408.15 & \pm & .05 & .04 & 3 & 96 & 2 & -90.1 & \pm \\
\hline
\end{tabular}


and used to record the UT for each scan, is essential in determining the scan-associated topocentric line of sight velocity of Venus. However, IRTF generator departures from $60 \mathrm{~Hz}$ cause clock drift. Continuous clock update (with the aid of IRTF-generated UT) is thus essential, but was unfortunately forgotten for scan sets v01 and v02. Comparison of IRTF-generated hour angle (recorded in the observing log at the beginning of each scan) and the hour angle associated with LSI $11 / 23$ UT at scan start, yielded a clock offset error of $\pm 1 \mathrm{~m} / \mathrm{s}$. This is reflected in the $\pm 2 \mathrm{~m} / \mathrm{s}$ ephemeris systematic error given in the upper half of Table 5.4 .

The UT and Local Times associated with October 1986 scan set acquisition are provided on Figure 5.8. Lunar scan sets L1 and L2 were used for terrestrial absorption removal.

The upper half of Table 5.5 provides the number of scans, and total integration time, per scan set. The integration time per scan was $120^{\mathrm{s}}$.

The October 1986 video camera field-of-view precluded locating more than -258 of the Venus crescent on the TV monitor. Guiding by template overlay was therefore not possible (see Section 3.5), allowing only crude visual alignment of the monitor crosshair against the imaged portion of the crescent. The crescent's cusps were each visually aligned on the monitor cross hair, thereby providing the IRTF tracking computer the cusps' coordinates. Computer offsetting from the 1 ine connecting the cusps was then used to position the beam. However, the visible cusps did not extend to the Venusian poles. The resulting positional uncertainty perpendicular to the cusp line was estimated at 


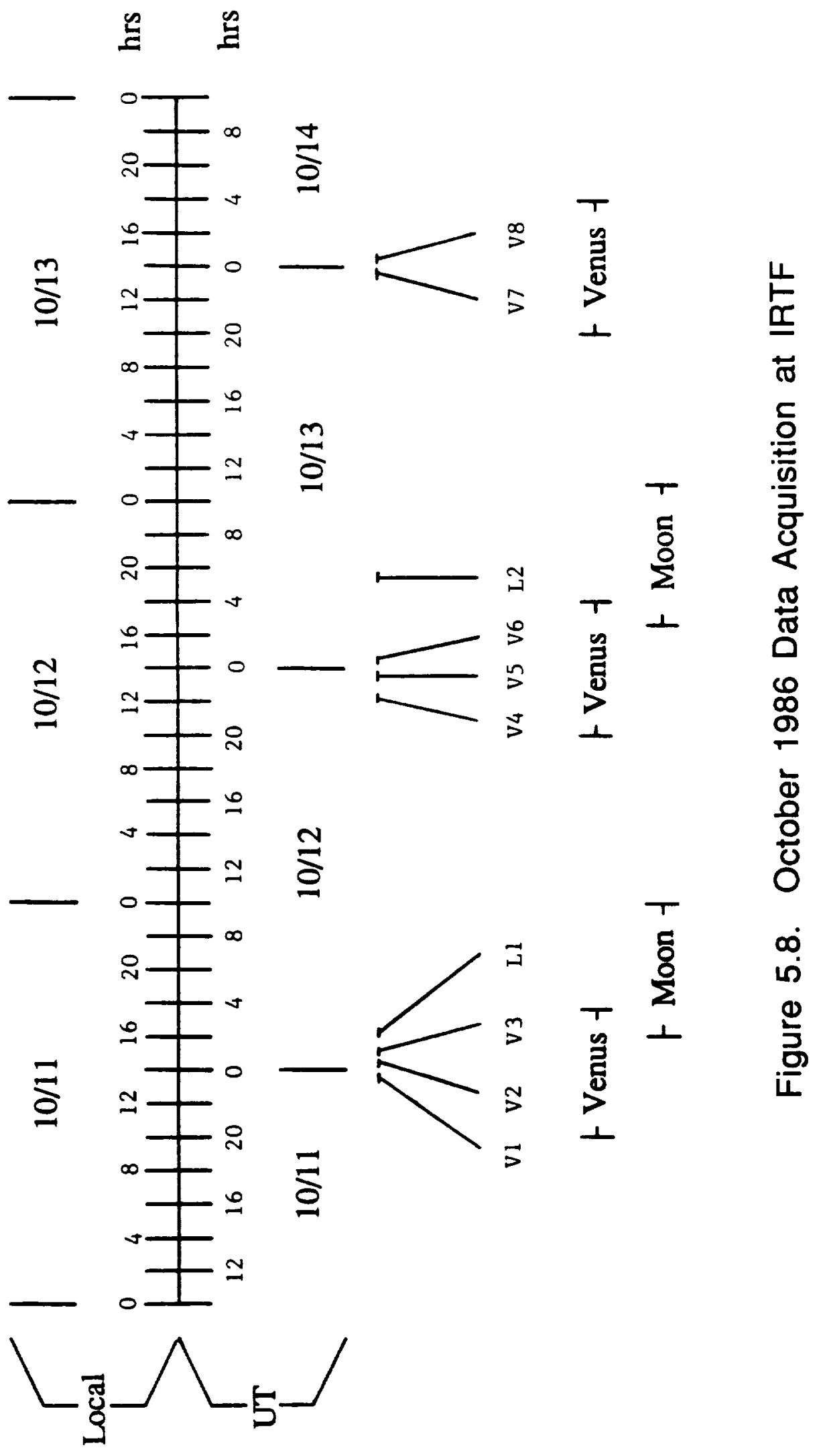




\section{Table 5.5. Integration Time for Venus Scan Sets}

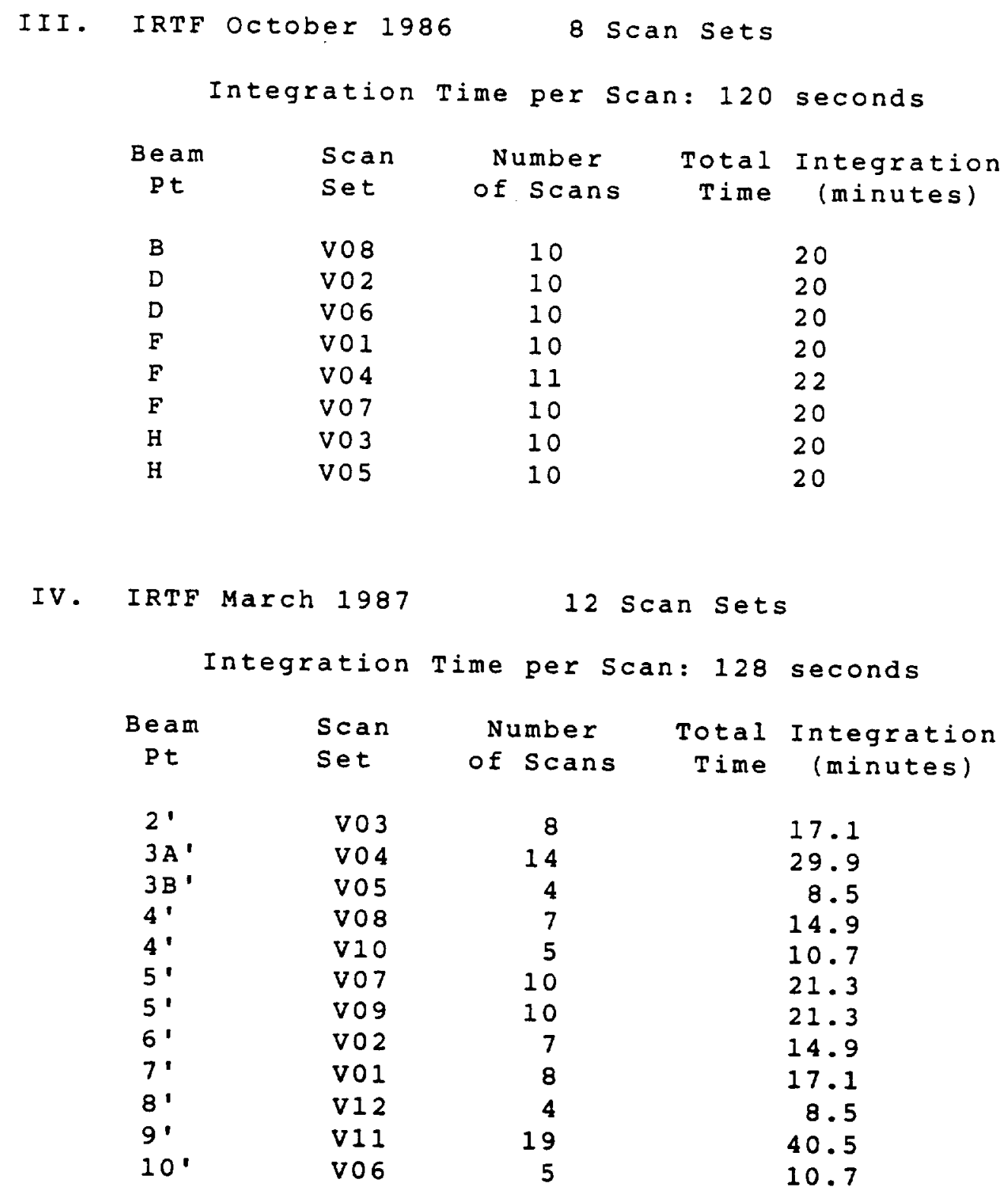


-1.5 arc-seconds. (Clear detection of the Venusian limb in the broadband heterodyne signal, an excellent means of reducing positional uncertainty, was not possible due to beam dilution from consistent 3 arc-second seeing.) Cusp coordinates were determined with the wobbling secondary turned off to provide enhanced Venus/sky contrast. It was later determined that powering up the wobbling secondary introduced a -1.5 arc-second unpredictable coordinate offset. As well, prior to March 1987, Venus tracking at Coude was poor. A drift of -0.2 arc-seconds per minute was determined during the October observing period, warranting frequent recalibration of the tracking computer zero point. Overall, worst case pointing uncertainty (the point at which the TV monitor crosshair viewed against the crescent definitively indicated pointing offset) was estimated at -2 arc-seconds. Seeing was -3 arc-seconds throughout data acquisition.

Since the illuminated portion of the planet was only a thin crescent, and the disk was 48 arc-seconds, 2 arc-second pointing uncertainty and 3 arc-second seeing was still thought sufficient for meaningful spatial coverage (with minimal beam overlap) if four beam positions (nominally centered as shown on Fig. 5.7) were used.

\subsubsection{March 1987}

Observations 22 and 23 March 1987 ( $38^{\circ} \mathrm{W}$ elongation, 0.76 phase - nearly identical to the June 1986 eastern aspect), starting 66 days after greatest western elongation, yielded twelve beam-integrated line of sight wind velocities, distributed over the ten beam positions indicated on Figure 5.9. A 14.5 arc-second planetary diameter, and 


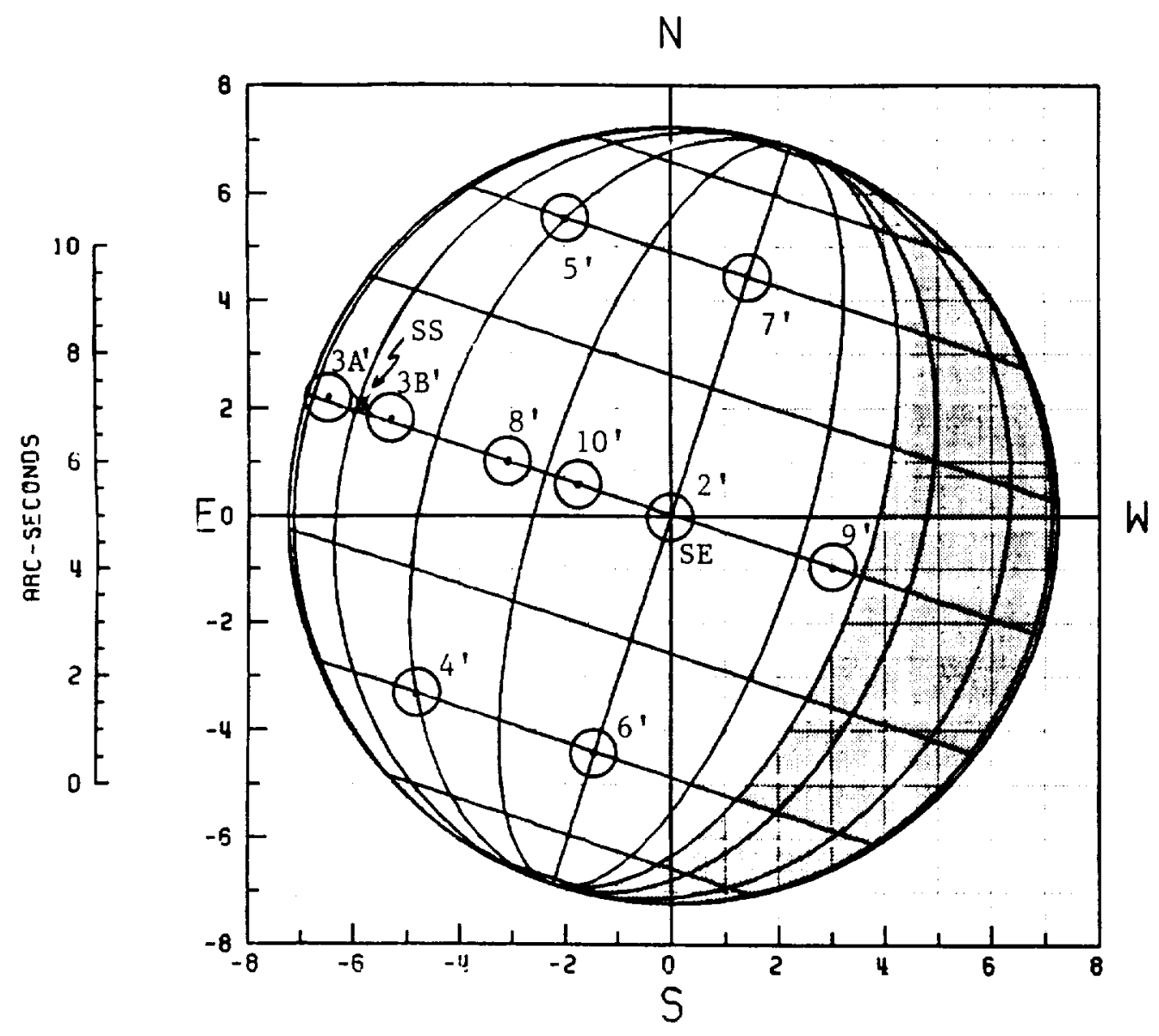

2' Subearth Point

$3 \mathrm{~A}^{\prime} \sim$ Equator $-70^{\circ} \mathrm{W}$ of Subearth Meridian

$3 B^{\prime} \sim$ Equator $-50^{\circ} \mathrm{W}$ of Subearth Meridian

4' $40^{\circ} \mathrm{S}-40^{\circ} \mathrm{W}$ of Subearth Meridian

$5^{\prime} 40^{\circ} \mathrm{N}-40^{\circ} \mathrm{W}$ of Subearth Meridian

6' $40^{\circ} \mathrm{S}$ - Subearth Meridian

$7^{\prime} \quad 40^{\circ} \mathrm{N}$ - Subearth Meridian

$8^{\prime} \sim$ Equator $-25^{\circ} \mathrm{W}$ of Subearth Meridan

9' $\sim$ Equator $-25^{\circ} \mathrm{E}$ of Subearth Meridian

$10^{\prime} \sim$ Equator $-15^{\circ} \mathrm{W}$ of Subearth Meridian

Figure 5.9. Venus Beam Positions March 1987 NASA IRTF 
0.9 arc-second (HPBW) IRTF diffraction-limited beam, yielded a seeing-excluded disk-to-beam ratio of 18 . Beam pointing uncertainty was $<1$ arc-second.

The Subearth Point was located at $0.2^{\circ} \mathrm{S}$ latitude. The Subsolar Point was located at $1.2^{\circ} \mathrm{N}$ latitude, $59^{\circ} \mathrm{W}$ longitude. Beam position $2^{\prime}$ was centered on SE. Positions $3 \mathrm{~A}^{\prime}, 3 \mathrm{~B}^{\prime}, 8^{\prime}, 10^{\prime}$, and $9^{\prime}$ were centered on the equator at: $70^{\circ} \mathrm{W}, 50^{\circ} \mathrm{W}, 25^{\circ} \mathrm{W}, 15^{\circ} \mathrm{W}$, and $25^{\circ} \mathrm{E}$ longitude, respectively. Positions $6^{\prime}$ and $7^{\prime}$ were centered at $40^{\circ} \mathrm{S}$ and $40^{\circ} \mathrm{N}$ latitude along the Subearth Meridian. Positions 4' and 5' were centered at $40^{\circ} \mathrm{S}$ and $40^{\circ} \mathrm{N}$ latitude along the $40^{\circ} \mathrm{W}$ longitude meridian. Positions $2^{\prime}, 8^{\prime}, 9^{\prime}, 6^{\prime}, 7^{\prime}, 4^{\prime}$ and $5^{\prime}$ correspond directly to their unprimed counterparts June 1986 (see Fig. 5.4).

Table 5.6 provides the 12 beam-integrated wind velocities for March 1987. The UT and Local Times associated with March 1987 scan set acquisition are provided on Figure 5.10. Mercury scan sets ME1 and ME2 were used for terrestrial absorption removal.

Scan sets V1 through V6 were associated with 2 arc-second seeing. Seeing for V7 through V12 was 1 arc-second.

The lower half of Table 5.5 provides the number of scans, and total integration time, per scan set. The integration time per scan was $128^{\mathrm{s}}$. 


\section{Table 5.6}

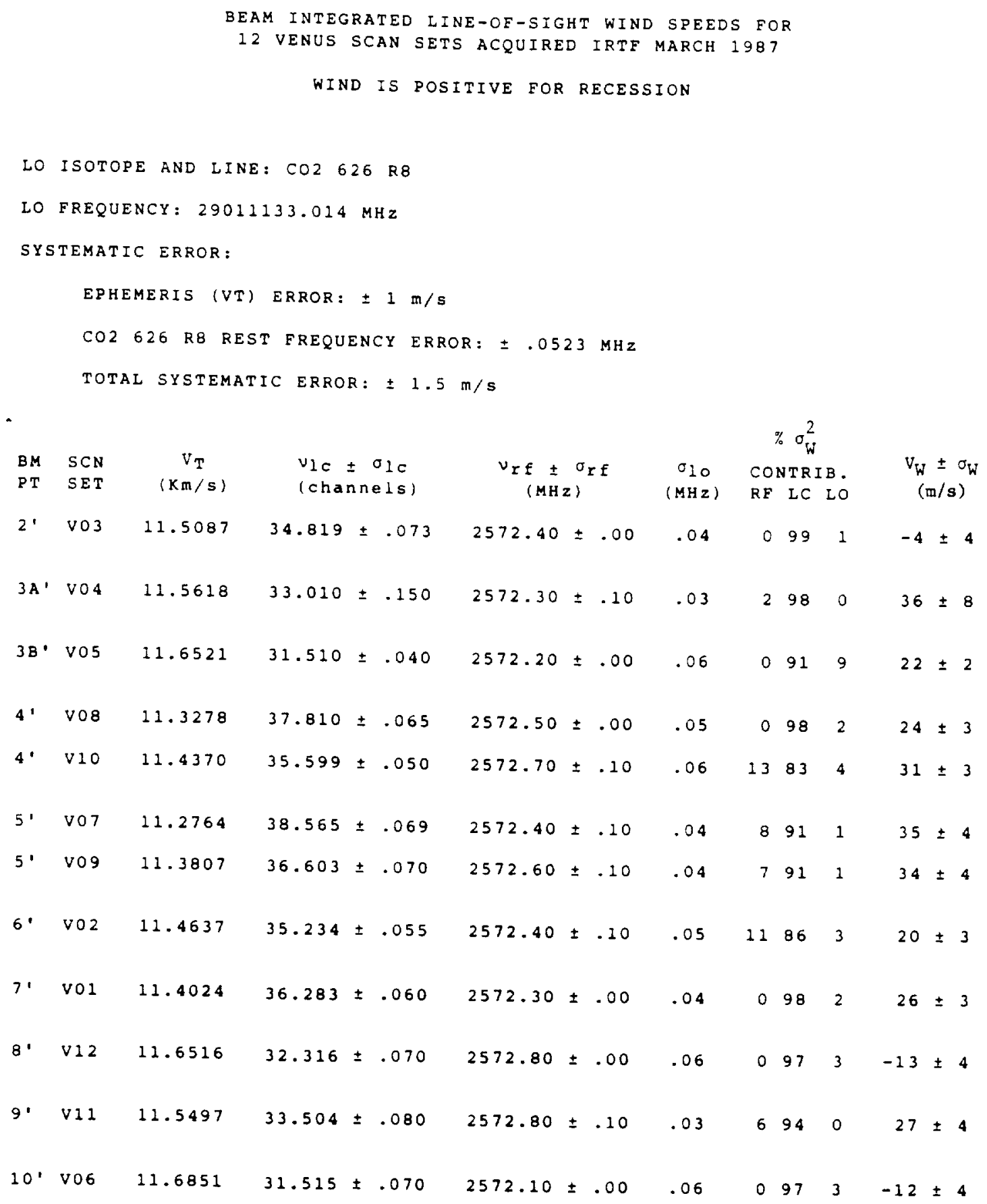




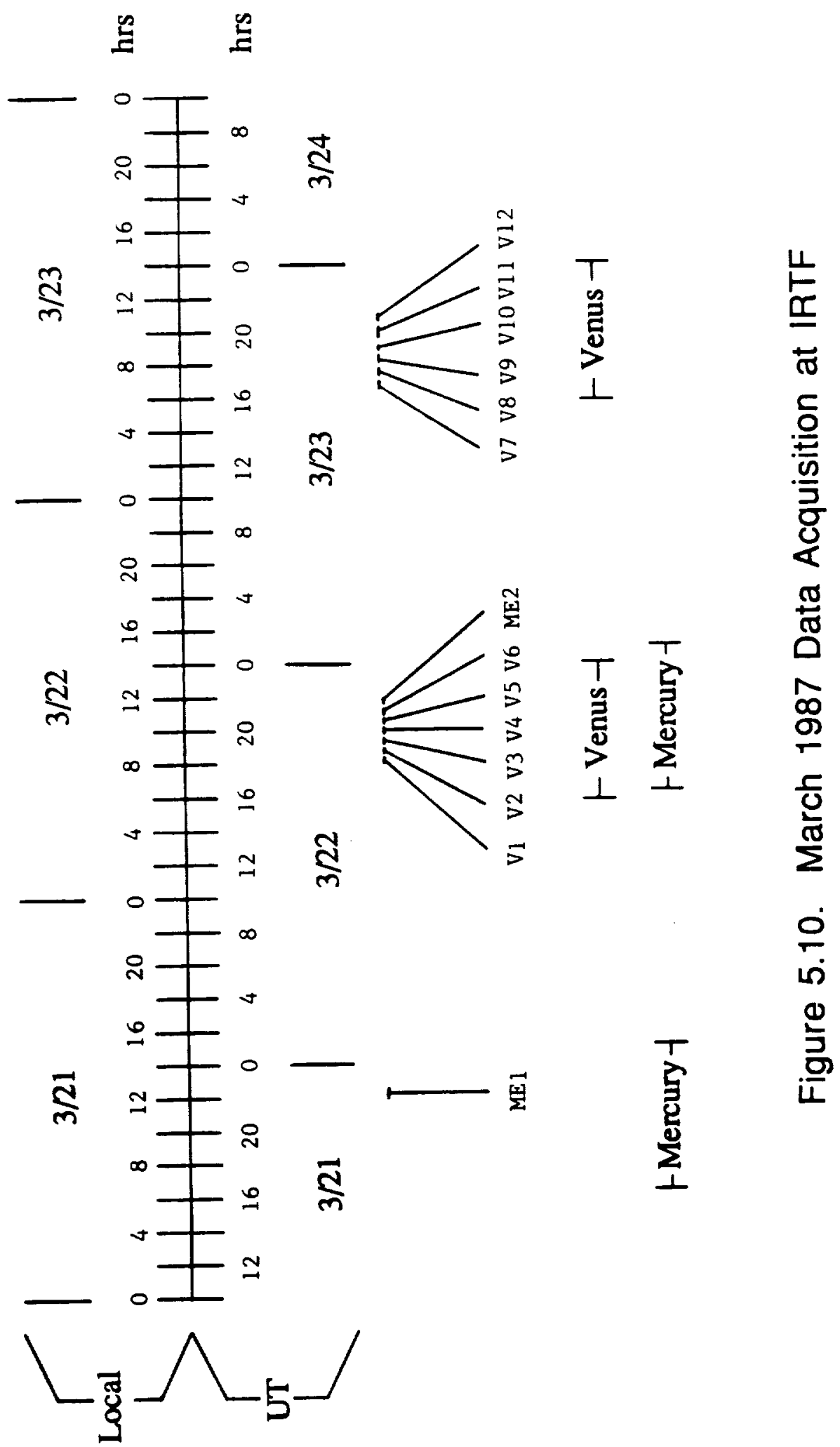


CHAPTER 6: RESULTS I - QUALITATIVE INTERPRETATION OF THE OBSERVED

\section{BEAM-INTEGRATED WIND VELOCITIES}

\subsection{VELOCITY SHIFT EFFECTS}

Prior to data interpretation, a number of frequency (velocity) shift mechanisms need to be explored.

Model atmospheres (see Seiff 1983) yield a $0.026 \mathrm{mb}$ maximal

pressure for the ${ }^{12} \mathrm{C}^{16} \mathrm{O}_{2}$ non-thermal emitting shell (100-120 km altitude), inducing a pressure shift in line-center frequency. A $\mathrm{CO}_{2}-\mathrm{CO}_{2}-48 \mathrm{KHz} / \mathrm{mb}$ blueward pressure shift has been experimentally determined by SooHoo (1984; see Chapter 4) for pressures <0.13 mb. The expected shift at line forming altitude is thus $<0.012 \mathrm{~m} / \mathrm{s} \quad(<1.2 \mathrm{KHz})$, and may be neglected.

Travelling from Venus to Earth, photons suffer a solar-induced gravitational redshift $\mathrm{V}_{\mathrm{gr}}$ given by:

$$
v_{g r}=\frac{c}{2}\left[r_{e}^{-1}-r_{o}^{-1}\right],
$$

where $V_{g r}$, in $m / s$, is positive for a redward shift, $r_{e}$ and $r_{o}$ are the Sun/Venus and Sun/Earth distances, respectively, in units of the solar Schwarzschild radius $R_{S}$, and $c$ is the speed of light (see Goldstein 1987). $R_{S}$ is given by:

$$
R_{s}=\frac{2 G M}{c^{2}},
$$

where $G$ is the gravitational constant, and $M$ is the Sun's mass. (Eq. 6.1 assumes the weak field limit where $r_{e}$ and $r_{o} \gg R_{s}$.)

$\mathrm{V}_{\mathrm{gr}}=+1.1 \mathrm{~m} / \mathrm{s}$ and is virtually independent of Venus/Earth/Sun alignment - orbital-induced variations in $r_{e}$ and $r_{o}$ are only 0.78 and 
1.78, respectively. All beam-integrated wind velocities should therefore be $1.1 \mathrm{~m} / \mathrm{s}$ bluer than observed, and have henceforth been gravitational redshift corrected. (A negligible $0.03 \mathrm{~m} / \mathrm{s}$ gravitational blueshift results from the local gravity fields of Venus and Earth as photons fall into a slightly deeper local potential well than from which they were emitted).

Use of the classical Doppler shift in the data reduction (Eq. A1.36), as opposed to the special relativistic formulation, introduces a negligible $<0.167 \mathrm{~m} / \mathrm{s}$ offset in the beam-integrated winds.

The most appropriate reference frame for the derived global wind field is a Venus-centered, solar-fixed coordinate system co-rotating with the subsolar-antisolar axis (hereafter BF1). Relative to the frame co-rotating with the solid planet, BF1 rotates prograde with a period of $116.75^{\mathrm{d}}$ (the Venusian solar day) corresponding to an equatorial velocity of $3.8 \mathrm{~m} / \mathrm{s}$ at $110 \mathrm{~km}$ altitude (neglecting the $2.6^{\circ}$ Venusian obliquity).

The data reduction yields line of sight wind velocities referenced to a frame (hereafter BF2) centered on the Venusian barycenter and fixed to the Earth/Venus line (see Appendix 2). Converting observed dynamics in $B F 2$ to $B F 1$ requires determination of the relative angular velocity $\Omega_{T}$ between both frames, the latter dictated by two effects:

1. Rotation of the SS-AS axis (about a perpendicular to the orbital plane), over the $584^{\mathrm{d}}$ synodic period $\left(\mathrm{P}_{\text {syn }}\right)$, causes a $B F 1$ prograde rotation with respect to $B F 2$, at a uniform angular velocity $\Omega_{0}$ corresponding to an equatorial velocity 
of $0.77 \mathrm{~m} / \mathrm{s}$ at $110 \mathrm{~km}$ altitude.

2. The sweep in the Venus/Earth line of sight over $P_{\text {syn' from }}$ maximum eastern to maximum western elongation, and back again, introduces a periodic component $\left(\Omega_{p}\right)$ to $\Omega_{T}$.

$\Omega_{\mathrm{T}}=\Omega_{\mathrm{o}}+\Omega_{\mathrm{p}}$ is uniquely specified for a given planetary elongation, varying from $\Omega_{\mathrm{T}}=0.58 \Omega_{\mathrm{o}}$ at superior conjunction to $\Omega_{\mathrm{T}}=3.6 \Omega_{\mathrm{o}}$ at inferior conjunction (at maximum elongation $\Omega_{T}=\Omega_{0}$ ). This corresponds to an equatorial velocity of 0.5 to $2.8 \mathrm{~m} / \mathrm{s}$ at $110 \mathrm{~km}$ altitude (Venus and Earth orbital eccentricities and the $3.4^{\circ}$ Venusian orbital inclination to the ecliptic have been neglected).

$\Omega_{\mathrm{T}}$ produces a prograde zonal velocity field, as seen by an Earth-bound observer in BF2, which should be subtracted from the observed beam-integrated wind velocities for conversion to BFI. Within the beam, however, different latitudes will exhibit different zonal velocities, and a gradient in line of sight projection geometry exists, implying that a beam-specific systematic correction should not be made. Rather, $\Omega_{\mathrm{T}}$ has been built into the model wind field used (in Chapter 7) to generate model beam-integrated wind velocities referenced to BF2.

For the following qualitative interpretation of beam-integrated winds, wind velocity differences between $B F 1$ and $B F 2$ are slight enough to be neglected, buried by effects on beam-integrated wind velocity owing to variation in diffraction-limited beam shape, seeing, and pointing uncertainty. 


\subsection{JUNE $1986 /$ MARCH 1987}

Based on already presented observational and theoretical evidence (see Chapters 1 and 2), a negligible lower thermospheric vertical wind component may be assumed. All wind velocity vectors are therefore tangent to the sphere of the planet.

The June 1986 and March 1987 observing geometries, shown on Figure 6.1, were symmetrically located about the Earth/Sun Iine, allowing decoupling of subsolar-antisolar flow (hereafter SS-AS) and any zonal component. Pure SS-AS flow, at a given altitude, is assumed solely a function of SZA. Thus, corresponding unprimed (June 1986) and primed (March 1987) beam positions (e.g., positions 5 and 5') should yield identical line of sight SS-AS components, yet equal but opposite zonal components. If the beam-integrated wind velocities for a June 1986 beam position and its March 1987 primed counterpart are $V_{w}$ and $\mathrm{V}_{\mathrm{w}^{\prime}}$, respectively, then the magnitudes of the (beam-integrated) line of sight SS-AS and zonal (Z) components are given by:

$$
\mathrm{V}_{\mathrm{SS}-\mathrm{AS}}=\left[\mathrm{V}_{\mathrm{w}}+\mathrm{V}_{\mathrm{w}},\right] / 2
$$

and,

$$
v_{z}=\left[v_{w}-v_{w^{\prime}}\right] / 2
$$

This expectation, however, is moderated by effects owing to differences in beam shape and pointing uncertainty (in general, non-negligible and addressed in Chapter 7). In addition, the wind field may be temporally variable, yet a priori admission of this possibility limits meaningful conclusions from data spanning 828 of a synodic period. 

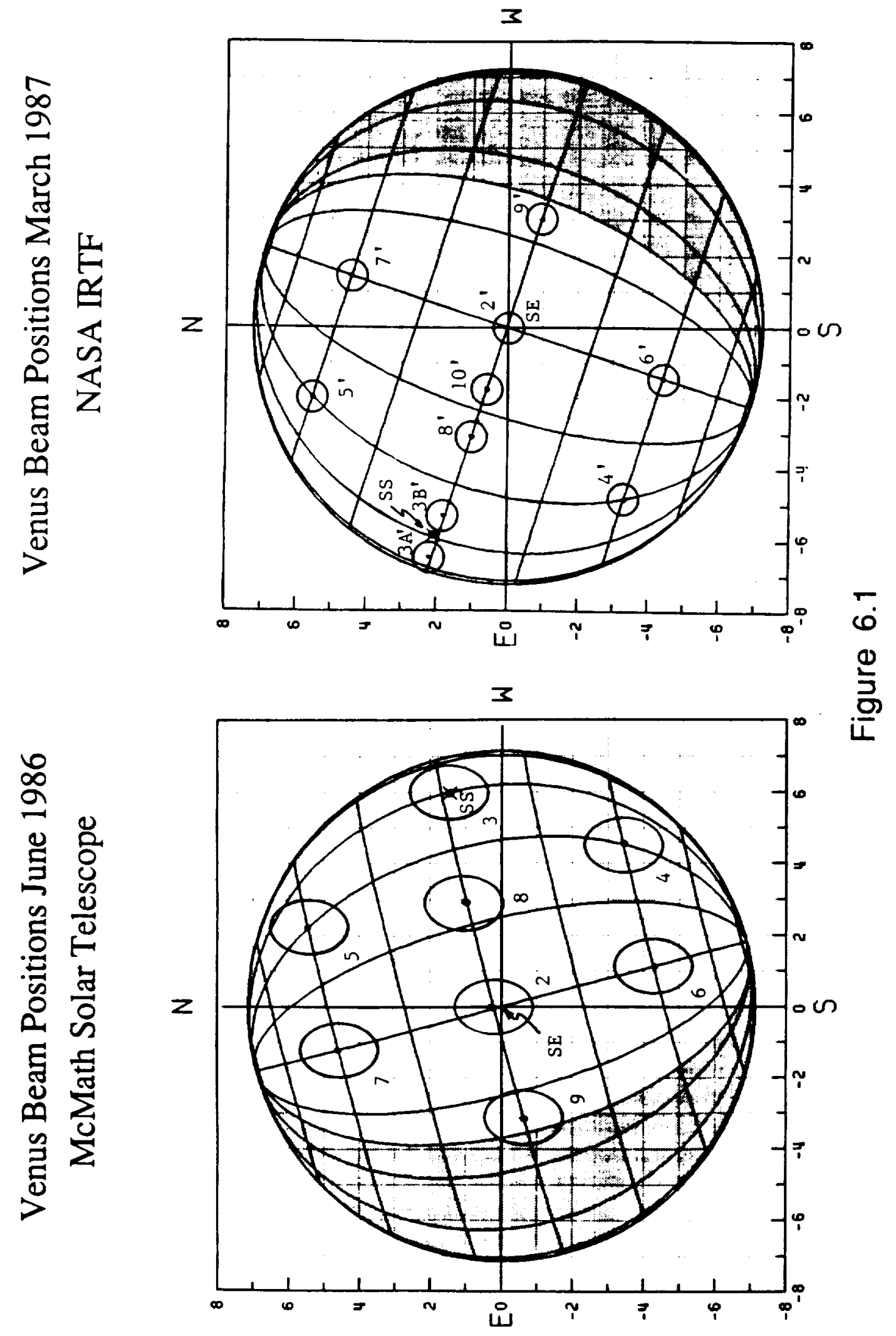
The June 1986 and March 1987 geometries are again shown on Figure 6.2 (the June beams have been represented as circles with the R.A. HPBW). The lower portion of the Figure is a plot of beam-integrated line of sight velocity versus beam position (circles - June 1986 , crosses - March 1987). The velocities (gravitational redshift corrected) are positive for recession, and are shown with their $1 \sigma$ beam-specific error bars.

A dominant zonal circulation can be immediately ruled out by inspection of Figure 6.2. In general, such circulation requires that the June and March data, at corresponding beam positions, have equal magnitudes but lie on opposite sides of the line $v_{w}=0$. Just the reverse seems apparent: Positions 4,5,6,7, and 9 show the same behavior as their primed counterparts. From Eq. 6.4, such behavior implies a negligible zonal component. More specifically, and regardless of beam shape, a retrograde zonal circulation would yield approaching beam-integrated velocities for beam positions (sky) westward of the subearth meridian (positions 3,4,5,8 June 1986; 9' March 1987). Yet all such positions (except 8) are indicative of the strongest recessional velocities measured. Likewise, positions (sky) eastward of the subearth meridian should exhibit recession, yet positions $8^{\prime}$ and 10' are seen in approach. A prograde zonal circulation is as well ruled out.

Consider a dominant SS-AS circulation with the same general behavior as modelled by Bougher et al. (1986; see Figure 2.2). Such circulation may be observationally established, based solely on a qualitative analysis of beam-integrated winds, if: 


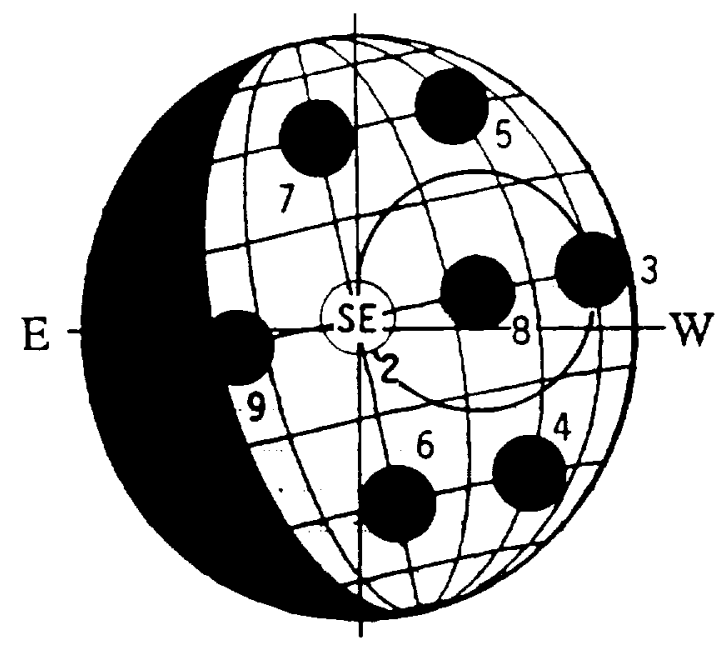

o- $6 / 86$

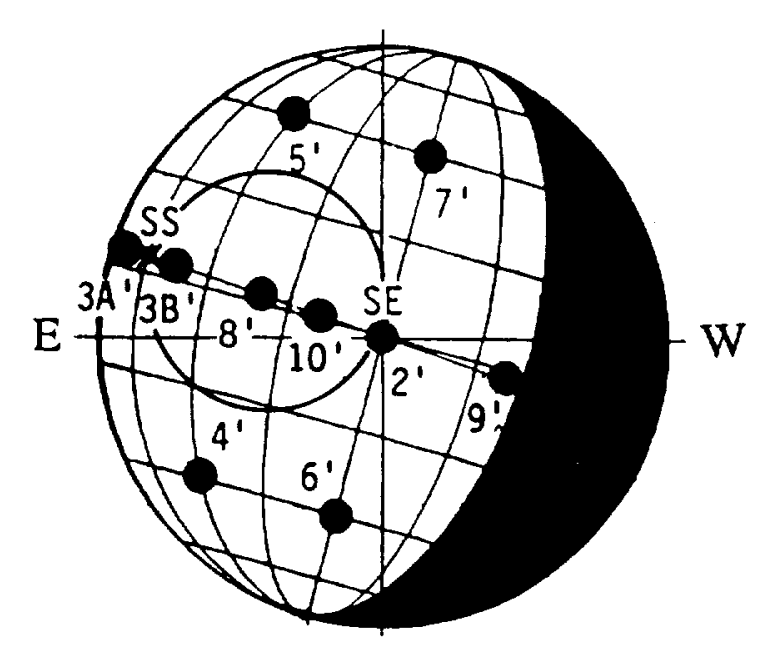

$\mathrm{x}-3 / 87$

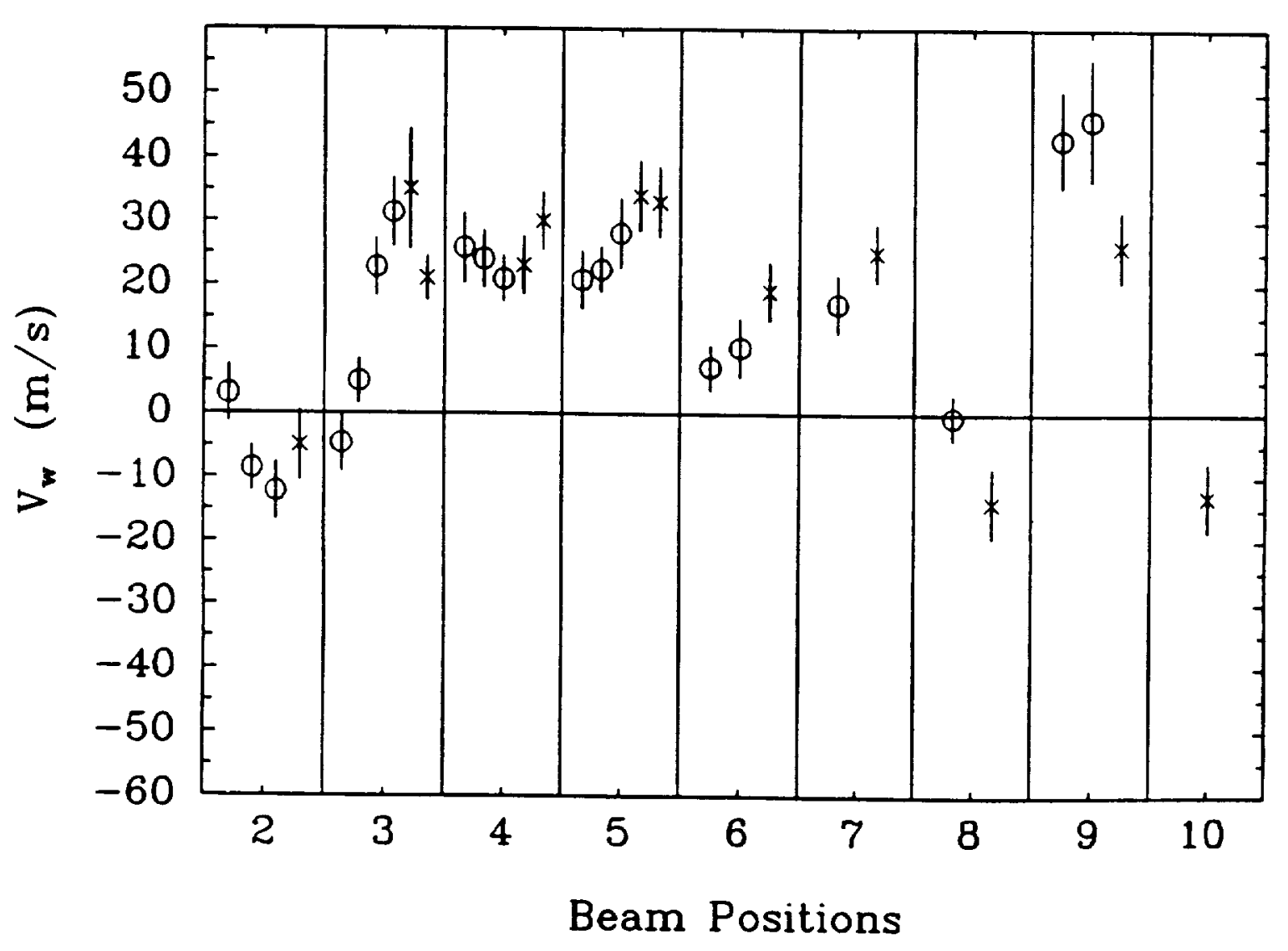

Figure 6.2. Beam-Integrated Wind Velocities June 1986/March 1987 
1. corresponding primed and unprimed positions exhibit the same behavior, and;

2. positions within the large circles intercepting $S S$ and $S E$ on the Figure 6.2 planetary aspects exhibit blueshifts, while positions outside exhibit redshifts.

By inspection, both these criteria are met (with the exception of position 8). Regarding specific dynamical indicators:

i. The Subearth Point (positions 2 and $2^{\prime}$ ) should be associated with a $0 \mathrm{~m} / \mathrm{s}$ line of sight Doppler shift, regardless of wind field. A 1 arc-second pointing uncertainty for both observing periods should introduce scatter about $0 \mathrm{~m} / \mathrm{s}$. Such scatter is observed.

ii. A strong spatial gradient in line of sight Doppler shift should exist in the vicinity of the Subsolar Point (positions $\left.3,-3 A^{\prime},-3 B^{\prime}\right)$.

A beam slightly limbward of the Subsolar Point should sample the SS-AS field projected almost entirely along the line of sight, and in recession. If the beam intercepts portions of the limb, limb dynamics can dominate the beam-integrated wind (due to exceptionally strong limb non-thermal emission; see Chapter 7). The limb near the Subsolar Point has a SZA of $-30^{\circ}$, implying appreciable horizontal velocity (see Fig. 2.2). Conversely, a beam located 1 arc-second disk-centerward of the Subsolar Point will have a (disk-center) SZA of $-10^{\circ}$. The lack of appreciable wind velocity here (see Fig. 2.2), coupled with a smaller line of sight projection, implies a small beam-integrated blueshift. Multiple observations at the Subsolar Point, together with 1 
arc-second pointing uncertainty, should therefore yield a large Doppler scatter, heavily favoring strong recessional flow. Such behavior is observed on Figure 6.2.

iii. Positions 4 and 5, symmetrically located with respect to the Subsolar Point, show consistent recessional velocities as do their primed counterparts. The sense of the relative June/March (statistically marginal) velocity offset, is consistent with a small retrograde component. Other explanations include wind field temporal variability and pointing uncertainty. The offset cannot result from beam shape differences - the larger June beam, partially intercepting the limb (after folding in seeing effects), should provide the greater recessional velocities.

iv. Positions 9 and $9^{\prime}$, both indicative of strong recessional flow, exhibit a sizable velocity offset, probably too large to be explained away by beam shape/pointing uncertainty differences. The sense of the offset is indicative of a $-10 \mathrm{~m} / \mathrm{s}$ (1ine of sight) retrograde zonal component (from Eq. 6.4).

While positions $8^{\prime}$ and $10^{\prime}$ exhibit the requisite blueshift, the position 8 wind velocity, one of the most precise measurements, does not. If a retrograde zonal component is responsible for the 9-9' velocity offset, position 8 should have a significantly stronger blueshift than position $8^{\prime}$. The problem is somewhat alleviated if a retrograde zonal component exists only in the March 1987 data, which would imply true wind field variability. (Pointing uncertainty is probably not capable of accounting for a $0 \mathrm{~m} / \mathrm{s}$ position 8 beam-integrated velocity: the 
across-the-disk gradient in line of sight projection geometry for SS-AS flow is smallest at this location.)

v. Subearth meridian positions 6 and 7 , symmetrically located at \pm 40 latitude, are reasonably insensitive to zonal circulation and are therefore subsolar-antisolar flow specific indicators. Both primed and unprimed wind velocities exhibit similar behavior. The enhanced redshift for the primed positions may be indicative of SS-AS temporal variability, or beam shape/pointing uncertainty effects.

The June 1986/March 1987 data establish SS-AS flow as the dominant circulation in the $100-120 \mathrm{~km}$ altitude range. Modelling of the beam-integrated wind velocities, and fitting the modelled ensemble to the observed, is warranted for a quantitative description of the SS-AS circulation and the level of possible zonal involvement.

\subsection{DECEMBER 1985}

Figure 6.3 provides the beam positions and beam-integrated wind velocities for December 1985.

In the presence of a dominant retrograde zonal circulation, positions P4 and P5 would exhibit a strong blueshift and redshift, respectively. Positions $\mathrm{P} 1, \mathrm{P} 2$ and $\mathrm{P} 3$, located close to the subearth meridian, would exhibit only mild recession. Yet P2 and P3 exhibit strong redshifts, as does $\mathrm{P} 4$. The observed is clearly more consistent with SS-AS circulation, which calls for strong redshifts at all positions but $\mathrm{P} 1$. A $60 \mathrm{~m} / \mathrm{s}$ differential shift between $\mathrm{P} 4$ and $\mathrm{P} 5$ may be 

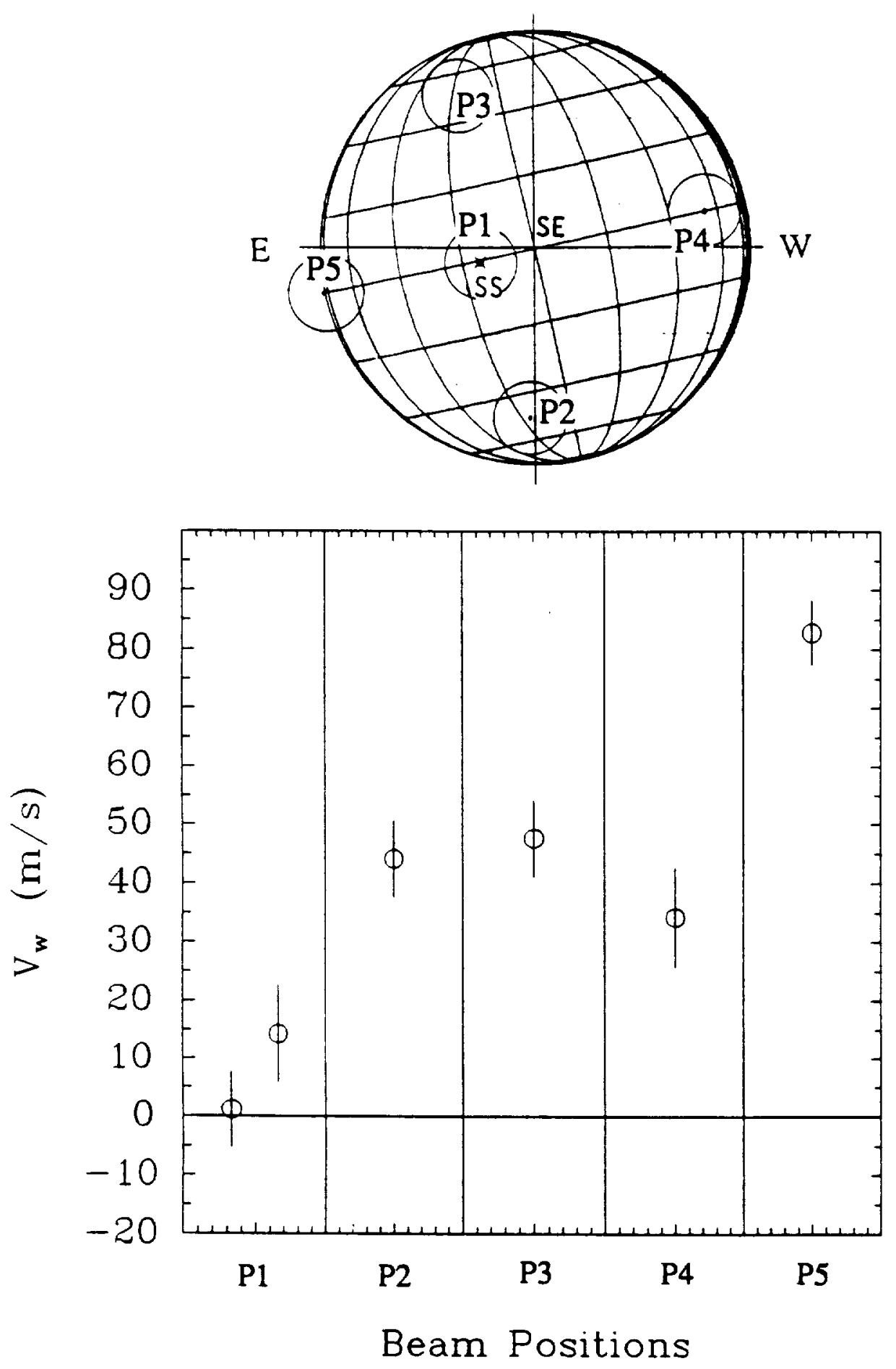

Figure 6.3. Beam-Integrated Wind Velocities December 1985 
indicative of a $-30 \mathrm{~m} / \mathrm{s}$ zonal retrograde component.

6.4. OCTOBER 1986

Figure 6.4 provides the October 1986 beam-integrated wind velocities. Observed blueshifts of $90-110 \mathrm{~m} / \mathrm{s}$, at the four probed locations, can be explained by either (or coupled) SS-AS and zonal retrograde circulations. The magnitudes of these line of sight winds are the largest observed over the four observing periods, and indicate near-terminator horizontal velocities in excess of $110 \mathrm{~m} / \mathrm{s}$. 


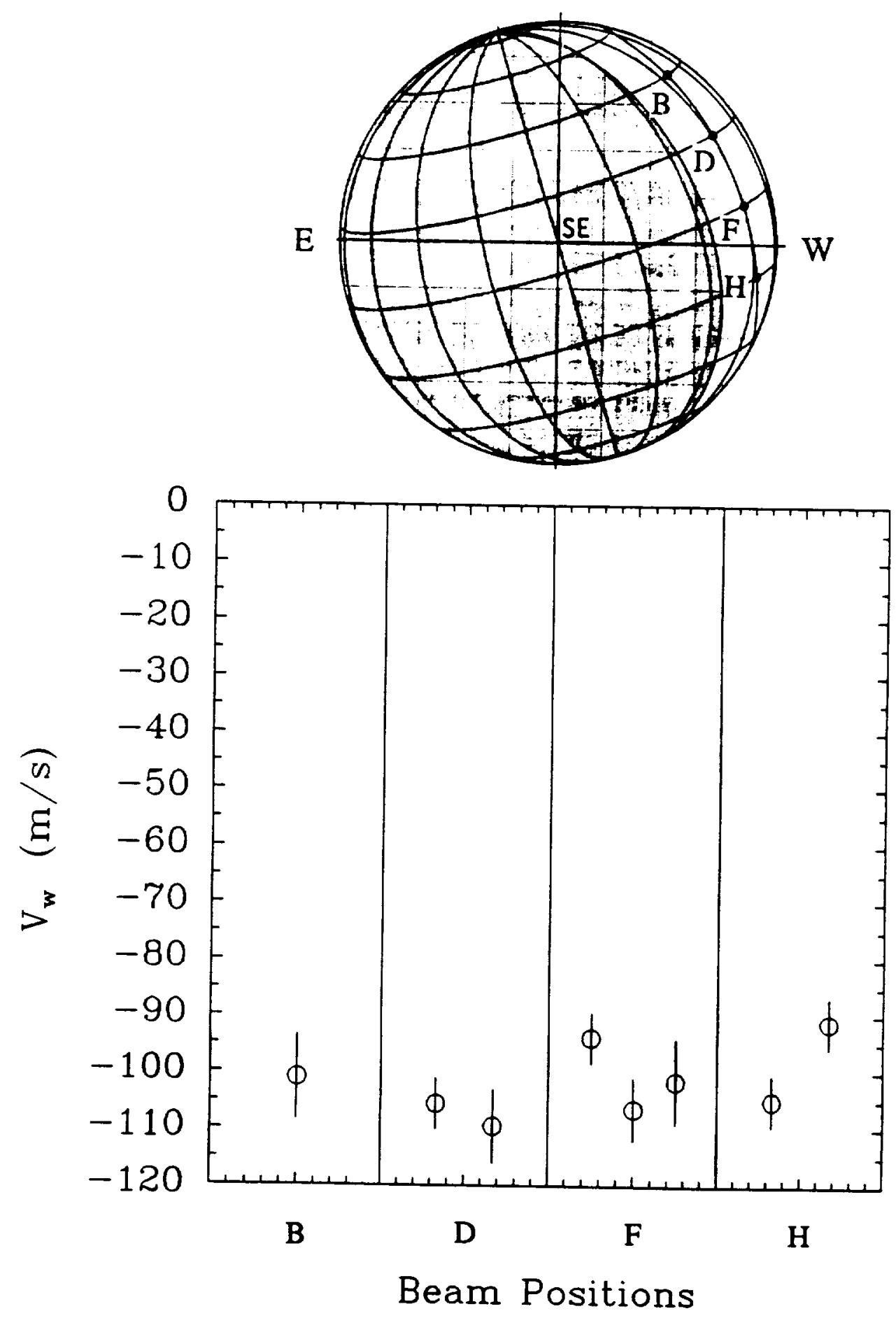

Figure 6.4. Beam-Integrated Wind Velocities October 1986 

CHAPTER 7: RESULTS II - WIND FIELD RETRIEVAL FROM KINEMATICAL FITS

\section{TO THE BEAM-INTEGRATED WIND VELOCITIES}

\subsection{PROGRAM BEAMINT}

The beam-integrated non-thermal emission sampled by the spectrometer - as a spectral probe of dynamics in the atmospheric parcel within the beam - often represents a convolution of dynamical contributions from a large portion of the planet. The complicated nature of the convolution precludes association of the derived beam-integrated wind velocity with dynamics at a specific location within the beam. Program BEAMINT was therefore developed to generate (BF2-referenced) beam-integrated wind velocities from a model global wind field. Comparison with the observed beam-integrated winds thereby allows model discrimination. The quantitative aspects of BEAMINT are covered at length in Appendix 2.

Figure 7.1 provides the beam-center Subsolar longitudes $(\alpha)$ and latitudes $(\beta)$ for all four observing periods. The Sun-lit hemisphere is sampled from $-50^{\circ}$ to $+50^{\circ}$ latitude. Each observing period is associated with a worst case pointing uncertainty $(\Gamma)$, in arc-seconds. Each beam position is associated with an effective beam shape, owing to both diffraction-limited spatial roll-off and seeing.

Depending upon planetary disk size, beam position, and pointing uncertainty, a given beam can sample vast portions of the planet, with a beam-center displaced from its nominal longitude and latitude.

\subsubsection{Pointing Uncertainty $(\Gamma)$ Considerations}

Figures 7.2 through 7.5 provide the uncertainty in beam-center $\alpha$ 


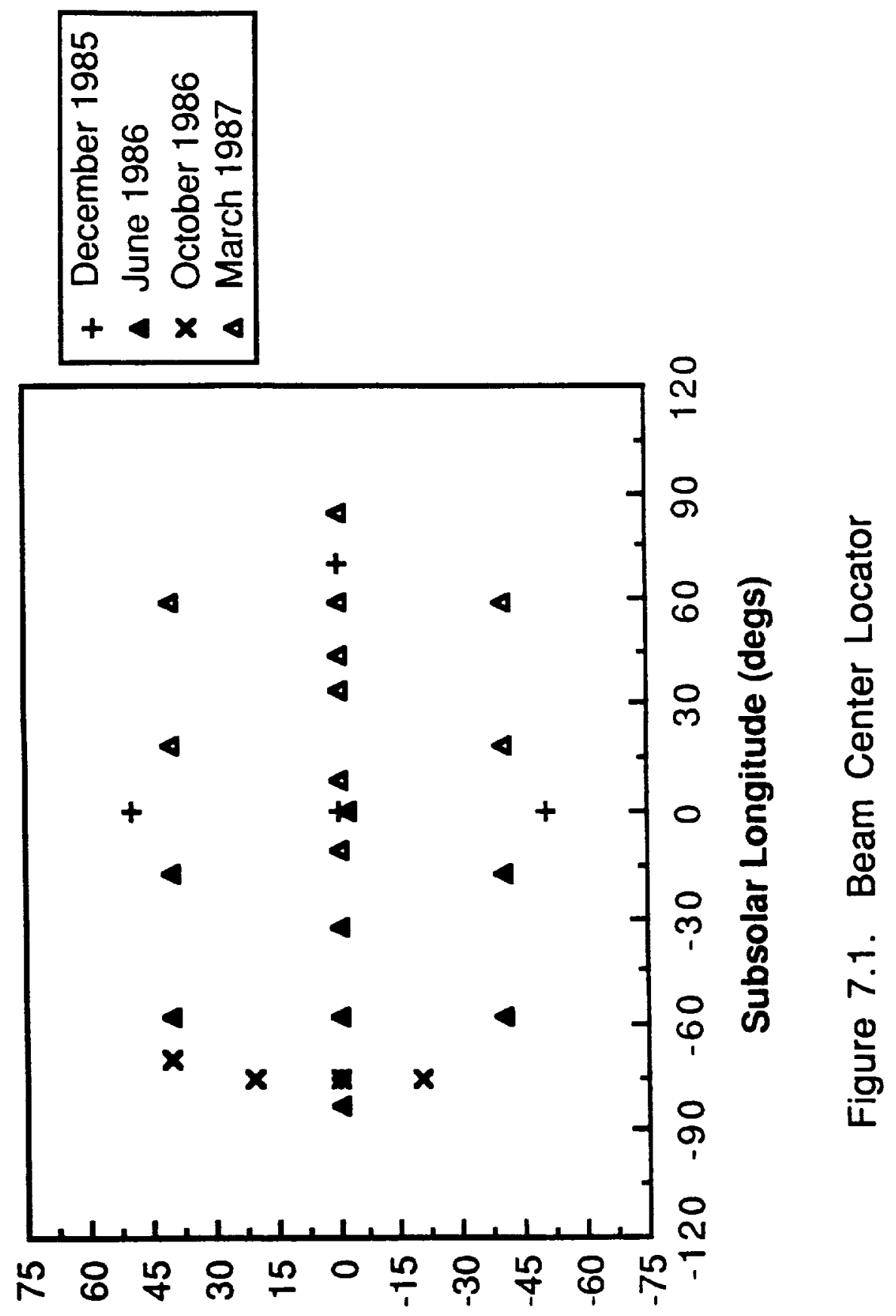

(s6อp) әрпษ!ฺอา 


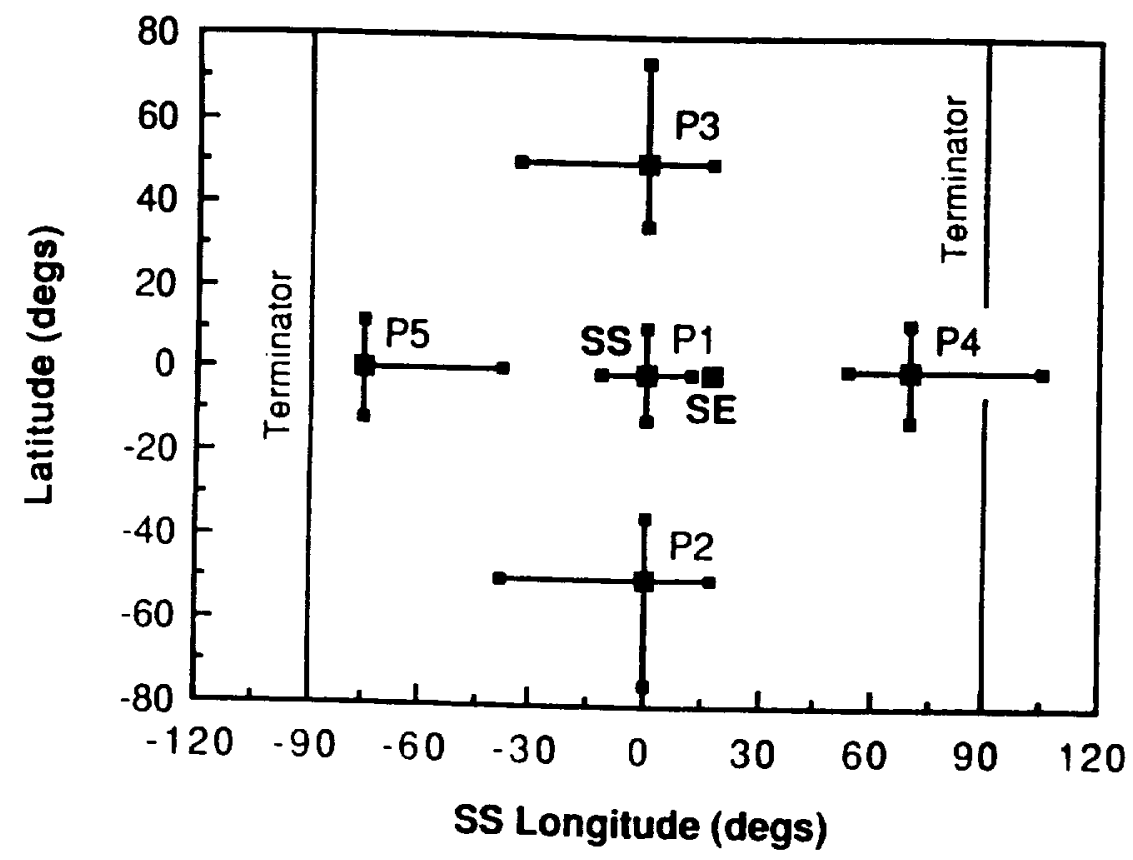

Figure 7.2. Beam Center Uncertainty December 1985

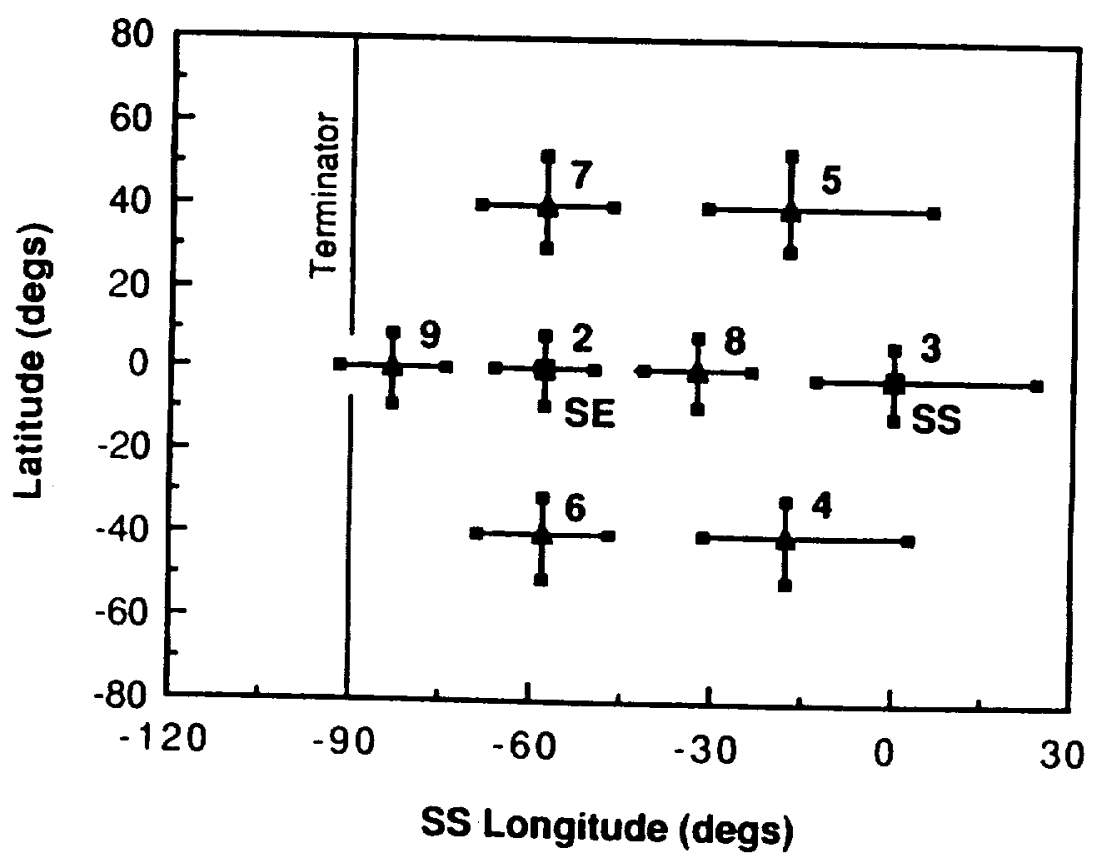

Figure 7.3. Beam Center Uncertainty June 1986 


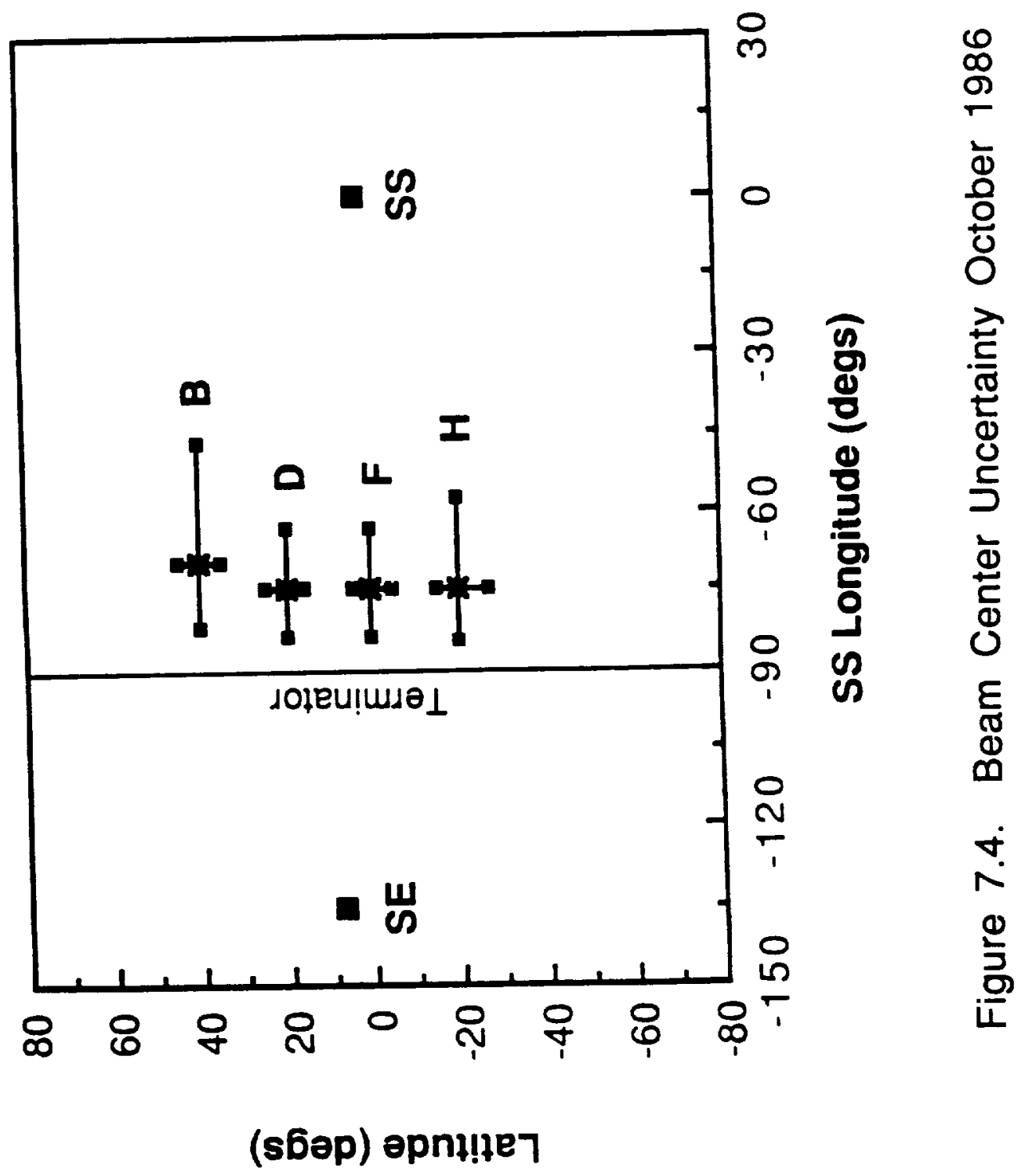




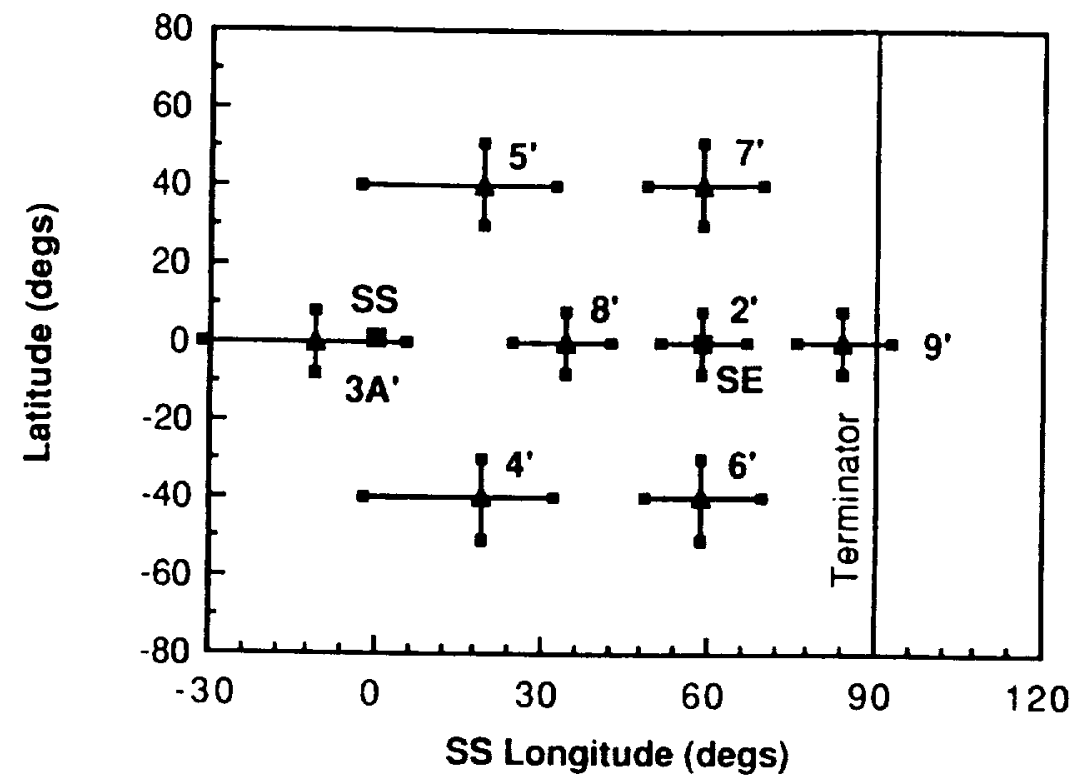

Figure 7.5a. Beam Center Uncertainty March 1987

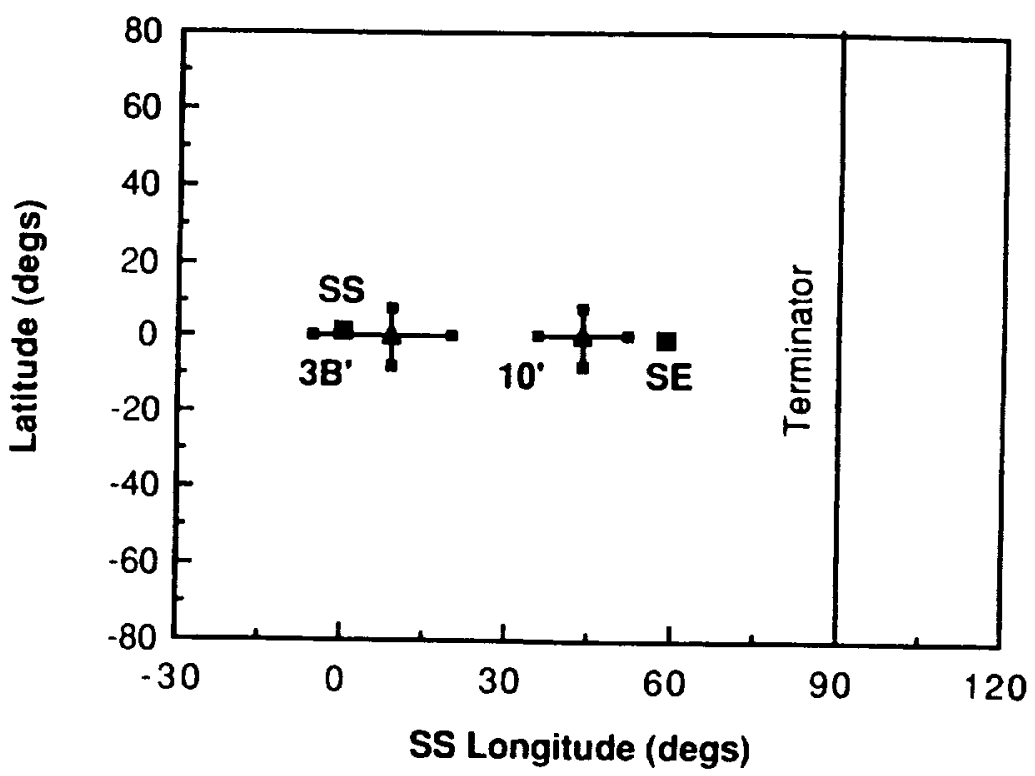

Figure 7.5b. Beam Center Uncertainty March 1987 (cont'd) 
and $\beta$ resulting from $\Gamma=1$ for December 1985, June 1986, and March 1987 , and $\Gamma=2$ for October 1986. The Subsolar Point (SS), Subearth Point $(\mathrm{SE})$, and terminator $(s)$ are labelled on each of the Figures. SE necessarily marks disk center for an Earth-bound observer. The $\Gamma$-induced uncertainties in $\alpha$ and $\beta$, for a given observing period, are solely a function of line of sight projection geometry, with beam positions near disk-center having the smallest $\alpha$ and $\beta$ uncertainties. These Figures establish, on the basis of pointing uncertainty alone, that an observed beam-integrated wind velocity should not be associated with beam-center position $(\alpha, \beta)$. (December 1985 positions P2 and P3 have $\alpha$ and $\beta$ uncertainties of $-60^{\circ}$ and $-40^{\circ}$, respectively.)

Individual sources of pointing uncertainty (and their systematic/random character) need to be isolated to properly assess effects on beam-integrated velocities. The worst case pointing uncertainty, determined in the field, owes to (when appropriate):

1. IR $(10 \mu \mathrm{m})$ and visible (tracking) beam misalignment Misalignment gives rise to a spatial systematic offset constant in magnitude, but varying in direction, from beam position to beam position (due to image rotation).

The IRTF has an eyepiece allowing alignment of the $10 \mu \mathrm{m}$ and visible (tracking) beams at the detector focal plane. The broadband heterodyne signal is maximized on a calibration blackbody point source (see Fig. 3.8) through X-Y motion of the detector. A star is then located via the eyepiece at the visible position of the blackbody in the detector focal plane. Centering the TV monitor cross hairs on the visible stellar image thereby 
insures visible/IR alignment. The advantage is the star need not generate a heterodyne signal for alignment - the calibration blackbody serves the purpose. (The blackbody is not imaged by the monitor and cannot be used for cross-hair calibration.)

At McMath no focal plane eyepiece as yet exists. While the blackbody heterodyne signal is maximized as before, the visible position of the blackbody on the detector is unknown. Alignment of $10 \mu \mathrm{m}$ and visible beams is accomplished using an external IR source (IR point source, e.g., VY CMa, or planetary limb) to generate a $10 \mu \mathrm{m}$ signal (see Section 3.5). In principle the IR source's broadband heterodyne response is maximized using the telescope drive paddle, insuring coincidence of the source's 10 $\mu \mathrm{m}$ beam with the spectrometer's IR axis as defined by the blackbody. The monitor cross-hairs are then aligned with the source's visible image.

While both IRTF and McMath approaches are equivalent, regarding the latter: 1) stellar sources suffer from a lack of definition in the broadband heterodyne signal, and 2) the visible limb of Venus can be poorly defined (particularly for daytime observations). In practice, alignment at McMath was accomplished by rastering the Venusian disk across the cross hairs and matching the limb-to-limb heterodyne response to the visible image. A planetary disk radially symmetric both in the visible and at $10 \mu \mathrm{m}$ allows alignment to $<1$ arc-second. Planetary phases with large elongations, however, exhibit visible and $10 \mu \mathrm{m}$ limb-to-terminator gradients, the latter highly non-linear (see 
Section 7.1.2), which can significantly limit visible/IR

alignment.

Both IRTF observing periods were associated with visible-IR alignment to $\ll<1$ arc-second. Alignment at McMath December 1985, near superior conjunction, should be significantly better than 1 arc-second. Alignment at McMath June 1986, near maximum elongation, is estimated at -1 arc-second due to the highly asymmetric E-W $10 \mu \mathrm{m}$ gradient.

2. Guiding template-associated offsets

Guiding by template overlay is subject to systematic offsets due to: 1) guiding subjectivity of the eye, and 2) image rotation between (frequent) template repositionings. Such offsets will appear pseudo-random in magnitude and direction from one wind measurement to the next. Continuous update of planetary position with respect to the template (via the telescope drive paddle), due to low frequency image motion, introduces an r.m.s. component. Template-associated offsets are estimated to be $<1$ arc-second.

3. Telescope tracking drift rates

The IRTF focal plane image scale precludes using guiding templates near inferior conjunction. Beam pointing must rely on the tracking computer. Tracking drift rates are different in R.A. and Declination, and dependent on Local Time. Tracking drift between zero point recalibrations was found large and unpredictable prior to March 1987 (in part due to wobbling secondary behavior), inducing systematic pointing offsets varying in magnitude and direction from one beam position to the next. 
Only the October 1986 data is so affected and its worst case 2 arc-second pointing uncertainty has been discussed at length in Section 5.3.3.

\subsubsection{Beam Character Considerations}

Figures 7.2-7.5 are also useful from the standpoint of beam size. The beam-specific error bars are equally representative of the planetary surface intercepted by a 2 arc-second diameter beam ( 4 arc-seconds for October 1986). The worst-case effective HPBW's (obtained by coupling diffraction-limited beamshape and worst-case seeing; see Appendix 2) for December 1985, June 1986, October 1986, and March 1987, were $3.7,3.7$ (in R.A.), 3.9 and 2.9 arc-seconds, respectively. The planetary regions intercepted by three of these beams are significantly larger than the already sizable regions delineated by the error bars on the Figures. Yet, if beam-sampled dynamics folds into the beam-integrated wind velocity symmetrically about beam center, one could still arguably associate observed wind velocity with beam-center $\alpha$ and $\beta$ ( \pm the $\alpha, \beta$ uncertainites). This is, however, far from the case:

1. The beam samples the wind field projected along the line of sight. The projection geometry has a non-linear gradient across the beam for small planetary disk-to-beam ratios, particularly regarding limb scans. In addition, the global wind field can exhibit a strong non-linear across-the-beam gradient in horizontal velocity.

2. The non-thermal emission strength, dependent on solar zenith 
angle (SZA) and line of sight column length (L) through the emitting shel1 (see Chapter 2), exhibits a strong non-linear gradient across the beam for beams even marginally intercepting the limb.

Figure 7.6 provides the non-thermal emission geometry (not to scale). The emitting shell has inner radius $R_{1}$ and outer radius $R_{2}$ (radii to half power; see Chapter 2). $R_{p}$ is the $10 \mu \mathrm{m}$ continuum radius (taken as the solid planet radius (R) plus 60 $\mathrm{km})$.

As the $10 \mu \mathrm{m}$ emission is optically thin, emission strength is proportional to the length (L) of the line of sight through the shell. L is plotted as a function of distance (D) from disk center at the bottom of Figure 7.6. While negligible variation occurs for $0<D<R_{p}$, dramatic variation can occur for $D>R_{p}$. Figure 7.7 represents the situation to scale. The non-thermal emission is centered $109 \mathrm{~km}$ above the solid surface and has a half power thickness of $19 \mathrm{~km}$ (see Chapter 2). The circular beam intercepting the limb represents a planetary disk-to-beam ratio of 4. (By comparison, the December 1985 effective HPBW gives a worst-case disk-to-beam ratio of 2.7.) The relative intensity at the bottom of the Figure has been smoothed over the mean beam element. The line of sight column length tangential to the emitting region $\left(R_{1}<D<R_{2}\right)$ is maximally 51 times the thickness of the shell! Thus a beam even partially probing the planetary 1 imb can be heavily weighted in favor of limb dynamics as opposed to conditions at beam-center. 

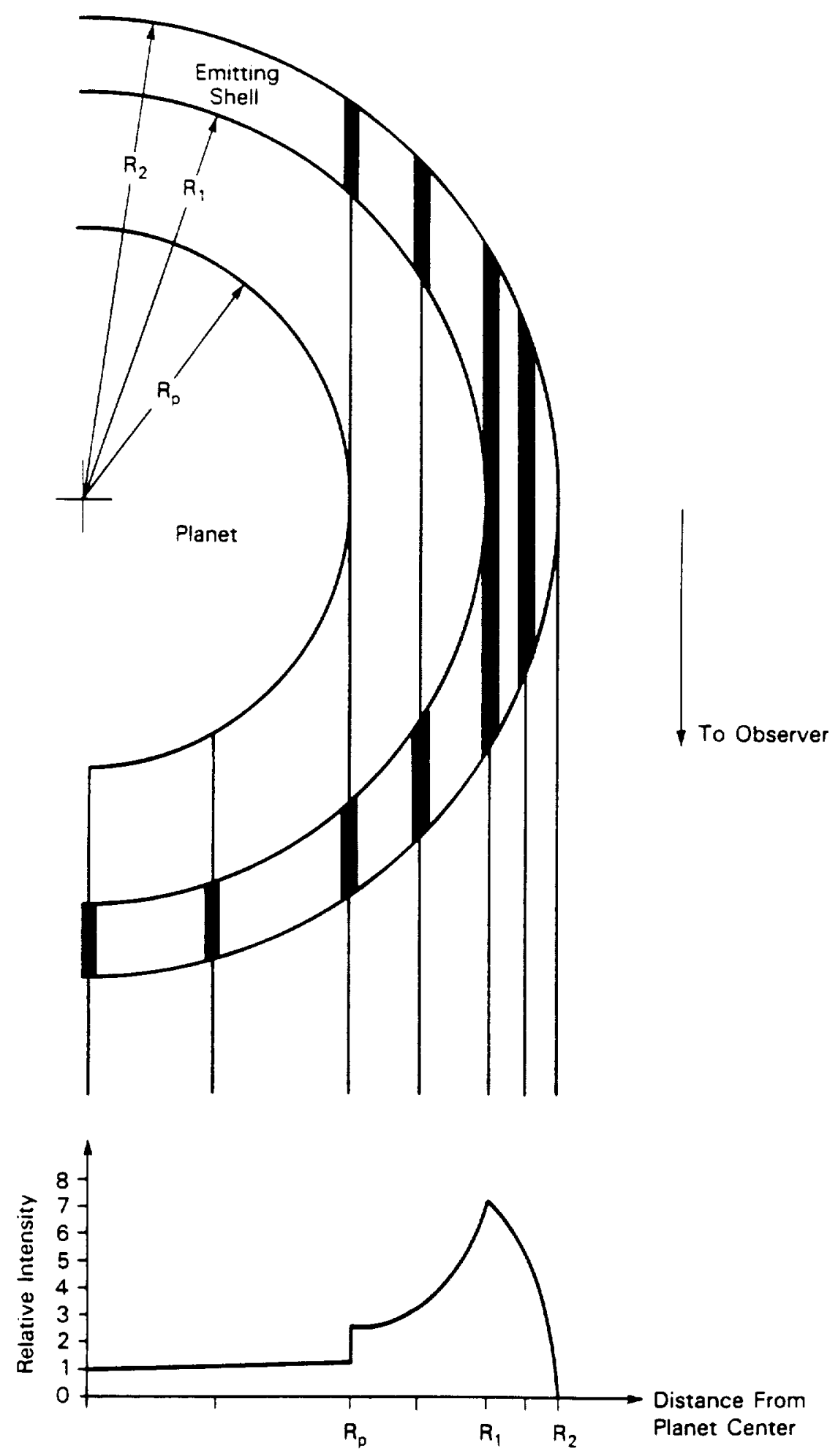

Figure 7.6. Non-Thermal Emission Intensity Variation

193 


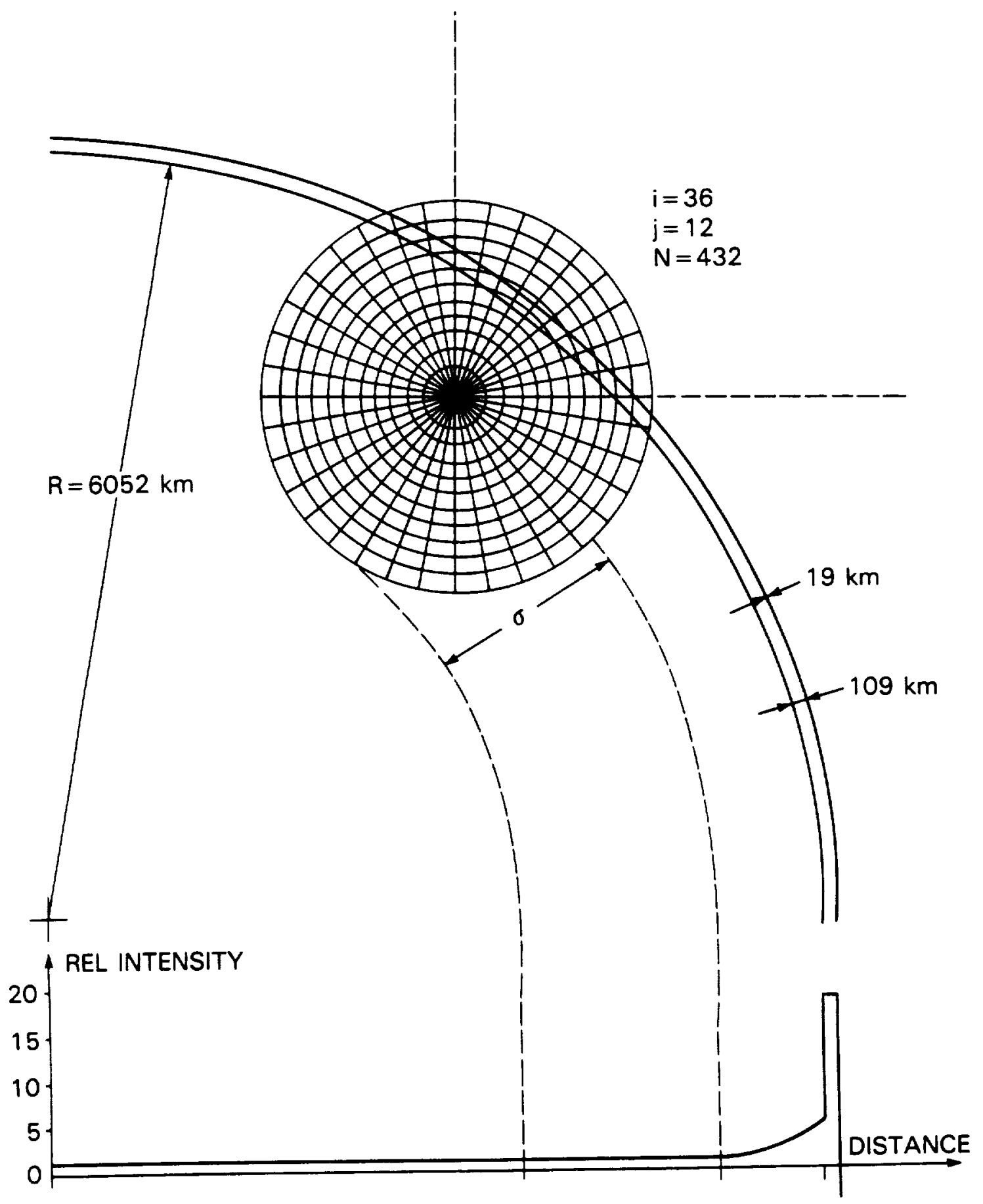

Figure 7.7. Program BEAMINT Geometry 
Additionally, emission intensity is moderated by

across-the-beam variation in solar zenith angle (SZA) (up to $55^{\circ}$ for December 1985) and the beam's spatial roll-off.

\subsubsection{BEAMINT Beam Modelling}

To facilitate derivation of model beam-integrated winds, BEAMINT partitions the beam into $i$ slices and $j$ annuli (the case $36 \times 12=432$ is shown on Figure 7.7). A $100 \times 100=10,000$ element beam is used, allowing resolution of the $20 \mathrm{~km}$ thick emitting shell at the 1 imb.

Within each beam element, all dynamically relevant variables (e.g., horizontal wind velocity, line of sight projection geometry, beam spatial roll-off, line of sight column length, solar zenith angle, and $B F 1$ rotational component) are taken as constant and evaluated at the element centroid. As the elements are not of equal size, their beam contributions are weighted by their relative areas. Each element produces a Gaussian non-thermal emission line, frequency translated by the appropriate line of sight velocity, as dictated by the model wind field. The line's relative intensity is determined from the beam roll-off and line of sight emission strength. Beam roll-off is derived from the diffraction-limited beam shape coupled with beam-specific seeing.

The beam-integrated non-thermal emission is constructed through superposition of all individual Gaussian line profiles, and the frequency centroid of the convolution is defined as the line of sight beam-integrated velocity. Elements off the planet, or located at SZA $>90^{\circ}$, are zero-weighted. The beam is then offset by the worst case 
pointing uncertainty $(\Gamma): E, N E, N, N W, W, S W, S$, and $S E$ (on the sky) to determine pointing uncertainty-induced error bars for the modelled beam-integrated winds.

The model wind field incorporates both SS-AS and zonal components, the former axi-symmetric about the SS-AS axis, the latter axi-symmetric about the planet's spin axis. Vertical velocities are assumed everywhere zero.

SS-AS horizontal velocity is taken proportional to SZA, with a maximum at $\mathrm{SZA}=90^{\circ}$ and zeros at $\mathrm{SZA}=0^{\circ}$, and $180^{\circ}$. This simple form is almost identically representative of the horizontal velocity field obtained by the Bougher et al. (1986) hydrodynamical model for $110 \mathrm{~km}$ altitude (with $121 \mathrm{~m} / \mathrm{s}$ cross-terminator winds; see Chapter 2).

Choice of the model's zonal field must rely on observed zonal behavior at lower altitudes. Pioneer Venus (PV) Orbiter UV imaging of the cloud decks (see Chapter 2) indicated solid-body zonal super-rotation from 0 to $\pm 60^{\circ}$ latitude. However, PV descent-probe wind measurements, cyclostrophic balance models, and Mariner $10 \mathrm{UV}$ imaging indicated strong departures exist, e.g., strong mid-latitude jets. Whether this discrepancy results from probing at different altitudes, or real variation in the Venusian wind field (see Chapter 2), is not relevant. Both interpretations hold that the zonal wind field can exhibit either a simple or complex latitudinal dependence.

Regarding an analytic form for the model's zonal component, a characterization somewhere between solid-body rotation (proportional to $\cos \beta$ ) and one dictated by conservation of angular momentum (proportional to $1 / \cos \beta$ ) certainly seems reasonable. The latter 
however, in its strictest sense, is probably not realistic. Through conservation of angular momentum, the cloud-deck mean-meridional circulation should enhance high latitude angular momentum at the expense of the equatorial region. The fact that a strong equatorial super-rotation is observed indicates the presence of other mechanisms effecting angular momentum redistribution.

Since little is known about latitudinal behavior of the high altitude zonal component, it is difficult to decide a priori on its best characterization. As this investigation represents only a first attempt at a quantitative analysis of high altitude winds, a solid-body zonal component is assumed. Future beam-integrated modelling can incorporate a different latitudinal dependence in the zonal component and a non-linear SS-AS circulation.

\subsection{KINEMATICAL FITS TO A TWO-COMPONENT CIRCULATION MODEL}

Figures 7.8a-e provide BEAMINT fits to the December 1985 data. Each fit is shown as a plot of observed $\left(\mathrm{V}_{\text {obs }}\right)$ versus modelled ( $\mathrm{V}_{\text {model }}$ ) beam-integrated wind velocity, in $\mathrm{m} / \mathrm{s}$ (positive for recession). The line $\mathrm{V}_{\text {obs }}=\mathrm{V}_{\text {model }}$ has been drawn on each of the plots. Model goodness of fit is determined from the dispersion about $\mathrm{V}_{\text {obs }}=\mathrm{V}_{\text {model }}$.

Each model is characterized by two parameters: SS-AS cross-terminator horizontal velocity $\left(\mathrm{V}_{\mathrm{Hmax}}\right)$ and the zonal component's equatorial velocity referenced to $B F 1\left(V_{e q}\right)$, both in $\mathrm{m} / \mathrm{s}$. Each of Figures $7.8 \mathrm{a}-\mathrm{e}$ contains a set of model fits with constant $V_{\text {eq }}$ (given at the Figure's upper right; negative for retrograde), and varied $V_{H m a x}$. $V_{\text {obs }}$ error bars are the $1 \sigma$ r.m.s. errors associated with the 


\section{December 1985}

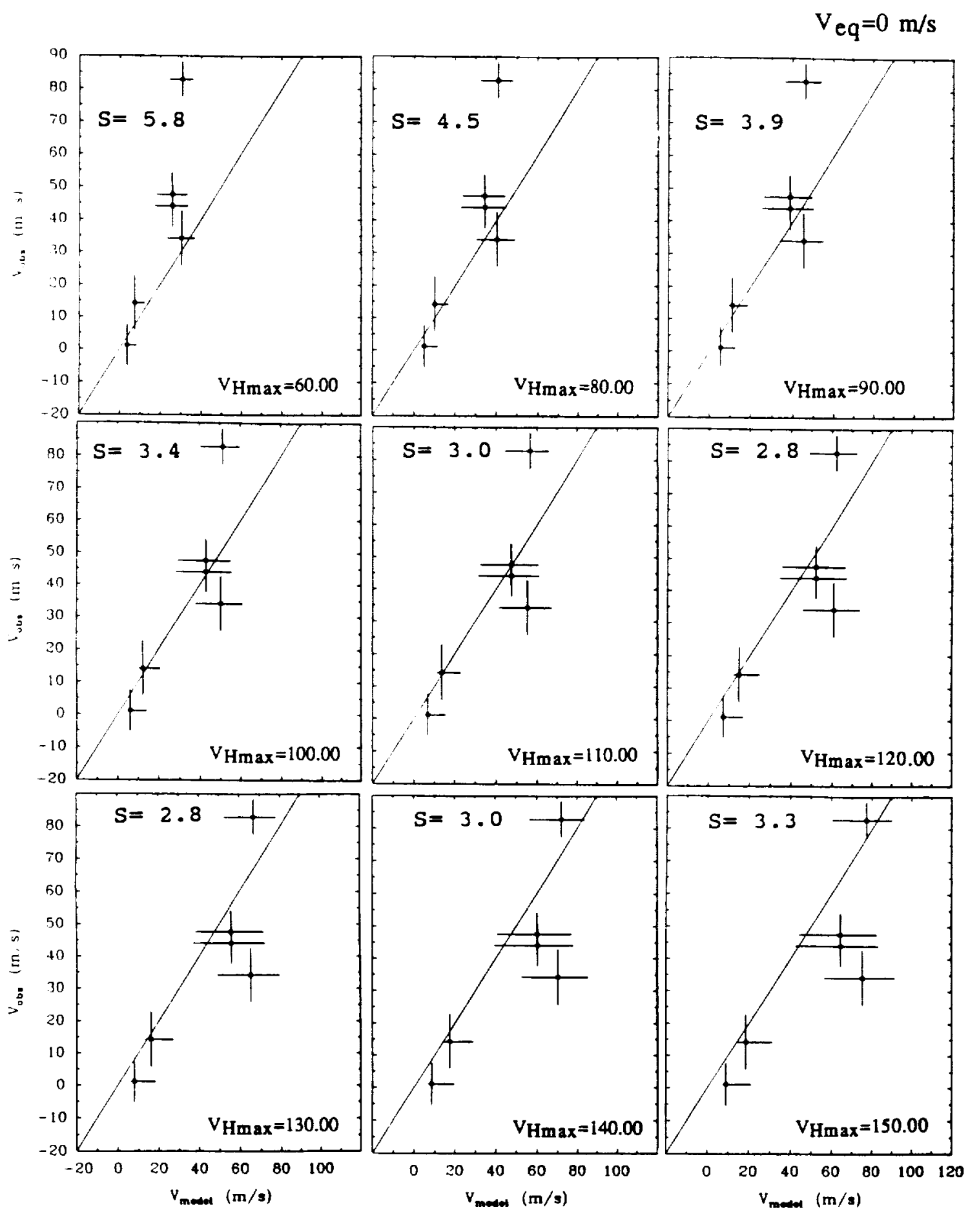

Figure $7.8 \mathrm{a}$ 


\section{December 1985}

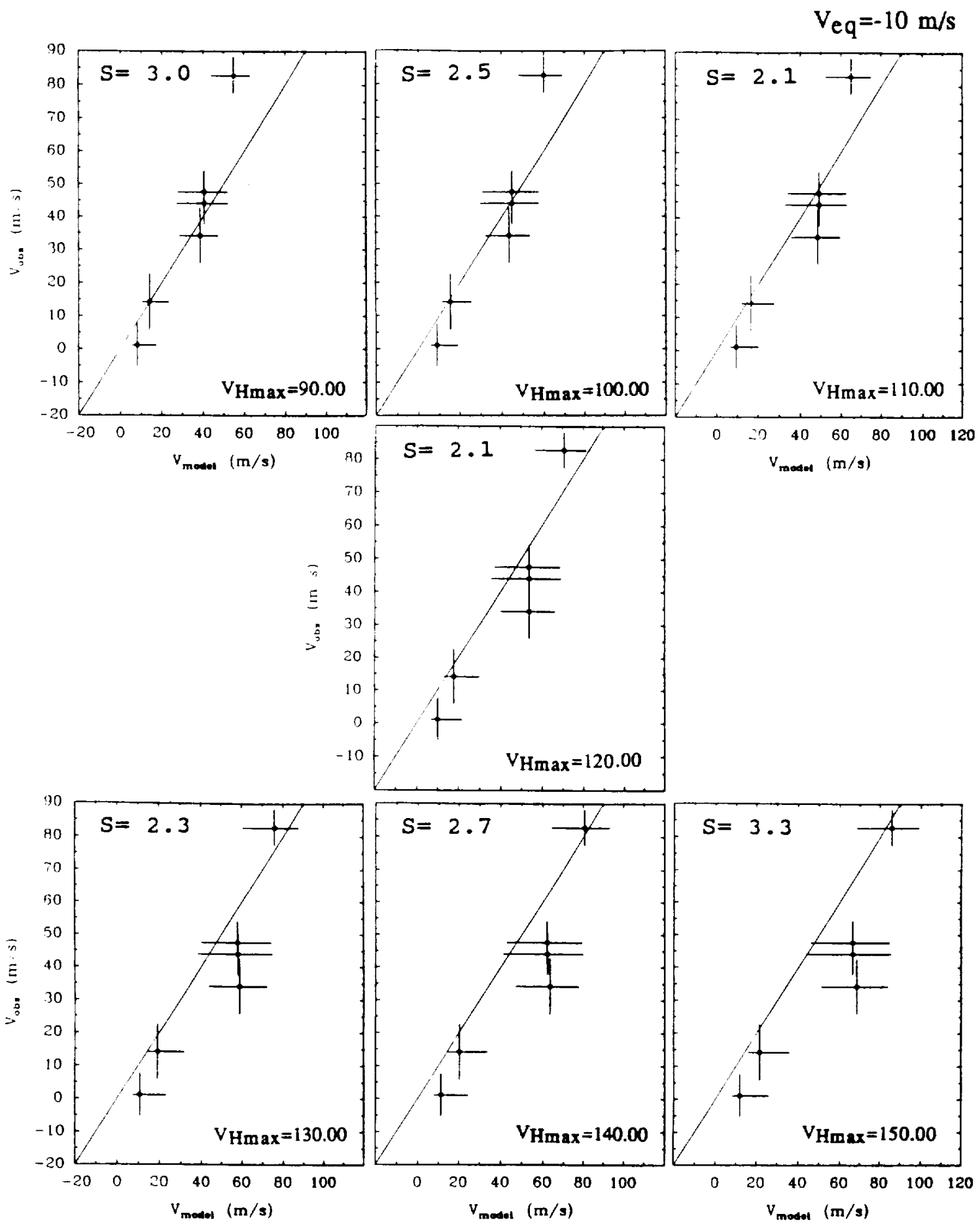

Figure $7.8 \mathrm{~b}$ 


\section{December 1985}

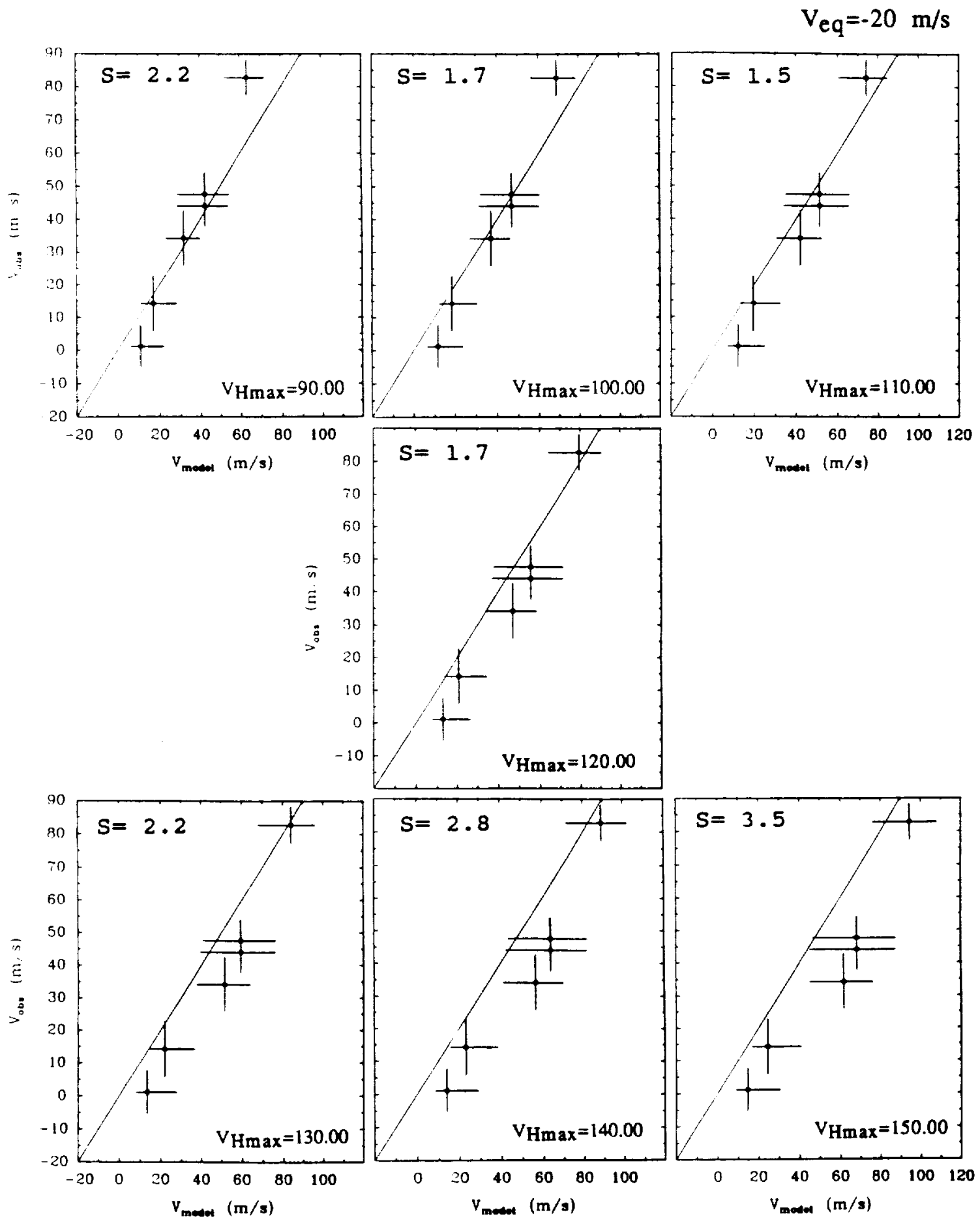

Figure $7.8 \mathrm{c}$ 


\section{December 1985}

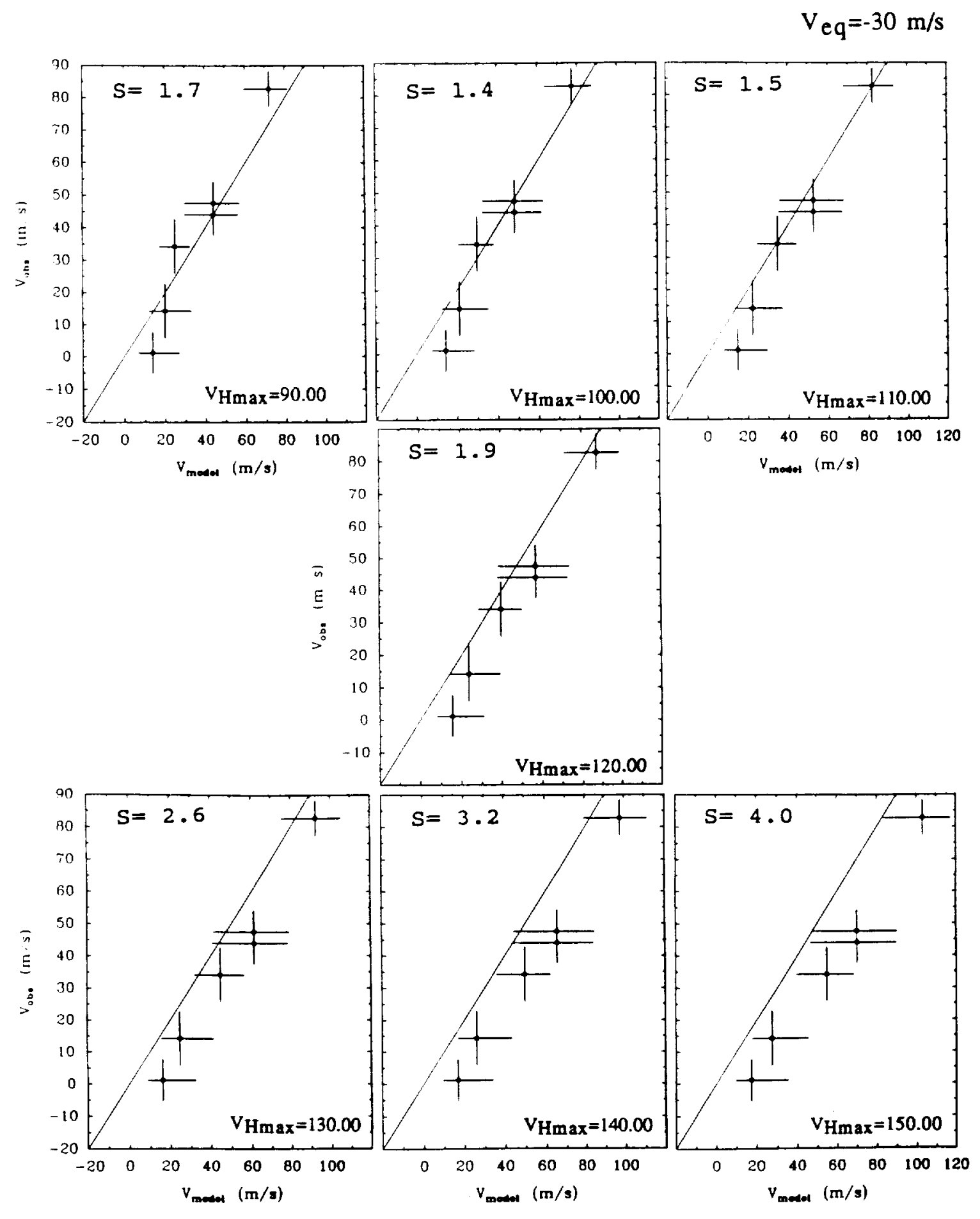

Figure $7.8 \mathrm{~d}$ 


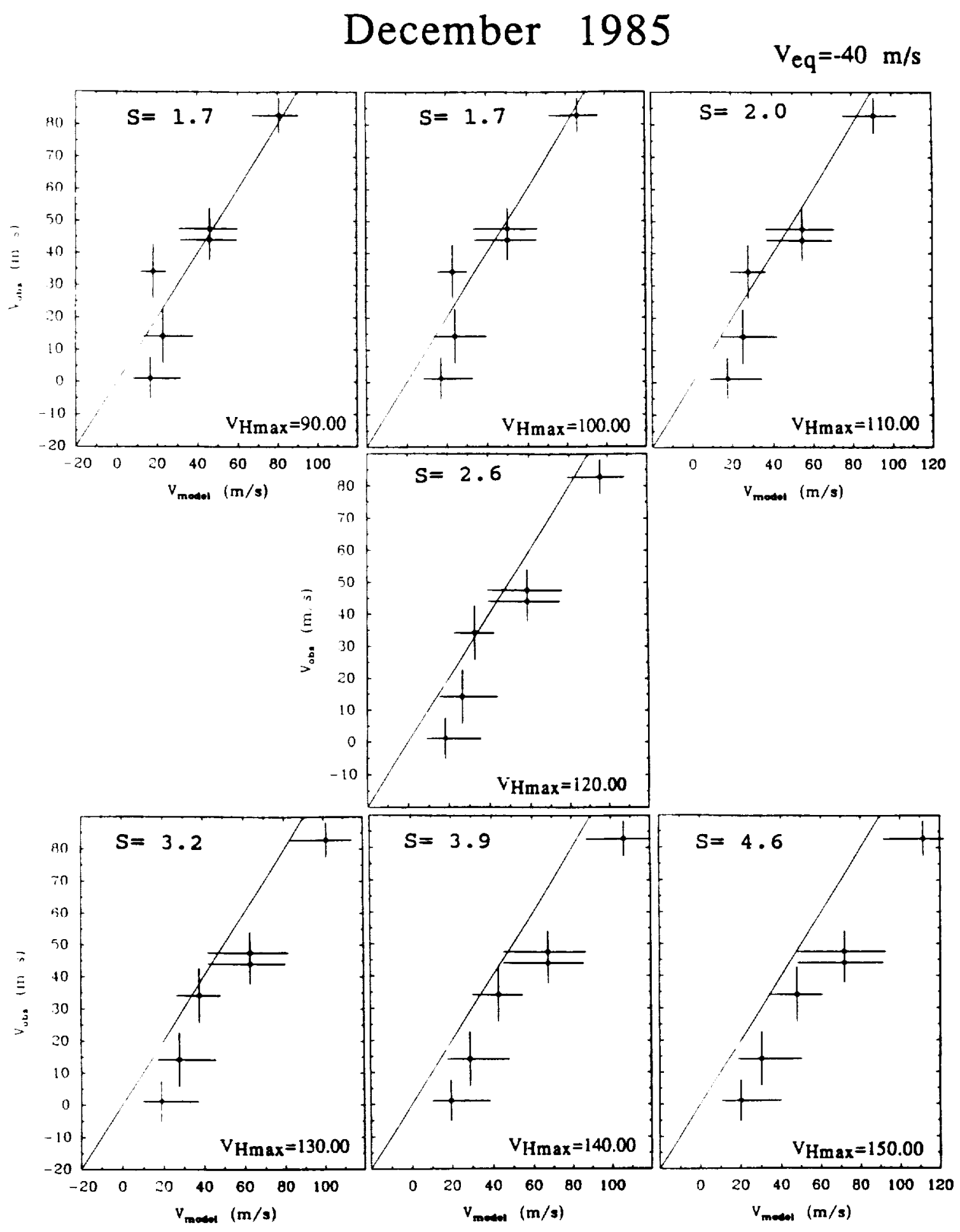

Figure $7.8 \mathrm{e}$ 
datum-specific scan sets (as given on Table 5.1). BEAMINT-generated $V_{\text {model }}$ error bars are not $1 \sigma$ errors but derive from the worst case observing period-specific pointing uncertainty - the latter a measure of how far a beam position may be from the nominal. As such, these error bars reflect only reasonable limiting magnitude effects on $\mathrm{V}_{\text {model }}$ from the combined pointing uncertainty systematic and pseudo-random components - both hard to quantitatively deconvolve. In addition, $V_{\text {model }}$ errors provide only the possible effect on model beam-integrated velocity as BEAMINT only considers 8 beam center offsets from the nominal beam position.

A systematic error in $V_{o b s}$ is specific to an observing period and would result in a constant vertical offset in the data on the model fits. Conversely, a pointing uncertainty systematic error, even one constant in magnitude and direction (e.g., 1 arc-second East) will in general appear as a set of non-linear offsets in the $v_{\text {model }}$ data and not necessarily in the same sense.

The pointing uncertainty for all observing periods should be dominated by a pseudo-random spatial offset varying from one wind velocity determination to the next, so that $V_{\text {model }}$ error bars can be considered to derive from random effects. However, a pseudo-random distribution in the pointing uncertainty offset about a given nominal beam position can generate a highly non-linear response in the modelled beam-integrated wind velocity. Thus a straightforward quantitative means of including the $v_{\text {model }}$ errors in a statistical measure of the model fits is not available.

For each model, the $\sigma^{2}$ (error bar weighted) dispersion $\mathrm{S}$ about 
$\mathrm{V}_{\text {obs }}=\mathrm{V}_{\text {model }}$ was calculated in $\mathrm{V}_{\text {obs }}$ (along lines of constant $\mathrm{V}_{\text {model }}$ ), in units of the mean error bar, using Eq. 7.1:

$$
S=\left(\sum_{i=1}^{N} \frac{\left[{ }^{i} v_{o b s}-m_{o b s}\right]^{2}}{N \sigma_{i}^{2}}\right]^{1 / 2}
$$

The quantity ${ }^{i} v_{\text {obs }}$ is the $i^{\text {th }}$ of $N$ observed beam-integrated wind velocities, $\mathrm{m}_{\mathrm{obs}}$ is the corresponding value on the line $\mathrm{v}_{\text {obs }}=\mathrm{v}_{\text {model }}$, and $\sigma_{i}$ is the $1 \sigma$ error associated with element $i$. While the unweighted scatter about $\mathrm{V}_{\text {obs }}=\mathrm{V}_{\text {model }}$ is the same whether calculated along lines of constant $\mathrm{V}_{\text {model }}$ or constant $\mathrm{V}_{\text {obs }}$, the weighted dispersion measure should include a means of weighting by the $\mathrm{V}_{\text {model }}$ error bars. However, as stated, interpretation of these error bars in a meaningful quantitative way is not possible. This problem is circumvented through realization that the dominant pseudo-random component in pointing uncertainty will yield poorer model fits as gauged by Eq. 7.1. Thus a good model fit ( $V_{\text {obs }}$ scatter limited) should a posteriori indicate negligible effects due to pointing uncertainty. In addition an observed $S<<1$, when evaluated along lines of constant $\mathrm{V}_{\text {obs }}$, can be used to establish overestimation of the $\mathrm{V}_{\text {model }}$ random component.

$S$ is given on each plot of Figures 7.8a-e. S near unity indicates a good model fit with a dispersion attributable to the r.m.s. noise in the data.

The grid on Table 7.1 provides $S$ over the entire December 1985 model space with grid element coordinates $\left(\mathrm{V}_{\mathrm{Hmax}}, \mathrm{V}_{\mathrm{eq}}\right)$. While a minimum $S\left(S_{\min }\right)$ occurs at $S=1.4$, a model space about the minimum can exist 


\section{Table 7.1. December 1985 Goodness-of-Fit Parameter S Over the Model Space}

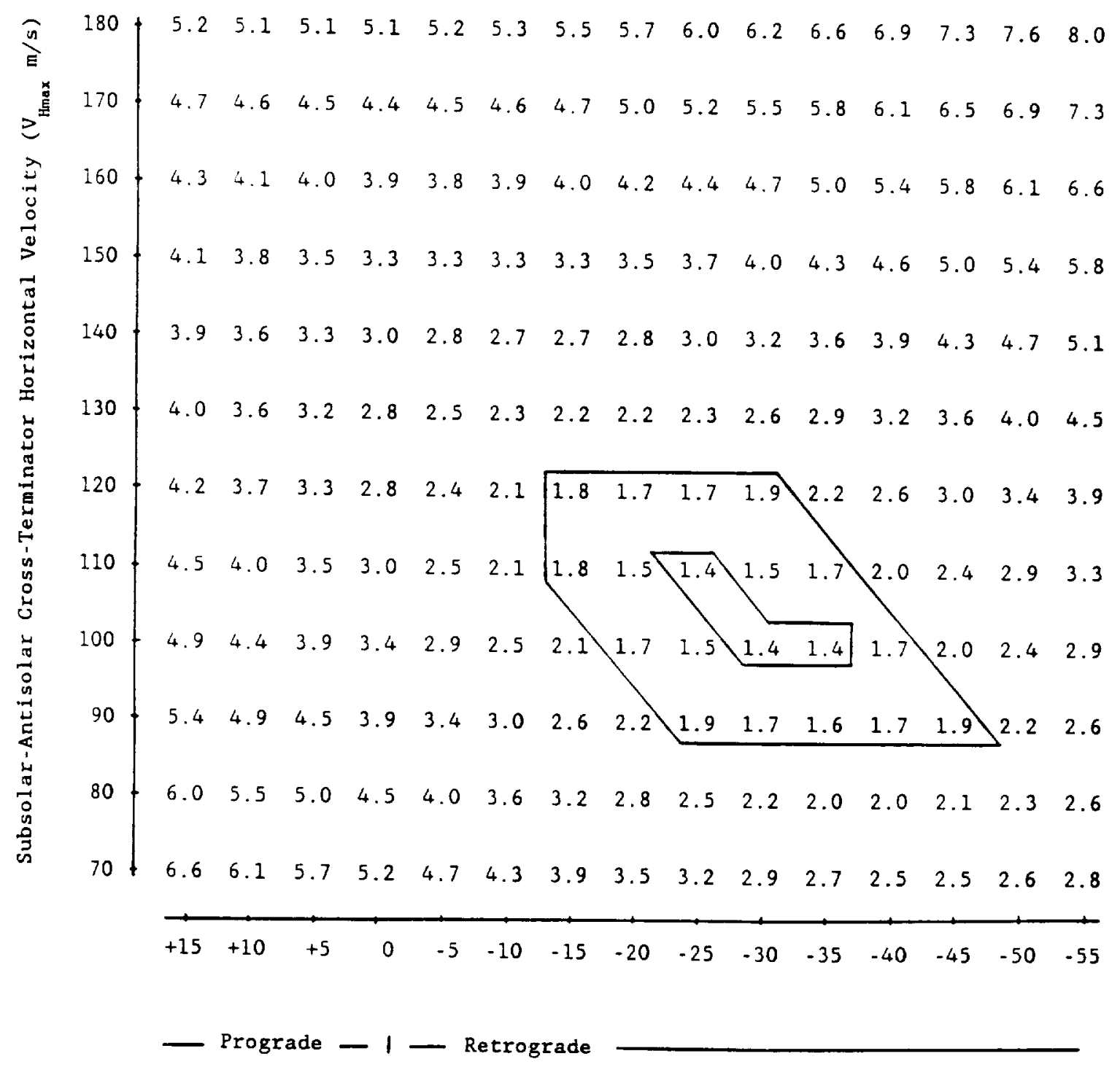

Zonal Equatorial Velocity (V $\mathrm{m} / \mathrm{s}$ ) 
within which models are statistically indistinguishable. Quantitative description of this "best-fit model space" may be obtained using the chi-squared $\left(\chi^{2}\right)$ test. $\mathrm{S}$ is related to $\chi^{2}$ through:

$$
x^{2}=\mathrm{NS}^{2}
$$

For a normally distributed data set, $\mathrm{S}$ approaches unity as $\mathrm{N} \rightarrow \infty$. However, all four observing periods are associated with a small number of data points, introducing the possibility that these data yield an $S$ different from the generating distribution. It is therefore possible that the best fit model to the generating distribution is not represented by $S_{\text {min }}$, due to perverse sampling, but rather resides within some domain about $\mathrm{s}_{\min }$. As one moves away from the minimum in the model space of Table 7.1, the observed $S$ becomes harder to explain through statistical sampling perversity, eventually $S$ variation becoming a true measure of the model fit. The convention is adopted whereby a boundary at $\mathrm{S}=\mathrm{S}_{0}$ is defined, along which the probability of obtaining $\mathrm{S}_{\mathrm{S}} \mathrm{S}_{\mathrm{o}}$ through statistical fluctuations is down to 0.18 . Using the $\chi^{2}$ tables we can find the value of $\chi^{2}$ for $N$ data such that $\mathrm{P}_{\mathrm{N}-1}\left(>\chi^{2}\right)=0.001$. Substitution into Eq. 7.2 allows determination of $\mathrm{S}_{\mathrm{o}}$. If $\mathrm{S}_{\text {min }}<\mathrm{S}_{0}$ then a "best fit model space" exists and all models with $\mathrm{S}<\mathrm{S}_{0}$ are said to fit the data. Conversely, $\mathrm{S}_{\min }>\mathrm{S}_{0}$ indicates that no subset of the model space is capable of fitting the data, implying either a model circulation inconsistent with the lower thermosphere or significant pointing uncertainty error.

On Table 7.1, observed model variation exterior to the outer polygonal border, with $\mathrm{S}_{0}=1.9$, is a true model discriminator. 
Equivalently, the region within the border is a region of model statistical indistinguishability. Existence of the border assures a model space capable of describing the lower thermosphere, and a posteriori indicates pointing uncertainty effects are negligible. A dominant subsolar-antisolar circulation with a cross-terminator horizontal velocity of 90 to $120 \mathrm{~m} / \mathrm{s}$ is indicated, as well as a retrograde zonal component with -15 to $-45 \mathrm{~m} / \mathrm{s}$ equatorial velocity.

The best fit model space has a range in $V_{\text {Hmax }}$ and $V_{\text {eq }}$ significantly larger than the few meter/sec precision of the observed beam-integrated velocities. These model parameters set the horizontal velocity field whose large acceptable range, when projected along the line of sight, is accommodated by the small $\mathrm{V}_{\text {obs }}$ error bars (particularly for near disk center beam positions).

$S$ was calculated independently in $\mathrm{V}_{\text {model }}$ (along lines of constant $\mathrm{V}_{\text {obs }}$ ). An $\mathrm{S}_{\min }=0.6$ was found coincident with the Table 7.1 minimum. The $\chi^{2}$ probability of $\mathrm{s}_{\min } \leq 0.6$ is only 158 , indicating probable $\mathrm{V}_{\text {model }}$ error bar overestimation of the pointing uncertainty random component.

Figure 7.8a, with $\mathrm{V}_{\mathrm{eq}}=0 \mathrm{~m} / \mathrm{s}$ (no zonal component), provides nine models for $V_{\text {Hmax }}=60$ to $150 \mathrm{~m} / \mathrm{s}$. The best fit case, with $V_{H \max }=120.00$ $\mathrm{m} / \mathrm{s}(\mathrm{S}=2.8)$, does not adequately fit east- and west-limb positions $\mathrm{P} 4$ and P5, which qualitatively had reflected a zonal component (see Section 6.3). $V_{\text {Hmax }}=120.00 \mathrm{~m} / \mathrm{s}$ and $v_{e q}=0 \mathrm{~m} / \mathrm{s}$ characterized the Bougher et al. (1986) circulation at $110 \mathrm{~km}$ altitude (see Figure 2.2).

Figures $7.8 \mathrm{~b}, 7.8 \mathrm{c}, 7.8 \mathrm{~d}$, and $7.8 \mathrm{e}$ are select slices through the Table 7.1 model space at $\mathrm{V}_{\mathrm{eq}}=-10,-20,-30$, and $-40 \mathrm{~m} / \mathrm{s}$, respectively. Figures 7.9a-d provide the BEAMINT fits to the October 1986 data. 


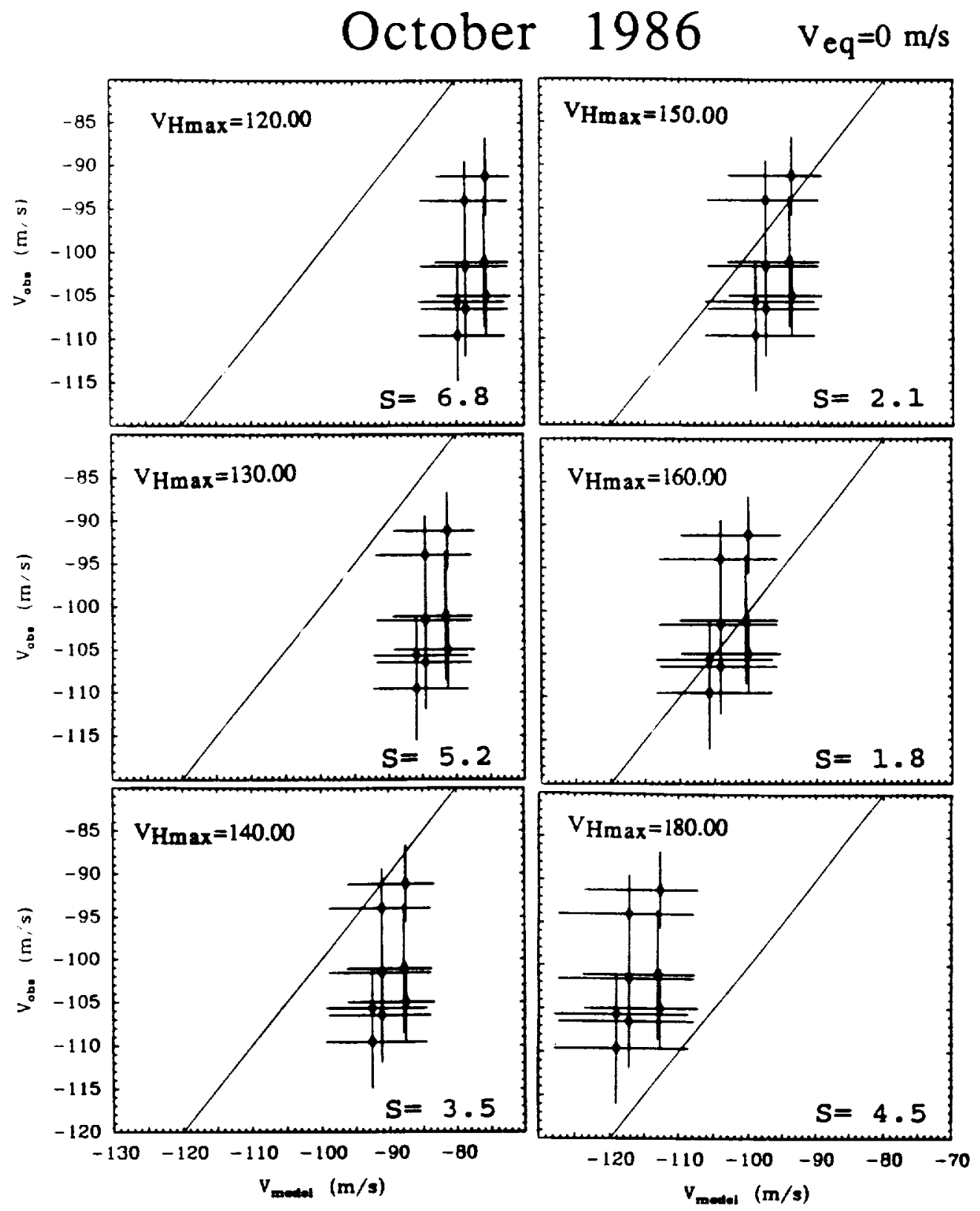

Figure $7.9 \mathrm{a}$ 


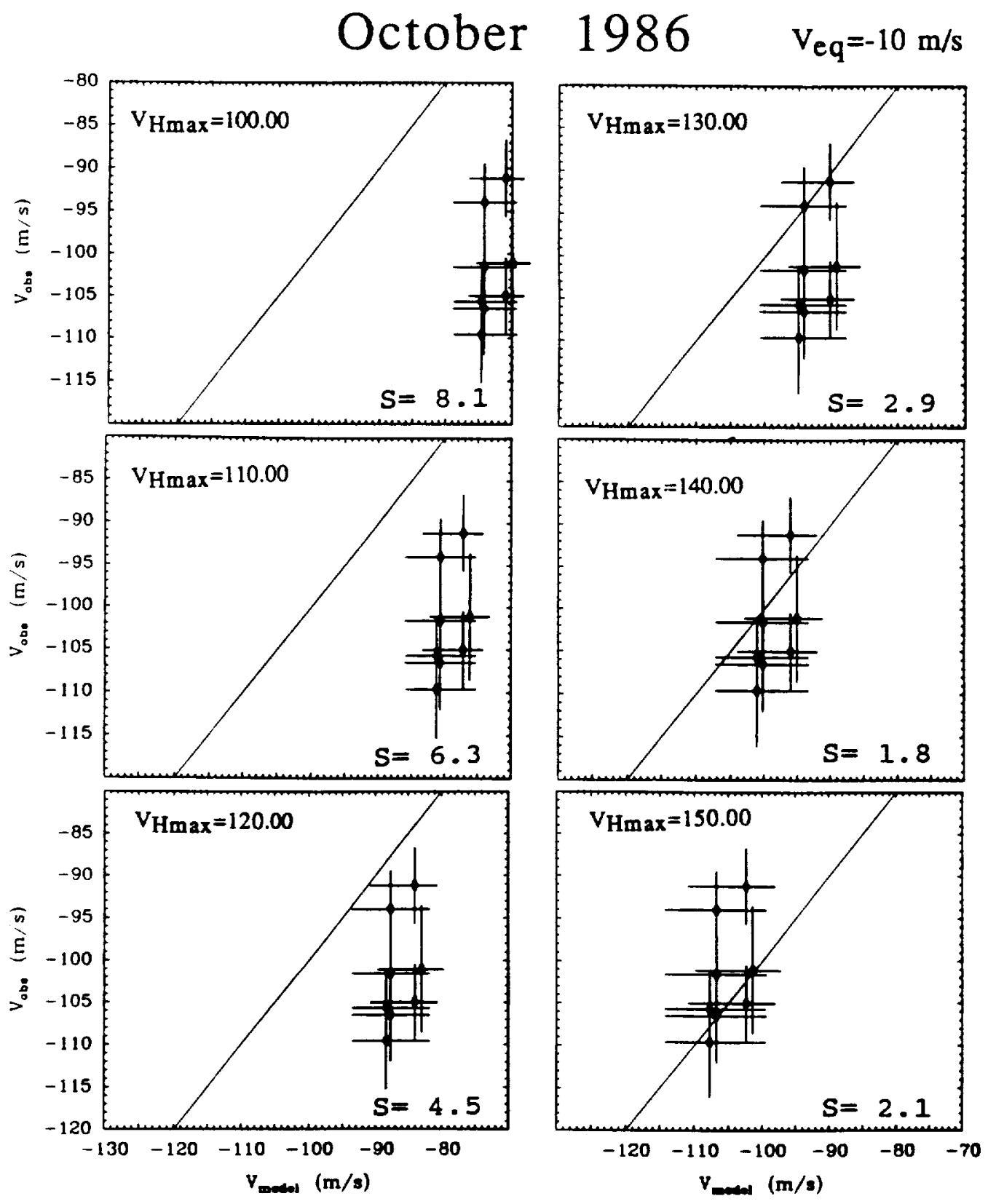

Figure $7.9 \mathrm{~b}$ 


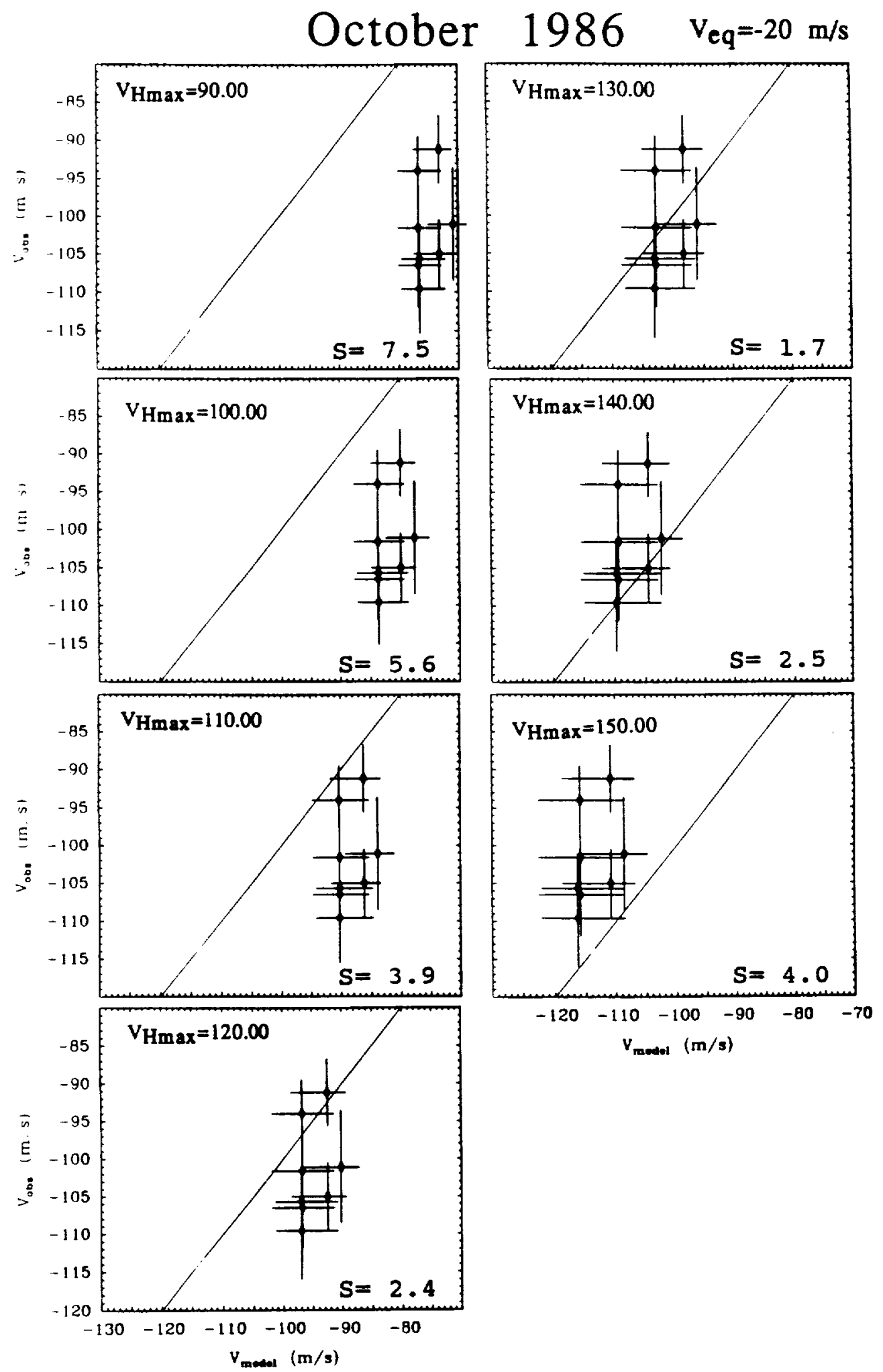

Figure $7.9 c$ 


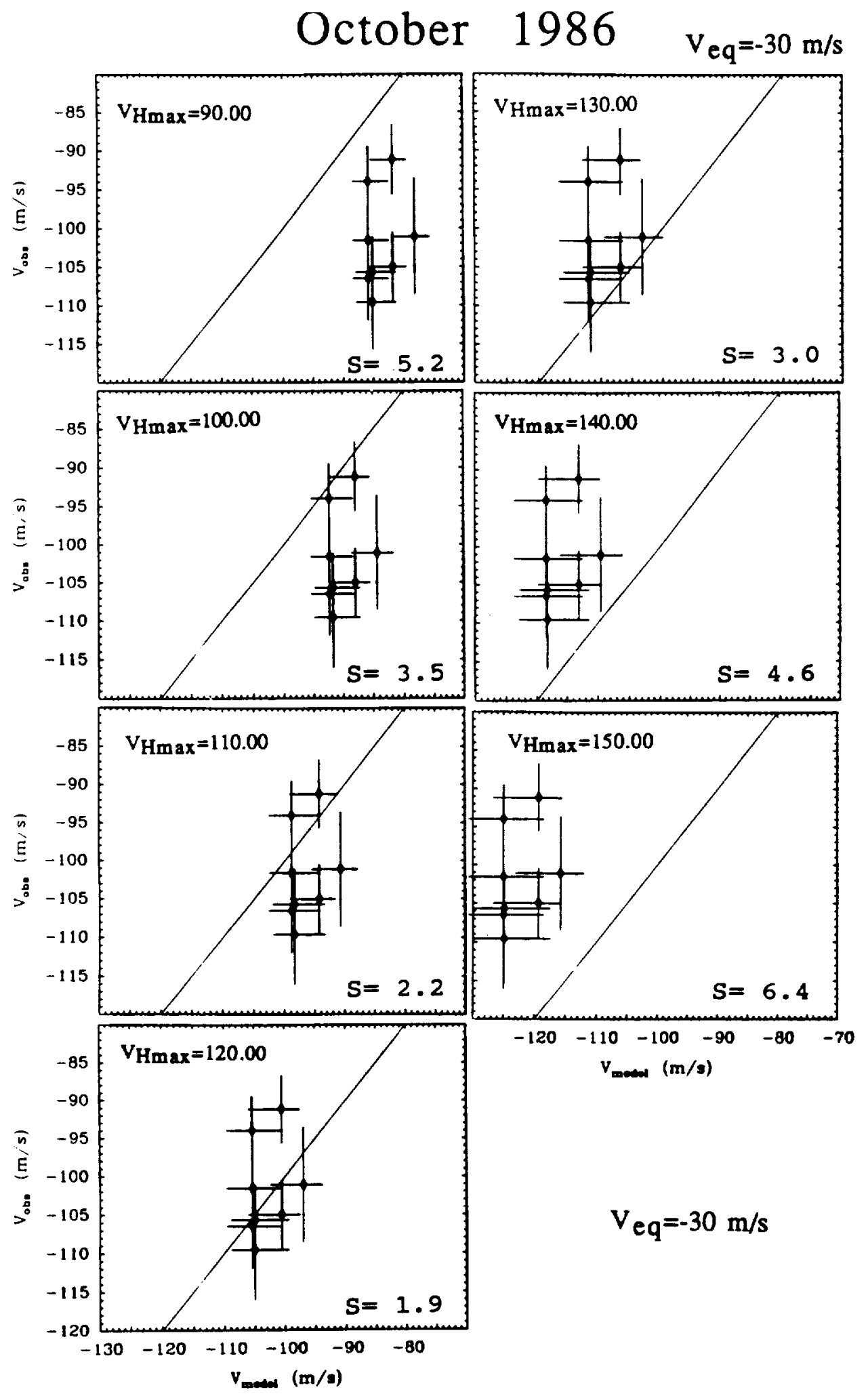

Figure $7.9 \mathrm{~d}$ 
As data were acquired just prior to inferior conjunction, both zonal retrograde and SS-AS components will produce strong blueshifts at probed positions along the crescent, as is observed. Expected latitudinal variation in beam-integrated wind velocity was not great enough to discriminate between the two components with confidence. (Just after inferior conjunction, zonal retrograde and SS-AS circulations would have exhibited strong red-and blueshifts, respectively, providing determination of the dominant circulation.) The data, however, rule out a strong prograde zonal circulation.

For the October 1986 data, BEAMINT fitting provides a quantitative description for the superposition of both components. A family of best fits exists whereby a given $V_{\text {eq }}$ has a complementary $\mathrm{V}_{\text {Hmax }}$ capable of yielding the observed beam-integrated winds. Table 7.2 provides $S$ over the October 1986 model space with $S_{\min }=1.7$. The channel from upper left to lower right is the border defined by $S_{0}=1.9$. Best fits are generated when $V_{H \max }-V_{\text {eq }}=\sim 140$ to $155 \mathrm{~m} / \mathrm{s}$. The narrowness of the channel (reflecting good circulation model horizontal velocity discrimination) is due to the large line of sight components at the probed beam positions. The lack of channel continuity at lower right may reflect rejection of very large retrograde zonal components. $\mathrm{S}_{\min }=0.7$ when calculated along lines of constant $\mathrm{V}_{\text {obs }}$ - also indicative of possible overestimation of the $V_{\text {model }}$ error bars.

BEAMINT fits to the March 1987 data are provided on Figures 7.10a-e. Table 7.3 provides $S$ over the March 1987 model space, with $\mathrm{S}_{\min }=1.3$. The polygonal border defined by $\mathrm{S}_{0}=1.6$ is consistent with $\mathrm{V}_{\mathrm{Hmax}}=110-150 \mathrm{~m} / \mathrm{s}$ and $\mathrm{V}_{\mathrm{eq}}=-20$ to $-25 \mathrm{~m} / \mathrm{s}$. Dramatic improvement in the 
Table 7.2. October 1986 Goodness-of-Fit Parameter S Over the Model Space

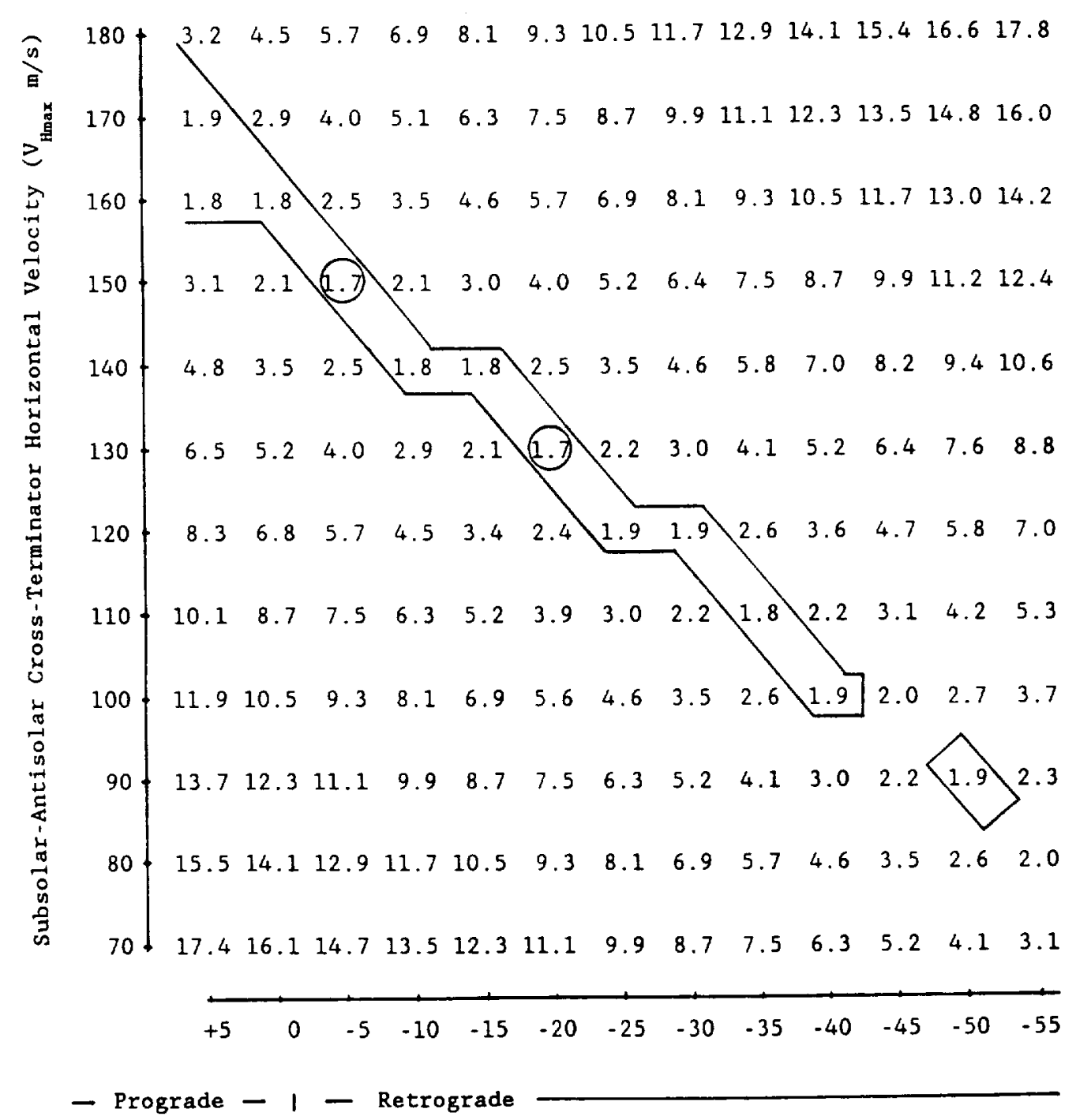

Zonal Equatorial Velocity (V $\mathrm{m} / \mathrm{s})$ 


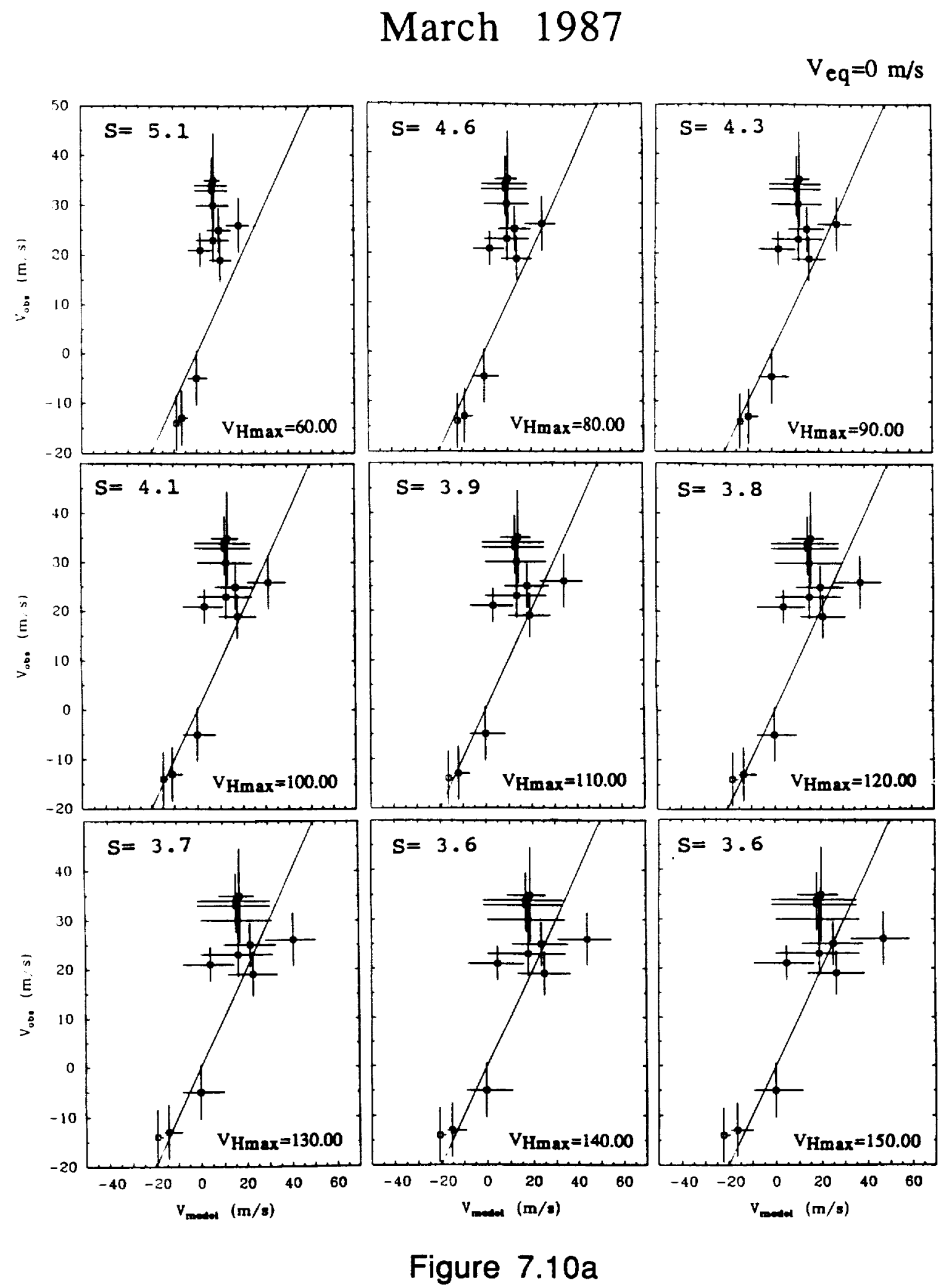




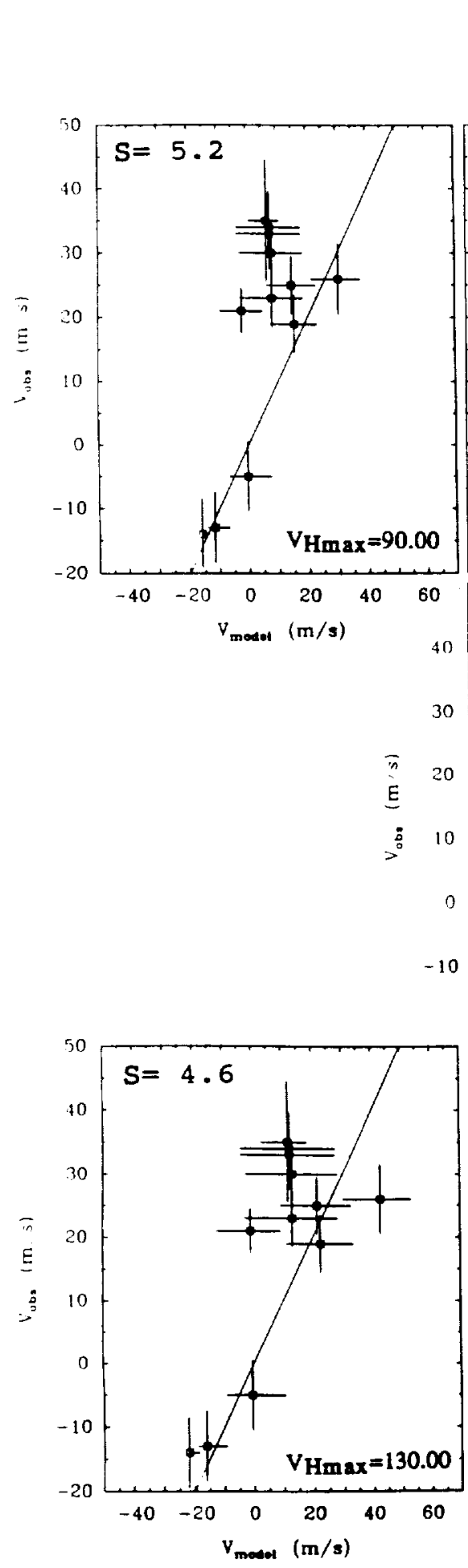

March 1987
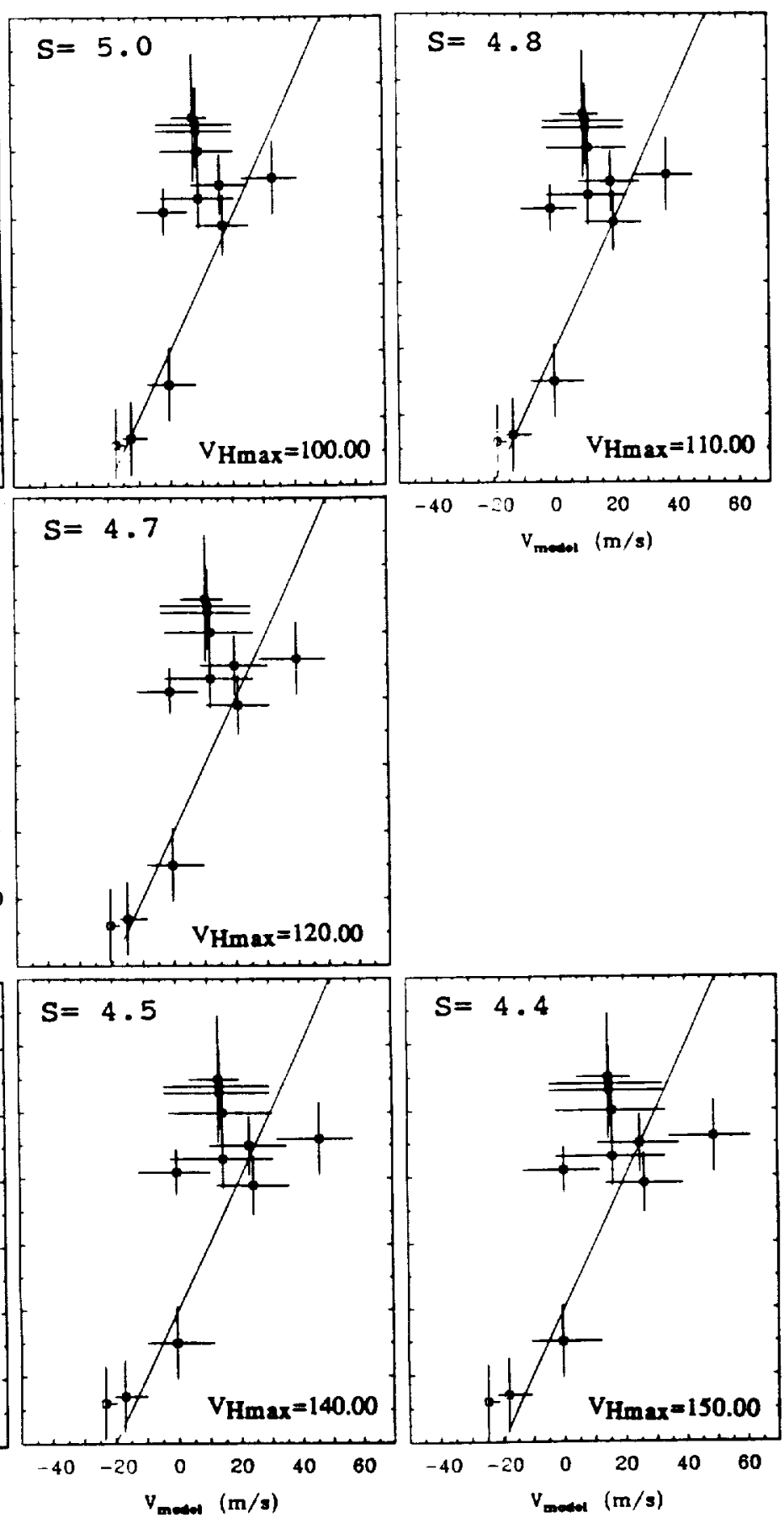

Figure $7.10 \mathrm{~b}$ 


\section{March 1987}

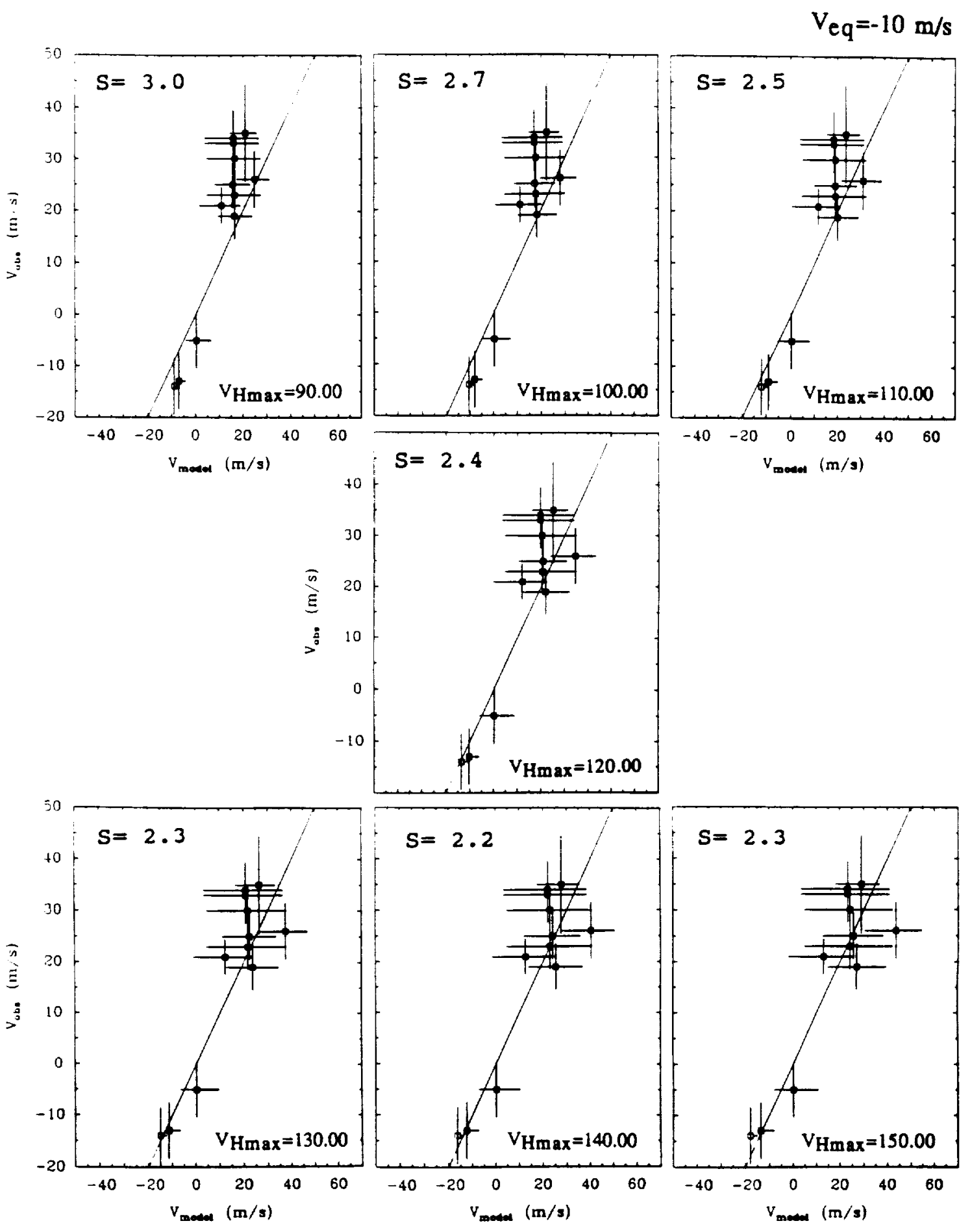

Figure $7.10 \mathrm{c}$ 


\section{March 1987}
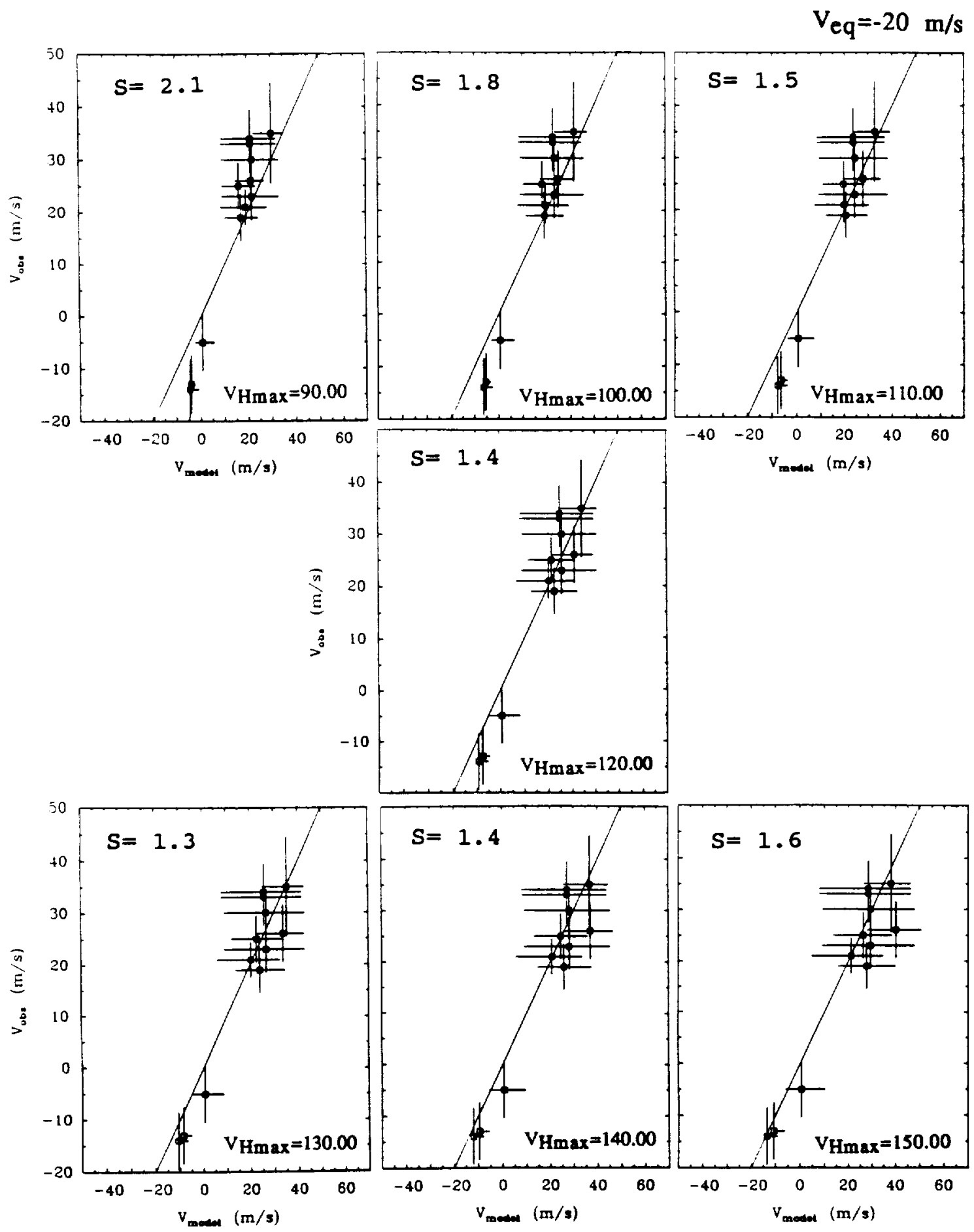

Figure $7.10 \mathrm{~d}$ 


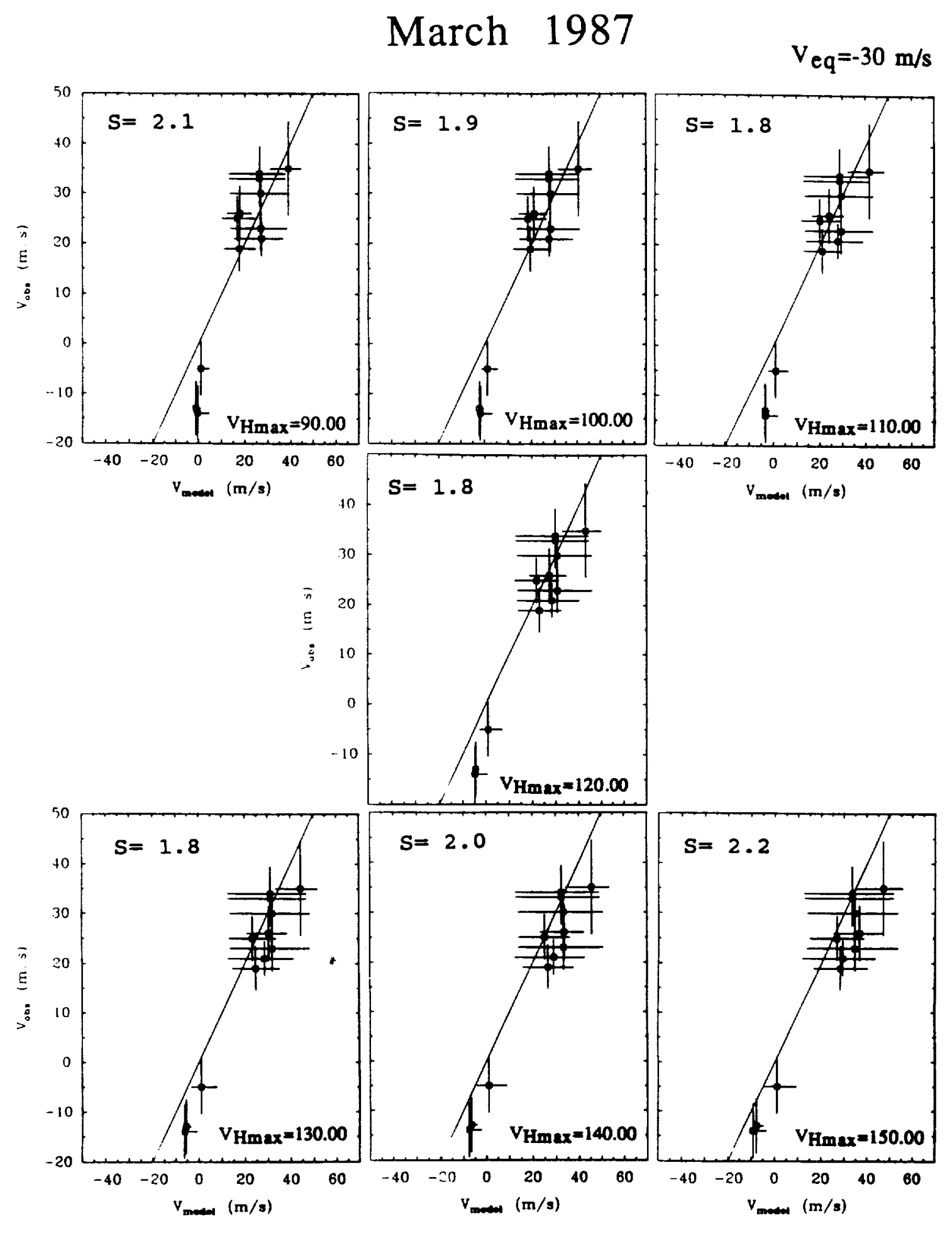

Figure $7.10 \mathrm{e}$ 


\section{Table 7.3. March 1987 Goodness-of-Fit Parameter S Over the Model Space}

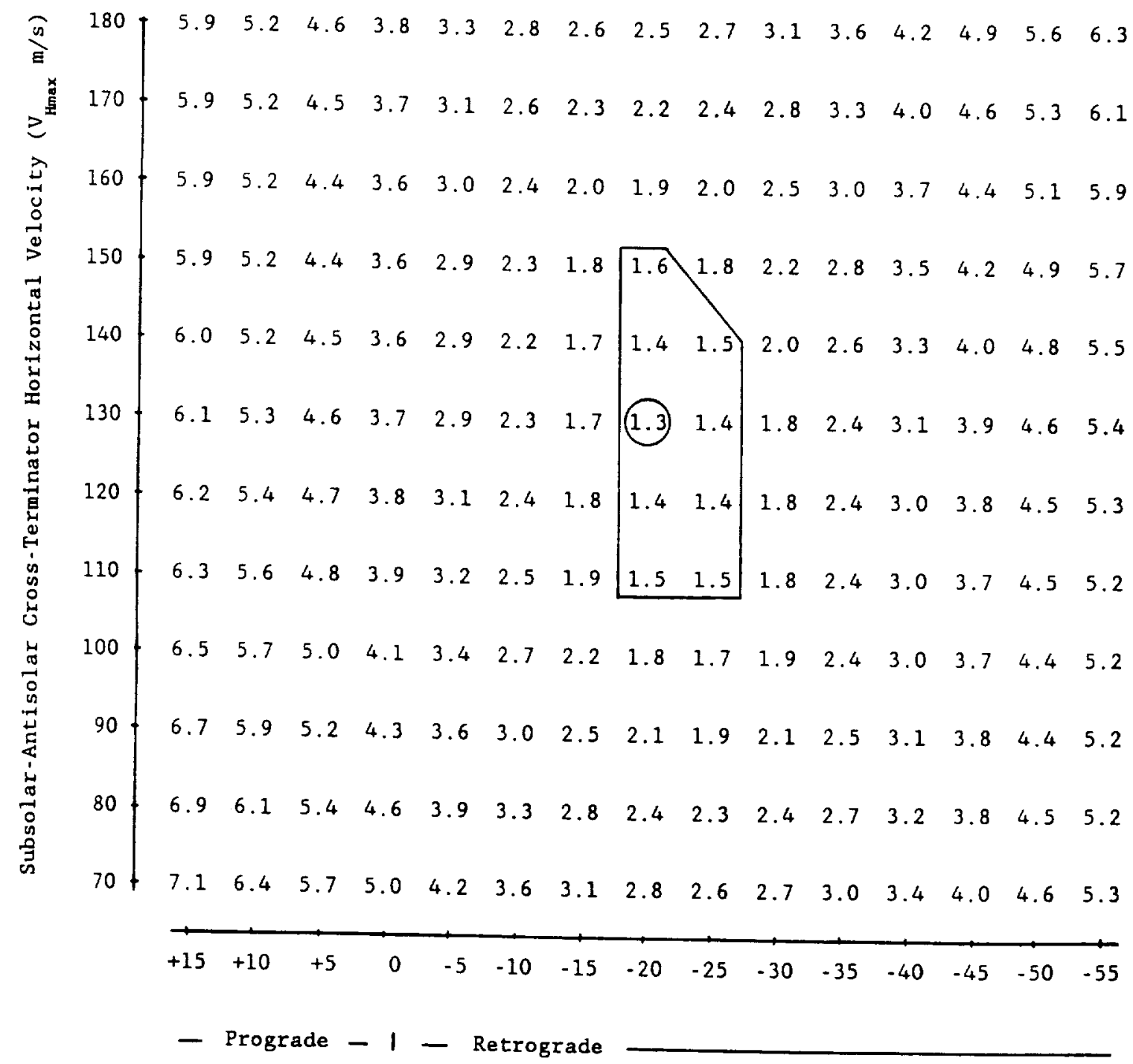

Zonal Equatorial Velocity $\left(V_{\bullet q} m / s\right)$ 
the model fits, on introduction of a zonal retrograde component, is evident through comparison of Figures $7.10 \mathrm{a}$ and $7.10 \mathrm{~d} . \mathrm{S}_{\min }=0.5$ when calculated in $\mathrm{V}_{\text {model }}$, nearly coincident with the Table 7.3 minimum, indicating probable $\mathrm{V}_{\text {model }}$ error bar overestimation of the pointing uncertainty random component.

June 1986 model fits are shown on Figures 7.11a-d. Table 7.4 provides $S$ over the June 1986 model space, with $\mathrm{S}_{\min }=3.4$. However, $S_{0}=1.5$, which renders the model fits unacceptable. Either the circulation model is inconsistent with the lower thermosphere (implying circulation departures from the other observing periods), or significant pointing uncertainty errors are present. Note, however, that quantitatively the June data is still best fit with a dominant subsolar-antisolar circulation as was determined through qualitative analysis in Chapter 6 .

To investigate possible alternative circulations, a more detailed look at the point by point dynamics is warranted.

The lower right plate in each of Figures $7.11 \mathrm{a}-\mathrm{d}$ indicates the beam positions associated with the data points (refer to Figure 5.4). On Figure 7.11a, points 6 and 7, virtually insensitive to a zonal component, are adequately fit for $\mathrm{V}_{\mathrm{Hmax}}<120 \mathrm{~m} / \mathrm{s}$. A BEAMINT model with $\mathrm{V}_{\text {Hmax }}>120 \mathrm{~m} / \mathrm{s}$ is therefore unreasonable regardless of zonal involvement. On Figure 7.11a, however, for $\mathrm{v}_{\mathrm{Hmax}}<120 \mathrm{~m} / \mathrm{s}$, points $4,5,9$, and the upper point 3 data, are too weakly redshifted. As well, point 8 is too strongly blueshifted.

Consider effects of the zonal component:

1. To enhance point 4 and 5 redshifts, and cancel the point 8 


\section{June 1986}

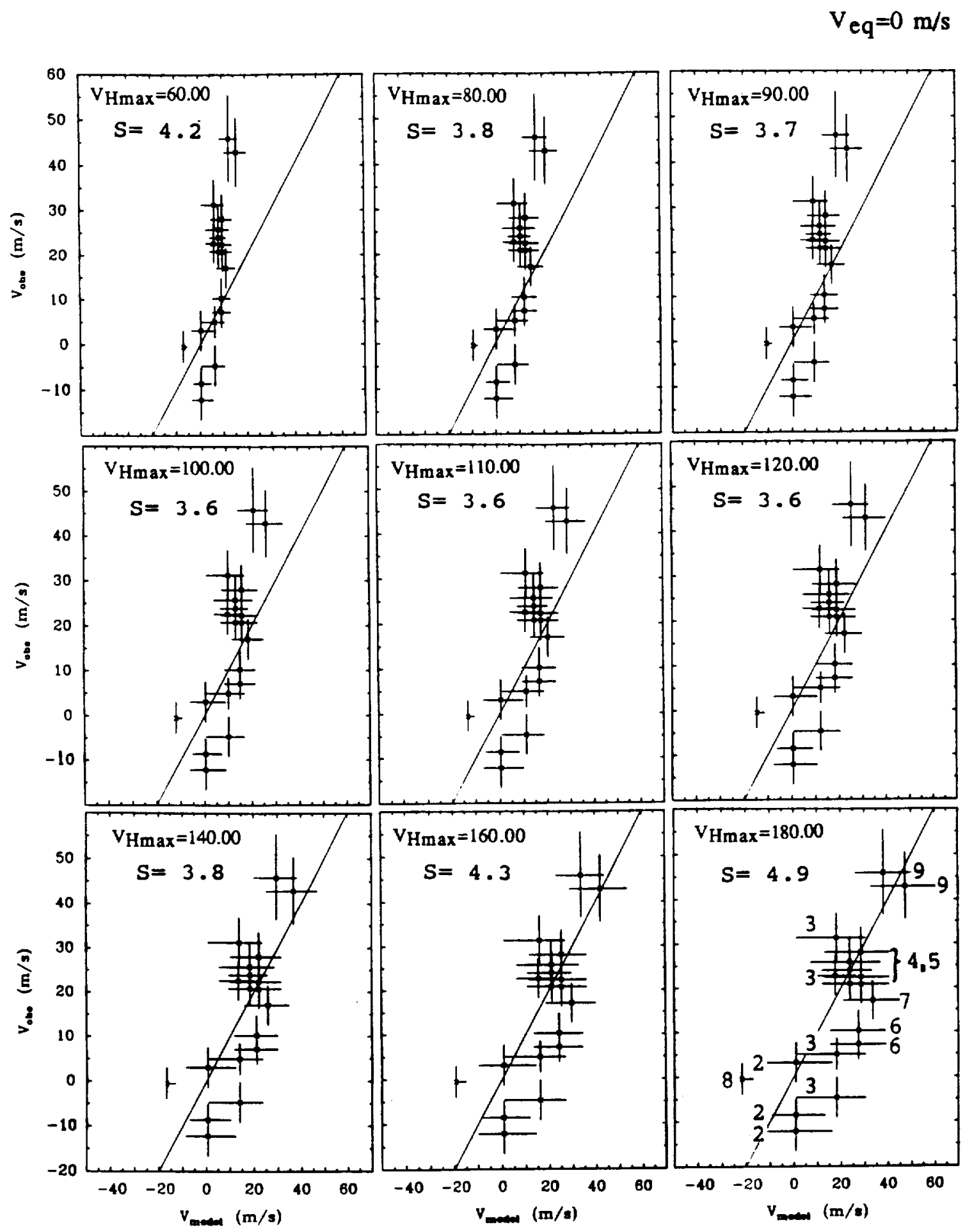

Figure $7.11 \mathrm{a}$ 


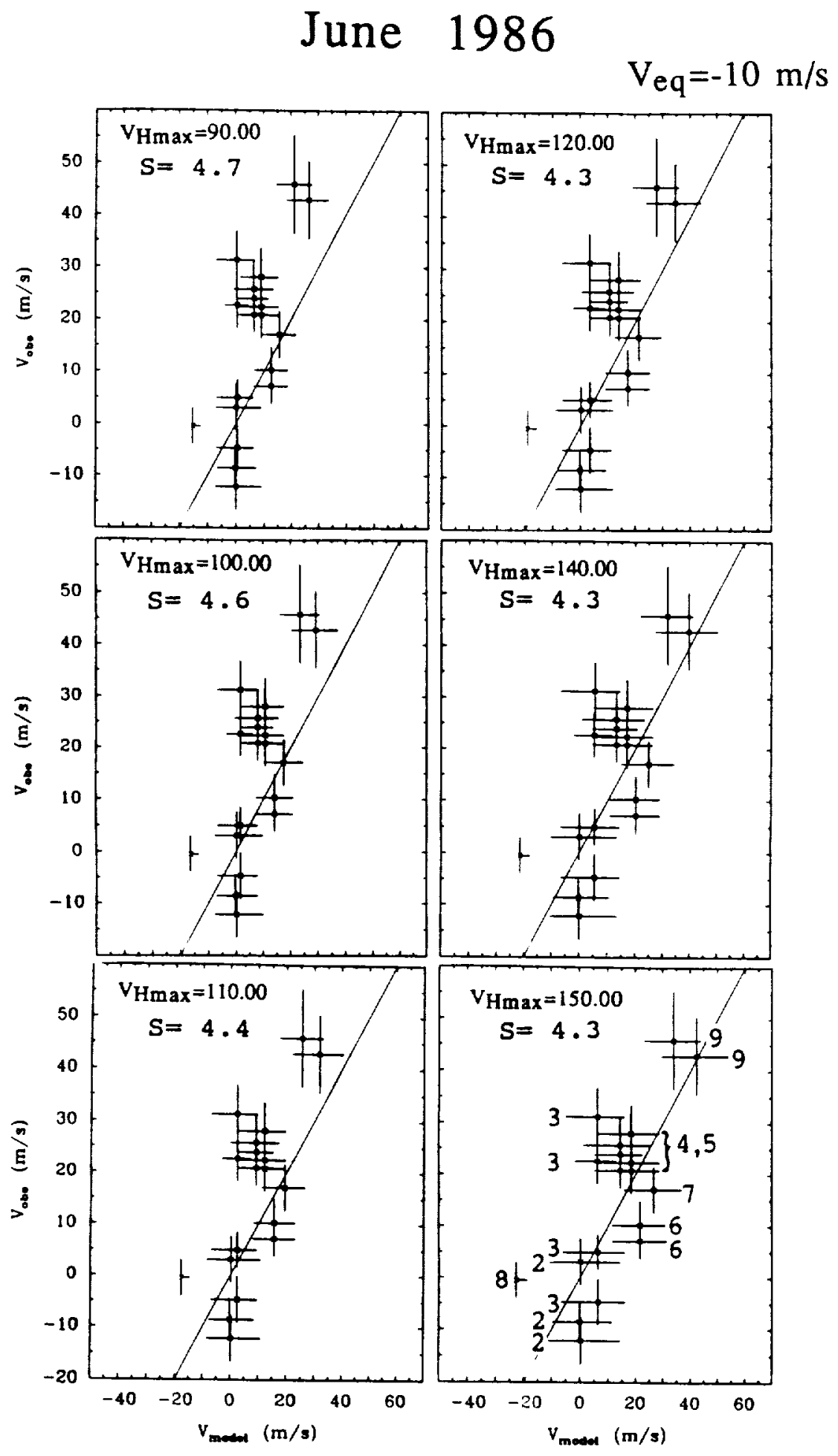

Figure $7.11 \mathrm{~b}$ 


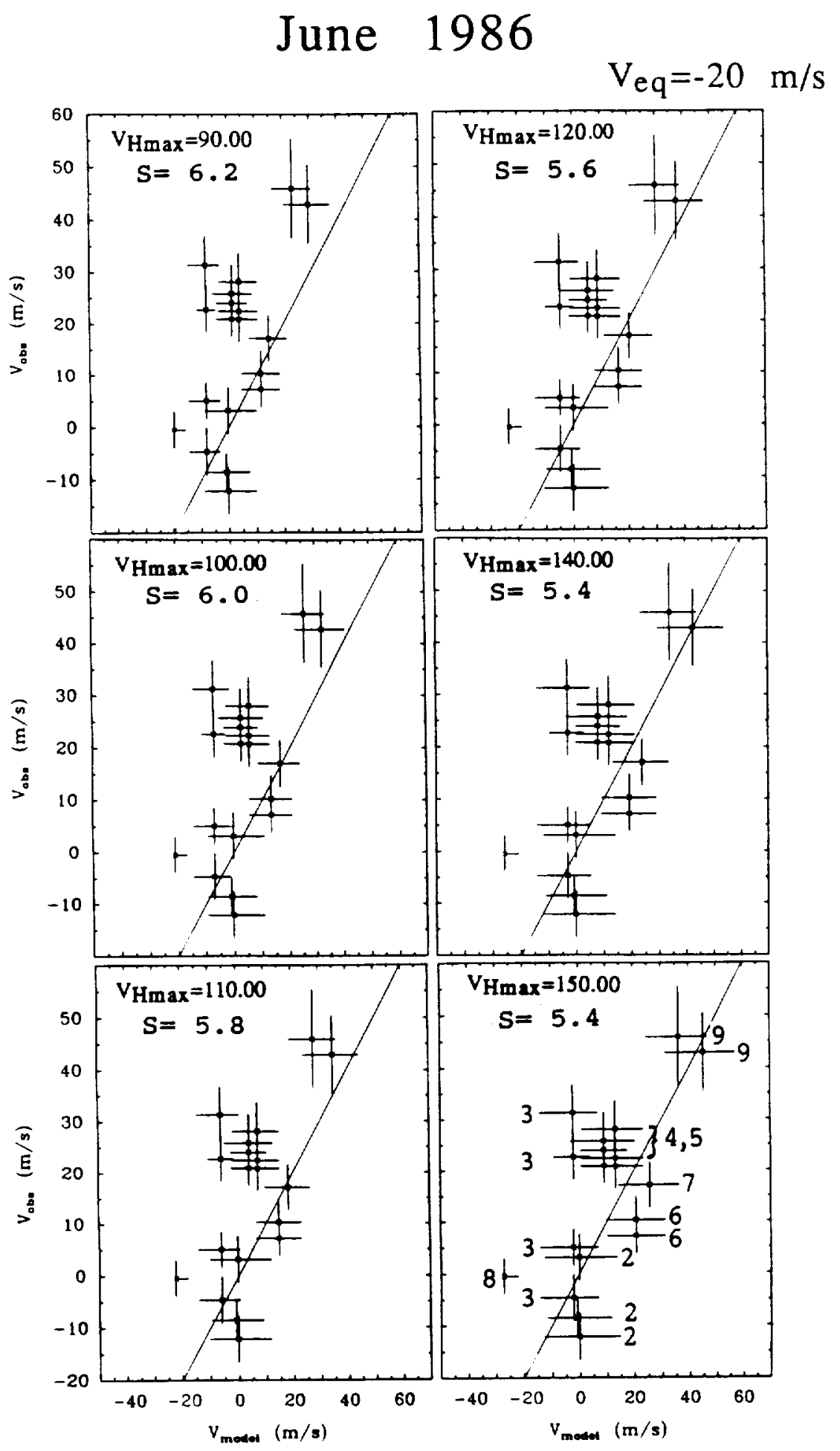

Figure $7.11 \mathrm{c}$ 
June 1986

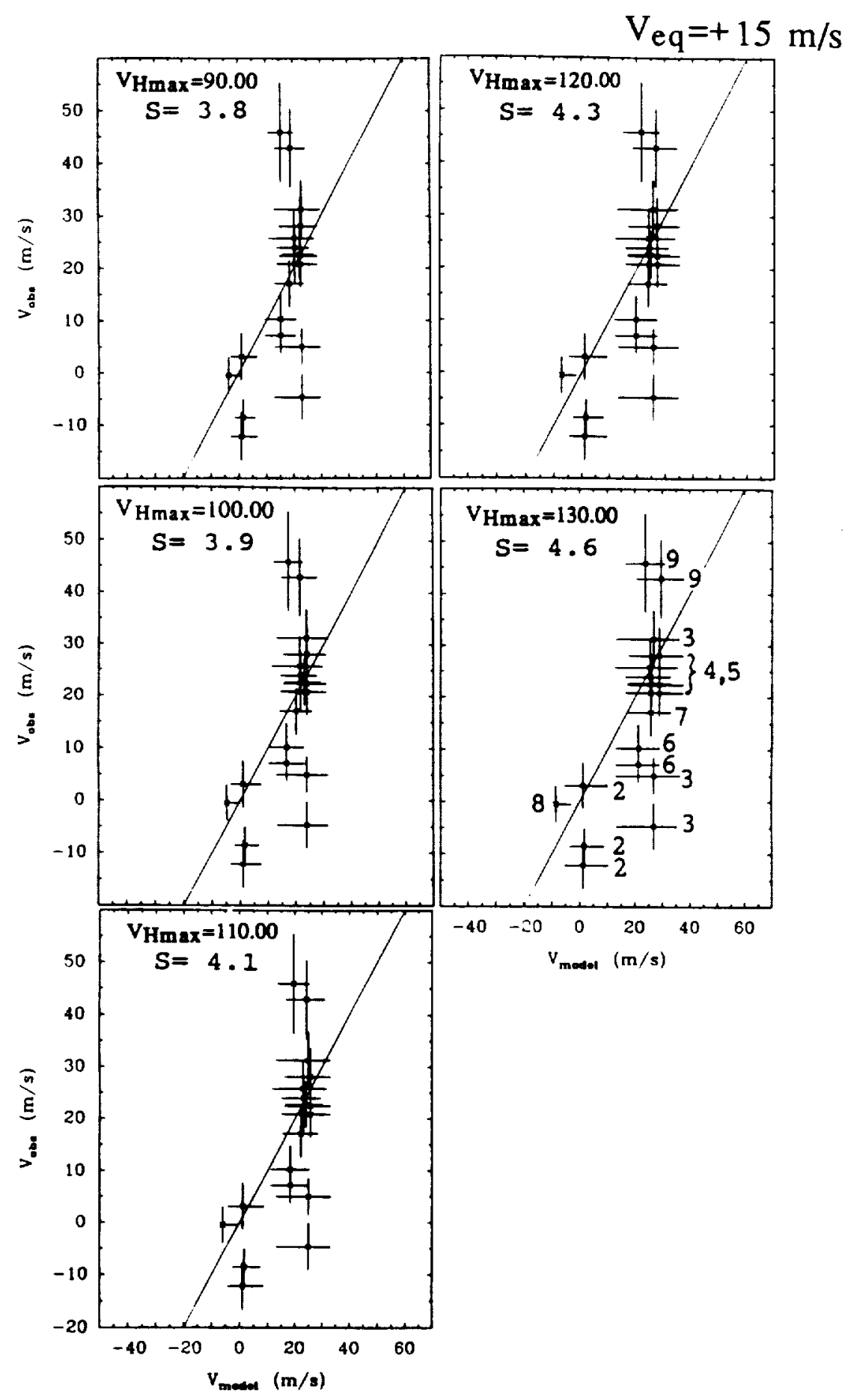

Figure $7.11 d$ 


\section{Table 7.4. June 1986 Goodness-of-Fit Parameter S Over the Model Space}

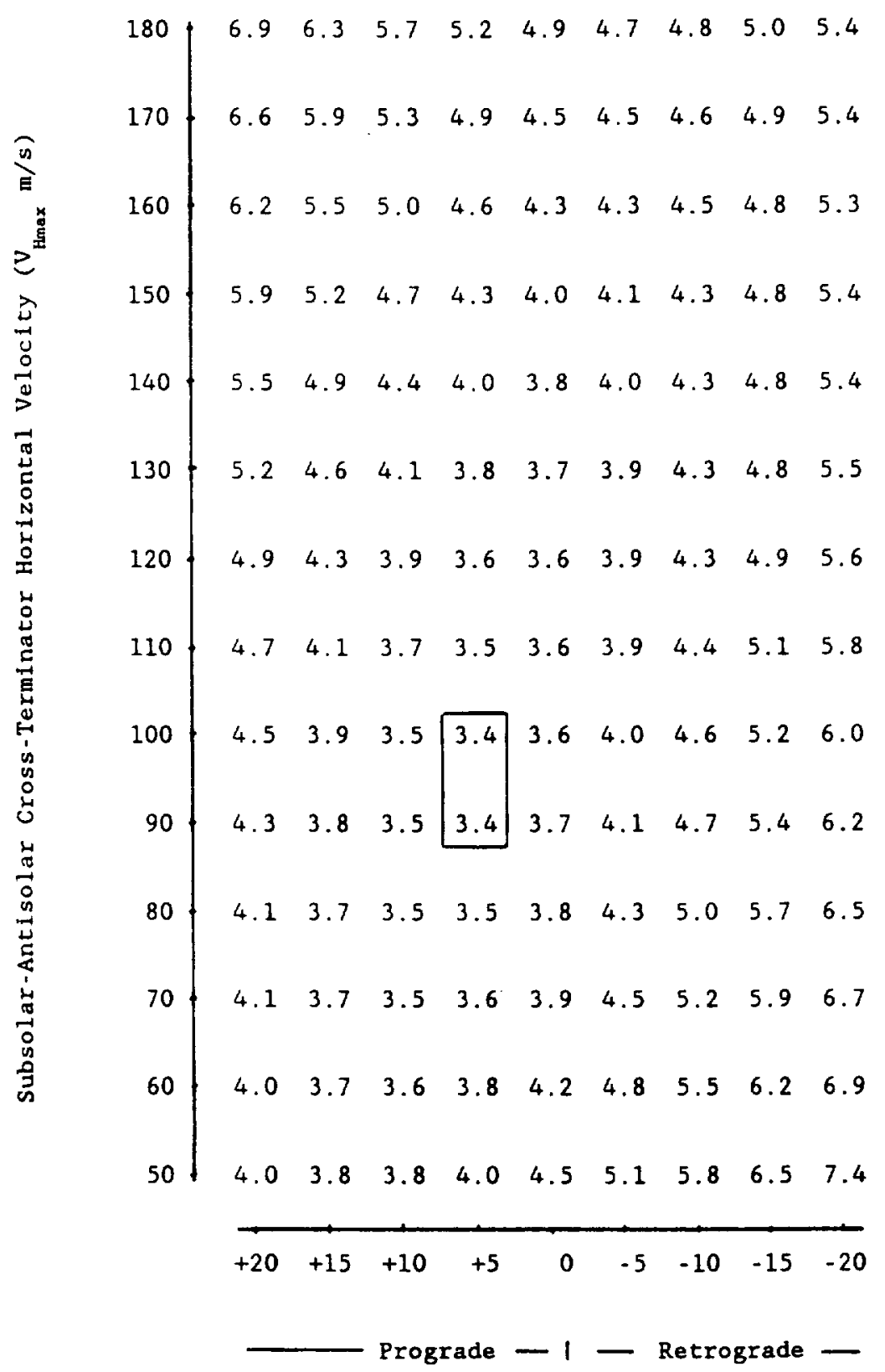

Zonal Equatorial Velocity (V $\mathrm{m} / \mathrm{s})$ 
blueshift, requires a small prograde zonal component. As shown on Figure $7.11 \mathrm{~d}$, points 4,5 , and 8 are indeed reasonably fit for $\mathrm{V}_{\mathrm{Hmax}}-<100 \mathrm{~m} / \mathrm{s}$ (as are points 6,7 ). However, point 9, possessing a weak redshift on Fig 7.11a, is further weakened by prograde rotation. In addition, point 2 (disk center) error bars have been so significantly reduced, that a non-zero beam-integrated wind velocity is no longer tolerated.

2. Adding a retrograde zonal component (Figs. $7.11 \mathrm{~b}, \mathrm{c}$ ) alleviates all problems caused by the prograde situation only to exacerbate problems a prograde rotation was to correct.

There are a number of possible changes to the BEAMINT circulation model:

1. Invoking a different latitudinal dependence in the zonal flow is not reasonable. The zonal " $\mathrm{fix}$ " for point 8 exacerbates problems with points 2 and 9 , yet all are at the same latitude.

2. A SS-AS circulation non-linear in SZA could be invoked such that horizontal velocity versus SZA on Figure 2.2 is concave upward. For smaller SZA, V $V_{\text {Hmax }}$ would be less than the BEAMINT-assumed case. For a SS-AS model with reasonably fitted points 6 and 7 , point 9 would have the requisite greater redshift, and point 8 a reduced blueshift. Yet this fix may not explain strong redshifting in points 3,4 , and 5 , and would create a well defined discontinuity in $\mathrm{V}_{\mathrm{Hmax}}$ at $\mathrm{SZA}=90^{\circ}$.

The apparent failure of reparameterized BEAMINT circulation models points toward significant June 1986 pointing uncertainty error, consistent with a possible systematic component introduced by 
IR/visible beam misalignment (see Section 7.1.1). This planetary

aspect should be redone at the IRTF.

Definitive BEAMINT best-fits are found for the December 1985, October 1986, and March 1987 data. Modelling for all four observing periods indicates a dominant SS-AS circulation, as was determined from qualitative analysis of beam-integrated winds.

Isolated planetary aspect geometries are limited discriminators of the global circulation. Assuming wind field temporal stability over the synodic period of the observations, the best fit model spaces for the individual observing periods may be overlapped. The sole model common to the December 1985, October 1986 and March 1987 best fit mode1 spaces is $V_{H \max }=120 \mathrm{~m} / \mathrm{s}$ and $V_{e q}=-25 \mathrm{~m} / \mathrm{s}$, consistent with the Bougher et al. (1986) $110 \mathrm{~km}$ model with a superimposed retrograde zonal component. 

CHAPTER 8: THE BETZ ET AL. HETERODYNE RESULTS - A REINTERPRETATION

\subsection{LASER STABILITY AND IMPLICATIONS}

The Betz et al. (1976, 1977b; Betz 1977, 1982) heterodyne wind measurements were made using a power peak stabilized laser local oscillator (LO). Short and long term frequency stability, in the absence of a stabilization system, were determined by beating the LO with a second laser. An observed beat frequency long term drift of 5 $\mathrm{MHz} /$ day $(50 \mathrm{~m} / \mathrm{s}$ per day at $10 \mu \mathrm{m})$ indicated the need for a stabilization system. (This, however, reflects only relative drift between lasers. Individual laser drift might be greater.)

The instantaneous position on the gain profile was found by sensing cavity impedance across a pickoff resistor (Betz 1977). A PZT-applied $1 \mathrm{KHz}$ dither provided $180 \mathrm{KHz} \mathrm{p}-\mathrm{p}$ modulation of laser output frequency. The impedance signal was fed to a lock-in amplifier, the latter triggered by a reference signal in phase with cavity modulation. The lock-in output thus provided a DC signal proportional to the slope of the gain profile. By beating the Lo with the second laser, the lock-in's output meter was calibrated in frequency departure from power peak.

Two basic differences exist between the Betz system and the GSFC power peak system (used for the laser stabilization tests outlined in Chapter 4):

1. The GSFC system employs a pyroelectric detector - found to be less noisy than a resistance pickoff.

2. The Betz system allowed only passive feedback - DC level corrections to the PZT were applied manually, whenever (in 
theory) a non-zero lock-in output signal became apparent. This allows the possibility of accidental frequency offset in the field due to either infrequent or overlooked PZT adjustment. Conversely, the GSFC system has an active servo feedback loop for continuous update of PZT biasing.

The Betz passive system was claimed to provide a laser output within $\pm 0.2 \mathrm{MHz}(2 \mathrm{~m} / \mathrm{s})$ of power peak, though no evidence for such stability was presented. By comparison, the GSFC ${ }^{13} \mathrm{C}^{16} \mathrm{O}_{2}$ power peak time series yielded best-case relative stability to $5 \mathrm{~m} / \mathrm{s}$ (see Section 4.8.2).

As stated in Chapter 4, there are inherent shortcomings associated with power peak stabilization. Betz (1977) used a saturated resonance (Lamb dip) cell to calibrate the power peak frequency offset (PPFO) absolutely with respect to the laser transition rest frequency.

Most important for interpretation of the Betz measurements, the PPFO was found to have minor dependence on laser gain cell pressure and major dependence on line competition within the cavity (Betz 1977). However, Betz interpreted both effects as constant for a given laser system, categorizable as a laser-specific pressure shift, which is quite erroneous. Summarizing relevant etalon characteristics (see Section 4.2):

1. The gain profile for a specific laser line will be measurably distorted if there is appreciable and simultaneous cavity support for additional laser transitions. The level of distortion is specific to the cavity, as well as critically dependant on the cavity alignment and the relative strengths of 
the involved laser lines.

2. Changes in cavity alignment result from long term thermal variation of the cavity rods, and adjustment of output coupler and diffraction grating verniers. SooHoo et al. (1985; SooHoo 1984) found diffraction grating tilt adjustment could shift the gain peak several $\mathrm{MHz}$ (tens of $\mathrm{m} / \mathrm{s}$ at $10 \mu \mathrm{m}$ ) to either side of the transition rest frequency. (GSFC LO vernier adjustments are made routinely in the field for LO performance optimization, predominantly during daily system power-up.)

3. The peak's wide frequency plateau, depending on $S / N$, can support a significant frequency offset before lock-in sensing of a non-zero slope.

The PPFO is therefore time variable, inextricably linked to inevitable changes in cavity alignment, and the laser output frequency may fluctuate about the PPFO-dictated power peak.

PPFO variability is strikingly evident in the GSFC power peak stability test (Fig. 4.26). A $1.2 \mathrm{MHz}(12 \mathrm{~m} / \mathrm{s})$ drift was observed over the first $40^{\mathrm{m}}$ of the $80^{\mathrm{m}}$ time series, with fluctuations characterized by a $0.5 \mathrm{MHz}(5 \mathrm{~m} / \mathrm{s}) 1 \sigma$ scatter for $2^{\mathrm{m}}$ integrations. A S/N-induced standard deviation of only $0.1 \mathrm{MHz}(1 \mathrm{~m} / \mathrm{s})$ indicated fluctuations were real. The Lo baseline frequency leveled off after -40 minutes, possibly reflecting post power-up thermal equilibration of the cavity. Clearly large and unpredictable (uncorrectable) frequency offsets can be associated with power peak stabilization.

For the November 1975 and April 1976 emission measurements, using 
${ }^{12} \mathrm{C}^{16} \mathrm{O}_{2} \mathrm{P}(16)$ at $948 \mathrm{~cm}^{-1}$, a $-50 \mathrm{kHz} /$ Torr redward PPFO was determined, or $0.7 \pm 0.1 \mathrm{MHz}(7 \pm 1 \mathrm{~m} / \mathrm{s})$ for a 14 Torr laser mix total pressure (Betz 1977). Yet, the validity of such a calibration over days seems unlikely. After wind measurement PPFO corrections, a total systematic error $<6 \mathrm{~m} / \mathrm{s}$ was claimed. This included PPFO correction uncertainty, as well as RFLO and ephemeris errors estimated to be $< \pm 2 \mathrm{~m} / \mathrm{s}$ (Betz et a1. 1976).

The October 1976 emission data (again using $948 \mathrm{~cm}^{-1} \mathrm{P}(16)$ ) lacked Lamb dip calibration of the LO, yet a maximum $10 \mathrm{~m} / \mathrm{s}$ systematic offset was determined (Betz 1977). LO calibration for the April/May 1977 emission and absorption measurements is not discussed (see Betz et a1. 1977b).

Other more subtle sources of systematic error may be present in the Betz data set. Earth rotation and Venus/Earth barycentric translation-induced Doppler shifts produce a continuous line-frequency drift during data acquisition. To remove such differential Doppler shifts, the radio frequency local oscillator (RFLO) was periodically varied in the field to keep the spectral line centered in the 50-250 MHz filter bank bandpass (Betz 1977). Details of how often the RFLO was repositioned are not presented. Yet the critical need for frequent RFLO repositioning is apparent from Equation A1.26, which yields an Earth rotation-induced Doppler shift of up to $102 \mathrm{~m} / \mathrm{s}$ per hr $(10.2$ $\mathrm{MHz} / \mathrm{hr}$ ) for the Betz observational circumstances: $31^{\circ} 57.8^{\prime}$ latitude, early May 1977 at meridian transit, and $5.5^{\circ}$ planetary declination. In addition, no correction for terrestrial absorption was made during data reduction, possibly introducing a non-negligible baseline 
slope to the target spectral feature (hence a centroid shift; see Appendix A1). Near superior and inferior conjunctions, two of the Betz observing periods, the Doppler shift between the spectral feature and LO is minimal, the situation characterized by maximal baseline distortion.

Finally, there is no mention of observing period-associated pointing uncertainty or atmospheric seeing, both important for interpretation of beam-integrated wind velocity.

The means by which systematic errors may have been introduced at the tens of $\mathrm{m} / \mathrm{s}$ level seem apparent. Such errors are consistent with dynamically unreasonable disk-center vertical wind velocities obtained at both superior (Betz 1977) and inferior (Betz et al. 1977b) conjunction. The observed vertical winds are a good indicator of LO calibration and stability, as a host of dynamical measurements and theoretical considerations indicate a maximum vertical wind field component of only a few $\mathrm{m} / \mathrm{s}$ (see Chapters 1 and 2).

\subsection{MESOSPHERIC WINDS}

The only direct mesospheric wind measurements, to date, were made by Betz et al. (1977b; Betz 1977, 1982) just after the 1977 inferior conjunction, using an IR heterodyne spectrometer mated to the McMath Solar Telescope $81-\mathrm{cm}$ (west) auxiliary. An $11 \mu \mathrm{m}{ }^{13} \mathrm{C}^{16} \mathrm{O}_{2}$ absorption line (892 $\mathrm{cm}^{-1} \mathrm{P}(26)$ ) was used as the dynamical probe. The line contribution function indicated line formation at 75 (Betz et al. $1977 \mathrm{~b}$ ) or $85 \mathrm{~km}$ (Betz 1982) altitude, for the region within $\pm 200 \mathrm{MHz}$ of line center (the frequency bandwidth used for centroid determination). 
Winds were monitored at three points along the equator (eastern limb, western $1 \mathrm{imb}$ and disk-center), April 17 to May 8 , from $17.3^{\circ}$ to $37^{\circ}$ western elongation. A $<6 \mathrm{~m} / \mathrm{s}$ systematic error was claimed, along with a $\mathrm{S} / \mathrm{N}$-induced $\sim \pm 25 \mathrm{~m} / \mathrm{s}$ statistical uncertainty for a $30^{\mathrm{m}}$ integration. A pressure shift in the data, due to 1 ine formation at $-20 \mathrm{mb}$ in the Venusian atmosphere (slightly less near the limbs), was removed assuming a $1 \mathrm{~m} / \mathrm{s}$ per mb redward shift as measured by Freed (1976). The wind velocity zero point was thereby shifted 15 to $20 \mathrm{~m} / \mathrm{s}$.

Betz viewed this favorably as it symmetrized the magnitude of the east-1imb recession and west-1imb approach. A $90 \pm 7 \mathrm{~m} / \mathrm{s}$ mean zonal wind was indicated - which is consistent with cyclostrophic balance model predictions. Unfortunately, the pressure shift correction created a serious dynamical problem concerning the disk-center winds - which must necessarily represent vertical velocities. The correction implied mean vertical upwelling at $45 \mathrm{~m} / \mathrm{s}$, which was attributed to dynamics at the antisolar point (Betz et al. 1977b). In addition, Betz reported up to $-40 \mathrm{~m} / \mathrm{s}$ "real" fluctuations about the mean at all three beam locations.

Figures $8.1 \mathrm{a}, \mathrm{b}$ and $c$ provide the GSFC-generated Venusian aspect geometries for April 17, May 1 and May 8, respectively. Based on planetary declination, $P(26)$ wavelength and telescope aperture, the approximate diffraction-limited elliptical beam ( 3.3 arc-second HPBW in R.A., -4.6 arc-second HPBW in dec.) has been located on the Figures at the probable beam positions, providing a seeing-excluded disk-to-beam ratio of 12.3 to 16.8 (in R.A.).

Inspection of Figures 8.1a-c shows that the Betz et al. (1977b) claimed disk-center sampling of the antisolar region is clearly 


\section{VENUS 17 APR 1977 0: O UT}

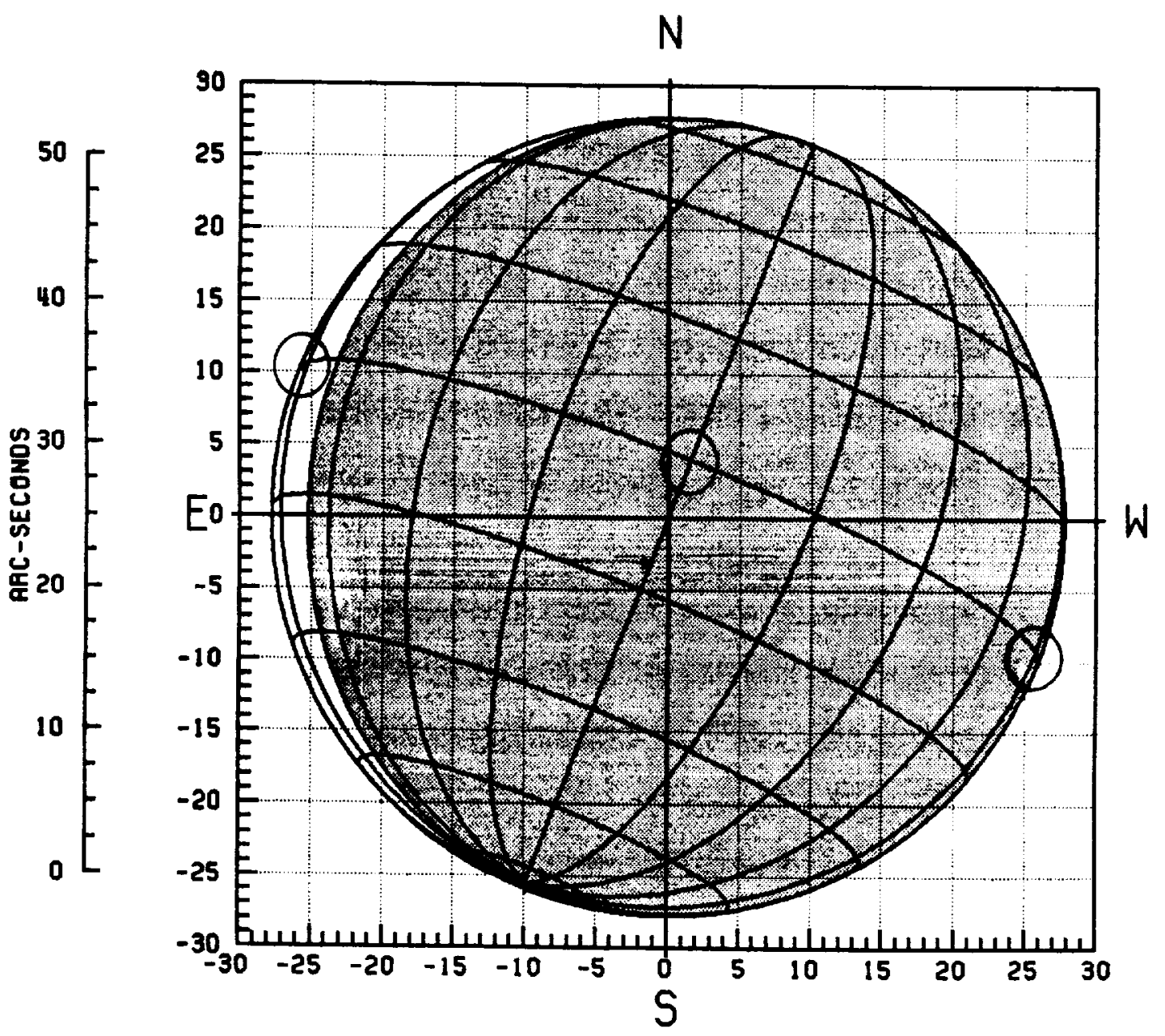

Figure $8.1 \mathrm{a}$ 


\section{VENUS 1 MAY 1977 O: O UT}

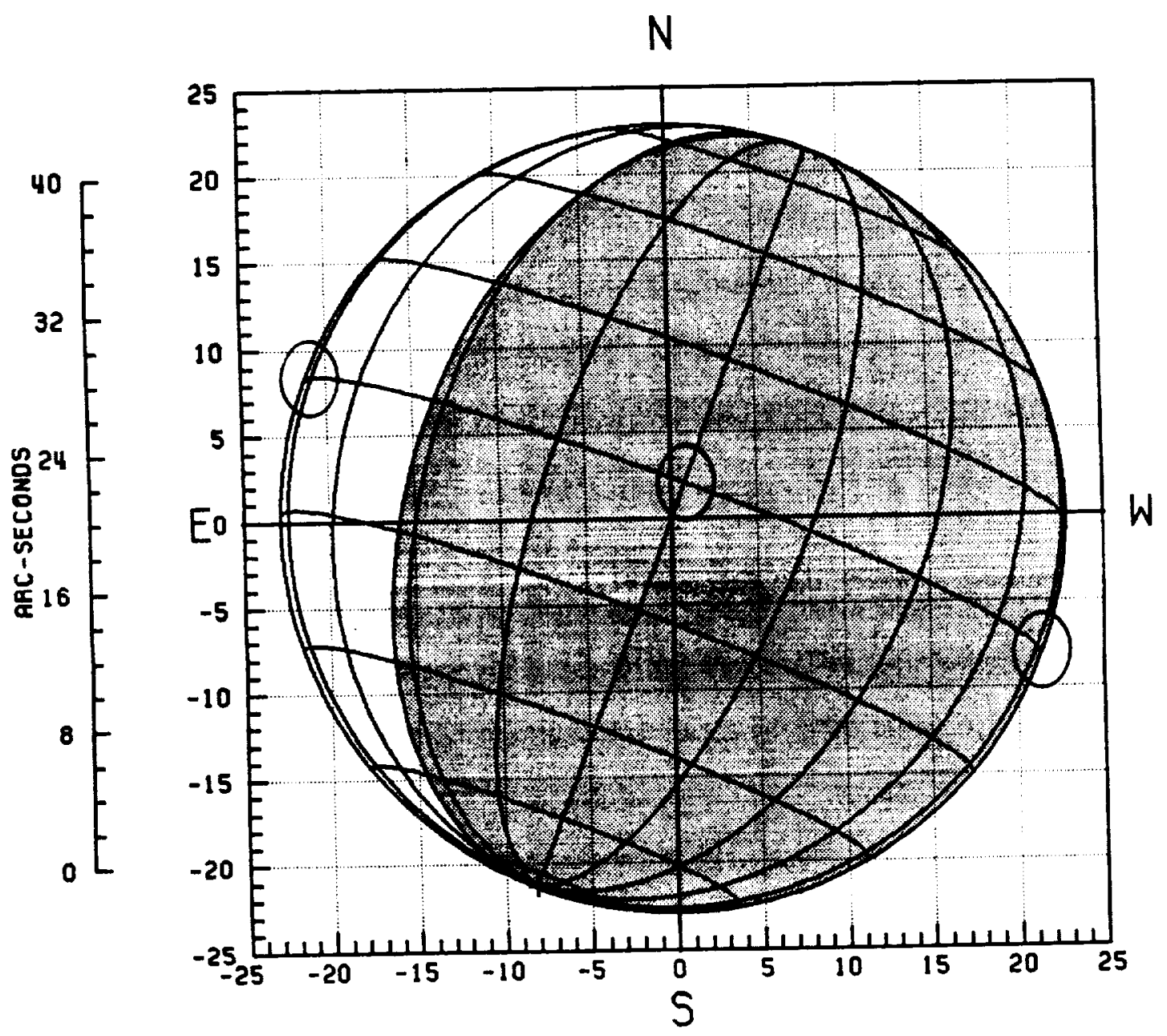

Figure $8.1 \mathrm{~b}$ 


\section{$\begin{array}{llllll}\text { VENUS } & 8 & \text { MAY } 1977 & 0: & 0 & \text { UT }\end{array}$}

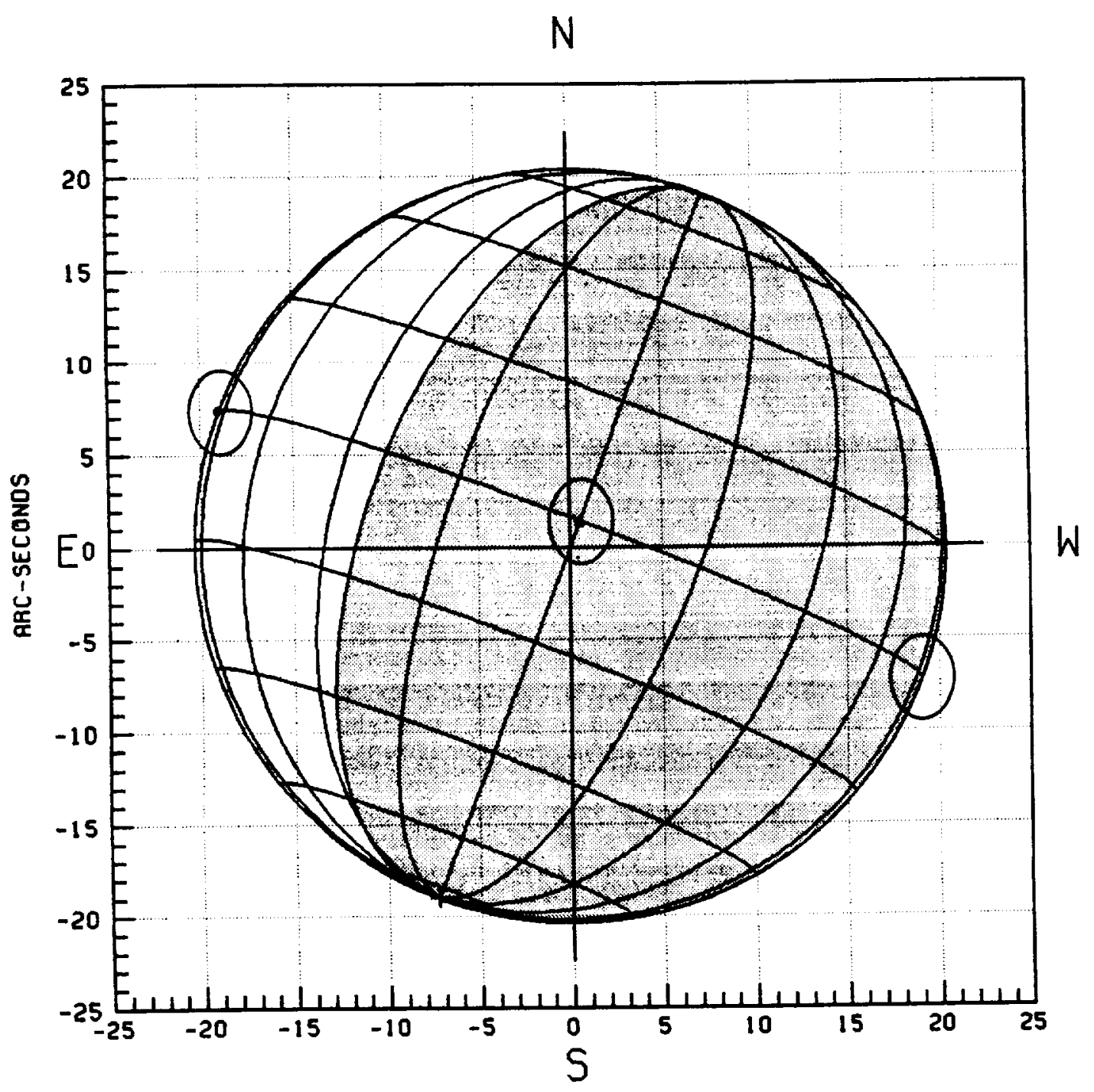

Figure $8.1 \mathrm{c}$ 
incorrect. Due to changing planetary aspect, the disk-center beam probed a $33.5^{\circ}$ longitudinal swath from $23.5^{\circ}$ (April 17) to $57^{\circ}$ longitude (May 8), as referenced to the antisolar meridian (assuming a beam at the subearth meridian/equator intersection). The strong vertical winds cannot be attributed to antisolar point-specific dynamics.

Based on the stability considerations discussed, the Betz mesospheric winds can be reinterpreted in a dynamically reasonable way. Table 8.1 lists the wind velocities determined for the 14 days of data acquisition during the observing period, along with their S/N-limited 1 $\sigma$ errors. These values were obtained from Figure 2 in Betz et al. (1977b), and are probably good to $\pm 2 \mathrm{~m} / \mathrm{s}$ (barring any Figure distortion). The data, essentially three time series of 3 weeks duration, yield mean ( $\sigma^{2}$-weighted) line of sight velocities of $+102.8 \pm 7$ $\mathrm{m} / \mathrm{s},-27.7 \pm 5 \mathrm{~m} / \mathrm{s}$, and $-76.8 \pm 6 \mathrm{~m} / \mathrm{s}$ for the east limb, disk-center and west $1 \mathrm{imb}$, respectively (positive denotes recession). Correction for a $17 \mathrm{mb}$ induced pressure shift (assuming $100 \mathrm{KHz} / \mathrm{mb}$; Freed 1976) produces $\sigma^{2}$-weighted means of $+85.8 \pm 7 \mathrm{~m} / \mathrm{s},-44.7 \pm 5 \mathrm{~m} / \mathrm{s}$ and $-93.8 \pm 6 \mathrm{~m} / \mathrm{s}$, respectively, consistent with the Betz results.

If wind-field vertical velocities are indeed nearly zero, the mean wind velocity at disk-center may be interpreted as LO miscalibration, as already outlined. If the disk-center $\sigma^{2}$-weighted mean is defined as the zero level, the new $\sigma^{2}$-weighted means for the east and west limbs are $+130.5 \mathrm{~m} / \mathrm{s}$ and $-49.1 \mathrm{~m} / \mathrm{s}$, respectively.

With subsolar-antisolar flow established as the dominant circulation at $109 \mathrm{~km}$ altitude, it is not unreasonable to assume a 
1977 Date East Limb $(\mathrm{m} / \mathrm{s})$ Center $(\mathrm{m} / \mathrm{s})$ West Limb $(\mathrm{m} / \mathrm{s})$

$\begin{array}{cccc}\text { April } 17 & +128.2 \pm 35.2 & +33.8 \pm 32.4 & -74.7 \pm 33.8 \\ 20 & +118.3 \pm 23.2 & +7.0 \pm 19.7 & -36.6 \pm 18.3 \\ 21 & +107.0 \pm 23.2 & -14.1 \pm 18.3 & -88.7 \pm 18.3 \\ 25 & +84.5 \pm 28.2 & -26.8 \pm 18.3 & -70.4 \pm 26.8 \\ 26 & +69.0 \pm 21.1 & +14.1 \pm 18.3 & -49.3 \pm 15.5 \\ 28 & +112.7 \pm 21.1 & -57.7 \pm 14.8 & -94.4 \pm 18.3 \\ 29 & +94.4 \pm 23.9 & +5.6 \pm 22.5 & -98.6 \pm 16.9 \\ 30 & +85.9 \pm 32.4 & -70.4 \pm 24.6 & -91.6 \pm 31.0 \\ 1 & +52.1 \pm 21.1 & -26.8 \pm 29.6 & -36.6 \pm 28.2 \\ 2 & +147.9 \pm 28.2 & -28.2 \pm 30.3 & -94.4 \pm 43.0 \\ 4 & +119.7 \pm 38.7 & -35.2 \pm 19.7 & -49.3 \pm 30.3 \\ 5 & +80.3 \pm 28.9 & -14.1 \pm 17.6 & -73.2 \pm 26.1 \\ 7 & +176.1 \pm 27.5 & -39.4 \pm 18.3 & -156.3 \pm 28.9 \\ 8 & +136.6 \pm 35.9 & -83.1 \pm 18.3 & -150.7 \pm 40.1\end{array}$

Average: $+102.8 \pm 7 \mathrm{~m} / \mathrm{s} \quad-27.7 \pm 5 \mathrm{~m} / \mathrm{s} \quad-76.8 \pm 6 \mathrm{~m} / \mathrm{s}$

Table 8.1: Mesospheric wind time series of ${ }^{13} \mathrm{C}^{16} \mathrm{O}_{2} 892 \mathrm{~cm}^{-1}$ $P(26)$, as measured from Figure 2 Betz et al. (1977b). Positive denotes recession. Winds do not reflect corrections for the pressure shift at the $-20 \mathrm{mb}$ line-forming altitude. Averages are $\sigma^{2}$-weighted. 
superposition of zonal and subsolar-antisolar flow in the $70-100 \mathrm{~km}$ transition zone. While the new east-and west-limb means are not interpretable in this manner, they are interpretable as a superposition of a $90 \mathrm{~m} / \mathrm{s}$ zonal super-rotation and a $40 \mathrm{~m} / \mathrm{s}$ antisolar-to-subsolar return flow.

Figure 8.2 shows the recalibrated mesospheric time series. The dotted lines are the $\sigma^{2}$-weighted means. Day-to-day large-scale fluctuations may reflect variation in the PPFO, as indicated by the disk-center vertical winds. A disk-center $32 \mathrm{~m} / \mathrm{s} 1 \sigma$ scatter about zero velocity is 508 greater than the typical $22 \mathrm{~m} / \mathrm{s} \mathrm{S} / \mathrm{N}$-induced standard deviation per measurement. The scatter is therefore plausibly real, interpretable as variation in line of sight wind velocity, or equivalently, a day-to-day variation in PPFO. The latter is most likely, however, as the implied magnitude of vertical wind variation is, again, unreasonable.

Speculating further, the disk-center wind velocities given on Figure 8.2 are consistent with saturated resonance calibration of the LO at the beginning of the observing run. The Betz claim of a $-45 \mathrm{~m} / \mathrm{s}$ higher zero point (thus a $\sigma^{2}$-weighted disk-center mean vertical wind velocity of $\sim-45 \mathrm{~m} / \mathrm{s}$ ) would indeed place the initial disk-center measurements close to zero. PPFO drift (see Fig. 8.2) might then be responsible for dragging the $\sigma^{2}$-weighted disk-center average down to $-45 \mathrm{~m} / \mathrm{s}$.

A better time series recalibration fixes each disk-center point at the zero level. Day-to-day PPFO variation is thereby removed, provided the new limb measurement standard deviations are appropriately 


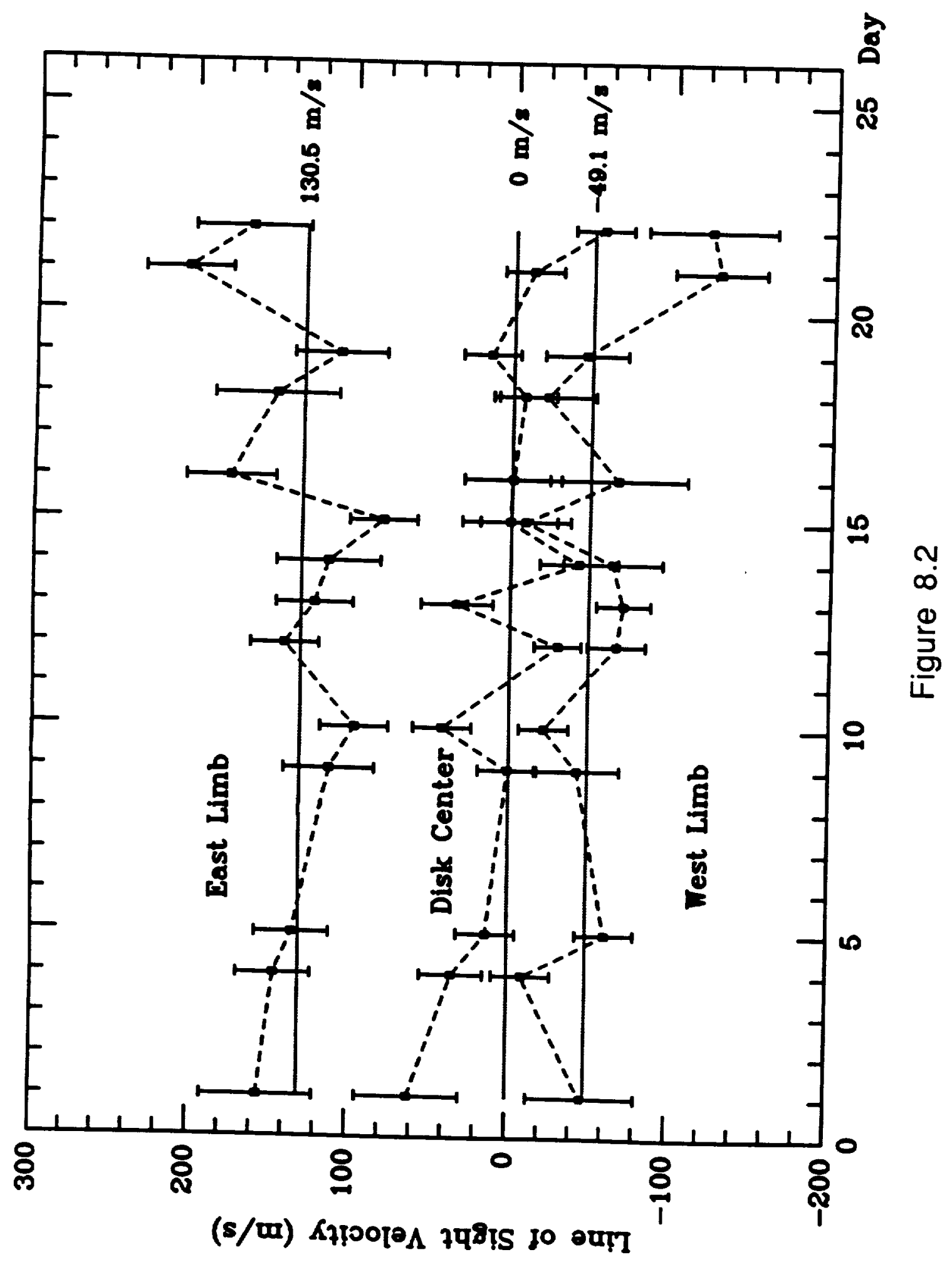


scaled. Fixing the disk-center measurements at zero is equivalent to plotting the limb/disk-center differences. The standard deviations associated with the latter are therefore obtained by adding (in quadrature) the limb and disk-center standard deviations. Figure 8.3 depicts this situation. The $\sigma^{2}$-weighted means (dotted lines) are $+128.7 \pm 9.1 \mathrm{~m} / \mathrm{s}$ and $-59.2 \pm 8.5 \mathrm{~m} / \mathrm{s}$ for the east-and west-1imb/diskcenter differences respectively. This corresponds to superposition of a $94.0 \pm 6.2 \mathrm{~m} / \mathrm{s}$ zonal circulation, and a $34.8 \pm 6.2 \mathrm{~m} / \mathrm{s}$ antisolar-tosubsolar return branch. The dynamical implications of such a strong return flow are addressed in Section 8.3 .

It should be noted in passing that, from day 12 to day 19, the east-limb/disk-center differences weakly hint at a $-2^{d}$ periodicity. A $94 \pm 6 \mathrm{~m} / \mathrm{s}$ equatorial zonal component is equivalent to a $4.7 \pm .3^{\mathrm{d}}$ period. A wavenumber-2 planetary-scale wave drifting slowly with respect to the atmospheric bulk motion (see Chapters 1 and 2 ) could produce a $-2^{d}$ periodicity in wind velocity. The periodicity, however, is not evident in the west-limb/disk-center differences. It is also not evident in the data prior to recalibration, as pointed out by Betz (1982).

If the antisolar-subsolar return flow is real, one might expect maximum wind velocities across the terminator. During the course of the time series, limb beam positions sampled regions progressively farther from the terminator (see Figures 8.1a-c), hence should have observed a diminishing antisolar-to-subsolar component. However, an expected negative slope in the limb/disk-center differences (Fig. 8.3) is not indicated.

A significant flaw in the reasoning thus far makes 


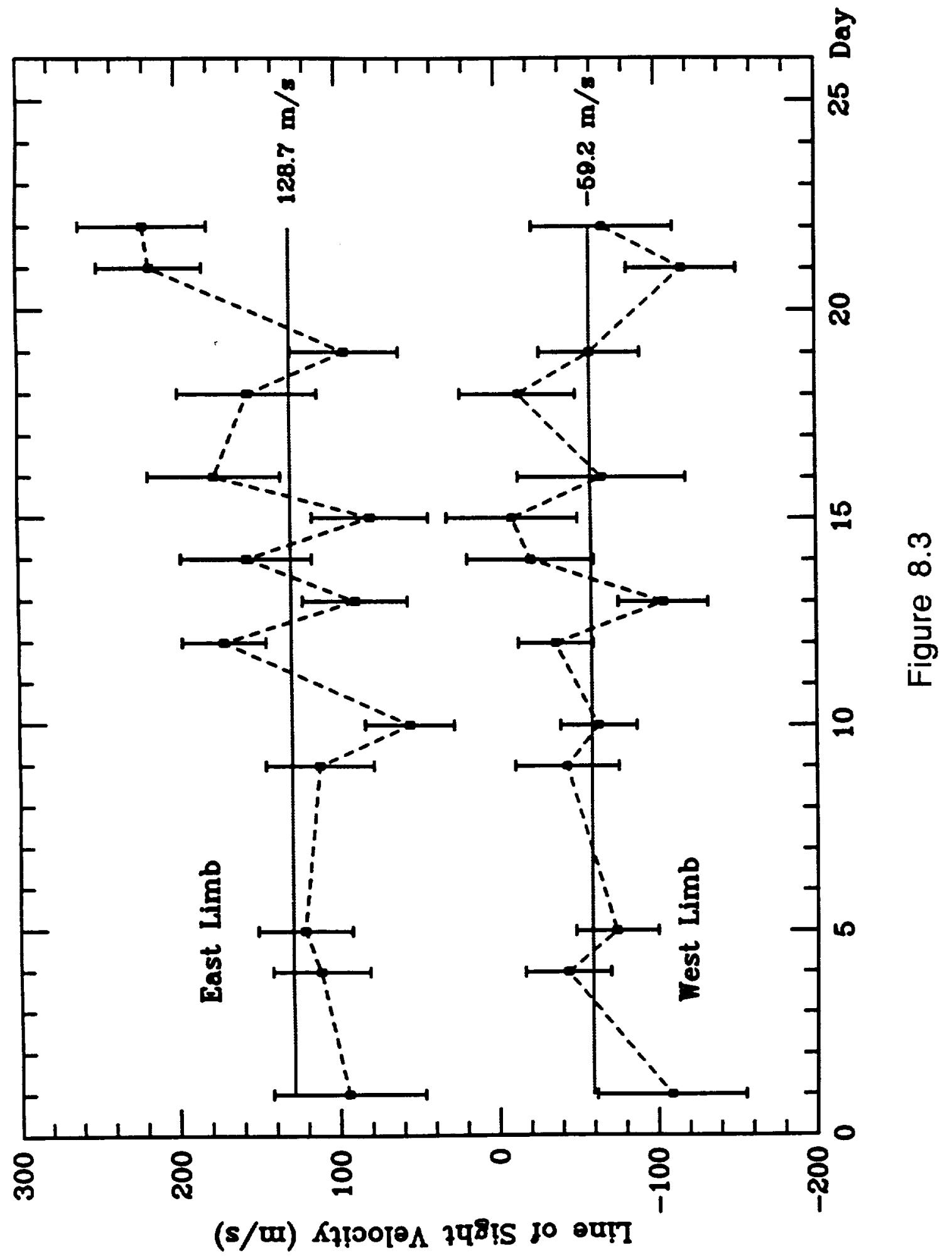


reinterpretation of the Betz data even more speculative. It has been assumed relative Doppler shifts between limb and disk-center, on a given date, are purely wind-induced - thus PPFO variation, on a given date, is negligible. This may be quite incorrect, yet day-to-day Lo optimization adjustments can cause dramatic PPFO variation, while a day's continuous operation, with an undisturbed laser, should not. While the GSFC short duration $\left(80^{\mathrm{m}}\right)$ power peak time series did show a PPFO baseline drift, an equilibration may have taken place after $-35^{\mathrm{m}}$. A many-hour-constant PPFO for an undisturbed power peak stabilized laser (barring significant misalignment through thermal variation of the cavity rods), seems reasonable, and has been observed at the -10 $\mathrm{m} / \mathrm{s}$ level (Deming et al. 1986).

Based on a more recent model atmosphere (for $0^{\circ}-30^{\circ}$ latitude; Seiff 1983), the ${ }^{13} \mathrm{C}^{16} \mathrm{O}_{2} 892 \mathrm{~cm}^{-1} \mathrm{P}(16)$ contribution function was generated by F. Espenak at GSFC and is shown on Figure 8.4. The altitude zero level is at $64 \mathrm{~km}$. Line formation $( \pm 200 \mathrm{MHz}$ from line center) occurs in the 69-79 $\mathrm{km}$ altitude range (40-6 mb). The best experimental determination of the $\mathrm{CO}_{2}-\mathrm{CO}_{2}$ pressure shift, $80 \pm 50$ $\mathrm{KHz} /$ Torr $(0.6 \pm 0.38 \mathrm{~m} / \mathrm{s}$ per $\mathrm{mb})$ redward, for pressures greater than 1 Torr (SooHoo 1985), yields a total pressure shift of $15 \pm 9.5 \mathrm{~m} / \mathrm{s}$ in the center of the line forming region $(25 \mathrm{mb})$. This is consistent with Betz's pressure shift correction.

Winds at both poles were also intermittently monitored (Betz et a1. 1977b), but no data or $\mathrm{S} / \mathrm{N}$ estimates were presented. Nothing beyond a short qualitative discussion of polar wind behavior was given, precluding any serious consideration here. 
CO2 (636) 1 P 26 $\quad W N=891.573944 \quad \mathrm{CM}-1$

TEMPERATURE PROFILE AND CONTRIBUTION FUNCTIONS

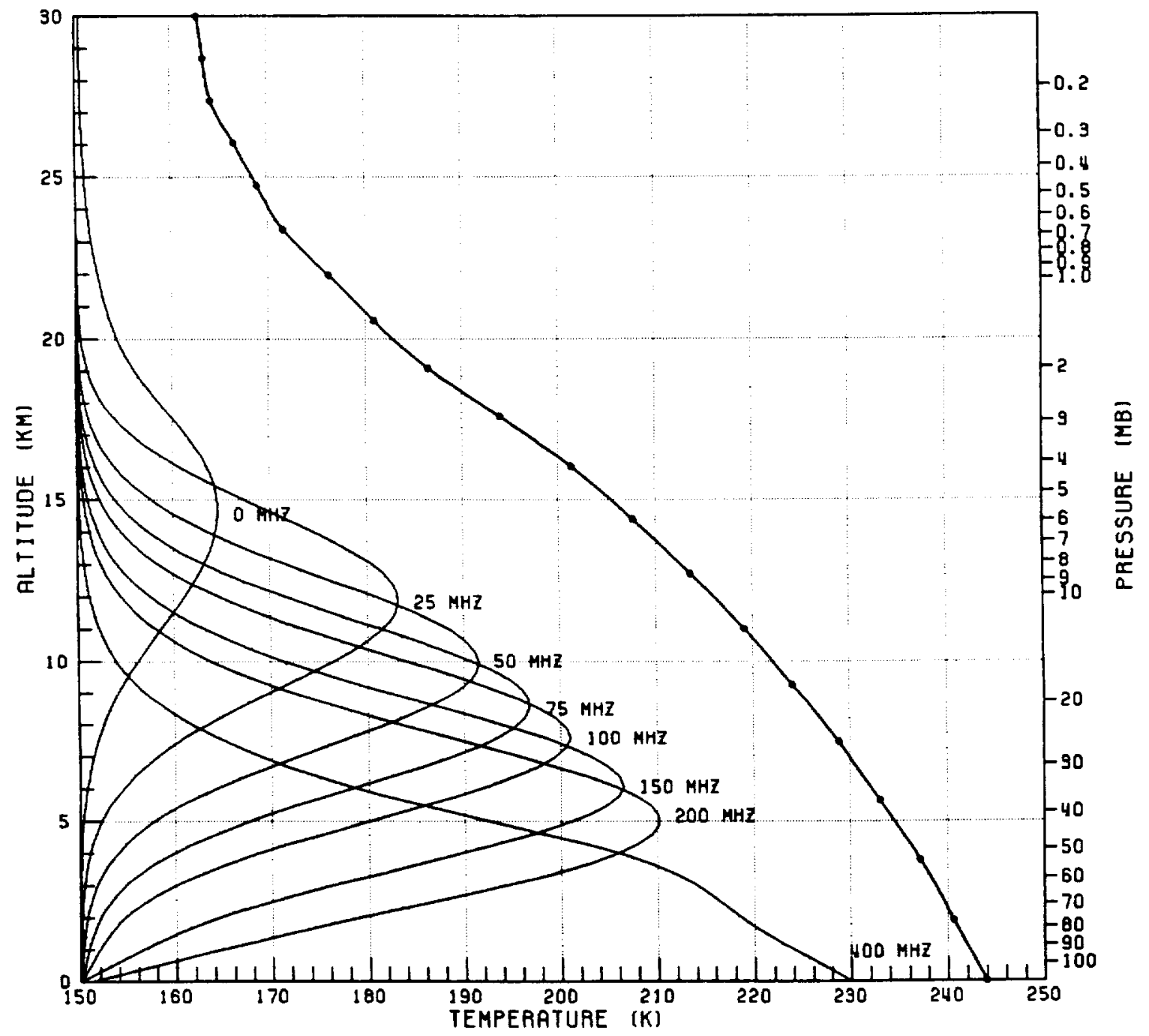

Figure 8.4 
In summary, by constraining the disk-center vertical winds to be dynamically reasonable, the Betz $90 \mathrm{~m} / \mathrm{s}$ super-rotation is preserved, and the SS-AS cell's lower return branch is possibly detected for the first time. However, as the reinterpretation of the Betz data a priori assumes Lo miscalibration, a reinvestigation employing a Lamb dip stabilized LO is warranted (see Chapter 10).

\subsection{MASS CONTINUITY IN THE SUBSOLAR-ANTISOLAR CELL}

The viability of a large $35 \mathrm{~m} / \mathrm{s}$ return branch velocity may be tested by invoking mass continuity. Figure 8.5 shows a cross-section through the terminator of the subsolar-antisolar cell. The central circle is solid Venus with the Subsolar Point, North Pole, and South Pole denoted by SS, NP, and SP, respectively. The direct zone (subsolar-to-antisolar flow) is represented by the outer shaded annulus. The return zone (antisolar-to-subsolar flow) is represented by the smaller shaded annulus. The cross-terminator mass flux in both zones must be equal for steady state circulation, and is given by:

$$
\int_{\text {Direct }} \rho(r) v(r) 2 \pi r d r=\int_{\text {Return }} \rho(r) v(r) 2 \pi r d r \text {, }
$$

where $v(r)$ is the cross-terminator velocity at distance $r$ from the center of Venus, and $\rho(r)$ is the atmospheric density at position $r$. Assuming a constant return velocity $\left(v_{R E T}\right)$ throughout the return zone, Eq. 8.1 reduces to: 


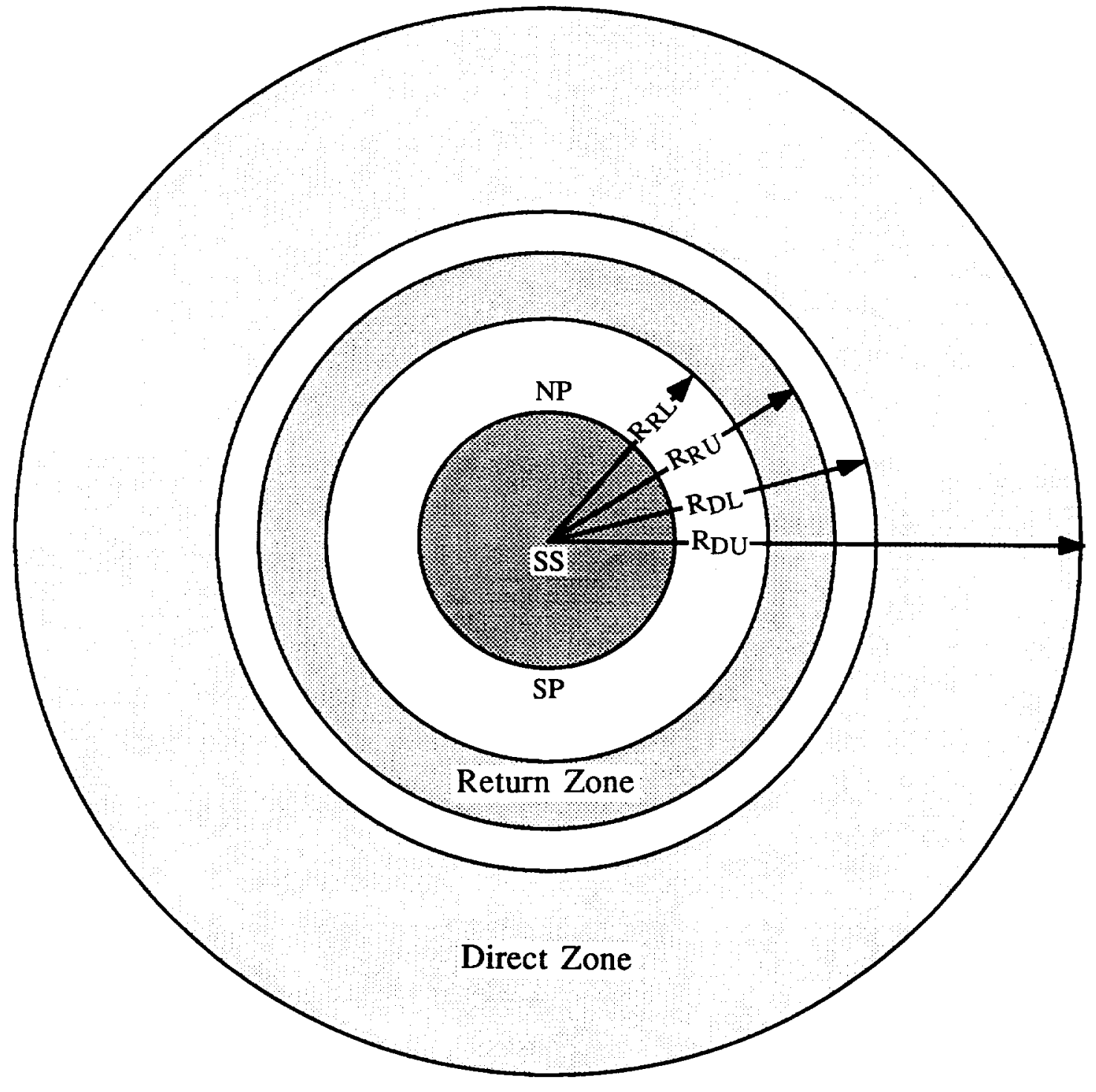

Figure 8.5. Cross-Section of the Subsolar-Antisolar Cell 


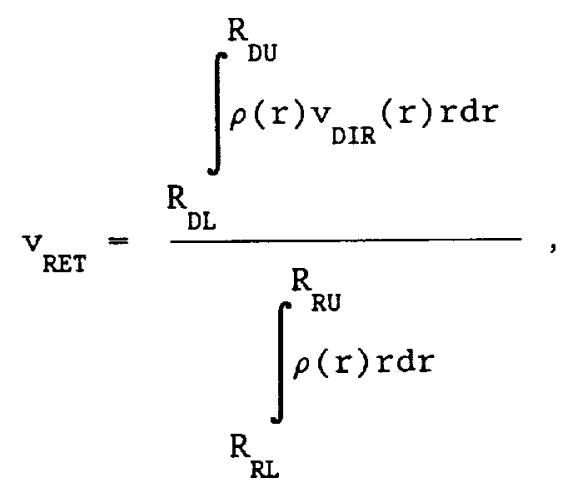

where $R_{D U}$ and $R_{D L}$ are the distances (in $\mathrm{km}$ ), from planet center, of the direct zone upper and lower boundaries, and $R_{R U}$ and $R_{R L}$ are the corresponding boundaries for the return zone (See Fig. 8.5).

The atmospheric density from 60 to $130 \mathrm{~km}$ (in $\mathrm{kg} / \mathrm{m}^{3}$ ) may be reasonably represented by the form:

$$
\rho(r)=51.64 \exp \left(-.0013\left(r-R_{V}\right)^{2}\right)
$$

where $R_{V}=6052 \mathrm{~km}$ is the radius of solid Venus. Eq. 8.3 was empirically generated using atmospheric models by Seiff (1983) for the middle atmosphere, 60 to $100 \mathrm{~km}$ altitude ( 0 to $30^{\circ}$ latitude), and for the upper atmosphere, 100 to $180 \mathrm{~km}$ altitude (midnight model: $\mathrm{SZA}>120^{\circ}$ ).

Two velocity profiles were considered for the direct zone: i) $v_{\text {DIR }}$ constant with altitude, and ii) $v_{D I R}$ linear with $r$, as given by:

$$
v_{D I R}(r)=\frac{v_{\max }}{\left[R_{D U}-R_{D L}\right]}\left(r-R_{D L}\right)
$$

where $v_{D I R}$ is zero at $r=R_{D L}$, and is maximum $\left(v_{D I R}=v_{\max }\right.$ ) at $r=R_{D U}$. Return velocity $v_{\text {RET }}$ is obtained, for varied boundary conditions, through numerical integration of Eq. 8.2. Figure 8.6, a plot of direct 


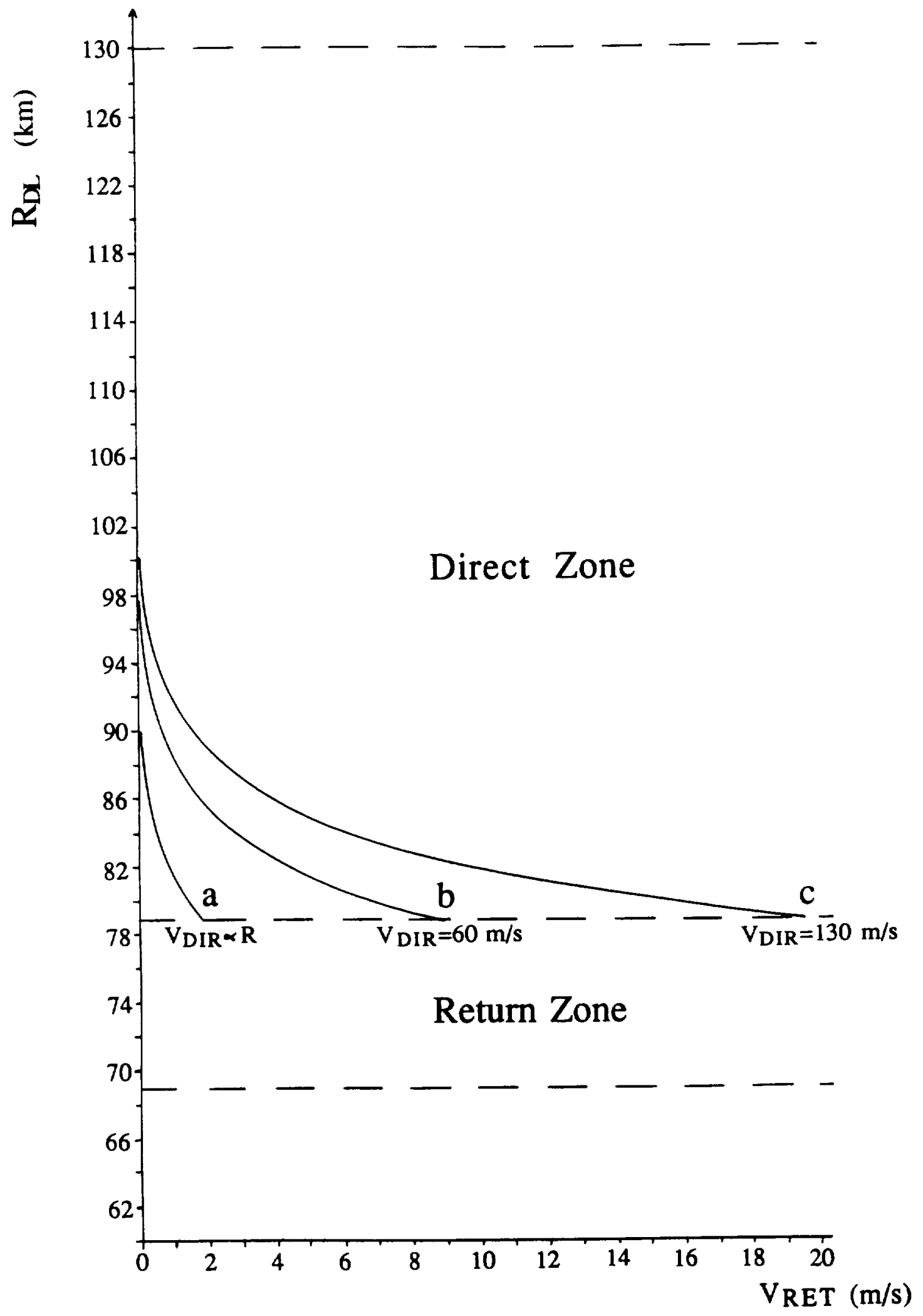

Figure 8.6. Antisolar-to-Subsolar Return Velocity 
zone lower boundary $\mathrm{R}_{\mathrm{DL}}$ versus $\mathrm{v}_{\mathrm{RET}}$, summarizes the results. The direct zone upper boundary has been taken at $130 \mathrm{~km}$ altitude - contributions from higher altitudes are negligible due to the small atmospheric density. Curve "a" assumes $v_{D I R}$ is linear with $r$, as given by Eq. 8.4, with $v_{\max }=130 \mathrm{~m} / \mathrm{s}$. Curves $\mathrm{b}$ and $\mathrm{c}$ assume a constant velocity throughout the direct zone, with $v_{D I R}=60 \mathrm{~m} / \mathrm{s}$, and $130 \mathrm{~m} / \mathrm{s}$, respectively. Maximum return velocities, for all three models, occur when the direct zone lower boundary is coincident with the return zone upper boundary $\left(R_{D L}=R_{R U}\right)$. Yet, a zone separating the upper and lower branches seems necessary, though its required vertical extent is unknown.

The largest return velocity, $19.4 \mathrm{~m} / \mathrm{s}$, is provided by model $\mathrm{c}$, which is almost certainly an over estimate. Thus models invoking mass continuity (as summarized on Figure 8.6) provide maximal return velocities of $-10 \mathrm{~m} / \mathrm{s}$, and cannot account for $35 \mathrm{~m} / \mathrm{s}$ return flow as indicated by the reinterpreted Betz data. To boost return velocity, the width of the return zone could be narrowed - a return zone only 5 $\mathrm{km}$ thick ( $74 \mathrm{~km}$ altitude lower boundary) triples the return velocity for all three Figure 8.6 models. Yet the ${ }^{13} \mathrm{C}^{16} \mathrm{O}_{2}$ line used by Betz et al. (1977b) has a weighting function indicating significant contributions from as low as $69 \mathrm{~km}$ altitude (see Fig. 8.4). An integrated picture of circulation from the surface to $150 \mathrm{~km}$ altitude, based on all present dynamical indicators, is presented in Chapter 9. Evidence is discussed supporting a variant of the mode1 circulation considered above which is consistent with a large return velocity. 


\subsection{LOWER THERMOSPHERIC WINDS}

The literature concerning the early IRHS lower thermospheric wind measurements (Betz 1977, 1982; Betz et a1. 1976, 1977b) does not address seeing conditions and beam pointing uncertainty, and does not adequately establish beam positions, though small planetary disk-to-beam ratios are involved. No attempt will therefore be made at quantifying the Betz data using beam-integrated wind modelling. Instead, a qualitative look at the lower thermospheric wind results is provided.

Observations of ${ }^{12} \mathrm{C}^{16} \mathrm{O}_{2}$ non-thermal emission were carried out Nov/Dec 1975, April 1976, October 1976, and April/May 1977 (Betz 1977, Betz et al. 1977b). GSFC-generated aspect geometries for the first three observing periods, along with the approximate diffraction-limited beams and beam locations, are shown on Figure 8.7. The April/May 1977 aspects are given on Figures 8.1a-c. Seeing-excluded spatial resolution for December 1975 was about the same as the present investigation's worst-case (December 1985; see Figure 5.2). Spatial resolution for April 1976 and October 1976 was significantly poorer. As seeing is not discussed (see Betz 1977, 1982; Betz et al. 1976, 1977b), the effective beam size is unknown.

Based on beam-integrated velocities from the first three observing periods, Betz (1977) hypothesized a $20 \mathrm{~m} / \mathrm{s}$ SS-AS component and a $10 \mathrm{~m} / \mathrm{s}$ zonal retrograde rotation. Beam modelling was not undertaken.

Observations April 1976 on the east 1imb, disk-center and west 1 imb yielded beam-integrated velocities of $65 \mathrm{~m} / \mathrm{s}, 33 \mathrm{~m} / \mathrm{s}$ and $39 \mathrm{~m} / \mathrm{s}$, 


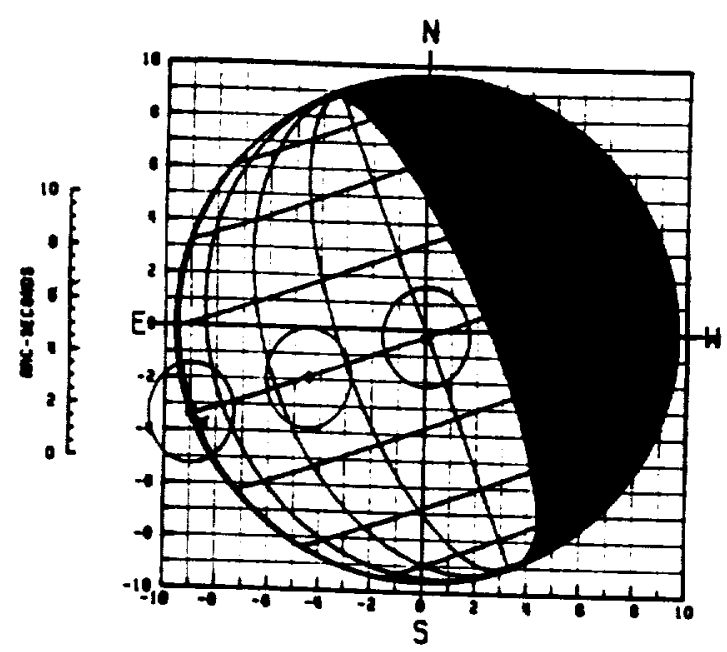

Decenber 1, 1975
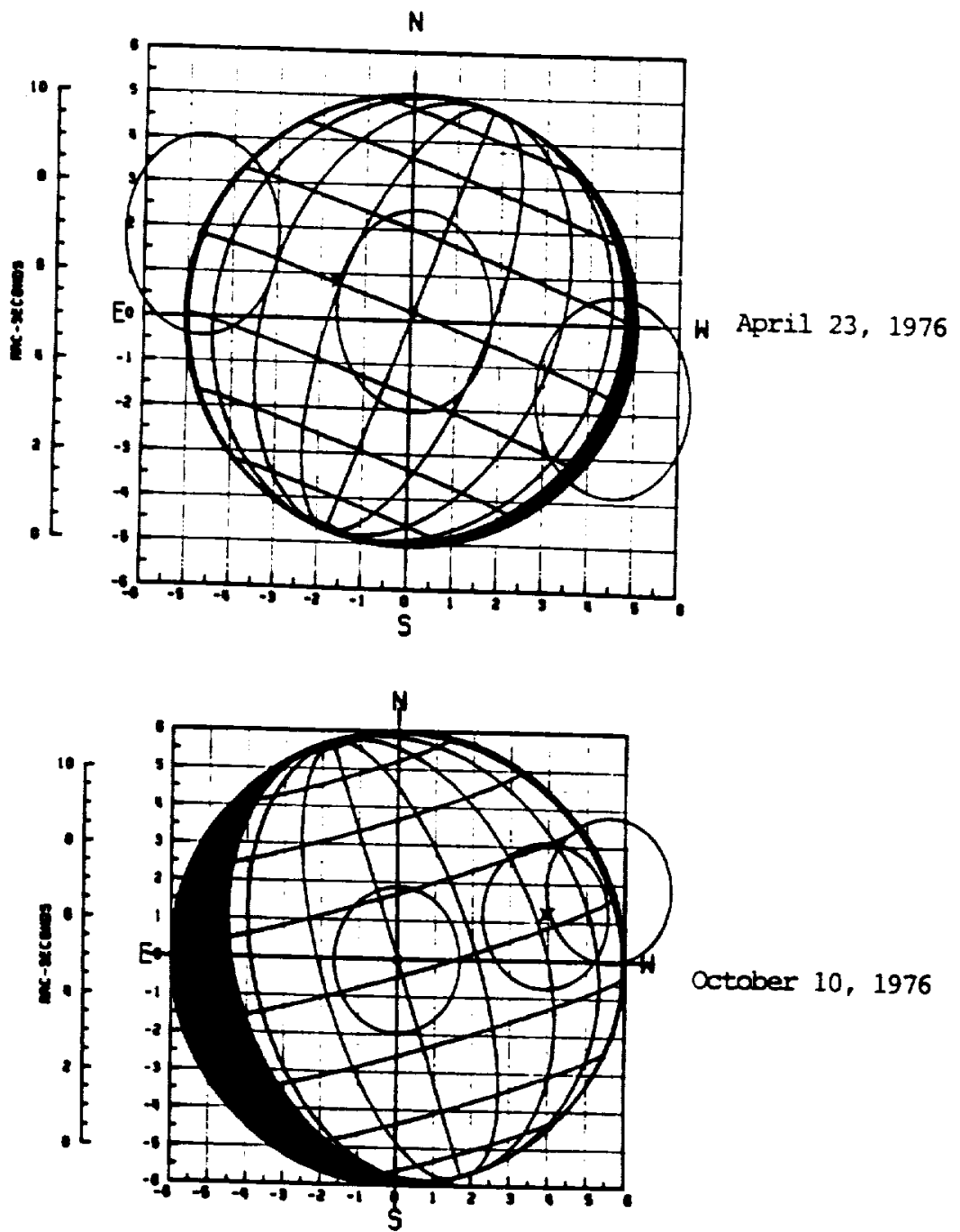

Figure 8.7. Betz Lower Thermospheric Aspect Geometries 
respectively, all in recession (these are $\sigma^{2}$-weighted means; Betz 1977). Disk-center was most precisely measured, with 5 wind determinations, all within $2 \mathrm{~m} / \mathrm{s}$ of the mean. Disk-center is also most troublesome, indicating vertical downwelling near the subsolar point at $120 \mathrm{~km} / \mathrm{hr}$. Whether beam-integrated effects can account for this is unknown. If not, LO frequency calibration is seriously called into question. Subtracting $33 \mathrm{~m} / \mathrm{s}$ from each mean (fixing disk-center at 0 $\mathrm{m} / \mathrm{s}$ ) gives roughly 30 and $10 \mathrm{~m} / \mathrm{s}$ recession on the east and west limbs respectively. This yields the Betz (1977) model circulation: $20 \mathrm{~m} / \mathrm{s}$ SS-AS and $10 \mathrm{~m} / \mathrm{s}$ zonal retrograde components. Thus a $33 \mathrm{~m} / \mathrm{s}$ arbitrary offset in the wind field was introduced to generate the model circulation. (Comparison to the almost identical December 1985 planetary aspect, on Figure 5.2 , is useful.)

April/May 1977 beam positions on the eastern equatorial limb (the only positions available; see Fig. 8.1) exhibited a strong blueshift, decreasing from 125 to $75 \mathrm{~m} / \mathrm{s}$ over several weeks (Betz et al. 1977b). Positions at $\pm 45^{\circ}$ exhibited the same magnitude winds as the equator (consistent with the present IRHS observations made October 1986). Spatial resolution should have been high enough (barring excessively poor seeing) to attribute beam-integrated winds to local dynamics (see Figs. 8.1a-c).

In 1ight of the April/May 1977 observations, Betz et al. (1977b) dramatically revised the model wind field, reporting winds were distinctly subsolar-antisolar with $125 \mathrm{~m} / \mathrm{s}$ cross-terminator velocities. While such an interpretation from the April/May 1977 data is reasonable, there are a number of problems: 
1. The large mesospheric vertical wind velocities, which raise serious questions concerning LO frequency calibration, were recorded during this same April/May 1977 period.

2. No lower thermospheric beam position was available April/May 1977 to serve as a test of Lo frequency calibration (disk-center was on the night hemisphere). Yet winds obtained at such a position April 1976 led to the introduction of a $33 \mathrm{~m} / \mathrm{s}$ systematic offset.

3. No data were ever presented for the April/May 1977 lower thermospheric observations.

4. A dominant prograde zonal rotation is not ruled out.

5. An underlying zonal component is not ruled out.

Until now, the sole evidence for strong SS-AS flow in the lower thermosphere of Venus rested on this single April/May 1977 set of observations, the implied dynamics not borne out by results from three other observing periods. 
CHAPTER 9: CONCLUSION

9.1. THE LOWER THERMOSPHERIC HETERODYNE RESULTS

The first absolute wind velocity measurements above the cloud-tops were successfully made using an IR heterodyne spectrometer with an absolute frequency calibration system. The line of sight projection of the lower thermospheric wind field at $109 \mathrm{~km}$ alt. $( \pm 10 \mathrm{~km}$ HPW) was sampled at four phases covering 828 of a synodic period. Qualitative comparison of beam-integrated winds obtained June 1986 and March 1987 established a dominant SS-AS circulation in the lower thermosphere. December 1985 beam-integrated winds, again established dominant SS-AS circulation, and pointed to possibly $-30 \mathrm{~m} / \mathrm{s}$ (equatorial) retrograde zonal involvement. The October 1986 data, though not capable of distinguishing between strong zonal retrograde and SS-AS circulations established winds in excess of $110 \mathrm{~m} / \mathrm{s}$ across the evening terminator.

BEAMINT modelling of beam-integrated winds provided a quantitative view of the wind field. A two-component horizontal wind field was assumed, comprised of a subsolar-antisolar component parameterized by cross-terminator velocity $V_{H m a x}$, and a zonal solid body rotation parameterized by equatorial velocity $\mathrm{V}_{\text {eq }}$. The best fit model space for each observing period was:

$$
\begin{array}{ll}
\text { December } 1985 & \mathrm{~V}_{\mathrm{H} \max }=90 \text { to } 120 \mathrm{~m} / \mathrm{s}, \mathrm{V}_{\mathrm{eq}}=-15 \text { to }-45 \mathrm{~m} / \mathrm{s} \\
\text { June } 1986 & \text { no best fit model space - model space minima at } \\
& \mathrm{V}_{\mathrm{H} \max }=90 \text { to } 100 \mathrm{~m} / \mathrm{s}, \mathrm{V}_{\mathrm{eq}}=-0 \mathrm{~m} / \mathrm{s} \\
\text { October } 1986 & \mathrm{~V}_{\mathrm{H} \max }-\mathrm{V}_{\mathrm{eq}}=140-155 \mathrm{~m} / \mathrm{s} \\
\text { March } 1987 & \mathrm{~V}_{\mathrm{H} \max }=110 \text { to } 150 \mathrm{~m} / \mathrm{s}, V_{\text {eq }}=-20 \text { to }-25 \mathrm{~m} / \mathrm{s}
\end{array}
$$


Model fits to three of the four observing periods were in good agreement with the observed. The poor June modelling, most probably resulting from pointing uncertainty associated errors, neverless indicated dominant SS-AS circulation.

Summarizing the major heterodyne results:

1. A dominant SS-AS circulation with $90-150 \mathrm{~m} / \mathrm{s}$ cross-terminator horizontal velocities, and a $20-40 \mathrm{~m} / \mathrm{s}$ (equatorial) zonal retrograde component, has been found to characterize the ${ }^{12} \mathrm{C}^{16} \mathrm{O}_{2}$ non-thermal emitting layer centered at $109 \mathrm{~km}$ altitude $( \pm 10 \mathrm{~km} \mathrm{HPW})$.

2. The investigation provides the first direct evidence of a dominant subsolar-antisolar cell in the lower thermosphere.

3. The circulation is not pure subsolar-antisolar - discovery of a zonal retrograde component (reasonably modelled as a solid body super-rotation) was not expected, and is not predicted by hydrodynamical models at $110 \mathrm{~km}$ altitude.

4. Assuming temporal stability over the synodic period of the observations, overlay of December 1985, October 1986, and March 1987 best fit model spaces revealed a single common model circulation: $\mathrm{V}_{\mathrm{Hmax}}=120 \mathrm{~m} / \mathrm{s}, \mathrm{V}_{\mathrm{eq}}=-25 \mathrm{~m} / \mathrm{s}$

5. The Bougher et al. (1986) hydrodynamical model (BEAM) for 110 $\mathrm{km}$ altitude is almost identically characterized by BEAMINT SS-AS circulation with $V_{H \max }=120 \mathrm{~m} / \mathrm{s}$ - consistent with the common model subsolar-antisolar component. 
6. The Mayr et al. $(1980,1985)$ circulation models, predicting $\mathrm{V}_{\mathrm{Hmax}}-20 \mathrm{~m} / \mathrm{s}$ at $110 \mathrm{~km}$, are inconsistent with the heterodyne results (see Chapter 2).

\subsection{AN INTEGRATED PICTURE}

\subsubsection{Summary of Dynamical Indicators}

The heterodyne-derived lower thermospheric absolute wind measurements may be coupled with other tracers of dynamics:

- cyclostrophic balance models $(65-90 \mathrm{~km} \mathrm{alt.})$

- Co distribution ( $80-100 \mathrm{~km}$ alt.)

- PV Orbiter upper atmospheric sounding ( $>150 \mathrm{~km} \mathrm{alt.)}$ to provide a coherent dynamical picture for the $70-200 \mathrm{~km}$ altitude region. Reinterpretation of the Betz IR heterodyne-determined mesospheric winds (69-79 $\mathrm{km}$ alt.) may also be incorporated. By way of summary:

1. Cyclostrophic Balance Mode1s (65-100 km; see Sect. 2.1):

Circulation models invoking cyclostrophic balance, and based on VORTEX (Taylor et al. 1980) and ORO (Newman et al. 1984) derived thermal fields, predict increasing zonal velocities above the cloud decks reaching a maximum of $-130 \mathrm{~m} / \mathrm{s}$ near $-70 \mathrm{~km}$ altitude. The observed poleward warming above this level implies decreasing zonal speeds with altitude, becoming negligible by $-90-100 \mathrm{~km}$. A concomitant increase in the mean meridional component with possibly $120 \mathrm{~m} / \mathrm{s}$ speeds near $90-100 \mathrm{~km}$ altitude has also been predicted (Taylor et al. 1980, Elson 1979).

2. The CO Distribution ( $80-100 \mathrm{~km}$; see Sections $1.1,2.1$ ): 
Clancy and Muhleman (1985a,b) have interpreted an observed $\left[{ }^{12} \mathrm{C}^{16} \mathrm{O}\right] 80-90 \mathrm{~km}$ altitude dayside enhancement, near $8.5^{\mathrm{h}}$ Local Venusian Time (LVT), as circulation-induced, resulting from a superposition of subsolar-to-antisolar circulation and a $-50 \mathrm{~m} / \mathrm{s}$ (equatorial) zonal retrograde component. An observed $\left[{ }^{12} \mathrm{C}^{16} \mathrm{O}\right]$ -95 $\mathrm{km}$ altitude nightside enhancement, near $0^{\mathrm{h}}$ LVT, was interpreted as due to nearly pure subsolar-to-antisolar circulation.

3. PV Orbiter Upper Atmospheric Sounding (>150 km; see Section 1.1):

An observed post-noon temperature maximum (Keating et al. 1980) and post-midnight maxima in neutral $\mathrm{H}$ density (Brinton et a1. 1980), He density (Niemann et al. 1980), and UV airglow (Stewart and Barth 1979), above $150 \mathrm{~km}$, suggest a retrograde zonal component with $-50-100 \mathrm{~m} / \mathrm{s}$ equatorial winds (see Mayr et a1. 1980).

4. Reinterpretation of the Early Heterodyne Mesospheric Winds $(69-79 \mathrm{~km}$; see Chapter 8$)$ :

Laser stability tests performed on IRHS power peak and Lamb dip local oscillator stabilization systems indicate the former can be associated with a variable and unpredictable systematic frequency offset at the tens of $\mathrm{m} / \mathrm{s}$ level. Such is consistent with Betz et al.(1977b) heterodyne-derived mesospheric winds (69-79 km alt.), obtained April/May 1977, which are dynamically unreasonable if interpreted as absolute winds. Based on numerous in situ measurements and theoretical considerations, the assumption of negligible wind field vertical velocity was used to 
justify fixing disk-center derived winds at the zero level. The resulting equatorial east-limb and west-limb mean wind velocities were $128.7 \pm 9.1$ and $-59.2 \pm 8.5 \mathrm{~m} / \mathrm{s}$, respectively, interpretable as superposition of a $94 \pm 6 \mathrm{~m} / \mathrm{s}$ retrograde zonal and a $35 \pm 6 \mathrm{~m} / \mathrm{s}$ antisolar-to-subsolar component. The latter would constitute a low-level return branch for the SS-AS cell characterizing the lower thermosphere. However, generation of a return flow >-10 $\mathrm{m} / \mathrm{s}$ is inconsistent with mass continuity as applied to the SS-AS cell.

\subsubsection{Reassessing the CO Observations}

The Clancy and Muhleman (1985a) derived diurnal [C0] variation was based on a survey of CO microwave spectra taken by a host of observers over five Venusian phase cycles. These were full-disk spectra - requiring acquisition at different planetary phases to obtain diurnal variability. Line center absorption occurs at -95-105 $\mathrm{km}$ while the pressure-broadened wings probe the $80-90 \mathrm{~km}$ altitude region. Typical nightside spectra were deep with narrow wings. Conversely, typical dayside spectra were shallower with extensive wings. The [CO] is thus enhanced on the nightside (by a factor of 2-4) near $100 \mathrm{~km}$ and equally enhanced on the dayside between $80-90 \mathrm{~km}$. Both the magnitude of the enhancement and the $-180^{\circ}$ phase shift at $-90 \mathrm{~km}$ are quite convincing. The locations of the [CO] maxima and minima, however, are not as well determined.

Based on percent absorption at line center, a least squares fit to the form $A \cos \theta+B \sin \theta$ indicated a $0.6^{\mathrm{h}} \pm \sim .6^{\mathrm{h}}$ LVT [CO] maximum at 
-95-105 km (Clancy and Muhleman 1985a). However no data were available in the critical $0-4^{\mathrm{h}}$ LVT period. Additionally, the fit may constrain [CO] diurnal variability unrealistically, and used the entire data set

- thus possibly introducing a component due to long-term temporal variation. (The eight full-disk spectra taken during the 1978-79 phase cycle, provide a reasonably short-term baseline. These data appear very symmetric about LVT=-0.5 $5^{\mathrm{h}}$, consistent with the Clancy and Muhleman (1985a) fit).

The near-midnight [CO] bulge was thought reasonable due to compatibility with the Dickinson and Ridley (1977) model (Clancy and Muhleman 1985a; DRM had a $95 \mathrm{~km}$ lower cut off altitude - see Chapter 2). Yet DRM was incompatible with Pioneer Venus (PV) observations. Discrepancies with PV data prompted reformulation of the DRM 2-D hydrodynamical model by Bougher et al. (1986) (BEAM; see Chapter 2). The BEAM results are consistent with the PV-derived [CO] dayside enhancement at higher altitudes $(>150 \mathrm{~km})$, and seem to imply a $180^{\circ}$ [CO] phase reversal near $130 \mathrm{~km}$ - in agreement with the Co microwave spectra. A heuristic explanation for the phase reversal may be obtained using the framework of the Mayr et al. (1980, 1985) dynamical models (see Chapter 2).

At a given altitude, the presence of a strong (and symmetric) diurnal temperature gradient will tend to produce a noon density enhancement, for all atmospheric species, due to atmospheric expansion. However, introduction of a strong SS-AS circulation will tend to carry the lighter species to midnight. If vertical diffusion is rapid, these species will diffuse into the mesosphere before being transported to 
the night side. As vertical diffusion is least efficient for the lightest species (due to their correspondingly larger scale-heights), these are the most likely to exhibit nightside enhancements. Such enhancements are observed for $\mathrm{H}$ and $\mathrm{He}$ in the Venus thermosphere above $150 \mathrm{~km}$ altitude. CO, however, exhibits a noon maximum at these altitudes.

Consider again the Clancy and Muhleman (1985a,b) $100 \mathrm{~km}$ results. As $C O$ is a photodissociation product, it must have been transported to the night side by the global-scale winds. Its enhancement near midnight (as opposed to noon higher up) can result from the absence of significant diurnal temperature contrast at $100 \mathrm{~km}$ altitude. The effect of atmospheric expansion no longer exists, hence, vertical diffusion is greatly reduced. A near midnight bulge might then be expected from a dominant SS-AS circulation, consistent with both the Clancy and Muhleman (1985b) interpretation and the present $10 \mu \mathrm{m}$ heterodyne results.

For pure SS-AS circulation, the subsolar and antisolar points are wind field stagnation points where horizontal velocity is zero. Introducing a small wind field retrograde zonal component, causes both stagnation points to move toward the morning terminator, e.g., $3^{\text {h }}$ and $9^{h}$ LVT. Light species allowed to migrate to the night side will thus pile up near the stagnation point after midnight - which is again what is observed for $\mathrm{H}$ and $\mathrm{He}$ at $150 \mathrm{~km}$. The slight post-midnight [CO] maximum at $100 \mathrm{~km}$ points to such a small retrograde zonal component. As a benchmark, for a $100 \mathrm{~km}$ altitude SS-AS field consistent with the BEAM-predicted field at $110 \mathrm{~km}$, a $20 \mathrm{~m} / \mathrm{s}$ zonal component would cause a 
stagnation point at $\sim 1^{\mathrm{h}}$ LVT, as observed.

In the 80-90 km region, also characterized by negligible diurnal temperature contrast, an $8.5^{h} \pm 1^{h}$ LVT [CO] maximum was obtained from absorption $5 \mathrm{MHz}$ and $10 \mathrm{MHz}$ off line center, probing roughly 85 and 80 $\mathrm{km}$ altitude, respectively ( $\mathrm{Clancy}$ and Muhleman 1985a). A heuristic model was presented yielding the observed phase shift through horizontal transport of the midnight [CO] maximum by a wind field zonal component (Clancy and Muhleman 1985b). During transport, the CO was assumed to track the mixing profile lower cutoff which was depressed on the dayside $10 \mathrm{~km}$ relative to the nightside (as indicated by the observed diurnal variability). Adopting a canonical vertical velocity of $4 \mathrm{~cm} / \mathrm{sec}$ (consistent with DRM), $10 \mathrm{~km}$ CO subsidence defined a vertical time constant. The zonal speed necessary to transport the [CO] maximum from $0^{\mathrm{h}}$ to $8.5^{\mathrm{h}}$ LVT, in the requisite time, was found to be a reasonable $-50 \mathrm{~m} / \mathrm{s}$. This model, however, is incompatible with the global wind field. As the zonal component displaces the [CO] enhancement toward the morning terminator, the SS-AS horizontal component will increase in magnitude opposing the effect of zonal transport. The Co should thus pile up at the zonally-induced early morning stagnation point - just as observed at $100 \mathrm{~km}$ - not across the morning terminator at $8.5^{\mathrm{h}}$ LVT. In fact, the Clancy and Muhleman (1985b) model considers a [CO] bulge at midnight which is subsequently transported back to the dayside where it was originally produced.

The 80-90 km [CO] maximum (determined using the same technique as for the $>95 \mathrm{~km}$ data - assuming a priori functional dependence) is poorly established - no survey data exist for $9.5-15^{\mathrm{h}}$ LVT (Clancy and 
Muhleman 1985a). Even qualitative inspection of the data fails to establish the location of the [CO] maximum. The distribution of data in LVT provides far better coverage in the vicinity of the [CO] minimum which seems to occur between 19 and $23^{\mathrm{h}}$ LVT.

Rather than ask why a [CO] maximum occurs at $-8.5^{\mathrm{h}}$ LVT, it seems more reasonable to address the location of the minimum. How can such pronounced diurnal [CO] variation be maintained in a region with negligible diurnal thermal contrast, and with a minimum a few hours before midnight? One possibility - $C O$ is not permitted to migrate from the site of formation, possibly achieved through a diminished SS-AS wind field component. Yet the observed asymmetry (the $-19-23^{\text {h }}$ LVT [CO] minimum) would remain unexplained. In addition, the presence of a zonal component (whose magnitude is expected to increase with decreasing altitude) would work to reduce the strong day/night contrast.

The reinterpreted Betz mesospheric winds, $69-79 \mathrm{~km}$ altitude, are indicative of a dominant zonal retrograde super-rotation, and a superimposed antisolar-to-subsolar return flow. Such a wind field convolution is also consistent with observed diurnal [CO] variation in the 80-90 km region, if, at these higher altitudes, the return flow dominates .

A dominant return branch with a superimposed zonal component will cause the wind field stagnation points to migrate toward the evening terminator, e.g., $15^{\mathrm{h}}$ and $21^{\mathrm{h}}$ LVT. Such circulation will both steepen [CO] diurnal contrast and produce a minimum at the late evening stagnation point - consistent with observation. The position of the dayside [CO] maximum, expected to be a strong function of 
photochemistry, may not reside at the dayside stagnation point.

\subsubsection{Gross Circulation Above the Cloud Tops}

A self-consistent picture of circulation begins to emerge. In the $95-120 \mathrm{~km}$ region a weak retrograde zonal component is superimposed on a dominant SS-AS circulation. Near $-90 \mathrm{~km}$ the SS-AS component reverses phase. Below $90 \mathrm{~km}$ the zonal component increases in intensity until it becomes dominant, as implied by the Betz reinterpretation, in the $69-79 \mathrm{~km}$ region.

An overview of the integrated model circulation is shown on Figure 9.1 superimposed on an equatorial slice through the atmosphere. Positions of the north pole (NP), subsolar point (SS) and antisolar point (AS) are given. The table at upper right summarizes all investigations indicating zonal retrograde super-rotation. Entries marked with an asterisk reflect the more speculative tracers of dynamics. Zonal super-rotation is indicated by the speckled area on the diagram.

The present investigation's detection of a zonal retrograde component at 100-120 km altitude implies such circulation exists throughout the atmosphere, though not everywhere dominant. The super-rotation at $-90 \mathrm{~km}$ and below may therefore be coupled to the super-rotation at $150 \mathrm{~km}$ and above. Such continuity in the zonal component (as predicted by Schubert 1983) implies upper levels may be pumped from below. The need for an independent mechanism for generation of upper atmospheric super-rotation may thereby be removed (Schubert 1983). 


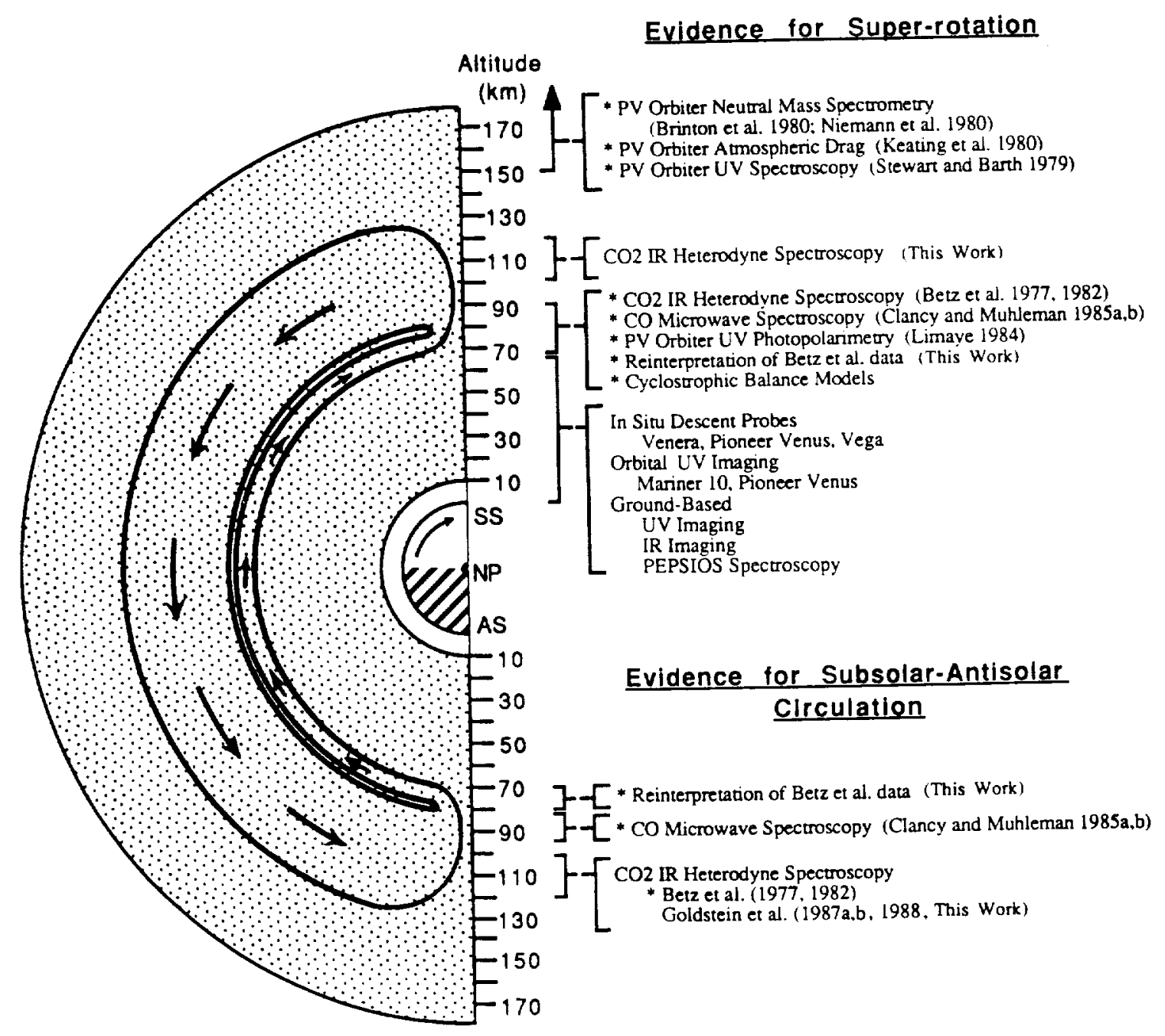

Figure 9.1. Proposed Circulation Model for Venus 
On Figure 9.1, the table at lower right summarizes the evidence for SS-AS circulation. Whether the SS-AS cell penetrates to levels $>120 \mathrm{~km}$ altitude is unknown. Yet, the even more pronounced diurnal temperature gradient at higher altitudes (see Fig. P.1) should drive SS-AS flow.

If the reinterpretation of the [CO] microwave observations is correct, generation of a high velocity sS-AS return flow $(-35 \mathrm{~m} / \mathrm{s})$ becomes even more difficult to explain. Invoking mass continuity (see Section 8.3 ) yields $<10 \mathrm{~m} / \mathrm{s}$ (cross-terminator) return velocities, assuming return only in the $-70-80 \mathrm{~km}$ region. Maximizing return velocity requires locating the SS-AS direct zone directly above the return zone, and minimizing return zone vertical extent.

1. The fact that the [CO] phase reverses so abruptly near $90 \mathrm{~km}$ may indeed indicate a SS-AS direct zone located just above the return zone.

2. The microwave observations point to a more extensive return zone extending to $-90 \mathrm{~km}$, hence a correspondingly lower return velocity. Yet it is possible return zone width, as derived from long time-base observations, significantly reflects temporal variation in return zone altitude (the Betz et al. (1977) mesospheric wind investigation predated the microwave data comprising the Clancy and Muhleman (1985a) study).

Support for such variability is provided by [CO] microwave data obtained by Schloerb and Good in 1981-82, post-dating the survey-included data, but neverless discussed by Clancy and Muhleman $(1985 a, b)$. These data indicate a $5^{\text {h }}$ LVT [CO] maximum 
at $80-90 \mathrm{~km}$, inconsistent with the survey results. A lower return zone altitude (entirely below $80 \mathrm{~km}$ ) during the $1981-82$ phase cycle would yield $80-90 \mathrm{~km}$ probing of the direct zone, and an early morning [CO] maximum consistent with $5^{\mathrm{h}}$ LVT provided the presence of a significant zonal component.

These considerations, however, attempt to maintain a return zone velocity near the $10 \mathrm{~m} / \mathrm{s}$ maximum. The means of generating $-35 \mathrm{~m} / \mathrm{s}$ return velocities remains to be addressed.

Limaye (1988) has reported a cloud-level horizontal mass divergence near the subsolar point from PV Orbiter Cloud Photopolarimetry. This may be consistent with a second weaker cloud-level SS-AS cell below the primary cell indicated on Figure 9.1. SS-AS return in the $70-90 \mathrm{~km}$ region could mutually feed both the cloud-level divergence and the lower-thermospheric circulation. Even low divergence velocities at cloud-level would significantly boost return zone velocities.

Deposition of solar energy in the cloud decks, maximally at the subsolar point, drives the lower cell, while the substantial thermosphere/cryosphere temperature gradient at higher altitudes drives the upper cell. The $70-90 \mathrm{~km}$ region, associated with negligible solar energy deposition and negligible diurnal thermal contrast, is ideally suited for a SS-AS return branch.

Models based on cyclostrophic balance predict decreasing zonal flow with altitude above $-70 \mathrm{~km}$ (consistent with the above integrated picture) and a possible concomitant increase in the meridional component. To date, the existence of the latter has still not been investigated. 


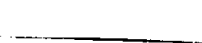




\section{CHAPTER 10: FUTURE DIRECTIONS}

The integration of a Lamb dip laser stabilization system into existing IR heterodyne spectrometers has opened a new avenue for the investigation of planetary atmospheric dynamics. Mapping of the Venusian lower thermospheric wind field is but a single application. Tractable problems still remain concerning Venus while Mars' dynamics is virgin territory.

10.1. VENUS - A SEARCH FOR THE SUBSOLAR-ANTISOLAR RETURN BRANCH AND A MESOSPHERIC MERIDIONAL COMPONENT

Venusian dynamical models for the $70-90 \mathrm{~km}$ transition region predict decreasing zonal winds with altitude with a possible concomitant dramatic increase in mean meridional circulation (maximally $-120 \mathrm{~m} / \mathrm{s}$; Taylor et a1. 1980, Limaye 1985). The transition region may also contain the lower return branch of the subsolar-antisolar cell.

The mesospheric wind velocities obtained by Betz et al. (1977b) comprise the sole data set for the transition zone. The reinterpretation of these data (Chapter 8 ) while exciting, is by no means conclusive as it a priori assumes spectrometer frequency instability. Additionally, as a predominantly equatorial investigation, it was not a suitable probe of the meridional circulation. With the Lamb dip system, a high spatial resolution map using ${ }^{13} \mathrm{C}^{16} \mathrm{O}_{2}$ at inferior and superior conjunction would allow deconvolution of all three possible components.

other IRHS-related Venus investigations include: 1) continued monitoring of the lower thermospheric circulation for possible (solar 
cycle-induced) temporal variability, 2) a search for mesospheric mid-1atitude zonal jets whose cores may not yet have been observed (Limaye 1985), and 3) investigation of high latitude $\left(>60^{\circ}\right.$ ) circulation, inadequately addressed by even space-based UV imaging due to a lack of trackable UV features (Limaye 1985).

As described in Appendix A4, visually observed phase-locked radial structure is strangely consistent with subsolar-antisolar circulation symmetry. Never detected photographically, it may truly be illusory. Yet, the circumstantial evidence for its existence is considerable. A CCD investigation in the visible, the best modern means to document such structure, would allow easy removal of the limb-to-terminator gradient and stacking of multiple exposures through image processing. These data reduction capabilities are considered crucial if such structure is to be imaged (Dollfus 1955). A CCD study could be done concomitantly with photopolarimetry using the Pioneer Venus Orbiter.

\subsection{MARS}

The dynamics of the Martian atmosphere, to date, are derived almost entirely from the planetary-scale thermal field obtained by Mariner 9 , mid-November 1971 through October 1972 (Conrath 1981). Thermal winds have been modelled for the two recognized dynamical regimes - near dust-free periods (Pollack et al. 1981, Conrath 1981) and periods of enhanced dust-loading near perihelion (Haberle et al. 1982). Yet thermal wind fields rely on boundary value wind measurements to determine the wind velocity zero offset.

To date, absolute wind velocities have only been obtained near the 
surface by the Viking landers (Ryan 1985) and through analysis of Viking orbiter imaging of exceedingly sparse cloud cover (Kahn 1984). Without additional absolute wind measurements over latitude, solar longitude and altitude, the nature of Martian circulation will remain only speculative.

Models predict mean zonal wind velocities possibly as high as 160 $\mathrm{m} / \mathrm{s}$ and $270 \mathrm{~m} / \mathrm{s}$ at the $0.1 \mathrm{mb}$ level $(-40 \mathrm{~km}$ alt. - approximate model cutoff alt.) for dust-free (Conrath 1981) and dust loaded ( $r=5$ ) (Haberle et al. 1982) cases respectively. For both models, these maximal zonal velocities characterize the core of a jet near $60^{\circ} \mathrm{N}$ latitude, representing conditions between winter solstice and spring equinox in the northern hemisphere $\left(L_{s}=270-360\right)$, starting 30 days after perihelion. The derived maximal meridional component below $40 \mathrm{~km}$ altitude is of order $10 \mathrm{~m} / \mathrm{s}$ (Pollack et al. 1981, Haberle et al. 1982). Polar warming has been observed by the Viking IRTM near $25 \mathrm{~km}$ altitude (Barnes and Hollingsworth 1987), consistent with dust loading models (Haberle et al. 1982), and by ground-based Doppler-limited IR spectroscopy (Deming et al. 1986) in the $50-85 \mathrm{~km}$ altitude range. The latter implies possible penetration of strong mean meridional circulation to higher altitudes, with polar warming due to polar subsidence-induced adiabatic heating.

On Mars, ${ }^{12} \mathrm{C}^{16} \mathrm{O}_{2} 10 \mu \mathrm{m}$ nonthermal emission peaks at $75 \mathrm{~km}$ altitude falling to half power by 50 and $80 \mathrm{~km}$ (Deming and Mumma 1983). Unlike Venus, the thermal absorption feature off line core fits comfortably within the IRHS high resolution bandwidth, allowing simultaneous sampling of atmospheric dynamics at lower altitudes $(0-40 \mathrm{~km})$. Such 
wind measurements were made at McMath during the 1986 Martian opposition (Figure 10.1; see also Figs. 5.5 and 5.6 ) and indicate a $\mathrm{S} / \mathrm{N}$ allowing independent centroiding of both emission and absorption features to a few $\mathrm{m} / \mathrm{s}$. An observed displacement between the centroids, implying a differential wind-induced Doppler shift, is evident in Figure 10.2 representing a stack of $102^{\mathrm{m}}$ scans.

The ongoing Mars wind investigation also includes 1988 opposition measurements at IRTF. The exceptionally large 25 arc-second Martian disk (not again realized until 2003), coupled with the $<1$ arc-second seeing and 0.8 arc-second beam attained at IRTF, provided a rare opportunity for 2-level mapping of the Martian wind field. Opposition 10 days after northern hemisphere winter solstice corresponded to the period for which models predict zonal winds of 160 or $270 \mathrm{~m} / \mathrm{s}$, depending upon the presence of a global dust storm.

These data are the only IRHS-derived Mars absolute wind measurements made to date. (Earlier measurements were made with a power peak stabilized laser local oscillator (Mumma 1988)). At the time of this writing, absolute wind velocities were not yet available.

\subsection{TITAN}

Initial work on Titan's atmospheric dynamics by Leovy and Pollack (1973) and Golitsyn (1975) indicated a possible analog to the Venusian $4^{\mathrm{d}}$ super-rotation. Titan, like Venus, is a slow rotator (a $1: 1$ spin-orbit resonance with a period of $15.95^{\mathrm{d}}$ is assumed) implying circulation in the cyclostrophic regime. The latitudinal temperature gradient in the Titanian stratosphere, derived from Voyager 1 IR 


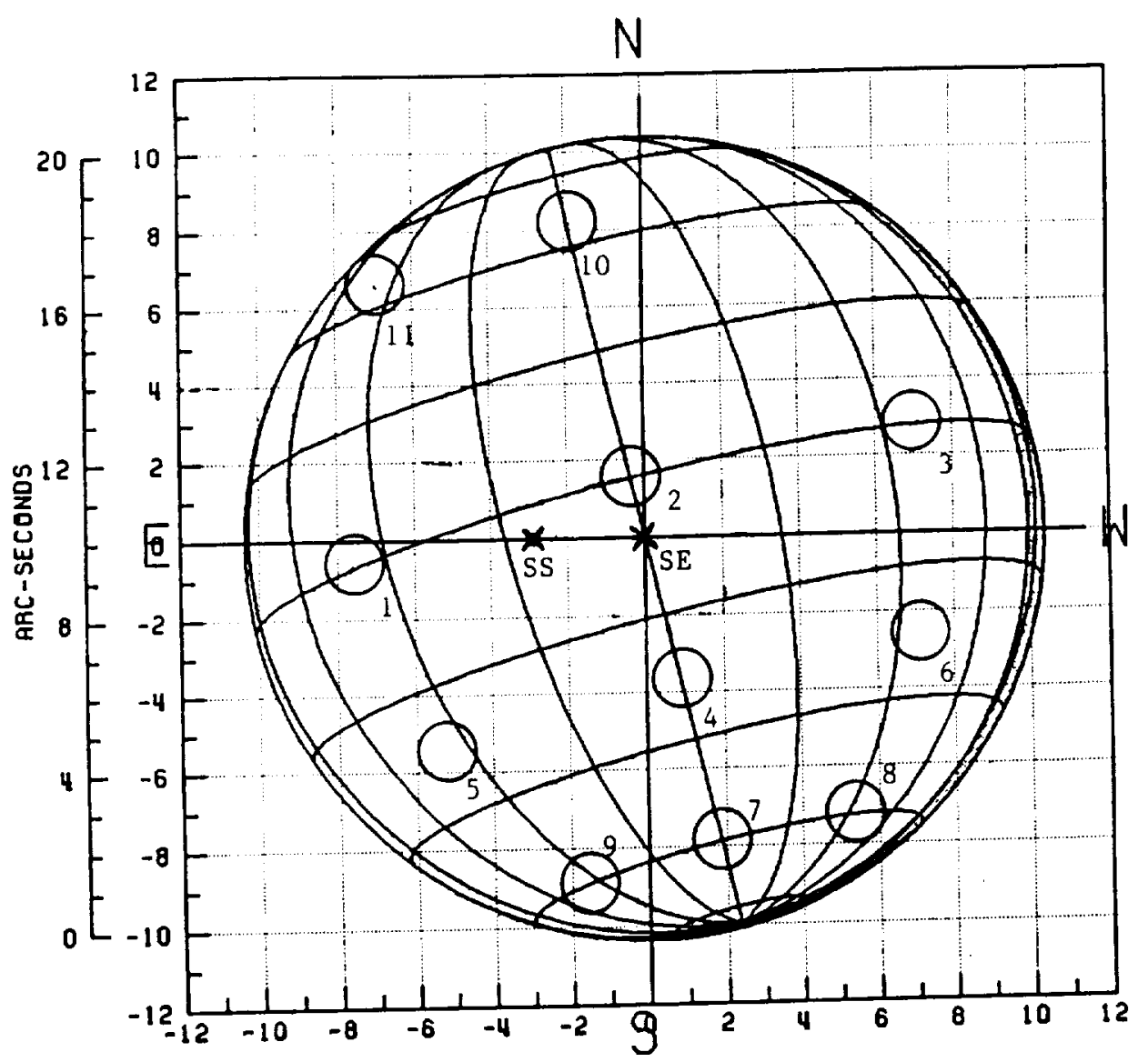

1 Equator $-45^{\circ} \mathrm{W}$ of Subearth Meridian

2 Equator-Subearth Meridian

3 Equator $-45^{\circ} \mathrm{E}$ of Subearth Meridian

$430^{\circ} \mathrm{S}$ - Subearth Meridian

$530^{\circ} \mathrm{S}-45^{\circ} \mathrm{W}$ of Subearth Meridian

$630^{\circ} \mathrm{S}-45^{\circ} \mathrm{E}$ of Subearth Meridian

$760^{\circ} \mathrm{S}$ - Subearth Meridian

$860^{\circ} \mathrm{S}-45^{\circ} \mathrm{E}$ of Subearth Meridian

$960^{\circ} \mathrm{S}-45^{\circ} \mathrm{W}$ of Subearth Meridian

$1045^{\circ} \mathrm{N}$ - Subearth Meridian

$1145^{\circ} \mathrm{N}-45^{\circ} \mathrm{W}$ of Subearth Meridan

Figure 10.1. Mars Beam Positions June 1986 McMath Solar Telescope 


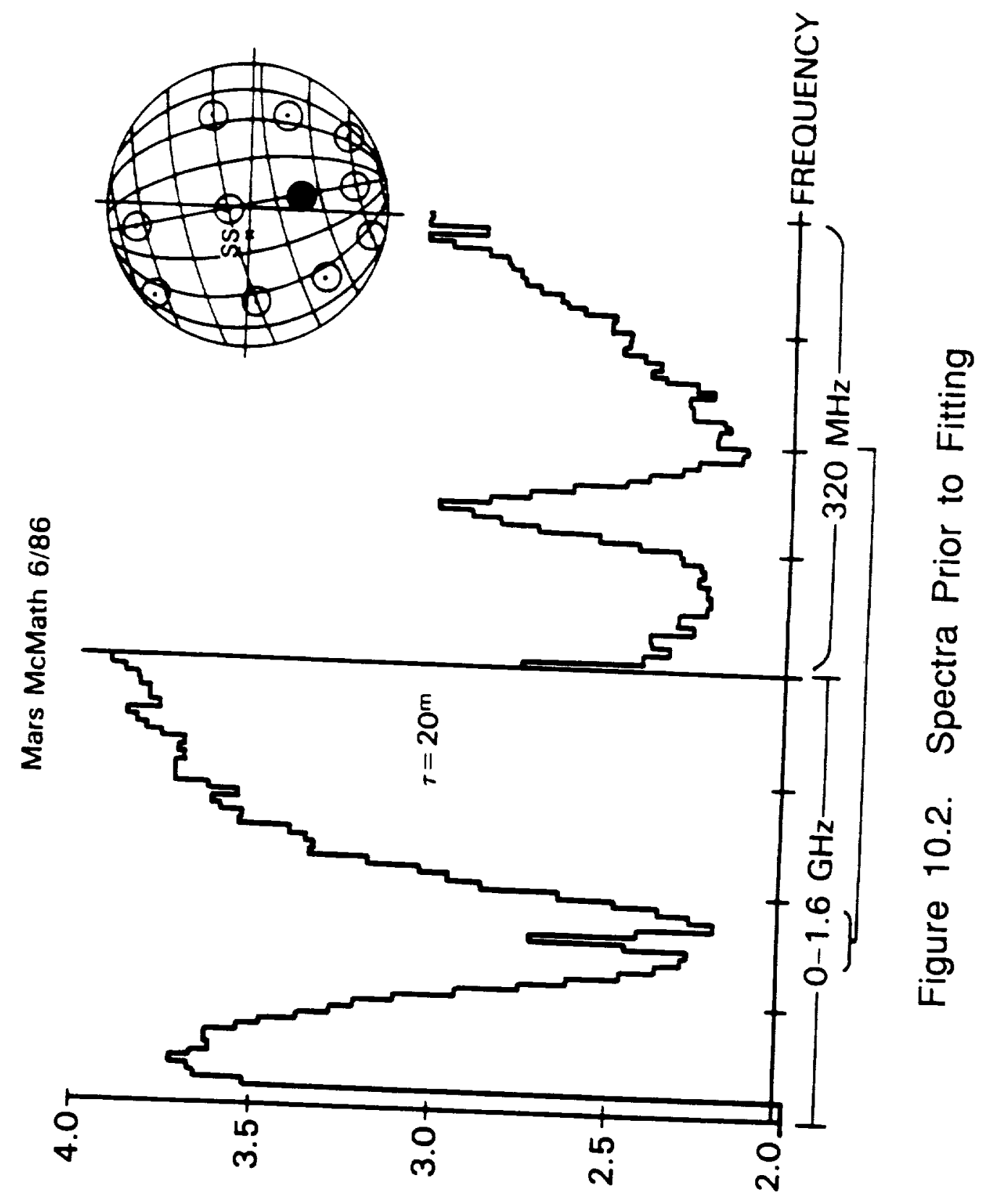

นISN $\exists \perp N I ~ \exists \wedge \perp \forall 7 \exists Y$ 
Interferometer Spectrometer (IRIS) data (a -20 K equator-to-pole decrease at $1 \mathrm{mb}$ ), and the absence of a longitudinal effect are consistent with such a dynamical state (Hanel et al. 1981, Flasar et al. 1981). Invoking cyclostrophic balance dictates a characteristic zonal velocity of $75-100 \mathrm{~m} / \mathrm{s}$ consistent with a $1.9-2.5^{\mathrm{d}}$ period (compared to an equatorial solid-planet rotation of $12 \mathrm{~m} / \mathrm{s}$; Hunten et al. 1984, Hanel et al. 1981). Due to a Titanian obliquity of $-26.7^{\circ}$, a strong seasonal variation in stratospheric zonal flow is expected unlike Venus.

As the only planetary satellite possessing a significant atmosphere (surface pressure -1.5 bar), Titan provides a fourth comparative planetological test case for terrestrial global circulation models. The dynamical constraints on Titan's atmosphere are borrowed from both Venus and Earth, defining Titanian circulation as a transitional case and therefore an important key to a comprehensive understanding of global circulation.

Direct confirmation of a seasonally varying Titanian zonal super-rotation would go far to support dynamics theory in the cyclostrophic regime. Unfortunately, there has been no direct detection of Titanian atmospheric motion. The absence to near absence of structure in Voyager visible images (Smith et al. 1981, 1982) precludes dynamical detection visually, and no spectroscopic studies with the necessary Doppler-limited spectral resolution have been undertaken.

Earth-based spectroscopic detection is limited to the determination of rotational broadening - atmospheric seeing coupled 
with a 0.67 to 0.83 arc-second Titanian disk all but exclude resolution of differential line shifts across the equator.

A number of molecular species present in the Titanian atmosphere $\left(\mathrm{C}_{2} \mathrm{H}_{6}, \mathrm{CH}_{4}, \mathrm{HCN}, \mathrm{C}_{2} \mathrm{H}_{4}, \mathrm{C}_{2} \mathrm{H}_{2}\right.$; Hunten et a1. 1984) have bands in the GSFC/IRHS 9-12.5 $\mu \mathrm{m}$ range (Park et al. 1987). The best candidate spectral probe, $\mathrm{C}_{2} \mathrm{H}_{6}$, is seen strongly in emission in Voyager IRIS spectra (Hunten et al. 1984). At $10 \mu \mathrm{m}$, an overall differential rotational broadening of $175-225 \mathrm{~m} / \mathrm{s}(17.5-22.5 \mathrm{MHz}$ ), (assuming a wind in the direction of solid-body rotation) dictates the need for a spectral resolving power of $10^{6}$. The GSFC/IRHS resolving power of $10^{7}$ and 0.8 arc-second beam - well matched to Titan's disk - are uniquely suited to the investigation. The $<1$ arc-second atmospheric seeing attained at IRTF in the past would produce minimal beam dilution. A series of scans first frequency normalized to one ephemeris time and then stacked would provide uniform coverage of the seeing disk and the $\mathrm{S} / \mathrm{N}$ necessary for line width determination.

An attempt at heterodyne detection of Titanian super-rotation, as suggested by Hunten et a1. (1984), has been targeted as a near term project.

\subsection{OTHER PLANETARY ATMOSPHERES}

Ground-based investigations of atmospheric dynamics on Jupiter and Saturn may be feasible in the near future. Presently, the low temperature environments of the outer planets require excessive integration time at $10 \mu \mathrm{m}$ to obtain $\mathrm{S} / \mathrm{N}$-limited wind velocities accurate to a few $\mathrm{m} / \mathrm{s}$ (see chapter 3 ). 
At $10 \mu \mathrm{m}, 230 \mathrm{~K}$ is a typical Venus continuum temperature ( $\mathrm{T}_{\mathrm{c}}$ ) (Kostiuk and Mumma 1983). From Figure 3.7, this implies a best-case (low resolution bank) $S / N=100$, assuming a $1^{h}$ integration. In the $300-900 \mathrm{~cm}^{-1}(11-30 \mu \mathrm{m})$ region, $\mathrm{T}_{c}=120-130 \mathrm{~K}$ for Jupiter (Hanel et al. 1979) and $T_{c}=90-100 \mathrm{~K}$ for Saturn (Hanel et al. 1983). At $10 \mu \mathrm{m}$ Figure 3.7 implies a $\mathrm{S} / \mathrm{N} \leq 1$ for both cases. Yet, a laser local oscillator longward of $10 \mu \mathrm{m}$ will sample higher intensity regions of the associated blackbody curve (see Fig. 3.7). A $\mathrm{S} / \mathrm{N}=100$ may be realized for Jupiter and Saturn, with a $1^{\text {h }}$ integration, if local oscillators at $-18 \mu \mathrm{m}$ and $\sim 23 \mu \mathrm{m}$ are employed, respectively. Spatial resolution will suffer only slightly. Assuming $30 \mu \mathrm{m}$ operation, a 2.4 arc-second diffraction limited beam (HPBW) at IRTF yields maximum seeing-excluded disk-to-beam ratios of -20 and -8 for Jupiter and Saturn, respectively.

Such longer wavelength local oscillators and mixers are under development (Kostiuk and Spears 1987) and Lamb dip stabilization is being explored.

\subsection{LABORATORY IR HETERODYNE SPECTROSCOPY}

Transition rest frequencies of species viable as probes of Titan, Jupiter and Saturn dynamics must be known to $0.1 \mathrm{MHz}$ at $10 \mu \mathrm{m}(0.05 \mathrm{MHz}$ at $20 \mu \mathrm{m}$ ) for wind accuracy at the $1 \mathrm{~m} / \mathrm{s}$ level. To date, this accuracy has only been realized for $\mathrm{CO}_{2}$ (Freed et al. 1980). In general, the Lamb dip system permits such measurements for any transition producible in the laboratory, provided it falls within the $1.6 \mathrm{GHz}$ photomixer bandwidth about an accessible laser line. 


\section{APPENDIX 1: DATA ACQUISITION AND ANALYSIS ALGORITHMS}

\section{A1.1. CORRECTION FOR SYSTEM OFFSET}

The different optical paths associated with the $s$ and $r$ beams within the telescope cause them to intercept either different portions of intervening optical elements or different elements completely. The corresponding variation in the emissivities and reflectivities of the optical surfaces within each beam leads to a non-negligible system offset. In general, system offsets are characterized by a significant slope across the filter bank bandpass. The resulting frequency shift in the observed spectral feature makes system offset removal mandatory for the generation of planetary wind fields at the $\mathrm{m} / \mathrm{s}$ level. Figure A1.1 is a schematic representation of the beam paths through the telescope optics with the optical elements numbered from the telescope focal plane outward toward the primary. Let $e_{i j}$ be the double sideband (DSB) emission contribution in watts/Hz and $k_{i j}$ the dimensionless reflection or transmission coefficient for element $i$ in beam $j$. Both $e_{i j}$ and $k_{i j}$ are assumed constant over the bandwidth of the filter bank. In addition, let $I\left(\nu^{\prime}\right)$ represent the DSB source spectral distribution and $b\left(\nu^{\prime}\right)$ the DSB background sky emission in the IF in units of watts/Hz. $b\left(\nu^{\prime}\right)$ is assumed to be due to un-Doppler shifted sky self-emission at $\nu_{10}$ and is therefore folded symetrically about $\nu^{\prime}=0$ in the IF. Given detector-preamp roll-off $\phi\left(\nu^{\prime}\right)$, post-detection noise level $\mathrm{N}\left(\nu^{\prime}\right)$, and post-detection zero level $\mathrm{z}\left(\nu^{\prime}\right)$, then the spectral distribution of power in the IF for both beams is: 


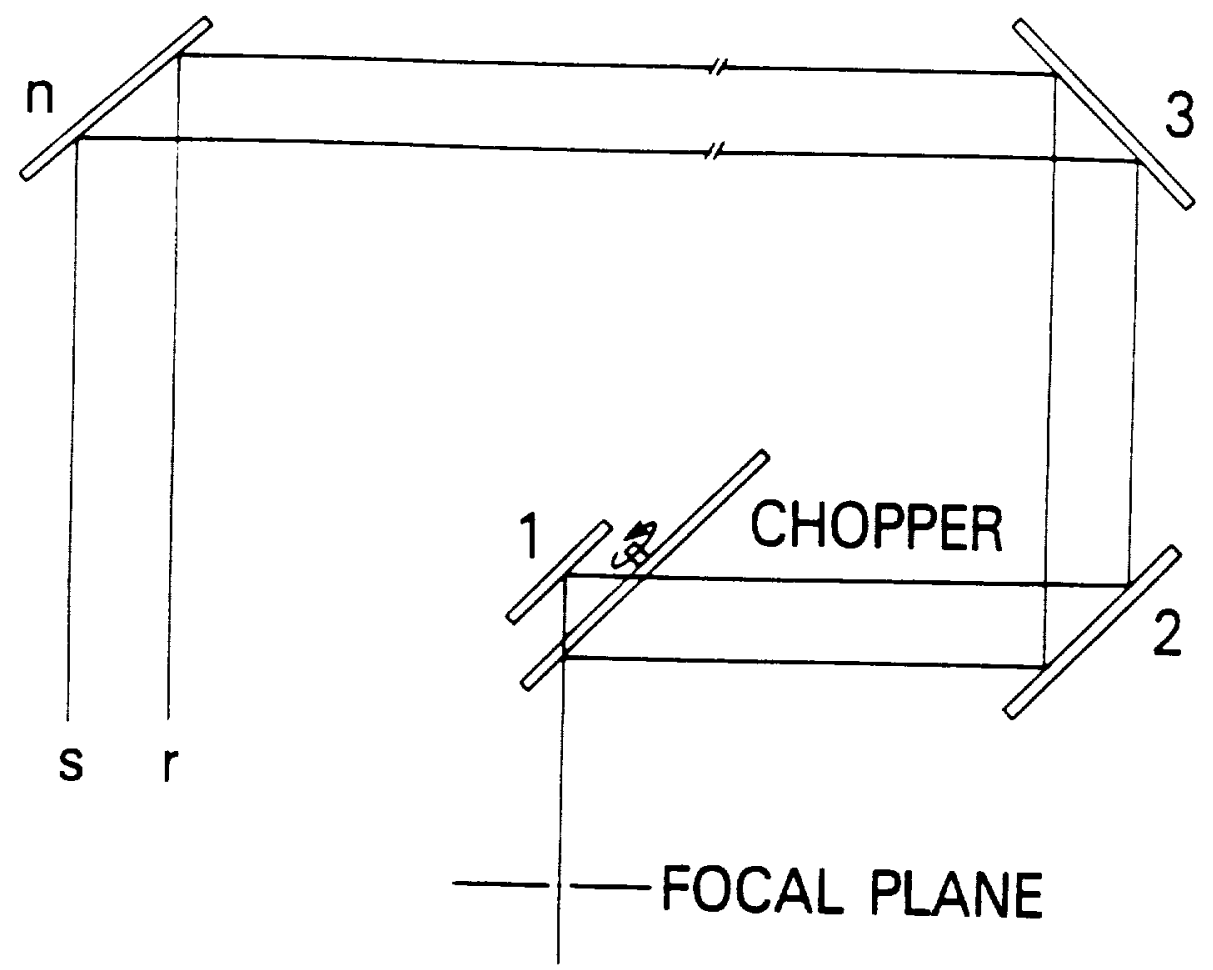

Figure A1.1. Beam Configuration for a Planetary Target

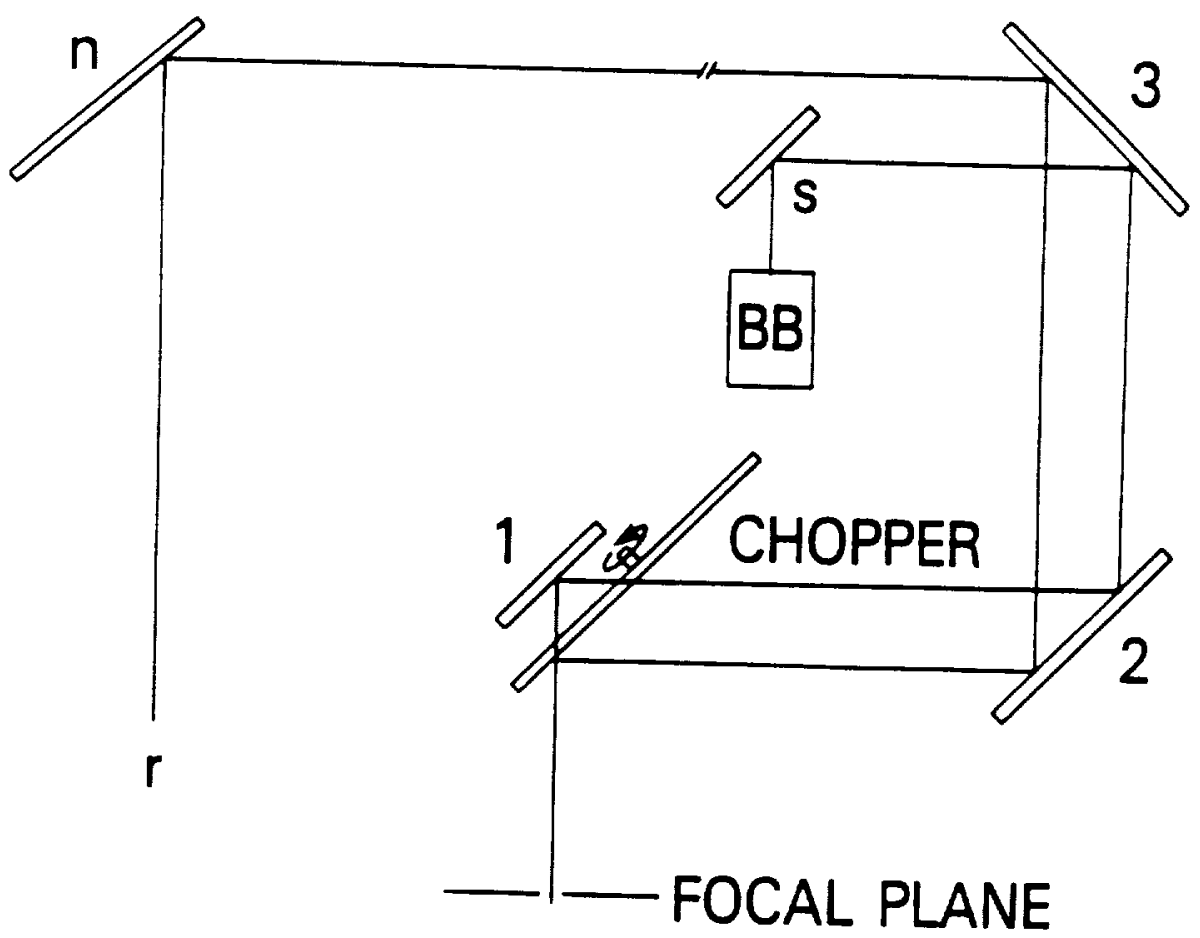

Figure A1.2. Beam Configuration for Blackbody Calibration 


$$
\begin{aligned}
& s\left(\nu^{\prime}\right)=
\end{aligned}
$$

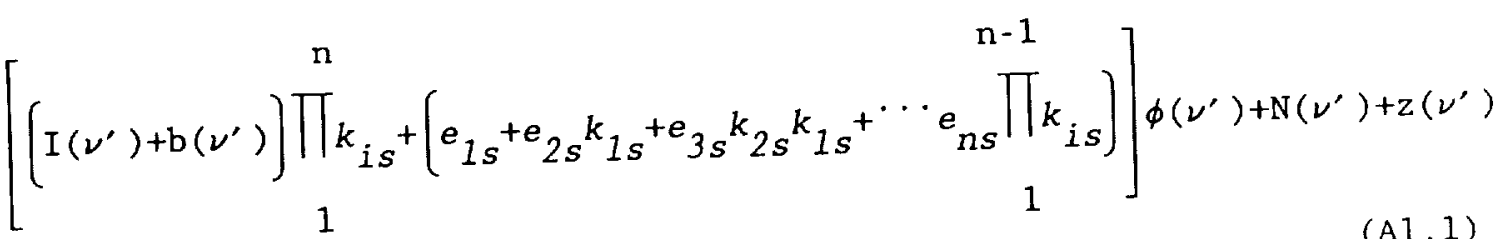

$$
\begin{aligned}
& r\left(\nu^{\prime}\right)=
\end{aligned}
$$

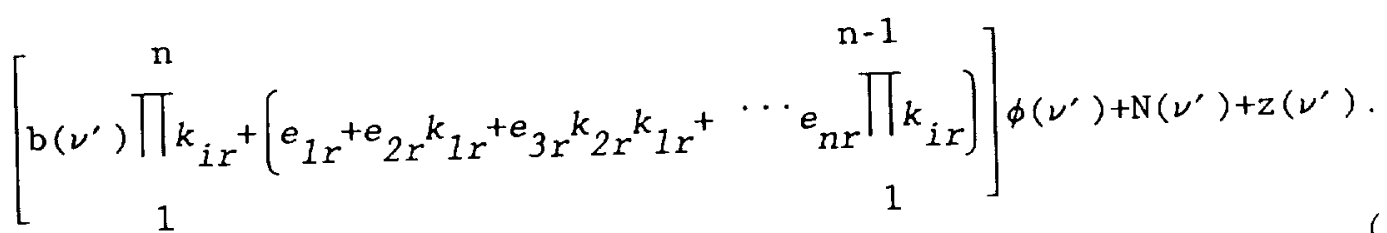

Eqs. A1.1 and A1.2 may be rewritten as:

$$
\begin{gathered}
s\left(\nu^{\prime}\right)=\left[\left(\mathrm{I}\left(\nu^{\prime}\right)+\mathrm{b}\left(\nu^{\prime}\right)\right) k_{s}+e_{s}\right] \phi\left(\nu^{\prime}\right)+\mathrm{N}\left(\nu^{\prime}\right)+\mathrm{z}\left(\nu^{\prime}\right), \\
r\left(\nu^{\prime}\right)=\left[\mathrm{b}\left(\nu^{\prime}\right) k_{r}+e_{r}\right] \phi\left(\nu^{\prime}\right)+\mathrm{N}\left(\nu^{\prime}\right)+\mathrm{z}\left(\nu^{\prime}\right)
\end{gathered}
$$

where $k_{s}, k_{r}$ and $e_{s}, e_{r}$ are the overall transmission-like and emission-like coefficients which define the telescope optics from the primary to the focal plane. In general, $k_{s} \neq k_{r}$ and $e_{s} \neq e_{r}$ which results in the system offset. The optical effects from the focal plane to the detector are applicable equally to the $s$ and $r$ beams for a properly aligned system, and can be embedded in $\phi\left(\nu^{\prime}\right)$ and $N\left(\nu^{\prime}\right)$.

The first task of the data reduction is to isolate the form of $I\left(\nu^{\prime}\right)$. Define an $S$ scan as:

$$
S=\frac{\left[s\left(\nu^{\prime}\right)-z\left(\nu^{\prime}\right)\right]-\left[r\left(\nu^{\prime}\right)-z\left(\nu^{\prime}\right)\right]}{r\left(\nu^{\prime}\right)-z\left(\nu^{\prime}\right)}
$$


or

$$
S=\frac{s\left(\nu^{\prime}\right)-r\left(\nu^{\prime}\right) .}{r\left(\nu^{\prime}\right)-z\left(\nu^{\prime}\right)}
$$

where $z\left(\nu^{\prime}\right)$ in A1.5 is independently determined at the beginning of each scan with the IF fully attenuated. Substituting Eqs. A1.3 and A1.4 into A1. 5 yields:

$$
S=\frac{\left[\left[\mathrm{I}\left(\nu^{\prime}\right)+\mathrm{b}\left(\nu^{\prime}\right)\right] k_{s}-\mathrm{b}\left(\nu^{\prime}\right) k_{r}+e_{s}-e_{r}\right] \phi\left(\nu^{\prime}\right)}{\left[\mathrm{b}\left(\nu^{\prime}\right) k_{r}+e_{r}\right] \phi\left(\nu^{\prime}\right)+\mathrm{N}\left(\nu^{\prime}\right)}
$$

For a weak source such as a planetary target, $I\left(\nu^{\prime}\right) k_{s} \phi\left(\nu^{\prime}\right)$ can be of the same magnitude as the other terms in the numerator. The latter terms may be removed in the following manner.

In the field, periodically moving the $s$ beam off the target and integrating on sky versus sky, in the vicinity of the target, allows one to generate a discretely continuous record of the system offset. A sky versus sky scan $s_{s k y}$ is of the form:

$$
s_{s k y}=\frac{\left[\mathrm{b}\left(\nu^{\prime}\right) k_{s}-\mathrm{b}\left(\nu^{\prime}\right) k_{r}+e_{s}-e_{r}\right] \phi\left(\nu^{\prime}\right)}{\left[\mathrm{b}\left(\nu^{\prime}\right) k_{r}+e_{r}\right] \phi\left(\nu^{\prime}\right)+\mathrm{N}\left(\nu^{\prime}\right)} .
$$

Subtracting Eq. A1.7 from A1.6 yields:

$$
S-S_{s k y}=\frac{I\left(\nu^{\prime}\right) k_{s} \phi\left(\nu^{\prime}\right)}{\left[\mathrm{b}\left(\nu^{\prime}\right) k_{r}+e_{r}\right] \phi\left(\nu^{\prime}\right)+\mathrm{N}\left(\nu^{\prime}\right)} .
$$


To finally isolate $I\left(\nu^{\prime}\right)$, a blackbody calibration scan $S_{B B}$ is used. Figure A1.2 is a schematic representation of the optical paths associated with the blackbody calibration configuration. If $\mathrm{I}_{\mathrm{BB}}$ is the DSB constant continuum level of the blackbody in the IF then:

$$
S_{B B}=\frac{\left[\mathrm{I}_{\mathrm{BB}} k^{\prime} s^{\left.-\mathrm{b}\left(\nu^{\prime}\right) k_{r}+\mathrm{e}^{\prime} s^{-} e_{r}\right] \phi\left(\nu^{\prime}\right)}\right.}{\left[\mathrm{b}\left(\nu^{\prime}\right) k_{r}+e_{r}\right] \phi\left(\nu^{\prime}\right)+\mathrm{N}\left(\nu^{\prime}\right)} .
$$

Note that $\mathrm{k}^{\prime}{ }_{s}$ and $\mathrm{e}^{\prime}{ }_{s}$ are different from $k_{s}$ and $e_{s}$ due to the different optical path for the $s$ beam during blackbody calibration. Dividing Eq. A1. 8 by A1. 9 gives:

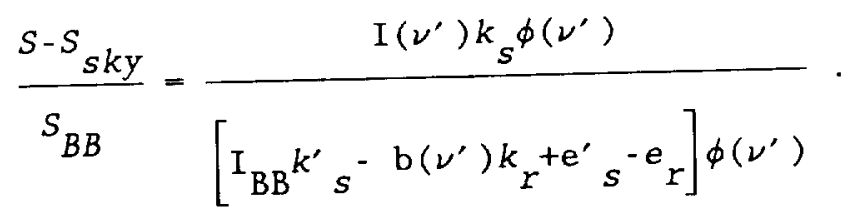

The blackbody calibration scan must necessarily be taken at the same time the source and sky versus sky scans are taken to insure the same system offset. To this end, a typical set of measurements for the wind investigation consisted of 10 two minute integrations on the source followed by a sky versus sky and a blackbody calibration scan. The GSFC IRHS employs calibration blackbodies at temperatures $\geq 1000 \mathrm{~K}$. At these temperatures the blackbody term in the denominator of Eq. A1.10 dominates so that Eq. Al.10 reduces to: 


$$
\frac{S-S_{s k y}}{S_{B B}}=\frac{I\left(\nu^{\prime}\right)}{\mathrm{I}_{\mathrm{BB}}} \frac{k_{s}}{k^{\prime}{ }_{s}} .
$$

With $k_{s} / k^{\prime} s^{-1}$, Eq. Al.11 provides the DSB source spectral distribution in the IF in units of the DSB blackbody continuum level. If the temperature of the blackbody is known, $I\left(\nu^{\prime}\right)$ can be absolutely calibrated. Since sky is associated with large noise fluctuations, a quadratic fit to $S_{s k y} / S_{B B}$ is actually employed in Eq. Al.11.

\section{A1.2. CORRECTION FOR TERRESTRIAL ABSORPTION}

Consider a source with a spectral intensity distribution $\grave{I}(\nu)$ prior to transmission through Earth's atmosphere. The spectral intensity distribution post transmission $I(\nu)$ is given by the equation of radiative transfer:

$$
I(\nu)=\grave{I}(\nu) \exp \left[-\int S(x) f(x, \nu) d x\right)
$$

where $S(x)$ and $f(x, \nu)$ are the terrestrial integrated absorption coefficient and normalized line shape function as functions of position $\mathrm{x}$ along the beam path. (Atmospheric self-emission has already been addressed.)

If one assumes a plane parallel atmosphere where the above quantities vary only with altitude $r$, then:

$$
\mathrm{dx}=\frac{\mathrm{dr}}{\cos \theta}
$$

where $\theta$ is the zenith angle of the beam. Substituting Eq. A1.13 into Eq. A1. 12: 


$$
I(\nu)=\grave{I}(\nu) \exp \left(-\int \frac{S(r) f(r, \nu) d r}{\cos \theta}\right),
$$

or:

$$
I(\nu)=\grave{I}(\nu) \exp \left(-\tau_{r}(\nu) / \cos \theta\right)
$$

where $\tau_{r}(\nu)$ is the optical depth at zenith as a function of $\nu$.

The optical depth along the beam path, $\tau_{x}(\nu)$, is given by:

$$
\tau_{\mathrm{x}}(\nu)=\frac{\tau_{\mathrm{r}}(\nu)}{\cos \theta}=\left(\frac{{ }_{\mathrm{x}}^{\mathrm{a}}}{\mathrm{a}_{\mathrm{r}}}\right) \tau_{\mathrm{r}}(\nu)
$$

where $a_{r}$, defined as unity, is the air mass at zenith and $a_{x}$ is the air mass along the beam path.

The source spectral distribution is mapped into the IF via Eq. 3.1:

$$
I(\nu)+I\left(2 \nu 10^{-\nu}\right) \rightarrow I\left(\nu^{\prime}\right)
$$

Substituting Eqs. Al.15 and Al.16 into 3.1 gives:

$$
\grave{I}(\nu) \exp \left(-a_{x^{\tau} r}(\nu)\right)+\grave{I}\left(2 \nu 10^{-\nu)} \exp \left(-a_{x^{\tau} r(2 \nu} 10^{-\nu)}\right) \rightarrow I\left(\nu^{\prime}\right)\right.
$$

If terrestrial absorption is due solely to the terrestrial $\mathrm{CO}_{2}$ line at $\nu_{10}$ then its effects are symmetrically folded about $\nu_{10}$ and $\tau_{\mathrm{r}}(\nu)=\tau_{\mathrm{r}}\left(2 \nu_{10^{-\nu}}\right)$ so that Eq. A1.17 becomes:

$$
\left(\grave{I}(\nu)+\grave{I}\left(2 \nu_{10}-\nu\right)\right) \exp \left(-a_{x}{ }^{\tau} r^{(\nu)}\right) \rightarrow I\left(\nu^{\prime}\right)
$$

Noting $\tau_{\mathrm{r}}(\nu)=\tau \mathrm{r}^{\left(\nu^{\prime}\right)}$ in the IF and rearranging yields: 


$$
\grave{I}(\nu)+\grave{I}\left(2 \nu 10^{-\nu}\right) \rightarrow \mathrm{I}\left(\nu^{\prime}\right) \exp \left(\mathrm{a}^{r} \mathrm{r}^{\left(\nu^{\prime}\right)}\right) \equiv \grave{\mathrm{I}}\left(\nu^{\prime}\right)
$$

Eq. Al.19 represents the DSB mapping into the IF of the source spectral distribution at the top of Earth's atmosphere, or equivalently, $I\left(\nu^{\prime}\right) \exp \left[a_{x}{ }^{T}{ }\left(\nu^{\prime}\right)\right]$ represents the DSB source spectrum corrected for terrestrial absorption. While $\mathrm{I}\left(\nu^{\prime}\right)$ is given by Eq. Al.11 in calibrated blackbody units, and $a_{x}$ may be determined for the source from the ephemeris, ${ }^{\tau}{ }_{\mathrm{r}}\left(\nu^{\prime}\right)$ must still be determined to correct for terrestrial absorption.

${ }_{r}\left(\nu^{\prime}\right)$ may be found by observing a continuum source with good $S / N$ at two different air masses. Such a source is characterized by a constant continuum level across the heterodyne bandpass which includes both single sidebands $\left(S S^{\prime} s\right)$. The Moon or Mercury are ideal. If $I_{\mathcal{L}}$ represents the lunar continuum level at the top of Earth's atmosphere, then from Eq. Al.19,

$$
2 I_{L}=I_{L}\left(\nu^{\prime}\right) \exp \left(a_{L}{ }^{T}\left(\nu^{\prime}\right)\right)
$$

Solving for $\tau_{\mathrm{r}}\left(\nu^{\prime}\right)$ :

$$
{ }_{r}{ }\left(\nu^{\prime}\right)=-\frac{\ln I_{L}\left(\nu^{\prime}\right)}{a_{L}}+\frac{\ln 2 I_{L}}{a_{L}} .
$$

From Eq. Al.11, assuming $k_{s} / k_{s}^{\prime}=1$,

$$
I_{\mathcal{L}}\left(\nu^{\prime}\right)=\left(\frac{S_{\mathcal{L}}^{-S_{\mathcal{L} k K y}}}{S_{B B \mathcal{L}}}\right) I_{\mathrm{BBL}} .
$$

Substituting A1.22 into Al.21 yields: 


$$
\begin{gathered}
r_{\mathrm{r}}\left(\nu^{\prime}\right)=\frac{-1}{\mathrm{a}_{\mathcal{L}}}\left(\frac{S_{\mathcal{L}}^{-S_{\mathcal{L} k \mathrm{k}}}}{S_{B B \mathcal{L}}}\right)+\mathrm{K}, \\
\mathrm{K}=\frac{-1}{\mathrm{a}_{\mathcal{L}}} \ln \left(\frac{\mathrm{I}_{\mathrm{BB} \mathcal{L}}}{2 \mathrm{I}_{\mathcal{L}}}\right) .
\end{gathered}
$$

where:

The value of $K$ is indeterminate for a single lunar observation since the lunar blackbody continuum leve $I_{L}$ is unknown. $I_{\mathcal{L}}$ strongly depends upon the aspect geometry of the Earth, Moon and Sun as well as the beam position on the Moon. If, however, a second lunar observation at a quite different air mass is made at the same beam position, a second independent equation of the form of Eq. A1.23 results, a1lowing both $\mathrm{K}$ and $r_{\mathrm{r}}\left(\nu^{\prime}\right)$ to be determined.

If a second lunar scan is unavailable, solar Fourier Transform Spectrometer (FTS) data provides an alternate means of determining $K$. FTS solar spectra taken at the McMath Solar Telescope at an atmospheric pressure of $785 \mathrm{mb}$ yield an optical depth of 0.199 at zenith, $850 \mathrm{MHz}$ from the center of the ${ }^{12} \mathrm{C}^{16} \mathrm{O}_{2} \Sigma_{\mathrm{u}}{ }^{+} 00^{\circ} 1-\Sigma_{\mathrm{g}}{ }^{+}\left[10^{\circ} 0,02^{\circ} 0\right]_{\mathrm{I}}$ R8 terrestrial line (from an unpublished spectrum taken by D.A. Glenar), which is the line used to probe the lower thermosphere of venus. $\tau_{r}(850)$ for a specific observing station may be obtained from the FTS derived value by scaling linearly with atmospheric pressure. By substituting the appropriate $\tau_{\mathrm{r}}(850)$ and the value of $\left(\mathrm{S}_{\mathcal{L}}-\mathrm{S}_{\mathcal{L S k y}}\right) / \mathrm{S}_{\mathcal{L B B}}$ at $850 \mathrm{MHz}$ into Eq. Al.23, $\mathrm{K}$ may be found. While the latter method is a less precise determination of $\mathrm{K}$, only the scale factor associated with the source spectral distribution is affected. The line center frequency, which is 
the crucial parameter needed for wind velocity determination, remains unchanged .

Since ${ }^{\tau} r^{\left(\nu^{\prime}\right)}$ (given by Eq. A1.23) is generated from lunar or Mercurian data, it is necessarily noisy. Fitting it to a cubic, prior to generation of $\dot{\mathrm{I}}\left(\nu^{\prime}\right)$ (see Eq. A1.19), provides a more accurate model of $r_{Y}\left(\nu^{\prime}\right)$, as long as the region of interest in the IF is a few hundred MHz from the local oscillator. Figure Al.3 is typical of a lunar scan in the form $\left(\mathrm{S}_{\mathcal{L}}-\mathrm{S}_{\mathcal{L} \text { sky }}\right) / \mathrm{S}_{\mathcal{L B B}}$. The ordinate is in calibrated blackbody units. The Figure's left side provides the spectrum in the low resolution bank (LRB), with an abscissa marked off in units of sixteen $25 \mathrm{MHz}$ channels and spanning 0 to $1.6 \mathrm{GHz}$. The LO is mapped into the origin. The vertical dotted lines provide the high resolution bank (HRB) "window". The spectrum in the HRB appears on the Figure's right side. Here the abscissa is marked off in units of sixteen 5 $\mathrm{MHz}$ channels, its sense reversed, as shown in Fig. 3.10 (see Section 3.4).

The cubic fit to $r_{r}\left(\nu^{\prime}\right)$ is shown in Figure A1.4.

\section{A1.3. CORRECTION FOR DIFFERENTIAL DOPPLER SHIFTS}

IRHS measurement of a planetary wind field is limited by the line of sight wind velocity resolution $\Delta \mathrm{Vm} / \mathrm{s}$ (or $0.1 \Delta \mathrm{V} \mathrm{MHz}$ at $10 \mu \mathrm{m}$ ). $\Delta \mathrm{V}$ is constrained by $\mathrm{S} / \mathrm{N}$-induced uncertainty in IF line center frequency, and uncertainties in LO frequency, emission rest frequency and the ephemeris-derived line of sight velocity between the Earth station and target planet. For this investigation a $\Delta V \sim 1 \mathrm{~m} / \mathrm{s}$ was achieved.

If $\Delta T$ is the integration time necessary for adequate $S / N$ at a 


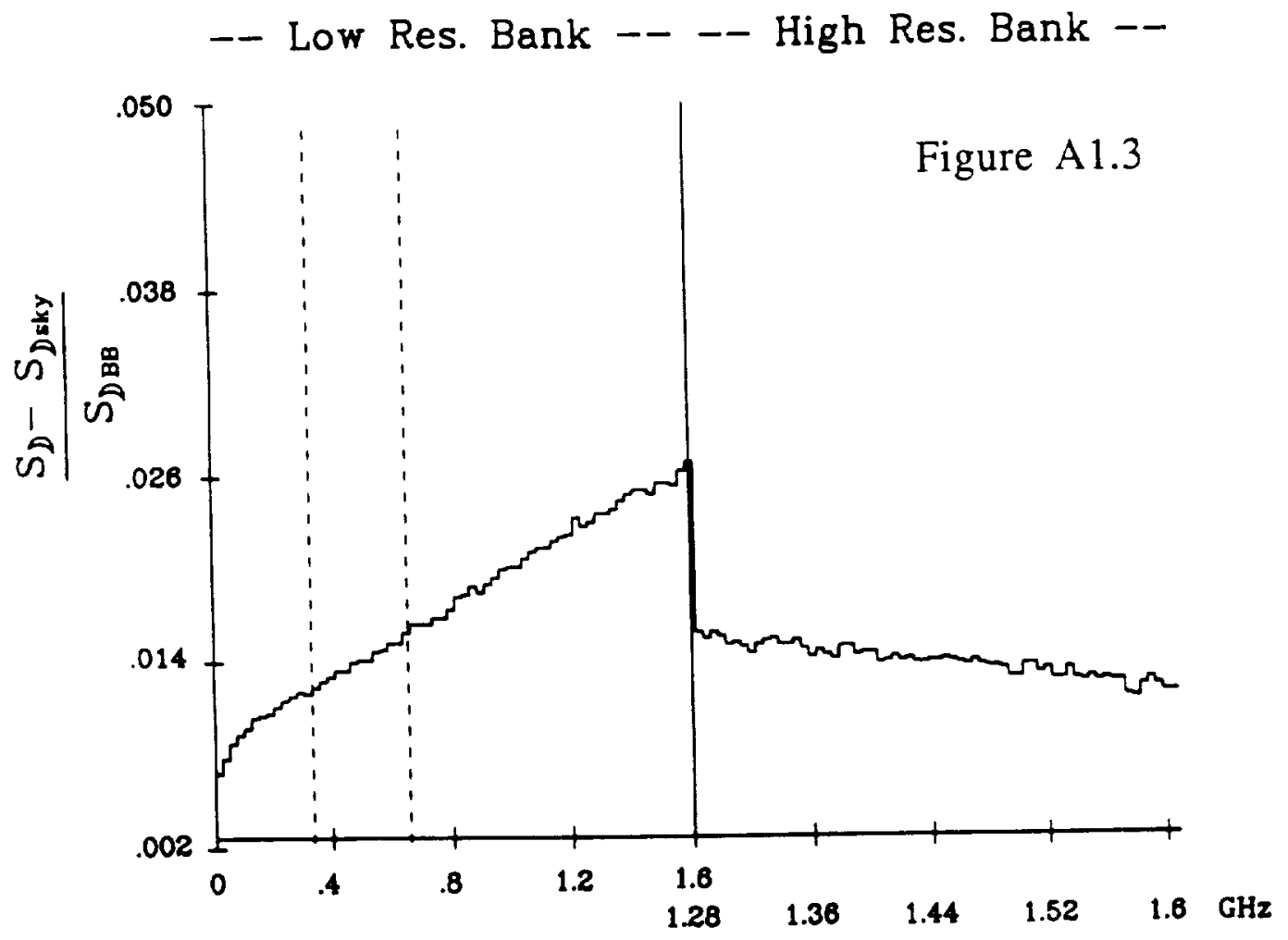

Figure A1.3

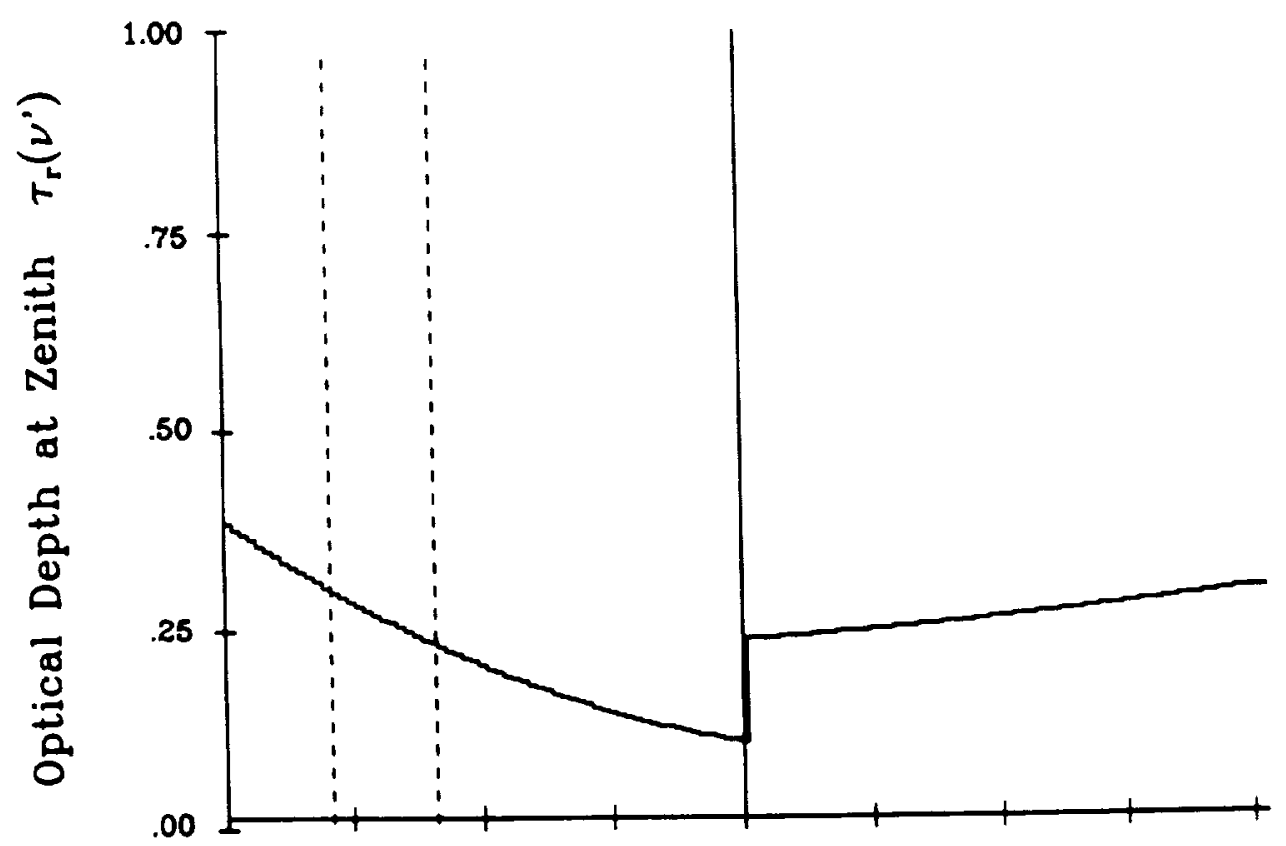

Figure $\mathrm{A} 1.4$ 
given beam position, and $\Delta \nu_{E}$ and $\Delta \nu_{P}$ are the Doppler shifts associated with the line of sight velocity components of Earth rotation $\left(V_{E}\right)$ and planet-planet center-of-mass translation $\left(V_{P}\right)$ respectively, then the associated differential Doppler shifts over $\Delta \mathrm{T}$ are:

$$
\int_{0}^{\Delta \mathrm{T}} \frac{\delta \Delta \nu_{\mathrm{E}} \mathrm{dt}}{\delta \mathrm{t}}-0.1 \int_{0}^{\Delta \mathrm{T}} \frac{\delta \mathrm{V}_{\mathrm{E}} \mathrm{dt}}{\delta \mathrm{t}}
$$

and

$$
\int_{0}^{\Delta \mathrm{T}} \frac{\delta \Delta{ }_{\mathrm{P}} \mathrm{dt}}{\delta t}-0.1 \int_{0}^{\Delta \mathrm{T}} \frac{\delta \mathrm{V}_{\mathrm{P}} \mathrm{dt}}{\delta \mathrm{t}}
$$

For Venus, $\delta \mathrm{V}_{\mathrm{P}} / \delta \mathrm{t}<0.4 \mathrm{~m} / \mathrm{s}$ per minute, where the maximum line of sight acceleration occurs at inferior and superior conjunction.

The line of sight velocity component due to Earth rotation is given to first order by:

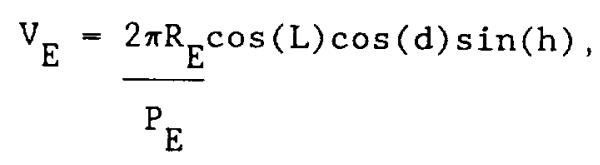

where $R_{E}$ is the radius of Earth, $P_{E}$ is the sidereal rotation period of Earth, L is the observing station latitude, $d$ is the target planet declination, and $h$ is the hour angle of the planet. Differentiating Eq. Al.26, assuming constant $L$ and $d$, yields the approximate line of sight acceleration $\delta \mathrm{V}_{\mathrm{E}} / \delta \mathrm{t}$. For a given $\mathrm{L}$ and $\mathrm{d}, \delta \mathrm{V}_{\mathrm{E}} / \delta \mathrm{t}$ is maximal at transit such that $\delta V_{E} / \delta t \leq 2 \mathrm{~m} / \mathrm{s}$ per minute.

An integration time $\Delta \mathrm{T}$ from $\sim 10^{\mathrm{m}}$ to $\sim 1^{\mathrm{h}}$ was necessary for IF 1 ine center determination to order $1 \mathrm{~m} / \mathrm{s}$. From Eqs. A1.24 and A1.25, with 
$\Delta \mathrm{T}=1 \mathrm{~h}$, the differential Doppler shifts are maximally $24 \mathrm{~m} / \mathrm{s}(2.4 \mathrm{MHz})$ and $120 \mathrm{~m} / \mathrm{s}(12.0 \mathrm{MHz})$ for planet-planet translation and Earth rotation respectively. The result is a broadening of the spectral feature by up to two orders of magnitude over the desired wind velocity resolution.

If line of sight accelerations and emission intensity are constant over $\Delta \mathrm{T}$, the spectral feature's centroid can be associated with the ephemeris-derived $V_{E}$ and $V_{P}$ at the mid-time of the integration period, allowing wind velacity acquisition at the desired resolution. In general, however, for $\Delta \mathrm{T}=1^{\mathrm{h}}$ :

1) accelerations cannot be taken as constant (especially for Earth rotation), and

2) temporally random variation in emission intensity due to, e.g., variation in terrestrial aerosol column density in the beam, can occur on timescales of seconds,

thereby prohibiting association of specific $V_{E}$ and $V_{P}$ with the integrated spectral feature.

To circumvent this problem, $\Delta T$ necessary for a specific wind measurement was obtained by stacking a set of consecutive scans, each of $2^{\mathrm{m}}$ duration. Over $2^{\mathrm{m}}$, line of sight accelerations can be taken as constant and if unacceptably large variation in emission strength is observed, the effected scan can be omitted from the stack without significant loss of time on the source. The spectral contribution from each scan can be associated with the Doppler shifts corresponding to the scan midpoint time. Defining the first scan in the stack as a normalization scan, the known differential Doppler shifts between each scan and the normalization scan may be removed. Coadding of the scan 
set results in a spectral feature with a centroid characterized by the normalization Doppler shift and a $\mathrm{S} / \mathrm{N}$ yielding the desired wind resolution. Since high resolution filter bank channels are $5 \mathrm{MHz}$ wide or $-50 \mathrm{~m} / \mathrm{s}$ at $10 \mu \mathrm{m}$, differential Doppler shift removal requires shifting each scan by fractions of a channel.

The upper graph of Figure A1.5 represents an emission line in the IF obtained from the first of a series of consecutive $2^{m}$ scans. The center frequencies of the filter bank channels are $\nu_{1}, \nu_{2}, \nu_{3} \ldots \nu_{n}$. The observed line center Doppler shift $\Delta \nu_{1}$ from the LO at $0 \mathrm{MHz}$ contains $\Delta \nu_{E}, \Delta \nu_{P}$ as well as the target planet's line of sight rotational and wind velocity components within the beam. Assuming the wind component remains constant over the entire scan set, the next consecutive scan, shown in the second graph, will be associated with a different overall Doppler shift $\Delta \nu_{2}$ due to variation in $\Delta \nu_{E}$ and $\Delta \nu_{P}$. We can define the differential Doppler shift between the first scan and the $j^{\text {th }}$ as:

$$
\delta \Delta \nu_{1 j}-\Delta \nu_{1}-\Delta \nu_{j}
$$

To coadd the second scan to the first requires shifting the second scan by $\delta \Delta \nu_{12}$, as shown in the third graph of Figure Al.5, thereby normalizing it to the ephemeris circumstances associated with the first scan. To add the scans now requires a proper accounting of the second scan's two contributions to each channel in the discrete frequency space of the first scan (shaded and unshaded regions in the last graph).

Let $f_{j}\left(\nu_{i}\right)$ be the histogram distribution function for scan $j$, 

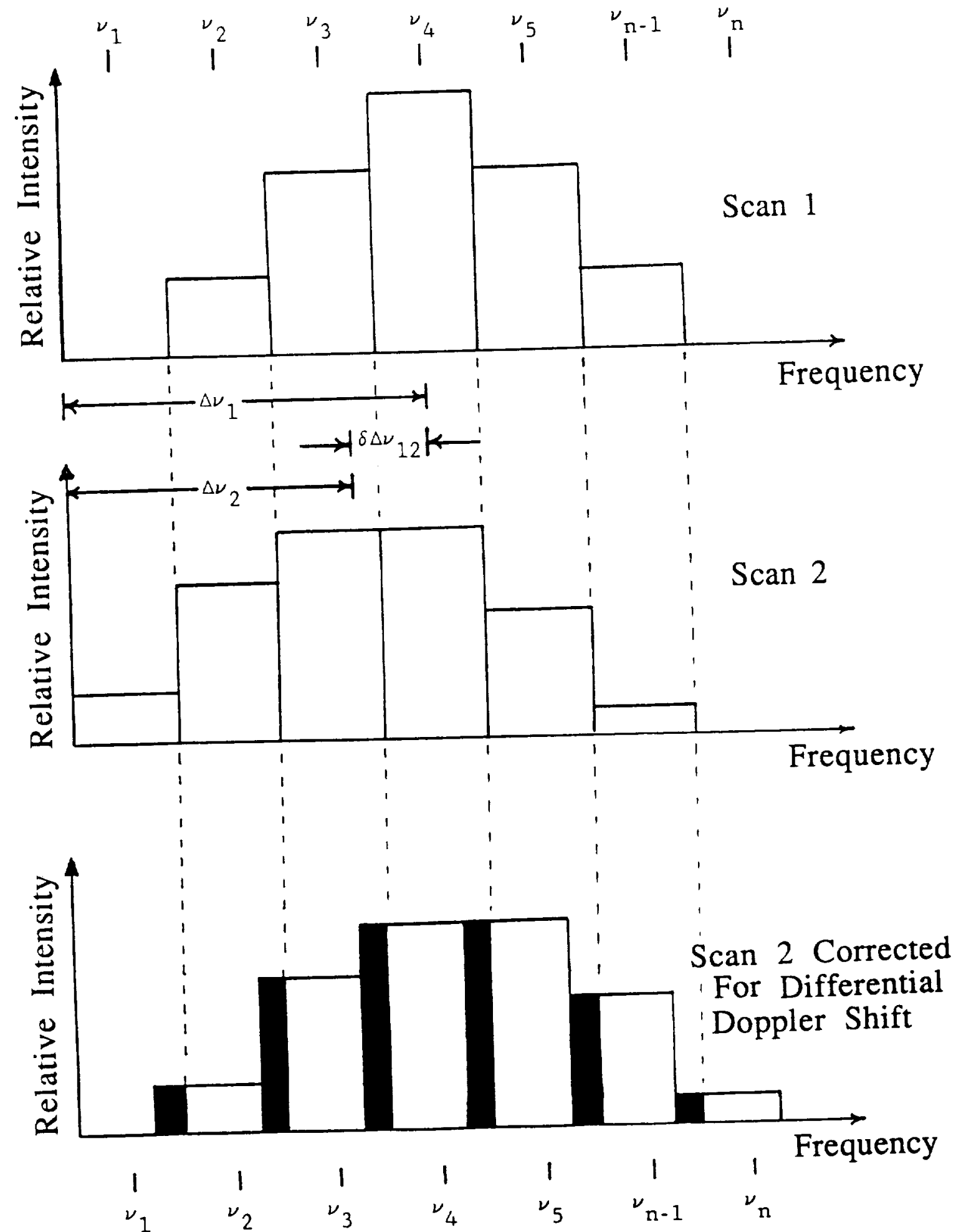

Figure A1.5. Differential Doppler Shift Removal 
$g\left(\nu_{i}\right)$ the distribution function of the sum of the scans and $B$ the channel bandwidth in $\mathrm{MHz}$. Scan $\mathrm{f}_{j}\left(\nu_{i}\right)$, appropriately shifted by $\delta \Delta \nu_{1 j}$ to correct for its differential Doppler shift, may be viewed from the discrete frequency space of the normalization scan as the sum:

$$
\alpha f_{j}\left(\nu_{i}\right)+\beta f_{j}\left(\nu_{i}-k B\right)
$$

where $k=1$ for $\Delta \nu_{j} / \Delta \nu_{1}<1$ and $k=-1$ for $\Delta \nu_{j} / \Delta \nu_{1}>1$, and $\alpha, \beta$ are constants. From the last graph of Fig. A1.5 one can see that:

and

$$
\alpha=\frac{B-\left|\delta \Delta \nu_{1 j}\right|}{B},
$$

$$
\beta=\frac{\left|\delta \Delta \nu_{1 j}\right|}{B} \text { or } \beta=1-\alpha \text {. }
$$

Summing Eq. Al.28 over all $\mathrm{N}$ scans in the set gives:

$$
g\left(\nu_{i}\right)=f_{1}\left(\nu_{i}\right)+\sum_{j=2}^{N}\left((1-\beta) f_{j}\left(\nu_{i}\right)+\beta f_{j}\left(\nu_{i}-k B\right)\right) .
$$

Substituting Eq. A1.30 into A1.31 and rearranging yields:

$$
g\left(\nu_{i}\right)=f_{1}\left(\nu_{i}\right)+\sum_{j=2}^{N} f_{j}\left(\nu_{i}\right)+\sum_{-}^{1} \sum_{j=2}^{N}\left|\delta \Delta \nu_{1 j}\right|\left(f_{j}\left(\nu_{i}-k B\right)-f_{j}\left(\nu_{i}\right)\right) .
$$

This procedure is easily extended for the case where $\delta \Delta \nu_{1 N}>B$.

Figure Al. 6 provides an overview of the data reduction up to this point as it pertains to typical Venus data. The ordinates are in units of the calibrated blackbody. In Figure A1.6a the linear plot is the 

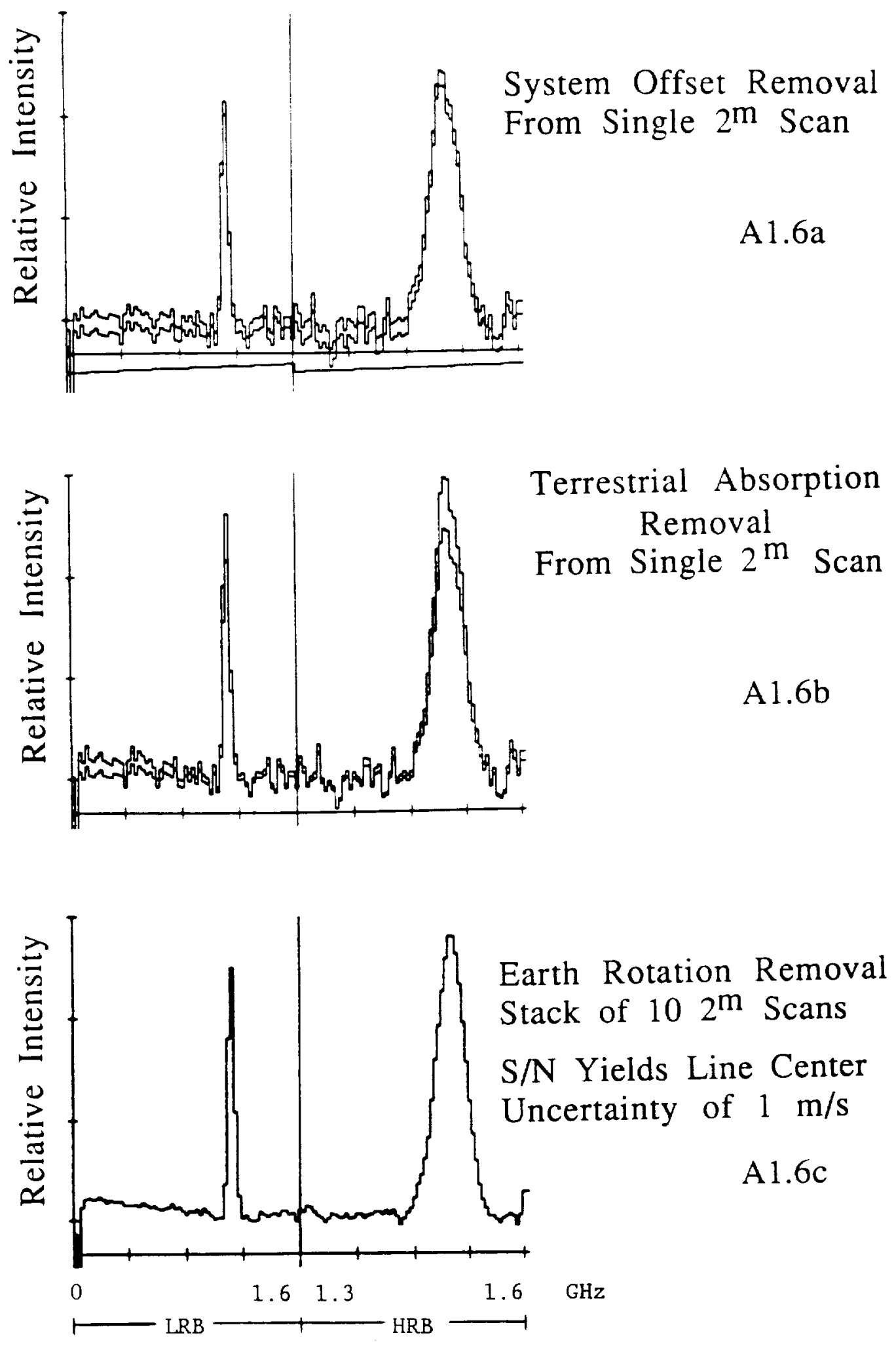

Figure A1.6. Data Reduction - Venus 
system offset, the lower emission feature is the result of a single $2^{\text {m }}$ integration, and the upper emission feature is the same $2^{\mathrm{m}}$ scan after system offset removal. The system offset corrected feature is in turn the lower plot in Figure A1.6b while in the upper plot it has been corrected for terrestrial absorption. Shifting and stacking 10 consecutive scans finally yields the emission feature illustrated in Figure $\mathrm{A} 1.6 \mathrm{C}$ which has a $\mathrm{S} / \mathrm{N}$ allowing line center determination in the IF to order $1 \mathrm{~m} / \mathrm{s}$. The data shown have not been smoothed. Note the shallow absorption feature in Fig. A1.6c.

\section{A1.4. LINE FITTING}

After differential Doppler shift removal the Venusian data may be fit to a Voigt profile. Models for the non-thermal emission in the cores of the Venusian 9.4 and $10.4 \mu \mathrm{m} \mathrm{CO}$ bands imply a thickness of only $19 \mathrm{~km}$ for the emitting region at a pressure of $5 \mu \mathrm{b}$ (Johnson et al. 1976, Betz 1977, Deming et a1. 1983). Gaussian profiles for the emissivity $j_{\nu}$ and absorption coefficient $k_{\nu}$ may be assumed at this pressure. Integration of the radiative transfer equation along a normal path, holding $\mathrm{j}_{\nu} / \mathrm{k}_{\nu}$ constant over the narrow emitting layer, gives:

$$
\mathrm{I}_{\nu}=\mathrm{I}_{\nu}{ }^{\mathrm{o}} \exp \left(-\tau_{\nu}\right)-\frac{\mathrm{j}_{\nu}}{\mathrm{k}_{\nu}}\left(\exp \left(-\tau_{\nu}\right)-1\right)
$$

where $I_{\nu}$ and $I_{\nu}{ }^{\circ}$ are the intensities at the top and bottom of the layer respectively. Near the core of the absorption feature temperatures are 
low enough so that $\mathrm{I}_{\nu}{ }^{\circ} \sim 0$. In addition, the emitting region is optically thin allowing the approximation $\exp \left(-\tau_{\nu}\right)-\left(1-\tau_{\nu}\right)$.

Substituting into Eq. A1.33 gives:

$$
\mathrm{I}_{\nu}=\frac{\mathrm{j}_{\nu}{ }^{\top} \mathrm{k}_{\nu}}{} .
$$

Noticing that $\tau_{\nu}=k_{\nu} r$ where $r$ is the normal path length reduces Eq. A1. 34 to:

$$
I_{\nu}=j_{\nu} r=r J f_{D}
$$

where $J$ is the line integrated emissivity and $f_{D}$ is the normalized line shape function. Eq. Al.35 provides the rationale for a Voigt fit to $I_{\nu}$

A program called PLOTOBS, based on the routines given in Bevington (1969), was employed to provide a non-linear fit to a Voigt profile. Specifically, the fit to the HRB data, with 5 fold better resolution than the LRB, was used for wind velocity determination.

For most of the data, PLOTOBS used a flat baseline to represent the continuum level. For the case where a shallow underlying absorption profile was evident, a quadratic baseline centered on the emission was employed. The output from PLOTOBS included the line center frequency $\nu_{1 c}$ in channels, the Doppler halfwidth to $1 / e$, the line intensity, and the ratio of Doppler to pressure broadening. All fits were found to have Gaussian profiles indicative of the low pressure regime associated with the emitting layer in the Venusian thermosphere. Figure Al.7 shows a PLOTOBS fit to Venus data acquired June 1986. The intensity is in units of the calibration blackbody intensity . 


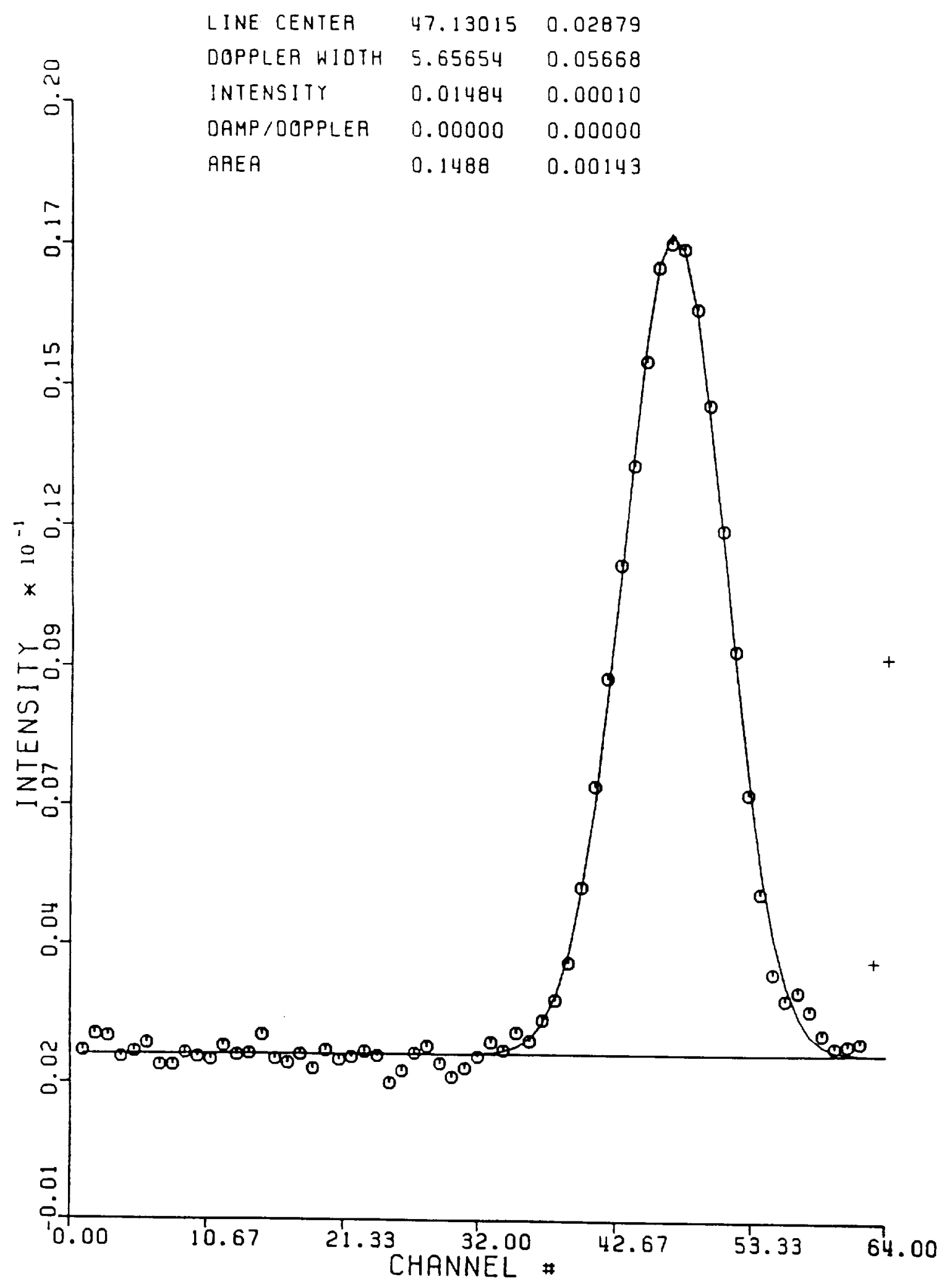

Figure A1.7. A Venus Line Fit 
To obtain the standard deviation in the output variables, the channel by channel residuals were used to calculate the standard deviation of the scan stack from the modelled Voigt profile, $\sigma_{\mathrm{N}}$. Random noise with the obtained $\sigma_{N}$ level was then generated, superimposed on the modelled Voigt profile and the resulting distribution was refit. After 20 repetitions, the standard deviations in the output variables were computed.

\section{A1.5. BEAM-INTEGRATED LINE OF SIGHT WIND VELOCITY DETERMINATION}

Define $V_{T}$ as the ephemeris-derived line of sight velocity component of the target planet's center of mass as seen from the observing station. The emitting parcel of gas within the beam is necessarily moving with respect to the center of mass frame due to both the target planet's rotation and the local wind field. Let $V_{w}$ equal the sum of the parcel's line of sight wind and planetary rotation velocity components. The line center frequency of the emission in the parcel's rest frame, $\nu_{r}$, is known (Freed et al. 1980) and corresponds to the rest frequency of the laser transition for this investigation. If $\nu_{0}$ is the observed line center frequency and $c$ is the speed of light, then:

$$
\nu_{\mathrm{r}}=\nu_{\mathrm{o}}\left(\frac{\mathrm{V}_{\mathrm{w}}+\mathrm{V}_{\mathrm{T}}}{\mathrm{c}}+1\right)
$$

or,

$$
V_{w}=\frac{c \nu_{r}}{\nu_{0}}-\left(V_{T}+c\right)
$$

The value of $\nu_{0}$ may be obtained from $\nu_{\mathrm{HRB}}$, the RFLO frequency $\nu_{r f}$, and 
the laser local oscillator frequency $\nu_{10}$. The Lamb dip laser stabilization system insures $\nu_{10}=\nu_{\mathrm{r}}$. From heterodyne principles discussed in Chapter 3 :

$$
\nu_{0}=\nu_{10} \pm\left(\nu_{\mathrm{rf}^{-\nu_{\mathrm{HRB}}}}\right)
$$

The term in parenthesis is added to $\nu_{10}$ for a target with an overall blueshift and subtracted for an overall redshift.

PLOTOBS fits to the HRB channel center frequencies. Define $\nu_{1 c}$ as the PLOTOBS-derived $\mathrm{HRB}$ line center frequency in $5 \mathrm{MHz}$ channels, as measured from the center of the first channel at $1290 \mathrm{MHz}$ (see Section A1.4). The line center frequency $\nu_{\mathrm{HRB}}$, in $\mathrm{MHz}$, is then given by:

$$
\nu_{\mathrm{HRB}}=1285+5 \nu_{1 \mathrm{c}} .
$$

Substituting Eq. A1.38 into A1.37 provides $\nu_{0}$ in terms of the measured quantities:

$$
\nu_{0}=\nu_{10} \pm\left[\nu_{\mathrm{rf}}-\left(1285+5 \nu_{1 \mathrm{c}}\right)\right]
$$

The standard deviation in $V_{w}$ derives from the achieved $S / N$ and fluctuations in the laser output frequency and RFLO. All sources of random error may be assumed uncorrelated. In Eq. A1.36, $\nu_{\text {o provides }}$ the sole source of random error, with uncertainties in both $\mathrm{V}_{\mathrm{T}}$ and $\nu_{\mathrm{r}}$ yielding only systematic offsets. Therefore, following from Eq. A1.36:

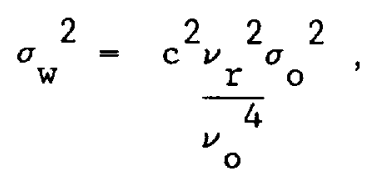

where $\sigma_{\mathrm{w}}$ and $\sigma_{\mathrm{o}}$ are the standard deviations in $\mathrm{V}_{\mathrm{w}}$ and $\nu_{\mathrm{o}}$ respectively. All three contributions to $\nu_{0}$ in Eq. A1.39 are sources of random 
error, thus:

$$
\sigma_{0}^{2}=\sigma_{10}^{2}+\sigma_{\mathrm{rf}}^{2}+25 \sigma_{1 \mathrm{c}}^{2}
$$

where $\sigma_{10}, \sigma_{\mathrm{rf}}$ and $\sigma_{1 \mathrm{c}}$ are likewise the standard deviations in $\nu_{10}$ ' $\nu_{\mathrm{rf}}$, and $\nu_{1 \mathrm{c}}$ respectively. Substituting Eq. Al.41 into A1.40 gives:

$$
\sigma_{\mathrm{w}}^{2}=\frac{c^{2} \nu_{\mathrm{r}}^{2}}{\nu_{\mathrm{o}}^{4}}\left(\sigma_{10}^{2}+\sigma_{\mathrm{rf}}^{2}+25 \sigma_{1 \mathrm{c}}^{2}\right) .
$$

From results of the $\mathrm{CO}_{2}$ isotopic overlap time series discussed in Chapter 4, $\sigma_{10}=0.13 /(\tau / 2)^{1 / 2} \mathrm{MHz}$, where $\tau$ is the integration time in minutes for the scan set. The RFLO frequency was recorded for each scan in the field allowing determination of $\sigma_{\mathrm{rf}}$ for a particular scan set. Finally, the PLOTOBS line fit program provided $\sigma_{1 \mathrm{c}}$.

Comparison between the ephemeris used for the investigation and the JPL Developmental Ephemeris 200 indicated a systematic uncertainty in $\mathrm{V}_{\mathrm{T}}$ of $\sim \pm 1 \mathrm{~m} / \mathrm{s}$. From Freed et al. (1980) the uncertainty in the ${ }^{12} \mathrm{C}^{16} \mathrm{O}_{2} \mathrm{R} 8$ transition rest frequency provided an additional systematic error of $\pm 0.0523 \mathrm{MHz}$. A total systematic error of $\pm 1.5 \mathrm{~m} / \mathrm{s}$ may therefore be expected in $V_{w}$.

It is important to note $v_{w}$ is a beam-integrated line of sight velocity. As three of the four Venus observing periods were characterized by a large beam to planetary disk ratio $(>0.05), \mathrm{V}_{\mathrm{w}}$ cannot be directly associated with local dynamics, (e.g., with the nominal beam center position on the planet). Program BEAMINT was thus developed to couple $v_{w}$ to a best-fit model wind field. 


\section{A1.6. REDUCTION OF TIME SERIES DATA}

The $\mathrm{CO}_{2}$ time series measurements carried out to test LDSS absolute stability were described in Chapter 4. Figure A1.8 shows the modified optical layout in front of the IRHS focal plane for these measurements. The $s$ beam consisted of blackbody BB1 focused at $\mathrm{f} / 20$ by lens LI into the Spectra Physics LO-3 White cell (see ref.). Lens L2 matched the $f / 20$ exit beam to the heterodyne field of view. The $s$ beam was chopped against a second blackbody BB2 whose intensity was adjusted so that the broadband heterodyne signals from each beam were equal. Such operation in "balanced mode" allows the RF electronics to operate in the linear regime maximizing system signal-to-noise.

As in the case of the Venusian data, program PLOTOBS was used to fit to the line shape function characterizing the white cell generated absorption feature. Isolation of the line shape function from the time series data will now be discussed.

From Figure A1.8, following the same notation as developed in Section A1.1:

$$
s\left(\nu^{\prime}\right)=\mathrm{I}_{\mathrm{BB} 1}\left(\nu^{\prime}\right) k_{s} \phi\left(\nu^{\prime}\right)+\mathrm{N}\left(\nu^{\prime}\right)+\mathrm{z}\left(\nu^{\prime}\right),
$$

and

$$
r\left(\nu^{\prime}\right)=\mathrm{I}_{\mathrm{BB} 2}\left(\nu^{\prime}\right) k_{r} \phi\left(\nu^{\prime}\right)+\mathrm{N}\left(\nu^{\prime}\right)+z\left(\nu^{\prime}\right),
$$

where $I_{B B 1}\left(\nu^{\prime}\right)$ and $I_{B B 2}\left(\nu^{\prime}\right)$ are the DSB blackbody spectral distributions and the emission-1ike coefficients $e_{s}, e_{r}$ have been neglected. The corresponding $S$ scan is of the form: 


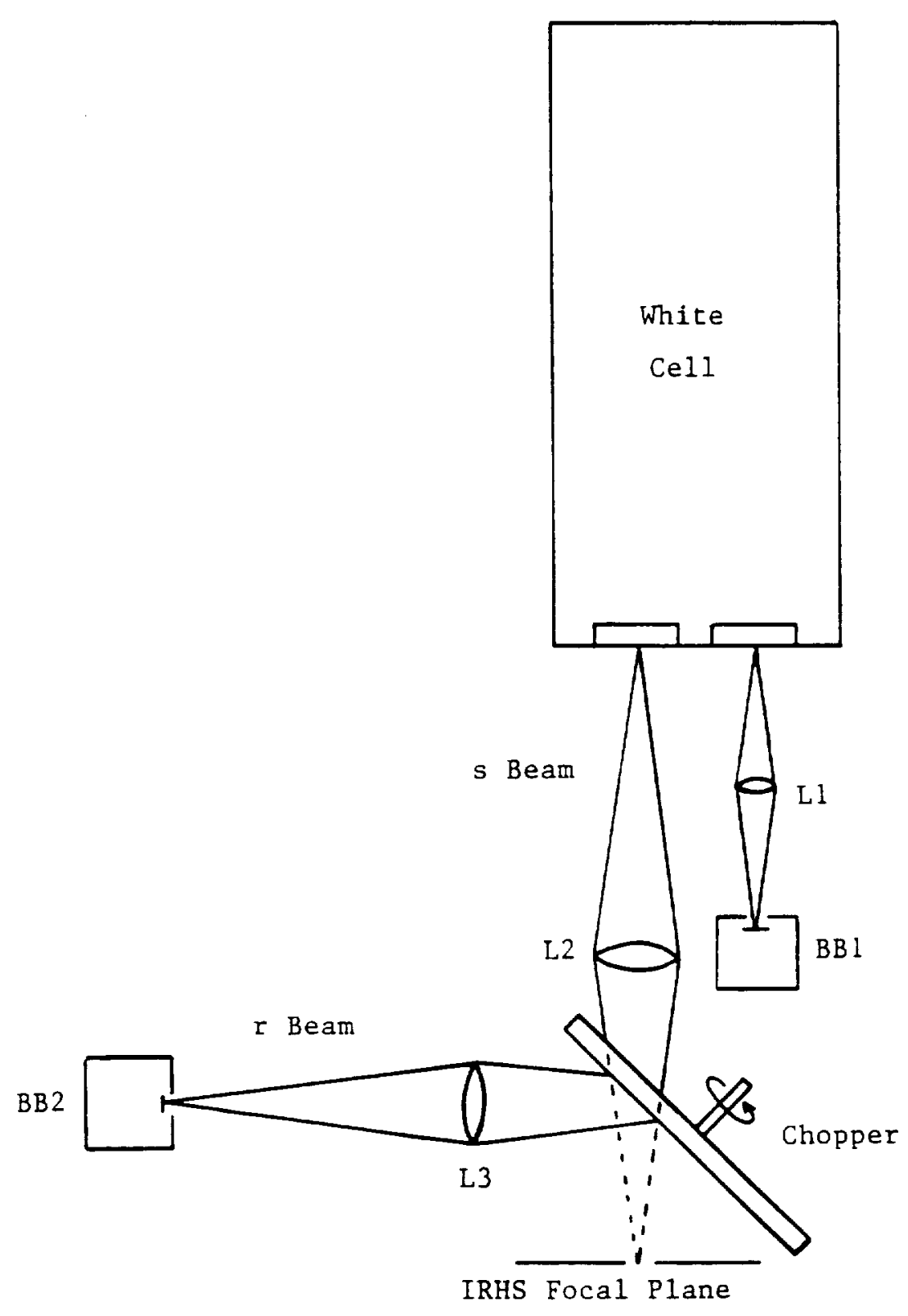

Figure A1.8. Time Series Optical Front End Layout 


$$
S=\frac{\left[\mathrm{I}_{\mathrm{BB} 1}\left(\nu^{\prime}\right) k_{s}-\mathrm{I}_{\mathrm{BB} 2}\left(\nu^{\prime}\right) k_{r}\right] \phi\left(\nu^{\prime}\right)}{\mathrm{I}_{\mathrm{BB} 2}\left(\nu^{\prime}\right) k_{r} \phi\left(\nu^{\prime}\right)+\mathrm{N}\left(\nu^{\prime}\right)} .
$$

The blackbody calibration scan $S_{B B}$ is generated by blocking BB1 so that:

$$
S_{B B}=\frac{-\mathrm{I}_{\mathrm{BB} 2}\left(\nu^{\prime}\right) k_{r} \phi\left(\nu^{\prime}\right)}{\mathrm{I}_{\mathrm{BB} 2}\left(\nu^{\prime}\right) k_{r} \phi\left(\nu^{\prime}\right)+\mathrm{N}\left(\nu^{\prime}\right)}
$$

Dividing Eq. A1.45 by Eq. A1.46 and assuming $k_{s} / k_{r}-1$ :

$$
\frac{s}{S_{\mathrm{BB}}}=1-\frac{\mathrm{I}_{\mathrm{BB} 1}\left(\nu^{\prime}\right)}{\mathrm{I}_{\mathrm{BB} 2}\left(\nu^{\prime}\right)}
$$

Let $I_{B B 1}$ and $I_{B B 2}$ represent the constant continuum levels of $B B 1$ and $B B 2$ respectively, across the IF. The DSB blackbody spectral distributions are given by:

$$
I_{B B 1}\left(\nu^{\prime}\right)=I_{B B 1} \exp \left[-A_{T} f_{D}\left(\nu^{\prime}-\nu^{\prime}{ }_{0}\right) x\right]+I_{B B 1}
$$

and

$$
\mathrm{I}_{\mathrm{BB} 2}\left(\nu^{\prime}\right)=2 \mathrm{I}_{\mathrm{BB} 2} \text {, }
$$

where $A_{T}$ is the integrated absorption coefficient for the $9.6 \mu \mathrm{m}$ ${ }^{13} \mathrm{C}^{16} \mathrm{O}_{2} \mathrm{R} 32$ line at $296 \mathrm{~K}, \mathrm{f}_{\mathrm{D}}\left(\nu^{\prime}-\nu^{\prime}{ }_{\mathrm{O}}\right)$ is the normalized line shape function and $x$ is the path length in the white cell. The first term in $\mathrm{I}_{\mathrm{BBl}}\left(\nu^{\prime}\right)$ can be recognized as Eq. A1.33, assuming no self-emission in the White cell. Substituting Eqs. A1.48 and A1.49 into A1.47 and rearranging gives: 


$$
A_{T} f_{D}\left(\nu^{\prime}-\nu^{\prime}{ }_{0}\right) x=\ln \left[2\left(1-\frac{S}{S_{B B}}\right) \frac{I_{B B 2}}{I_{B B 1}}-1\right]^{-1} .
$$

The right side of Eq. Al.50 is input to PLOTOBS where the ratio $\mathrm{I}_{\mathrm{BB} 1} / \mathrm{I}_{\mathrm{BB} 2}$, from $\mathrm{Eq}$. $\mathrm{A} 1.47$, is the continuum level associated with $1-\left(S / S_{\mathrm{BB}}\right)$.

Figure A1.9 is a PLOTOBS DSB line fit to a ${ }^{13} \mathrm{C}^{16} \mathrm{O}_{2}$ R32 line 2 minute integration. The $\mathrm{S} / \mathrm{N}$-induced standard deviation in line center position $\sigma_{1 c}$, is $-0.1 \mathrm{MHz}$.

The laser local oscillator frequency $\nu_{10}$ is finally obtained by substituting the PLOTOBS derived line center frequency $\nu_{1 c}$ in channels, the RFLO frequency $\nu_{\mathrm{rf}}$ in $\mathrm{MHz}$, and the white cell generated ${ }^{13} \mathrm{C}^{16} \mathrm{O}_{2} \mathrm{R} 32$ line frequency $\nu_{\text {wc }}=31159883.6641 \mathrm{MHz}$ (Freed et al. 1980) into Eq. A1.39. Noting $\nu_{w c}>\nu_{10}$, and that observed frequency $\nu_{0}$ is given by $\nu_{\mathrm{o}}=\nu_{\mathrm{wc}}{ }^{-\nu_{\mathrm{ps}}}$, where $\nu_{\mathrm{ps}}$ is the magnitude of the White cell associated redward pressure shift, Eq. Al.39 becomes:

$$
\nu_{10}=\left(\nu_{\mathrm{wc}}-\nu_{\mathrm{ps}}\right)+\left(1285+5 \nu_{1 c^{-\nu_{\mathrm{ff}}}}\right) \text {. }
$$

For small $\nu_{\mathrm{ps}}$, the local oscillator departure from the ${ }^{12} \mathrm{C}^{16} \mathrm{O}_{2} \mathrm{P} 28$ laser transition rest frequency $\nu_{1 t}=31159508.1635 \mathrm{MHz}$ (Freed et al. 1980) may be given by:

$$
\Delta \nu=\nu_{10^{-\nu}} 1 t=\left(\nu_{w c}-\nu_{1 t}\right)+\left(1285+5 \nu_{1 c^{-\nu}} \mathrm{rf}\right)
$$

As $\nu_{p s}$ is not precisely known (0.08 $05 \mathrm{MHz}$ /Torr redward; SooHoo 1984), it has been taken as a systematic error in $\Delta \nu$. For the time series specific lines, the systematic errors in $\Delta \nu$ associated with $\nu_{w c}$ and $\nu_{1 t}$ 


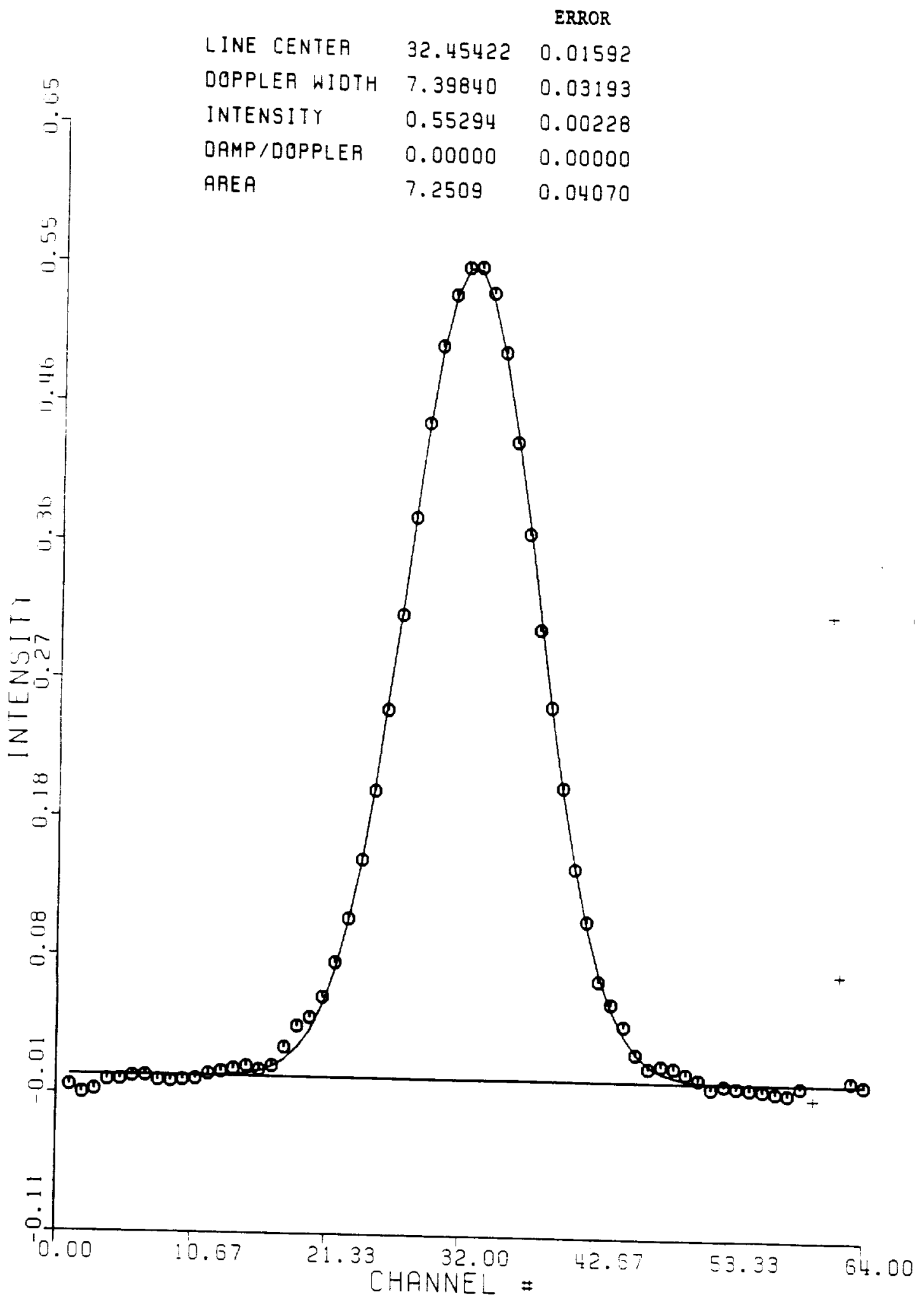

Figure A1.9. A White Cell Line Fit 
are $\pm 0.05 \mathrm{MHz}$ and $\pm 0.06 \mathrm{MHz}$, respectively (Freed et al. 1980). With a White cell pressure of 0.7 Torr, an overall systematic error of $0.06 \pm .14 \mathrm{MHz}$ is therefore expected in $\Delta \nu$.

The standard deviation in $\Delta \nu$ due to uncorrelated fluctuations in $\nu_{\mathrm{rf}}$ and $\nu_{1 \mathrm{c}}$ is given by:

$$
\sigma_{\Delta \nu}=\left(25 \sigma_{1 c}^{2}+\sigma_{r f}\right)^{3 / s} .
$$

Table A1.1 provides the details of the Lamb dip stabilization time series consisting of 33 scans spanning 1.5 hours. The $2^{\mathrm{m}}$ integration time per scan is the same used for Venusian data acquisition. The last four columns of the table provide $\nu_{\mathrm{rf}}, \nu_{1 \mathrm{c}}$ in $5 \mathrm{MHz}$ channels, $\nu_{10}$ and $\Delta \nu$ respectively, for each of the scans. From scan to scan, $\sigma_{\text {rf }}$ and $\sigma_{1 c}$ were nearly constant at $0.03 \mathrm{MHz}$ and 0.023 channels which, from Eq. A1.53, gives $\sigma_{\Delta \nu}=0.12 \mathrm{MHz}$ for an individual scan (lower left corner of Table A1.1).

In the lower right corner of the Table are provided the linear least squares fit coefficients, their standard deviations, $\langle\Delta \nu\rangle$ and, from the residuals about the fit, $\sigma_{\Delta \nu}$.

Table Al.2 provides the same details for the power peak stabilization time series. The white cell was held at 1 Torr resulting in an overall systematic error of $0.08 \pm .16 \mathrm{MHz}$. Linear least squares fits for scans 1 to 30,10 to 30 , and 13 to 30 were carried out, with sucessive fits better characterizing the steady state behavior of a power peak stabilized local oscillator. 


\section{Table A1.1. Lamb Dip Stabilization Time Series Analysis}

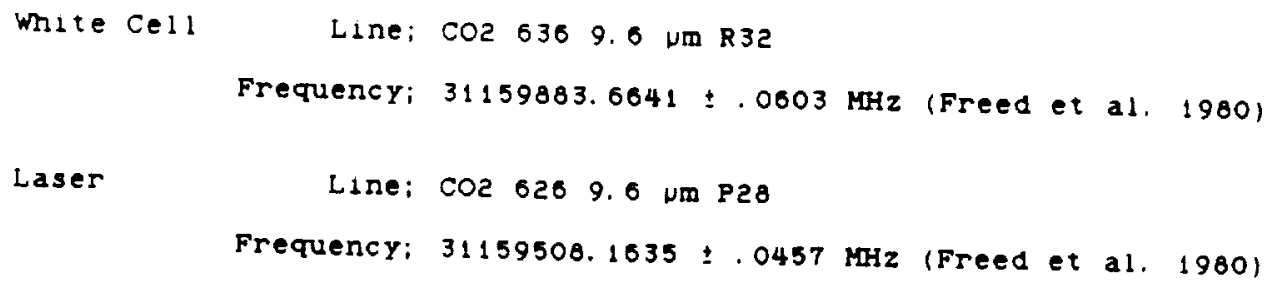

\begin{tabular}{|c|c|c|c|c|c|c|}
\hline SCAN & (Hrs & $\begin{array}{l}\text { TIME } \\
\text { Mins) }\end{array}$ & $\begin{array}{c}\text { vrf } \\
\text { (MHz) }\end{array}$ & $\left(\begin{array}{c}v 1 c \\
(\operatorname{cnls})\end{array}\right.$ & $\begin{array}{c}v 10+v P s \\
(\text { MHZ) }\end{array}$ & $\underset{(\mathbf{M H Z})}{\Delta v}$ \\
\hline 1 & 0 & 0.00 & 1825.86 & 33.096 & 31159508.28 & 0.12 \\
\hline 2 & 0 & 2. 62 & 1025.84 & 33.131 & 31159508.48 & 0.32 \\
\hline 3 & 0 & 5.15 & 1825.84 & 33.094 & 31159500.29 & 0.13 \\
\hline 4 & 0 & 7.67 & 1825.84 & 33.105 & 31159508.35 & 0.19 \\
\hline 5 & 0 & 10.25 & 1825.83 & 33.082 & 31159508.24 & 0.08 \\
\hline 6 & 0 & 12. 75 & 1825.84 & 33.069 & 31159508.17 & 0.01 \\
\hline 7 & 0 & 15. 30 & 1825.77 & 33.049 & 31159508.14 & -0.02 \\
\hline 8 & 0 & 17.90 & 1825.77 & 33.041 & 31159508.10 & -0.06 \\
\hline 9 & 0 & 20.43 & 1825.75 & 33.036 & 31159508.09 & -0.07 \\
\hline 10 & 0 & 23.00 & 1825.68 & 33.057 & 31159508.27 & 0.11 \\
\hline 11 & 0 & 25.55 & 1825.68 & 32. 982 & 31159507.89 & -0.27 \\
\hline 12 & 0 & 28.10 & 1825.70 & 32.998 & 31159507.94 & -0.22 \\
\hline 13 & 0 & 30.63 & 1025.72 & 33.033 & 31159508.11 & -0.05 \\
\hline 14 & 0 & 33. 13 & 1825.73 & 33.011 & 31159507.99 & -0.17 \\
\hline 15 & 0 & 35. 70 & 1825.73 & 33.040 & 31159508.13 & -0.03 \\
\hline 16 & 0 & 38. 30 & 1825.72 & 33.069 & 31159508.29 & 0.13 \\
\hline 17 & 0 & 40.82 & 1825.74 & 33.096 & 31159508.40 & 0.24 \\
\hline 18 & 0 & 43.40 & 1825.74 & 33. 070 & 31159508.27 & 0.11 \\
\hline 19 & 0 & 45.95 & 1825.70 & 33. 047 & 31159506.20 & 0.04 \\
\hline 20 & 0 & 48.67 & 1825.65 & 33.074 & 31159508.38 & 0.22 \\
\hline 21 & 0 & 51.17 & 1825.08 & 33.053 & 31159508.25 & 0.09 \\
\hline 22 & 0 & 53.73 & 1825.68 & 33.028 & 31159500.12 & -0.04 \\
\hline 23 & 1 & 3. 80 & 1825.60 & 33.056 & 31159508.28 & 0.12 \\
\hline 24 & 1 & 6. 30 & 1825.66 & 33.036 & 31159508.18 & 0.02 \\
\hline 25 & 1 & 9.02 & 1825.60 & 33.070 & 31159508,35 & 0.19 \\
\hline 26 & 1 & 11.57 & 1025.64 & 33.077 & 31159508.41 & 0.25 \\
\hline 27 & 1 & 14.05 & 1825.67 & 33. 042 & 31159508.20 & 0.04 \\
\hline 28 & 1 & 10.73 & 1825.63 & 33.055 & 31159508.31 & 0.15 \\
\hline 29 & 1 & 19.28 & 1825.64 & 33.069 & 31159508.37 & 0.21 \\
\hline 30 & 1 & 21.82 & 1825.68 & 33.057 & 31159508.27 & 0.11 \\
\hline 31 & 1 & 24.33 & 1825.62 & 33. 032 & 31159508.20 & 0.04 \\
\hline 32 & 1 & 26.87 & 1825.84 & 33.054 & 31159508.29 & 0.13 \\
\hline 33 & 1 & 29.42 & 1825.84 & 33.021 & 31159508.13 & -0.03 \\
\hline
\end{tabular}

Error Bar Determination

orf $=.03 \mathrm{MHz}$

$\sigma l c=.023$ channels

$\sigma_{\Delta V}=.12 \mathrm{MHz}$

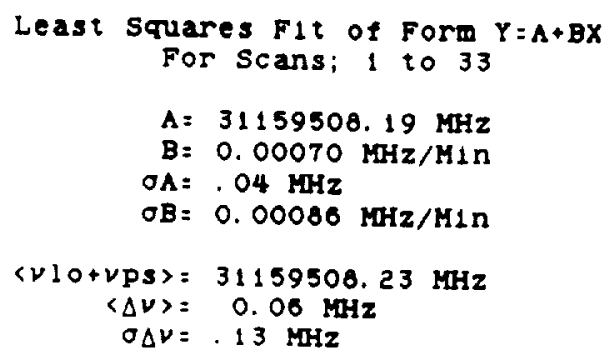




\section{Table A1.2. Power Peak Stabilization Time Series Analysis}

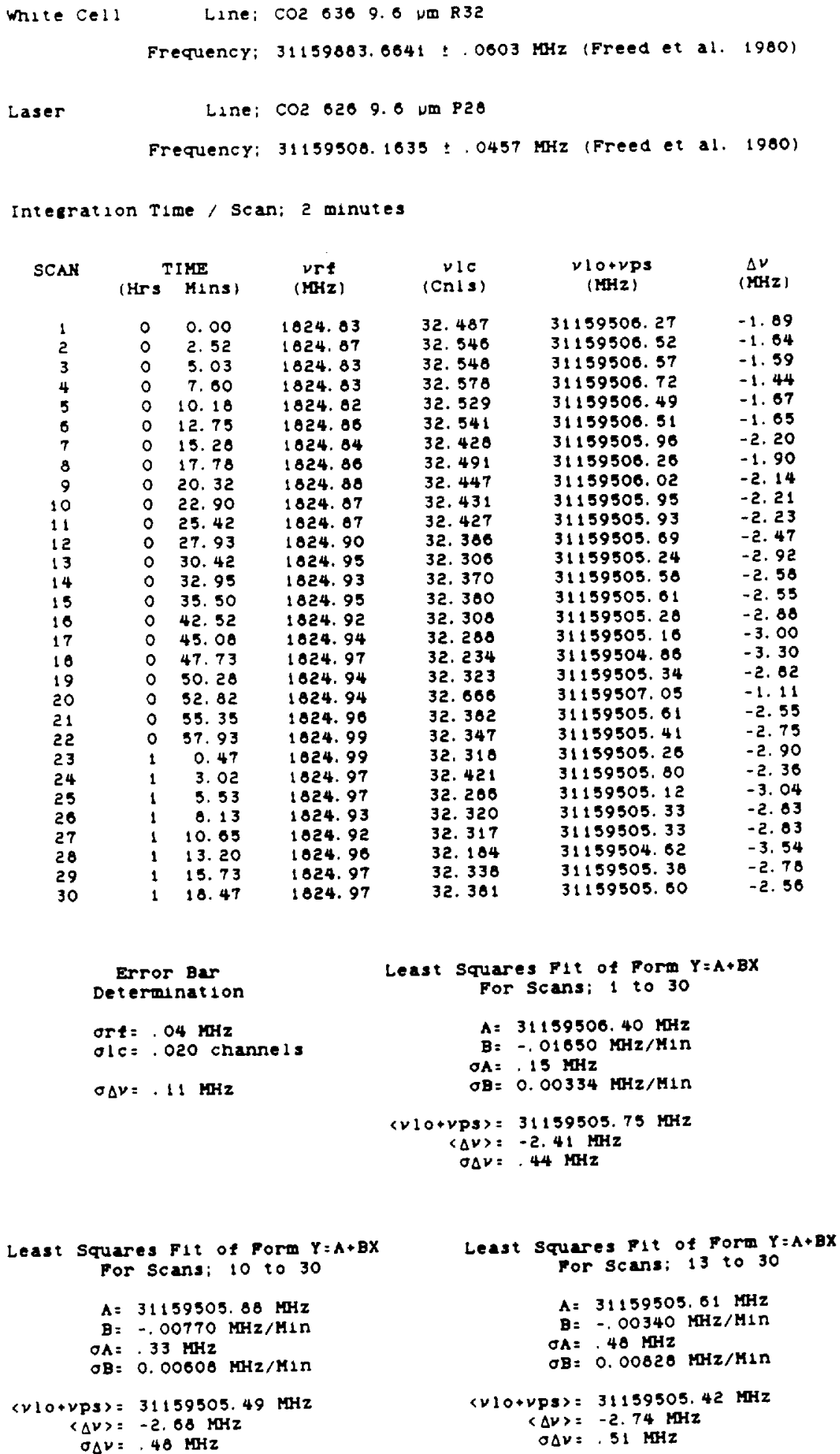




\section{APPENDIX 2: PROGRAM BEAMINT THEORETICAL DEVELOPMENT}

\section{A2.1. INTRODUCTION}

Typical Venus beam aspect geometry is indicated on Figure A2.1. The Subearth Point (SE) is the mutual origin of the $Y-Z$ and $Y "-Z "$ coordinate grids. The $Y^{\prime \prime}$ and $Z^{\prime \prime}$ axes are aligned with celestial $E-W$ and $\mathrm{N}-\mathrm{S}$, respectively. The $\mathrm{Z}$ axis, inclined an angle $\rho$ to the $Z^{\prime \prime}$ axis, is the sky projection of the Venusian Subearth Meridian and necessarily contains the sky projection of the Venusian North and South Poles. In general, the planet's equator is inclined to the $\mathrm{Y}$ axis.

For this investigation, planetary positions were most readily expressed in planetary longitude and latitude, themselves easily transformed into $\mathrm{Y}-\mathrm{Z}$ coordinates. However, as on Figure A2.1, the probe beam may be elliptical with a N-S semi-major axis (see Section $3.5)$, and thus most readily characterized in $Y^{\prime \prime}-Z^{\prime \prime}$ coordinates. Hence, the motivation for both coordinate systems is clear.

Consider a beam centered at coordinates $(y, z)$ on the Venusian disk and divided into $N$ slices and $M$ annuli (Figure A2.1 provides the case $\mathrm{N}=16, \mathrm{M}=4$, yielding $16 \times 4=64$ beam elements). The element associated with slice $i$ (numbered counterclockwise from celestial west) and annulus $j$ (numbered from beam center outward) may be characterized by a Gaussian non-thermal source spectral distribution $I_{i j}(\nu)$ (see Section A1.4) given by:

$$
I_{i j}(\nu)=\left(S_{i j} \exp \left[-\left(\nu-\nu_{i j}\right)^{2} / \nu_{D}{ }^{2}\right]+B_{i j}\right) \Phi_{i j} A_{i j},
$$

where $A_{i j}$ is the element area, $\Phi_{i j}$ is the beam weighting as a function 


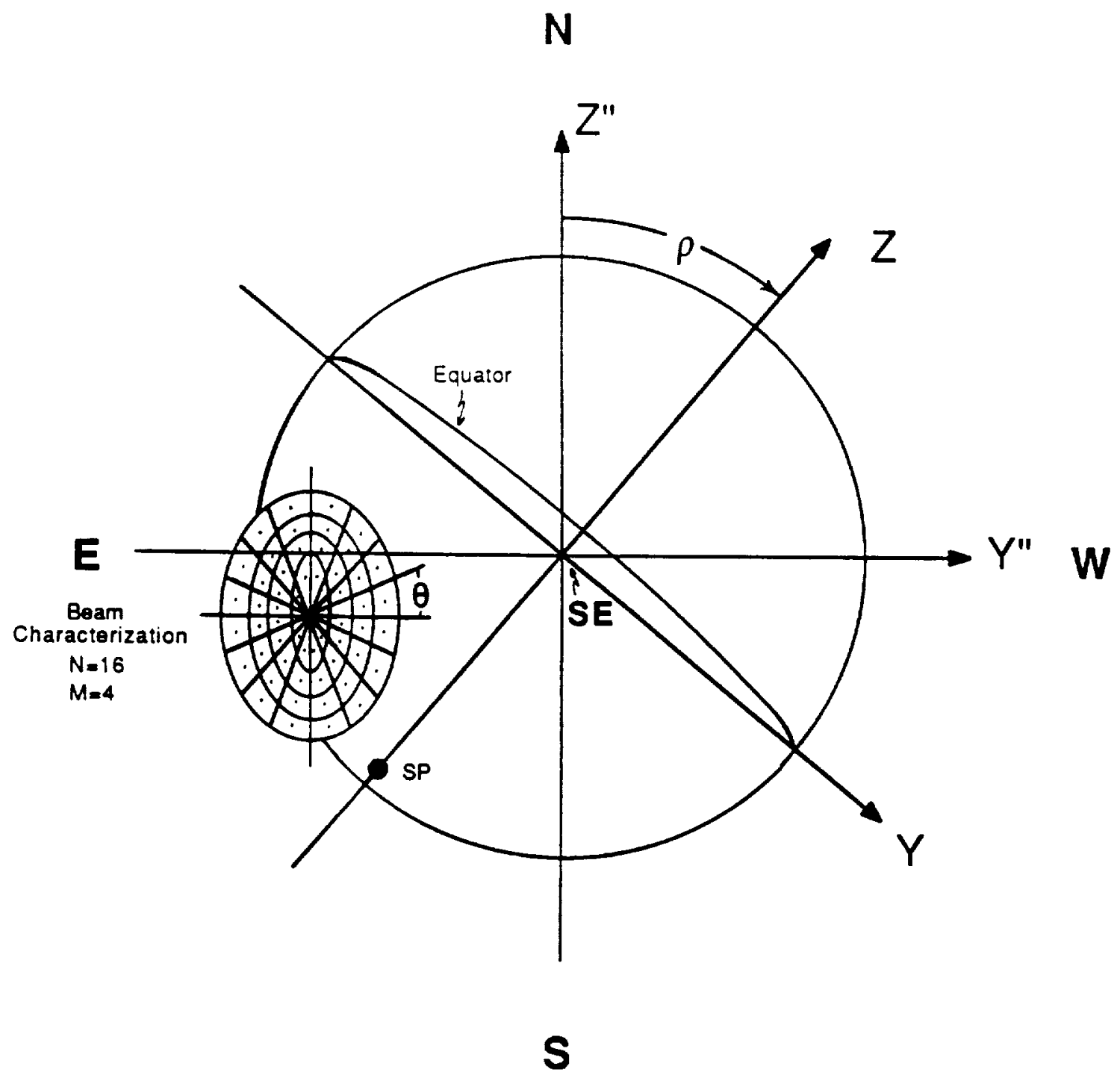

Figure A2.1. Venus Beam Aspect Geometry 
of element distance from beam center, $\nu_{i j}$ is the line center frequency, $\nu_{D}$ is the Doppler width (1/e HW), and $S_{i j}$ is the line-center relative intensity above virtual continuum level $\mathrm{B}_{i j}$, the latter characterizing the center of the corresponding pressure broadened absorption feature. Within element $(i, j)$ these Beam Parameters are assumed constant and evaluated at the element centroid. The beam-integrated source spectral distribution $I_{T}(\nu)$ is thus given by:

$I_{T}(\nu)=\sum_{j=1}^{M} \sum_{i=1}^{N} S_{i j} \exp \left[-\left(\nu-\nu_{i j}\right)^{2} / \nu_{D}^{2}\right] \Phi_{i j} A_{i j}+\sum_{j=1}^{M} \sum_{i=1}^{N} B_{i j} \Phi_{i j} A_{i j}$

In general, the probe beam intercepts a large portion of the planetary disk (see Fig. A2.1). Limb measurements, in particular, are associated with large across-the-beam variation in SZA, latitude, longitude and projection geometry. The resulting strong across-the-beam gradients in the beam parameters are the primary impetus for a high resolution (100x100 element) modelling of the beam-integrated winds.

\section{Sources of Beam Parameter Gradients:}

1. Horizontal wind velocity, for a coupled subsolar-antisolar and zonal retrograde circulation, is a function of SZA, latitude and longitude. In addition, the wind's line of sight component is a function of projection geometry. A strong Doppler-induced across-the-beam gradient in $\nu_{i j}$ is therefore expected.

2. $s_{i j}$ varies across the beam due to variation in both $S Z A$ and 1 ine 
of sight column density within the non-thermal emitting region (see Section 7.1).

3. $\Phi_{i j}$ varies due to the beam's diffraction-limited $\operatorname{sinx} / x$ spatial roll-off coupled with the Gaussian roll-off associated with the seeing disk (Hufnagel 1978).

4. $\nu_{D}$ varies with SZA due to temperature variation in the non-thermal emitting layer. However, $\nu_{D}$ is assumed constant over the beam to keep the beam modelling tractable. At $110 \mathrm{~km}$ altitude, a Subearth-to-terminator $\left(\triangle \mathrm{SZA}=90^{\circ}\right) \triangle \mathrm{T}-15 \mathrm{~K}$ has been observed (Taylor et al. 1983; also see Fig. P.1), with a mean $\mathrm{T}=185 \mathrm{~K}$, corresponding to an 88 temperature variation. Only 48 variation in $\nu_{D}$ is therefore expected as $\nu_{D} \alpha T^{1 / 2}$. In general, the beam spans a significantly smaller range in SZA rendering across-the-beam variation in $\nu_{D}$ negligible.

For large $N$ and $M$, the centroid of $I_{T}(\nu)$ identifies the beam-integrated line-center frequency hence the beam-integrated line of sight wind velocity. Comparison of the latter (generated for all 45 beams, assuming a model wind field) with the observed beam-integrated winds allows model discrimination.

The centroid of $I_{T}(\nu)$ may be defined by:

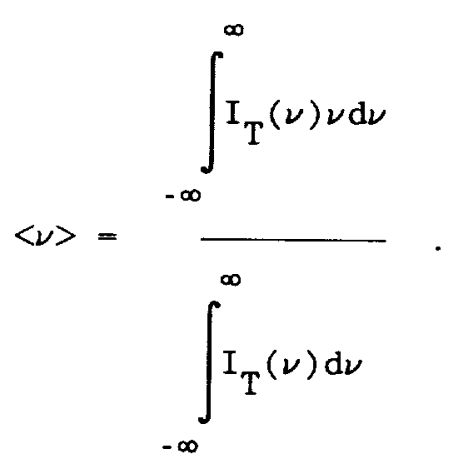


Substituting Eq. A2.2 into A2.3 and integrating yields:

$$
\langle\nu\rangle=\frac{\sum_{j=1}^{M} \sum_{i=1}^{N} \nu_{i j} S_{i j} \Phi_{i j} A_{i j}}{\sum_{j=1}^{M} \sum_{i=1}^{N} s_{i j} \Phi_{i j} A_{i j}}
$$

where the second term in Eq. A2.2, independent of $\nu$, has been neglected. Further substitution of Eq. 5.37 into Eq. A2.4, and rearranging, yields the beam-integrated line of sight wind velocity $\left\langle\mathrm{V}_{\mathrm{w}}>\right.$ :

$$
\left\langle V_{w}>=\left(\frac{\sum_{j=1}^{M} \sum_{i=1}^{N} s_{i j} \Phi_{i j} A_{i j}}{\sum_{j=1}^{M} \sum_{i=1}^{N}\left(\frac{s_{i j} \Phi_{i j} A_{i j}}{1+V_{i j} / c}\right)}-1\right) c\right.
$$

The determination of beam parameters $s_{i j}, \Phi_{i j}, A_{i j}$, and $V_{i j}$ will now be addressed. In the following discussion note the model beam radius is defined as the effective beam HPBW (twice the 1 /e radius), and all distances (unless otherwise stated) are in units of the planetary radius $\left(R_{2}\right)\left(R_{2}\right.$, the radius of the non-thermal emitting she11's outer boundary, is taken as the solid planet radius plus 120 $\mathrm{km}$, or $6172 \mathrm{~km}$; see Figs. 7.6 and 7.7 ).

\section{A2.2. BEAM ELEMENT AREA $A_{i j}$}

As discussed in Section 3.5, the heterodyne diffraction-1imited beam is circular for the McMath $1.52 \mathrm{~m}$ ( $1.7 \mathrm{arc}-\mathrm{sec} \mathrm{HPBW}$ at $10.33 \mu \mathrm{m}$ ) for planetary dec1inations $\delta<-7.18 \mathrm{deg}$, and circular for the NASA/IRTF 
$3 \mathrm{~m}(0.9$ arc $-\sec \mathrm{HPBW}$ at $10.33 \mu \mathrm{m})$. For $\delta>-7.18 \mathrm{deg}$, the McMath beam elongates N-S (e.g. June 1986), with declination HPBW (a), in arc-seconds, given by:

$$
a=\frac{1.279}{\sin (45-\delta / 2)}
$$

The right ascension HPBW (b) remains 1.7 arc-sec.

The presence of 1 to 2 arc-second seeing causes the planetary disk to rapidly traverse the sky-fixed diffraction-limited beam. The resulting effective beam may be approximated as a Gaussian with a HPBW equal to the sum of the diffraction-limited HPBW and the seeing FWHM. The Gaussian profile derives from the random walk in deflection angle suffered by photons as they traverse the terrestrial atmosphere.

$A_{i j}$ for a circular effective beam with HPBW $\sigma$ is given by:

$$
A_{i j}=\pi \sigma^{2}(2 j-1) / M^{2} N
$$

Analogously, $A_{i j}$ for an elliptical effective beam with right ascension HPBW a and declination HPBW $b$ is given by:

$$
A_{i j}=\frac{a b(2 j-1)}{2 M^{2}}\left(\operatorname{Tan}^{-1}[(b / a) \tan (2 \pi i / N)]-\operatorname{Tan}^{-1}[(b / a) \tan (2 \pi(i-1) / N)]\right)
$$

\section{A2.3. BEAM ELEMENT CENTROID DETERMINATION}

The beam element centroid coordinates $\left(y{ }_{i j}, z^{\prime \prime}{ }_{i j}\right)$, or equivalently the centroid's planetary longitude and latitude $\left(\alpha_{i j}, \beta_{i j}\right)$, dictate $\Phi_{i j}$, 
$s_{i j}$, and $v_{i j}$. For a circular beam:

$$
\begin{aligned}
& y{ }_{i j}=y "{ }_{b c}+(\sigma / M)(j-0.5) \cos [(i-0.5) 2 \pi / N] \\
& z "{ }_{i j}=z " b_{b c}+(\sigma / M)(j-0.5) \sin [(i-0.5) 2 \pi / N]
\end{aligned}
$$

where $\left(y^{\prime \prime}{ }_{b c}, z^{\prime \prime}\right)$ are the coordinates of the beam center. For an elliptical beam:

$$
\begin{aligned}
& y{ }_{i j}=y "_{b c}+a b(j-0.5) \cos [2 \pi(i-0.5) / N] /(M \Delta) \\
& z{ }_{i j}=z_{b c}+a b(j-0.5) \sin [2 \pi(i-0.5) / N] /(M \Delta), \\
& \text { where } \Delta=\left(b^{2}+\left(a^{2}-b^{2}\right) \cos ^{2}[2 \pi(i-0.5) / N]\right)^{1 / 2} .
\end{aligned}
$$

( $y{ }_{b c}, z "{ }_{b c}$ ) may be obtained from the known beam center longitude and latitude $\left(\alpha_{\mathrm{bc}}, \beta_{\mathrm{bc}}\right)$. The $\mathrm{Y}-\mathrm{Z}$ coordinate system on Figure A2.1 is shown 3-dimensionally on Figure A2.2. The primed system $X^{\prime}-Y^{\prime}-Z^{\prime}$ is obtained by rotating $X-Y-Z$ through angle $\phi$, about the $Y$-axis. The $Z^{\prime}$-axis intersects the North Pole. The location of a point $P$ may be expressed in planetary longitude $(\alpha)$ and latitude $(\beta)$ as defined with respect to the primed coordinate system. The coordinates $(x, y, z)$ of point $P$ are then given by:

$$
\begin{aligned}
& \mathrm{x}=\cos (\alpha) \cos (\beta) \cos (\phi)-\sin (\beta) \sin (\phi), \\
& \mathrm{y}=\sin (\alpha) \cos (\beta), \\
& \mathrm{z}=\sin (\beta) \cos (\phi)+\cos (\alpha) \cos (\beta) \sin (\phi) .
\end{aligned}
$$

The transformation $\left(y^{\prime \prime}, z^{\prime \prime}\right) \rightarrow(y, z)$ (see Fig. A2.1) is given by: 


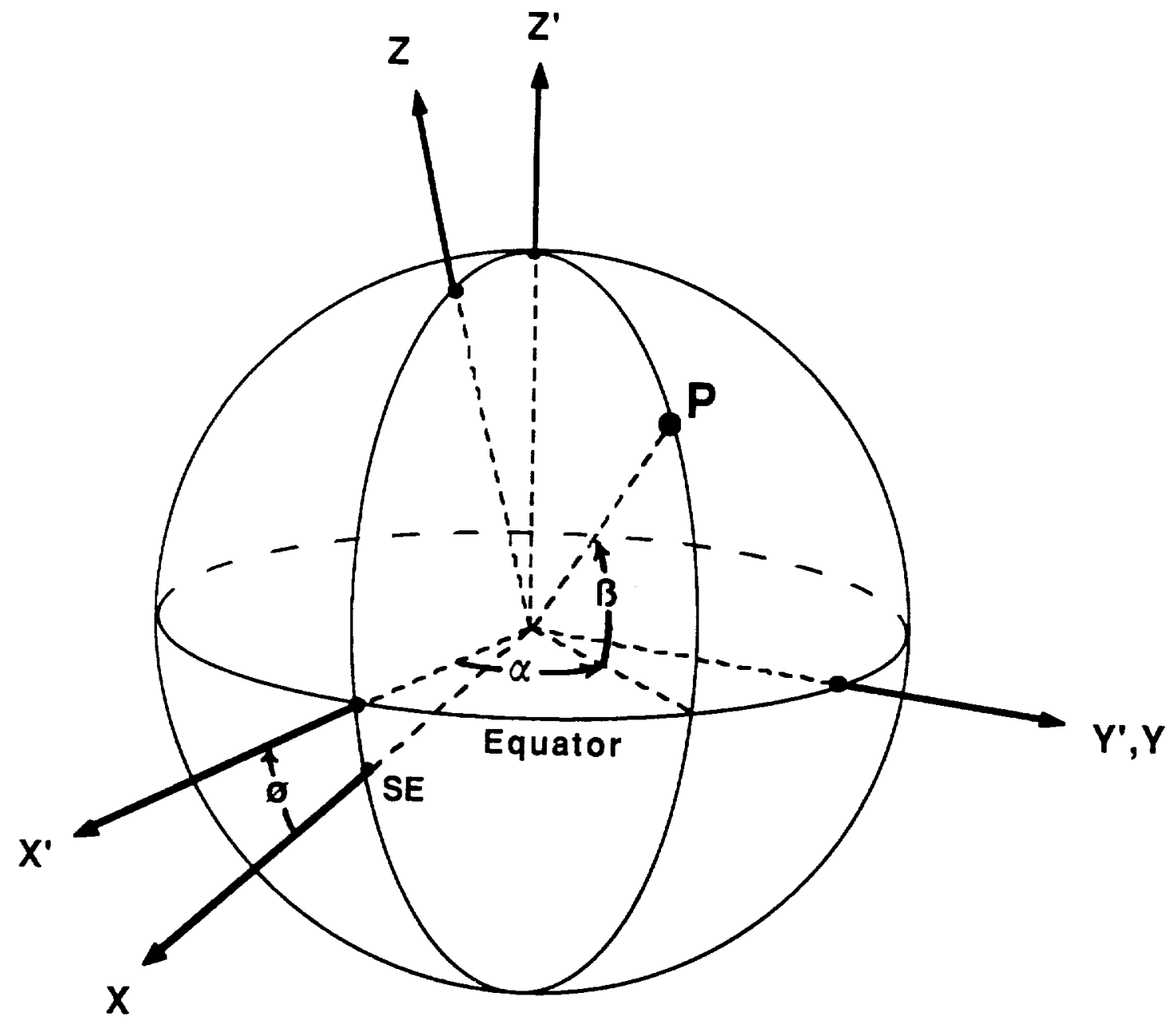

Figure A2.2. Transformation from Planetary Latitude and Longitude to Plane-of-Sky Coordinates 


$$
\begin{aligned}
& y=y^{\prime \prime} \cos (\rho)-z^{\prime \prime} \sin (\rho), \\
& z=y^{\prime \prime} \sin (\rho)+z^{\prime \prime} \cos (\rho),
\end{aligned}
$$

with inverse transform $(y, z) \rightarrow\left(y^{\prime \prime}, z^{\prime \prime}\right):$

$$
\begin{aligned}
y^{\prime \prime} & =y \cos (\rho)+z \sin (\rho), \\
z^{\prime \prime} & =-y \sin (\rho)+z \cos (\rho) .
\end{aligned}
$$

Substituting the second and third of Eqs. A2.11 into Eqs. A2.13 yields the beam center coordinates $\left(y^{\prime \prime}{ }_{b c}, z^{\prime \prime}{ }_{b c}\right)$ as required by Eqs. A2.10:

$$
\begin{aligned}
& y^{\prime \prime}= \sin \left(\alpha_{b c}\right) \cos \left(\beta_{b c}\right) \cos (\rho)+\sin \left(\beta_{b c}\right) \cos (\phi) \sin (\rho) \\
&+\cos \left(\alpha_{b c}\right) \cos \left(\beta_{b c}\right) \sin (\phi) \sin (\rho) \\
& z^{\prime \prime}{ }_{b c}=-\sin \left(\alpha_{b c}\right) \cos \left(\beta_{b c}\right) \sin (\rho)+\sin \left(\beta_{b c}\right) \cos (\phi) \cos (\rho) \\
&+\cos \left(\alpha_{b c}\right) \cos \left(\beta_{b c}\right) \sin (\phi) \cos (\rho) .
\end{aligned}
$$

By employing Eqs. A2.12, the beam element centroid coordinates ( $\left.y "{ }_{i j}, z "{ }_{i j}\right)$ may be transformed to $\left(y_{i j}, z_{i j}\right)$, so the centroid's planetary longitude and latitude $\left(\alpha_{i j}, \beta_{i j}\right)$ may be obtained using:

$$
\begin{gathered}
\beta_{i j}=\sin ^{-1}\left[z_{i j} \cos (\phi)-\left[1-y_{i j}{ }^{2}-z_{i j}{ }^{2}\right]^{1 / 2} \sin (\phi)\right), \\
\alpha_{i j}=\sin ^{-1}\left(y_{i j} / \cos \left(\beta_{i j}\right)\right) .
\end{gathered}
$$

\section{A2.4. BEAM ELEMENT WEIGHTING $\Phi_{i j}$}

The Gaussian beam weight for a circular beam is given by:

$$
\Phi_{i j}=\exp \left[-\ln 2\left(y "{ }_{i j}-y "{ }_{b c}\right)^{2} / \sigma^{2}-\ln 2\left(z "{ }_{i j}-z "{ }_{b c}\right)^{2} / \sigma^{2}\right]
$$


For an elliptical beam:

$$
\Phi_{i j}=\exp \left[-\ln 2\left(y "{ }_{i j}-y "{ }_{b c}\right)^{2} / b^{2}-\ln 2\left(z{ }_{i j}-z "{ }_{b c}\right)^{2} / a^{2}\right]
$$

Both cases reduce to:

$$
\Phi_{i j}=\exp \left[-\ln 2(j-0.5)^{2} / M^{2}\right]
$$

after substituting the equation of the $j^{\text {th }}$ circle into Eq. A2.16, and the $j^{\text {th }}$ ellipse into Eq. A2.17.

\section{A2.5. LINE OF SIGHT VELOCITY $v_{i j}$}

A two-component wind field is assumed, comprised of a solid-body zonal super-rotation and a subsolar-antisolar (SS-AS) circulation, both defined with respect to solar-fixed frame BFl (see Section 6.1). For an observer in frame BF2, fixed to the line of sight (see Section 6.1), the beam element centroid-associated line of sight velocity component $\left(V_{i j}\right)$ is given by:

$$
v_{i j}=\left[v_{x 1}(i, j)+v_{x 2}(i, j)\right]+T_{x}(i, j),
$$

where $V_{x 1}(i, j)$ and $V_{x 2}(i, j)$ are the zonal and SS-AS $x$-axis (see Fig. $A 2.2)$ components, respectively, referenced to $B F 1$, and $T_{x}(i, j)$ is the line of sight contribution due to relative rotation between $B F 1$ and BF2.

\section{A2.5.1. Zonal Components $V_{x 1}(i, j)$ and $T_{x}(i, j)$}

In $B F 1$, a solid-body zonal rotation with equatorial velocity $v_{\text {eq }}$ will yield a centroid-associated line of sight component $v_{x 1}(i, j)$ given 
by:

$$
v_{x 1}(i, j)=v_{e q} \sin \left(\alpha_{i j}\right) \cos \left(\beta_{i j}\right) \cos (\phi)
$$

with $V_{\text {eq }}$ negative for retrograde rotation. (The $2.6^{\circ}$ obliquity has been neglected.)

Beam-integrated wind velocities were obtained in BF2. To a BF2-fixed observer, even pure SS-AS circulation exhibits a prograde zonal component, resulting from SS-AS axial rotation with respect to the 1 ine of sight (over the $583.96^{d}$ synodic period $P_{\text {syn }}$ ). $T_{x}(i, j)$ is the requisite line of sight correction to the BFl-defined wind field, and given by:

$$
T_{x}(i, j)=T_{e q} \sin \left(\alpha_{i j}\right) \cos \left(\beta_{i j}\right) \cos (\phi)
$$

where $\mathrm{T}_{\text {eq }}$ is the BF2-observed zonal velocity for a BF1-fixed equatorial parcel at $110 \mathrm{~km}$ altitude. $\mathrm{T}_{\mathrm{eq}}$ is given by:

$$
T_{e q}=R \Omega_{0}+\frac{R \Omega_{o} r_{v}}{r_{e}-r_{v} \cos \theta}\left(\cos \theta-\frac{r_{v} r_{e} \sin ^{2} \theta}{\left(r_{v e}\right)^{2}}\right),
$$

where $R$ is the radius of Venus to $110 \mathrm{~km}$ altitude, $\Omega_{0}$ is angular velocity $2 \pi / \mathrm{P}_{\text {syn }}, \theta$ is the heliocentric angle between Earth and Venus, and $r_{v}, r_{e}$ and $r_{v e}$ are the Sun/Venus, Sun/Earth, and Venus/Earth distances in A.U. (Regarding the derivation of $\mathrm{T}_{\mathrm{eq}}$, Venusian orbital inclination to the ecliptic and Venus/Earth orbital eccentricities are negligible and have been taken as zero.)

$$
\mathrm{T}_{\text {eq }} \text { varies from } 0.45 \mathrm{~m} / \mathrm{s} \text { at superior conjunction to } 2.7 \mathrm{~m} / \mathrm{s} \text { at }
$$


inferior conjunction. $\mathrm{T}_{\text {eq }}$ for the December 1985, June 1986, October 1986, and March 1987 observing periods was $0.45,0.51,1.90$, and 0.52 m/s, respectively. Only the October 1986 correction was significant.

\section{A2.5.2. Subsolar-Antisolar Component $V_{x 2}(i, j)$}

A generalized empirical SS-AS model circulation was sought, consistent with existing hydrodynamical circulation models (e.g., Bougher et al. 1986). The basic constraints were: subsolar-antisolar axial symmetry, an everywhere negligible vertical component (see Chapters 1 and 2), zero horizontal velocity at the subsolar and antisolar points, and maximum horizontal velocity across the terminator. As the non-thermal emission probes only the illuminated hemisphere, the model needed address only $\mathrm{SZA}<90^{\circ}$. The requisite conditions were fulfilled by the 2 -parameter form:

$$
v_{h}(i, j)=v_{\max }-v_{\max }\left(\frac{90-\Omega_{i j}}{90}\right)^{N}
$$

where $V_{h}(i, j)$ is the beam element centroid-associated horizontal velocity, $\Omega_{i j}$ is the centroid SZA, $V_{\max }$ is the cross-terminator horizontal velocity, and $\mathrm{N}$ is the model index (1-linear, 2-parabolic, etc.). The Bougher et al. (1986) hydrodynamical model, at $110 \mathrm{~km}$ altitude, is represented almost identically by the case $N=1$, $\mathrm{V}_{\max }=121 \mathrm{~m} / \mathrm{s}$ (see Fig. 2.2). The element centroid SZA is given by:

$$
\Omega_{i j}=(180 / \pi) \cos ^{-1}\left(x_{i j} x_{s s}+y_{i j} y_{s s}+z_{i j} z_{s s}\right) \text {, }
$$


where $\left(\mathrm{x}_{S S}, \mathrm{y}_{\mathrm{sS}}, \mathrm{z}_{\mathrm{ss}}\right)$ are the coordinates of the subsolar point obtained from the subsolar longitude and latitude $\left(\alpha_{s s}, \beta_{s s}\right)$ using Eqs. A2.11.

Figure $A 2.3$ provides the geometry for $V_{x 2}(i, j)$ determination. The subsolar point (SS) is located by displacement vector $\mathbf{S}$ with components $\left(x_{s s}, y_{s s}, z_{s s}\right)$, and the beam element centroid is located by vector $P$ with components $\left(\mathrm{x}_{i j}, \mathrm{y}_{i j}, \mathrm{z}_{i j}\right)$. As distances are represented in planetary radii, $\mathbf{S}$ and $\mathbf{P}$ are necessarily unit vectors. $\mathbf{S}$ and $\mathbf{P}$ define a great circle with unit normal $U$ given by:

$$
U=\frac{S \times P}{|S \times P|}=\frac{S \times P}{\sin \Omega_{i j}} .
$$

The SS-AS horizontal velocity $v_{h}(i, j)$ is tangent to the great circle at $P$ in the direction of unit vector $V$. $V$ is mutually orthogonal to $U$ and $P$, and always directed away from the SS point, thus:

$$
V=U \times P .
$$

The $x$-component of $V_{h}(i, j)$, the quantity of interest, is therefore given by:

$$
v_{x 2}(i, j)=v_{h}(i, j)\left(z_{i j} U_{y}(i, j)-y_{i j} U_{z}(i, j)\right),
$$

where $U_{y}(i, j)$ and $U_{z}(i, j)$, the $y$ - and $z$-components of $U$, are obtained from Eq. A2.25:

$$
u_{y}(i, j)=\frac{x_{i j} z_{s s}-z_{i j} x_{s s}}{\sin \Omega_{i j}}
$$




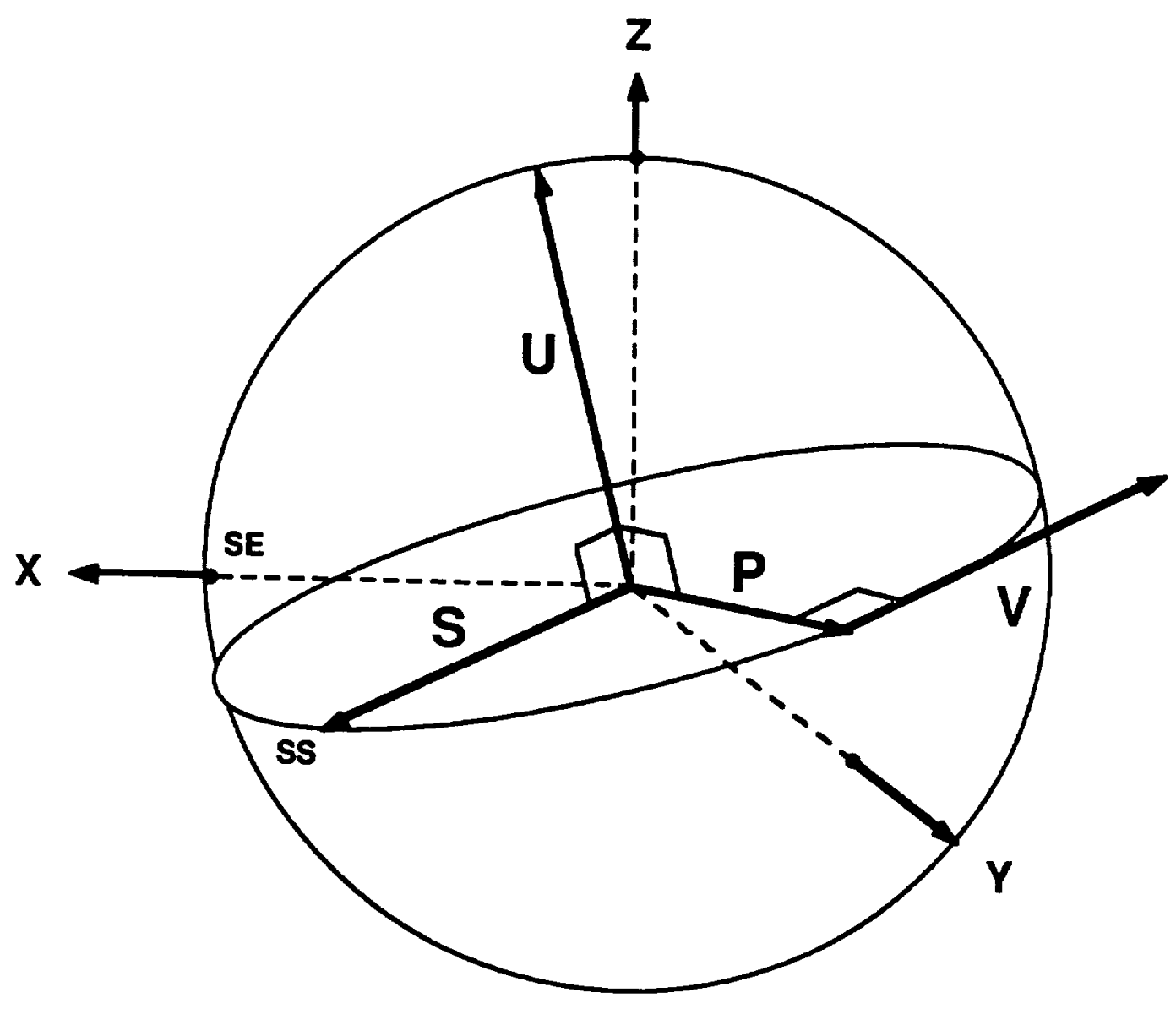

Figure A2.3. SS-AS Circulation Aspect Geometry 


$$
U_{z}(i, j)=\frac{y_{i j} x_{s s}-x_{i j} y_{s s}}{\sin \Omega_{i j}} .
$$

A2.6. RELATIVE INTENSITY $S_{i j}$

Relative intensity $s_{i j}$ is given by:

$$
s_{i j}=L_{i j} \cos \Omega_{i j}
$$

where $\Omega_{i j}$ is the solar zenith angle (SZA) of the element centroid (Eq. A2.24), and $L_{i j}$ is the width of the emitting shell projected along the line of sight (see Section 7.1). $L_{i j}$ is in units of the shell width. Consider Figure 7.6 with $10 \mu \mathrm{m}$ continuum radius $\mathrm{R}_{\mathrm{p}}$ (solid planet radius plus $60 \mathrm{~km}=6112 \mathrm{~km}$ ), non-thermal emitting shell inner radius $R_{1}(6153 \mathrm{~km})$ and outer radius $R_{2}(6172 \mathrm{~km})$. On the plane of the sky, the element centroid distance from disk center $\left(D_{i j}\right)$ is given by:

$$
D_{i j}=\left[y_{i j}{ }^{2}+z_{i j}{ }^{2}\right]^{1 / 2}
$$

An element centroid may reside in one of four regions:

$\begin{array}{cc}\text { Region } & \text { Condition } \\ 1 & D_{i j} \leq R_{P} \\ 2 & R_{P}<D_{i j} \leq R_{1} \\ 3 & R_{1} \leq D_{i j} \leq R_{2} \\ 4 & D_{i j}>R_{2}\end{array}$

Denoting $L_{i j}$ for region $N$ as $L_{i j}(N)$, then: 


$$
\begin{aligned}
& L_{i j}(1)=\frac{1}{R_{2}-R_{1}}\left(R_{2}^{2}-D_{i j}^{2}\right)^{1 / 2}-\frac{1}{R_{2}-R_{1}}\left(R_{1}^{2}-D_{i j}{ }^{2}\right)^{1 / 2} \\
& L_{i j}(2)=2 L_{i j}(1) \\
& L_{i j}(3)=\frac{2}{R_{2}-R_{1}}\left(R_{2}^{2}-D_{i j}\right)^{1 / 2} \\
& L_{i j}(4)=0
\end{aligned}
$$

$L_{i j}$ can attain a value of 51 for an element centroid intercepting the emitting shell tangentially (Region 3). The emitting shell's thickness maximally subtends only 0.1 arc-seconds at the limb. Thus a beam partitioned such that an element in Region 3 always resolves the emitting shel1 ( $100 \times 100$ elements) will exhibit a dramatic across-the-beam gradient in $L_{i j}$, hence in $s_{i j}$.

\section{A2.7. BEAM INTEGRATION}

Substitution of $\Phi_{i j}, S_{i j}, A_{i j}$, and $v_{i j}$ into Eq. A2.5 and summing over $i$ and $j$ yields the modelled beam-integrated line of sight wind velocity. A beam element whose centroid is off the planet or associated with a $S Z A>90^{\circ}$ is zero-weighted.

A non-zero pointing uncertainty $(\Gamma)$ may be associated with beam center. To generate the model's pointing uncertainty-induced line of sight wind velocity error bars, eight beam center offsets were made (of magnitude $\Gamma>E, N E, N, N W, W, S W, S$, and SE. The corresponding beam center uncertainties in $\alpha_{b c}$ and $\beta_{b c}$ are represented on Figures 7.2 to 7.5. 


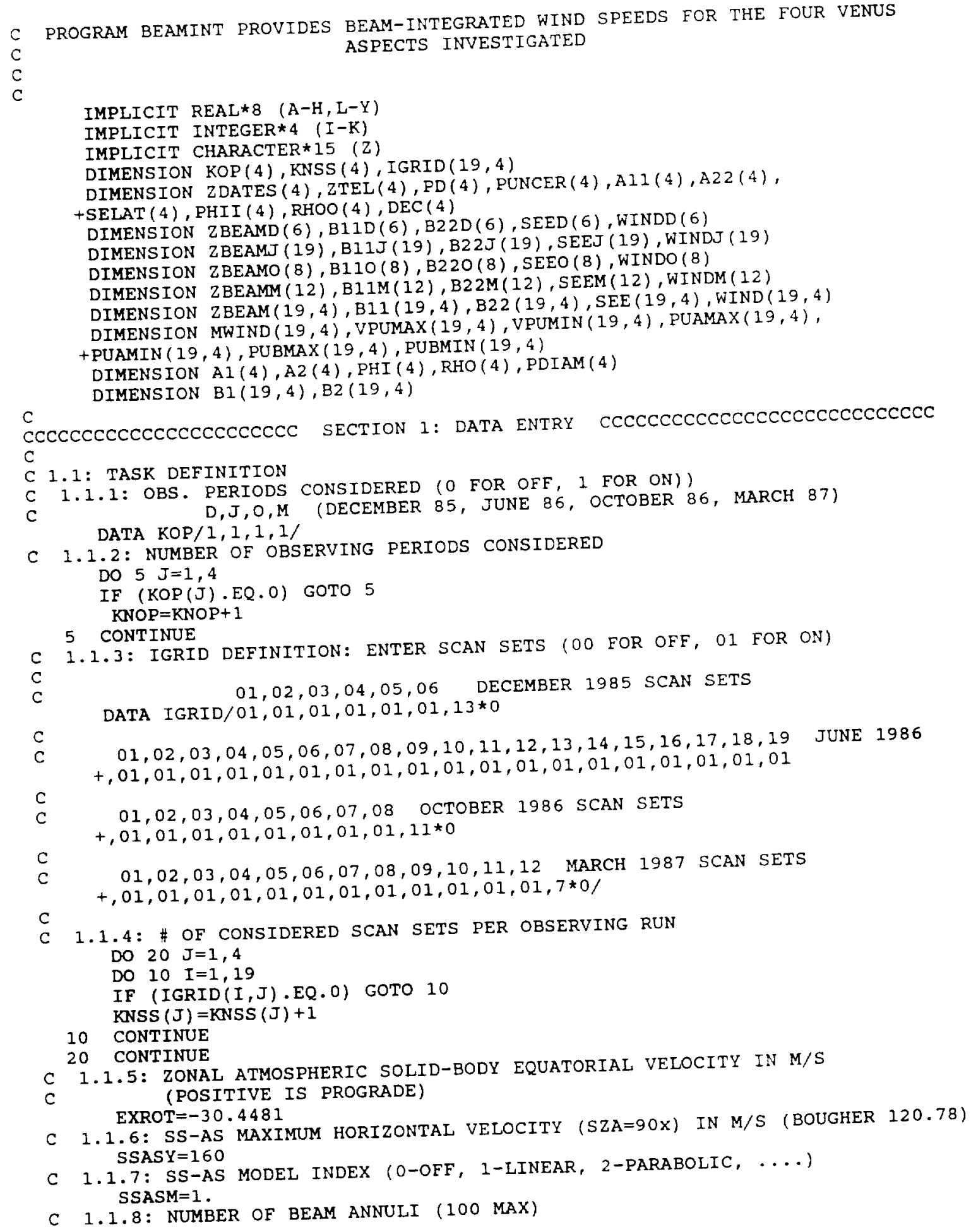




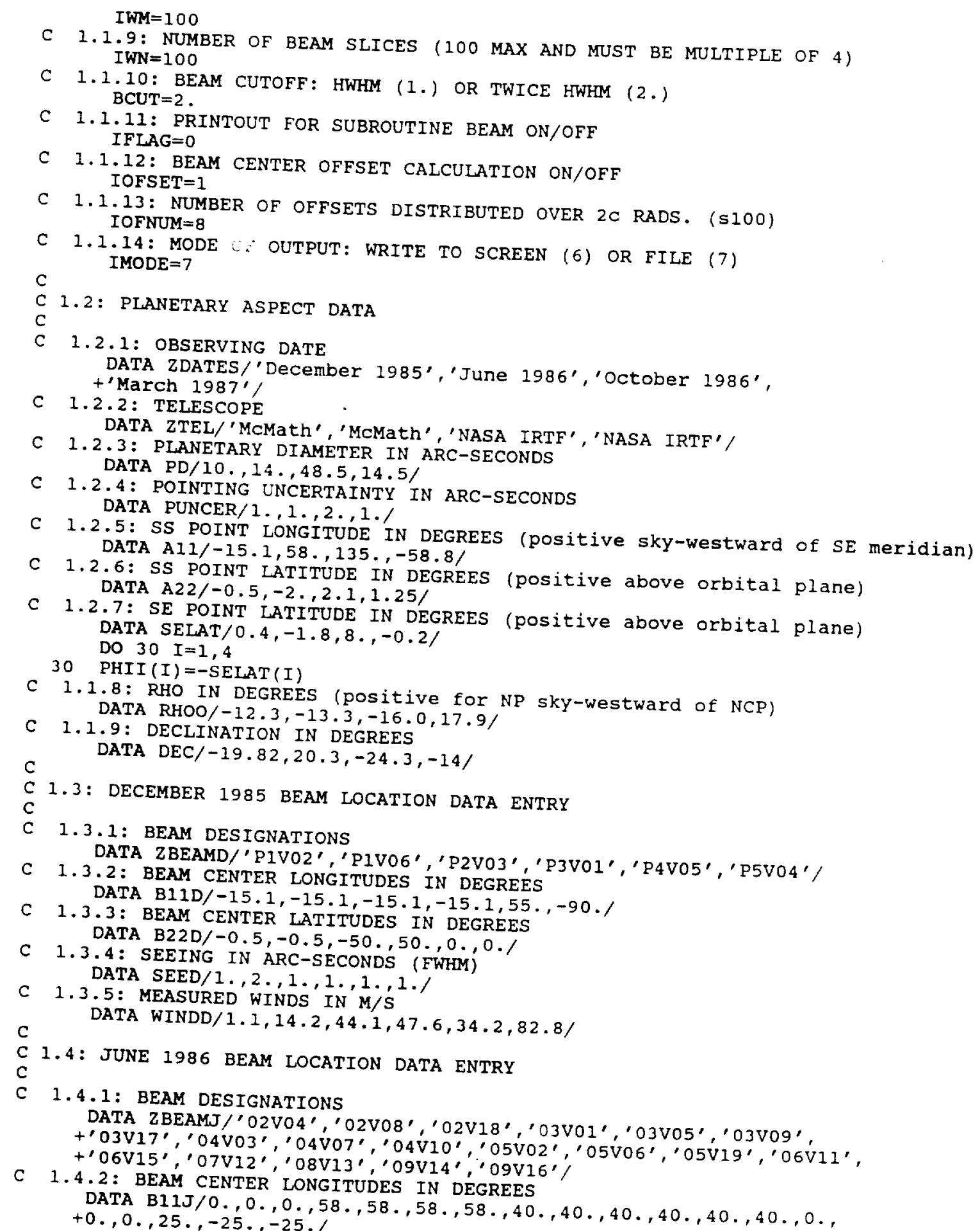




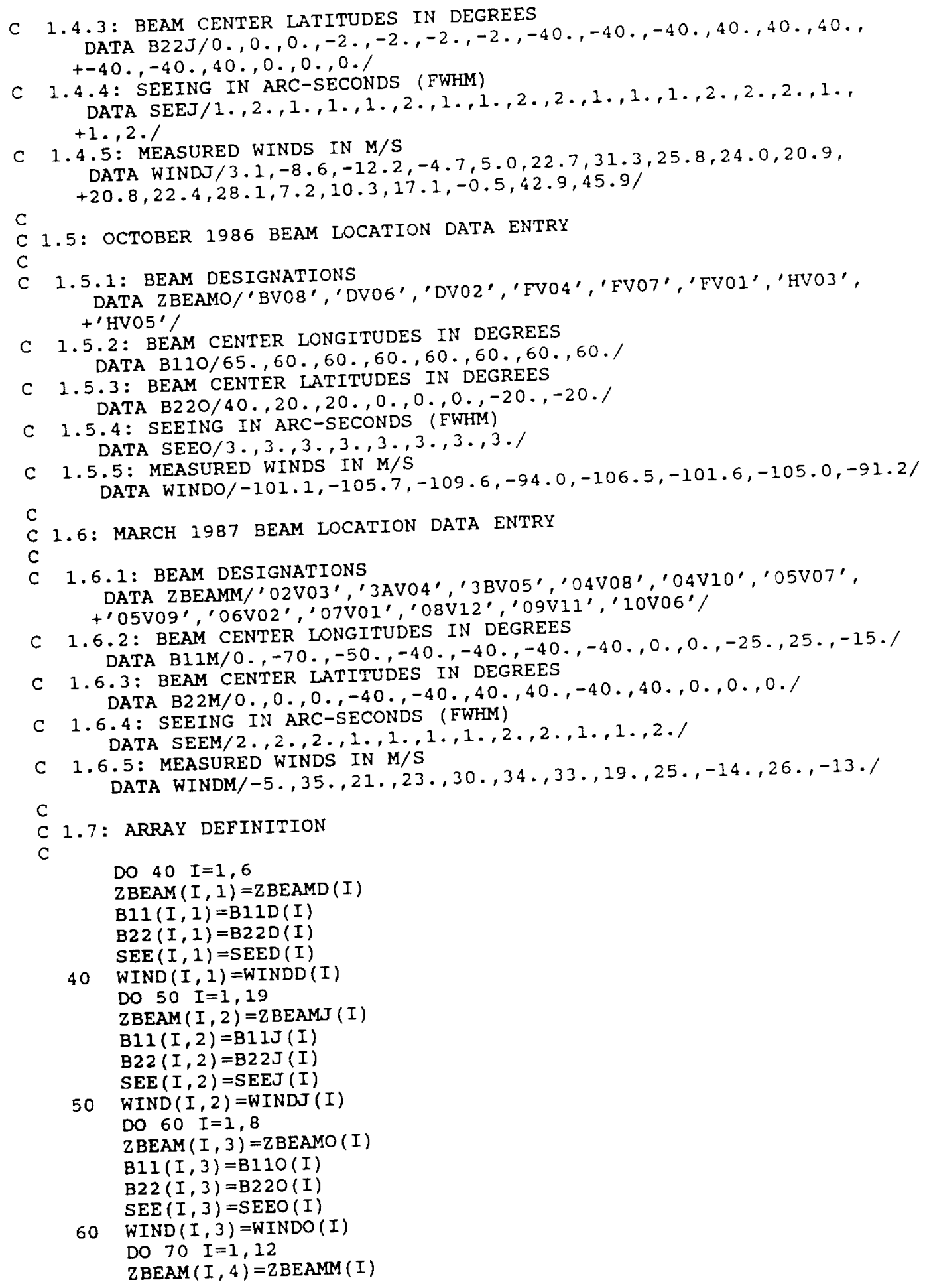




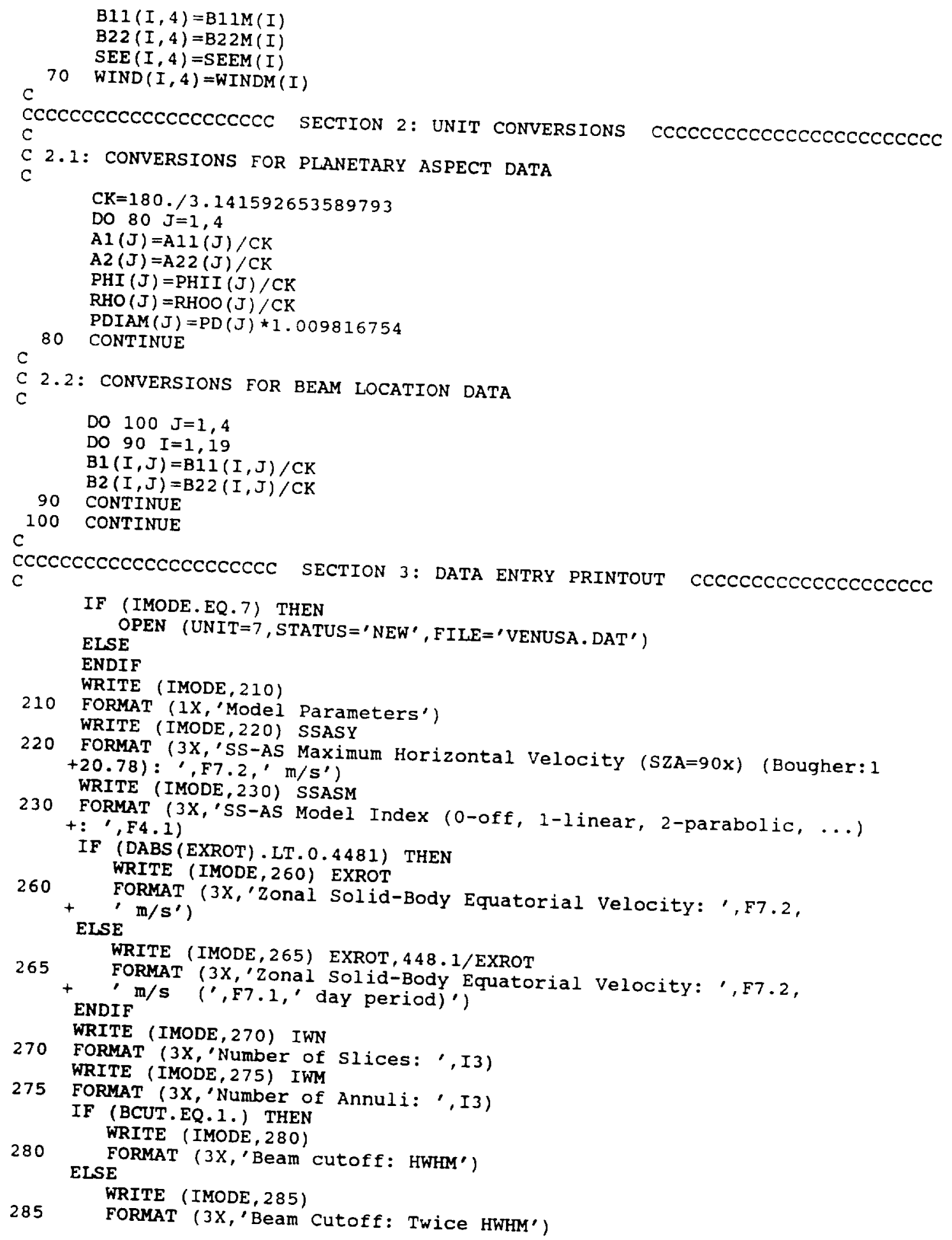




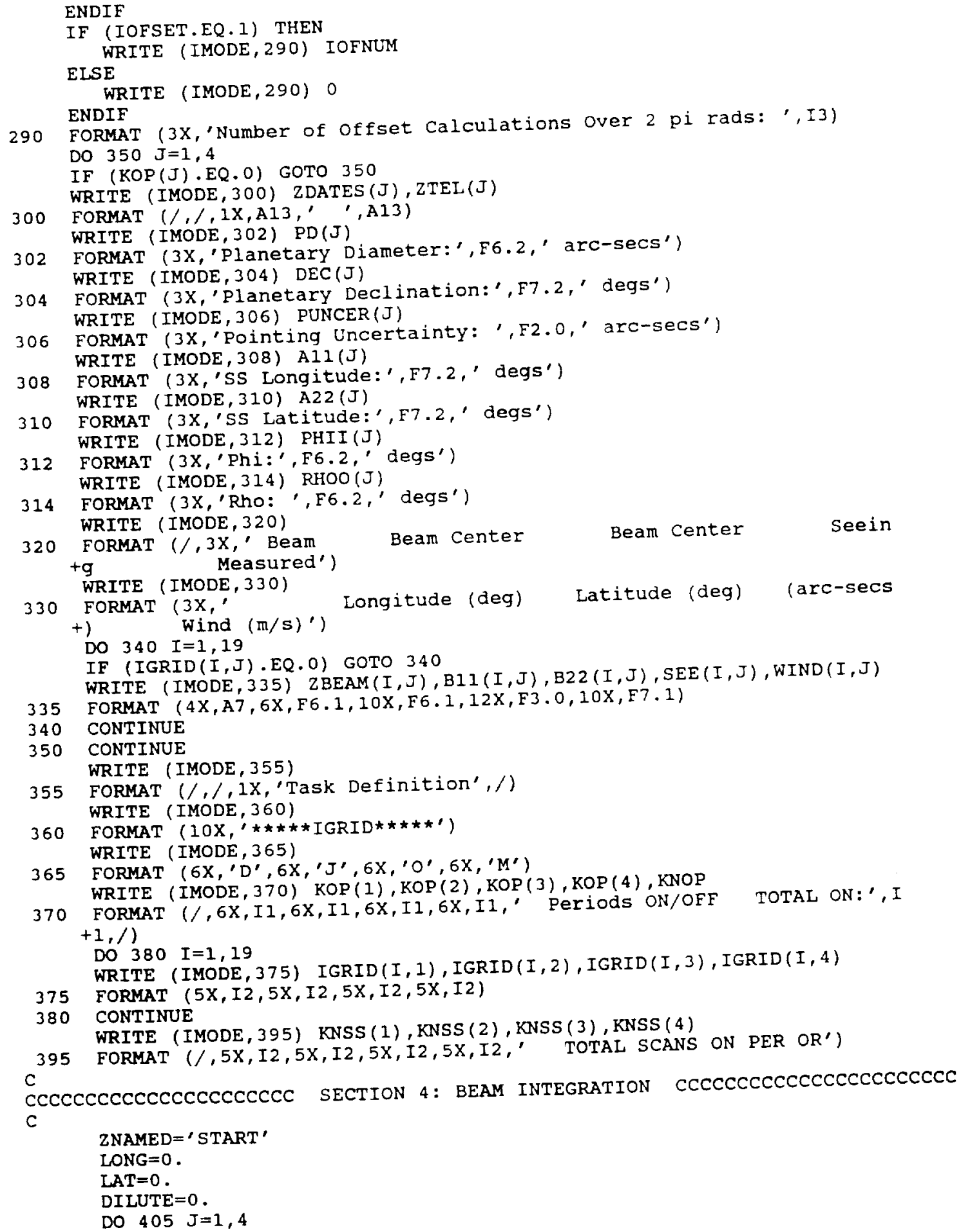




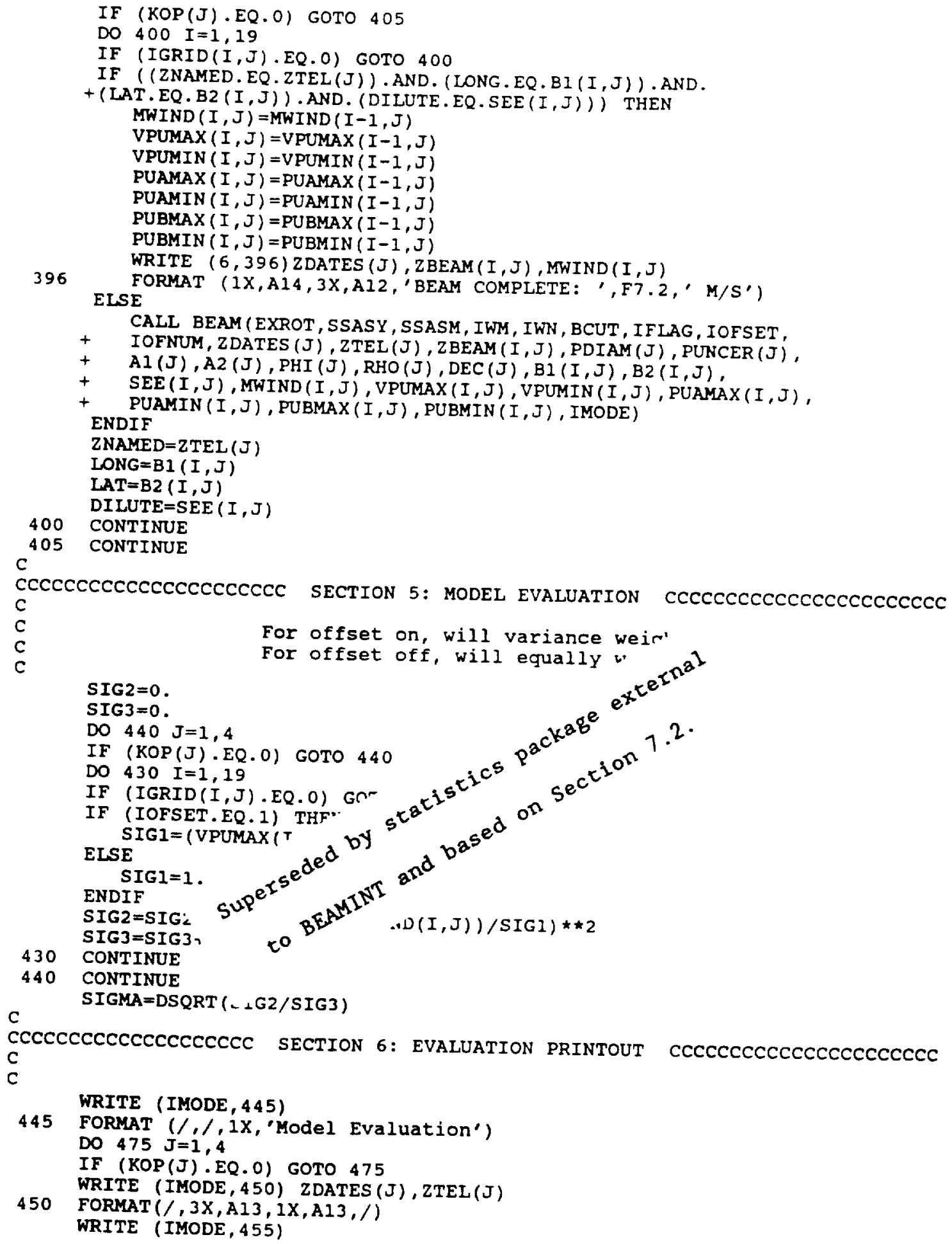




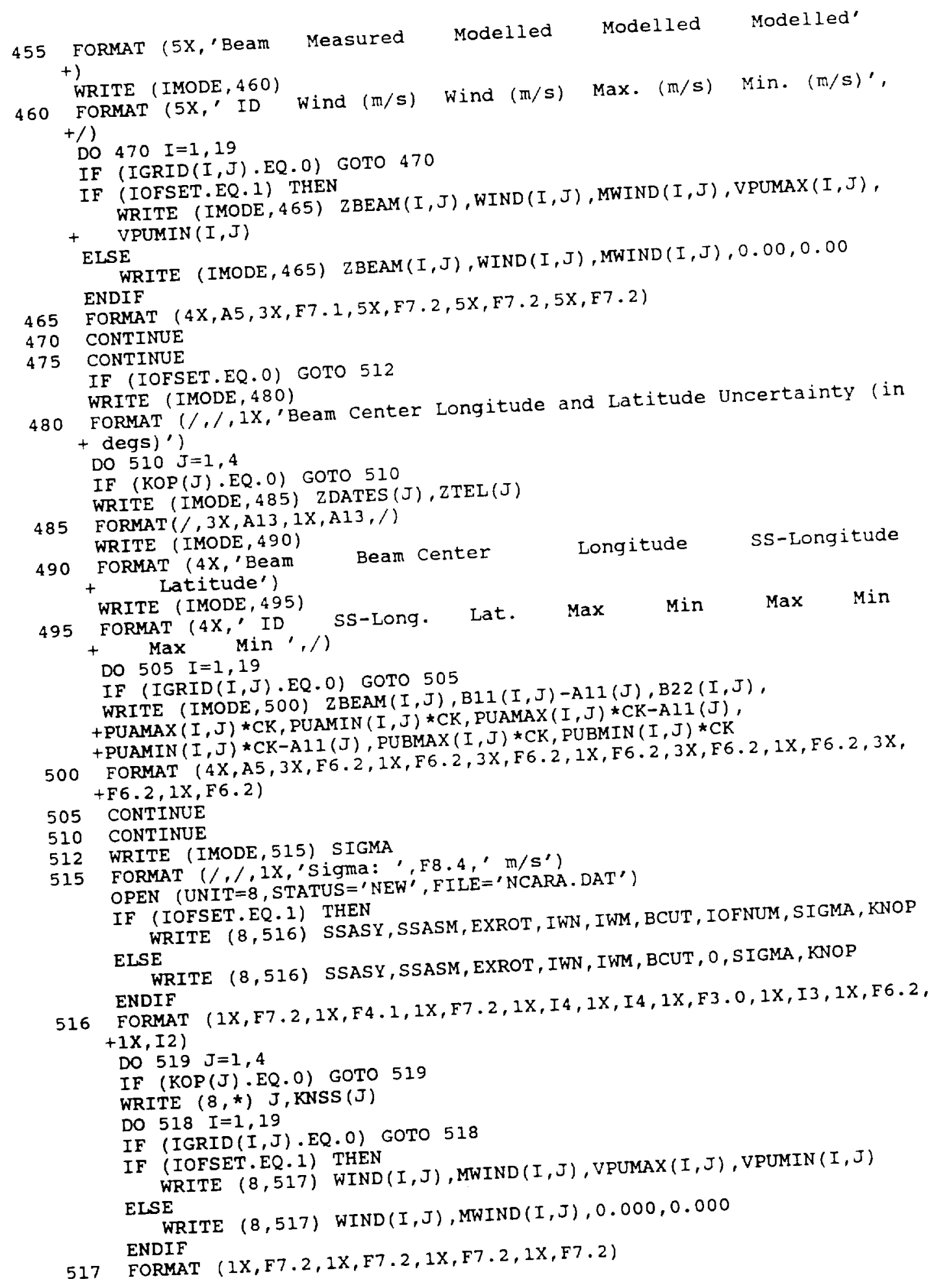




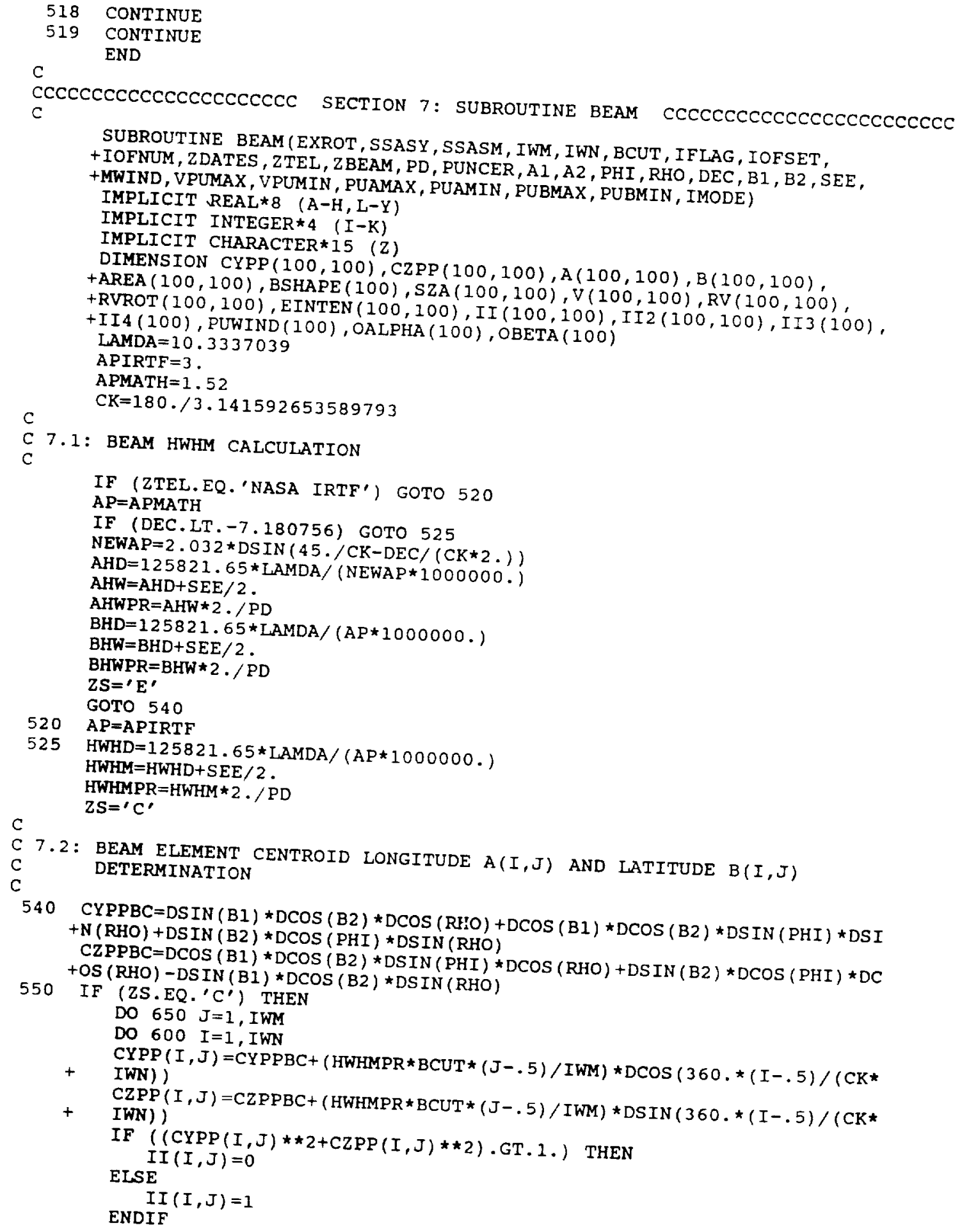




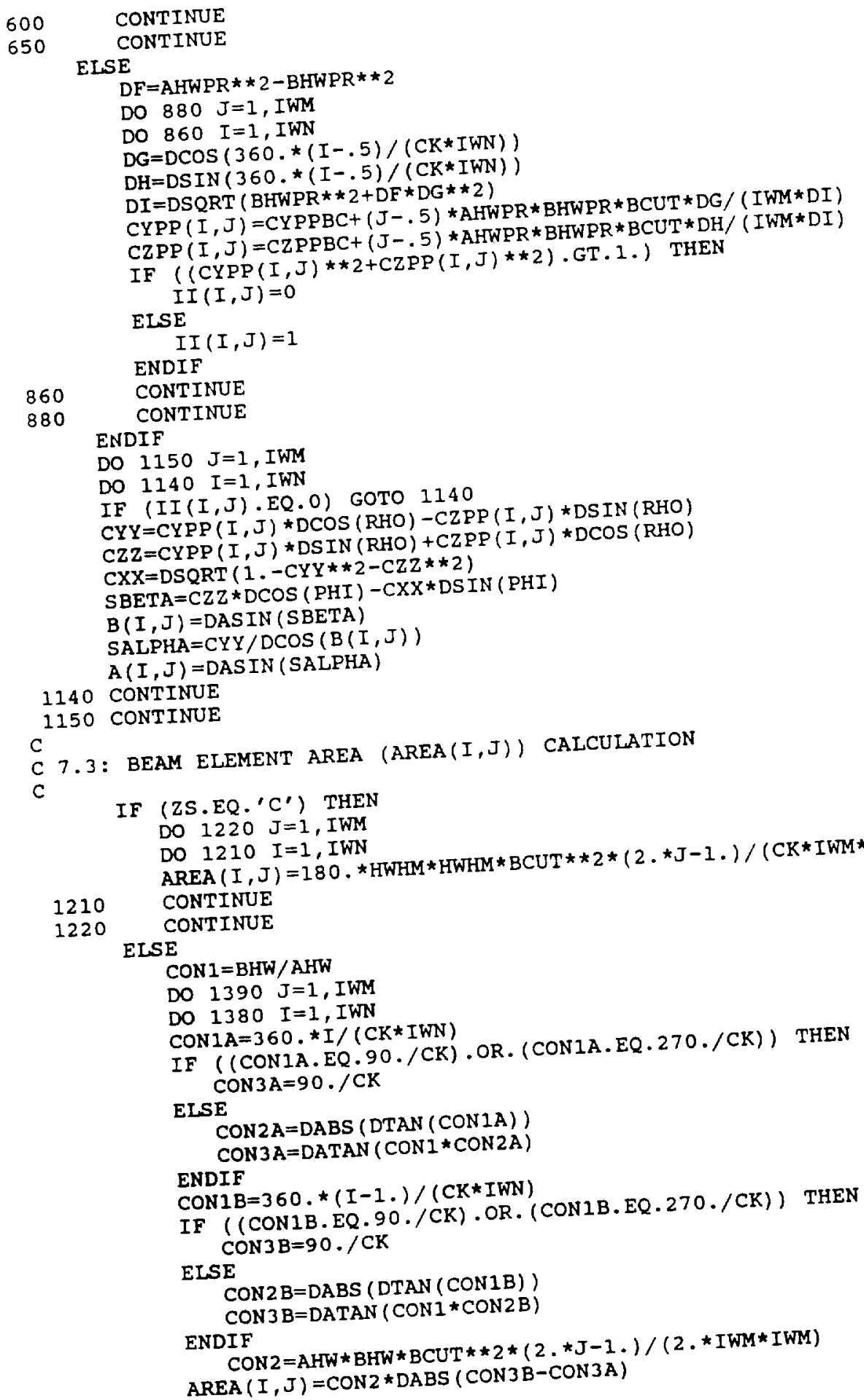




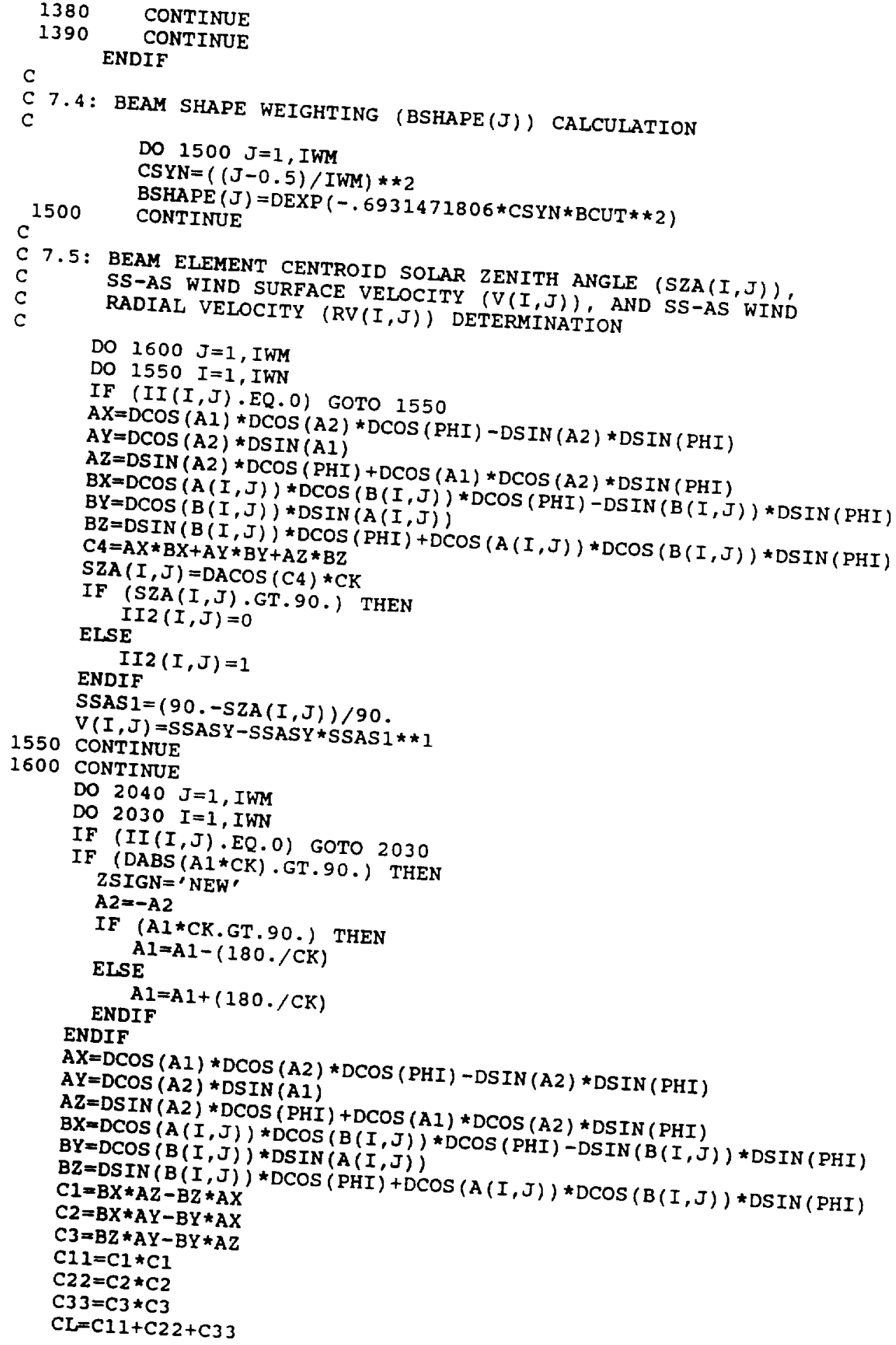




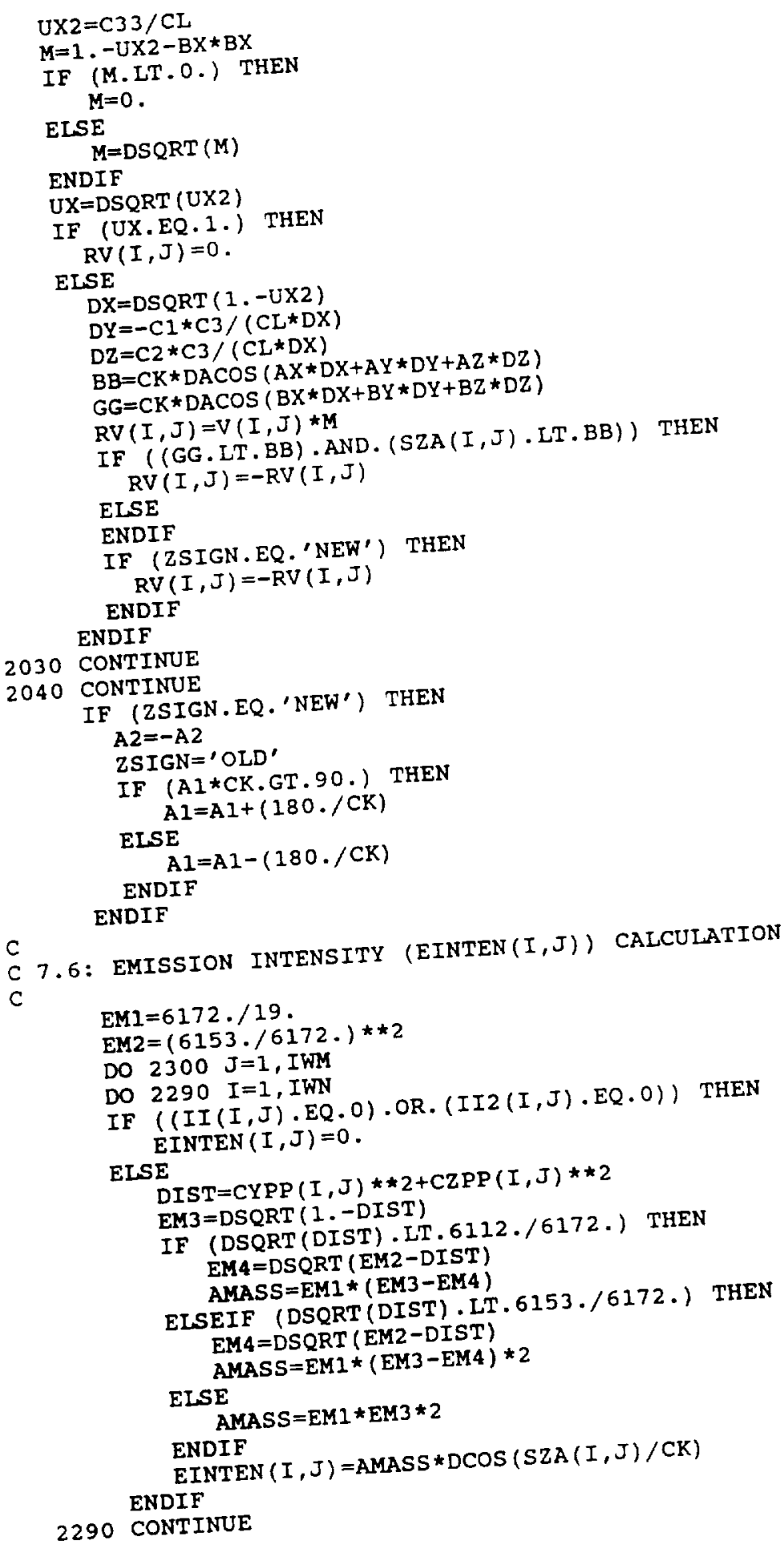




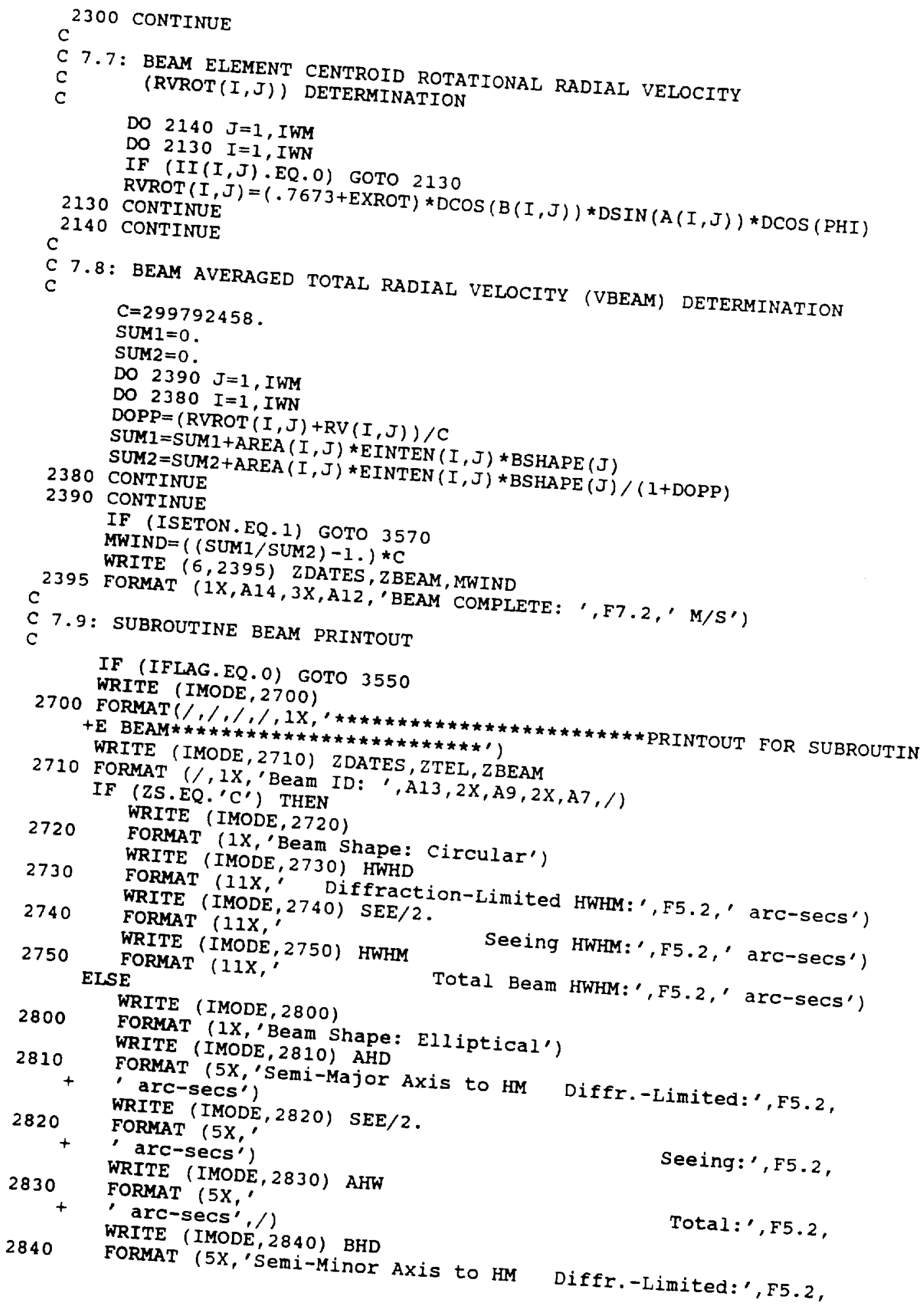




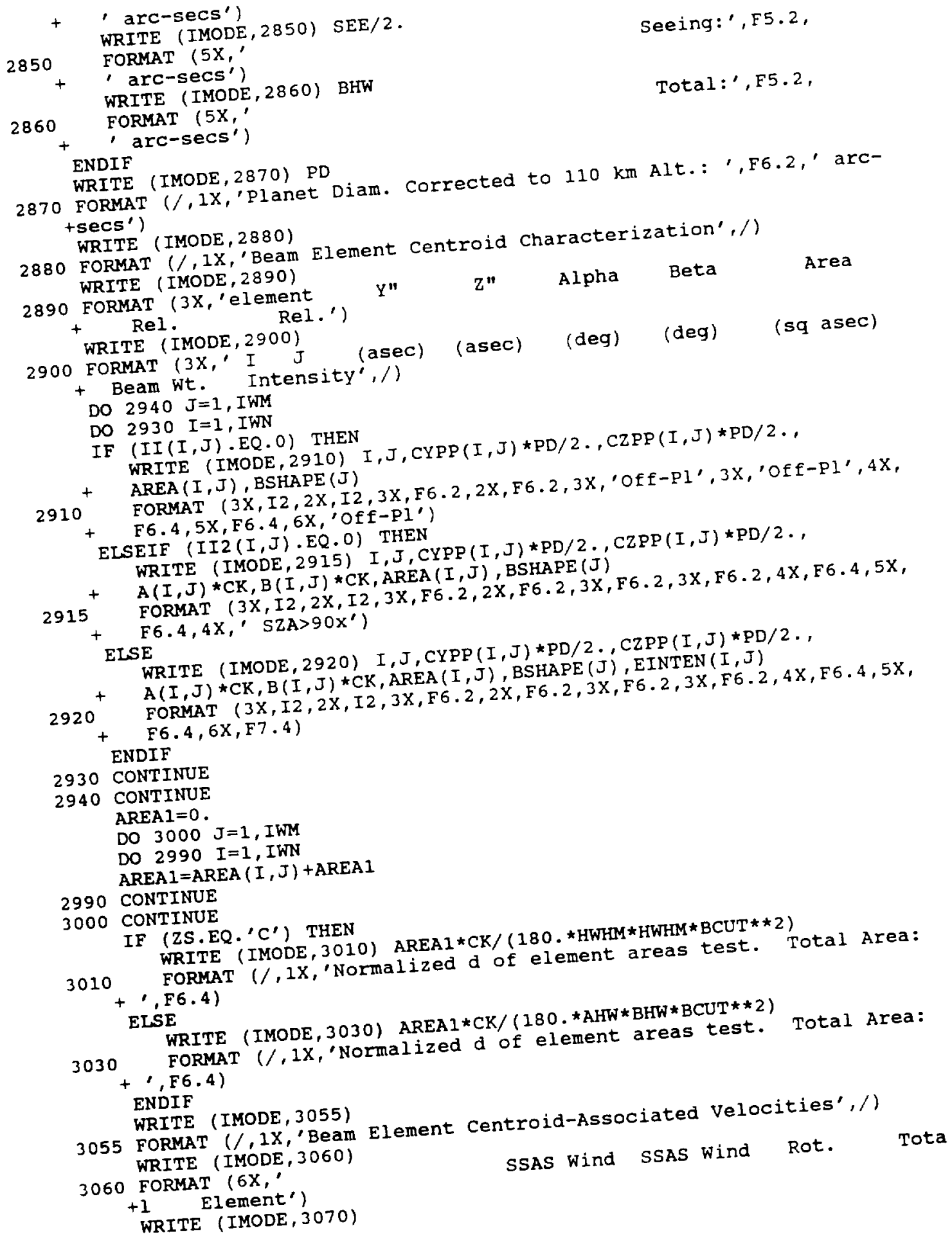




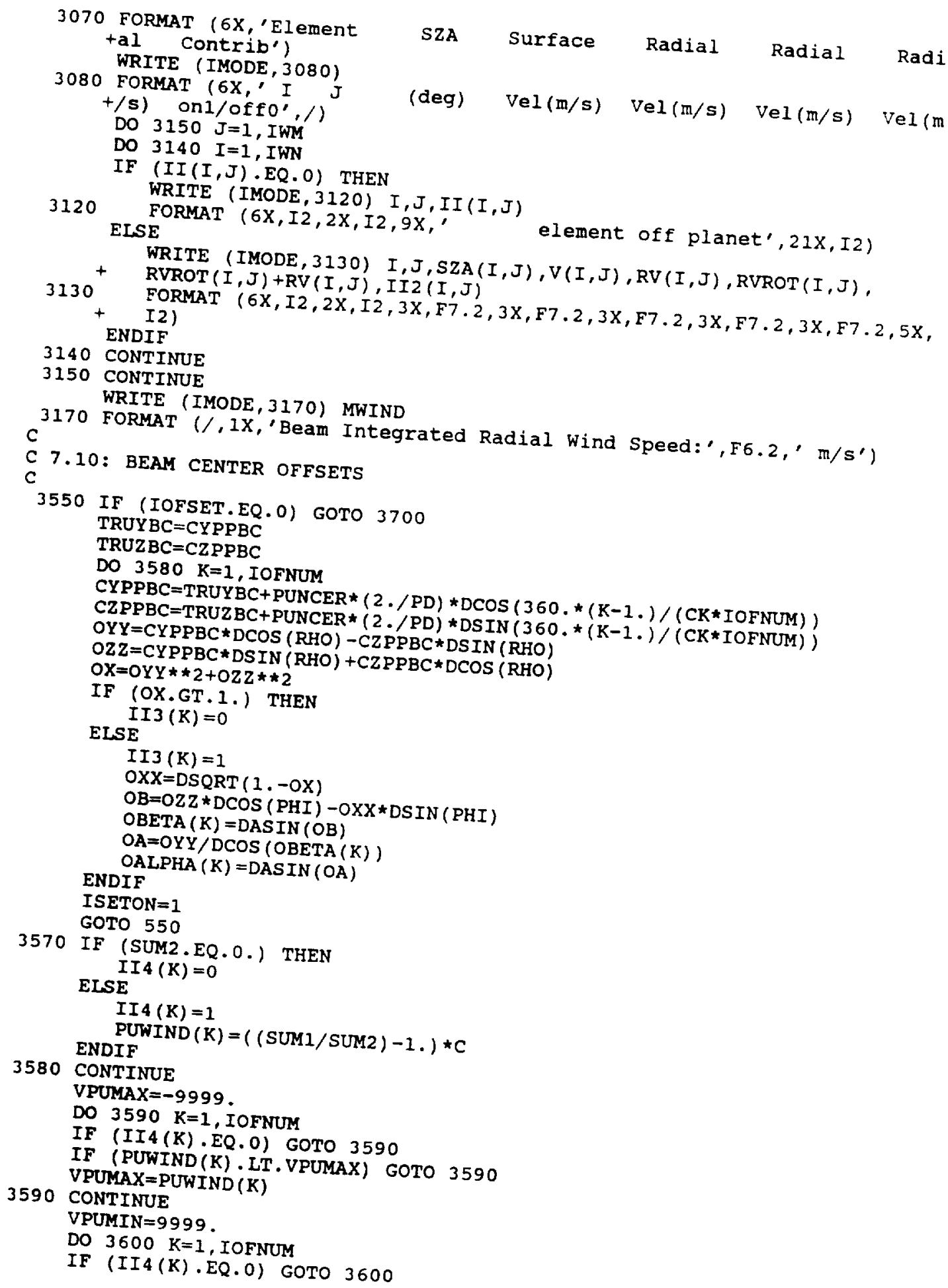


IF (PUWIND(K).GT.VPUMIN) GOTO 3600

VPUMIN=PUWIND $(K)$

3600 CONTINUE

PUAMAX $=-9999$.

DO $3605 \mathrm{~K}=1$, IOFNUM

IF (II3 (K) . EQ.0) THEN

IF (B1.GT.0.) THEN

PUAMAX $=90 . / \mathrm{CK}$

ELSE

GOTO 3605

ENDIF

ELSE

IF (OALPHA $(K)$.

ENDIF

3605 CONTINUE

PUAMIN $=9999$.

DO $3610 \mathrm{~K}=1$, IOFNUM

IF (II3 (K) .EQ.0) THEN

IF (BI.LT.O.) THEN

PUAMIN $=-90 . / \mathrm{CK}$

EISE

GoTO 3610

ENDIF

EISE

IF (OALPHA (K) GT.PUAMIN) GOTO 3610

PUAMIN $=O A L P H A(K)$

ENDIF

3610 CONTINUE

PUBMAX $=-9999$.

DO $3615 \mathrm{~K}=1$, IOFNUM

DO $(\mathrm{K})$.EO.0) GOTO 3615

IF (II3(K) K . LT.PUBMAX) GOTO 3615

IF (OBETA $(K)$ PUBMAX $=$ OBETA $(K)$

3615 CONTINUE

PUBMIN $=9999$.

DO $3620 \mathrm{~K}=1$, IOFNUM

(K) EO O) GOTO 3620

IF (II3 (K) EQ.OT. PUBMIN) GOTO 3620

IF (OBETA $(K) \cdot G$

3620 CONTINUE

ISETON $=0$. EQ.0) GOTO 3700

WRITE (IMODE, 3630 ) PUNCER of sets', 3X,'Pointing Uncertainty:

3630 FORMAT $(/, 1 X, '$ Beam

,$+ F 3.1$, arc-secs') $^{\prime}$

WRITE (IMODE, 3635 )

3635 FORMAT (/,5X,' Bea

3640 FORMAT ( $5 \mathrm{X}$, ' LONG.

DO $3660 \mathrm{~K}=1$, IOFN) THEN

IF (II3 (K) (IMODE, 3645) OALPHA (K) WRITE (IN

3645 FORMAT (9X,F6.2, IOX,

EISEIF (II4 (K).EQ.1) THEN PUWIND(K)

WRITE (IMODE, 3650) PUW off Planet', 10X, F7.2)

3650 ISE

FORMAT ( $9 \mathrm{X}$, 'Beam Center off Planet',

3655

WRITE (IMODE, 3655) offset Beam off Planet')

3660 CONTINUE

3700 END 
$-$ 
APPENDIX 3: THE VENUSIAN ATMOSPHERE - A HISTORICAL REVIEW TO 1962

Since 1962 no less than 15 Soviet and 6 American spacecraft have been sent to probe the Venusian environs. From this veritable armada were dispatched 17 entry probes, 11 of which transmitted data through descent with continued operation on the surface, 6 orbiters and 2 balloon-borne probes. Yet prior to the space age, astonishingly little was learned, in 300 years of ground-based observation, about our nearest planetary neighbor. In fact, until the advent of radar and radio astronomy in the late $1950^{\prime} \mathrm{s}$, the solid planet remained hidden from view, all Venus studies necessarily probing the atmospheric cloak above the cloud tops at $-65 \mathrm{~km}$ altitude. As such were by definition studies of atmospheric dynamics, they complement well the subject of this work.

What follows is a comprehensive review of Venus dynamical studies up to the flight of the first space probes. It is ironic testimony to human exploration of the cosmos - indicative of the dramatic strides in our understanding - yet humbling, for it demonstrates that our journey has only begun.

\section{A3.1. EARLY TELESCOPIC OBSERVATIONS}

Telescopic observations as early as 1645, by Francesco Fontana, gave rise to reports of both bright and dusky ill-defined spots on the brilliant disk of Venus (Fontana 1646, Riccioli 1651). The spots' periodic reappearance was regarded as a key to the planet's rotation period, though much of the literature is historically inaccurate as to who made what claim (Chandler 1897). The first to attempt such a 
determination was Jean Dominique Cassini at Bologna, in 1666 and 1667. Cassini was quite hesitant to derive a rotation period from features so vague and variable in form that their motion often seemed illusory, and which, during his best observation, could only be tracked for little more than an hour. He wrote simply, "... that in less than one day [the spot] completes its motion - be it revolution or libration - so that in about 23 days it returns at about the same time to the same location on the planet..." (J.D. Cassini 1667a,b). In a similar undertaking from Rome in 1726, Francesco Bianchini (1728) obtained the considerably longer period of $24^{\mathrm{d}} 8 \mathrm{~h}$.

After a failed attempt to determine Venus' rotation period with Maraldi in 1729, Jacques Cassini (1732a,b, 1740) combined the observations of his father and Bianchini to obtain a period of $23^{\mathrm{h}} 20^{\mathrm{m}}$, arguing that Bianchini unknowingly reported a sub-harmonic of the true period. To the younger Cassini, both sets of observations were mutually consistent if, in fact, Venus completed 25 rotations in the span of Bianchini's $24^{\mathrm{d}} 8^{\mathrm{h}}$ period. It is the son's $23^{\mathrm{h}} 20^{\mathrm{m}}$ rotation period which incorrectly frequents the literature as J.D. Cassini's original result of 1667 (Chandler 1897, see also Moore and Hunt 1983, Flammarion and Gore 1894, Hind 1852).

During the transit of 1761 in St. Petersburg (Leningrad), Mikhail Vasilyevich Lomonosov (1955a) found Venus' limb off the solar disk well defined by a luminous halo. He correctly attributed this effect to Venus' atmosphere and is credited with its discovery (Lomonosov 1955a,b). He wrote, "... the planet Venus is surrounded by a considerable atmosphere equal to, if not greater than, that which 
envelops our Earthly sphere" (Kudryavtsev 1954, Menshutkin 1952). Lomonosov's observations following first contact were confirmed by David Rittenhouse during the transit of 1769 (Hindle 1980, Herrmann 1984).

Concurrent observations of Venus made by Johann Schroter (1792, 1795) at Lilienthal in Germany, and William Herschel (1793) in England, from 1777 to 1793, convinced both men they were seeing predominantly light reflected from Venus' extensive atmosphere and not the true surface of the planet. "Replete with matter that reflects and refracts light copiously in all directions", as described by Herschel, such an atmosphere would account for the observed anomalously strong decrease in brilliance from limb to terminator due to the corresponding foreshortening of Venus' atmospheric column along the line of sight. As we11, nearly all surface definition derived from sunlight reflected at the surface would be obliterated by the strongly scattering atmosphere, consistent with the virtual absence of structure on the observed disk. While Herschel felt these effects implied extensive cloud cover, Schroter viewed them as resulting naturally from a thick atmosphere, "generally clear and transparent, like our own". Schroter adding, "... I cannot think ... that Providence would bless the inhabitants of Venus, incomparably less than ourselves, with the happiness of seeing the works of almighty power, and of discovering, like a Herschel, still more distant regions of the universe".

A more direct indication of an extensive Venusian atmosphere lay in Schroter's observation that the horns of Venus' crescent extended well beyond the line of the diameter. Schroter wrote, "... this phaenomenon 
is occasioned by a light reflected by the atmosphere of Venus into the dark hemisphere of the planet, ... being in fact, a real twilight". Schroter found this twilight to extend some 309 miles across the terminator, from which he calculated a perpendicular height of at least 3 miles for "the inferior and more dense part of the atmosphere".

Both observers also reported seeing the bright and dusky spots. To Herschel the variable nature of the spots always precluded a period determination. Schroter, in 1786, perceived spot motions indicative of a rotation period near 24 hours.

Schroter further claimed the discovery of a new periodic phenomenon - which considerably irked his illustrious colleague in England. Venus' horns near maximum elongation, particularly the southern horn, appeared sometimes blunted, other times with small luminous projections into the night hemisphere. He attributed both effects to extensive mountain ridges, with peaks rising 23 miles above the surface.

Schroter's initial observations of the new phenomenon from 1789-91 yielded a rotation period of $23^{\mathrm{h}} 21^{\mathrm{m}}$. Coupling these with a subsequent series of observations in 1793, Schroter obtained a period of $23^{\mathrm{h}} 20^{\mathrm{m}} 19^{\mathrm{s}}$.

Meanwhile, Herschel, after trying in vain to see the telltale irregularities in Venus' horns wrote; "... as to the mountains on Venus, I may venture to say that no eye, which is not considerably better than mine, or assisted by much better instruments, will ever get a sight of them". The reflecting telescope schroter principally used for his observations was built by Herschel.

The derived rotation periods were remarkably consistent - notably 
close to the terrestrial day. Yet the all-important bright and dusky spots could not be seen by many observers, could not be recovered by even the elder Cassini after his initial observations, and no hard evidence attested to their reality ( $\mathrm{J}$. Cassini $1732 \mathrm{~b}, 1740$, Schiaparelli 1890). The value, however, stood firm one hundred years beyond Schroter with, for example, Francesco de Vico reporting in 1841 a period of $23^{\mathrm{h}} 21^{\mathrm{m}} 22^{\mathrm{s}}$ after what was at the time considered a painstaking effort (Chandler 1897).

Giovanni Schiaparelli (1890, 1895) met considerable opposition in 1890 when his observations led him to claim a solar-synchronous rotation period of $224.7^{\mathrm{d}}$. While he felt the observational constraints weak enough to allow a rotation period from 6 to 9 months, solar-synchronicity seemed naturally appealing. Far from precedent setting - the Moon exhibited such a dynamical lock with Earth Schiaparelli's view implied either perpetual day or perpetual night for an observer on Venus.

By the turn of the twentieth century enough observational momentum had been gained by the Schiaparelli bandwagon, with Terby, Perrotin, Tacchini, Cerulli (1896) and Mascari (1896) firmly on board, and none other than Percival Lowell (1896b, 1903) at Flagstaff as one of the leading proponents, that the true rotation period of Venus was admittedly uncertain (Chandler 1897, Macpherson 1913, See 1910). On a somewhat facetious note, L. Brenner, a member of the $24^{\mathrm{h}}$ camp, reported in 1895 a period of $23^{h_{5}} 7^{\mathrm{m}} 7.5459^{\mathrm{s}}$, reflecting a level of precision certainly ahead of our time (Rotation of Venus 1896).

Taken together, a Venus diameter nearly identical to Earth's (known 
since the transit of 1761), an extensive atmosphere with ubiquitous cloud cover suggesting abundant water vapor, and a rotation period nearly $24^{\text {h }}$ laid a firm groundwork for the "twin planet" concept with strong implications for life on Venus (Mumford 1909).

A very contrary view, based on a solar-synchronous rotation, was forwarded by Lowell (1896a). He envisioned the sunlit hemisphere as a vast desert, scorched by a Sun motionless in Venus' sky, and the night hemisphere covered by the planet's almost entire complement of water in the form of glacial ice. All hope for life seemed to reside in the zone of perpetual twilight, a circumferential band centered on the terminator, which separated the two climatic extremes (Mumford 1909). Lowell (1896a) also held the observed visual markings to be surface structure, consistent with an atmosphere devoid of clouds, all moisture long ago having diffused across the terminator and frozen out on the dark side.

\section{A3.2. PHOTOGRAPHIC DETECTION OF ROTATION}

While visual attempts to determine Venus' rotation period continued well into the twentieth century, the critical need for far less subjective means of attacking the problem was clear.

The photographic record for Venus dates back to at least 1890 with work done at Lick Observatory by E.S. Holden and W.W. Campbell (1890). F. Quenisset at Flammarion Observatory near Paris, in 1911 and 1921, laid claim to the first successful photographs of Venus' markings though it appears that A. Rordame, observing in Salt Lake City, Utah, should be credited with the first published photographic evidence in 
1922 (Quenisset 1911, 1921; Rordamme 1922a,b).

In 1924 W.H. Wright at the Lick Observatory obtained photographs in the near-IR $(7600 \AA)$ and violet $(4400 \AA)$ with structure only evident in the latter group (Wright 1925, 1927). A series of UV photographs at $3600 \AA$, taken by F.E. Ross at Mt. Wilson in 1927, exhibited remarkably detailed structure over Venus' entire disk (Ross 1928). Ross felt the observed band structure could not be reconciled with a slow rotation. Consistent with Wright's observations, Ross' photographs in the blue-violet, blue, red and IR showed diminishing detail with increasing wavelength. The red and IR photos exhibited no detail at all.

Ross' original plates were unfortunately lost, with serious photographic studies not undertaken again until 1941 by $H$. Camichel and A. Dollfus at Pic-du-Midi (Dollfus 1953, 1955), and 1950 by G.P. Kuiper at McDonald Observatory (Richardson 1955). Both Kuiper and R.S. Richardson, carrying out a similar investigation in 1955 at Mt. Wilson, tried to deduce Venus' obliquity from its banded appearance on their UV photographs, obtaining $32^{\circ}$ and $14^{\circ}$, respectively (Kuiper 1954 , Richardson 1955). The results, as inconsistent as those by early telescopic observers (Chandler 1897), failed to deduce the true obliquity of $2.6^{\circ}$ (Colin 1983). Kuiper's observations led him to a fairly short rotation period of at most a few weeks. Dollfus (1955), carrying the Schiaparelli view into the second half of the twentieth century, concluded a solar-synchronous rotation period, based on his visual and photographic observations.

Detailed UV photographs made 1957-59 at Brazzaville, Republic of the Congo, by amateur astronomer $C$. Boyer, provided the first evidence 
of a $4^{d}$ sidereal retrograde rotation period (Boyer 1958a,b). Initially reported to the Académie des Sciences in 1957 (Smith 1967), Boyer tracked the large-scale, dark, Y-shaped feature now famous from spacecraft imaging, determining a spin axis nearly perpendicular to the orbital plane. Boyer asked Camichel at Pic-du-Midi to verify the result, which the latter found to be consistent with UV photographs taken at the Pic in 1953 (Boyer and Camichel 1961, Boyer et al. 1960). Venus' atmosphere, and by logical extension, the planet itself seemed to be rotating "backwards". Uranus, the only known retrograde rotator, had an obliquity of $98^{\circ}$ and was thus only marginally retrograde (Abell et al. 1987). If correct, Venus' case was the most extreme departure from solar system formation theory, which held all spin and orbital angular momenta for the Sun and the planets should be nearly aligned. The need for an ad hoc explanation for venus (and Uranus) was unpalatable (see Lago and Cazenave 1979).

While numerous UV photographic follow-up investigations at Pic-du-Midi and New Mexico State University Observatory confirmed the $4^{\mathrm{d}}$ retrograde result (Boyer and Camichel 1965, 1967; Boyer and Guerin 1966, 1969; Smith 1967; Scott and Reese 1972), concerns that the UV large-scale markings were tracers of planetary waves rather than of actual mass motion still remained (Young 1975).

As a historical note, Richardson's (1955) UV photographic study may have given the markings on Venus seen by visual observers their first foothold in reality. H.P. Squyres, an amateur astronomer from E1 Monte, California, had made detailed drawings of Venus' disk from blue filter visual observations. His observations were, by chance, made 
simultaneously with Richardson's (which were still underway at Mt. Wilson when Squyres appeared drawings in hand). Richardson found the visual and UV photographic detail in clear agreement.

Furthermore, to specifically address whether the visually observed markings had some basis in reality, W.K. Hartmann (1962) compared simultaneous and independent observations made by members of the Association of Lunar and Planetary Observers (ALPO). His conclusion; "while strict agreement among observers is not often found, observers frequently agree as to the general structure or pattern of the markings over periods of several hours, and occasionally ... up to several days". The study also implied visual and UV photographic agreement during periods of unusually prominent visual markings.

\section{A3.3. SPECTROSCOPIC DETECTION OF ROTATION}

Spectroscopic attempts to measure Venus' rotation in the reflected solar spectrum were made as early as 1900 by A. Belopolsky. Observing at Pulkovo Observatory in Russia, he reported a rotation period of less than $1^{\mathrm{d}}$ (Belopolsky 1900). Such investigations were recognized as direct indicators of mass motion, while UV photographic techniques assumed the markings were fixed in the atmosphere, that they had reasonably long temporal stability, and that observer subjectivity associated with photographic interpretation was minimal. Subsequent more accurate spectroscopic investigations by V.M. Slipher (1903a,b;

Lowel1 1903), St. John and Nicholson (1922, Ross 1928) and Richardson (1958) yielded a period of two weeks or longer and weakly implied a retrograde rotation. 
The first spectroscopic detection of a $4^{d}$ retrograde rotation period, consistent with the UV imaging results, was reported by $B$. Guinot (1965, Boyer 1965) at Haute-Provence Observatory. Subsequent work by Guinot and M. Feissel (1968) yielded a period of $4.3 \pm 0.4^{\mathrm{d}}$. W.A. Traub and N.P. Carleton (1975, 1979), using the PEPSIOS spectrometer on Mt. Hopkins, have provided additional confirmation and the possible detection of a time varying component.

On a related note, M.J.S. Belton et al. have concluded from Mariner 10 imaging that the large-scale UV markings are indeed planetary waves. Their speed with respect to the atmospheric bulk motion is small enough, $20-30 \mathrm{~m} / \mathrm{s}$ upstream, that the early UV derived $4^{\mathrm{d}}$ periodicities were nearly correct (Belton et al. 1976a,b).

\section{A3.4. RADIOMETRIC OBSERVATIONS OF THE CLOUD LAYER}

In 1923, the first radiometric determination of Venus' temperature by D.H. Menzel (1923), from water cell transmission measurements in the 8-13 $\mu \mathrm{m}$ band made at Lowell observatory by W.W. Coblentz and C.O. Lampland (1922), gave a rather uncertain disk averaged value of $323 \mathrm{~K}$ During the inferior conjunction of 1924, disk-resolved measurements by E. Pettit and Nicholson (1924a,b, 1955) at Mt. Wilson yielded a temperature of $250 \mathrm{~K}$ for the unilluminated portion of the disk. Most striking was that the temperature appeared uniform over the entire planet. There was also evidence from data taken before and after inferior conjunction suggesting direct rotation. Coblentz and Lampland (1924, 1925) at Lowell quickly confirmed the temperatures on the day and night sides were virtually identical. F.E. Ross (1928) noted a 
uniform temperature was consistent with rapid planetary rotation as was his observation of latitudinal bands in the UV.

Very precise radiometric measurements were made by W.M. Sinton and

J. Strong (1960) at Mt. Wilson and Mt. Palomar from 1952 to 1954. They

found a disk averaged temperature of $226 \mathrm{~K}$ with the night side only $2-3$ $\mathrm{K}$ lower than the day side. With an assumed albedo of 0.78 , and rapid rotation, $226 \mathrm{~K}$ corresponded to Venus' expected blackbody temperature (Mintz 1961). Consistent with these radiometric results, N.A. Kozyrev (1954) at the Crimean Astrophysics Observatory had obtained an integrated albedo of 0.80 in the $3800-6500 \AA$ band.

\section{A3.5. RADAR OBSERVATIONS}

Though not known with much more certainty than in 1610 , by 1961 it may have seemed that evidence for a rapid Venusian rotation was mounting. The most detailed UV photographic study (Boyer and Camichel) indicated a $4^{\mathrm{d}}$ retrograde rotation, though unconfirmed. Richardson's spectroscopic evidence for a $14^{\mathrm{d}}$ rotation was an order of magnitude more accurate than Slipher's 1903 results - which Lowell used to support solar-synchronicity. Finally, radiometric data obtained by Sinton and strong corroborated the 1924 measurements pointing to a nearly uniform temperature planetwide, consistent with rapid rotation. Yet all three means of scientific inquiry could not penetrate the cloud-tops at $65 \mathrm{~km}$ altitude.

As investigations of the cloud-top rotation period, and by inference, the solid planet rotation period seemed about to restore the short period hypothesis, results of the first successful Venus radar 
echo experiments were being reported.

During the inferior conjunction of 1961, researchers at Cal Tech's Jet Propulsion Laboratory, using the Deep Space Instrumentation Facility at Goldstone, and at MIT Lincoln Laboratory, using the Millstone Hill Radar Observatory, both reported a very slow solid body rotation (Victor and Stevens 1961, Pettengill et a1. 1962), and possibly retrograde, according to a subsequent MIT report (Smith 1963). During the inferior conjunction of 1962 two independent JPL measurements yielded a retrograde rotation period of $250 \pm 40^{\mathrm{d}}$ with an axis nearly perpendicular to the orbital plane (Carpenter 1964, Goldstein 1964), while concurrent observations in the Soviet Union established a $200-300^{d}$ retrograde rotation period (Kotelnikov et al. 1963). Measurements at sucessive inferior conjunctions yielded ever more precise values with groups at Jodrell Bank, JPL and MIT/Cornell reporting retrograde periods of $100-300^{\mathrm{d}}, 250 \pm 9^{\mathrm{d}}$ and $245.1 \pm 2 \mathrm{~d}$ respectively, for the inferior conjunction of 1964 (Ponsonby et al. 1964, Goldstein 1965, Carpenter 1966, Dyce et al. 1967).

The turn of the century dichotomy was back in an exciting new form - a slow retrograde solid body rotation and a retrograde super-rotating atmosphere. The confusion was best summed up by B.A. Smith (1967) who wrote; "the greatly different rotation periods... are difficult to reconcile with one another, for such would require a persistent and widespread planetary wind system having speeds in excess of $300 \mathrm{~km} / \mathrm{hr}$ with respect to the solid surface; ... The study of the rotation of Venus continues to produce inconsistencies."

This situation is today recognized as real. Still warranting what 
appears to be some ad hoc theory to account for the retrograde solid planet rotation (Lago and Cezenave 1979), Venus is as well an enigma with regard to its atmospheric zonal super-rotation.

While Schiaparelli's dynamically appealing solar-synchronous lock did not stand the test of time, an unexpected interaction does indeed exist. Every inferior conjunction Venus presents the same face to Earth-bound observers. Such a Venus-Earth lock dictates a sidereal retrograde rotation period of $243.16^{\mathrm{d}}$. The true period's present best value is $243.025 \pm .006^{\mathrm{d}}$ (Campbe11 1989). Coupling the retrograde rotation with the $224.7^{\mathrm{d}}$ revolution period results in a $116.75^{\mathrm{d}}$ Venus solar day (Colin 1983).

\section{A3.6. RADIOMETRIC OBSERVATIONS AT LONG WAVELENGTHS}

During the inferior conjunction of 1956 researchers at the Naval Research Laboratory observed Venus at 3.15 and $9.4 \mathrm{~cm}$ (Mayer et al. 1958, Mayer 1959). The two average brightness temperatures derived from the $3.15 \mathrm{~cm}$ data, $620 \pm 110 \mathrm{~K}$ and $560 \pm 73 \mathrm{~K}$, were more than double the $226 \mathrm{~K}$ cloud layer temperature obtained from the 8-13 $\mu \mathrm{m}$ radiometric observations. The $9.4 \mathrm{~cm}$ data, though associated with a $50 \%$ uncertainty, yielded average brightness temperatures of $430 \mathrm{~K}$ and $740 \mathrm{~K}$.

Confirmation came during the inferior conjunction of 1958 with NRL/Columbia reporting brightness temperatures of $518 \pm 50 \mathrm{~K}$ and $575 \pm 50 \mathrm{~K}$ at $3.4 \mathrm{~cm}$ and $410 \pm 160 \mathrm{~K}$ at $8.6 \mathrm{~mm}$ (Alsop et al. 1958, Gibson and McEwan 1959, Mayer 1959). Whether the surface or ionosphere was associated with these temperatures was unknown.

By 1961 there were three competing models: 
1) the hot ionosphere model proposed by D.E. Jones (1961, Sagan et al. 1961),

2) the aeolosphere model proposed by E.J. Opik (1961), providing for solar heating of high altitude dust with subsequent heating of the surface through wind friction, and

3) the greenhouse effect, reintroduced by C. Sagan (1960, 1961, 1962) after it was first proposed for Venus by R. Wildt (1940).

Presumably based on pioneering work by F.V. Very (1908) which hypothesized a mechanism for heat entrapment by the Jovian atmospheres, Wildt proposed an extensive $\mathrm{CO}_{2}$ atmosphere on Venus could raise surface temperature by $50 \mathrm{~K}$ over the expected blackbody value. Sagan generated a far higher surface temperature $(-600 \mathrm{~K})$ by introducing a small water vapor component (total $\mathrm{H}_{2} \mathrm{O}$ column density $1-10 \mathrm{~g} / \mathrm{cm}^{2}$ ) allowing absorption longward of $20 \mu \mathrm{m}$. The clouds were thought composed of $\mathrm{H}_{2} \mathrm{O}$ ice crystals, and associated with the 8-13 $\mu \mathrm{m}$ temperatures. Assuming an adiabatic lapse rate and a terrestrial partial pressure of $\mathrm{N}_{2}$, Sagan (1960, 1961) obtained a surface pressure of 4 atmospheres.

Opik's aeolosphere model maintained the atmospheric aerosol component through convective equilibrium. Yet convection, far more efficient in transporting heat than the action of wind friction, removed the need for the latter mechanism (Pollack 1969b).

Initial attempts to discriminate between the hot ionosphere and hot surface models gave conflicting results. The former predicted limb brightening and a larger planetary radius at centimeter wavelengths while the latter, just the reverse. Experiments performed to measure 
these effects by Korol'kov et al. (1963) and Clark and Spencer (1964) favored a hot surface and hot ionosphere model, respectively. In 1962 , brightness measurements made from Mariner 2 at $1.9 \mathrm{~cm}$ indicated $1 \mathrm{mb}$ darkening. Yet, measurements at $1.35 \mathrm{~cm}$ failed to detect it, though the latter data had five-fold poorer S/N (Barath et al. 1964).

Evidence for a hot surface began to mount with the detection of $10.6 \mathrm{~cm}$ polarization near Venus' 1 imb by Kuz'min and Clark (1965) and in situ measurements by the Venera 4 entry probe and Mariner 5 flyby, both in 1967 . Originally thought to have transmitted down to the surface, the Venera 4 probe was apparently crushed at $-26 \mathrm{~km}$ altitude, reporting a final temperature of $544 \mathrm{~K}$ (Avduevsky et a1. 1970, Sagan and Pollack 1969). The Mariner 5 radio occultation measurements probed down to the 5 bar $(35 \mathrm{~km}$ ) level (Sagan and Pollack 1969). Coupling the probe results, Sagan and Pollack (1969) derived temperature and pressure profiles yielding values of $750 \mathrm{~K}$ and 90 atmospheres $(91.2$ bar) when adiabatically extrapolated to the surface. Greenhouse models assuming $\mathrm{CO}_{2}, \mathrm{H}_{2} \mathrm{O}$ and $\mathrm{N}_{2}$ as IR opacity sources, water-ice clouds and an $\mathrm{H}_{2} \mathrm{O}$ mixing ratio of 0.58 were found consistent with the derived profiles (Pollack 1969b; see also Sagan 1969, Pollack 1969a). In 1969, measurements by the Venera 5 and 6 entry probes down to $-20 \mathrm{~km}$ altitude confirmed the earlier probe results (Avduevsky et al. 1970), and in 1970 the first soft landing by Venera 7 directly indicated a surface temperature and pressure of $747 \pm 20 \mathrm{~K}$ and $90 \pm 15 \mathrm{~kg} / \mathrm{cm}^{2}(88.2 \pm 14.7 \mathrm{bar})$ (Avduevsky et a1. 1971). The present values for the surface temperature and pressure are $735 \pm 3 \mathrm{~K}$ and $90 \pm 2$ bar (Colin 1983). It is interesting to note Earth surface pressure is found $50 \mathrm{~km}$ above Venus' 
surface.

More recent one-dimensional radiative-convective equilibrium greenhouse models have been developed by Pollack et al. (1980). Based on Pioneer Venus Data, they include: $\mathrm{CO}_{2}, \mathrm{H}_{2} \mathrm{O}, \mathrm{SO}_{2}, \mathrm{CO}$ and $\mathrm{HCl}$ as gaseous sources of IR opacity, an aerosol opacity based on an $858 \mathrm{H}_{2} \mathrm{SO}_{4}$ cloud composition, the vertical distribution and size of cloud particles, and the globally averaged solar net flux vertical profile from the Pioneer Venus sounder probe. $\mathrm{HCl}$ and $\mathrm{CO}$ were found to be minor sources of IR opacity (as was $\mathrm{N}_{2}$ in the earlier work), their omission inducing a $<28$ change in surface temperature. 
APPENDIX 4: HISTORICAL OBSERVATIONS OF PHASE-LOCKED STRUCTURE

This section's intent is to focus attention on one class of visual observations reported 1896 to 1955, and indicating solar phase-locked structure, whose reality may have been dismissed prematurely. This structure, though never recorded photographically, was until the mid-1960's seriously considered indicative of Venusian atmospheric dynamics (see Mintz 1961, Cruikshank 1965). It was, however, incompatible with a finally definitively established en masse atmospheric super-rotation.

These early observations are of present interest due to a shared symmetry with the IRHS-deduced Venusian lower thermospheric circulation. A reconsideration of the circumstances surrounding these early observations, the nature of the observed phase-locked structure, and the historical arguments for and against its reality, is thus warranted. If real, such structure points to a level of atmospheric dynamical complexity as yet uninvestigated.

As the content and principal players in the following discussion are clearly controversial, every effort has been made to provide a balanced presentation.

A4.1. THE LOWELLIAN MARKINGS ON VENUS

It is little known that the Lowellian canals on Mars were less controversial, regarding their reality, than the Venus observations carried out at Flagstaff 1896-1903. Three months after observations using Flagstaff's new 24 -inch Alvan Clark refractor began, Percival Lowell reported the discovery of Venusian markings whose solar 
phase-fixed appearance corroborated Schiaparelli's 1890 claim of a Venus 1:1 spin-orbit lock (Boston Transcript 1896). The spin axis was found to be perpendicular to the orbital plane. Jumping on the Schiaparelli bandwagon was not a problem - many observers were (see Appendix 3) - but the nature of Lowell's reported markings was a source of much professional consternation - particularly for E. S. Holden, director of the Lick Observatory on Mt. Hamilton (see, e.g., Holden 1897). Until Lowell's time, all who reported seeing Venusian markings (including Schiaparelli) found them vague and variable in form. Lowell, however, found a network of long and narrow well-defined linear features apparently dark against the Venusian disk (Lowell 1896a,b). While reminiscent of the Martian canals, Lowell (1896b) stated:

"... unlike the finer markings on Mars, they have the appearance permanently visible" - Earth atmospher only permanent, but lowell

Lowell reasoned that the $1: 1$ spin-orbit lock, and small libration about the Venus-Sun line, resulting from a nearly circular orbit, fixed the terminator on the Venusian surface. It was Lowell (1896a) who then first introduced the notion of subsolar-to-antisolar flow (see Chapter 2) stating that:

"[With the Sun-1it side of the planet] exposed in perpetuity to the full blaze of the Venusian Sun, it would seem that a then an umbrella-like return from the darkside to the bright and almost all water upon the unilluminated be the depositing of ice it would substantially remain."

Lowell's model did not admit the possibility of cloud cover, thick or otherwise (assuming an $\mathrm{H}_{2} \mathrm{O}$ aerosol), as was widely held by his contemporaries. He attributed the brightness of the disk to an 
extensive yet transparent atmosphere. His were therefore surface markings, having the appearance of "ground or rock".

It is the arrangement of Lowell's markings that appeal to the present heterodyne results. Figure A4.1 shows nine of Lowe1l's drawings made during the August through November 1896 observing period (Lowell 1896b). The linear features radiate from a central point (the radiant) very close to the subsolar point - a symmetry consistent with subsolar-to-antisolar flow. Lowell used the five drawings associated with October 15 , spanning $5^{\mathrm{h}}$ (the last drawing should read $4^{\mathrm{h}} 57^{\mathrm{m}}$ ), to indicate the phase-stationary character of the structure. A $24^{\mathrm{h}}$ Venusian period would have rotated the structure through $75^{\circ}$ (Lowell 1896a). Figure A4.2 contains five drawings by Lowell's assistant D.A. Drew and three by Lowell, at nearly concurrent periods (Lowell 1896a). Drawings acquired August through October 1896 were used to construct a map of Venus as shown in Figure A4.3 (Lowel1 1986a,b). East and west refer to the Venusian surface and not the observer's sky. The central meridian passes through the subsolar point, $37^{\circ}$ eastward of the radiant. An absence of structure in the east was taken as resulting from a poor viewing geometry. Superimposed on the radial structure, or spokes as Lowel1 (1897c) called them, were linear features forming a crude hub about the radiant. By February 1897, 227 drawings by various Flagstaff observers had been secured (Lowell 1897a).

Attempts by the astronomical community to see the Lowellian markings met with failure. Holden, at Lick Observatory, publicly stated such markings were due to either observer or instrument error, 
VENUS, 1896

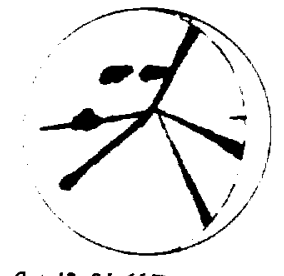

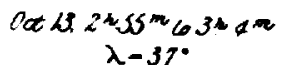
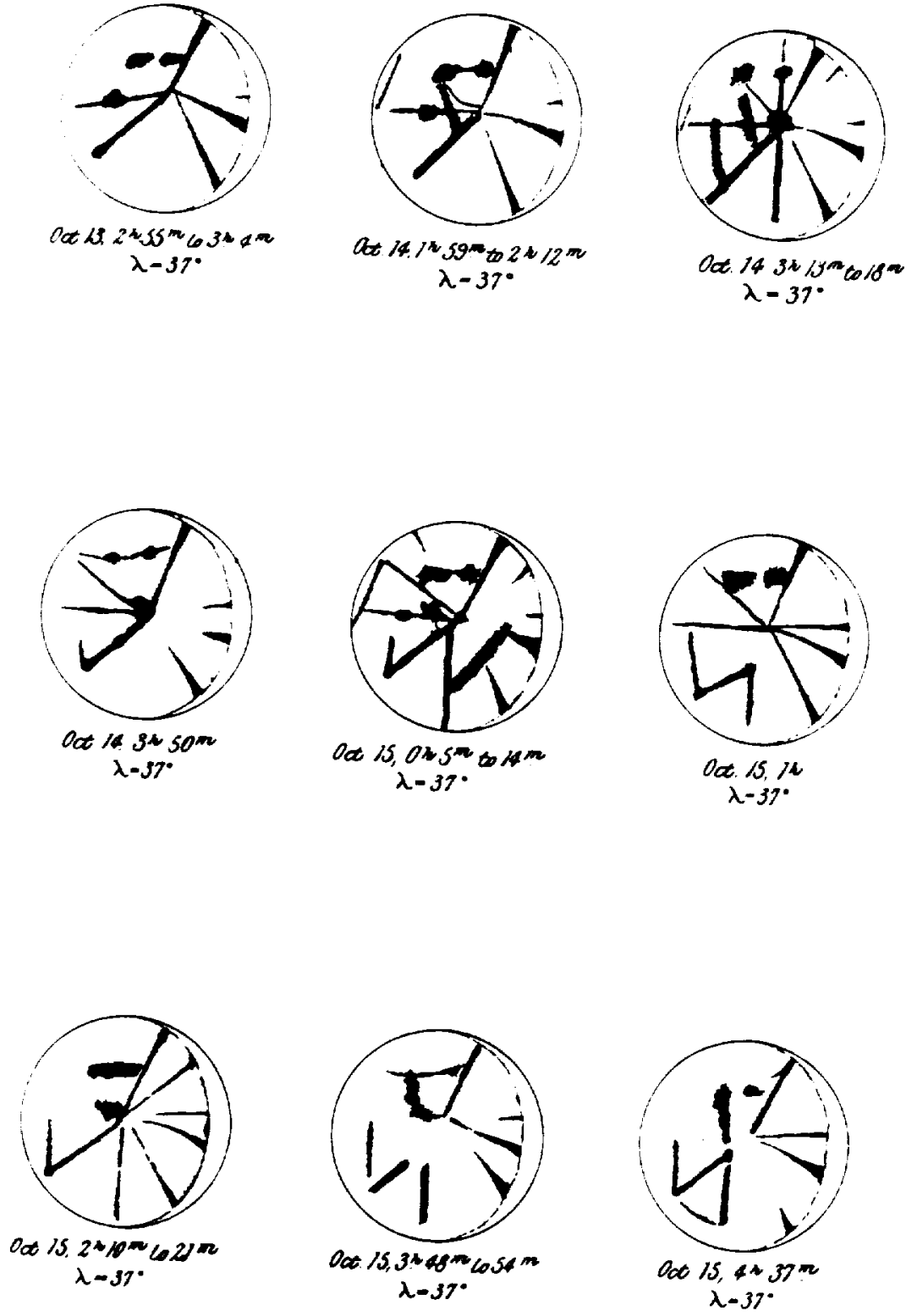

Arand Lamell, ald

Figure A4.1 


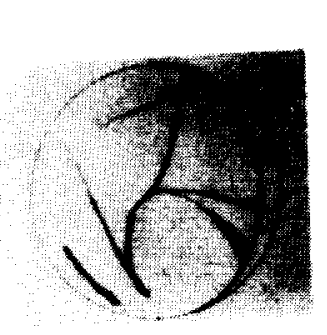

$\operatorname{sept} .294^{\mathrm{h}} 10^{\mathrm{m}}$

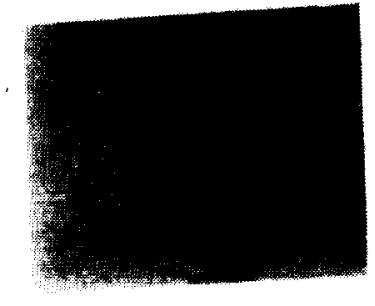

Oct. $75^{\text {h }} 5^{\mathrm{m}}$

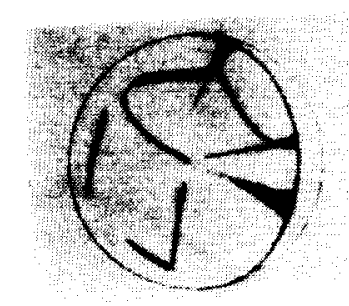

Oet. $3^{\text {h }} 15^{\mathrm{m}}$

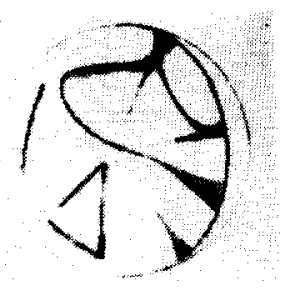

Oet. $80^{\text {h }}$

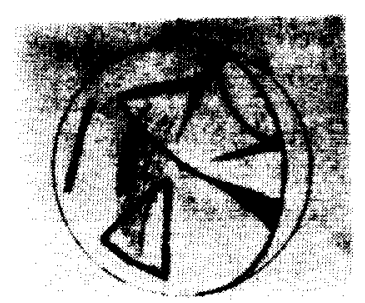

Oet $9210^{m}$

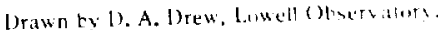

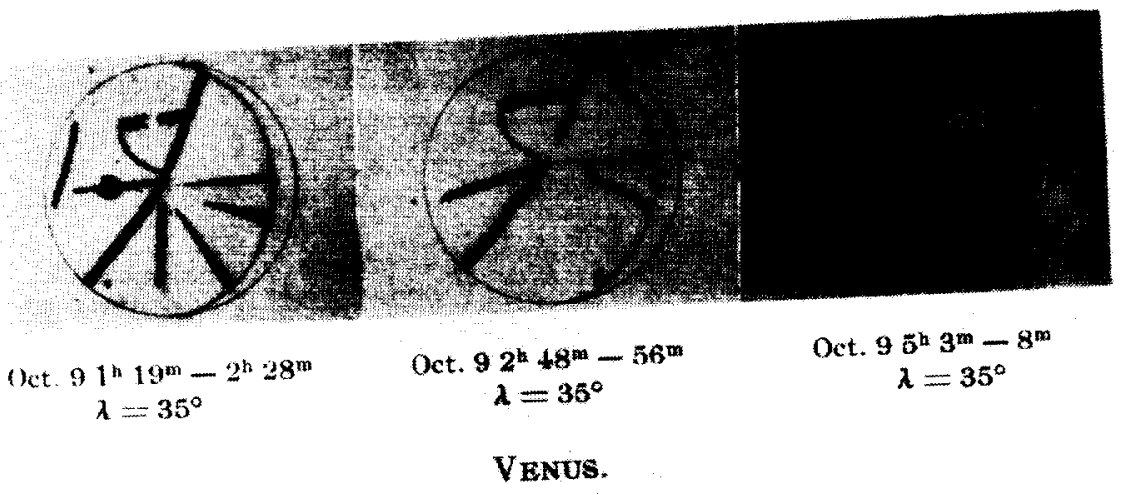

Jrawn by Percival Lowell, Lowell Observatory.

Figure $\mathrm{A} 4.2$ 
CHART OF VENUS.

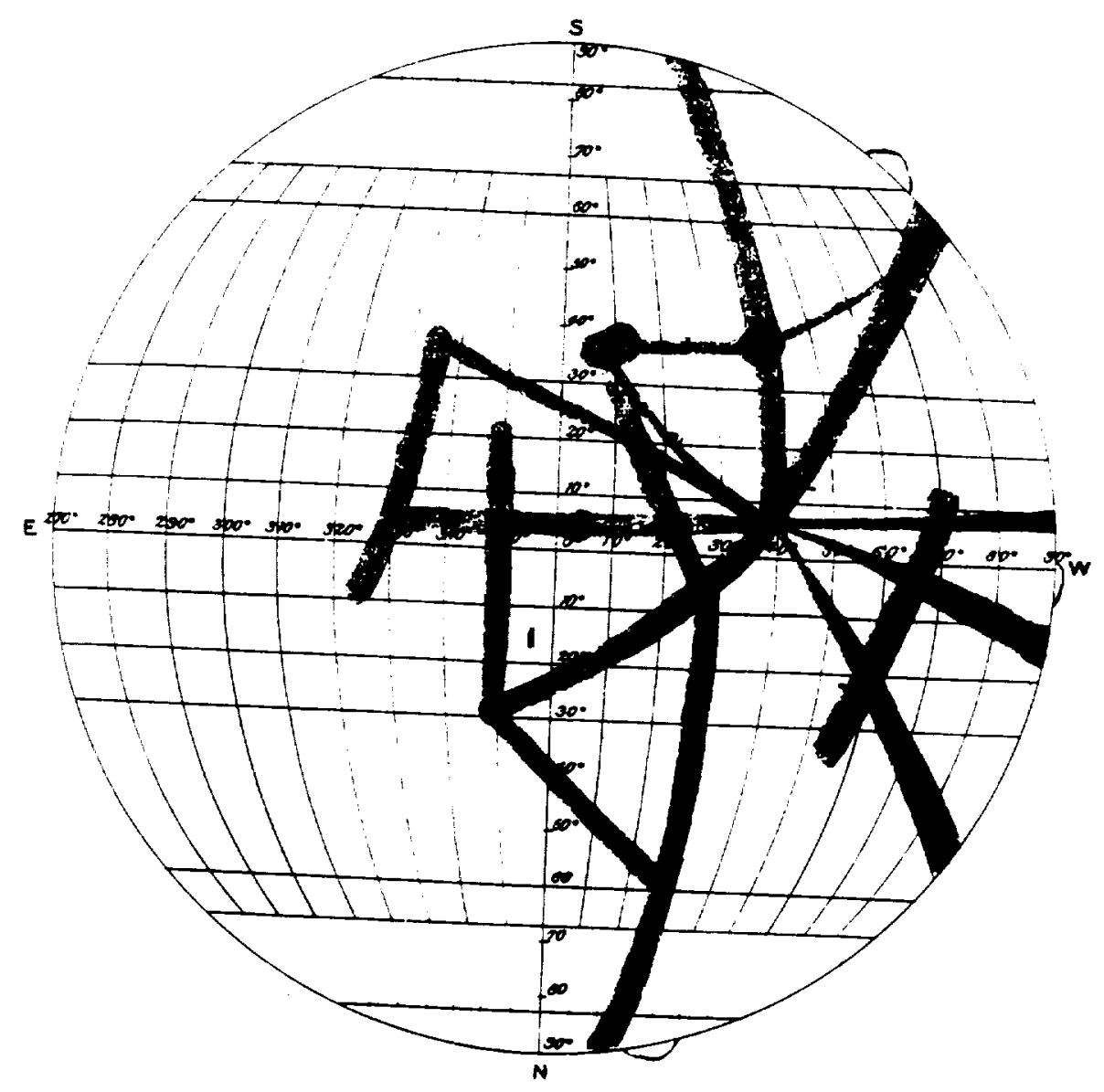

Figure $\mathrm{A} 4.3$

Proind tande ded. 
though the more vague features noted by Schiaparelli were easily recoverable (Holden 1897, Hoyt 1976). The renowned E.E. Barnard (1901) at Lick was unable to discern anything beyond large dusky spots whose permanence he questioned. Privately, in an 1897 letter to A.E. Douglass at Flagstaff, Holden wrote (Hoyt 1976):

"I have been rather skeptical, as you know, about the markings on Venus, and I still am ...

How do you personally explain [E. Schaeberle's and my inability to see ankilled observers. I began on Venus? Barnard and Schaeberle are no chance for several years. to observe Venus in 1873 and mis have any of us suspected your class Not once, as far as I know, have any of us su of markings."

The charges did not go unanswered. Alvan Clark defended the Flagstaff 24 -inch as being of the same quality as the 36 -inch at Lick (Hoyt 1976). Clark built both telescopes. Tests performed to vindicate the 24 -inch were reported by Douglass (1898a,b), while newly acquired independent and concurrent drawings made by Douglass and Drew were found consistent in detail. Figure A4.4 provides three drawings of Venus indicating, respectively: 1) the detail common to all of Douglass' observations April 1898, 2) a typical observation by Douglass and, 3) a concurrent observation by Drew.

Beyond the claimed exceptional seeing at Flagstaff, Lowell summarized his view of the controversy as follows:

"By a sensitive eye is designated one which is peculiarly susceptible to the action of light. This eye has the quality to perceive faint luminous effects or distinguish delicate differences of illumination.

On the other hand what is called an acute eye is one particularly perceptive to form. Definition plays with it the like part that illumination plays with the other. The trait is what is commonly known as keenness of sight... This, then, is the eye necessary to planetary work. What to the sensitive eye appears as a confused and blurred mass is by the acute eye resolved into its 


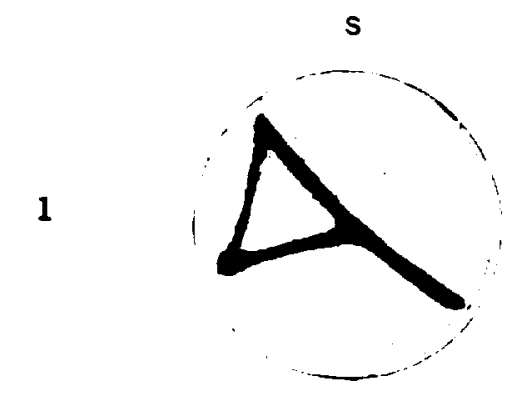

Detail seen in each combination of aperture and focus.
eave one, 1898 April 19

2

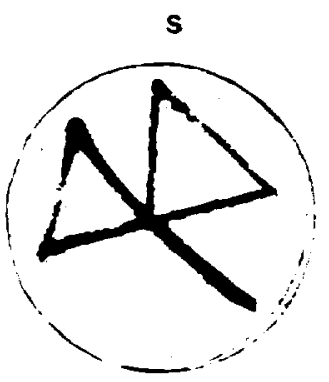

Detail drawn 1898 April 19 10b 47" G.M.T.

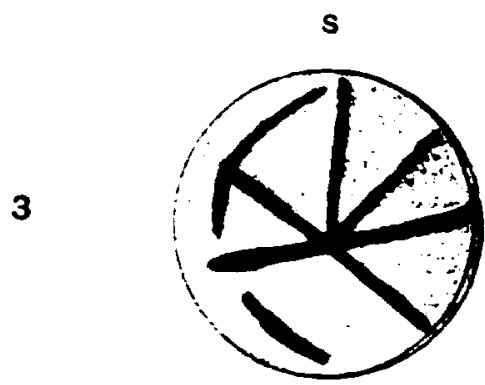

Me. Draw' drawing of 1898 April $19,13^{\text {h }} \mathrm{gm}^{\mathrm{m}}$

VENUS. -Lowell Observatory, Flagetarr. Arizona

Figure A4.4

366 
constituent parts. That the detail thus deciphered should to the possessor of the sensitive eye seem illusory is a merciving it natural wonder for not only is he incapalf with an eye superior to himself but has been led to be believed against others' most people, and therefore
assertions (Hoyt 1976)."

In 1902, after four years of illness, Lowell reassessed his views concerning the spokes in a short note to Astronomiche Nachrichten (Lowel1 1902). He became convinced that during the progression of the Venusian phase cycle, the radiant moved so as to maintain itself at the center of the disk (see Figs. A4.1 and A4.2). He now considered the spokes a physiological-induced effect.

In yet another change of opinion, Lowell (1904a,b) claimed careful observations, conducted February through July 1903, had reaffirmed his original belief that the spokes were real (Figure A4.5). Structure was found most defined near disk center - ever more of it revealed during the phase cycle as new portions of the Sun-lit hemisphere passed across the center of the disk. Lowell then considered but rejected an atmospheric source for the spokes due to their apparent permanence.

Lowe11's apparently last published reference to the spokes

(Lowe11 1914) states observations westward of the Sun (up to $8^{\circ}$ elongation) showed the spoke system to characterize the entire Sun-lit hemisphere.

Lowe11's reasoning concerning the dynamics of the Venusian atmosphere was sound. Yet, Lowell afforded the spokes (ascribed by him to the surface) and his model subsolar-antisolar circulation the same axial symmetry. Clearly, a coherent picture warranted associating the 


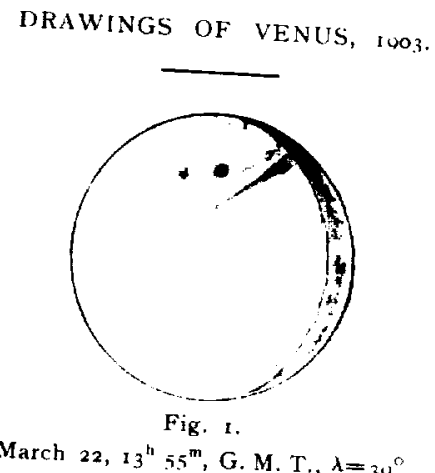

March 22, $13^{\mathrm{h}} 5.5^{\mathrm{m}}$, G. M. T., $\lambda=34^{\circ}$
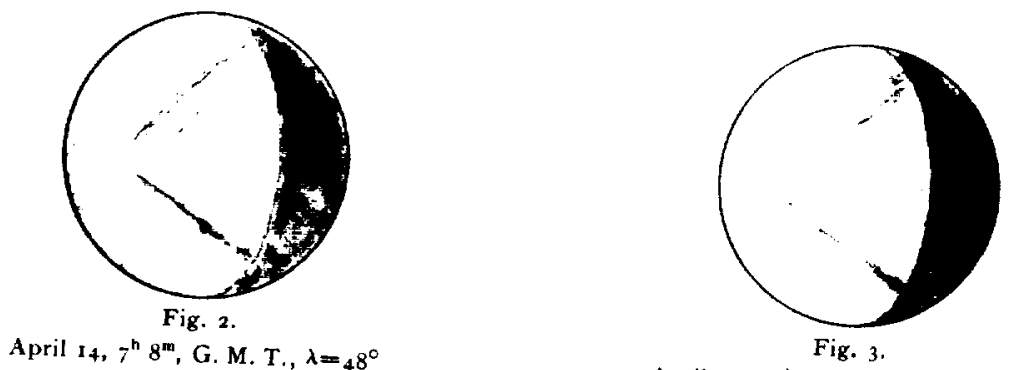

April $14,12^{\mathrm{ll}} 52^{\mathrm{m}}$, G. M. T., $\lambda=48^{\circ}$

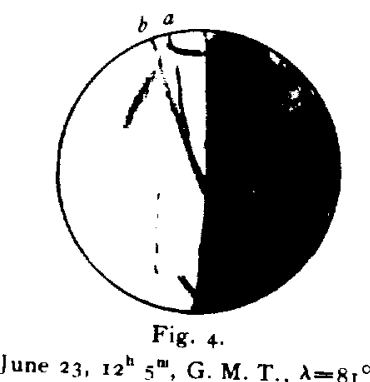

Figure A4.5 
spokes with the atmosphere or hypothesizing a ground/wind interaction. Lowell's (1910) solution - dust storms raised by the low-level antisolar-to-subsolar return branch were directed into streams by Venusian topography thereby creating the spokes (see also Maxwell 1916). Why the radiant was displaced westward of the subsolar point was not addressed.

44.2. THE MODERN RESURRECTION OF THE LOWELLIAN MARKINGS

A controversial and much publicized observation is sure to be followed by claims of "I see it too", as was the case with the spokes (see Maxwel1 1916, Wilson 1916, 1917, Seagrave 1919). Yet a number of independent observers resurrected a variant of the Lowellian spoke system more consistent with the diffuse nature of visual features observed historically.

J. Camus (1932a), Director of the Société Astronomique de France, published a set of sixteen drawings secured from visual observations made April 30 to september 3, 1930 (between superior conjunction and maximum eastern elongation). A spoke system was clearly evident and persisted until approximately mid-July ( $38^{\circ}$ eastern elongation). Unlike the narrow linear features reported by Lowell, Camus' spokes were diffuse. Nine of the sixteen drawings are shown in the upper plate of Figure A4.6. North is to the bottom. An additional set of drawings (Camus 1932b), acquired February to March 1932 near maximum eastern elongation, is shown in the lower plate of Figure A4.6. North is to the top.

A. Dollfus began visual observations of Venus at Pic du Midi 

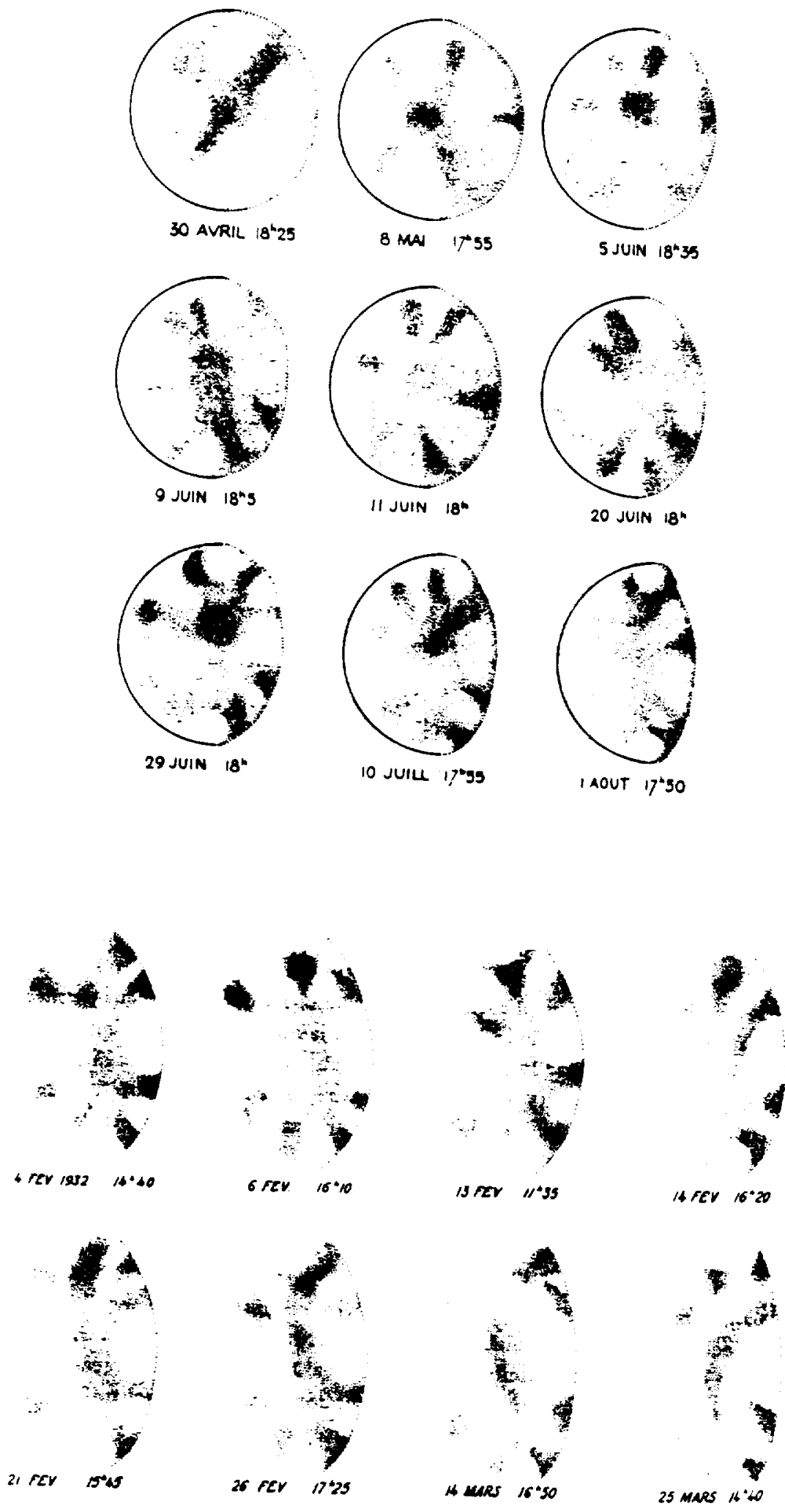

Figure A4.6 
Observatory in 1943 (Dollfus 1953). Low contrast ill-defined features were evident. Dollfus attributed their poor definition to the strong limb-to-terminator gradient in brightness (Dollfus 1961). The features were found to be variable, with the disk sometimes featureless and at other times associated with considerable detail. It was not uncommon for specific features to maintain their location from one day to the next, or conversely, disappear completely.

Approximately 100 drawings were made from visual observations with a $60 \mathrm{~cm}$ refractor, March 1948 through August 1953 (Dollfus 1955 , 1961). Drawings secured over the course of two to three weeks (characterized by nearly constant Venusian phase) were superimposed by first transfering each to a transparency and then stacking. The resulting composite accentuated features the drawings had in common. This technique de-emphasized time variable structure and allowed permanent or semi-permanent features to stand out.

The upper half of Figure A4.7 provides five Venus composites, and their mean observation dates, characterizing different epochs in the maximum western to maximum eastern elongation portion of the phase cycle. North is to the bottom. Each composite was produced from eight to ten individual drawings. Phase-locked structure showing a diffuse spoke system is clearly evident. An individual drawing was found to depart from its associated composite by lacking specific, or even large regions of structure.

Dollfus constructed the map (planisphere) shown in the lower half of Figure $\mathrm{A} 4.7$ from the five different composites. North is to the bottom. The horizontal axis is aligned with the orbital plane. The 


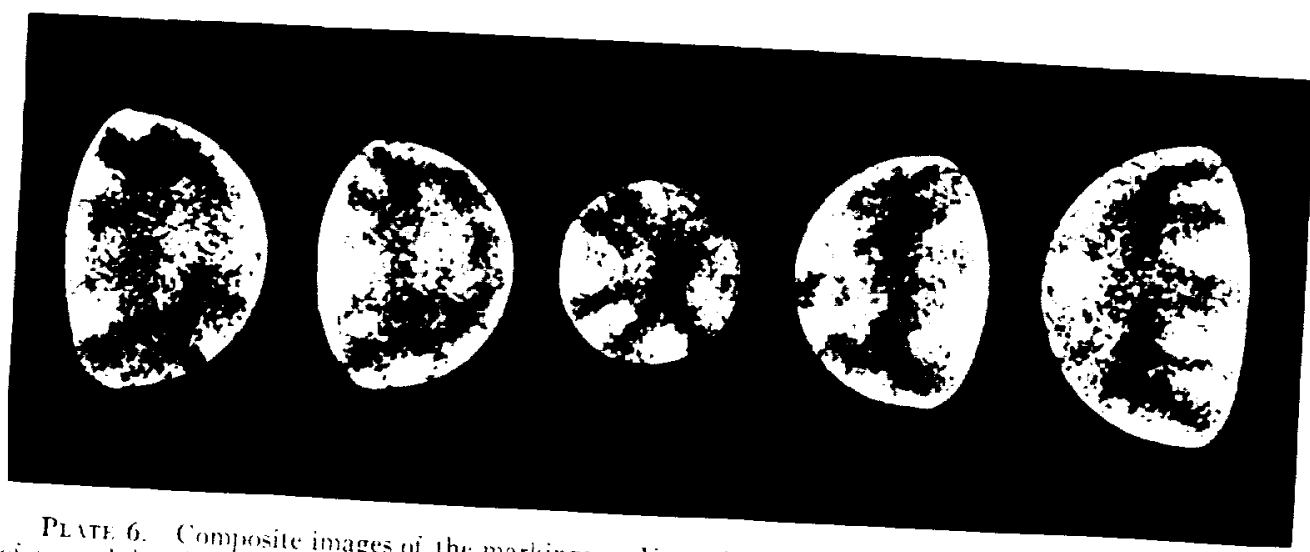

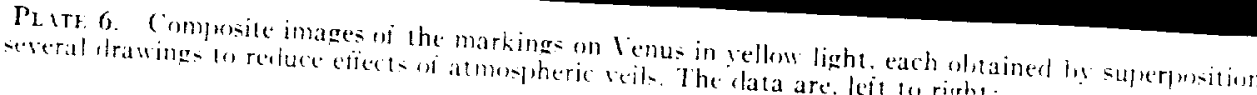

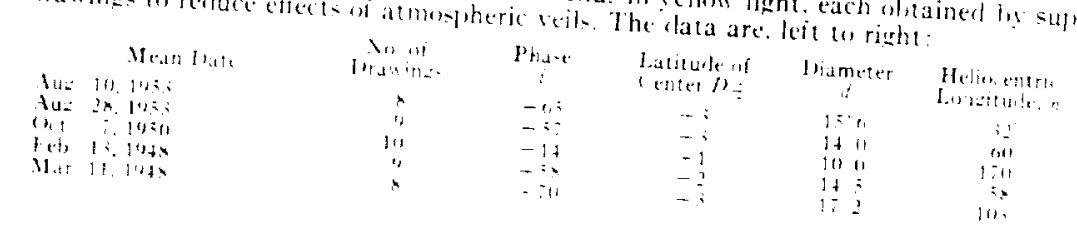

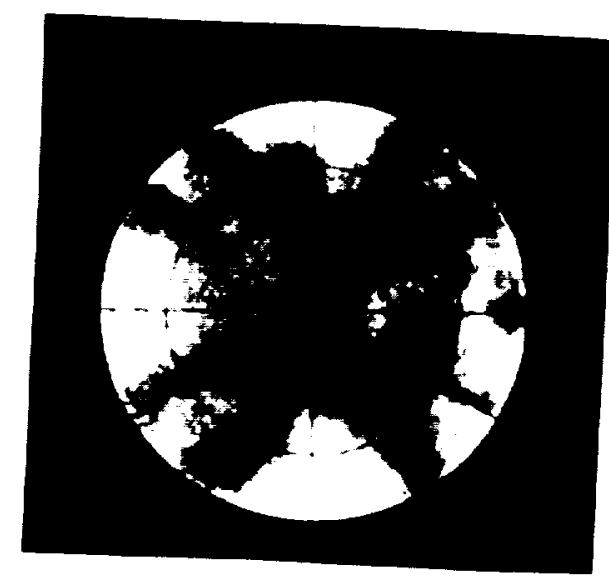

Pilit:

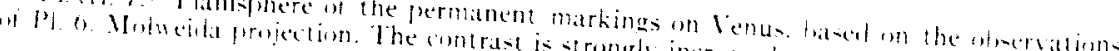

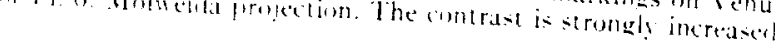

Figure A4.7 
grid center locates the mean subsolar point, and the concentric grid circles define solar zenith angles of $30^{\circ}, 60^{\circ}$ and $90^{\circ}$. Consistent with Lowell's spokes, a hub is evident encircling the radiant at a distance of $-50^{\circ}$. The radiant appears displaced slightly westward with respect to the subsolar point.

Photographic studies of Venus in visible light were begun at the Pic in 1942 by H. Camichel. Few plates showed structure. Dollfus' (1953, 1961) explanation - superposition of low contrast features onto a strong limb-to-terminator gradient results in only small perturbations of the isophotes. Gradient subtraction, allowing closure of the isophotes around low contrast features, was considered necessary to bring out the photographic detail. After applying a gradient subtraction technique, involving stacking of 15 to 20 plates (obtained nearly simultaneously at least for the published examples), the resulting photographic composite invariably showed structure in good agreement with individual drawings derived from concurrent visual observations (Dollfus 1955). Figure A4.8 (Dollfus 1955) provides a comparison between blue light photographic composites (right column) and drawings derived from concurrent visual observations (left column), August to September 1946. Note the striking similarity between the photographic composite of August 30 and the drawings of August 31 and September 6. Compare these with Figure A4.9 showing selected drawings by A. Mascari (1896) in 1892-3 and G. Mottoni (1926) in 1921.

If a definitive spoke pattern was to be obtained, the photographic composites should have been derived from plates spanning many days, as were the visual observation composites of Figure A4.7. 


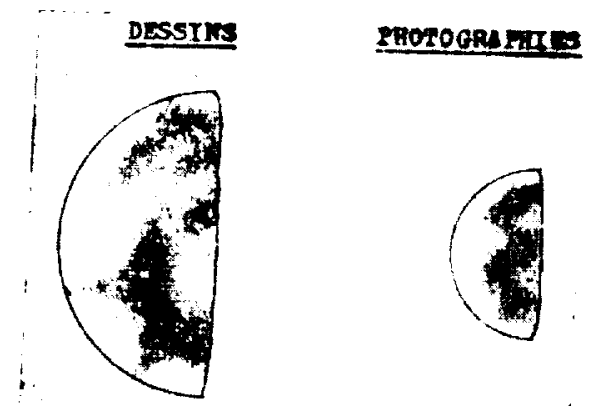

30 soet 1946
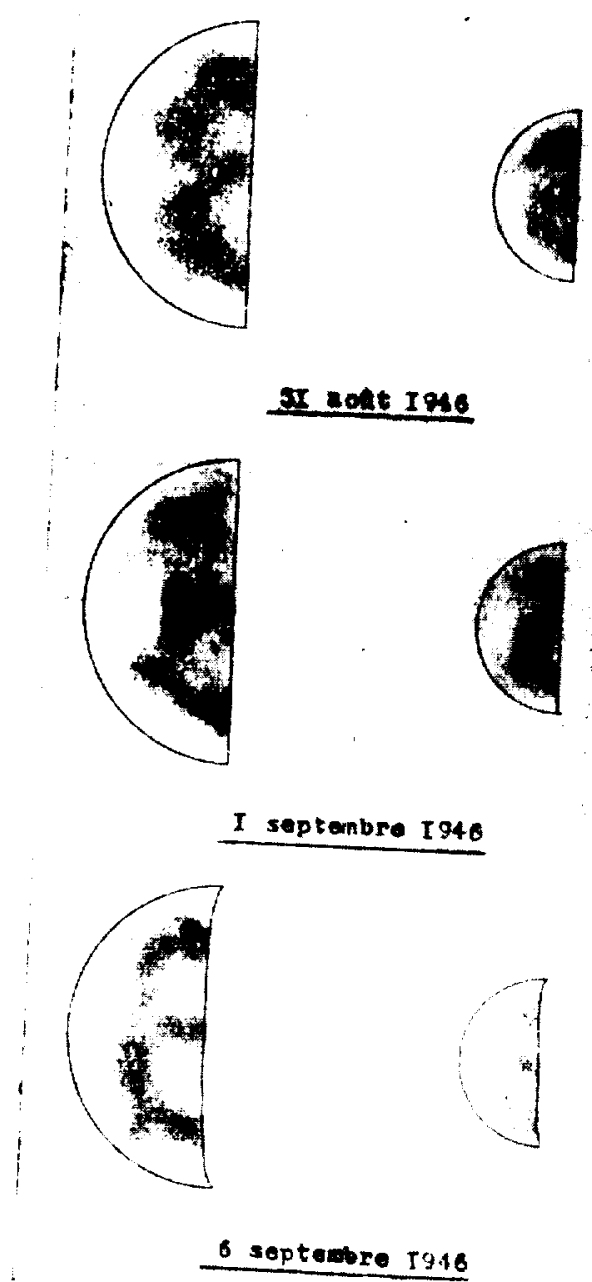

Fig. 179. - Comparaison

des observations visucles en lumiere jaun

et phutographiques en lumiere bleue.

similitucle genérale de: l'asprect des taches l,rillantes.

Ohservateire de Hougat, lunette de $20 \mathrm{~cm}$.

Figure $\mathrm{A} 4.8$ 


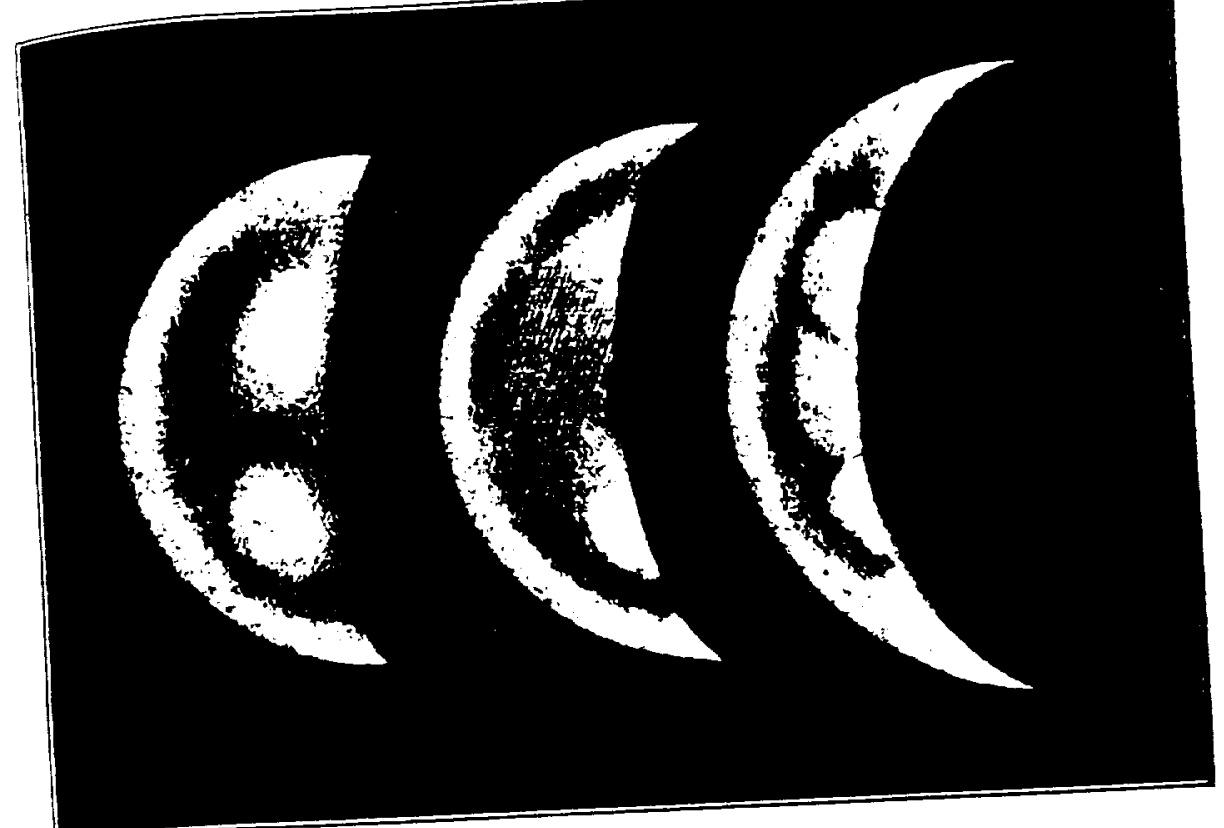

5 mars 1921, 19:20m

12 mars $1921,19^{n}$

23 mars $1921,19^{\circ}$

Observations de la planete Vénus laites par M. Glauco de Mottoni, à Milan Itatiel.

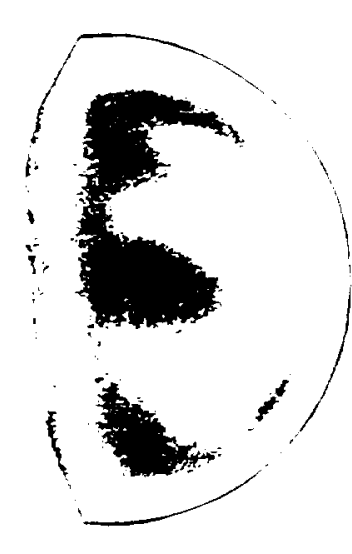

2I Novemóne

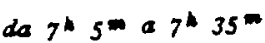

189

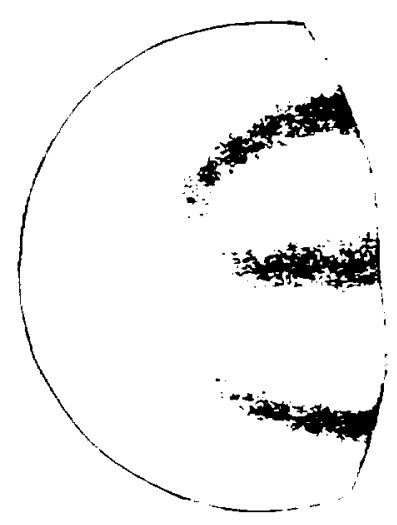

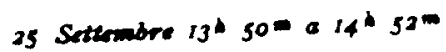

1893

\section{A. Mascari.}

all'osservatorio di Catania e sul M. Etna

Figure A4.9 
Probably more significant, the photographic composites were made at phases near maximum elongation (at least those published). As in the upper plate of Figure A4.7, structure evident during such phases did not definitively establish a spoke pattern. Clearly a phase near superior conjunction was needed.

Dollfus attributed the spokes to either surface topography or a topography-forced low-level cloud distribution. The observed variability was associated with transient high altitude clouds in rapid motion or undergoing large-scale formation/dissolution above the spoke system. Why such an axisymmetric pattern (the spokes) should characterize the Venusian surface and why it was aligned with the subsolar-antisolar axis was not explained.

Two sets of UV photographs were made by Dollfus in 1948 and Camichel in 1953. Agreement was found between UV-bright regions and regions of high albedo seen visually (attributed by Dollfus to the high altitude clouds), but there was no indication of the spoke system (Dollfus 1961).

A lively debate concerning the Lowellian markings may be found in Strolling Astronomer 1955-56. Prior to publication of Dollfus' planisphere, R.M. Baum (1952, see Bartlett 1955) claimed to have recovered Lowe11's spokes. P. Moore (1955), a long time observer and unconvinced, countered by reviewing the nondetections by such notables as E.E. Barnard. Interestingly, both Baum and Moore had their color sensitivity tested in a 1953 experiment (Baum 1955). Baum had far better sensitivity blueward. Note it was structure in Dollfus' blue light photographs that correlated with visual drawings. 
The upper plate of Figure A4.10 (Bartlett 1955) is a drawing made by Baum, April 21, 1951, with spokes labelled 1-10. The least defined spoke, between 7 and 8 , has not been labelled. The lower plate is Dollfus' later published planisphere. Note the correlation between the spokes. Note specifically: i) spoke 10 does not intercept the limb in either drawing, ii) the linear "hub" feature, connecting spokes 3,4 and 5 on Baum's drawing, is clearly defined on the planisphere, and iii) Baum's poorly defined spoke is absent on the planisphere. Observations by Baum and Dollfus were made during the same period.

In conclusion, however, there still exists no definitive photographic evidence, in any bandpass, for a spoke-like pattern in the Venusian atmosphere.

The correlation found between: i) independent visual observations (Hartmann 1962, see Appendix 3), and ii) visual observations and blue light photographic composites (Dollfus 1955), speak to the reality of visually observed structure. The lesser correlation between visual observations and UV photographs (Dollfus 1961, Hartmann 1962) might indicate sampling at different atmospheric levels.

A4.3. THE LOWELLIAN MARKINGS AND THE HETERODYNE RESULTS - A POSSIBLE CONNECTION

The facts as they stand:

i) The cloud decks and below ( $<65 \mathrm{~km}$ altitude) are characterized by a definitively established super-rotation whose effect would be to rapidly disrupt any phase-locked structure. This zonal circulation is dramatically apparent 


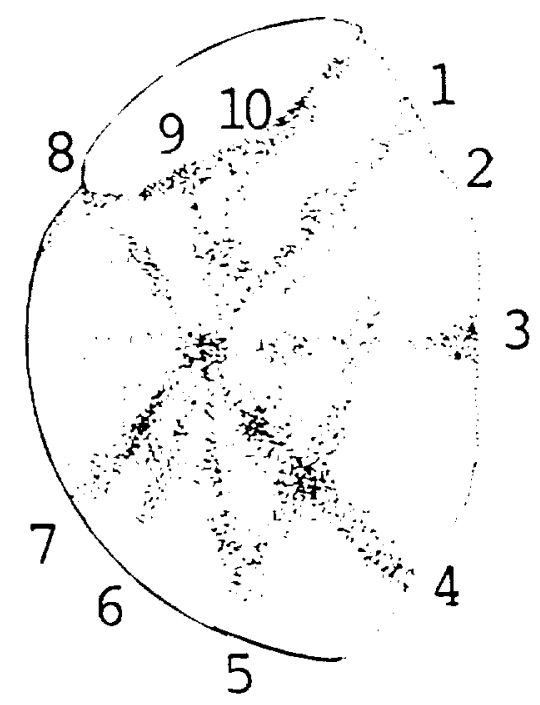

Drawing by R.M. Baum
April 26, 1951

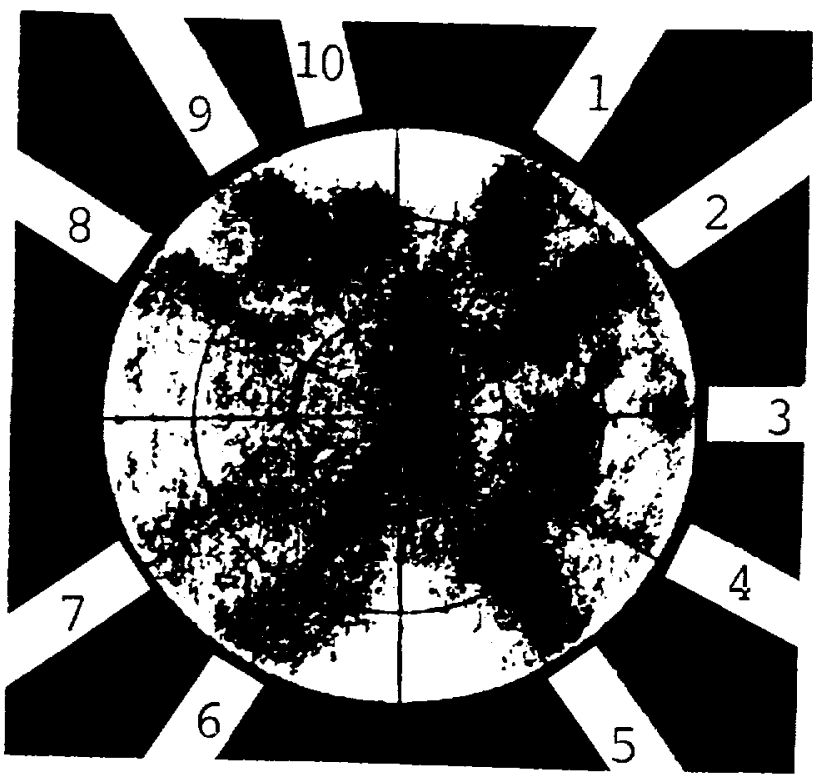

Planisphere from Visual

Observations by A. Dollfus 1948-1953

Figure A4.10 
in UV imaging. Visual photographs show only vague structure.

ii) The atmosphere at $109 \mathrm{~km}$ altitude is characterized by a phase-locked subsolar-antisolar circulation with a small zonal component.

Consider the possibility that the Lowellian markings are real and atmospheric. Instead of Dollfus' low level source for the spokes and upper level atmospheric veil in rapid motion, assume the reverse.

Identify the spoke system with subsolar-antisolar flow whose effect is to concentrate some gaseous or aerosol absorber along specific radial trajectories (a mechanism will be discussed below). As phase-locked structure is not immediately evident in UV imaging, such an absorber must have a small optical depth in the UV. The strong spatial and temporal variability in cloud-deck UV albedo would then overwhelm any phase-locked structure impressed upon the reflected solar radiation.

In the visible, however, a non-zero absorber optical depth, and uniform cloud deck albedo (unlike the UV), would yield a spoke system superimposed against a smooth background gradient. If the reflected visible radiation carries some trace of the cloud circulation, then visible observations would be characterized by a permanent (or semipermanent) phase-locked spoke system superimposed on a time variable background - consistent with observation. Put in a historical context, elements necessary for observers to claim either a rapid rotation or a $1: 1$ spin-orbit lock would therefore be present (see Appendix 3) 
A radiant position westward of the subsolar point, as reported by a number of observers (see Figs. A4.3, A4.6, A4.7), might be indicative of a zonal component associated with the spoke generation region. (It is interesting that a radiant eastward of the subsolar point is noticeably absent in the literature.) Consistent with this interpretation there is now evidence for a superposition of zonal and subsolar-antisolar flow from 80 to $120 \mathrm{~km}$ altitude (see Fig. 9.1). Assuming the dark spokes to be tracers of atmospheric dynamics, they may delineate individual convection cells within a more generalized subsolar-to-antisolar flow. Based on Dollfus' planisphere, just such a model was proposed by Y. Mintz (1961), and is shown in Figure A4.11. It represents one dynamical interpretation of the observations, based on then known characteristics of terrestrial dynamics as applied to a slowly rotating planet. Whether such an interpretation was indeed dynamically possible was not addressed. Mintz had in mind a global hydrocarbon ocean, as proposed by Hoyle (1955), or equivalently, a water ocean with a hydrocarbon surface layer. A thin layer of high albedo hydrocarbon fog was located above the ocean. While Mintz did not argue specifically for cyclonic or anticyclonic cells, for illustration, he chose the latter. The fog layer was dissipated within the intracell shear zones causing a darker appearance, hence the spokes. The banded appearance of Venus in the UV was thought due to zonal circulation of high altitude $\mathrm{CO}_{2}$ cirrus clouds. (Note the subrotating equatorial region.)

Mintz's Venus was not to be, yet the concept of a multicellular subsolar-to-antisolar flow might still be workable. The Lowellian 

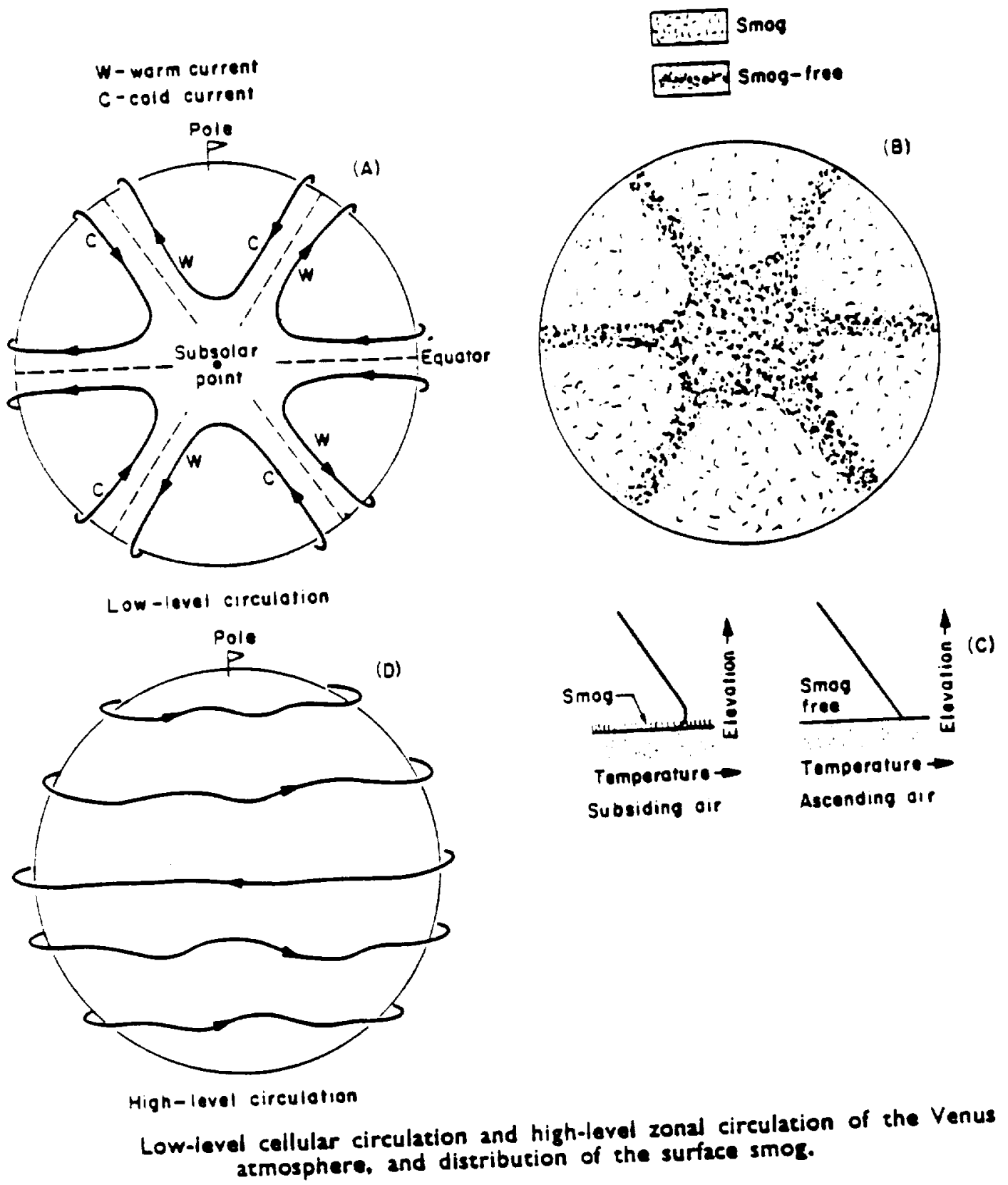

Figure A4.11 
spokes, if convection cell boundaries, may characterize either the upper or lower branch of a SS-AS cell. If dust-loading is responsible for the albedo variation, the spokes would need to be located at lower levels. Yet spoke generation in the $70-80 \mathrm{~km}$ region (if the Betz reinterpretation holds true) seems unlikely due to the dominant super-rotation.

The lower thermospheric heterodyne results (this work) do not support multicellular circulation at $-109 \mathrm{~km}$ altitude if such circulation warrants significant antisolar-to-subsolar flow between cells. The derived wind field is very compatible with a single sS-AS ce11. However, in the $80.90 \mathrm{~km}$ range, zonal winds at the $-50(?) \mathrm{m} / \mathrm{s}$ level might be introduced, possibly inducing multicellular (as opposed to unicellular) SS-AS circulation. At higher levels $(90-120 \mathrm{~km})$, a reduced zonal flow might be conducive to single cell SS-AS circulation. Multicellular SS-AS circulation might result from long-term variation of the wind field, and thus may not be a long-lived phenomenon. Coordinated observations of Venus by the Association of Lunar and Planetary Observers (ALPO), were made $-1960-72$. During this period Cruikshank (1965) reported a decreasing frequency of observations showing radial structure. For the eastern apparition of 1960-61, 213 drawings were secured, with 78 indicating radial structure. For the 1963-64 eastern apparition, only 18 of 315 drawings, and by the 1971-72 eastern apparition, $<18$ of 105 drawings showed such structure (Cruikshank 1965, Benton 1975). One of many interpretations is a waning of whatever mechanism might generate the spokes. An admittedly more plausible explanation - observations of radial structure fell from 
grace as UV-observed zonal super-rotation became ever more established. Super-rotation was not commensurate with phase-locked structure.

Venusian atmospheric $\mathrm{SO}_{2}$ has suffered a tenfold decline from 1978

to 1983 (Prinn and Fegley 1987). Esposito (1984) has suggested

volcanic atmospheric injection of $\mathrm{SO}_{2}$, followed by decreasing $\mathrm{SO}_{2}$ concentrations, is responsible. An absorber causing a circulation induced radial pattern might be equally affected. In addition, a deeply penetrating (down to $\sim 75 \mathrm{~km}$ alt.) subsolar-antisolar cell could provide a mechanism for carrying newly injected gases to higher altitudes. There is, however, no conclusive evidence of Venusian vulcanism (Taylor and Cloutier $1986 \mathrm{a}, \mathrm{b}$ ).

The purpose of this discussion was not to convince the reader Lowellian markings are real, but that they are curiously consistent with observed dynamical symmetry. Clearly, a search for these features using verifiable means is now warranted.

In closing, the reality of the spokes was considered plausible in 1965, when D.P. Cruikshank (1965) wrote:

"... the radial circulation pattern may prove to be real at least in certain levels of the Venus atmosphere."

Thus, the curious story of the Lowellian markings on Venus did not die with Lowe11. As Lowell put it:

"It is merely a question of time when [the Venusian markings] and their consequences shall be generally recognized" (Lowell 1897b). 


\section{REFERENCES}

Abbas, M.M., Mumma, M.J., Kostiuk, T., and Buh1, D. 1976, Appl. Opt. $15,427$.

Abe11, G.O., Morrison, D. and Wolff, S.C. 1987, Exploration of the Universe $5^{t h}$ Ed., Saunders, Philadelphia.

Ainsworth, J.E. and Herman, J.R. 1978, J. Geophys. Res. 83, 3113.

Allen, D.A. 1987, Icarus 69, 221.

Allen, D.A. and Crawford, J.W. 1984, Nature 307, 222.

Alsop, L.E., Giordmaine, J.A., Mayer, C.H. and Townes, C.H. 1958, A.J. 63,301 . Alvarez, W. , Alvarez, L.W., Asaro, F. and Miche1, H.V. 1980, Science
208, 1095 .

Alvarez, W., Kauffman, E.G., Surlyk, F., Alvarez, L.W., Asaro, F. and Miche1, H.V. 1984, Science 223, 1135.

Andreev, R.A., Altunin, V.I., Kerzhanovich, V.V., Kogan, L.R., Kostenko, V.I., Kustodiev, V.D., Linkin, V.M., Matveenko, L.I., Pogrebenko, S.V., Puchkov, V.I., Tarnoruder, I. Ya., Armand, N.A., Bakit'ko, R.V., Molotov, E.P., Vyshlov, A.S., Akim, E.L., Blamont, J.E., Boloh, L., Laurans, G., Gorshenkov, Yu. N., Hildebrand, C.E., Preston, R.A., Stelzried, C.T., Ivanov, N.M., Tikhonov, V.F., Morales, J., Petit, G. and Selivanov, A.S. 1986, Soviet Astronomy Letters 12, 17.

Apt, J. and Goody, R.M. 1979, J. Geophys. Res. 84, 2529.

Apt, J., Brown, R.A. and Goody, R.M. 1980, J. Geophys. Res. 85, 7934. Avduevsky, V.S., Marov, M.Ya. and Rozhdestvensky, M.K. 1970, J. Atmos. Sci, 27, 561 .

Avduevsky, V.S., Marov, M.Ya., Rozhdestvensky, M.K., Borodin, N.F. and Kerzhanovitch, V.V. 1971, J. Atmos. Sci. 28, 263.

Avduevsky, V.S., Marov, M.Ya., Kulikov, Yu.N., Shari, V.P., Gorbachevsky, A.Ya., Uspensky, G.R. and Cheremukhina, Z.P. 1983, Venus, D.M. Hunten, L. Colin, T.M. Donahue and V.I. Moroz Eds., University of Arizona Press, Tucson.

Avduevsky, V.S., Vishnevetskii, S.L., Golov, I.A., Karpeishkii, Yu.Ya., Lavrov, A.D., Kikhuskin, V.Ya., Marov, M.Ya., Melnikov, D.A., Pomagin, N.I., Pronina, N.N., Razin, K.A. and Fokin, V.G. 1976, Cosmic Res. 14, 622 .

Baker, N.L. and Leovy, C.B. 1987, Icarus 69, 202.

Baker, W.E. and Curran, R.J. Eds, 1985, NASA Workshop on Global Wind Measurements, July 29 - August 1, Columbia, MD.

Barath, F.T., Barrett, A.H., Copeland, J., Jones, D.E. and Lilley, A.E. 1964, A. J. 69, 49.

Barnard, E.E. 1901, Astronomiche Nachrichten 3760, 261.

Barnes, J.R. and Hollingsworth, J.L. 1987, Icarus 71, 313.

Bartlett, J.C. Jr. 1955, Strolling Astronomer 9, 2.

Baum, R.M. 1955, Strolling Astronomer 9, 82.

Baum, R.M. 1952, Urania 229, 1.

Belopolsky, A. 1900, Astronomiche Nachrichten 3641, 263.

Belton, M.J.S., Smith, G.R., Elliott, D.A., Klaasen, K. and Danielson, G.E. 1976a, J. Atmos. Sci. 33, 1383.

Belton, M.J.S., Smith, G.R., Schubert, G. and Del Genio, A.D. 1976b, J. Atmos. Sci. 33, 1394. 
Benton, J.L. 1975, Strolling Astronomer 25, 151.

Betz, A.L. 1982, Vibrational-Rotational Spectroscopy for Planetary
Atmospheres, NASA CP-2223.

Betz, A.L. 1977, Infrared Heterodyne Spectroscopy of Carbon Dioxide in Planetary Atmospheres, Ph.D. Thesis, University of California at Berkley. Betz, A.L., Johnson, M.A., McLaren, R.A. and Sutton, E.C. 1976, Ap.J.
208, Li41. Betz, A.L., McLaren, R.A., Sutton, E.C. and Johnson, M.A. 1977a, Icarus
30, 650.

Betz, A.L., Sutton, E.C., McLaren, R.A. and McAlary, C.W. 1977b, Proceedings of the Symposium on Planetary Atmospheres, A.V. Jones, ed., Royal Soc. Canada, Ottawa.

Bevington, P.R. 1969, Data Reduction and Error Analysis for the Physical Sciences, McGraw-Hill.

Bianchini, F. 1728, Hesperi et Phosphori Nova Phenomena.

Blamont, J.E., Young, R.E., Seiff, A., Ragent, B., Sagdeev, R.Z., Linkin, V.M., Kerzhanovich, V.V., Ingersol1, A.P., Crisp, D., Elson, L.S., Preston, R.A., Golitsyn, G.S. and Ivanov, V.N. 1986, Science 231, 1422.

Blaney, T.G. 1975, Space Sci. Rev. 17, 5, 691. Borde, C.J., Hall, J.L., Kunasz, C.V. and Hummer, D.G. 1976, Phys. Rev.
A 14, 236.

Boston Transcript, October, 1896.

Bougher, S.W. 1987, Private Communication.

Bougher, S.W., Dickinson, R.E., Ridley, E.C. and Roble, R.G. 1988,
Icarus 73, 545.

Bougher, S.W., Dickinson, R.E., Ridley, E.C., Roble, R.G., Nagy, A.F. and Cravens, T.E. 1986, Icarus 68, 284.

Boyer, C. 1973, Planet. Space Sci. 21, 1559.

Boyer, C. 1965, L'Astronomie 79, 223.

Boyer, C. 1958a, L'Astronomie 72, 73.

Boyer, C. 1958b, L'Astronomie 72, 328.

Boyer, C. and Camiche1, H. 1967, Compt. Rend. Acad. Sci. Paris 264,

Boyer, C. and Camichel, H. 1965, Compt. Rend. Acad. Sci. Paris 260,

Boyer, C. and Camichel, H. 1961, Anneles D'Astrophysique 24, 531.

Boyer, C., Camichel, H. and Dollfus, A. 1960, L'Astronomie 74, 375.

Boyer, C. and Guerrin, P. 1969, Icarus 11, 338

Boyer, C. and Guerrin, P. 1966, Compt. Rend. Acad. Sci. Paris 263, 253.

Bradley, L.C., SooHoo, K.L. and Freed, C. 1986, IEEE J. Quant. Electronics QE-22, 234.

Brinton, H.C., Taylor, H.A. Jr., Niemann, H.B., Mayr, H.G., Nagy, A.F., Cravens, T.E. and Strobe1, D.F. 1980, Geophys. Res. Lett. 7, 865

Campbe11, D.B. 1989, Private Communication.

Camus, J. 1932a, L'Astronomie 46, 145.

Camus, J. 1932b, L'Astronomie 46, 434.

Carpenter, R.L. 1966, A.J. 71, 142.

Carpenter, R.L. 1964, A.J. 69, 2.

Carr, M.H., Saunders, R.S., Strom, R.G. and Wilhelms, D.E. 1984, The Geology of the Terrestrial Planets, NASA SP -469. 
Cassini, J. 1740, Elements D'Astronomie, Paris.

Cassini, J. 1732a, Mem. Acad. Sci. Paris, 73.

Cassini, J. 1732b, Mem. Acad. Sci. Paris, 197.

Cassini, J.D. 1667a, Journal des Savans, 261.

Cassini, J.D. 1667b, Phil. Trans. Royal Soc. London, 615.

Cerulli, V. 1896, Astronomiche Nachrichten 3329, 263.

Chandler, S.C. 1897, Popular Astronomy 4, 393.

Clark, B.G. and Spencer, C.I. 1964, A.J. 69, 59.

Clancy, R.T. and Muhleman, D.0. 1989, Private Communication.

Clancy, R.T. and Muhleman, D.0. 1985a, Icarus 64, 157.

Clancy, R.T. and Muhleman, D.0. 1985b, Icarus 64, 183.

Coblentz, W.W. and Lampland, C.O. 1925, J. Franklin Inst. 199, 804.

Coblentz, W.W. and Lampland, C.0. 1924, Pub1. Astron. Soc. Pacific 36, 274.

Coblentz, W.W. and Lampland, C.0. 1922, Popular Astronomy 30, 551.

Cohen, S.C. 1975, Appl. Opt. 14, 1953.

Colin, L. 1983, Venus, D.M. Hunten, L. Colin, T.M. Donahue and V.I. Moroz Eds., University of Arizona Press, Tucson.

Conrath, B.J. 1981, Icarus 48, 246 .

Considine, D.M., Ed. 1976, Van Nostrand's Scien
Ed., Van Nostrand Reinhold Co., New York.

Counselman, C.C. III, Gourevitch, S.A., King, R.W. and Loriot, G.B. 1980, J. Geophys. Res. 85, 8026.

Covey, C., Walterscheid, R.L. and Schubert, G. 1986, J. Atmos. Sci. 43, 3273 .

Covey, C., Pitcher, E.J. and Brown, J.P. 1986, Icarus 66, 380.

Cruikshank, D.P. 1984, Mauna Kea, A Guide to the Upper Slopes and Observatories, University of Hawaii Press.

Cruikshank, D.P. 1983, Venus, D.M. Hunten, L. Colin, T.M. Donahue and V.I. Moroz Eds., University of Arizona Press, Tucson.

Cruikshank, D.P. 1965, Strolling Astronomer 19, 132.

Curran, R.J., Chairman, EOS Laser Atmospheric Wind Sounder Pane1, 1987, Instrument Panel Report, NASA.

Davies, M.E., et al. 1983, Celestial Mechanics 29, 309.

Deming, D., Glenar, D.A., Kauf1, H.U., Hill, A.A. and Espenak, F. 1986, Nature 322, 232. Nature 322, 232 .
Deming, D. Espenak, F., Jennings, D., Kostiuk, T. and Mumma, M. 1983,
Icarus 55, 347.

Deming, L.D., Espenak, F., Jennings, D., Kostiuk, T. and Mumma, M. 1982, Icarus 49, 35.

Deming, D. and Mumma, M.J. 1983, Icarus 55, 356.

Deming, D., Mumma, M.J., Espenak, F. and Kostiuk, T. 1986, Icarus 66, 366.

Dickinson, R.E. 1976, Icarus 27, 479.

Dickinson, R.E. and Bougher, S.W. 1986, J.G.R. 91, 70.

Dickinson, R.E. and Ridley, E.C. 1977, Icarus 30, 163.

Dobrovolskis, A.R. and Ingersol1, A.P. 1980, Icarus 41, 1.

Dollfus, A. 1961, Planets and Satellites III: The Solar System, G.P.

Kuiper and M. Middlehurst eds., U. of Chicago Press, Chicago.

Dollfus, A. 1955, L'Astronomie 69, 413.

Dollfus, A. 1953, L'Astronomie 67, 61.

Douglass, A.E. 1898a, M.N.R.A.S. 58, 382. 
Douglass, A.E. 1898b, Popular Astronomy 6, 253.

Dunne, J.A and Burgess, E. 1978, The Voyage of Mariner 10, NASA SP-424.

Dushman, S. 1962, Scientific Foundations of Vacuum Technique, J.M. Lafferty, ed., John Wiley and Sons, New York.

Dyce, R.B., Pettengil1, G.H. and Shapiro, I.I. 1967, A.J. 72, 351.

Eisberg, R. and Resnick, R. 1974, Quantum Physics of Atoms, Molecules, Solids, Nuclei and Particles, John Wiley and Sons, 121.

Elson, L.S. 1979, Geophys. Res. Lett. 6, 720. Espenak, F. 1986, Ten Year Planetary Ephemeris: 1986-1995, NASA
RP-1176.

Esposito, L. 1984, Science 223, 1072.

Esposito, L.W. 1980, J. Geophys. Res. 85, 8151.

Esposito, L.W., Knollenberg, R.G., Marov, M. Ya., Toon, O.B. and Turco, R.P. 1983, Venus, D.M. Hunten, L. Colin, T.M. Donahue and V.I.

Evenson, K.M. University of Arizona Press, Tucson.

G.W. 1973, App1. Phys. Lett. 22, 192. , Danielson, B.L. and Day,

Fels, S.B. and Lindzen, R.S. 1974, , 192.

Fimmel, R.0., Colin, L a .S. 1974, Geophys. Fluid Dyn. 6,149. SP-461. Flammarion, C. and Gore, J.E. 1894, Popular Astronomy, D. Appleton and
Company, New York.

Flasar, F.M., Samuelson, R.E. and Conrath, B.J. 1981, Nature 292, 693.
Fontana, F. 1646, Novae Observationes, Naples.
Fox, J.L

Fox, J.L. and Dalgarno, A. 1981, J.G.R. 86, 629.

Elect. QE-16, 1195.

Freed, C. and Javan, A. 1970, App. Phys. Lett. 17, 53.

Freed, C. and O'Donne11, R.G. 1976, Proceedings of the $2^{\text {nd }}$ Frequency
Standards and Metrology Symposium Copper 1976 .

Gibson, J.E. and McEwan, R.J. 1959, Proc. URI-IAU Radio Astron. Symp.
Paris 1958.

Gierasch, P.J. 1975, J. Atmos. Sci. 32, 1038. Glenar, D. Deming, D., Espenak, F., Kostiuk, T., and Mumma, M.J. 1986,
App1. Opt. 25, 58.

Glenar, D., Kostiuk, T., Jennings, D.E., Buhl, D., and Mumma, M.J.
1982, Appl. Opt. 21, 253 .

Gold, T. and Soter, S. 1971, Icarus 14, 16.

Goldstein, J. 1987, Am. J. Phys. 55,476.

Goldstein, J., Mumma, M., Zipoy, D. Kostiuk, T., Deming, D. and
Espenak, F. 1988, B A

Goldstein, J., Mumma, M. . Zipoy $20,833$.

Espenak, F. 1987a, EOS Trans. A.G.U. 68, 343, Deming, D. and

Goldstein, J., Mumma, M., Zipoy, D. Kostiuk, 343.

Espenak, F. 1987b, B.A.A.S. 19, 869.

Goldstein, J., Zipoy,

Glenar, D. and Kauf1, H.U. 1985, B.A.A. M. Kostiuk, T., Deming, D.,

Goldstein, R.M. 1965, J. Res. Nat1. Bur. St.S. 17, 725.

Goldstein, R.M. 1964, A.J. 69, 12. Bur. Stds. Radio Sci. 69D, 1623.

Golitsyn, G.S. 1975, Icarus $24,70$.

Goody, R.M and Robinson, A.R. 1966, Ap. J. 146, 339. 
Guinot, B. 1965, Compt. Rend. Acad. Sci. Paris 260, 431.

Guinot, B. and Feisse1, M. 1968, Publs. Obs. Haute-Provence 9, 36. Haberle, R.M., Leovy, C.B. and Pollack, J.B. 1982, Icarus 50, 322.

Halley, E. 1686, Phil. Trans. Royal Soc. 16, 153.

Hanel, R.A., Conrath, B.J., Flasar, M., Herath, L., Kunde, V., Lowman, P., Maguire, W., Pearl, J.C., Pirraglia, J.A., Samuelson, R., Gautier, D., Gierasch, P., Kumar, S. and Ponnamperuma, C. 1979, Science 206, 952.

Hane1, R.A., Conrath, B.J., Kunde, V.G., Pear1, J.C. and Pirraglia, J.A. 1983, Icarus 53, 262.

Hane1, R., Conrath, B., Flasar, F.M., Kunde, V., Maguire, W., Pearl, J., Pirraglia, J., Samuelson, R., Herath, L., Allison, M. Cruikshank, D., Gautier, D., Gierasch, P., Horn, L., Koppany, R. and Ponnamperuma, C. 1981, Science 212, 192.

Hartmann, W.K. 1962, Strolling Astronomer 16, 171.

Head, J.W. III 1981, The New Solar System, J.K. Beatty, B. O'Leary and A. Chaikin eds., Sky Publishing Corp., Cambridge.

Hedin, A.E., Niemann, H.B., Kasparzak, W.T. and Seiff, A. 1983, J.G.R. $88,73$.

Herrmann, D.B. 1984, The History of Astronomy from Herschel to Hertzsprung, Cambridge University Press, London.

Herschel, W. 1793, Phil. Trans. Royal Soc. London, 201.

Hess, S.I. 1959, Introduction to Theoretical Meteorology, Holt, Rinehart and Winston, New York.

Hide, R., Birch, N.T., Morrison, L.V., Shea, D.J. and White, A.A. 1980, Nature 286, 114.

Hillman, J.J., Kostiuk, T., Buhl, D., Faris, J.L., Novaco, J.C. and Mumma, M.J. 1977, Opt. Lett. 1, 81.

Hind, J.R. 1852, The Solar System, G.P. Putnam, New York.

Hindle, B. 1980, The Scientific Writings of David Rittenhouse, Arno Press, New York.

Holden, E.S. 1897, P.A.S.P. 9, 92.

Holden, E.S. and Campbel1, W.W. 1890, Publ. Astron. Soc. Pacific 2, 249.

Hollenbach, D.J., Prasad, S.S. and Whitten, R.C. 1985, Icarus 64, 205. Hou, A.Y. and Goody, R.M. 1985, J. Atmos. Sci. 42, 413.

Hoyle, F. 1955, Frontiers of Astronomy, Harper and Row Bros., New York. Hoyt, W.G. 1976, Lowell and Mars, University of Arizona Press, Tucson.

Hufnagel, R.E. 1978, The Infrared Handbook, Chapter 6, W.L. Wolfe, Ed. office of Naval Research, Department of the Navy, Washington, D.C.

Hunten, D.M., Colin, L., Donahue, T.M. and Moroz, V.I., eds. 1983, Venus, University of Arizona Press, Tucson.

Hunten, D.M., Tomasko, M.G., Flasar, F.M., Samuelson, R.E., Strobel, D.F. and Stevenson, D.J. 1984, Saturn, T. Gehrels and M.S. Matthews Eds., University of Arizona Press, Tucson.

Johnson, M.A., Betz, A.L., McLaren, R.A., Sutton, E.C. and Townes, C.H. 1976, Ap. J. 208, L145.

Jones, D.E. 1961, Planet. Space Sci. 5, 166.

Kahn, R. 1984, J. Geophys. Res. 89, 6671.

Kahn, R. 1982, Application of Planetary Studies to Problems of the Terrestrial Atmosphere, NASA Internal Memorandum to $\mathrm{H}$. Brinton, Code EL, NASA HQ. 
Keating, G.M., Nicholson, J.Y. III and Lake, L.R. 1980, J. Geophys.
Res. 85, 7941.

Kerzhanovich, V.V. and Limaye, S.S. 1985, Adv. Space Res. 5, 11, 59.

Kerzhanovich, V.V. and Marov, M. Ya. 1983, Venus, D.M. Hunten, L. Colin, T.M. Donahue and V.I. Moroz Eds., University of Arizona Press, Tucson.

Kliore, A.J. and Patel, I.R. 1980, J. Geophys. Res. 85, 7957.

Knollenberg, R.G. 1984, Icarus, 57, 161.

Knollenberg, R.G. and Hunten, D.M. 1980, J. Geophys. Res. 85, 8039.

Korolkov, D.V., Pariyskiy, Ya.N., Timofeeva, Ya.N. and Kharykiu, S.E. 1963, Dok1. AN SSSR 149, 65.

Kostiuk, T., and Mumma, M.J. 1983, Appl. Opt. 22, 2644.

Kostiuk, T., Mumma, M.J., Hillman, J.J., Buh1, D., Brown, L.W., and Faris, J.L. 1977, IR Phys. 17, 431. Kostiuk, T. and Spears. D.L. 1987, Int. J. Infrared and Millimeter
Waves 8, 1269 .

Kotelnikov et al. 1963, Dok1. Akad. Nauk 151, 532.

Kozyrev, N.A. 1954, Astrophys. Obsns. Crimea 12, 177.

Kremnev, R.S., Linkin, V.M., Lipatov, A.N., Pichkadze, K.M., Shurupov, B.A. , Terterashvili, A.V., Bakitko, R.V., Blamont, J.E., Malique, C. , Ragent, B., Preston, R.A., Elson, L.S. and Crisp, D. 1986,

Kudryavtsev, B.B. 1954, Mikhail Vasilyevich Lomonosov, Foreign Languages Publishing House, Moscow.

Kuiper, G.P. 1954, Ap. J. 120, 603.

Kuz'min, A.D. and Clark, B.G. 1965, A.J. 43, 595.

Lago, B. and Cazenave, A. 1979, Moon and Planets 21, 127.

Lang, K.R. 1980, Astrophysical Formulae - A Compendium for the Physicist and Astrophysicist, $2^{\text {nd }}$ Ed., Springer-Verlag, New York.

Leovy, C.B 1973, J. Atmos. Sci. 30, 1218.

Leovy, C.B. and Pollack, J.B. 1973, Icarus 19, 195.

Letokhov, V.S. and Chebotayev, V.P. 1977, Nionlinear Laser Spectroscopy, Springer-Verlag, New York

Limaye, S.S. 1988, Icarus 73, 212.

Limaye, S.S. 1985, Adv. Space Res. 5, 9, 51.

Limaye, S.S. 1984, Icarus 57, 362.

Limaye, S.S., Grund, C.J. and Burre, S.P. 1982, Icarus 51, 416.

Limaye, S.S. and Suomi, V.E. 1981, J. Atmos. Sci., 38, 1220.

Lomonosov, M.V. 1955a, Polnoe Sobranie Sochinenii 4, 361, USSR Acad. Sci., Moscow. Lomonosov, M.V. 1955b, Polnoe Sobranie Sochinenii 4, 767, USSR Acad.
Sci., Moscow.

Lowe11, P. 1914, Lowe11 Observatory Bulletin 59, Flagstaff, Arizona.

Lowell, P. 1910, The Evolution of Worlds, McMillan Co., New York.

Lowe 11, P. 1904a, Lowell Observatory Bulletin 6, Flagstaff, Arizona.

Lowel1, P. 1904b, Popular Astronomy 12, 184.

Lowe11, P. 1903, Popular Astronomy 11, 426.

Lowe11, P. 1902, Astronomiche Nachrichten 3823, 129.

Lowe11, P. 1897a, M.N.R.A.S. 57, 402.

Lowe11, P. 1897b, The Observatory 20, 112.

Lowe 11, P. 1897c, Astronomiche Nachrichten 3406, 361.

Lowe 11, P. 1896a, Popular Astronomy 4, 281. 
Lowe11, P. 1896b, M.N.R.A.S. 57, 148 .

Macpherson, H. 1913, The Observatory 36, 221.

Mahoney, J.R. 1962, A Dynamical Study of the Venus Atmosphere, Space

Tech. Lab Report 9990-6332-KU-000.

Marov, M.Ya., Avduevsky, V.S., Kerzhanovich, V.V., Rozhdestvensky,

M.K., Borodin, N.F. and Ryabov, 0.L. 1973, J. Atmos. Sci. 30, 1210

Mascari, A. 1896, Astronomiche Nachrichten 3329, 257.

Maxwe11, S. 1916, J.B.A.S. 26, 138.

Mayer, C.H. 1959, A.J. 64, 43. and Sloanaker, R.M. 1958, Ap. J. 127, 1.

Mayer, C.G. 1989, Private Communication.

Mayr, H.G. 1989, Private Commen, H.B., Brinton, H.C., Spencer, N.W.,

Mayr, H.G., Harris, I., Niemann, Hartle, R.E., Hoegy, W.R. and Hunten, D.M. 1980 , J.G.R. 85,7841

Mayr, H.G., Harris, I., Stevens-Rayburn, D.R., Niemann, H.B., Taylor, H.A., Jr. and Hartle, R.E. 1985, Adv. Space Res. 5, 109.

McClatchey, R.A., Benedict, W.S., Clough, S.A., Burch, D.E., Calfee, R.F., Fox, K., Rothman, L.S. and Garing, J.S. 1973, AFCRL

Atmospheric Absorption Line Parameters Compilation,

AFCRL-TR-73-0096, Reinhold, New York.

Menshutkin, B.N. 1952, Russia's Lomonosov, Princeton University Press, Princeton.

Menze1, D.H. 1923, Ap. J. 58, 65.

Mintz, Y. 1962, Icarus 1, 172.

Mintz, Y. 1961, Planet. Space Sci. 5, 141.

Molina, M.J. and Rowland, F.S. 1974, Nature 249, 810.

Moore, P. 1955, Strolling Astronomer 950 . Solar System, Rand McNally

Moore, P. and Hunt, G.
Company, New York.

Moroz, V.I. 1983, Venus, D.M. Hunten, L. Colin, T.M. Donahue and V.I. Moroz Eds., University of Arizona Press, Tucson.

Mottoni, G. 1926, L'Astronomie 40, 36.

Mumford, N.W. 1909, Popular Astronomy 17, 497.

Mumma, M.J. 1988, Private communication.

Mumma, M.J., Buh1, D., Chin, G., Deming, D., Espenak, F., Kostiuk, T. and Zipoy, D. 1981, Science 212, 45.

Mumma, M.J., Kostiuk, T., Buhl, D. 1978, Opt. Eng. 17, 50

Mumma, M.J., Kostiuk, T., Cohen, S., Buhl, D., and Von Thuna, P.C. 1975, Nature 253, 516.

Murray, E.R., Kruger, C. and Mitchner, M. 1974, App1. Phys. Lett. 24, 180.

Newman, M., Schubert, G., K1iore, A.J. and Patel, I.R. 1984, J. Atmos. Sci. 41, 1901. Sci. 41, 1901.
Niemann, H.B., Kasprzak, W.T., Hedin, A.E., Hunten, D.M. and Spencer,
N.W. 1980, J. Geophys. Res. 85,7817 .

Opik, E.J. 1961, J. Geophys. Res. 66, 2807 . Pickett, H.M., Richardson, D.J. and Namkung, J.S. 1987, Atlas of Absorption Lines From 0 to $17900 \mathrm{~cm}^{-1}$, NASA RP-1188.

Petersen, F.R., Beaty, E.C. and Pollack, C.R. 1983, J. Molec. Spectroscopy 102, 112 .
Pettengill, G.H., Briscoe, H.W., Evans, J.V., Gehrels, E., Hyde, G.M., 
Kraft, L.G., Price, R. and Smith, W.B. 1962, A.J. 67, 181.

Pettit, E. and Nicholson, S.B. 1955, Publ. Astron. Soc. Pacific 67,
293.

Pettit, E. and Nicholson, S.B. 1924a, Pub1. Astron. Soc. Pacific 36,
227.

Pettit, E. and Nicholson, S.B. 1924b, Popular Astronomy 32, 614.
Pierce, A.K. 1964, App1. Opt. 3, 1337.
Pollack, J.B. 1969.

Pollack, J.B. 1969a, Icarus 10, 301 .

Pollack, J.B. 1969b, Icarus 10, 314.

Pollack, J.B., Leovy, C.B., Greiman, P.W. and Mintz, Y. 1981, J.

Pollack, J.B., Toon, 8223.

. 1980, J. Geophys. Res. 85, Tomasko, M.G., Esposito, Whitten, R.C., Boese, R., Ragent, B., Geophys. Res. 85, 8141. L.W., Travis, L. and Wiedman, D. 1980b, J.

Ponsonby, J.E.B., Thomson, J.H. and Imerie, K.S. 1964, Nature 204, 63.

Preston, R.A., Hildebrand, C.E., Purcell G.H. Jr., E1lis, J.,
Stelzried, C.T. Finley, Stelzried, C.T., Finley, S.G., Sagdeev, R.Z., Linkin, V.M.,

Matveenko, L.I., Pogrebenko, V.I., Kogan, L.R., Kostenko, V.I., Alexandrov, Yu.N., Armand, S.V., Strukov, I.A., Akim, E.L., Bogomolov, A.F., Gorchankov. A., Bakitko, R.N., Vyshlov, A.S., Tichonov, V.F., Blamont, J.E Yu.N., Selivanov, A.S., Ivanov, N.M., A., Biraud, F., Ortega-Molina, Boloh, L., Laurans, G., Boischot, Science 231, 1414. Ga-Molina, A., Rosolen, C. and Petit, G. 1986, Prinn, R.G. 1971, J. Atm. Sci. 28, 1058.

Prinn, R.G., and Fegley. Sc1. 28, 1058. 171.

Quenisset, F. 1911, Compt. Rend. Acad. Sci. Paris 1721645.

Riccioli, G.B. 1651, Almagest. Acad. Sci. Paris 1531208.

Richardson, R.S. 1958, Publ. Astrong .

Richardson, R.S. 1955, Publ. Astron. Soc. Pacific 70, 251.

Rordame, A. 1922a, Popular Astronon. Soc. Pacific 67, 304.

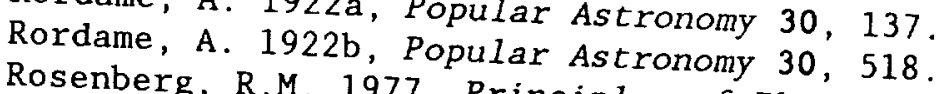

University Press, New York.

Ross, F.E. 1928, Ap. J. 68 57 .

P.H. 1980, J. Geophys. Res. $85,8107$. . , Travis, L.D. and Stone,

Rotation of Venus 1896, ams, G.P. 1979, J. Atmos. Sci. 36, 377.

Ryan, J.A. 1985, J. Geophys Res $90,19,116$.

Sagan, C. 1969, Icarus 10, 290.

Sagan, C. 1962, Icarus 1, 151.

Sagan, C. 1961, Science 133, 849.

Sagan, C. 1960, A.J. 65, 352 .

Sagan, C. and Kellogg, W.W. 1963, Ann. Rev. Astron. Astrophys. 1, 235.

Sagan, C. Siegel, K.M. and Jones, D.E. 1961, A.J. 66, 52.

Sagdeev, R.Z., Linkin, V.M., Kremnev, R.S., Blamont, J.E., Preston, 
R.A. and Selivanov, A.S. 1986, Soviet Astronomy Letters 12, 3. Sagdeev, R.Z., Linkin, V.M., Kerzhanovich, V.V., Lipatov, A.N.,

Schurupov, A.A., Blamont, J.E., Crisp, D., Ingersol1, A.P., Elson, L.S., Preston, R.A., Hildebrand, C.E., Ragent, B., Seiff, A.,

Young, R.E., Petit, G., Boloh, L., Alexandrov, Yu.N., Armand, N.A., Bakitko, R.V. and Selivanov, A.S. 1986b, Science 241, 1411.

Sagdeev, R.Z., Linkin, V.M., Blamont, J.E. and Preston, R.A. 1986c, Science 241, 1407.

St. John, C.E. and Nicholson, S.B. 1922, Ap. J. 56, 380.

Schiaparelli, G.V. 1895, Astronomiche Nachrichten 3304, 249.

Schiaparelli, G.V. 1890, Pub1. Astron. Soc. Pacific 2, 246.

Schofield, J.T. and Tayler, F.W. 1983, Quart. J. Royal Meteorol. Soc. $109,57$.

Schr8ter, J.H. 1795, Phil. Trans. Royal Soc. London 117.

Schroter, J.H. 1792, Phil. Trans. Royal Soc. London 309.

Schubert, G. 1983, Venus, D.M. Hunten, L. Colin, T.M. Donahue and V.I. Moroz eds., University of Arizona Press, Tucson.

Schubert, G., Covey, C., Del Genio, A., Elson, L.S., Keating, G., Seiff, A., Young, R.E., Apt, J., Counselman, C.C. III, Kliore, A.J., Limaye, S.S., Revercomb, H.E., Sromovsky, L.A., Suomi, V.E., Taylor, F., Woo, R. and von Zahn, U. 1980, J. Geophys. Res. 85, 8007.

Scott, A.H. and Reese, E.J. 1972, Icarus 17, 589.

Seagrave, F.E. 1919, Popular Astronomy 27, 406.

See, T.J.J. 1910, Popular Astronomy 18, 1.

Seiff, A. 1983, Venus, D.M. Hunten, L. Colin, T.M. Donahue and V.I. Moroz eds., University of Arizona Press, Tucson.

Seiff, A. 1982, Icarus 51, 574.

Seiff, A., Kirk, D.B., Young, R.E., Blanchard, R.C., Findlay, J.T., Kelly, G.M. and Sommer, S.C. 1980, J. Geophys. Res. 85, 7903.

Siegman, A.E. 1966, Proc. IEEE 54, 1350.

Sinton, W.M. and Strong, J. 1960, Ap. J. 131, 470.

Slipher, V.M. 1903a, Astronomiche Nachrichten 3891, 35.

Slipher, V.M. 1903b, Bul1. Lowell Obs. 1, 9, 19.

Smith, B. 1967, Science 158, 114.

Smith, B.A., et al. 1981, Science 212, 163.

Smith, B.A., et al. 1981, Science 215, 504.

Smith, W.B. 1963, A.J. 68, 15.

SooHoo, K.L. 1988, Private communication.

SooHoo, K.L. 1984, Ph.D. Thesis, Massachussetts Institute of Technology.

SooHoo, K.L., Freed, C., Thomas, J.E. and Haus, H.A. 1985, IEEE J. Quant. Elect. QE-21, 1159.

Spears, D.L. 1981, Proc. Soc. Photo-Opt. Instrum. Eng. 300, 174.

Spears, D.L. 1977, IR Phys., 17, 5.

Spectra Physics Laser Analytic Div., Operator's Manual Lo-3 Long Path Ce11, 1980.

Stewart, A.I. and Barth, C.A. 1979, Science 205, 59.

Taylor, F.W., Beer, R., Chahine, M.T., Diner, D.J., Elson, L.S., Haskins, R.D., McCleese, D.J., Martonchik, J.V., Reichley, P.E., Bradley, S.P., Delderfield, J., Schofield, J.T., Farmer, C.B., Froidevaux, L., Leung, J., Coffey, M.T. and Gille, J.C. 1980, J. 
Geophys. Res 85, 7963.

Taylor, F.W., Hunten, D.M. and Ksanfomality, L.V. 1983, Venus, D.M. Hunten, L. Colin, T.M. Donahue and V.I. Moroz Eds., University of Arizona Press, Tucson.

Taylor, H.A., Jr. and Cloutier, P.A. 1986a, Science 234, 1087.

Taylor, H.A., Jr. and Cloutier, P.A. 1986b, B.A.A.S. 18, 634.

Taylor, R.L. and Bitterman, S. 1969, Rev. Mod. Phys. 41, 26.

The Infrared Telescope Facility Observers' Manual February 1985, University of Hawaii.

Toon, O.B., Ragent, B., Colburn, D., Blamont, J. and Cot, C. 1984, Icarus 57, 143.

Traub, W.A. and Carleton, N.P. 1979, Ap. J. 227, 329.

Traub, W.A. and Carleton, N.P. 1975, J. Atmos. Sci. 32, 1045.

Travis, L.D. 1978, J. Atmos. Sci. 35, 1584.

Very, F.W. 1908, Philosophical Magazine 16, 462.

Victor, W.K. and Stevens, R. 1961, Science 134, 46.

von Zahn, U., Fricke, K.H., Hunten, D.M., Krankowsky, D., Mauersberger, K. and Nier, A.0. 1980, J. Geophys. Res. 85, 7829.

von Zahn, U., Kumar, S., Niemann, H. and Prinn, R. 1983, Venus, D.M. Hunten, L. Colin, T.M. Donahue and V.I. Moroz eds., University of
Arizona Press, Tucson.

Walterscheid, R.L., Schubert, G., Newman, M. and Kliore, A.J. 1985, J. Atmos. Sci. 42, 1982.

Wildt, R. 1940, Ap. J. 91, 266.

Williams, G.P. and Holloway, J.L. 1982, Nature 297, 295.

Wilson, D.H. 1917, Popular Astronomy 25, 336.

Wilson, D.H. 1916, Popular Astronomy 24, 571.

Woods, P.T. and Jolliffe, B.W. 1976, J. Phys. E. 9395.

Wright, W.H. 1927, Publ. Astron. Soc. Pacific 39, 220.

Wright, W.H. 1925, Lick Obs. Bull. 12, 48.

Yakovlev, O.I., Efimov, A.I., Timofeeva, T.S., Yakovleva, G.D., Chub, E.V., Tikhonov, V.F. and Shtrykov, V.K. 1976, Cosmic Res. 14, 632.

Young, A.T. 1975, Icarus 24, 1.

Young, R.E. and Pollack, J.B. 1975, J. Atmos. Sci. 32, 1025. 


\section{GLOSSARY OF ACRONYMS}

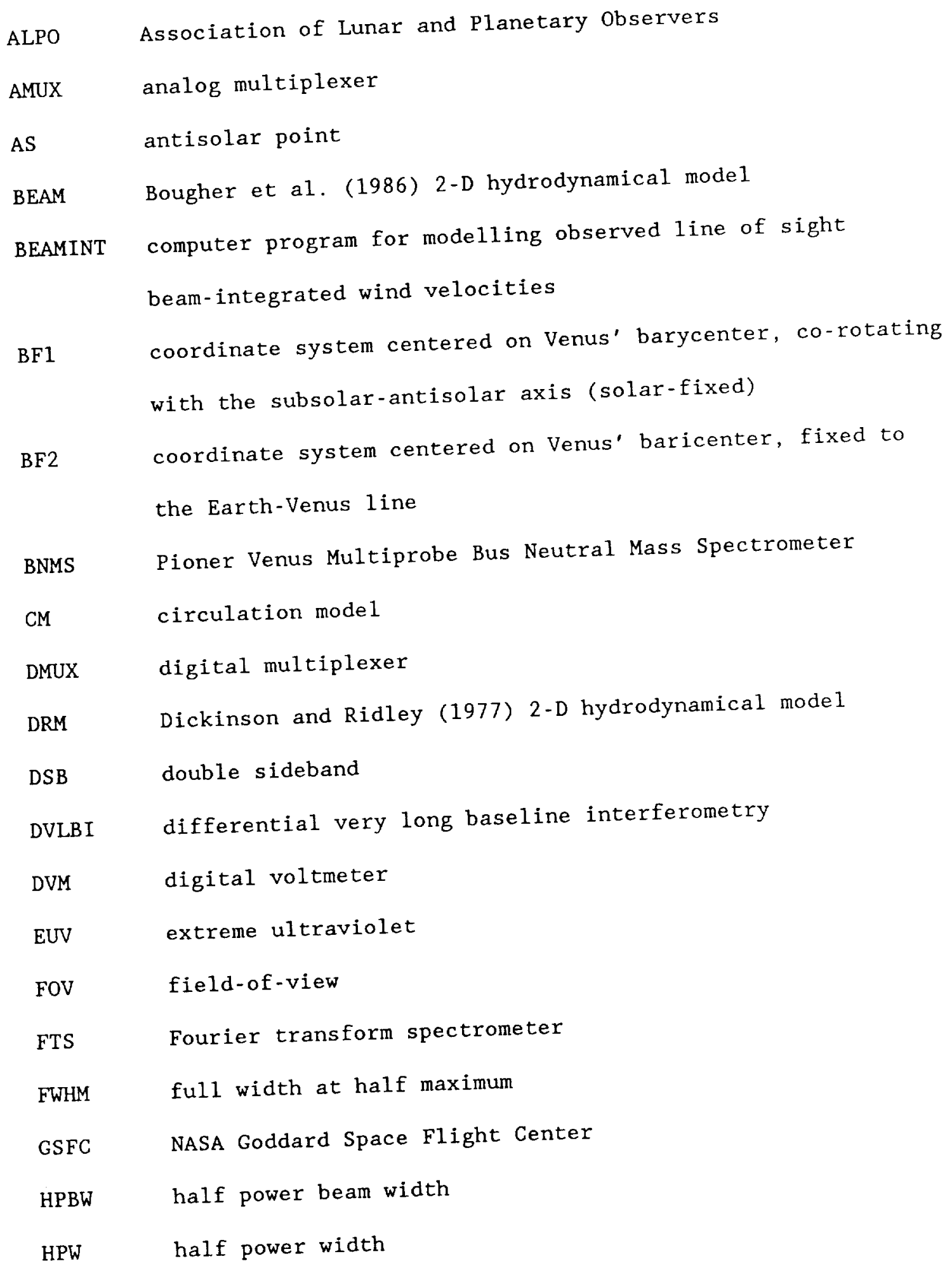




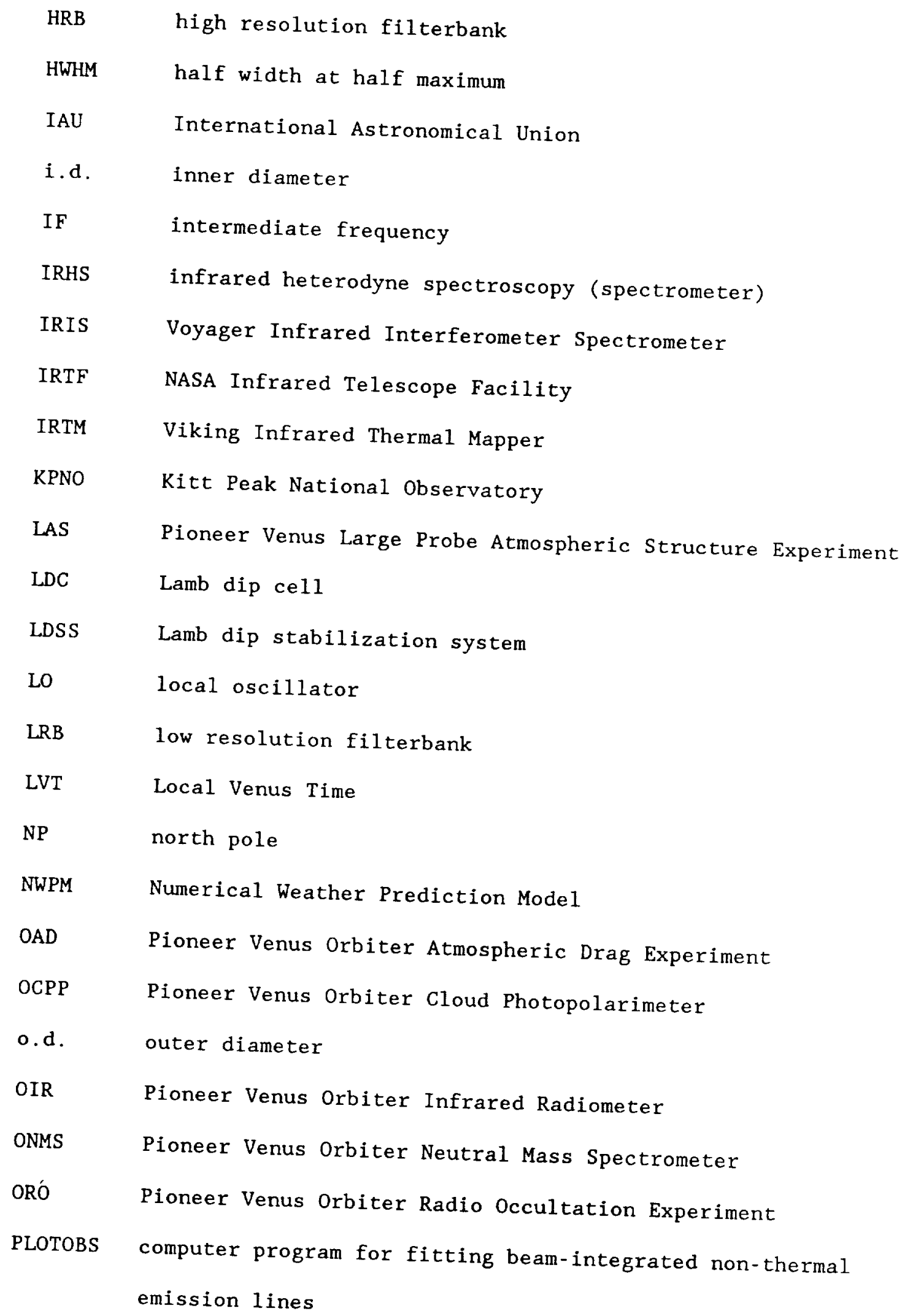




$\begin{array}{ll}\text { P-p } & \text { peak to peak } \\ \text { PPFO } & \text { power peak frequency offset } \\ \text { PSE } & \text { power slope error } \\ \text { PSW } & \text { planetary-scale wave } \\ \text { PV } & \text { Pioneer Venus } \\ \text { PZT } & \text { piezoelectric translator } \\ \text { R.A. } & \text { right ascension } \\ \text { RF } & \text { radio frequency } \\ \text { RFLO } & \text { radio frequency local oscillator } \\ \text { SAS } & \text { Pioneer Venus Small Probe Atmospheric Structure Experiment } \\ \text { SE } & \text { sub-Earth point } \\ \text { S/N } & \text { signal-to-noise } \\ \text { SS } & \text { subsolar point } \\ \text { SS-AS } & \text { subsolar-to-antisolar circulation } \\ \text { SSB } & \text { single sideband } \\ \text { SZA } & \text { solar zenith angle } \\ \text { TDL } & \text { tunable diode laser } \\ \text { UT } & \text { Universal Time } \\ \text { VORTEX } & \text { Pioneer Venus Orbiter Radiometric Temperature Experiment } \\ & \end{array}$


acronyms, glossary of...

atmospheric dynamics, early observations

mesosphere

CO observations

$6,23,257$

imaging. .

9

spectroscopic, early heterodyne

$5,11,22,233$

thermosphere/cryosphere.

6,29

CO observations.

7,257

$\mathrm{H}, \mathrm{He}$ asymmetries.

7,258

spectroscopic, early heterodyne.

$6,11,251$

thermal asymmetries

7,258

troposphere

4,17

cyclostrophic balance................... 20

Doppler tracking.

$9,18,20$

imaging.

$6,8,18,20$

latitudinal variation.

longitudinal (diurnal) variation.

mean meridional (Hadley) circulation.

spectroscopic

11,18

atmospheric dynamics, modelling

mesosphere..

4,20

cyclostrophic balance models............ 5, 21, 257 thermosphere/cryosphere

SS-AS circulation. 
Bougher et al. model (BEAM) ............. 34

Dickinson and Ridley model (DRM) ........... 32

early models....................... 29

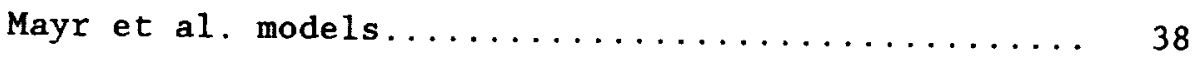

troposphere. $\ldots \ldots \ldots \ldots \ldots \ldots \ldots \ldots \ldots \ldots \ldots \ldots \ldots \ldots \ldots, 17$

cyclostrophic balance models............... 20

maintaining zonal super-rotation.............. 24

atmospheric dynamics, present investigation

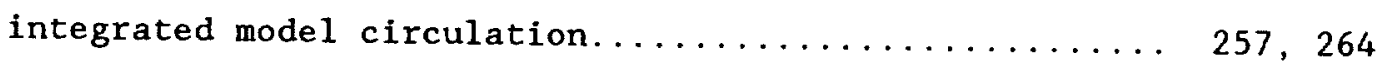

mesosphere

SS-AS return branch

mass continuity................... 246

reinterpretation of Betz data......... 242, 258

reinterpretation of $\mathrm{CO}$ data.............. 259

super-rotation................. 233, 242, 258

thermosphere/cryosphere............ 146, 172, 197, 255

atmospheric thermal structure $\ldots \ldots \ldots \ldots \ldots \ldots \ldots \ldots \ldots \ldots \ldots \ldots \ldots$

beam-integrated wind modelling

beam character considerations ................. 191

kinematical fits...................... 197

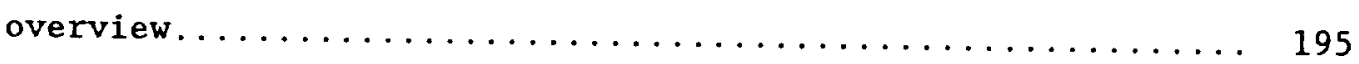

pointing uncertainty considerations.............. 183

theoretical development (program BEAMINT)........ 195, 311

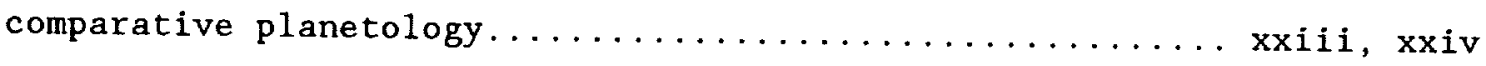
data reduction

differential Doppler shifts................. 288 
line fitting

system offset correction.

terrestrial absorption correction.

time series

wind velocity determination

future directions

heterodyne spectrometer operation.

diffraction-limited spatial resolution.

front end alignment / target pointing.

intermediate frequency generation.

optical front end.

radio frequency back end

signal-to-noise

historical observations

phase locked structure.

photographic

$$
\text { radar }
$$

radiometric

spectroscopic

visual.

Jovian atmospheres

laboratory spectroscopy

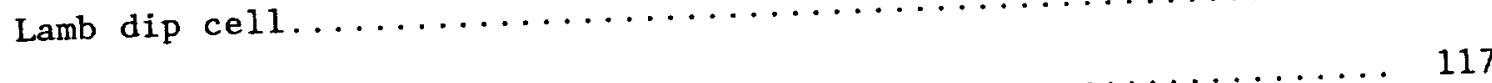

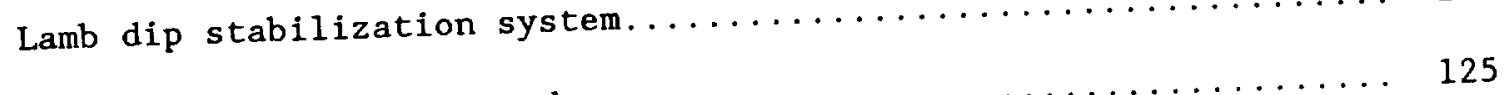

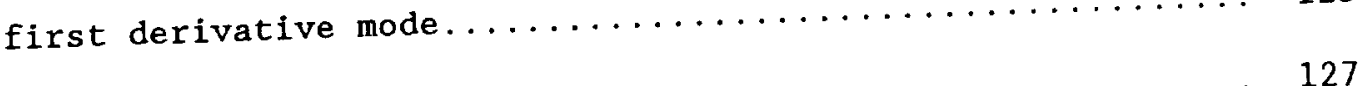

lock mode. 
power peak stabilization. . . . . . . . . . . . . . 132

undifferentiated mode $\ldots \ldots \ldots \ldots \ldots \ldots \ldots \ldots \ldots \ldots \ldots \ldots \ldots$

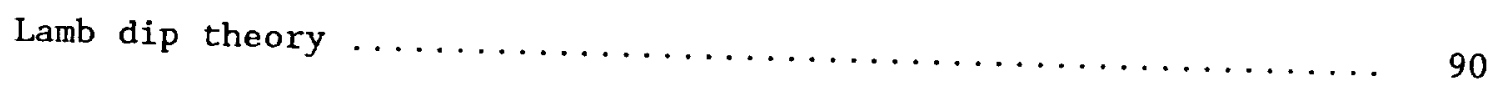

Lamb Dip Frequency Shift Mechanisms.............. 100

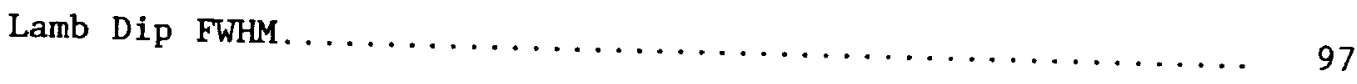

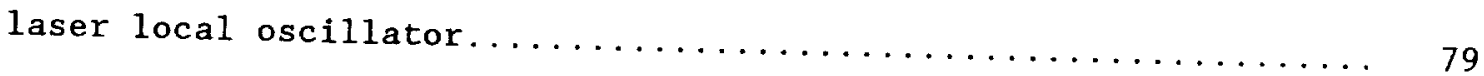

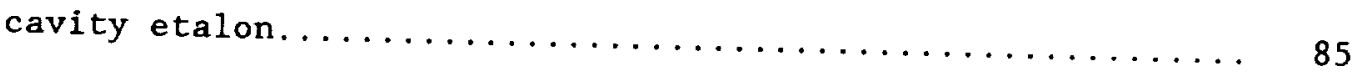

gain curve characteristics.................. 82

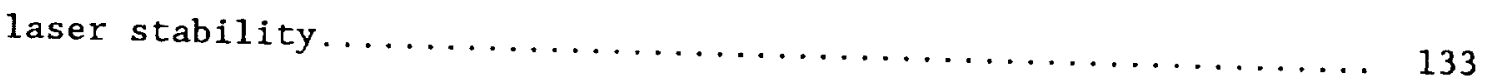

Lamb dip time series..................... 138

power peak time series................. 138, 231

power peak stabilization. $\ldots \ldots \ldots \ldots \ldots \ldots \ldots \ldots \ldots \ldots$ 8, 132, 229

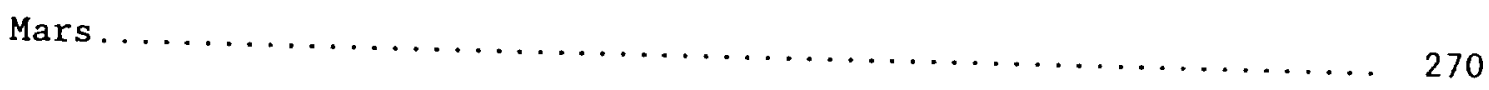

nomenclature

planetary aspect.......................... 145

thermal structure $\ldots \ldots \ldots \ldots \ldots \ldots \ldots \ldots \ldots \ldots \ldots \ldots \ldots \ldots \ldots$ xxii

non-thermal emission

choice of emission line as dynamical probe........... 143

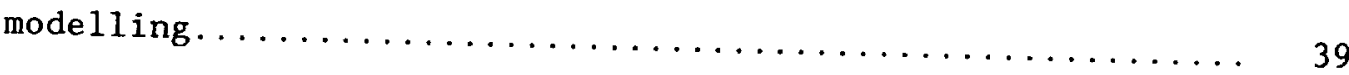

observations

beam-integrated wind velocities............... 146

overview. . . . . . . . . . . . 143

qualitative interpretation................... 172

saturated resonance $\operatorname{dip} \ldots \ldots \ldots \ldots \ldots \ldots \ldots \ldots \ldots$ see Lamb dip

Titan. . . . . . . . . . . . .

velocity shift effects...................... 169 


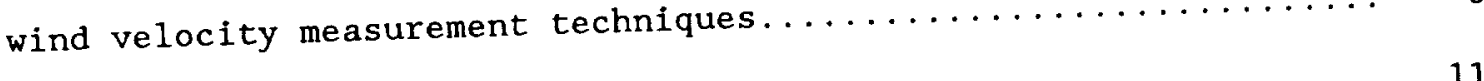

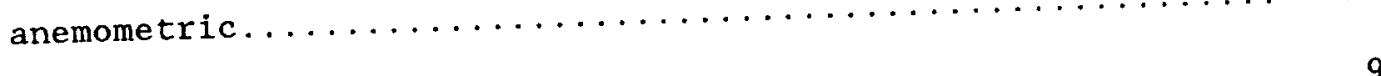

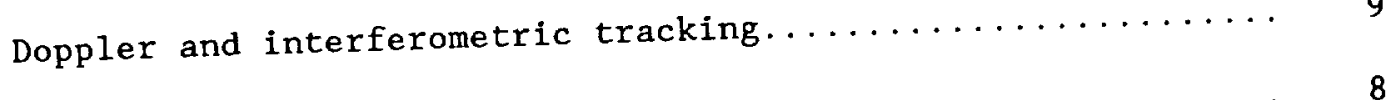
imaging. spectroscopic

general.

IR heterodyne 
$-$ 


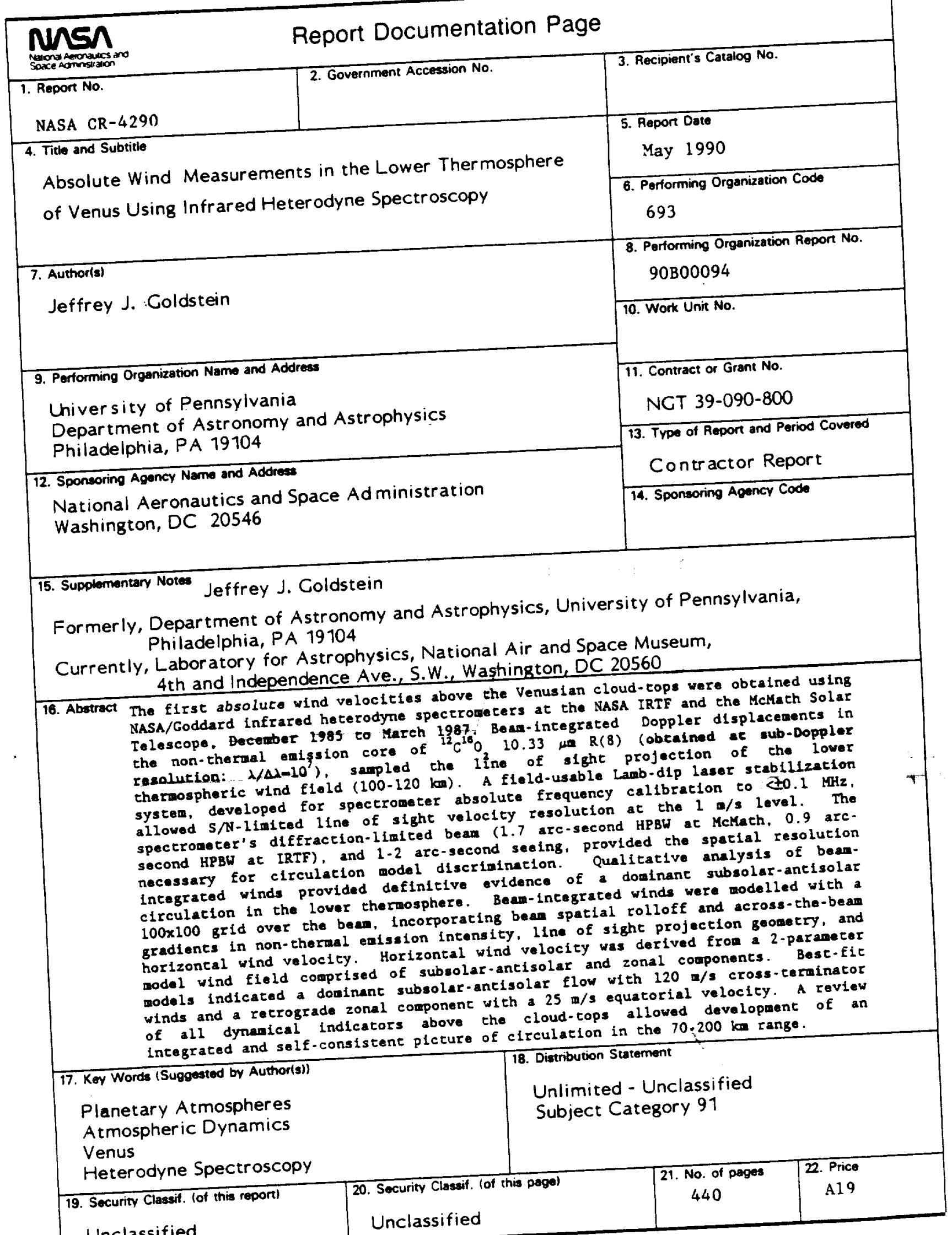

Unclassified

Unclassified 
